\title{
Data Summary Report for Savannah River Integrator Operable Unit Fish Tissue
}

(U)

January 2000 


\title{
Data Summary Report for Savannah River Integrator Operable Unit Fish Tissue
}

(U)

\author{
Prepared by: \\ Environmental Protection Department \\ Environmental Geochemistry Group \\ Westinghouse Savannah River Company \\ Aiken, SC \\ and \\ Exploration Resources, Inc. \\ Athens, GA
}

January 27, 2000 
This document was prepared in conjunction with work accomplished under Contract No.

DE-AC09-96SR18500 with the U.S. Department of Energy.

\section{DISCLAIMER}

This report was prepared as an account of work sponsored by an agency of the United States Government. Neither the United States Government nor any agency thereof, nor any of their employees, makes any warranty, express or implied, or assumes any legal liability or responsibility for the accuracy, completeness, or usefulness of any information, apparatus, product or process disclosed, or represents that its use would not infringe privately owned rights. Reference herein to any specific commercial product, process or service by trade name, trademark, manufacturer, or otherwise does not necessarily constitute or imply its endorsement, recommendation, or favoring by the United States Government or any agency

thereof. The views and opinions of authors expressed herein do not necessarily state or reflect those of the United States Government or any agency thereof.

This report has been reproduced directly from the best available copy.

Available for sale to the public, in paper, from: U.S. Department of Commerce, National Technical Information Service, 5285 Port Royal Road, Springfield, VA 22161, phone: (800)

553-6847, fax: (703) 605-6900, email: orders@ntis.fedworld.gov online ordering: http://www.ntis.gov/ordering.htm

Available electronically at http://www.doe.gov/bridge

Available for a processing fee to U.S. Department of Energy and its contractors, in paper, from: U.S. Department of Energy, Office of Scientific and Technical Information, P.O. Box 62, Oak Ridge, TN 37831-0062, phone: (865 ) 576-8401, fax: (865) 576-5728, email: reports@ adonis.osti.gov 


\section{Table of Contents}

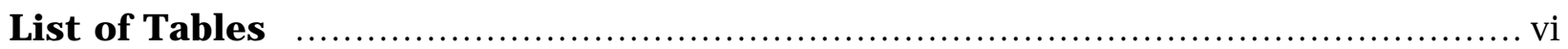

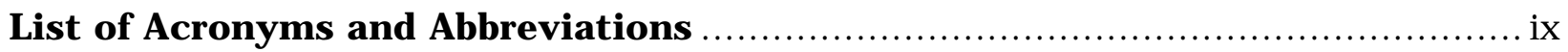

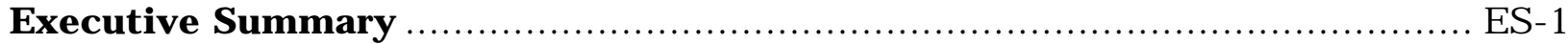

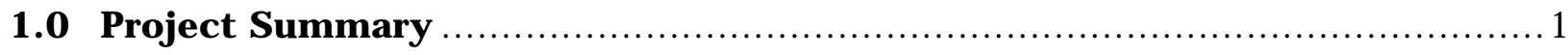

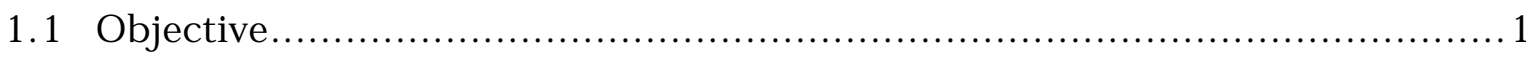

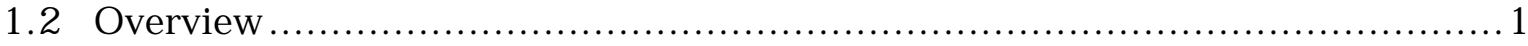

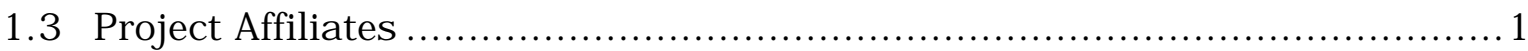

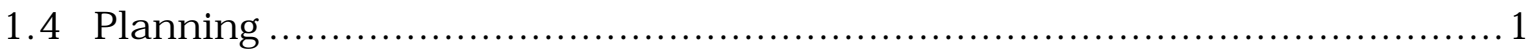

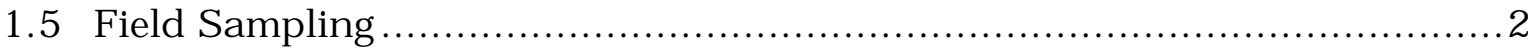

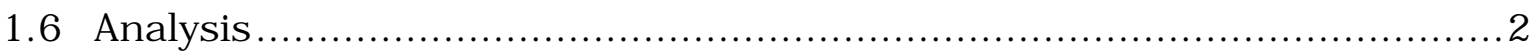

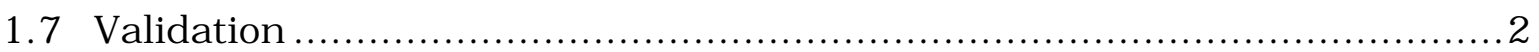

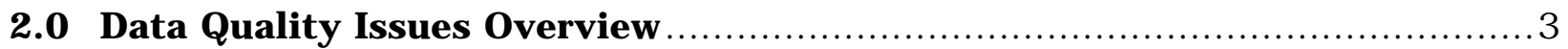

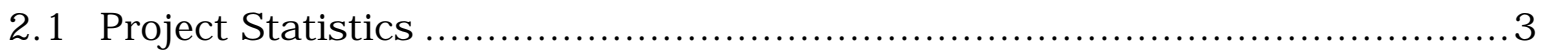

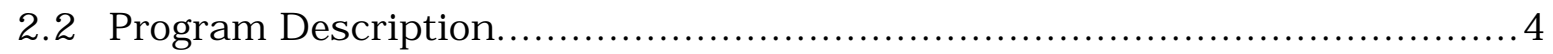

2.2.1 Verification and Validation Objectives ........................................ 4

2.2.2 Verification and Validation Functions ........................................5

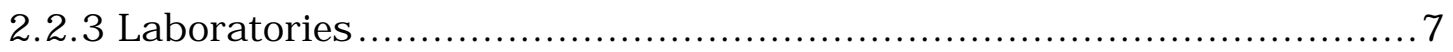

2.3 Data Quality Issues Summary ......................................................

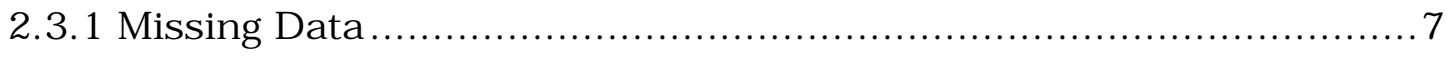

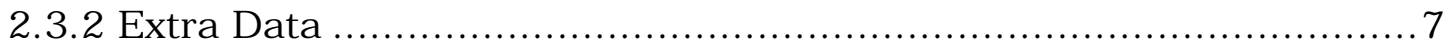

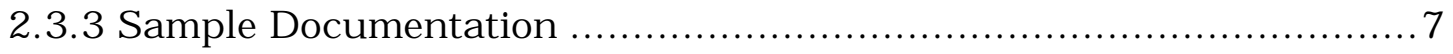

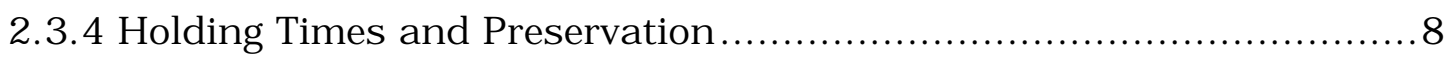

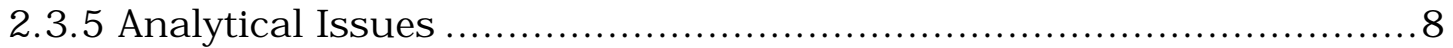

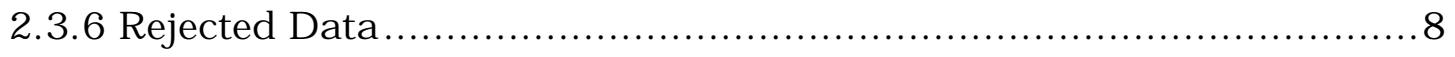

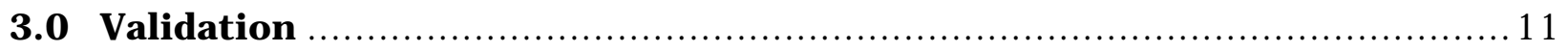

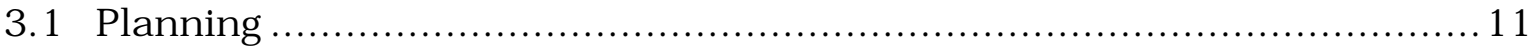

Data Summary Report for Savannah River Integrator Operable Unit Fish Tissue iii (WSRC-TR-99-00386) 
3.1.1 Planned Work............................................................. 11

3.1.2 Production of Chain-of-Custody Forms...................................... 11

3.1.3 Sample Set.............................................................. 11

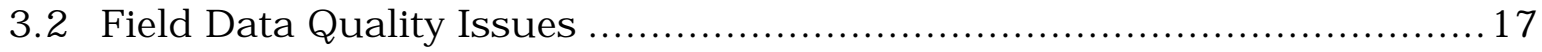

3.2.1 Discrepancies from the Planned Work ................................. 17

3.2.2 Sample Logbook Observations ...................................... 17

3.2.3 Chain-of-Custody Observations .................................... 17

3.3 Analytical Data Quality Issues .......................................... 17

3.3.1 The Laboratories ............................................................ 17

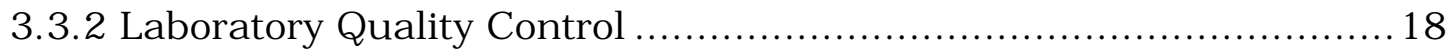

3.3.3 List of Analytical Suites ............................................ 18

3.3.4 Analytical Data Observations ........................................ 18

3.4 Verification and Validation Issues........................................... 19

3.4.1 Verification and Validation Process ..................................... 19

3.4.2 Analytical Narrative Review............................................ 20

3.4.3 Laboratory Data Records Review ...................................20

3.4.4 Radiological Data Review ............................................ 21

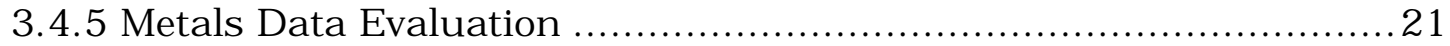

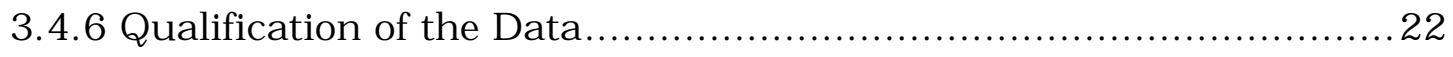

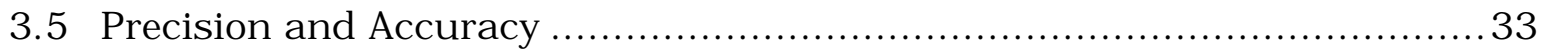

3.5.1 Precision .................................................................... 34

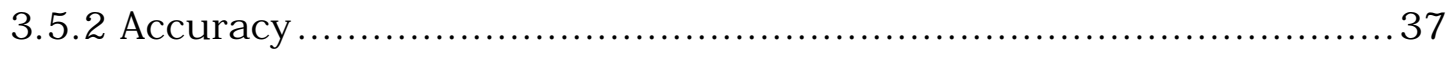

3.5.3 Contamination in Quality Control Samples............................43

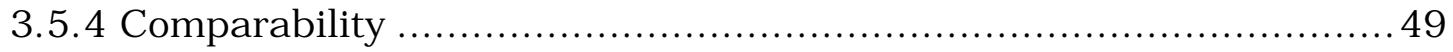

4.0 References ....................................................................... 51

Appendix A: Analytical Methods and Method Detection Limits ................. A-1

Appendix B: Laboratory Performance Evaluation Results ..................... B-1

B. 1 EPA Performance Evaluation Study Results ............................... B-1

Data Summary Report for Savannah River Integrator Operable Unit Fish Tissue (WSRC-TR-99-00386) 
B.2 EMS Quality Control Standards Results............................... B-12

B.3 Mixed Analyte Performance Evaluation Program.......................... B-22

B.4 EPA Radiological Performance Evaluation Studies ........................ B-25

Appendix C: Formats of the Field and Laboratory $\mathbf{8}$ C Codes ................... C-1

Appendix D: Data Tables ..........................................................

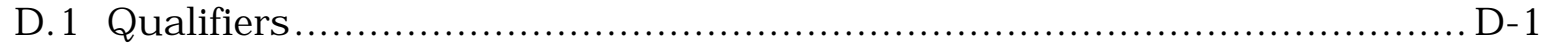

D.2 Data Summaries ...................................................... D 3

Appendix E: Data Management/Administrative Files.......................... E-1

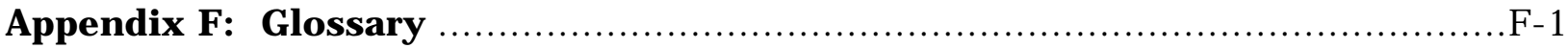




\section{List of Tables}

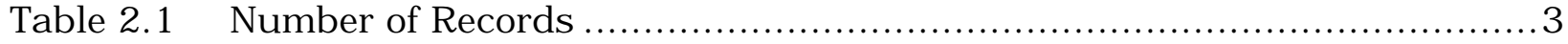

Table 2.2 Number of Records Qualified as Rejected, Estimated, or Not

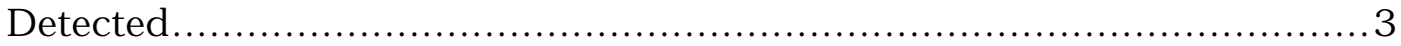

Table 2.3 Environmental Sample Analytical Record Qualification Summary...........3

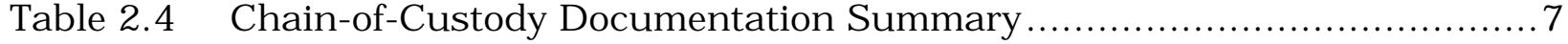

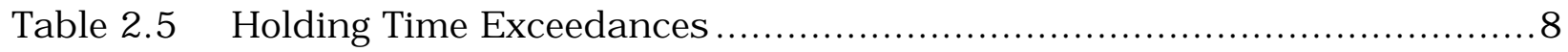

Table 2.6 Summary of Rejected Records ................................................9

Table $3.1 \quad$ Fish Tissue Sample Details ................................................ 12

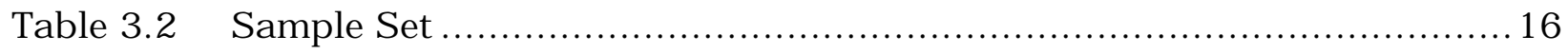

Table 3.3 Trip Blanks and Associated Samples ....................................... 17

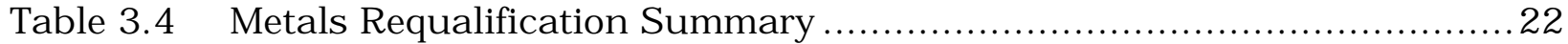

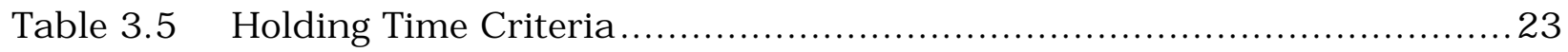

Table 3.6 Surrogate Recoveries Outside EPA Recovery Limits ........................26

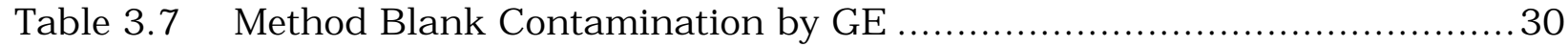

Table 3.8 Method Blank Contamination by EP ........................................ 30

Table 3.9 Matrix Spike Recoveries Outside the EPA Recovery Limits by GE..........32

Table 3.10 Matrix Spikes Rejected Due to Dilution Effects or Other Reason by

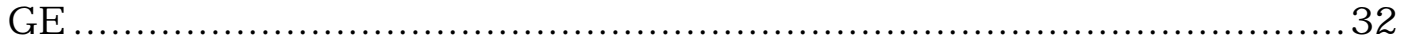

Table 3.11 Laboratory Duplicate Results Outside RPD Limits for GE.................33

Table 3.12 Relative Percent Differences of Laboratory Duplicates by GE ..............34

Table 3.13 Relative Percent Differences of Laboratory Duplicates by EP...............35

Table 3.14 Relative Percent Differences of Field Duplicates by GE ......................36

Table 3.15 Relative Percent Differences of Field Duplicates by EP.......................36

Table 3.16 EPA Quality Control Limits for Laboratory Control Samples ................37

Table 3.17 Laboratory Control Sample Recoveries by GE .............................39

Table 3.18 Laboratory Control Sample Recoveries by EP .............................39

Table 3.19 EPA Quality Control Limits for Surrogates ...............................40

Data Summary Report for Savannah River Integrator Operable Unit Fish Tissue vi (WSRC-TR-99-00386) 
Table 3.20 Surrogate Recoveries by GE ……................................................ 4

Table 3.21 Matrix Spike Recoveries by GE .................................................... 42

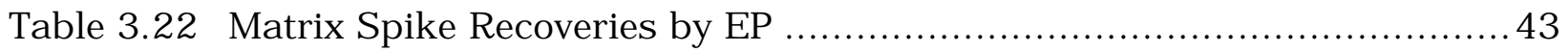

Table 3.23 Analytes Detected in Method Blanks by GE ...................................44

Table 3.24 Analytes Detected in Method Blanks by EP ...................................46

Table 3.25 Analytes Detected in Trip Blanks by GE.......................................48

Table A.1 Analytical Methods and Detection Limits for Liquid Matrices by GE..... A-1

Table A.2 Analytical Methods and Detection Limits for Solid Matrices by GE....... A-2

Table A.3 Analytical Methods and Minimum Detectable Activities for Solid Matrices by EP ..................................................................... A

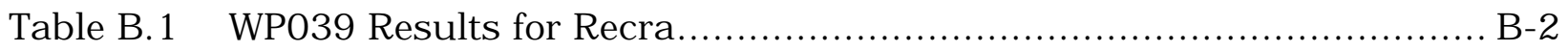

Table B.2 WP040 Results for EMAX, GE, and Recra ................................. B-4

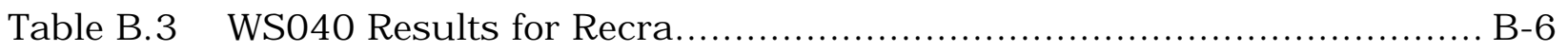

Table B.4 WS041 Results for EMAX and GE............................................. B

Table B.5 Guality Control Standards for Selected Analyses for EMAX …........... B-12

Table B.6 Guality Control Standards for Selected Analyses for GE .................. B-16

Table B.7 Guality Control Standards for Selected Analyses for Recra ............... B-19

Table B.8 Mixed Analyte Performance Evaluation Program Results for MAPEP-96-S3 for GE and Recra ........................................... B-23

Table B.9 Mixed Analyte Performance Evaluation Program Results for MAPEP-96-S3 for EP and TNU ............................................ B-24

Table B.10 Mixed Analyte Performance Evaluation Program Results for MAPEP-96-W4 for GST …................................................ B-24

Table B. 11 Blind-A Results for EP and QR (10/15/96) .............................. B-26

Table B. 12 Blind-B Results for EP and QR (10/15/96) ............................... B-26

Table B.13 Gamma in Water Results for EP and QR $(11 / 8 / 96) \ldots \ldots \ldots \ldots \ldots \ldots \ldots \ldots$ B-26

Table B.14 Strontium in Water Results for EP and QR (1/17/97) ................... B-26

Table B.15 Gross Alpha-Beta in Water Results for EP and QR (1/31/97) ........... B-27

Table B. 16 Uranium-Radium in Water Results for EP and GR (2/14/97) ........... B-27

Table C. $1 \quad$ AN98 Laboratory QC Samples Codes .............................................. C-1 
Table C.2 AN98 Field QC Codes........................................................

Table D.1 AN98 EPA Functional Guideline Codes ...................................... D-1

Table D.2 AN98 EPA STORET Codes used by EMS ................................ D-1

Table D.3 AN98 EMS Codes ......................................................... D

Table D.4 Number of Analytical Records by Method and Type........................ D-4

Table D.5 Analytes Not Detected in Fish Samples ................................. D-5

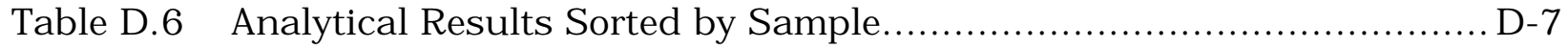

Table D.7 Results for Blanks Sorted by Sample.................................... D-60

Table D.8 Analytical Results Sorted by Analyte.................................... D-63

Table D.9 Results for Blanks Sorted by Analyte.............................. D-1 12 


\section{List of Acronyms and Abbreviations}

The following acronyms and abbreviations are commonly used in Data Summary Reports:

\begin{tabular}{|c|c|}
\hline${ }^{\circ} \mathrm{C}$ & degrees Celsius \\
\hline$\mu \mathrm{S}$ & microSiemens \\
\hline$\mu g / L$ & micrograms per liter \\
\hline $\mathrm{AA}$ & atomic absorption \\
\hline $\mathrm{Bq}$ & Becquerel \\
\hline BRA & baseline risk assessment \\
\hline $\mathrm{CCB}$ & continuing calibration blank sample \\
\hline $\mathrm{CCV}$ & continuing calibration verification sample \\
\hline CERCLA & $\begin{array}{l}\text { Comprehensive Environmental Response, Compensation, and } \\
\text { Liability Act (Superfund) }\end{array}$ \\
\hline CFR & Code of Federal Regulations \\
\hline CLP & Contract Laboratory Program \\
\hline $\mathrm{cm}$ & centimeter(s) \\
\hline $\mathrm{CMS} / \mathrm{FS}$ & Corrective Measures Study/Feasibility Study \\
\hline $\mathrm{COC}$ & chain of custody; chain-of-custody form \\
\hline $\mathrm{CPT}$ & cone penetrometer test \\
\hline CVAA & cold vapor atomic absorption analysis \\
\hline DI & deionized (water) \\
\hline DOE & United States Department of Energy \\
\hline DPT & direct push technology \\
\hline DQOs & data quality objectives \\
\hline EDD & electronic data deliverable \\
\hline EGG & Environmental Geochemistry Group \\
\hline $\mathrm{EM}$ & EMS' Environmental Chemistry and Analysis Group (laboratory) \\
\hline
\end{tabular}

Data Summary Report for Savannah River Integrator Operable Unit Fish Tissue ix (WSRC-TR-99-00386) 
EMSL

EMS

EP

EPA

EPD/EMS

EQL

ERA

ERD

ESC

ExR

FG

$\mathrm{ft}$

GC

$\mathrm{GC} / \mathrm{MS}$

GE

GFAA

GIMS

GP

GPR

ICB

ICP

ICS

ICSA

ICV

ID

IDL
EPA's Environmental Monitoring and Support Laboratory

Environmental Monitoring Section; also, EMS codes are used to qualify data

Environmental Physics, Inc.

United States Environmental Protection Agency

Environmental Protection Department/Environmental Monitoring Section

estimated quantitation limit (for non-radiochemistry analyses)

Environmental Resource Associates

Environmental Restoration Department

expedited site characterization

Exploration Resources, Inc.

Functional Guideline (FG codes are used to qualify analytical data)

feet

gas chromatography

gas chromatography/mass spectrometry

General Engineering Laboratories, Inc.

graphite furnace atomic absorption (metals analysis)

Geochemical Information Management System

Environmental Physics

ground penetrating radar

initial calibration blank

inductively coupled plasma (metals analysis)

interference check sample

ICP interference check sample - interferents only

initial calibration verification

identification

instrument detection limit 


\begin{tabular}{|c|c|}
\hline $\mathrm{km}$ & kilometer \\
\hline LCS & laboratory control sample(s) \\
\hline LDRR & laboratory data records review \\
\hline LIMS & laboratory information management system \\
\hline MAPEP & Mixed Analyte Performance Evaluation Program \\
\hline MCL & maximum contaminant level \\
\hline MDA & minimum detectable activity \\
\hline MDL & method detection limit \\
\hline $\mathrm{mg} / \mathrm{kg}$ & milligrams per kilogram \\
\hline $\mathrm{mg} / \mathrm{L}$ & milligrams per liter \\
\hline ML & General Engineering's Mobile Laboratory, Aiken, SC \\
\hline MS & matrix spike \\
\hline MSA & method of standard addition \\
\hline MSD & matrix spike duplicate \\
\hline msl & mean sea level \\
\hline $\mathrm{ng} / \mathrm{kg}$ & nanograms per kilogram \\
\hline $\mathrm{ng} / \mathrm{L}$ & nanograms per liter \\
\hline NPDES & National Pollutant Discharge Elimination System \\
\hline OVA & organic vapor analyzer \\
\hline $\mathrm{PAH}$ & polynuclear aromatic hydrocarbons \\
\hline PAL & performance acceptance limit \\
\hline $\mathrm{PE}$ & performance evaluation \\
\hline PHA & pulse-height analysis; e.g., gamma PHA \\
\hline PID & photoionization detector \\
\hline $\mathrm{r}$ & correlation coefficient \\
\hline RCRA & Resource Conservation and Recovery Act \\
\hline Recra & $\begin{array}{l}\text { Recra LabNet Philadelphia (formerly Roy F. Weston, Inc., Analytics } \\
\text { Division) }\end{array}$ \\
\hline
\end{tabular}


RFI/RI RCRA Facility Investigation/Remedial Investigation

RPD relative percent difference

SAFER Streamlined Approach for Environmental Restoration

SAIC Science Applications International Corporation

SCDHEC South Carolina Department of Health and Environmental Control

SGS Site Geotechnical Services

SQL sample quantitation limit

SRF Savannah River Fish

SRS Savannah River Site

SRTC Savannah River Technology Center

ssDL sample-specific detection limit

ssEQL sample-specific estimated quantitation limit

ssMDL sample-specific method detection limit

STORET data coding system developed by EPA; STORET codes are used to qualify analytical results

SW-846

EPA's Test Methods for Evaluating Solid Waste

TAL target analyte list

TCL target compound list

TCLP toxicity characteristic leaching procedure

TIC tentatively identified compound

TNU Thermo NUtech, a laboratory that is a subcontractor for Recra LabNet Philadelphia

USEPA $\quad$ see EPA

UTM Universal Transverse Mercator Zone 17 Coordinate System

VOA volatile organic analyses

WA laboratory code for Recra; used in data formats

WHC Westinghouse Hanford Company

WPA work plan assessment 
WSRC Westinghouse Savannah River Company 


\section{Executive Summary}

\section{Purpose}

This report presents the results of the verification and validation of the analytical data for the Savannah River Fish (SRF) investigation. The data were validated to determine if the records conform to the technical criteria associated with definitive data. The investigation was conducted under the direction of the WSRC ERD. This report was prepared under the direction of EPD/EMS.

\section{Data Usability}

Sixteen environmental sample records and four QC records were rejected. Five radiological sample records were rejected due to fluctuations in the background count rate. Ten radiological sample records were rejected because of low abundance, and one cerium-144 sample record was rejected because of interference. Three radiological QC records (a method blank and two laboratory duplicates) were rejected because of low abundance and interference. One total recoverable mercury matrix spike was rejected due to low recovery. A detailed discussion may be found in subsection 2.3.6, Rejected Data.

Otherwise, the data from this project are considered to be representative of actual conditions at the unit being investigated.

\section{Process and Documentation}

Sample documentation and COC maintenance were reviewed by examining the EPD/EMS sample logbooks and COC forms. See subsections 2.3, Data Quality Issues Summary; 3.1.2, Production of Chain-of-Custody Forms; 3.2.2, Sample Logbook Observations; and 3.2.3, Chain-of-Custody Observations.

Sample holding times were checked by comparing the time between sample collection, extraction, and analysis with required holding times. Preservation was evaluated by reviewing cooler temperatures upon receipt at the laboratory. See subsections 2.3.4, Holding Times and Preservation, and 3.4, Verification and Validation Issues.

Analyte identification and analyte quantitation were verified as part of the computerized checking of the EDDs and during review of the analytical narratives. Anomalies were resolved with the laboratories whenever possible, and records not meeting specific criteria were qualified. See subsection 3.4, Verification and Validation Issues.

In evaluating analytical error, percent recoveries for QC samples were reviewed, and the QC sample and associated project sample data were qualified where necessary. RPDs between laboratory-generated duplicate sample analyses were calculated. The criteria for qualification are described in subsection 3.4.6, Qualification of the Data.

Field-generated blanks (field blanks, rinsates, and trip blanks) and laboratory-generated blanks (e.g., method blanks) were examined. See section 3.1, Planning, for an overview of the sampling plan for fieldgenerated blanks and subsection 3.4.6, Qualification of the Data, for a discussion of the constituents detected in field-generated blanks. Laboratory-generated blanks were analyzed in ratios of at least one for every 20 samples. 
Quantitation limits and methods used for the project are listed in Appendix A of this report, Analytical Methods and Method Detection Limits. Evaluations of laboratory performance against the standards of various certification programs are discussed in Appendix B, Laboratory Performance Evaluation Results. Appendix C, Formats of the Data Files, describes the formats used for the project data. Appendix D, Data Tables, contains summary statistics for the analytical data and the results sorted by sample location and by analyte. Appendix E, Data Management/Administrative Files, discusses the generation, processing, and storage of hard copy and computer records associated with the project. Appendix F, Glossary, defines key terms used in the report. 


\subsection{Project Summary}

\subsection{Objective}

The objective of the Savannah River Fish project is to provide definitive-level data from the analysis of whole-body fish tissue in support of the SRS CERCLA program.

\subsection{Overview}

The Savannah River is defined under the South Carolina Pollution Control Act as a freshwater system. Freshwater systems are bodies of surface water that are suitable for primary- and secondary-contact recreation; a drinking water source after treatment; industrial and agricultural uses; and fishing, survival and propagation of a balanced indigenous aquatic community of fauna and flora. It borders SRS for a 35mile stretch and is analyzed periodically to determine what effects, if any, the site's effluents have on the river water. Surveillance is performed along the Savannah River at points above and below SRS and below the point at which Plant Vogtle liquid discharges enter the river.

\subsection{Project Affiliates}

The project was completed by the following persons and/or organizations:

- Ron Malanowski was the WSRC ERD project team lead.

- Susan Dyer was the WSRC SGS project technical representative.

- Bob Craig was the WSRC EPD/EMS project technical member.

- EMS Environmental Sampling Group collected the samples.

- General Engineering Laboratories (GE), Charleston, SC, the primary laboratory, provided definitive-level inorganic and organic analyses under contract AA07208N. In the data set, the code assigned by EPD/EMS to identify this laboratory is GE. GE's affiliate, Environmental Physics (EP), Charleston, SC, performed definitive-level radiological analyses. In the data set, the code assigned to identify EP is GP.

- ExR of Athens, GA, validated the data and prepared this report.

\subsection{Planning}

Twenty-one fish samples were planned to be collected from seven sites along the Savannah River. The sites were planned as follows: above Steven's Creek Dam, river mile 157 (mouth of Upper Three Runs Creek), river mile 151 (mouth of Fourmile Branch), the Vogtle plant, river mile 141 (mouth of Steel Creek), river mile 129 (mouth of Lower Three Runs), and river mile 120. Each site was to be electrofished to collect three composite fish samples representing a benthic species, a predator species, and

Data Summary Report for Savannah River Integrator Operable Unit Fish Tissue 1

(WSRC-TR-99-00386) 
a regional species. Each composite was to consist of a minimum of two fish and was to weigh at least $400 \mathrm{~g}$, which is the minimum mass for analytical purposes. At least one sample was to contain sufficient mass (600-1000g wet weight) to conduct duplicate analyses. The number of duplicate analyses and QA samples required was to be determined by the laboratory. Benthic composites were to be used for the duplicate analyses.

\subsection{Field Sampling}

Twenty-four regular fish samples and two duplicates were collected for analysis, and three trip blanks were included in the project for a total of 29 samples.

See subsection 3.1.3, Sample Set, for a complete list of the samples collected and analyzed.

\subsection{Analysis}

All fish samples were analyzed for RCRA metals, TCL volatiles, and the following radionuclides: gross alpha, nonvolatile beta, gamma spectroscopy, plutonium239/240, strontium-89/90, tritium, and uranium-233/234. Duplicate samples were to be analyzed for the same constituents as the parent samples. Trip blanks were to be analyzed for TCL VOA.

See subsections 3.3.3, List of Analytical Suites, and Appendix A, Analytical Methods and Method Detection Limits.

\subsection{Validation}

Validation and verification activities, using definitive data requirements (EPA, 1993), assessed the quality of the definitive level data for the following issues:

- sample documentation

- maintenance of chain of custody

- adherence to sample holding time requirements

- achievement of contract-required quantitation limits

- analytical instrument calibration

- analyte identification

- analyte quantitation

Evaluation of each laboratory's performance in analyte identification and quantitation included examination of the following:

- analytical error

- contamination of blanks

- laboratory performance evaluation samples

Data Summary Report for Savannah River Integrator Operable Unit Fish Tissue

(WSRC-TR-99-00386) 
- quantitation limit determination, if applicable

See section 3.0, Validation. 


\subsection{Data Quality Issues Overview}

This section provides a summary of the major points affecting the quality of the data for this project.

\subsection{Project Statistics}

All of the 29 COCs generated for this project were used. Twenty-four fish samples and two field duplicates were collected; three trip blanks were also included. The total number of records for this project is given in Table 2.1.

Table 2.1 Number of Records

\begin{tabular}{|l|l|l|l|}
\hline & Chemical Records & Radiochemical Records & Totals \\
\hline Environmental Sample Records & 1,376 & 884 & 2,260 \\
\hline QC Records & 513 & 162 & 675 \\
\hline Calibration Records & 0 & 0 & 0 \\
\hline Totals & 1,889 & 1,046 & 2,935 \\
\hline
\end{tabular}

Table 2.2 lists the number of sample and QC records that were qualified with $R, U, J$, or $U J$ Functional Guideline (FG) codes after verification and validation were performed.

Table 2.2 Number of Records Qualified as Rejected, Estimated, or Not Detected

\begin{tabular}{|l|l|l|l|}
\hline Data Validation Records & Sample Records & QC Records & Total \\
\hline Total Number of Analytical Records & 2,260 & 675 & 2,935 \\
\hline Qualified Data & \multicolumn{2}{l}{} \\
\hline Rejected Analytical Records & 16 & 4 & 20 \\
\hline FG Code $U$ & 1,716 & 321 & 2,037 \\
\hline FG Code $J$ & 143 & 21 & 164 \\
\hline FG Code $U J$ & 29 & 34 & 63 \\
\hline
\end{tabular}

Table 2.3 summarizes the numbers of environmental samples assigned STORET (SC) or EMS Codes for the reasons listed in the table.

Table 2.3 Environmental Sample Analytical Record Qualification Summary

\begin{tabular}{|l|l|l|}
\hline Reason for Qualification & $\begin{array}{l}\text { Number of Records } \\
\text { Qualified }\end{array}$ & $\begin{array}{l}\text { Percent of Total Sample } \\
\text { Records }\end{array}$ \\
\hline Holding time exceedances & 0 & 0.0 \\
\hline Sample preservation criteria not met & 0 & 0.0 \\
\hline
\end{tabular}


Table 2.3 Environmental Sample Analytical Record Qualification Summary (cont.)

\begin{tabular}{|l|l|l|}
\hline Reason for Qualification & $\begin{array}{l}\text { Number of Records } \\
\text { Qualified }\end{array}$ & $\begin{array}{l}\text { Percent of Total Sample } \\
\text { Records }\end{array}$ \\
\hline $\begin{array}{l}\text { Instrument calibration, analyte identification, analyte } \\
\text { quantitation criteria not met }\end{array}$ & 0 & 0.0 \\
\hline Trip blank contamination & 0 & 0.0 \\
\hline Rinsate blank contamination & 0 & 0.0 \\
\hline Field blank contamination & 0 & 0.0 \\
\hline Method blank contamination & 14 & 0.62 \\
\hline Laboratory duplicate precision limit exceeded & 3 & 0.13 \\
\hline Matrix spike criteria not met & 5 & 0.22 \\
\hline Laboratory control sample criteria not met & 0 & 0.0 \\
\hline Surrogate or tracer criteria not met & 114 & 5.04 \\
\hline Totals & $\mathbf{1 3 6}$ & $\mathbf{6 . 0 2}$ \\
\hline
\end{tabular}

\subsection{Program Description}

This section discusses the validation of the data and gives a brief overview of the laboratories.

\subsubsection{Verification and Validation Objectives}

\section{Data Quality Objectives}

Definitive data, as defined in EPA, 1993, have been determined as most appropriate to meet the DQOs of this investigation. Validation and verification activities assessed the data in relation to these standards, especially with regard to the following issues:

- sample documentation

- maintenance of $\mathrm{COC}$

- adherence to sample holding time and preservation requirements

- achievement of contract-required detection limits

- analyte identification

- analyte quantitation

Evaluation of each laboratory's performance for analyte identification and quantitation included examination of analytical error, blank contamination, and laboratory performance in the analysis of independent standards. 
Each analytical record in the computer data files contains three qualifier fields: functional guideline (FG) code, STORET code, and EMS code. The FG code describes the analytical result. The STORET code describes issues arising during the analytical process. The EMS code contains additional information to aid the validators in qualifying results. The laboratories use these fields in reporting the data. During validation and verification of the analytical data, additional qualifiers may be applied to provide additional information about data quality.

\section{Data Quality Levels}

Definitive-level data are used for data collection activities that require a high degree of qualitative and quantitative accuracy for all findings. Rigorous methods of analysis and quality assurance are used for those samples considered essential in making a decision. This data level is intended to give the decision maker a level of confidence to make decisions regarding the following:

- treatment

- disposal

- site remediation and/or removal of pollutants

- health risk or environmental impact

- cleanup verification, pollutant source identification, delineation of contaminants

- other significant decisions where an action level is of concern

Only analyte-specific methods can be used for definitive-level data.

\subsubsection{Verification and Validation Functions}

The data verification process confirms that the required samples were collected and documented, the required analyses were performed on the samples, and the analytical results were reported correctly. The information evaluated during this process includes work plan sample lists, work plan analyte/compound lists, field logbooks, COC forms, and analytical results files.

Data reviewers use computer programs and look-up tables to review the computer data files of sample collection, shipping, and analytical data. Where applicable, the data were examined as follows:

- The following fields were checked to ensure that they were not blank: customer sample identification number, COC number (for groundwater samples) or soils sample number, sample collection date, laboratory receipt date, analysis date, analysis time, analytical method, laboratory code, laboratory sample identification number, analyte code (test name), ssMDL, ssEQL, analytical result, result units, counting uncertainty (for radionuclide analyses), nominal concentration (if applicable), wet weight or dry weight, dilution factor, instrument, analyst's initials, percent solids and sample fraction (soil/sediment only), sample matrix, preparation factor, and subcontract number.

- The following fields were checked to ensure that reported data were within an acceptable range of values: customer sample identification number, COC number, soils sample number, sample collection date, sample collection time, lab receipt date, extraction date, extraction time, analysis date, analysis time, analytical result, counting uncertainty, residual weight, nominal concentration, dilution factor, percent solids, and bottle label number.

- The following fields were checked against a look-up table or a list of valid codes: field QC code; extraction, digestion, or preparation method; analytical method; laboratory code; laboratory QC sample 
code; analyte code; ssMDL; ssEQL; FG code; STORET code; EMS code; result units; percent recovery; sample fraction; sample matrix; and subcontract number.

The data validation process determines the usefulness of each analytical result based on QC and method requirements. The information evaluated during this process includes $\mathrm{COC}$ forms, analytical narrative summaries, and analytical result data files.

Data validation involves evaluating requirements in the following QC categories: holding times, sample preservation, instrument calibration, analyte identification/quantitation, blanks (trip, method, rinsate, and field blanks), laboratory control samples, duplicates (field and laboratory), matrix spikes, surrogates, and internal standards.

Data validation and qualification policies were developed from the following sources: quality control requirements in the analytical methods, data review guidelines provided by the US EPA, contract-specific WSRC requirements, and discussions with laboratory personnel.

\section{Data Qualifiers}

When analytical data are validated, the analytical results and the associated QC information are reviewed using criteria specific to the analysis performed. Data qualifiers are used during the data validation process to classify sample data as conforming to QC requirements.

The qualifiers used for data associated with this project are defined in Appendix D.

\section{Precision}

Precision measures the reproducibility of measurements under a given set of conditions. It is a quantitative measure of the variability of a group of measurements compared to its average value. Precision is reported in terms of the relative percent difference. The analytical results from the field replicate samples provide data on overall measurement precision; analytical results from the laboratory duplicates provide data on analytical precision. See Section 3.5.1.

\section{Accuracy}

Accuracy measures the bias in a measurement system; it is difficult to measure for the entire data collection activity. Sources of error are the sampling process, field contami-nation, preservation, handling, sample matrix, sample preparation, and sample analysis techniques. Sampling accuracy may be assessed by evaluating the results of rinsates, field blanks, and trip blanks. Analytical accuracy may be assessed through the use of known and unknown QC samples, matrix spikes, and method blanks. See Section 3.5.2.

\section{Representativeness}

Representativeness expresses the degree to which sample data accurately and precisely represent a characteristic of a population, parameter variations at a sampling point, or an environmental condition. Representativeness is a qualitative parameter that is most concerned with the proper design of the sampling program and is best satisfied by making certain that sampling locations are selected properly and that a sufficient number of samples are collected.

\section{Completeness}

Completeness is defined as the percentage of measurements made that are judged to be valid measurements. It is important that critical samples are identified and that plans are made to achieve valid data for them. Completeness was not determined for this report. 


\section{Comparability}

Comparability is a qualitative parameter expressing the confidence with which one data set can be compared with another. Sample data should be comparable with other measurement data for similar samples and sample conditions. This goal is achieved through using standard techniques to collect and analyze representative samples and through reporting analytical results in appropriate units. See Section 3.5.4.

\subsubsection{Laboratories}

GE of Charleston, SC, provided definitive-level inorganic and organic analyses under contract AA07208N. GE's affiliate, EP, of Charleston, SC, performed definitive-level radiological analyses.

\subsection{Data Quality Issues Summary}

This section provides a summary of the major data quality issues identified during the verification and validation process.

\subsubsection{Missing Data}

All planned data for collected environmental samples were received.

\subsubsection{Extra Data}

No data that had not been requested were received.

\subsubsection{Sample Documentation}

Because fish samples were sent to the laboratory frozen solid $\left(0^{\circ} \mathrm{C}\right)$, cooler temperatures were not expected to have an impact on sample quality.

Table 2.4 Chain-of-Custody Documentation Summary

\begin{tabular}{|l|c|c|}
\hline Description & Number & Percent \\
\hline Missing transfer dates, times, and signatures & 0 & 0 \\
\hline Missing transfer dates and/or times only & 0 & 0 \\
\hline Missing transfer signatures only & 0 & 0 \\
\hline Missing cooler number & 0 & 0 \\
\hline Missing temperature & 0 & 0 \\
\hline Missing COCs & 0 & 0 \\
\hline
\end{tabular}

Table 2.4 Chain-of-Custody Documentation Summary (cont.)

\begin{tabular}{|l|c|c|}
\hline Description & Number & Percent \\
\hline No problems & 29 & 100 \\
\hline Total number of COCs used $=\mathbf{2 9}$ & \\
\hline
\end{tabular}




\subsubsection{Holding Times and Preservation}

The holding time for the fish samples commenced at the preparation stage, not at the collection stage. As a result, all samples were analyzed within the holding time.

Table 2.5 Holding Time Exceedances

\begin{tabular}{|l|l|l|}
\hline & Number of Records & Percentage of Total Sample Records \\
\hline $\begin{array}{l}\text { Detects Exceeding Holding } \\
\text { Time }\end{array}$ & 0 & 0.0 \\
\hline $\begin{array}{l}\text { Non-detects Exceeding } \\
\text { Holding Time }\end{array}$ & 0 & 0.0 \\
\hline $\begin{array}{l}\text { Total Analyses Exceeding } \\
\text { Holding Time }\end{array}$ & 0 & 0.0 \\
\hline
\end{tabular}

\subsubsection{Analytical Issues}

As discussed in section 3.4.5, Metals Data Evaluation, nine antimony and eleven cadmium records were converted to nondetects due to interference. No other significant analytical issues were encountered during verification and validation of the analytical data.

\subsubsection{Rejected Data}

Fluctuations in the background count rate resulted in the laboratory rejecting one uranium-233/234 and four uranium-238 samples.

The laboratory rejected five lead-212, three cesium-137, one actinium-228, and one potassium-40 sample records due to low abundance and one cerium-144 sample record due to interference.

Additionally, an antimony-125 method blank, one lead-212 laboratory duplicate, and one actinium-228 laboratory duplicate records were rejected by the laboratory due to low abundance and interference.

One total recoverable mercury matrix spike was rejected due to low recovery.

Table 2.6 Summary of Rejected Records

\begin{tabular}{|c|l|l|l|l|}
\hline & $\begin{array}{l}\text { Number of } \\
\text { Records }\end{array}$ & $\begin{array}{l}\text { Number } \\
\text { Rejected }\end{array}$ & $\begin{array}{l}\text { Percent of Type } \\
\text { Rejected }\end{array}$ & $\begin{array}{l}\text { Percent of Total Records } \\
\text { Rejected }\end{array}$ \\
\hline Chemical & 513 & 1 & 0.19 & 0.03 \\
\hline QC Records & 1,376 & 0 & 0.0 & 0.0 \\
\hline $\begin{array}{l}\text { Environmental } \\
\text { Sample Records }\end{array}$ & \multicolumn{5}{l}{} \\
\hline Radiochemical & 3 & 1.85 & 0.10 \\
\hline $\begin{array}{l}\text { QC Records } \\
\text { Environmental } \\
\text { Sample Records }\end{array}$ & 162 & 1.81 & 0.55 \\
\hline Totals & 884 & 16 & & \\
\hline All Records & 2,935 & 20 & 0.68 & 0.68 \\
\hline
\end{tabular}


This page was left blank intentionally. 


\subsection{Validation}

\subsection{Planning}

There was no formal work plan for this project. A sampling and analysis plan, the Savannah River Fish Tissue Task Plan, was provided to EMS for project mobilization.

\subsubsection{Planned Work}

Sampling and analysis were conducted according to the Savannah River Fish Tissue Task Plan except as described in section 3.2.1.

Fish samples were to be collected from seven sampling sites via electrofishing, with three composite samples (one benthic, one predatory, and one regional species) to be taken from each location.

In order to fulfill EPA criteria for field sampling activities, trip blanks are used to monitor volatile contaminants during shipping and handling. Trip blanks consist of preserved DI water and are placed in the coolers with the VOA samples at the rate of one trip blank per cooler. The number of duplicate and other QA samples was to be determined by the laboratory; no split samples were planned for this project. Duplicate samples were to be analyzed for the same parameters as the associated samples.

Fish samples, consisting of composites of whole fish, were to be analyzed for TCL VOA, radionuclides (gross alpha, nonvolatile beta, gamma spectroscopy, plutonium 239/240 [plutonium series], strontium 89/90, tritium, and uranium 233/234 [uranium series]) and RCRA metals (antimony, arsenic, barium, cadmium, chromium, copper, lead, manganese, mercury, nickel, selenium, silver, and zinc, plus iron and aluminum as required to confirm the presence of interferences). Trip blanks were to be analyzed for TCL VOA only.

\subsubsection{Production of Chain-of-Custody Forms}

COC forms containing the list of requested analyses were printed for each planned sample. Information completed on the twenty-nine COC forms generated included the following:

- EMS sample identification number (logbook page number for that sample)

- laboratory sample identification number (to be completed by the laboratory)

- sample collection date and time

- shipping information (including dates and signatures)

- cooler number and temperature upon arrival at the analytical laboratory

A peel-off section of each bottle label, printed with the bottle number, was affixed to the analysis request line on the COC form.

\subsubsection{Sample Set}

The following table provides details about the location and composition of the fish tissue samples. 
Table 3.1 Fish Tissue Sample Details

\begin{tabular}{|c|c|c|c|c|c|c|c|c|c|c|}
\hline $\begin{array}{l}\text { Composited } \\
\text { Sample }\end{array}$ & $\begin{array}{l}\text { Log-book } \\
\#\end{array}$ & Sample & Location & UTM-E & UTM-N & Species & $\begin{array}{l}\text { Trophic } \\
\text { Level }\end{array}$ & $\begin{array}{l}\text { Length } \\
(\mathrm{mm})\end{array}$ & $\begin{array}{l}\text { Weight } \\
\text { (g) }\end{array}$ & $\begin{array}{l}\text { K } \\
\text { Factor }^{1}\end{array}$ \\
\hline SRF-06-02 & 117038 & 1 & L3R Creek Mouth² & 452033 & $\begin{array}{l}3654459 . \\
8\end{array}$ & Redbreast SF & Regional & 188 & 120.9 & 1.8195 \\
\hline SRF-06-02 & 117038 & 2 & L3R Creek Mouth & 452033 & $\begin{array}{l}3654459 . \\
8\end{array}$ & Redbreast SF & Regional & 192 & 143.5 & 2.0274 \\
\hline SRF-06-02 & 117038 & 3 & L3R Creek Mouth & 452033 & $\begin{array}{l}3654459 . \\
8\end{array}$ & Redbreast SF & Regional & 120 & 30.8 & 1.7824 \\
\hline SRF-06-02 & 117038 & 5 & L3R Creek Mouth & 452033 & $\begin{array}{l}3654459 . \\
8\end{array}$ & Redbreast SF & Regional & 190 & 123.6 & 1.802 \\
\hline SRF-06-01 & 117037 & 1 & L3R Creek Mouth & 452033 & $\begin{array}{l}3654459 . \\
8\end{array}$ & $\begin{array}{l}\text { Spotted } \\
\text { Sucker }\end{array}$ & Benthic & 367 & 550 & 1.1127 \\
\hline SRF-06-01 & 117037 & 2 & L3R Creek Mouth & 452033 & $\begin{array}{l}3654459 . \\
8\end{array}$ & $\begin{array}{l}\text { Spotted } \\
\text { Sucker }\end{array}$ & Benthic & 262 & 218.7 & 1.216 \\
\hline SRF-07-02 & 117042 & 1 & US 301 Bridge $^{3}$ & 452924 & 3644207 & Redear SF & Regional & 120 & 30.9 & 1.7882 \\
\hline SRF-07-02 & 117042 & 2 & US 301 Bridge & 452924 & 3644207 & Redear SF & Regional & 187 & 116.4 & 1.78 \\
\hline SRF-07-02 & 117042 & 3 & US 301 Bridge & 452924 & 3644207 & Redear SF & Regional & 179 & 120.2 & 2.0958 \\
\hline SRF-07-02 & 117042 & 4 & US 301 Bridge & 452924 & 3644207 & Redear SF & Regional & 208 & 140 & 1.5557 \\
\hline SRF-07-03 & 117043 & 1 & US 301 Bridge & 452924 & 3644207 & Bowfin & Predator & 570 & 1767.7 & 0.9545 \\
\hline SRF-07-03 & 117043 & 2 & US 301 Bridge & 452924 & 3644207 & Bowfin & Predator & 650 & 3039.3 & 1.1067 \\
\hline SRF-07-01 & 117041 & 1 & US 301 Bridge & 452924 & 3644207 & Spotted & Benthic & 395 & 623.6 & 1.0118 \\
\hline
\end{tabular}

${ }^{1} \mathrm{~K}=\left(\mathrm{W} \times 10^{5}\right) / \mathrm{L}^{3}$ where $\mathrm{K}$ is the condition factor; $\mathrm{W}=$ weight in $\mathrm{g}$, and $\mathrm{L}=$ total length in $\mathrm{mm}$. A low condition factor often indicates that a fish is in poor health.

2 RM-129 Lower Three Runs Mouth

${ }^{3}$ Highway 301 at State Line

Data Summary Report for Savannah River Integrator Operable Unit Fish Tissue (WSRC-TR-99-00386) 


\begin{tabular}{|c|c|c|c|c|c|c|c|c|c|c|}
\hline & & & & & & Sucker & & & & \\
\hline SRF-07-01 & 117041 & 2 & US 301 Bridge & 452924 & 3644207 & $\begin{array}{l}\text { Spotted } \\
\text { Sucker }\end{array}$ & Benthic & 340 & 467.2 & 1.1887 \\
\hline SRF-05-02 & 117035 & 1 & $\begin{array}{l}\text { Steel Creek } \\
\text { Mouth4 }\end{array}$ & 442307.2 & $\begin{array}{l}3661941 . \\
1\end{array}$ & Redear SF & Regional & 260 & 316.8 & 1.8025 \\
\hline SRF-05-02 & 117035 & 2 & Steel Creek Mouth & 442307.2 & $\begin{array}{l}3661941 . \\
1\end{array}$ & Redear SF & Regional & 250 & 263.7 & 1.6877 \\
\hline SRF-05-01 & 117034 & 1 & Steel Creek Mouth & 442307.2 & $\begin{array}{l}3661941 . \\
1\end{array}$ & $\begin{array}{l}\text { Spotted } \\
\text { Sucker }\end{array}$ & Benthic & 363 & 507 & 1.06 \\
\hline
\end{tabular}

Table 3.1 Fish Tissue Sample Details (cont.)

\begin{tabular}{|c|c|c|c|c|c|c|c|c|c|c|}
\hline $\begin{array}{l}\text { Composited } \\
\text { Sample }\end{array}$ & $\begin{array}{l}\text { Log-book } \\
\#\end{array}$ & Sample & Location & UTM-E & UTM-N & Species & $\begin{array}{l}\text { Trophic } \\
\text { Level }\end{array}$ & $\begin{array}{l}\text { Length } \\
\text { (mm) }\end{array}$ & $\begin{array}{l}\text { Weight } \\
\text { (g) }\end{array}$ & K Factor \\
\hline SRF-05-03 & 117036 & 1 & $\begin{array}{l}\text { Steel Creek } \\
\text { Mouth }\end{array}$ & $\begin{array}{l}442307 . \\
2\end{array}$ & $\begin{array}{l}3661941 . \\
1\end{array}$ & Bowfin & Predator & 593 & 1918.1 & 0.9198 \\
\hline SRF-05-03 & 117036 & 2 & $\begin{array}{l}\text { Steel Creek } \\
\text { Mouth }\end{array}$ & $\begin{array}{l}442307 . \\
2\end{array}$ & $\begin{array}{l}3661941 . \\
1\end{array}$ & Bowfin & Predator & 620 & 2346.3 & 0.9845 \\
\hline SRF-04-02 & 117032 & 1 & FM Creek Mouth5 & $\begin{array}{l}429879 . \\
1\end{array}$ & $\begin{array}{l}3667695 . \\
4\end{array}$ & Redbreast SF & Regional & 165 & 90.1 & 2.0057 \\
\hline SRF-04-02 & 117032 & 2 & FM Creek Mouth & $\begin{array}{l}429879 . \\
1\end{array}$ & $\begin{array}{l}3667695 . \\
4\end{array}$ & Redbreast SF & Regional & 110 & 28.8 & 2.1638 \\
\hline SRF-04-02 & 117032 & 3 & FM Creek Mouth & $\begin{array}{l}429879 . \\
1\end{array}$ & $\begin{array}{l}3667695 . \\
4\end{array}$ & Redbreast SF & Regional & 140 & 52.3 & 1.906 \\
\hline SRF-04-02 & 117032 & 4 & FM Creek Mouth & $\begin{array}{l}429879 . \\
1\end{array}$ & $\begin{array}{l}3667695 . \\
4\end{array}$ & Redbreast SF & Regional & 130 & 42.8 & 1.9481 \\
\hline SRF-04-02 & 117032 & 5 & FM Creek Mouth & $\begin{array}{l}429879 . \\
1\end{array}$ & $\begin{array}{l}3667695 . \\
4\end{array}$ & Redbreast SF & Regional & 150 & 66.9 & 1.9822 \\
\hline
\end{tabular}

\footnotetext{
4 Steel Creek/Pen Branch Mouth
}

5 R-3B (Monthly Grab) 
Table 3.1 Fish Tissue Sample Details (cont.)

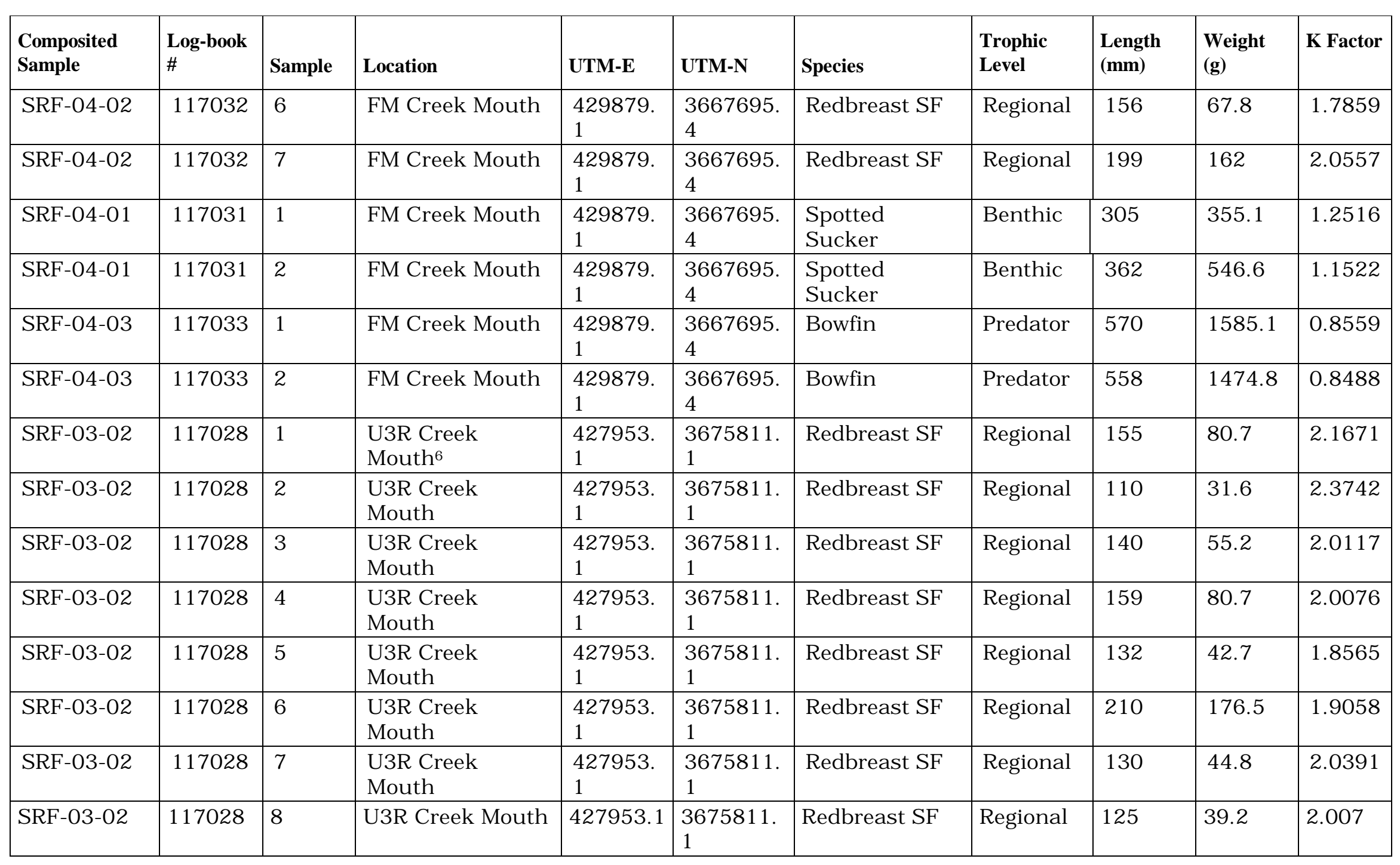

${ }^{6}$ RM 157.2 Upper Three Runs Mouth

Data Summary Report for Savannah River Integrator Operable Unit Fish Tissue (WSRC-TR-99-00386) 
Table 3.1 Fish Tissue Sample Details (cont.)

\begin{tabular}{|c|c|c|c|c|c|c|c|c|c|c|}
\hline $\begin{array}{l}\text { Composited } \\
\text { Sample }\end{array}$ & $\begin{array}{l}\text { Log-book } \\
\#\end{array}$ & Sample & Location & UTM-E & UTM-N & Species & $\begin{array}{l}\text { Trophic } \\
\text { Level }\end{array}$ & $\begin{array}{l}\text { Length } \\
(\mathrm{mm})\end{array}$ & $\begin{array}{l}\text { Weight } \\
\text { (g) }\end{array}$ & K Factor \\
\hline SRF-03-01 & 117027 & 1 & U3R Creek Mouth & 427953.1 & $\begin{array}{l}3675811 . \\
1\end{array}$ & Spotted Sucker & Benthic & 468 & 1006.3 & 0.9817 \\
\hline SRF-03-01 & 117027 & 2 & U3R Creek Mouth & 427953.1 & $\begin{array}{l}3675811 . \\
1\end{array}$ & Spotted Sucker & Benthic & 422 & 724.3 & 0.9638 \\
\hline SRF-03-03 & 117029 & 1 & $\begin{array}{l}\text { U3R Creek } \\
\text { Mouth }\end{array}$ & $\begin{array}{l}427953 . \\
1\end{array}$ & $\begin{array}{l}3675811 . \\
1\end{array}$ & Bowfin & Predator & 552 & 1622.2 & 0.9645 \\
\hline SRF-03-03 & 117029 & 2 & $\begin{array}{l}\text { U3R Creek } \\
\text { Mouth }\end{array}$ & $\begin{array}{l}427953 . \\
1\end{array}$ & $\begin{array}{l}3675811 . \\
1\end{array}$ & Bowfin & Predator & 622 & 2482.9 & 1.0318 \\
\hline SRF-03-04 & 117030 & 1 & $\begin{array}{l}\text { U3R Creek } \\
\text { Mouth }\end{array}$ & $\begin{array}{l}427953 . \\
1\end{array}$ & $\begin{array}{l}3675811 . \\
1\end{array}$ & Bluegill SF & Regional & 114 & 30.3 & 2.0452 \\
\hline SRF-03-04 & 117030 & 2 & $\begin{array}{l}\text { U3R Creek } \\
\text { Mouth }\end{array}$ & $\begin{array}{l}427953 . \\
1\end{array}$ & $\begin{array}{l}3675811 . \\
1\end{array}$ & Bluegill SF & Regional & 187 & 134.2 & 2.0522 \\
\hline SRF-03-04 & 117030 & 3 & $\begin{array}{l}\text { U3R Creek } \\
\text { Mouth }\end{array}$ & $\begin{array}{l}427953 . \\
1\end{array}$ & $\begin{array}{l}3675811 . \\
1\end{array}$ & Bluegill SF & Regional & 132 & 44.2 & 1.9218 \\
\hline SRF-03-04 & 117030 & 4 & $\begin{array}{l}\text { U3R Creek } \\
\text { Mouth }\end{array}$ & $\begin{array}{l}427953 . \\
1\end{array}$ & $\begin{array}{l}3675811 . \\
1\end{array}$ & Bluegill SF & Regional & 125 & 36.2 & 1.8534 \\
\hline SRF-03-04 & 117030 & 5 & $\begin{array}{l}\text { U3R Creek } \\
\text { Mouth }\end{array}$ & $\begin{array}{l}427953 . \\
1\end{array}$ & $\begin{array}{l}3675811 . \\
1\end{array}$ & Bluegill SF & Regional & 126 & 41.6 & 2.0796 \\
\hline SRF-03-04 & 117030 & 6 & $\begin{array}{l}\text { U3R Creek } \\
\text { Mouth }\end{array}$ & $\begin{array}{l}427953 . \\
1\end{array}$ & $\begin{array}{l}3675811 . \\
1\end{array}$ & Bluegill SF & Regional & 125 & 36.4 & 1.8637 \\
\hline SRF-03-04 & 117030 & 7 & $\begin{array}{l}\text { U3R Creek } \\
\text { Mouth }\end{array}$ & $\begin{array}{l}427953 . \\
1\end{array}$ & $\begin{array}{l}3675811 . \\
1\end{array}$ & Bluegill SF & Regional & 121 & 32.1 & 1.812 \\
\hline SRF-03-04 & 117030 & 8 & $\begin{array}{l}\text { U3R Creek } \\
\text { Mouth }\end{array}$ & $\begin{array}{l}427953 . \\
1\end{array}$ & $\begin{array}{l}3675811 . \\
1\end{array}$ & Bluegill SF & Regional & 107 & 24.5 & 1.9999 \\
\hline SRF-03-04 & 117030 & 9 & $\begin{array}{l}\text { U3R Creek } \\
\text { Mouth }\end{array}$ & $\begin{array}{l}427953 . \\
1\end{array}$ & $\begin{array}{l}3675811 . \\
1\end{array}$ & Bluegill SF & Regional & 114 & 28.8 & 1.9439 \\
\hline SRF-03-04 & 117030 & 10 & $\begin{array}{l}\text { U3R Creek } \\
\text { Mouth }\end{array}$ & $\begin{array}{l}427953 . \\
1\end{array}$ & $\begin{array}{l}3675811 . \\
1\end{array}$ & Bluegill SF & Regional & 90 & 12.1 & 1.6598 \\
\hline SRF-03-04 & 117030 & 11 & $\begin{array}{l}\text { U3R Creek } \\
\text { Mouth }\end{array}$ & $\begin{array}{l}427953 . \\
1\end{array}$ & $\begin{array}{l}3675811 . \\
1\end{array}$ & Bluegill SF & Regional & 110 & 25.8 & 1.9384 \\
\hline
\end{tabular}


Table 3.1 Fish Tissue Sample Details (cont.)

\begin{tabular}{|c|c|c|c|c|c|c|c|c|c|c|}
\hline $\begin{array}{l}\text { Composited } \\
\text { Sample }\end{array}$ & $\begin{array}{l}\text { Log-book } \\
\#\end{array}$ & Sample & Location & UTM-E & UTM-N & Species & $\begin{array}{l}\text { Trophic } \\
\text { Level }\end{array}$ & $\begin{array}{l}\text { Length } \\
(\mathrm{mm})\end{array}$ & $\begin{array}{l}\text { Weight } \\
\text { (g) }\end{array}$ & K Factor \\
\hline SRF-03-04 & 117030 & 12 & $\begin{array}{l}\text { U3R Creek } \\
\text { Mouth }\end{array}$ & $\begin{array}{l}427953 . \\
1\end{array}$ & $\begin{array}{l}3675811 . \\
1\end{array}$ & Bluegill SF & Regional & 97 & 16 & 1.7531 \\
\hline SRF-03-04 & 117030 & 13 & $\begin{array}{l}\text { U3R Creek } \\
\text { Mouth }\end{array}$ & $\begin{array}{l}427953 . \\
1\end{array}$ & $\begin{array}{l}3675811 . \\
1\end{array}$ & Bluegill SF & Regional & 97 & 15.5 & 1.6983 \\
\hline SRF-02-02 & 117024 & 1 & Augusta L\&D7 & $\begin{array}{l}411924 . \\
8\end{array}$ & $\begin{array}{l}3692668 . \\
7\end{array}$ & Redear SF & Regional & 204 & 164.3 & 1.9353 \\
\hline SRF-02-02 & 117024 & 2 & Augusta L\&D & $\begin{array}{l}411924 . \\
8\end{array}$ & $\begin{array}{l}3692668 . \\
7\end{array}$ & Redear SF & Regional & 219 & 218.6 & 2.0812 \\
\hline SRF-02-02 & 117024 & 3 & Augusta L\&D & $\begin{array}{l}411924 . \\
8\end{array}$ & $\begin{array}{l}3692668 . \\
7\end{array}$ & Redear SF & Regional & 270 & 454 & 2.3066 \\
\hline SRF-02-01 & 117023 & 1 & Augusta L\&D & $\begin{array}{l}411924 . \\
8\end{array}$ & $\begin{array}{l}3692668 . \\
7\end{array}$ & $\begin{array}{l}\text { Spotted } \\
\text { Sucker }\end{array}$ & Benthic & 422 & 888.2 & 1.1819 \\
\hline SRF-02-01 & 117023 & 2 & Augusta L\&D & $\begin{array}{l}411924 . \\
8\end{array}$ & $\begin{array}{l}3692668 . \\
7\end{array}$ & $\begin{array}{l}\text { Spotted } \\
\text { Sucker }\end{array}$ & Benthic & 451 & 1006.7 & 1.0974 \\
\hline SRF-02-03 & 117025 & 1 & Augusta L\&D & $\begin{array}{l}411924 . \\
8\end{array}$ & $\begin{array}{l}3692668 . \\
7\end{array}$ & Bowfin & Predator & 493 & 1232.6 & 1.0287 \\
\hline SRF-02-03 & 117025 & 2 & Augusta L\&D & $\begin{array}{l}411924 . \\
8\end{array}$ & $\begin{array}{l}3692668 . \\
7\end{array}$ & Bowfin & Predator & 551 & 1562.5 & 0.934 \\
\hline SRF-01-02 & 117019 & 1 & NA Rapids $^{8}$ & $\begin{array}{l}407789 . \\
7\end{array}$ & $\begin{array}{l}3688856 . \\
8\end{array}$ & Redear SF & Regional & 242 & 327.8 & 2.3129 \\
\hline SRF-01-02 & 117019 & 2 & NA Rapids & $\begin{array}{l}407789 . \\
7\end{array}$ & $\begin{array}{l}3688856 . \\
8\end{array}$ & Redear SF & Regional & 155 & 94.1 & 2.5269 \\
\hline SRF-01-02 & 117019 & 3 & NA Rapids & $\begin{array}{l}407789 . \\
7\end{array}$ & $\begin{array}{l}3688856 . \\
8\end{array}$ & Redear SF & Regional & 126 & 50.5 & 2.5245 \\
\hline SRF-01-02 & 117019 & 4 & NA Rapids & $\begin{array}{l}407789 . \\
7\end{array}$ & $\begin{array}{l}3688856 . \\
8\end{array}$ & Redear SF & Regional & 120 & 34.2 & 1.9792 \\
\hline
\end{tabular}

7 Augusta Lock \& Dam 614

${ }^{8}$ North Augusta Rapids 
Table 3.1 Fish Tissue Sample Details (cont.)

\begin{tabular}{|c|c|c|c|c|c|c|c|c|c|c|}
\hline $\begin{array}{l}\text { Composited } \\
\text { Sample }\end{array}$ & $\begin{array}{l}\text { Log-book } \\
\#\end{array}$ & Sample & Location & UTM-E & UTM-N & Species & $\begin{array}{l}\text { Trophic } \\
\text { Level }\end{array}$ & $\begin{array}{l}\text { Length } \\
(\mathrm{mm})\end{array}$ & $\begin{array}{l}\text { Weight } \\
\text { (g) }\end{array}$ & K Factor \\
\hline SRF-01-02 & 117019 & 5 & NA Rapids & $\begin{array}{l}407789 . \\
7\end{array}$ & $\begin{array}{l}3688856 . \\
8\end{array}$ & Redear SF & Regional & 104 & 26.5 & 2.3558 \\
\hline SRF-01-01 & 117018 & 1 & NA Rapids & $\begin{array}{l}407789 . \\
7\end{array}$ & $\begin{array}{l}3688856 . \\
8\end{array}$ & $\begin{array}{l}\text { Spotted } \\
\text { Sucker }\end{array}$ & Benthic & 436 & 955 & 1.1522 \\
\hline SRF-01-01 & 117018 & 2 & NA Rapids & $\begin{array}{l}407789 . \\
7\end{array}$ & $\begin{array}{l}3688856 . \\
8\end{array}$ & $\begin{array}{l}\text { Spotted } \\
\text { Sucker }\end{array}$ & Benthic & 432 & 1010.9 & 1.2539 \\
\hline SRF-01-03 & 117020 & 1 & NA Rapids & $\begin{array}{l}407789 . \\
7\end{array}$ & $\begin{array}{l}3688856 . \\
8\end{array}$ & Bowfin & Predator & 468 & 1132.9 & 1.1052 \\
\hline SRF-01-03 & 117020 & 2 & NA Rapids & $\begin{array}{l}407789 . \\
7\end{array}$ & $\begin{array}{l}3688856 . \\
8\end{array}$ & Bowfin & Predator & 551 & 1848.5 & 1.105 \\
\hline SRF-01-05 & 117022 & 1 & NA Rapids & $\begin{array}{l}407789 . \\
7\end{array}$ & $\begin{array}{l}3688856 . \\
8\end{array}$ & Redbreast SF & Regional & 158 & 81.8 & 2.0739 \\
\hline SRF-01-05 & 117022 & 2 & NA Rapids & $\begin{array}{l}407789 . \\
7\end{array}$ & $\begin{array}{l}3688856 . \\
8\end{array}$ & Redbreast SF & Regional & 179 & 102.3 & 1.7837 \\
\hline SRF-01-05 & 117022 & 3 & NA Rapids & $\begin{array}{l}407789 . \\
7\end{array}$ & $\begin{array}{l}3688856 . \\
8\end{array}$ & Redbreast SF & Regional & 127 & 39.6 & 1.9332 \\
\hline SRF-01-05 & 117022 & 4 & NA Rapids & $\begin{array}{l}407789 . \\
7\end{array}$ & $\begin{array}{l}3688856 . \\
8\end{array}$ & Redbreast SF & Regional & 120 & 33.2 & 1.9213 \\
\hline SRF-01-05 & 117022 & 5 & NA Rapids & $\begin{array}{l}407789 . \\
7\end{array}$ & $\begin{array}{l}3688856 . \\
8 \\
\end{array}$ & Redbreast SF & Regional & 117 & 38.8 & 2.4226 \\
\hline SRF-01-04 & 117021 & 1 & NA Rapids & $\begin{array}{l}407789 . \\
7\end{array}$ & $\begin{array}{l}3688856 . \\
8\end{array}$ & Bluegill SF & Regional & 189 & 173.9 & 2.5758 \\
\hline SRF-01-04 & 117021 & 2 & NA Rapids & $\begin{array}{l}407789 . \\
7\end{array}$ & $\begin{array}{l}3688856 . \\
8\end{array}$ & Bluegill SF & Regional & 157 & 89.9 & 2.3231 \\
\hline SRF-01-04 & 117021 & 3 & NA Rapids & $\begin{array}{l}407789 . \\
7\end{array}$ & $\begin{array}{l}3688856 . \\
8\end{array}$ & Bluegill SF & Regional & 163 & 104.1 & 2.4037 \\
\hline SRF-01-04 & 117021 & 4 & NA Rapids & $\begin{array}{l}407789 . \\
7\end{array}$ & $\begin{array}{l}3688856 . \\
8\end{array}$ & Bluegill SF & Regional & 91 & 14.2 & 1.8844 \\
\hline SRF-01-04 & 117021 & 5 & NA Rapids & $\begin{array}{l}407789 . \\
7\end{array}$ & $\begin{array}{l}3688856 . \\
8\end{array}$ & Bluegill SF & Regional & 152 & 85.8 & 2.4432 \\
\hline
\end{tabular}


Table 3.1 Fish Tissue Sample Details (cont.)

\begin{tabular}{|l|l|l|l|l|l|l|l|l|l|l|}
\hline $\begin{array}{l}\text { Composited } \\
\text { Sample }\end{array}$ & $\begin{array}{l}\text { Log-book } \\
\#\end{array}$ & Sample & Location & UTM-E & UTM-N & Species & $\begin{array}{l}\text { Trophic } \\
\text { Level }\end{array}$ & $\begin{array}{l}\text { Length } \\
(\mathbf{m m})\end{array}$ & $\begin{array}{l}\text { Weight } \\
(\mathbf{g})\end{array}$ & $\begin{array}{l}\text { K Factor } \\
\text { SRF-01-04 }\end{array}$ \\
\hline 117021 & 6 & NA Rapids & $\begin{array}{l}407789 . \\
7\end{array}$ & $\begin{array}{l}3688856 . \\
8\end{array}$ & Bluegill SF & Regional & 137 & 56 & 2.1778 \\
\hline SRF-01-04 & 117021 & 7 & NA Rapids & $\begin{array}{l}407789 . \\
7\end{array}$ & $\begin{array}{l}3688856 . \\
8\end{array}$ & Bluegill SF & Regional & 105 & 27.9 & 2.4101 \\
\hline
\end{tabular}


The following table lists each survey station ID, sample logbook ID, laboratory ID number, sample type, sample date, and the analyses requested for samples sent to the laboratories.

Table 3.2 Sample Set

\begin{tabular}{|l|l|l|l|l|l|}
\hline $\begin{array}{l}\text { Survey Station } \\
\text { ID }\end{array}$ & Sample ID & Laboratory ID & Sample Type & Sample Date & $\begin{array}{l}\text { Analyses } \\
\text { Requested }\end{array}$ \\
\hline Samples and Duplicates & \multicolumn{5}{l|}{} \\
\hline SRF-01-01 & 117018 & $9907977-23$ & Fish Tissue & $07 / 22 / 99$ & 1,2 \\
\hline SRF-01-02 & 117019 & $9907977-22$ & Fish Tissue & $07 / 22 / 99$ & 1,2 \\
\hline SRF-01-03 & 117020 & $9907977-17$ & Fish Tissue & $07 / 22 / 99$ & 1,2 \\
\hline SRF-01-04 & 117021 & $9907977-21$ & Fish Tissue & $07 / 22 / 99$ & 1,2 \\
\hline SRF-01-05 & 117022 & $9907977-20$ & Fish Tissue & $07 / 22 / 99$ & 1,2 \\
\hline SRF-02-01 & 117023 & $9907977-19$ & Fish Tissue & $07 / 21 / 99$ & 1,2 \\
\hline SRF-02-02 & 117024 & $9907977-18$ & Fish Tissue & $07 / 21 / 99$ & 1,2 \\
\hline SRF-02-03 & 117025 & $9907977-16$ & Fish Tissue & $07 / 21 / 99$ & 1,2 \\
\hline SRF-02-03-A & 117026 & $9907977-15$ & Duplicate & $07 / 21 / 99$ & 1,2 \\
\hline SRF-03-01 & 117027 & $9907977-14$ & Fish Tissue & $07 / 20 / 99$ & 1,2 \\
\hline SRF-03-02 & 117028 & $9907977-13$ & Fish Tissue & $07 / 20 / 99$ & 1,2 \\
\hline SRF-03-03 & 117029 & $9907977-12$ & Fish Tissue & $07 / 20 / 99$ & 1,2 \\
\hline SRF-03-04 & 117030 & $9907977-11$ & Fish Tissue & $07 / 20 / 99$ & 1,2 \\
\hline SRF-04-01 & 117031 & $9907977-10$ & Fish Tissue & $07 / 20 / 99$ & 1,2 \\
\hline SRF-04-02 & 117032 & $9907977-09$ & Fish Tissue & $07 / 20 / 99$ & 1,2 \\
\hline SRF-04-03 & 117033 & $9907977-08$ & Fish Tissue & $07 / 20 / 99$ & 1,2 \\
\hline SRF-05-01 & 117034 & $9907977-07$ & Fish Tissue & $07 / 19 / 99$ & 1,2 \\
\hline SRF-05-02 & 117035 & $9907977-06$ & Fish Tissue & $07 / 19 / 99$ & 1,2 \\
\hline SRF-05-03 & 117036 & $9907977-05$ & Fish Tissue & $07 / 19 / 99$ & 1,2 \\
\hline SRF-06-01 & 117037 & $9907977-03$ & Fish Tissue & $07 / 19 / 99$ & 1,2 \\
\hline SRF-06-02 & 117038 & $9907977-04$ & Fish Tissue & $07 / 19 / 99$ & 1,2 \\
\hline SRF-06-03 & 117039 & $9907977-01$ & Fish Tissue & $07 / 19 / 99$ & 1,2 \\
\hline SRF-06-03-A & 117040 & $9907977-02$ & Duplicate & $07 / 19 / 99$ & 1,2 \\
\hline SRF-07-01 & 117041 & $9907977-24$ & Fish Tissue & $07 / 19 / 99$ & 1,2 \\
\hline SRF-07-02 & 117042 & $9907977-25$ & Fish Tissue & $07 / 19 / 99$ & 1,2 \\
\hline SRF-07-03 & 117043 & $9907977-26$ & Fish Tissue & $07 / 19 / 99$ & 1,2 \\
\hline & & & \\
\hline
\end{tabular}

91 = RCRA metals (antimony, arsenic, barium, cadmium, chromium, copper, lead, manganese, mercury, nickel, selenium, silver, and zinc, plus iron and aluminum as required to confirm the presence of interferences); radionuclides (gross alpha, nonvolatile beta, gamma spectroscopy, plutonium-239/240 [plutonium series], strontium-89/90, tritium, uranium 233/234 [uranium series])

$2=$ TCL VOA 
Table 3.2 Sample Set (cont.)

\begin{tabular}{|l|l|l|l|l|l|}
\hline $\begin{array}{l}\text { Survey Station } \\
\text { ID }\end{array}$ & Sample ID & Laboratory ID & Sample Type & Sample Date & $\begin{array}{l}\text { Analyses } \\
\text { Requested }\end{array}$ \\
\hline Trip Blanks \\
\hline SRF-TB-01-B & 117044 & $9907977-27$ & Water & $07 / 28 / 99$ & 2 \\
\hline SRF-TB-02-B & 117045 & $9907977-29$ & Water & $07 / 28 / 99$ & 2 \\
\hline SRF-TB-03-B & 117046 & $9907977-28$ & Water & $07 / 28 / 99$ & 2 \\
\hline
\end{tabular}

The following table lists the trip blank ID numbers and associated sample ID numbers for the samples.

Table 3.3 Trip Blanks and Associated Samples

\begin{tabular}{|l|l|}
\hline Trip Blank ID & Associated Samples \\
\hline SRF-TB-01-B & $117037,117038,117039,117040,117041,117042,117043$ \\
\hline SRF-TB-02-B & $\begin{array}{l}117027,117028,117029,117030,117031,117032,117033,117034,117035, \\
117036\end{array}$ \\
\hline SRF-TB-03-B & $117018,117019,117020,117021,117022,117023,117024,117025,117026$ \\
\hline
\end{tabular}

\subsection{Field Data Quality Issues}

\subsubsection{Discrepancies from the Planned Work}

All of the 29 COCs generated for this project were used. This number includes three samples of regional fish not specified in the Savannah River Fish Tissue Task Plan. Three trip blanks - one for each of the three coolers required to ship samples to the laboratory - were used. Although the plan suggested that composites for the duplicate samples be taken from the benthic specimens, the actual duplicates were composed of predatory fish.

\subsubsection{Sample Logbook Observations}

There were no sample logbook observations that impacted data quality. Although one of three regional fish samples from the SRF-01 location was described as a "minimal weight sample; less than 300g," it was analyzed as planned.

\subsubsection{Chain-of-Custody Observations}

There were no significant COC observations

\subsection{Analytical Data Quality Issues}

\subsubsection{The Laboratories}

GE provided definitive-level inorganic and organic analyses under contract AA07208N. GE's affiliate, EP, performed definitive-level radiological analyses. Both laboratories have been certified by SCDHEC. 


\subsubsection{Laboratory Quality Control}

The laboratories follow their own QA/QC plans, which are designed to measure and document the accuracy and precision for the various sample control and analytical methods they perform. The laboratories are guided by published method-specific QC requirements as well as other regulatory and contract-required QC procedures.

Appendix B describes performance evaluation studies in which the laboratories and their subcontractors participate.

\subsubsection{List of Analytical Suites}

- RCRA metals (antimony, arsenic, barium, cadmium, chromium, copper, lead, manganese, mercury, nickel, selenium, silver, and zinc, plus iron and aluminum as required to confirm the presence of interferences)

- Radionuclides (gross alpha, nonvolatile beta, gamma spectroscopy, plutonium-239/40 [plutonium series], strontium-89/90, tritium, uranium 233/234 [uranium series])

- TCL VOA

See Appendix A for listings of the definitive-level suites, constituents analyzed, and analytical methods used.

\subsubsection{Analytical Data Observations}

The data are final and complete except as noted in this subsection. Data validation is also complete.

Some of the data did not meet all the criteria listed in EPA, 1993, as noted below:

- Analytical error determination (a measurement of method precision) was not performed; however, precision can be evaluated by referring to the tables in section 3.5, Precision and Accuracy, of this report.

- Total measurement error determination (measurement of overall precision from sample acquisition through analysis) was not performed because it requires independently collected samples from the same location, and this practice does not fit into the scope of the project work plan. Each sampling location was used during one sampling event.

- No adjustments to sample values were made for bias or precision.

- Using the criteria defined in EPA, 1993, matrix spike recoveries for an entire project are averaged, and all associated sample data are qualified on the basis of this average. Matrix spike-based qualification for this project, however, was done on a sample-by-sample basis, using the criteria in subsection 3.4.4, Qualification of the Data.

- One matrix spike could not be evaluated due to high concentration of the analyte in the sample, matrix interference, or dilution effects. 


\subsection{Verification and Validation Issues}

\subsubsection{Verification and Validation Process}

The data verification process confirms that the required samples were collected and documented, the required analyses were performed on the samples, and the analytical results were reported correctly. The information evaluated during this process includes work plan assessment sample lists, work plan assessment analyte/compound lists, sample logbooks, COC forms, and analytical results files.

Data reviewers use computer programs and look-up tables to review the computer data files of sample collection, shipping, and analytical data. The data were examined as follows:

- The following fields were checked to ensure that they were not blank: customer sample identification number, COC number (for groundwater samples) or sample number (for soil or biological samples), sample collection date, laboratory receipt date, analysis date, analysis time, analytical method, laboratory code, laboratory sample identification number, analyte code (test name), ssMDL, ssEQL, analytical result, result units, counting uncertainty (for radionuclide analyses), nominal concentration (if applicable), wet weight or dry weight, dilution factor, instrument, analyst's initials, percent solids and sample fraction (soil/sediment only), sample matrix, preparation factor, and subcontract number.

- The following fields were checked to ensure that reported data were within an acceptable range of values: customer sample identification number, COC number, soils sample number, sample collection date, sample collection time, lab receipt date, extraction date, extraction time, analysis date, analysis time, analytical result, counting uncertainty, residual weight, nominal concentration, dilution factor, percent solids, and bottle label number.

- The following fields were checked against a look-up table or a list of valid codes: field QC code; extraction, digestion, or preparation method; analytical method; laboratory code; laboratory QC sample code; analyte code; ssMDL; ssEQL; FG code; STORET code; EMS code; result units; percent recovery; sample fraction; sample matrix; and subcontract number.

The data validation process determines the usefulness of each analytical result based on QC and method requirements. The information evaluated during this process includes $\mathrm{COC}$ forms, analytical narrative summaries, analytical result data files, and raw result and calibration data.

Data validation evaluates requirements in the following QC categories: holding times, sample preservation, instrument calibration, analyte identification, analyte quantitation (surrogates and internal standards), blanks (trip, method, rinsate, and field), laboratory control samples, matrix spikes, and duplicates (field and laboratory).

Data validation and qualification policies were developed from the following sources: EPA, 1986; EPA, 1988a; EPA, 1988b; EPA, 1990; EPA, 1991b; EPA, 1994a; EPA, 1994b; WHC, not dated; contractspecific WSRC requirements; and discussions with laboratory personnel.

The qualifiers are defined in the beginning of Appendix $D$.

\subsubsection{Analytical Narrative Review}

The EDDs and analytical narratives arrive from the laboratories and are logged in together. If the analytical narrative is missing, it is requested from the analytical laboratory. The narratives are reviewed and used as a reference throughout the data validation process. Discrepancies between the narratives and 
the analytical data must be resolved by the analytical laboratory. The analytical narratives contain summaries of the following problems encountered by the laboratory:

- QC samples that do not meet the criteria specified by the analytical method

- matrix interference problems

- sample-specific adjustments to the method caused by high concentration of some analytes

- sample preservation and holding time problems

- instrument calibration problems

- contaminated blanks

- other laboratory QC issues

Additional information about the chain-of-custody data and analytical data is often found in the analytical narratives. Copies of the COC forms attached to the analytical narratives are used for data review.

\subsubsection{Laboratory Data Records Review}

Laboratory data records reviews (LDRRs) are conducted periodically at laboratories that perform environmental analyses for WSRC. The purpose of the reviews is to investigate technical validation issues discussed in the Data Quality Objectives for Superfund that are not adequately addressed by computer checking of the AN98 EDD, by review of analytical narratives, or by review of COC forms. These technical issues include instrument calibration, analyte identification, and analyte quantitation. The issues are addressed by examination of all initial calibration records for the period reviewed, examination of continuing calibration records for randomly selected dates within the period reviewed, and selected sample records from those dates.

The emphasis of the LDRR is on programmatic laboratory behavior; a judgment is formed on whether the laboratory is or is not in compliance with WSRC requirements. However, if during the review any QA/QC issues identified are judged to be significant enough to affect data usability ( $\mathrm{R}$ - and U-qualifier issues), then the affected data are appropriately qualified. QA/QC issues that do not affect data usability (J-qualifier issues) are noted in the report but do not result in requalification of data.

The LDRR covering the period of sample analysis for this project was conducted at GE on December 8-10, 1999, by Jenny Blankenship, Tom Coffey, and Trina Fortier of ExR. The LDRR report was issued as ESH-EMS-2000016. No technical issues affecting data usability for this project were identified during the LDRR, and all data should be accepted as reported.

\subsubsection{Radiological Data Review}

On September 9, 1999, Thomas Coffey of ExR reviewed radiological data from GE for selected radionuclides from fish samples in order to evaluate data for false positives. No requalification was required based on the review.

\subsubsection{Metals Data Evaluation}

Analytical results for metals analyzed for this project by GE were reviewed to determine sample-specific detection limits and, if necessary, to requalify the analytical results based on these detection limits. The review identified twenty false positives, which were converted to nondetects. 
Metals data are subject to low level false positives due to several factors, which apply in varying degrees to the ICP technology used for metals analyses for this project.

- First, the nominal method detection limit (MDL) obtained from an annual MDL study may not represent the true sensitivity of the instrument at the time of analysis. If the nominal MDL for a metal is $100 \mu \mathrm{g} / \mathrm{kg}$, but the actual instrument sensitivity six months later is $180 \mu \mathrm{g} / \mathrm{kg}$, then noise in the range of $100-180 \mu \mathrm{g} / \mathrm{kg}$ will be reported as detects.

- Second, the presence of dissolved solids or salts in the sample digestate may cause loss of instrument precision due to thermal effects. This precision loss is manifested by fluctuations of noise which can in turn be expressed as false low level analyte signals.

- Third, analyte-specific spectral interferences (AA, ICP/OES) or m/z interferences (ICP/MS) may result in a false positive of one metal in the presence of large concentrations of a second metal.

Algorithms were generated to estimate the magnitude of each of the three disturbances listed above, using laboratory quality control data and analysis of single-element standards as indicators.

- For the first mechanism, the true instrument sensitivity was estimated using interpretation of Initial Calibration Blanks (ICBs) that were analyzed by the lab on every analysis day. A true detection limit was calculated from the ICB data.

- For the second mechanism, an effective detection limit was obtained by interpretation of Interference Check Sample A (ICSA) analyses. These quality control standards contain high levels of dissolved solids and were analyzed by the lab on every analysis day.

- For the third mechanism, the sensitivity of the laboratory's instruments to selected spectral interferences was evaluated by submitting a population of single-element standards to the laboratory and evaluating the analytical results.

For each EPA6010B record, three different sample-specific detection limits were considered:

- the laboratory's reported ssMDL

- a sample-specific detection limit calculated from ICSA data (the ssICSA MDL)

- an sample-specific detection limit calculated from ICB data (the ssICB MDL)

At this point, two or three different sample-specific DLs were available for each record. The highest was chosen as the analytical record's sample-specific detection limit. For reported detects, if the new estimated ssDL exceeded the analytical result, the result was converted to a nondetect with UJ and 4 as the qualifiers, and the ssMDL was replaced with the new ssDL. If the new estimated ssDL also exceeded the reported ssEQL, the old ssEQL was replaced with the new estimated ssDL.

The twenty false positives determined by examination of the ssICSA MDLs and ssICB MDLs are listed in the following table.

Table 3.4 Metals Requalification Summary

\begin{tabular}{|l|l|l|l|l|}
\hline Analyte & $\begin{array}{l}\text { Number of } \\
\text { Sample } \\
\text { Records }\end{array}$ & $\begin{array}{l}\text { Number of } \\
\text { Detects }\end{array}$ & $\begin{array}{l}\text { Number of False } \\
\text { Positives Based on ss } \\
\text { ICB MDL }\end{array}$ & $\begin{array}{l}\text { Number of False Positives } \\
\text { Based on ss ICSA MDL }\end{array}$ \\
\hline Antimony & 26 & 9 & 9 & 0 \\
\hline
\end{tabular}




\begin{tabular}{|l|l|l|l|l|}
\hline Arsenic & 26 & 26 & 0 & 0 \\
\hline Barium & 26 & 26 & 0 & 0 \\
\hline Cadmium & 26 & 17 & 0 & 11 \\
\hline Chromium & 26 & 26 & 0 & 0 \\
\hline Copper & 26 & 26 & 0 & 0 \\
\hline Lead & 26 & 23 & 0 & 0 \\
\hline Nickel & 26 & 4 & 0 & 0 \\
\hline Selenium & 26 & 26 & 0 & 0 \\
\hline Silver & 26 & 10 & 0 & 0 \\
\hline Zinc & 26 & 26 & 0 & 0 \\
\hline Totals & $\mathbf{2 8 6}$ & $\mathbf{2 1 9}$ & $\mathbf{9}$ & $\mathbf{1 1}$ \\
\hline
\end{tabular}

\subsubsection{Qualification of the Data}

Each sample was evaluated on the following issues during validation:

- holding time

- sample preservation

- analyte identification

- analyte quantitation: surrogate recovery and internal standards

- trip blanks

- field blanks

- rinsates

- method blanks

- laboratory control samples

- matrix spikes

- field duplicates

- laboratory duplicates

- internal standards

- additional information

\section{Holding Time}

\section{Criteria}

Each analysis was evaluated according to the holding time limits for preparation (extraction or digestion) and analysis given in the following table.

Table 3.5 Holding Time Criteria

\begin{tabular}{|l|l|l|l|}
\hline Analytes & Reference & Extraction/Digestion & Analysis \\
\hline Cation exchange capacity & EGG OH & NA & NA \\
\hline
\end{tabular}




\begin{tabular}{|c|c|c|c|}
\hline Analytes & Reference & Extraction/Digestion & Analysis \\
\hline Chloride, fluoride & EGG OH & NR & 28 days from collection \\
\hline Cyanide & EGG OH & NA & 14 days from collection \\
\hline Dioxins/Furans & EGG OH & 30 days from collection & 45 days from extraction \\
\hline $\begin{array}{l}\text { Hexavalent chromium } \\
\text { (soil) } \\
\text { Hexavalent chromium } \\
\text { (water) }\end{array}$ & EGG OH & $\begin{array}{l}30 \text { days (soil) } \\
\text { NR (water) }\end{array}$ & $\begin{array}{l}7 \text { days from extraction } \\
\text { (soil) } \\
24 \text { hours from collection } \\
\text { (water) }\end{array}$ \\
\hline Mercury & EGG OH & NA & 28 days from collection \\
\hline Metals & EGG OH & NA & 6 months from collection \\
\hline Nitrate, nitrite & EGG OH & NA & 48 hours from collection \\
\hline Nitrate-nitrite & EGG OH & NR & 28 days from collection \\
\hline Nitrogen (Kjeldahl method) & EGG OH & NA & 28 days from collection \\
\hline Organic halogens, total & EGG OH & NR & 28 days from collection \\
\hline Orthophosphate & EGG OH & NA & 48 hours from collection \\
\hline Pesticide (soil) & EGG OH & 14 days from collection & 40 days from extraction \\
\hline Pesticide (water) & EGG OH & 7 days from collection & 40 days from extraction \\
\hline $\mathrm{pH}$ & EGG OH & $\mathrm{NR}$ & Immediate analysis \\
\hline Phenols & EGG OH & $\mathrm{NR}$ & 28 days from collection \\
\hline Radionuclides & EGG OH & NA & 6 months from collection \\
\hline Semivolatile organics (soil) & EGG OH & 14 days from collection & 40 days from extraction \\
\hline $\begin{array}{l}\text { Semivolatile organics } \\
\text { (water) }\end{array}$ & EGG OH & 7 days from collection & 40 days from extraction \\
\hline $\begin{array}{l}\text { Solids, total dissolved; } \\
\text { Solids, total suspended }\end{array}$ & EGG OH & NR & 7 days from collection \\
\hline Specific conductance & EGG OH & NR & 28 days from collection \\
\hline Sulfate & EGG OH & NA & 28 days from collection \\
\hline Sulfide & EGG OH & NR & 7 days from collection \\
\hline Total organic carbon & EGG OH & NA & 28 days from collection \\
\hline
\end{tabular}

Table 3.5 Holding Time Criteria (cont.)

\begin{tabular}{|c|c|c|c|}
\hline Analytes & Reference & Extraction/Digestion & Analysis \\
\hline Total phosphorus as $\mathrm{P}$ & EGG OH & NA & 28 days from collection \\
\hline Turbidity & EGG OH & NA & 48 hours from collection \\
\hline Volatile organics & EGG OH & NA & 14 days from collection \\
\hline \multicolumn{4}{|l|}{ TCLP Analytes } \\
\hline Mercury & EGG OH & 28 days from collection & 28 days from extraction \\
\hline Metals (except Mercury) & EGG OH & 180 days from collection & 180 days from extraction \\
\hline $\begin{array}{l}\text { Semivolatile organic } \\
\text { compounds }\end{array}$ & EGG OH & $\begin{array}{l}14 \text { days from collection to } \\
\text { TCLP extraction }+7 \text { days } \\
\text { from TCLP extraction to prep } \\
\text { extraction }\end{array}$ & 40 days from preparative extra \\
\hline
\end{tabular}


Table 3.5 Holding Time Criteria (cont.)

\begin{tabular}{|l|l|l|l|}
\hline Analytes & Reference & Extraction/Digestion & Analysis \\
\hline Volatile organic compounds & EGG OH & 14 days from collection & 14 days from extraction \\
\hline
\end{tabular}

\section{Action}

When holding times are exceeded, the following qualifiers are applied:

STORET Code: $Q$

FG Code: $\quad J$-for detects $\quad U J$-for nondetects

If holding time is exceeded by $200 \%$ or more, the following qualifiers are applied

STORET Code: $Q L$

FG Code: $\quad J$-for detects $\quad R$-for nondetects

\section{Findings}

None of 2,260 environmental sample records were qualified.

\section{Discussion}

Most methods specify limits on the time that can pass between sample collection and extraction or analysis. When holding times are exceeded, sample integrity may be compromised due to chemical and/or physical effects that can bias sample results either positively or negatively.

\section{Sample Preservation}

\section{Criteria}

Sample preservation requirements are determined by regulatory guidance, method specifications, and laboratory protocols. General requirements follow.

Soil

Inorganics: Inorganic analyses for metals require that soil samples be kept between $2^{\circ}$ and $6^{\circ} \mathrm{C}$.

Organics: Organic analyses for chlorinated hydrocarbons, haloethers, PCBs, pesticides, phenols, phthalate esters, purgeable aromatic hydrocarbons, purgeable halocarbons, tetrachlorodibenzo-p-dioxin isomers, and total organic carbon also require that soil samples be kept between $2^{\circ}$ and $6^{\circ} \mathrm{C}$. Soil samples to be analyzed for nitroaromatics and isophorone, nitrosamines, or polynuclear aromatic hydrocarbons must be stored in the dark at temperatures between $2^{\circ}$ and $6^{\circ} \mathrm{C}$.

\section{Water}

Inorganics: Water samples collected for metals analyses should have nitric acid added so that $\mathrm{pH}$ is less than 2.

Organics: Water samples for all organic analyses should be kept between $2^{\circ}$ and $6^{\circ} \mathrm{C}$. Purgeable aromatic hydrocarbon analyses require that hydrochloric acid be added so that $\mathrm{pH}$ is less than 2 , and total organic carbon analysis requires that hydrochloric acid or sulfuric acid be added so that $\mathrm{pH}$ is less than 2. Samples 
for nitroaromatics and isophorone must be stored in the dark. Samples for pesticides analyses should have $\mathrm{pH}$ adjusted to between 5 and 9 .

Soil and water samples for organic and inorganic analyses (excluding radiologicals and metals) must be shipped in coolers with temperatures of $4^{\circ} \mathrm{C} \pm 2^{\circ} \mathrm{C}$.

Biological Tissue

Refrigeration can be used for short-term storage of the samples. The samples should remain at $4{ }^{\circ} \mathrm{C} \pm 2{ }^{\circ} \mathrm{C}$. For long-term storage, the sample should be frozen as soon as possible after collection and remain frozen $\left(0^{\circ} \mathrm{C}\right.$ or less $)$ until the time of sample preparation and analysis.

For biological tissue samples, the holding time is dependent upon the preservation method. If the sample has been frozen, the suggested holding times are as follows:

- 180 days for organics, mercury, and cyanide

- 365 days for inorganics and radionuclides

For samples that have been preserved by refrigeration at $4^{\circ} \mathrm{C} \pm 2^{\circ} \mathrm{C}$, the suggested holding times are those listed in the table of holding time criteria above.

Action

When cooler temperature requirements are not met, a $Y$ STORET code is assigned.

If temperature is grossly exceeded ( $>12$ degrees), nondetects are rejected and both nondetects and detects get an L STORET code in addition to the Y.

Under the current contracts, the laboratories do not report $\mathrm{pH}$ of samples requiring preservation. Thus, no assessment of the $\mathrm{pH}$ preservation criterion can be made.

\section{Findings}

None of 2,260 environmental sample records were qualified.

\section{Discussion}

Temperatures greater than the established limit may compromise the integrity and representativeness of the samples due to increased biological activity, hydrolysis of organic compounds, thermal degradation, and the loss of volatile and semivolatile compounds. The analytical results are expected to be biased low, and false negative results are possible. False positive results also are possible due to the effects of biological and chemical degradation and would most likely appear as TICs.

\section{Analyte Quantitation: Surrogate Recovery}

\section{Criteria}

- Pesticides by Method 8080: 60-150\%, page D-56 PEST (EPA, 1991b)

- Semivolatiles by Method 8270: Table 8, page 8270-31 (EPA, 1986)

- Volatiles by Method 8240: Table 8, page 8240-38 (EPA, 1986)

- Volatiles by Method 8260: Table 9, page 8260-44 (EPA, 1986) 
Action

Pesticides: When one or more surrogate recoveries are above or below QC limits, all analytes in the sample are qualified as follows:

EMS Code: $\quad O$

FG Codes: $\quad J$-for detects $\quad U J$-for nondetects when surrogate is low

[null] - for nondetects when surrogate is high

$\mathrm{R}$ - for nondetects when surrogate is very low

and sample dilution is not a factor

Volatiles and/or semivolatiles: The data are qualified as above, and a STORET code is added as follows:

STORET Codes: $\quad K$-if failed surrogates have high recoveries

$L$-if failed surrogates have low recoveries

For volatiles and semivolatiles, if one failed surrogate was high and another low, no STORET code is assigned.

Findings

One hundred fourteen of 2,260 environmental sample records were qualified.

Table 3.6 Surrogate Recoveries Outside EPA Recovery Limits

\begin{tabular}{|l|l|l|l|l|}
\hline Sample ID & Analyte & Minimum (\%) & Maximum (\%) & Recovery (\%) \\
\hline \multicolumn{5}{|l|}{ Solids (method blanks) } \\
\hline LB & Toluene-d8 & 81 & 117 & 80.7 \\
\hline
\end{tabular}

Table 3.6 Surrogate Recoveries Outside EPA Recovery Limits (cont.)

\begin{tabular}{|l|l|l|l|l|}
\hline Sample ID & Analyte & Minimum (\%) & Maximum (\%) & Recovery (\%) \\
\hline \multicolumn{5}{|l|}{ Fauna } \\
\hline 117018 & p-Bromofluorobenzene & 74 & 121 & 129 \\
\hline 117019 & p-Bromofluorobenzene & 74 & 121 & 128 \\
\hline 117021 & p-Bromofluorobenzene & 74 & 121 & 122 \\
\hline 117021 & p-Bromofluorobenzene & 74 & 121 & 135 \\
\hline 117021 & p-Bromofluorobenzene & 74 & 121 & 128 \\
\hline 117022 & p-Bromofluorobenzene & 74 & 121 & 123 \\
\hline 117023 & p-Bromofluorobenzene & 74 & 121 & 128 \\
\hline 117024 & p-Bromofluorobenzene & 74 & 121 & 134 \\
\hline 117025 & p-Bromofluorobenzene & 74 & 121 & 149 \\
\hline 117031 & p-Bromofluorobenzene & 74 & 121 & 182 \\
\hline 117033 & p-Bromofluorobenzene & 74 & 121 & 143 \\
\hline 117034 & p-Bromofluorobenzene & 74 & 121 & 148 \\
\hline 117036 & p-Bromofluorobenzene & 74 & 121 & 126 \\
\hline
\end{tabular}

Data Summary Report for Savannah River Integrator Operable Unit Fish Tissue

(WSRC-TR-99-00386) 


\begin{tabular}{|l|l|l|l|l|}
\hline 117037 & Toluene-d8 & 81 & 117 & 121 \\
\hline 117037 & p-Bromofluorobenzene & 74 & 121 & 176 \\
\hline 117039 & Dibromofluoromethane & 80 & 120 & 79.1 \\
\hline 117039 & p-Bromofluorobenzene & 74 & 121 & 126 \\
\hline 117041 & p-Bromofluorobenzene & 74 & 121 & 154 \\
\hline 117042 & p-Bromofluorobenzene & 74 & 121 & 128 \\
\hline 117043 & p-Bromofluorobenzene & 74 & 121 & 178 \\
\hline 117043 & Toluene-d8 & 81 & 117 & 131 \\
\hline
\end{tabular}

\section{Discussion}

When the surrogate percent recovery is high, the results for associated data may be biased positively. When the surrogate percent recovery is low, the results for associated data may be biased negatively, and the nondetected results should be considered questionable.

\section{Trip Blanks}

\section{Criteria}

Trip blanks were used to determine the existence and magnitude of contamination from shipping activities. Only data for the volatile organic fraction were evaluated based on trip blank results. The CLP $5 \times / 10 \times$ rule was used for data qualification.

\section{Action}

When an analyte is detected in a trip blank, an EMS code is assigned to that analyte in the blank and to all samples that are shipped in the same cooler as the trip blank having positive results.

\section{EMS Code: $\quad 8$}

If the analyte concentration in a sample is less than five times that in the blank (less than 10 times for the common laboratory contaminants acetone, dichloromethane [methylene chloride], and methyl ethyl ketone), an FG code is assigned to that analyte in the sample.

FG Code: $\quad U$

\section{Findings}

None of 2,260 environmental sample records were qualified.

\section{Discussion}

Volatile organic contamination can be introduced into a trip blank in three ways: during blank preparation at the laboratory; during storage of the trip blank in the cooler; or by cross-contamination from other samples. The contamination can cause false positives in the trip blank and in the samples exposed to contamination.

\section{Field Blanks}

\section{Criteria}

Field blanks are used to determine the existence and magnitude of contamination from the atmosphere at the sampling location. No field blanks were used in this project. 


\section{Action}

When an analyte is detected in a field blank, an EMS code is assigned to that analyte in the blank and to all samples from the associated soil boring or well cluster having positive results. If the analyte concentration in a sample is less than five times that in the blank (less than 10 times for the common laboratory contaminants acetone, dichloromethane [methylene chloride], and methyl ethyl ketone), an FG code is assigned to that analyte in the sample.

The following qualifiers may be assigned:

EMS Code: 6

FG Code: $\quad U$

\section{Discussion}

Contamination can be introduced into a field blank while it is exposed to the atmosphere during the sampling process. The field blank is placed close to the sampling location, but far enough away so that the sampling activities do not affect it. The contamination can cause false positives in the field blank and in the samples exposed to atmospheric contamination.

\section{Rinsates}

\section{Criteria}

Rinsates are used to determine the existence and magnitude of contamination from field equipment cleaning activities. No rinsates were used in this project.

\section{Action}

When an analyte is detected in a rinsate, an EMS code is assigned to that analyte in the blank and in the associated sample having positive results. If the analyte concentration in a sample is less than five times that in the blank (less than 10 times for the common laboratory contaminants acetone, dichloromethane [methylene chloride], and methyl ethyl ketone), an FG code is assigned to that analyte in the sample.

The following qualifiers may be assigned:

EMS Code: 7

FG Code: $\quad U$

\section{Method Blanks}

\section{Criteria}

Method blanks are used to determine the existence and magnitude of contamination from laboratory activities. Method blanks with analytical results greater than the method detection limit are considered to be contaminated. The CLP $5 \times / 10 \times$ rule is used for data qualification.

\section{Action}

When an analyte is detected in a method blank, a STORET code is assigned to that analyte in the blank. If the analyte concentration in a sample is less than five times that in the blank (less than 10 times for common laboratory contaminants), an FG code and STORET cdoe are assigned to that analyte in the 
sample. For radionuclide analysis by alpha spectroscopy, an FG code is assigned to that analyte in the blank only if the result is greater than zero.

The following qualifiers may be assigned:

STORET Code: $V$

FG Code: $\quad U$

Findings

Fourteen of 2,260 environmental sample records were qualified.

Table 3.7 Method Blank Contamination by GE

\begin{tabular}{|l|l|}
\hline Analyte & Frequency Detected \\
\hline Metals & $1 / 2$ \\
\hline Nickel, total recoverable & $1 / 2$ \\
\hline Zinc, total recoverable & $1 / 6$ \\
\hline Volatile Organic Compounds & \multicolumn{2}{|l|}{} \\
\hline Dichloromethane (Methylene chloride) &
\end{tabular}

Table 3.8 Method Blank Contamination by EP

\begin{tabular}{|l|l|}
\hline Analyte & Frequency Detected \\
\hline Radionuclides & $1 / 2$ \\
\hline Antimony-125 & \multicolumn{2}{|l|}{} \\
\hline
\end{tabular}

Discussion

Laboratory contamination of samples during preparation and/or analysis is monitored through the method blank. Laboratory procedures may cause false positive results in the method blanks for common laboratory contaminants.

\section{Laboratory Control Samples}

\section{Criteria}

- Dioxins/furans: 40-120\% recovery (EPA, 1986)

- Inorganics: 80-120\% recovery

- Pesticides: Page D-61 PEST (EPA, 1991b)

- Radiologicals: 80-120\% (WHC, not dated)

- Semivolatiles: Table 7, page D-59 SV (EPA, 1991b)

- Volatiles: Table 7, page D-55 VOA (EPA, 1991b)

Action 
If an LCS was not included in the preparation batch, all spike compounds for the associated samples are qualified with an EMS code. When the percent recovery for an analyte in the LCS is outside the QC limits, records for that analyte in the LCS and that analyte in each sample of the preparation batch are qualified with an EMS code.

LCS recovery below lower QC limit:

EMS Code: $\quad C$

STORET Code $\quad L$ or none

FG Codes: $\quad R$ or $J$-for detects $\quad R$ or $U J$-for nondetects

LCS recovery above upper QC limit:

EMS Code: $\quad C$

STORET Code $\quad K$

FG Codes: $\quad R$ or $J$-for detects $\quad R$ or none-for nondetects

\section{Findings}

None of 2,260 environmental sample records were qualified.

\section{Discussion}

LCS percent recoveries that exceed the upper QC limit could indicate a high bias for samples with positive results. Percent recoveries that are less than the lower QC limit could indicate a low bias for samples with positive results, and nondetect results should be considered questionable.

\section{Matrix Spikes}

Criteria

- Dioxins: 40-120\% (EPA, 1986)

- Inorganics: Page 11 (EPA, 1988a)

- Pesticides: Page D-61 PEST (EPA, 1991b)

- Semivolatiles: Table 7, page D-59 SV (EPA, 1991b)

- Volatiles: Table 7, page D-55 VOA (EPA, 1991b)

\section{Action}

1. When an MS does not meet criteria, qualifiers are added only to the failed analyte in the MS, the parent sample, and any duplicates or re-runs of those samples. The data are qualified as follows:

EMS Code: $\quad I$

FG Codes: $\quad J$-for detects $\quad U J$ or $R$-for nondetects when MS is low

$$
\text { [null }] \text { —for chemical nondetects when MS is high }
$$

STORET Code: $\quad K$-if MS recoveries are above QC limits 


\section{$L$-if MS recoveries are below QC limits}

2. MS recovery limits do not apply when the spike concentration is less than $25 \%$ of the parent sample concentration. When this occurs, all samples in the batch are qualified as follows:

EMS Code: 5

and the MS receives the FG and STORET codes appropriate for the recovery.

3. If the laboratory did not perform matrix spikes for nonradiological analyses, the following EMS code is used:

EMS Code: $\quad I$

\section{Findings}

Five of 2,260 environmental sample records were qualified.

Table 3.9 Matrix Spike Recoveries Outside the EPA Recovery Limits by GE

\begin{tabular}{|l|l|l|l|l|}
\hline Sample ID & Analyte & $\begin{array}{l}\text { EPA Minimum } \\
(\%)\end{array}$ & $\begin{array}{l}\text { EPA Maximum } \\
(\%)\end{array}$ & Recovery (\%) \\
\hline Metals & \multicolumn{5}{l|}{} \\
\hline 117022 & Manganese, total recoverable & 75 & 125 & 128 \\
\hline 117022 & Mercury, total recoverable & 75 & 125 & 9.18 \\
\hline 117025 & Mercury, total recoverable & 75 & 125 & 71.8 \\
\hline 117043 & Silver, total recoverable & 75 & 125 & 72.9 \\
\hline Volatile Organic Compounds & 1,1-Dichloroethylene & 172 & 42 \\
\hline 117021 & 59 & \\
\hline
\end{tabular}

Table 3.10 Matrix Spikes Rejected Due to Dilution Effects or Other Reason by GE

\begin{tabular}{|l|l|l|}
\hline Sample ID & Laboratory ID & Analyte \\
\hline Metals & \\
\hline 117022 & $9907977-20$ & Mercury, total recoverable \\
\hline
\end{tabular}

\section{Discussion}

When the matrix spike percent recovery for an analyte is high, the results for associated data may be biased positively. When the matrix spike percent recovery for an analyte is low, the results for associated data may be biased negatively, and the nondetected results should be considered questionable.

\section{Field Duplicates}

No qualification is performed based on field duplicates. 


\section{Laboratory Duplicates and Matrix Spike Duplicates}

\section{Criteria}

- Inorganics: Page 10 (EPA, 1988a)

- Organics: The laboratories qualify on the basis of matrix spikes/duplicates. No additional qualification is done on this basis during validation.

- Radiologicals: (WHC, not dated); only soil samples are evaluated during validation.

The RPD of a sample and its associated laboratory duplicate must be $20 \%$ or less for water samples and $35 \%$ or less for soil samples. These method-required limits do not apply to results reported at levels less than five times the detection limit. When one result is less than $5 \times$ the ssEQL, the difference between the two results must be less than the ssEQL (aqueous samples) or twice the ssEQL (solid samples). For radiological analyses, the second criterion is the absolute difference between the samples.

\section{Action}

For soil samples, when duplicate analysis results for a particular analyte fall outside the QC limits for the RPD, records for that analyte in the parent and duplicate samples are qualified for inorganics, and the MS and MSD are qualified for organics.

For water samples, when the RPD for a particular analyte is outside the appropriate QC limits, FG and EMS codes are assigned to that analyte in the parent and duplicate samples for inorganics or to the MS and MSD for organics, and EMS codes are assigned to that analyte in every sample in the same preparation batch.

$$
\begin{array}{ll}
\text { EMS Code: } & X \\
\text { FG Codes: } & J \text { —for detects }
\end{array}
$$

If no duplicates were analyzed, that analyte in every sample of the preparation batch is assigned the EMS code $X$.

\section{Findings}

Three of 2,260 environmental sample records were qualified.

Table 3.11 Laboratory Duplicate Results Outside RPD Limits for GE

\begin{tabular}{|l|l|l|l|l|l|l|}
\hline $\begin{array}{l}\text { Sample } \\
\text { ID }\end{array}$ & Analyte & $\begin{array}{l}\text { Sample } \\
\text { Conc. }\end{array}$ & $\begin{array}{l}\text { Duplicate } \\
\text { Conc. }\end{array}$ & Unit & RPD (\%) & $\begin{array}{l}\text { Acceptance } \\
\text { Limit (\%) }\end{array}$ \\
\hline 117021 & 1,1-Dichloroethylene & 105 & 223 & $\mathrm{mg} / \mathrm{kg}$ & 71.9512 & 20 \\
\hline
\end{tabular}

\section{Discussion}


Poor precision (i.e., high RPDs) can be caused by poor instrument performance, inconsis-tent application of method procedures, the heterogeneous nature of soil samples, or low quantities of an analyte in the sample.

\subsection{Precision and Accuracy}

This section discusses the analytical data in terms of the precision and accuracy indicators of data quality. Precision is determined from the field and laboratory duplicate analyses and indicates the consistency of field and laboratory techniques. Accuracy is determined from the laboratory control samples, surrogates, matrix spikes, and the results of the method, field, trip, and equipment blanks or rinsates and indicates the ability of the laboratory to generate correct results. 


\subsubsection{Precision}

Precision is a measure of the repeatability of a measurement and is evaluated from the results of field and laboratory duplicate samples. Field duplicates measure the repeatability for the sampling and analytical techniques, and laboratory duplicates measure the ability of the laboratory to reproduce a result. Low precision can be caused by poor instrument performance, poor operator technique, inconsistent application of method protocols, laboratory environment, time between analyses, or by a difficult, heterogeneous sample matrix. Precision is especially important when the action limit approaches the quantitation limit. At least $5 \%$ of the samples are collected in duplicate.

The laboratory performs duplicate analyses on at least $10 \%$ of the samples received.

Precision is expressed in terms of the relative percent difference as follows:

$$
\operatorname{RPD}=\frac{|x-y|}{\left(\frac{x+y}{2}\right)} \times 100
$$

where $x$ is the original sample result and $y$ is the duplicate sample result. When one result of a duplicate pair is below the MDL, the ssEQL is used for that result in the calculation. For radiological analyses, when one result of a duplicate pair is below the MDA, the MDC is used for that result in the calculation. When both results are below the MDL, the RPD cannot be calculated.

The RPD should be less than $20 \%$ for water samples and less than $35 \%$ for soil samples when results are above the ssEQL. In the case where results are between the ssEQL and the MDL, the RPD should be less than $100 \%$ for water samples and less than $200 \%$ for soil samples.

The RPDs for the laboratory and field duplicates are listed in the following tables. The frequency out of range is the number of duplicates that had RPDs greater than the acceptance limit compared to the total number of pairs analyzed; the other columns provide the mean RPD, standard deviation, and the minimum and maximum RPDs. A standard deviation cannot be calculated when only one pair has at least one result above detection limits.

Table 3.12 Relative Percent Differences of Laboratory Duplicates by GE

\begin{tabular}{|l|l|l|l|l|l|}
\hline Analyte & $\begin{array}{l}\text { Frequency } \\
\text { Out of } \\
\text { Range }\end{array}$ & $\begin{array}{l}\text { Mean RPD } \\
(\%)\end{array}$ & Std. Dev. & $\begin{array}{l}\text { Minimum } \\
\text { RPD (\%) }\end{array}$ & $\begin{array}{l}\text { Maximum } \\
\text { RPD (\%) }\end{array}$ \\
\hline Water & \multicolumn{5}{l|}{} \\
\hline Volatile Organic Compounds & $0 / 1$ & 1.49733 & - & 1.497 & 1.497 \\
\hline Benzene & $0 / 1$ & 1.2848 & - & 1.285 & 1.285 \\
\hline Chlorobenzene & $0 / 1$ & 1.56775 & - & 1.568 & 1.568 \\
\hline 1,1-Dichloroethylene &
\end{tabular}


Table 3.12 Relative Percent Differences of Laboratory Duplicates by GE (cont.)

\begin{tabular}{|c|c|c|c|c|c|}
\hline Analyte & $\begin{array}{l}\text { Frequency } \\
\text { Out of } \\
\text { Range }\end{array}$ & $\begin{array}{l}\text { Mean RPD } \\
(\%)\end{array}$ & Std. Dev. & $\begin{array}{l}\text { Minimum } \\
\text { RPD (\%) }\end{array}$ & $\begin{array}{l}\text { Maximum } \\
\text { RPD (\%) }\end{array}$ \\
\hline Toluene & $0 / 1$ & 0.685714 & - & 0.686 & 0.686 \\
\hline Trichloroethylene & $0 / 1$ & 2.17865 & - & 2.179 & 2.179 \\
\hline \multicolumn{6}{|l|}{ Fauna } \\
\hline \multicolumn{6}{|c|}{ Metals (total recoverable) } \\
\hline Antimony & $0 / 2$ & 4.20148 & 5.012 & 0.657 & 7.746 \\
\hline Arsenic & $0 / 2$ & 5.02736 & 3.160 & 2.793 & 7.262 \\
\hline Barium & $0 / 2$ & 7.38176 & 6.106 & 3.064 & 11.699 \\
\hline Cadmium & $0 / 2$ & 4.39492 & 3.377 & 2.007 & 6.783 \\
\hline Chromium & $0 / 2$ & 4.42521 & 3.553 & 1.913 & 6.938 \\
\hline Copper & $0 / 2$ & 4.83398 & 3.968 & 2.028 & 7.640 \\
\hline Lead & $0 / 2$ & 4.32301 & 3.916 & 1.554 & 7.092 \\
\hline Manganese & $0 / 2$ & 13.7278 & 11.087 & 5.888 & 21.567 \\
\hline Mercury & $0 / 3$ & 4.27687 & 5.236 & 0.000 & 10.117 \\
\hline Nickel & $0 / 2$ & 4.39474 & 3.421 & 1.976 & 6.814 \\
\hline Selenium & $0 / 2$ & 4.6728 & 3.245 & 2.378 & 6.967 \\
\hline Silver & $0 / 2$ & 5.59085 & 1.621 & 4.444 & 6.737 \\
\hline Zinc & $0 / 2$ & 8.18684 & 1.545 & 7.095 & 9.279 \\
\hline \multicolumn{6}{|c|}{ Volatile Organic Compounds } \\
\hline Benzene & $0 / 2$ & 8.27667 & 11.069 & 0.449 & 16.104 \\
\hline Chlorobenzene & $0 / 2$ & 10.9628 & 13.012 & 1.762 & 20.163 \\
\hline 1,1-Dichloroethylene & $1 / 2$ & 42.4272 & 41.753 & 12.903 & 71.951 \\
\hline Toluene & $0 / 2$ & 9.50538 & 6.459 & 4.938 & 14.072 \\
\hline Trichloroethylene & $0 / 2$ & 10.2107 & 12.033 & 1.702 & 18.719 \\
\hline
\end{tabular}

Table 3.13 Relative Percent Differences of Laboratory Duplicates by EP

\begin{tabular}{|c|c|c|c|c|c|}
\hline Analyte & $\begin{array}{l}\text { Frequency } \\
\text { Out of } \\
\text { Range }\end{array}$ & $\begin{array}{l}\text { Mean RPD } \\
(\%)\end{array}$ & Std. Dev. & $\begin{array}{l}\text { Minimum } \\
\text { RPD (\%) }\end{array}$ & $\begin{array}{l}\text { Maximum } \\
\text { RPD (\%) }\end{array}$ \\
\hline \multicolumn{6}{|l|}{ Fauna } \\
\hline \multicolumn{6}{|l|}{ Radionuclides } \\
\hline Actinium-228 & $1 / 2$ & 83.9623 & & 83.962 & 83.962 \\
\hline Cesium-137 & $0 / 2$ & 17.3913 & & 17.391 & 17.391 \\
\hline Lead-212 & $1 / 2$ & 56.0334 & 65.863 & 9.461 & 102.606 \\
\hline Nonvolatile beta & $1 / 2$ & 79.2378 & 110.676 & 0.978 & 157.498 \\
\hline Potassium-40 & $0 / 2$ & 13.4358 & 13.968 & 3.559 & 23.313 \\
\hline Strontium-89/90 & $1 / 2$ & 119.886 & & 119.886 & 119.886 \\
\hline
\end{tabular}


Table 3.14 Relative Percent Differences of Field Duplicates by GE

\begin{tabular}{|c|c|c|c|c|c|}
\hline Analyte & $\begin{array}{l}\text { Frequency } \\
\text { Out of } \\
\text { Range }\end{array}$ & $\begin{array}{l}\text { Mean RPD } \\
(\%)\end{array}$ & Std. Dev. & $\begin{array}{l}\text { Minimum } \\
\text { RPD }(\%)\end{array}$ & $\begin{array}{l}\text { Maximum } \\
\text { RPD }(\%)\end{array}$ \\
\hline \multicolumn{6}{|l|}{ Fauna } \\
\hline \multicolumn{6}{|l|}{ Metals (total recoverable) } \\
\hline Arsenic & $0 / 2$ & 25.776 & 5.601 & 21.815 & 29.737 \\
\hline Barium & $2 / 2$ & 54.3272 & 18.925 & 40.945 & 67.709 \\
\hline Calcium & $1 / 2$ & 35.9061 & 48.668 & 1.493 & 70.320 \\
\hline Chromium & $0 / 2$ & 6.57183 & 4.702 & 3.247 & 9.897 \\
\hline Copper & $1 / 2$ & 81.2295 & 72.612 & 29.885 & 132.574 \\
\hline Lead & $1 / 2$ & 82.9219 & 100.738 & 11.690 & 154.154 \\
\hline Magnesium & $1 / 2$ & 53.1418 & 50.862 & 17.177 & 89.107 \\
\hline Manganese & $1 / 2$ & 40.0161 & 38.394 & 12.867 & 67.165 \\
\hline Mercury & $1 / 2$ & 23.829 & 28.225 & 3.871 & 43.787 \\
\hline Selenium & $1 / 2$ & 42.2456 & 48.724 & 7.792 & 76.699 \\
\hline Silver & $2 / 2$ & 147.017 & 7.164 & 141.951 & 152.083 \\
\hline Zinc & $2 / 2$ & 48.1336 & 5.750 & 44.068 & 52.199 \\
\hline \multicolumn{6}{|c|}{ Volatile Organic Compounds } \\
\hline Acetone & $1 / 2$ & 74.3854 & & 74.385 & 74.385 \\
\hline $\begin{array}{l}\text { Dichloromethane (Methylene } \\
\text { chloride) }\end{array}$ & $1 / 2$ & 68.025 & 95.022 & 0.834 & 135.215 \\
\hline Ethylbenzene & $0 / 2$ & 24.8687 & & 24.869 & 24.869 \\
\hline Toluene & $1 / 2$ & 114.163 & & 114.163 & 114.163 \\
\hline Xylenes & $2 / 2$ & 101.446 & 55.125 & 62.467 & 140.426 \\
\hline
\end{tabular}

Table 3.15 Relative Percent Differences of Field Duplicates by EP

\begin{tabular}{|l|l|l|l|l|l|}
\hline Analyte & $\begin{array}{l}\text { Frequency } \\
\text { Out of } \\
\text { Range }\end{array}$ & $\begin{array}{l}\text { Mean RPD } \\
(\%)\end{array}$ & Std. Dev. & $\begin{array}{l}\text { Minimum } \\
\text { RPD (\%) }\end{array}$ & $\begin{array}{l}\text { Maximum } \\
\text { RPD (\%) }\end{array}$ \\
\hline Fauna & \multicolumn{5}{l|}{} \\
\hline Radionuclides & $2 / 2$ & 166.228 & 6.286 & 161.783 & 170.672 \\
\hline Cesium-137 & $1 / 2$ & 90.0485 & 91.428 & 25.399 & 154.698 \\
\hline Nonvolatile beta & $1 / 2$ & 76.5763 & 82.582 & 18.182 & 134.971 \\
\hline Potassium-40 & $1 / 2$ & 49.505 & & 49.505 & 49.505 \\
\hline Strontium-89/90 & $0 / 2$ & 31.1688 & & 31.169 & 31.169 \\
\hline Tritium & & & & \\
\hline
\end{tabular}

\subsubsection{Accuracy}

Accuracy is defined as the closeness of agreement between an observed value and an accepted reference value. Accuracy is especially important when the concentration of concern approaches the quantitation 
limit and/or the action limit. When the concentration is underestimated near the quantitation limit, the analyte may be present but reported as not detected. When the concentration is underestimated near the action limit, the analyte may be at a concentration that would require remediation, but the remediation would not be performed. When the concentration is overestimated near the quantitation limit, the analyte may not be present but reported as detected. When the concentration is overestimated near the action limit, the analyte may not be at a concentration that would require remediation, but the remediation would be performed. The sample types used to evaluate accuracy are performance evaluation studies (see Appendix $B), \mathrm{LCS} / \mathrm{BSs}$, surrogate spikes, and matrix spikes.

\section{Laboratory Control Samples}

LCSs monitor the performance of all steps in the analysis process, including sample preparation, and are used to identify problems with the analytical procedure. LCSs for inorganic analyses are DI water that is spiked with the target analyte, digested, and analyzed with the regular samples. LCSs for organic analyses are DI water that is spiked with selected target analytes, extracted, and analyzed with the regular samples. The LCS spiking solutions or solid LCSs are obtained from EPA or a third-party supplier or are prepared in the laboratory from chemicals from a different source than the calibration standards. The following table lists the QC limits for each analyte.

Table 3.16 EPA Quality Control Limits for Laboratory Control Samples

\begin{tabular}{|c|c|c|}
\hline Analyte & $\begin{array}{l}\text { Limits for } \\
\text { Aqueous Samples (\%) }\end{array}$ & $\begin{array}{l}\text { Limits for } \\
\text { Soil Samples (\%) }\end{array}$ \\
\hline Dioxins/Furans ${ }^{1}$ & $40-120$ & 40-120 \\
\hline Inorganics $^{2}$ & 80-120 & 80-120 \\
\hline \multicolumn{3}{|l|}{ Pesticides $^{3}$} \\
\hline Aldrin & $40-120$ & $34-132$ \\
\hline gamma-Benzene hexachloride (Lindane) & $56-123$ & $46-127$ \\
\hline $4,4^{\prime}$-DDT & $38-127$ & $23-134$ \\
\hline Dieldrin & $52-126$ & $31-134$ \\
\hline Endrin & $56-121$ & $42-139$ \\
\hline Heptachlor & $40-131$ & $35-130$ \\
\hline Radionuclides $^{4}$ & $80-120$ & $80-120$ \\
\hline
\end{tabular}

Table 3.16 EPA Quality Control Limits for Laboratory Control Samples (cont.)

\begin{tabular}{|l|l|l|}
\hline Analyte & $\begin{array}{l}\text { Limits for } \\
\text { Aqueous Samples (\%) }\end{array}$ & $\begin{array}{l}\text { Limits for } \\
\text { Soil Samples (\%) }\end{array}$ \\
\hline Semivolatiles $^{5}$ & \\
\hline
\end{tabular}

1 Page 8280-17 (EPA, 1986)

2 Page E-24 (EPA, 1988a) or as supplied with purchased solid LCS

3 Page D-61 PEST (EPA, 1991b)

4 (WHC, not dated)

5 Table 7, page D-59 SV (EPA, 1991b) 
Table 3.16 EPA Quality Control Limits for Laboratory Control Samples (cont.)

\begin{tabular}{|l|l|l|}
\hline Analyte & $\begin{array}{l}\text { Limits for } \\
\text { Aqueous Samples (\%) }\end{array}$ & $\begin{array}{l}\text { Limits for } \\
\text { Soil Samples (\%) }\end{array}$ \\
\hline Acenaphthene & $46-118$ & $31-137$ \\
\hline 4-Chloro-3-methylphenol & $23-97$ & $26-103$ \\
\hline 2-Chlorophenol & $27-123$ & $25-102$ \\
\hline 1,4-Dichlorobenzene & $36-97$ & $28-104$ \\
\hline 2,4-Dinitrotoluene & $24-96$ & $28-89$ \\
\hline 4-Nitrophenol & $10-80$ & $11-114$ \\
\hline N-Nitrosodi-n-propylamine & $41-116$ & $41-126$ \\
\hline Pentachlorophenol & $9-103$ & $17-109$ \\
\hline Phenol & $12-110$ & $26-90$ \\
\hline Pyrene & $26-127$ & $35-142$ \\
\hline $1,2,4-T r i c h l o r o b e n z e n e$ & $39-98$ & $38-107$ \\
\hline Volatiles ${ }^{6}$ & \multicolumn{2}{|l|}{} \\
\hline Benzene & $76-127$ & $66-142$ \\
\hline Chlorobenzene & $75-130$ & $60-133$ \\
\hline 1,1-Dichloroethylene & $61-145$ & $59-172$ \\
\hline Toluene & $76-125$ & $59-139$ \\
\hline Trichloroethylene & $71-120$ & $62-137$ \\
\hline
\end{tabular}

The following tables list the statistical information for the percent recovery for LCSs by analyte. The frequency out of range is the number of LCSs that had percent recoveries outside the acceptance limits compared to the total number analyzed; the other columns provide the mean percent recovery, standard deviation, and the minimum and maximum percent recoveries.

The percent recovery is calculated as

$$
\% R=\frac{S R}{S A} \times 100
$$

where $\% \mathrm{R}=$ percent recovery

$$
\begin{aligned}
& \mathrm{SR}=\mathrm{LCS} \text { sample result } \\
& \mathrm{SA}=\text { spike-added result }
\end{aligned}
$$

${ }^{6}$ Table 7, page D-55 VOA (EPA, 1991b) 
Table 3.17 Laboratory Control Sample Recoveries by GE

\begin{tabular}{|c|c|c|c|c|c|}
\hline Analyte & $\begin{array}{l}\text { Frequency Out } \\
\text { of Range }\end{array}$ & $\begin{array}{l}\text { Mean } \\
\text { Recovery (\%) }\end{array}$ & Std. Dev. & $\begin{array}{l}\text { Minimum } \\
\text { Recovery } \\
(\%)\end{array}$ & $\begin{array}{l}\text { Maximum } \\
\text { Recovery } \\
(\%)\end{array}$ \\
\hline \multicolumn{6}{|l|}{ Fauna } \\
\hline \multicolumn{6}{|c|}{ Metals (total recoverable) } \\
\hline Antimony & $0 / 2$ & 104 & 2.82843 & 102 & 106 \\
\hline Arsenic & $0 / 2$ & 100.15 & 2.6163 & 98.3 & 102 \\
\hline Barium & $0 / 2$ & 100.7 & 4.6669 & 97.4 & 104 \\
\hline Cadmium & $0 / 2$ & 99.7 & 7.49533 & 94.4 & 105 \\
\hline Chromium & $0 / 2$ & 103.5 & 3.53553 & 101 & 106 \\
\hline Copper & $0 / 2$ & 102.5 & 2.12132 & 101 & 104 \\
\hline Lead & $0 / 2$ & 101.6 & 6.22254 & 97.2 & 106 \\
\hline Manganese & $0 / 2$ & 102.5 & 3.53553 & 100 & 105 \\
\hline Mercury & $0 / 2$ & 106.5 & 2.12132 & 105 & 108 \\
\hline Nickel & $0 / 2$ & 101.55 & 7.70746 & 96.1 & 107 \\
\hline Selenium & $0 / 2$ & 92.85 & 2.47487 & 91.1 & 94.6 \\
\hline Silver & $0 / 2$ & 105.5 & 3.53553 & 103 & 108 \\
\hline Zinc & $0 / 2$ & 98.75 & 4.59619 & 95.5 & 102 \\
\hline \multicolumn{6}{|c|}{ Volatile Organic Compounds } \\
\hline Benzene & $0 / 4$ & 89.875 & 3.06309 & 86.8 & 93.2 \\
\hline Chlorobenzene & $0 / 4$ & 91.125 & 3.35398 & 87.2 & 94.8 \\
\hline 1,1-Dichloroethylene & $0 / 4$ & 87.25 & 4.10325 & 82 & 91.8 \\
\hline Toluene & $0 / 4$ & 86.625 & 3.191 & 82.7 & 89.9 \\
\hline Trichloroethylene & $0 / 4$ & 101.875 & 4.13068 & 96.5 & 106 \\
\hline \multicolumn{6}{|l|}{ Water (trip blanks) } \\
\hline \multicolumn{6}{|c|}{ Volatile Organic Compounds } \\
\hline Benzene & $0 / 2$ & 92.6 & 2.26274 & 91 & 94.2 \\
\hline Chlorobenzene & $0 / 2$ & 92.25 & 3.3234 & 89.9 & 94.6 \\
\hline 1,1-Dichloroethylene & $0 / 2$ & 90.2 & 2.54558 & 88.4 & 92 \\
\hline Toluene & $0 / 2$ & 90.4 & 2.12132 & 88.9 & 91.9 \\
\hline Trichloroethylene & $0 / 2$ & 92.9 & 2.68701 & 91 & 94.8 \\
\hline
\end{tabular}

Table 3.18 Laboratory Control Sample Recoveries by EP

\begin{tabular}{|l|l|l|l|l|l|}
\hline Analyte & $\begin{array}{l}\text { Frequency } \\
\text { Out of } \\
\text { Range }\end{array}$ & $\begin{array}{l}\text { Mean } \\
\text { Recovery } \\
(\boldsymbol{\%})\end{array}$ & Std. Dev. & $\begin{array}{l}\text { Minimum } \\
\text { Recovery } \\
(\boldsymbol{\%})\end{array}$ & $\begin{array}{l}\text { Maximum } \\
\text { Recovery }(\boldsymbol{\%})\end{array}$ \\
\hline Cesium-137 & $0 / 2$ & 102.5 & 0.707107 & 102 & 103 \\
\hline Gross alpha & $0 / 2$ & 108.5 & 4.94975 & 105 & 112 \\
\hline Nonvolatile beta & $0 / 2$ & 117 & 1.41421 & 116 & 118 \\
\hline
\end{tabular}


Table 3.18 Laboratory Control Sample Recoveries by EP (cont.)

\begin{tabular}{|l|l|l|l|l|l|}
\hline Analyte & $\begin{array}{l}\text { Frequency } \\
\text { Out of } \\
\text { Range }\end{array}$ & $\begin{array}{l}\text { Mean } \\
\text { Recovery } \\
(\boldsymbol{\%})\end{array}$ & Std. Dev. & $\begin{array}{l}\text { Minimum } \\
\text { Recovery } \\
(\boldsymbol{\%})\end{array}$ & $\begin{array}{l}\text { Maximum } \\
\text { Recovery (\%) }\end{array}$ \\
\hline Plutonium-239/240 & $0 / 2$ & 100.3 & 8.06102 & 94.6 & 106 \\
\hline Strontium-89/90 & $0 / 2$ & 103.5 & 3.53553 & 101 & 106 \\
\hline Tritium & $0 / 2$ & 96.65 & 7.56604 & 91.3 & 102 \\
\hline Uranium-238 & $0 / 2$ & 88.6 & 1.41421 & 87.6 & 89.6 \\
\hline
\end{tabular}

\section{Surrogates}

Surrogates are analytes not normally found in environmental samples and are used to spike all samples, QC samples, and standards for organic analyses. Surrogates are added prior to analysis for VOAs and prior to extraction for semivolatiles, pesticides, and herbicides. Low surrogate recovery is a measure of the effect of the sample matrix, high analyte concentration, or poor instrument sensitivity on the sample analysis procedure. High surrogate recovery usually indicates instrument or sample preparation errors. The following table lists the recovery limits for each surrogate.

Table 3.19 EPA Quality Control Limits for Surrogates

\begin{tabular}{|c|c|c|}
\hline Analyte & $\begin{array}{l}\text { Limits for } \\
\text { Aqueous Samples (\%) }\end{array}$ & $\begin{array}{l}\text { Limits for } \\
\text { Soil Samples (\%) }\end{array}$ \\
\hline \multicolumn{3}{|l|}{ Pesticides $^{7}$} \\
\hline Decachlorobiphenyl & $30-150$ & $30-150$ \\
\hline Dibutylchlorendate & $30-150$ & $30-150$ \\
\hline Tetrachloro-m-xylene & $30-150$ & $30-150$ \\
\hline \multicolumn{3}{|l|}{ Semivolatiles $^{8}$} \\
\hline 2-Chlorophenol-d4 (advisory) & $33-110$ & $20-130$ \\
\hline 1,2-Dichlorobenzene-d4 (advisory) & $16-110$ & $20-130$ \\
\hline 2-Fluorobiphenyl & $43-116$ & $30-115$ \\
\hline 2-Fluorophenol & $21-100$ & $25-121$ \\
\hline Nitrobenzene-d5 & $35-114$ & $23-120$ \\
\hline Phenol-d5 & $10-94$ & $24-113$ \\
\hline Phenol-d6 & $10-94$ & $24-113$ \\
\hline p-Terphenyl-d14 & $33-141$ & $18-137$ \\
\hline 2,4,6-Tribromophenol & $10-123$ & $19-122$ \\
\hline
\end{tabular}

7 Page D-56 PEST (EPA, 1991a)

8 Table 8, page 8270C-31 (EPA, 1986)

Data Summary Report for Savannah River Integrator Operable Unit Fish Tissue

(WSRC-TR-99-00386) 
Table 3.19 EPA Quality Control Limits for Surrogates (cont.)

\begin{tabular}{|c|c|c|}
\hline Analyte & $\begin{array}{l}\text { Limits for } \\
\text { Aqueous Samples (\%) }\end{array}$ & $\begin{array}{l}\text { Limits for } \\
\text { Soil Samples (\%) }\end{array}$ \\
\hline \multicolumn{3}{|l|}{ Volatiles $(8260)^{9}$} \\
\hline 4-Bromofluorobenzene & $86-115$ & $74-121$ \\
\hline Dibromofluoromethane-d4 & $86-118$ & $80-120$ \\
\hline 1,2-Dichloroethane-d4 & $76-114$ & $70-121$ \\
\hline Toluene-d8 & $88-110$ & $81-117$ \\
\hline
\end{tabular}

The following tables list the statistical information for the percent recovery for the surro-gates by analyte and laboratory. The frequency out-of-range is the number of surrogates that had percent recoveries outside the acceptance limits compared to the total number analyzed; the other columns provide the mean percent recovery, standard deviation, and the minimum and maximum percent recoveries.

Table 3.20 Surrogate Recoveries by GE

\begin{tabular}{|c|c|c|c|c|c|}
\hline Analyte & $\begin{array}{l}\text { Frequency } \\
\text { Out of } \\
\text { Range }\end{array}$ & $\begin{array}{l}\text { Mean } \\
\text { Recovery } \\
(\%)\end{array}$ & Std. Dev. & $\begin{array}{l}\text { Minimum } \\
\text { Recovery }(\%)\end{array}$ & $\begin{array}{l}\text { Maximum } \\
\text { Recovery } \\
(\%)\end{array}$ \\
\hline \multicolumn{6}{|l|}{ Solids (laboratory blanks) } \\
\hline \multicolumn{6}{|c|}{ Volatile Organic Compounds } \\
\hline p-Bromofluorobenzene & $0 / 8$ & 94.1875 & 7.04464 & 85.7 & 107 \\
\hline Toluene-d8 & $1 / 8$ & 87.2625 & 4.91991 & 80.7 & 95.6 \\
\hline Dibromofluoromethane & $0 / 8$ & 100.213 & 6.70446 & 93 & 114 \\
\hline \multicolumn{6}{|l|}{ Water (trip blanks) } \\
\hline \multicolumn{6}{|c|}{ Volatile Organic Compounds } \\
\hline p-Bromofluorobenzene & $0 / 9$ & 92.6778 & 2.58736 & 88.9 & 96.3 \\
\hline Toluene-d8 & $0 / 9$ & 102.667 & 2 & 100 & 105 \\
\hline Dibromofluoromethane & $0 / 9$ & 100.967 & 1.47309 & 98.6 & 103 \\
\hline \multicolumn{6}{|l|}{ Fauna } \\
\hline \multicolumn{6}{|c|}{ Volatile Organic Compounds } \\
\hline p-Bromofluorobenzene & $18 / 30$ & 126.26 & 24.6799 & 85.3 & 182 \\
\hline Toluene-d8 & $2 / 30$ & 96.9133 & 10.9622 & 82.3 & 131 \\
\hline Dibromofluoromethane & $1 / 30$ & 93.7867 & 6.92993 & 79.1 & 113 \\
\hline
\end{tabular}

\section{Matrix Spikes}

Matrix spikes are used to evaluate the effect of the sample matrix on the analytical procedure. Matrix spikes for at least $5 \%$ of the samples are prepared by adding a known quantity of the target analyte to the samples prior to sample preparation. All target analytes are spiked for the inorganic analyses. Selected

9 Table 8, page 8260B-45 (EPA, 1986) 
target analytes are used in the spiking solution for the organic analyses. Results from the matrix spikes are used to evaluate the extent of matrix interference.

The QC limits for matrix spikes are the same as those for LCSs except for inorganics, which have limits of 75-125\%. Matrix spikes are rejected if the concentration of the analyte in the sample is more than four times the amount of the spike.

The following tables list the percent recovery for the matrix spikes. The frequency out of range is the number of matrix spikes that had percent recoveries outside the acceptance limits compared to the total number analyzed; the other columns provide the mean percent recovery, standard deviation, and the minimum and maximum percent recoveries.

Table 3.21 Matrix Spike Recoveries by GE

\begin{tabular}{|c|c|c|c|c|c|}
\hline Analyte & $\begin{array}{l}\text { Frequency } \\
\text { Out of } \\
\text { Range }\end{array}$ & $\begin{array}{l}\text { Mean } \\
\text { Recovery } \\
(\%)\end{array}$ & Std. Dev. & $\begin{array}{l}\text { Minimum } \\
\text { Recovery } \\
(\%)\end{array}$ & $\begin{array}{l}\text { Maximum } \\
\text { Recovery } \\
(\%)\end{array}$ \\
\hline \multicolumn{6}{|l|}{ Water (trip blanks) } \\
\hline \multicolumn{6}{|c|}{ Volatile Organic Compounds } \\
\hline Benzene & $0 / 2$ & 93.55 & 0.919239 & 92.9 & 94.2 \\
\hline Chlorobenzene & $0 / 2$ & 93.35 & 0.919239 & 92.7 & 94 \\
\hline 1,1-Dichloroethylene & $0 / 2$ & 89.3 & 1.13137 & 88.5 & 90.1 \\
\hline Toluene & $0 / 2$ & 87.5 & 0.282843 & 87.3 & 87.7 \\
\hline Trichloroethylene & $0 / 2$ & 91.85 & 1.3435 & 90.9 & 92.8 \\
\hline \multicolumn{6}{|l|}{ Fauna } \\
\hline \multicolumn{6}{|c|}{ Metals (total recoverable) } \\
\hline Antimony & $0 / 4$ & 99.7 & 5.55998 & 94.7 & 105 \\
\hline Arsenic & $0 / 4$ & 100.7 & 5.07412 & 95.6 & 106 \\
\hline Barium & $0 / 4$ & 100.85 & 5.12803 & 95.7 & 107 \\
\hline Cadmium & $0 / 4$ & 97.6 & 4.53358 & 93.3 & 102 \\
\hline Chromium & $0 / 4$ & 101.2 & 4.47512 & 97 & 106 \\
\hline Copper & $0 / 4$ & 103 & 2.94392 & 100 & 106 \\
\hline Lead & $0 / 4$ & 97.625 & 5.64764 & 92.5 & 103 \\
\hline Manganese & $1 / 4$ & 102.45 & 18.8647 & 85.8 & 128 \\
\hline Mercury & $2 / 6$ & 76.38 & 34.3831 & 9.18 & 101 \\
\hline Nickel & $0 / 4$ & 99.475 & 5.30118 & 94.5 & 105 \\
\hline Selenium & $0 / 4$ & 96.95 & 2.96929 & 93.8 & 100 \\
\hline Silver & $1 / 4$ & 91.475 & 19.1721 & 72.9 & 109 \\
\hline Zinc & $0 / 4$ & 100.35 & 10.1425 & 88.3 & 111 \\
\hline
\end{tabular}


Table 3.21 Matrix Spike Recoveries by GE (cont.)

\begin{tabular}{|l|l|l|l|l|l|}
\hline & $\begin{array}{l}\text { Frequency } \\
\text { Out of } \\
\text { Range }\end{array}$ & $\begin{array}{l}\text { Mean } \\
\text { Recovery } \\
(\%)\end{array}$ & Std. Dev. & $\begin{array}{l}\text { Minimum } \\
\text { Recovery } \\
(\%)\end{array}$ & $\begin{array}{l}\text { Maximum } \\
\text { Recovery } \\
(\%)\end{array}$ \\
\hline Volatile Organic Compounds & \multicolumn{5}{|l|}{} \\
\hline Benzene & $0 / 4$ & 82.525 & 8.24919 & 70.6 & 88.3 \\
\hline Chlorobenzene & $0 / 4$ & 82.075 & 11.7102 & 66 & 91.4 \\
\hline 1,1-Dichloroethylene & $1 / 4$ & 82.5 & 27.9537 & 42 & 106 \\
\hline Toluene & $0 / 4$ & 94.075 & 5.92192 & 86.1 & 99.1 \\
\hline Trichloroethylene & $0 / 4$ & 87.6 & 9.66678 & 73.6 & 94.7 \\
\hline
\end{tabular}

Table 3.22 Matrix Spike Recoveries by EP

\begin{tabular}{|l|l|l|l|l|l|}
\hline & $\begin{array}{l}\text { Frequency } \\
\text { Out of } \\
\text { Range }\end{array}$ & $\begin{array}{l}\text { Mean } \\
\text { Recovery } \\
(\%)\end{array}$ & Std. Dev. & $\begin{array}{l}\text { Minimum } \\
\text { Recovery } \\
(\%)\end{array}$ & $\begin{array}{l}\text { Maximum } \\
\text { Recovery } \\
(\%)\end{array}$ \\
\hline \multicolumn{5}{|l|}{ Fauna } & \multicolumn{3}{l|}{} \\
\hline Radionuclides & $0 / 2$ & 100.25 & 19.4454 & 86.5 & 114 \\
\hline Gross alpha & $0 / 2$ & 105 & 1.41421 & 104 & 106 \\
\hline Nonvolatile beta & $0 / 2$ & 98.1 & 0.141421 & 98 & 98.2 \\
\hline Plutonium-239/240 & $0 / 2$ & 92.7 & 13.1522 & 83.4 & 102 \\
\hline Strontium-89/90 & $0 / 2$ & 97.3 & 8.06102 & 91.6 & 103 \\
\hline Tritium & $0 / 2$ & 93.2 & 4.38406 & 90.1 & 96.3 \\
\hline Uranium-238 & & & & & \\
\hline
\end{tabular}

\subsubsection{Contamination in Quality Control Samples}

\section{Method Blanks}

Method blanks are used to determine the existence and magnitude of contamination resulting from the analytical process. Method blanks are analyte-free matrices (DI water for aqueous samples and sodium sulfate for solid samples) that are prepared in the same manner and at the same time as the samples. When method blanks have detectable concentrations of analytes, the laboratory must determine the cause and take corrective action to eliminate the contamination.

The following tables list the statistical information for the method blanks. The frequency detected column gives the number of method blanks analyzed for each analyte that had detectable concentrations compared to the total number analyzed. The other columns list the mean result, standard deviation, minimum and maximum results, and reporting unit. When the analyte is not detected, the mean and minimum and maximum results are the ssEQLs for the method blanks. 
Table 3.23 Analytes Detected in Method Blanks by GE

\begin{tabular}{|c|c|c|c|c|c|}
\hline Analyte & $\begin{array}{l}\text { Frequency } \\
\text { Detected }\end{array}$ & $\begin{array}{l}\text { Mean } \\
\text { Result }\end{array}$ & Std. Dev. & $\begin{array}{l}\text { Min./Max. } \\
\text { Result }\end{array}$ & Unit \\
\hline \multicolumn{6}{|l|}{ Solids } \\
\hline \multicolumn{6}{|l|}{ Metals (EPA6010B, except as noted) } \\
\hline Antimony & $0 / 2$ & 500 & 0 & $500 / 500$ & $\mu \mathrm{g} / \mathrm{kg}$ \\
\hline Arsenic & $0 / 2$ & 250 & 0 & $250 / 250$ & $\mu \mathrm{g} / \mathrm{kg}$ \\
\hline Barium & $0 / 2$ & 250 & 0 & $250 / 250$ & $\mu \mathrm{g} / \mathrm{kg}$ \\
\hline Cadmium & $0 / 2$ & 250 & 0 & $250 / 250$ & $\mu \mathrm{g} / \mathrm{kg}$ \\
\hline Chromium & $0 / 2$ & 250 & 0 & $250 / 250$ & $\mu \mathrm{g} / \mathrm{kg}$ \\
\hline Copper & $0 / 2$ & 250 & 0 & $250 / 250$ & $\mu \mathrm{g} / \mathrm{kg}$ \\
\hline Lead & $0 / 2$ & 250 & 0 & $250 / 250$ & $\mu \mathrm{g} / \mathrm{kg}$ \\
\hline Manganese & $0 / 2$ & 500 & 0 & $500 / 500$ & $\mu \mathrm{g} / \mathrm{kg}$ \\
\hline Mercury (EPA7471A) & $0 / 3$ & 33.3 & 0 & $33.3 / 33.3$ & $\mu \mathrm{g} / \mathrm{kg}$ \\
\hline Nickel & $1 / 2$ & 212.5 & 53.033 & $175 / 250$ & $\mu \mathrm{g} / \mathrm{kg}$ \\
\hline Selenium & $0 / 2$ & 250 & 0 & $250 / 250$ & $\mu \mathrm{g} / \mathrm{kg}$ \\
\hline Silver & $0 / 2$ & 250 & 0 & $250 / 250$ & $\mu \mathrm{g} / \mathrm{kg}$ \\
\hline Zinc & $1 / 2$ & 230 & 28.2843 & $210 / 250$ & $\mu \mathrm{g} / \mathrm{kg}$ \\
\hline \multicolumn{6}{|l|}{ Volatile Organic Compounds } \\
\hline Acetone & $0 / 4$ & 25 & 0 & $25 / 25$ & $\mu \mathrm{g} / \mathrm{kg}$ \\
\hline Benzene & $0 / 4$ & 1 & 0 & $1 / 1$ & $\mu \mathrm{g} / \mathrm{kg}$ \\
\hline Bromodichloromethane & $0 / 4$ & 1 & 0 & $1 / 1$ & $\mu \mathrm{g} / \mathrm{kg}$ \\
\hline Bromoform & $0 / 4$ & 1 & 0 & $1 / 1$ & $\mu \mathrm{g} / \mathrm{kg}$ \\
\hline Bromomethane (Methyl bromide) & $0 / 4$ & 1 & 0 & $1 / 1$ & $\mu \mathrm{g} / \mathrm{kg}$ \\
\hline Carbon disulfide & $0 / 4$ & 5 & 0 & $5 / 5$ & $\mu \mathrm{g} / \mathrm{kg}$ \\
\hline Carbon tetrachloride & $0 / 4$ & 1 & 0 & $1 / 1$ & $\mu \mathrm{g} / \mathrm{kg}$ \\
\hline Chlorobenzene & $0 / 4$ & 1 & 0 & $1 / 1$ & $\mu \mathrm{g} / \mathrm{kg}$ \\
\hline Chloroethane & $0 / 4$ & 1 & 0 & $1 / 1$ & $\mu \mathrm{g} / \mathrm{kg}$ \\
\hline Chloroethene (Vinyl chloride) & $0 / 4$ & 1 & 0 & $1 / 1$ & $\mu \mathrm{g} / \mathrm{kg}$ \\
\hline Chloroform & $0 / 4$ & 1 & 0 & $1 / 1$ & $\mu \mathrm{g} / \mathrm{kg}$ \\
\hline Chloromethane (Methyl chloride) & $0 / 4$ & 1 & 0 & $1 / 1$ & $\mu \mathrm{g} / \mathrm{kg}$ \\
\hline Dibromochloromethane & $0 / 4$ & 1 & 0 & $1 / 1$ & $\mu \mathrm{g} / \mathrm{kg}$ \\
\hline 1,1-Dichloroethane & $0 / 4$ & 1 & 0 & $1 / 1$ & $\mu \mathrm{g} / \mathrm{kg}$ \\
\hline 1,2-Dichloroethane & $0 / 4$ & 1 & 0 & $1 / 1$ & $\mu \mathrm{g} / \mathrm{kg}$ \\
\hline 1,1-Dichloroethylene & $0 / 4$ & 1 & 0 & $1 / 1$ & $\mu \mathrm{g} / \mathrm{kg}$ \\
\hline 1,2-Dichloroethylene & $0 / 4$ & 10 & 0 & $10 / 10$ & $\mu \mathrm{g} / \mathrm{kg}$ \\
\hline $\begin{array}{l}\text { Dichloromethane (Methylene } \\
\text { chloride) }\end{array}$ & $0 / 4$ & 5 & 0 & $5 / 5$ & $\mu \mathrm{g} / \mathrm{kg}$ \\
\hline 1,2-Dichloropropane & $0 / 4$ & 1 & 0 & $1 / 1$ & $\mu \mathrm{g} / \mathrm{kg}$ \\
\hline cis-1,3-Dichloropropene & $0 / 4$ & 1 & 0 & $1 / 1$ & $\mu \mathrm{g} / \mathrm{kg}$ \\
\hline
\end{tabular}


Table 3.23 Analytes Detected in Method Blanks by GE (cont.)

\begin{tabular}{|c|c|c|c|c|c|}
\hline Analyte & $\begin{array}{l}\text { Frequency } \\
\text { Detected }\end{array}$ & $\begin{array}{l}\text { Mean } \\
\text { Result }\end{array}$ & Std. Dev. & $\begin{array}{l}\text { Min./Max. } \\
\text { Result }\end{array}$ & Unit \\
\hline trans-1,3-Dichloropropene & $0 / 4$ & 1 & 0 & $1 / 1$ & $\mu \mathrm{g} / \mathrm{kg}$ \\
\hline Ethylbenzene & $0 / 4$ & 1 & 0 & $1 / 1$ & $\mu \mathrm{g} / \mathrm{kg}$ \\
\hline 2-Hexanone & $0 / 4$ & 5 & 0 & $5 / 5$ & $\mu \mathrm{g} / \mathrm{kg}$ \\
\hline Methyl ethyl ketone & $0 / 4$ & 5 & 0 & $5 / 5$ & $\mu \mathrm{g} / \mathrm{kg}$ \\
\hline Methyl isobutyl ketone & $0 / 4$ & 5 & 0 & $5 / 5$ & $\mu \mathrm{g} / \mathrm{kg}$ \\
\hline Styrene & $0 / 4$ & 1 & 0 & $1 / 1$ & $\mu \mathrm{g} / \mathrm{kg}$ \\
\hline 1,1,2,2-Tetrachloroethane & $0 / 4$ & 1 & 0 & $1 / 1$ & $\mu \mathrm{g} / \mathrm{kg}$ \\
\hline Tetrachloroethylene & $0 / 4$ & 1 & 0 & $1 / 1$ & $\mu \mathrm{g} / \mathrm{kg}$ \\
\hline Toluene & $0 / 4$ & 1 & 0 & $1 / 1$ & $\mu \mathrm{g} / \mathrm{kg}$ \\
\hline 1,1,1-Trichloroethane & $0 / 4$ & 1 & 0 & $1 / 1$ & $\mu \mathrm{g} / \mathrm{kg}$ \\
\hline 1,1,2-Trichloroethane & $0 / 4$ & 1 & 0 & $1 / 1$ & $\mu \mathrm{g} / \mathrm{kg}$ \\
\hline Trichloroethylene & $0 / 4$ & 1 & 0 & $1 / 1$ & $\mu \mathrm{g} / \mathrm{kg}$ \\
\hline Vinyl acetate & $0 / 4$ & 15 & 0 & $15 / 15$ & $\mu \mathrm{g} / \mathrm{kg}$ \\
\hline Xylenes & $0 / 4$ & 5 & 0 & $5 / 5$ & $\mu \mathrm{g} / \mathrm{kg}$ \\
\hline \multicolumn{6}{|l|}{ Water } \\
\hline \multicolumn{6}{|l|}{ Volatile Organic Compounds } \\
\hline Acetone & $0 / 2$ & 5 & 0 & $5 / 5$ & $\mu \mathrm{g} / \mathrm{L}$ \\
\hline Benzene & $0 / 2$ & 1 & 0 & $1 / 1$ & $\mu \mathrm{g} / \mathrm{L}$ \\
\hline Bromodichloromethane & $0 / 2$ & 1 & 0 & $1 / 1$ & $\mu \mathrm{g} / \mathrm{L}$ \\
\hline Bromoform & $0 / 2$ & 1 & 0 & $1 / 1$ & $\mu \mathrm{g} / \mathrm{L}$ \\
\hline Bromomethane (Methyl bromide) & $0 / 2$ & 1 & 0 & $1 / 1$ & $\mu \mathrm{g} / \mathrm{L}$ \\
\hline Carbon disulfide & $0 / 2$ & 5 & 0 & $5 / 5$ & $\mu \mathrm{g} / \mathrm{L}$ \\
\hline Carbon tetrachloride & $0 / 2$ & 1 & 0 & $1 / 1$ & $\mu \mathrm{g} / \mathrm{L}$ \\
\hline Chlorobenzene & $0 / 2$ & 1 & 0 & $1 / 1$ & $\mu \mathrm{g} / \mathrm{L}$ \\
\hline Chloroethane & $0 / 2$ & 1 & 0 & $1 / 1$ & $\mu \mathrm{g} / \mathrm{L}$ \\
\hline Chloroethene (Vinyl chloride) & $0 / 2$ & 1 & 0 & $1 / 1$ & $\mu \mathrm{g} / \mathrm{L}$ \\
\hline Chloroform & $0 / 2$ & 1 & 0 & $1 / 1$ & $\mu \mathrm{g} / \mathrm{L}$ \\
\hline Chloromethane (Methyl chloride) & $0 / 2$ & 1 & 0 & $1 / 1$ & $\mu \mathrm{g} / \mathrm{L}$ \\
\hline Dibromochloromethane & $0 / 2$ & 1 & 0 & $1 / 1$ & $\mu \mathrm{g} / \mathrm{L}$ \\
\hline 1,1-Dichloroethane & $0 / 2$ & 1 & 0 & $1 / 1$ & $\mu \mathrm{g} / \mathrm{L}$ \\
\hline 1,2-Dichloroethane & $0 / 2$ & 1 & 0 & $1 / 1$ & $\mu \mathrm{g} / \mathrm{L}$ \\
\hline cis-1,2-Dichloroethylene & $0 / 2$ & 1 & 0 & $1 / 1$ & $\mu \mathrm{g} / \mathrm{L}$ \\
\hline trans-1,2-Dichloroethylene & $0 / 2$ & 1 & 0 & $1 / 1$ & $\mu \mathrm{g} / \mathrm{L}$ \\
\hline 1,1-Dichloroethylene & $0 / 2$ & 1 & 0 & $1 / 1$ & $\mu \mathrm{g} / \mathrm{L}$ \\
\hline $\begin{array}{l}\text { Dichloromethane (Methylene } \\
\text { chloride) }\end{array}$ & $1 / 2$ & 3.905 & 1.54856 & $2.81 / 5$ & $\mu \mathrm{g} / \mathrm{L}$ \\
\hline 1,2-Dichloropropane & $0 / 2$ & 1 & 0 & $1 / 1$ & $\mu \mathrm{g} / \mathrm{L}$ \\
\hline cis-1,3-Dichloropropene & $0 / 2$ & 1 & 0 & $1 / 1$ & $\mu \mathrm{g} / \mathrm{L}$ \\
\hline trans-1,3-Dichloropropene & $0 / 2$ & 1 & 0 & $1 / 1$ & $\mu \mathrm{g} / \mathrm{L}$ \\
\hline Ethylbenzene & $0 / 2$ & 1 & 0 & $1 / 1$ & $\mu \mathrm{g} / \mathrm{L}$ \\
\hline
\end{tabular}


Table 3.23 Analytes Detected in Method Blanks by GE (cont.)

\begin{tabular}{|l|l|l|l|l|l|}
\hline Analyte & $\begin{array}{l}\text { Frequency } \\
\text { Detected }\end{array}$ & $\begin{array}{l}\text { Mean } \\
\text { Result }\end{array}$ & Std. Dev. & $\begin{array}{l}\text { Min./Max. } \\
\text { Result }\end{array}$ & Unit \\
\hline 2-Hexanone & $0 / 2$ & 5 & 0 & $5 / 5$ & $\mu \mathrm{g} / \mathrm{L}$ \\
\hline Methyl ethyl ketone & $0 / 2$ & 10 & 0 & $10 / 10$ & $\mu \mathrm{g} / \mathrm{L}$ \\
\hline Methyl isobutyl ketone & $0 / 2$ & 5 & 0 & $5 / 5$ & $\mu \mathrm{g} / \mathrm{L}$ \\
\hline Styrene & $0 / 2$ & 1 & 0 & $1 / 1$ & $\mu \mathrm{g} / \mathrm{L}$ \\
\hline $1,1,2,2-$ Tetrachloroethane & $0 / 2$ & 1 & 0 & $1 / 1$ & $\mu \mathrm{g} / \mathrm{L}$ \\
\hline Tetrachloroethylene & $0 / 2$ & 1 & 0 & $1 / 1$ & $\mu \mathrm{g} / \mathrm{L}$ \\
\hline Toluene & $0 / 2$ & 1 & 0 & $1 / 1$ & $\mu \mathrm{g} / \mathrm{L}$ \\
\hline $1,1,1-$ Trichloroethane & $0 / 2$ & 1 & 0 & $1 / 1$ & $\mu \mathrm{g} / \mathrm{L}$ \\
\hline $1,1,2$-Trichloroethane & $0 / 2$ & 1 & 0 & $1 / 1$ & $\mu \mathrm{g} / \mathrm{L}$ \\
\hline Trichloroethylene & $0 / 2$ & 1 & 0 & $1 / 1$ & $\mu \mathrm{g} / \mathrm{L}$ \\
\hline Xylenes & $0 / 2$ & 2 & 0 & $2 / 2$ & $\mu \mathrm{g} / \mathrm{L}$ \\
\hline
\end{tabular}

Table 3.24 Analytes Detected in Method Blanks by EP

\begin{tabular}{|c|c|c|c|c|c|}
\hline Analyte & $\begin{array}{l}\text { Frequency } \\
\text { Detected }\end{array}$ & $\begin{array}{l}\text { Mean } \\
\text { Result }\end{array}$ & Std. Dev. & Min./Max. Result & Unit \\
\hline \multicolumn{6}{|l|}{ Solids } \\
\hline \multicolumn{6}{|l|}{ Radionuclides } \\
\hline \multicolumn{6}{|l|}{ EPA905 } \\
\hline Strontium-89/90 & $0 / 2$ & -0.23935 & 0.220122 & $-0.395 /-0.0837$ & $\mathrm{pCi} / \mathrm{g}$ \\
\hline \multicolumn{6}{|l|}{ EPIA-001B } \\
\hline Gross alpha & $0 / 2$ & -0.0685 & 0.184555 & $-0.199 / 0.062$ & $\mathrm{pCi} / \mathrm{g}$ \\
\hline Nonvolatile beta & $0 / 2$ & 0.339 & 0.14425 & $0.237 / 0.441$ & $\mathrm{pCi} / \mathrm{g}$ \\
\hline \multicolumn{6}{|l|}{ EPIA-002B } \\
\hline Tritium & $0 / 2$ & -0.073 & 0.103238 & $-0.146 / 0$ & $\mathrm{pCi} / \mathrm{g}$ \\
\hline \multicolumn{6}{|l|}{ EPIA-011B } \\
\hline Uranium-233/234 & $0 / 2$ & 0.00297 & 0.0018809 & $0.00164 / 0.0043$ & $\mathrm{pCi} / \mathrm{g}$ \\
\hline Uranium-235 & $0 / 2$ & 0.000776 & 0.00153301 & $-0.000308 / 0.00186$ & $\mathrm{pCi} / \mathrm{g}$ \\
\hline Uranium-238 & $0 / 2$ & 0.00345 & 0.000113137 & $0.00337 / 0.00353$ & $\mathrm{pCi} / \mathrm{g}$ \\
\hline \multicolumn{6}{|l|}{ EPIA-012B } \\
\hline Plutonium-238 & $0 / 2$ & 0.000343 & 0.00155139 & $-0.000754 / 0.00144$ & $\mathrm{pCi} / \mathrm{g}$ \\
\hline Plutonium-239/240 & $0 / 2$ & 0.000422 & 0.00108612 & $-0.000346 / 0.00119$ & $\mathrm{pCi} / \mathrm{g}$ \\
\hline \multicolumn{6}{|l|}{ EPIA-013B } \\
\hline Actinium-228 & $0 / 2$ & 0.01535 & 0.00205061 & $0.0139 / 0.0168$ & $\mathrm{pCi} / \mathrm{g}$ \\
\hline Antimony-124 & $0 / 2$ & 0.01482 & 0.0136896 & $0.00514 / 0.0245$ & $\mathrm{pCi} / \mathrm{g}$ \\
\hline Antimony-125 & $1 / 2$ & 0.03664 & 0.059199 & $-0.00522 / 0.0785$ & $\mathrm{pCi} / \mathrm{g}$ \\
\hline
\end{tabular}


Table 3.24 Analytes Detected in Method Blanks by EP (cont.)

\begin{tabular}{|l|l|l|l|l|l|}
\hline Analyte & $\begin{array}{l}\text { Frequency } \\
\text { Detected }\end{array}$ & $\begin{array}{l}\text { Mean } \\
\text { Result }\end{array}$ & Std. Dev. & Min./Max. Result & Unit \\
\hline Barium-133 & $0 / 2$ & -0.007355 & 0.000869741 & $-0.00797 /-0.00674$ & $\mathrm{pCi} / \mathrm{g}$ \\
\hline Cerium-144 & $0 / 2$ & -0.01475 & 0.0542351 & $-0.0531 / 0.0236$ & $\mathrm{pCi} / \mathrm{g}$ \\
\hline Cesium-134 & $0 / 2$ & 0.00165 & 0.0239709 & $-0.0153 / 0.0186$ & $\mathrm{pCi} / \mathrm{g}$ \\
\hline Cesium-137 & $0 / 2$ & 0.0193 & 0.00707107 & $0.0143 / 0.0243$ & $\mathrm{pCi} / \mathrm{g}$ \\
\hline Cobalt-57 & $0 / 2$ & 0.0029305 & 0.00542987 & $-0.000909 / 0.00677$ & $\mathrm{pCi} / \mathrm{g}$ \\
\hline Cobalt-58 & $0 / 2$ & $-8.5 \mathrm{e}-05$ & 0.0115471 & $-0.00825 / 0.00808$ & $\mathrm{pCi} / \mathrm{g}$ \\
\hline Cobalt-60 & $0 / 2$ & 0.00744 & 0.00125865 & $0.00655 / 0.00833$ & $\mathrm{pCi} / \mathrm{g}$ \\
\hline Europium-152 & $0 / 2$ & 0.0032 & 0.0370524 & $-0.023 / 0.0294$ & $\mathrm{pCi} / \mathrm{g}$ \\
\hline Europium-154 & $0 / 2$ & 0.0571 & 0.0110309 & $0.0493 / 0.0649$ & $\mathrm{pCi} / \mathrm{g}$ \\
\hline Europium-155 & $0 / 2$ & 0.0263 & 0.00692965 & $0.0214 / 0.0312$ & $\mathrm{pCi} / \mathrm{g}$ \\
\hline Lead-212 & $0 / 2$ & 0.014085 & 0.0182646 & $0.00117 / 0.027$ & $\mathrm{pCi/g}$ \\
\hline Manganese-54 & $0 / 2$ & -0.001704 & 0.00220052 & $-0.00326 /-0.000148$ & $\mathrm{pCi/g}$ \\
\hline Neptunium-239 & $0 / 2$ & -0.0252 & 0.0118794 & $-0.0336 /-0.0168$ & $\mathrm{pCi/g}$ \\
\hline Potassium-40 & $0 / 2$ & 0.0622 & 0.0333754 & $0.0386 / 0.0858$ & $\mathrm{pCi} / \mathrm{g}$ \\
\hline Promethium-144 & $0 / 2$ & 0.002205 & 0.00709228 & $-0.00281 / 0.00722$ & $\mathrm{pCi/g}$ \\
\hline Promethium-146 & $0 / 2$ & 0.00534 & 0.00598212 & $0.00111 / 0.00957$ & $\mathrm{pCi/g}$ \\
\hline Ruthenium-106 & $0 / 2$ & 0.011295 & 0.0227759 & $-0.00481 / 0.0274$ & $\mathrm{pCi/g}$ \\
\hline Sodium-22 & $0 / 2$ & 0.02055 & 0.00388909 & $0.0178 / 0.0233$ & $\mathrm{pCi/g}$ \\
\hline Tin-113 & $0 / 2$ & -0.003625 & 0.0105712 & $-0.0111 / 0.00385$ & $\mathrm{pCi/g}$ \\
\hline Yttrium-88 & $0 / 2$ & 0.0121 & 0.0127279 & $0.0031 / 0.0211$ & $\mathrm{pCi/g}$ \\
\hline Zinc-65 & $0 / 2$ & 0.00485 & 0.036416 & $-0.0209 / 0.0306$ & $\mathrm{pCi/g}$ \\
\hline Zirconium-95 & $0 / 2$ & -0.011235 & 0.00178898 & $-0.0125 /-0.00997$ & $\mathrm{pCi/g}$ \\
\hline
\end{tabular}

\section{Field Blanks}

Field blanks are used to identify possible sources of contamination from the processing and shipping of samples. Field blanks consist of DI water or dry quartz sand sealed in sample bottles prior to sampling, opened in the field during sampling, resealed, and shipped to the laboratory with the samples. Positive results from field blanks can result from contami-nated sample bottles, contaminated DI water or quartz sand, vapors in the air during sampling, contamination during shipping or analysis, or analytical bias. The results from all samples in the sample delivery group are evaluated to determine the cause of the contamination and the corrective action to be taken.

This project did not use field blanks.

\section{Trip Blanks}

Trip blanks are used to detect contamination by volatile substances during shipping, primarily due to the breaking of the seal on the vial caused by depressurization during air transport. Trip blanks are analyzed for VOAs only. Trip blanks are prepared by the EPD/EMS laboratory for groundwater studies and by the analytical laboratory for other studies. If prepared by the analytical laboratory, trip blanks are shipped to EPD/EMS with the sample containers. The blanks are prepared by adding preservative to a $40-\mathrm{mL}$ vial, filling it completely with DI water (no air bubbles), and sealing the top with a Teflon-lined septum cap. If 
a trip blank is found to be contaminated, the results from all samples shipped in the same cooler are evaluated to determine the extent of the contamination and the corrective action to be taken.

The following tables list the statistical information for the trip blanks. The frequency detected is the number of trip blanks analyzed for each analyte that had detectable concentrations compared to the total number analyzed. The other columns list the mean result, standard deviation, minimum and maximum results, and reporting unit. When the analyte is not detected, the mean and minimum and maximum results are the ssEQLs for the trip blanks.

Table 3.25 Analytes Detected in Trip Blanks by GE

\begin{tabular}{|c|c|c|c|c|c|}
\hline Analyte & $\begin{array}{l}\text { Frequency } \\
\text { Detected }\end{array}$ & $\begin{array}{l}\text { Mean } \\
\text { Result }\end{array}$ & Std. Dev. & $\begin{array}{l}\text { Min./Max. } \\
\text { Result }\end{array}$ & Unit \\
\hline \multicolumn{6}{|l|}{ Volatile Organic Compounds } \\
\hline Acetone & $0 / 3$ & 5 & 0 & $5 / 5$ & $\mu \mathrm{g} / \mathrm{L}$ \\
\hline Benzene & $0 / 3$ & 1 & 0 & $1 / 1$ & $\mu \mathrm{g} / \mathrm{L}$ \\
\hline Bromodichloromethane & $0 / 3$ & 1 & 0 & $1 / 1$ & $\mu \mathrm{g} / \mathrm{L}$ \\
\hline Bromoform & $0 / 3$ & 1 & 0 & $1 / 1$ & $\mu \mathrm{g} / \mathrm{L}$ \\
\hline Bromomethane (Methyl bromide) & $0 / 3$ & 1 & 0 & $1 / 1$ & $\mu \mathrm{g} / \mathrm{L}$ \\
\hline Carbon disulfide & $0 / 3$ & 5 & 0 & $5 / 5$ & $\mu \mathrm{g} / \mathrm{L}$ \\
\hline Carbon tetrachloride & $0 / 3$ & 1 & 0 & $1 / 1$ & $\mu \mathrm{g} / \mathrm{L}$ \\
\hline Chlorobenzene & $0 / 3$ & 1 & 0 & $1 / 1$ & $\mu \mathrm{g} / \mathrm{L}$ \\
\hline Chloroethane & $0 / 3$ & 1 & 0 & $1 / 1$ & $\mu \mathrm{g} / \mathrm{L}$ \\
\hline Chloroethene (Vinyl chloride) & $0 / 3$ & 1 & 0 & $1 / 1$ & $\mu \mathrm{g} / \mathrm{L}$ \\
\hline Chloroform & $0 / 3$ & 1 & 0 & $1 / 1$ & $\mu \mathrm{g} / \mathrm{L}$ \\
\hline Chloromethane (Methyl chloride) & $0 / 3$ & 1 & 0 & $1 / 1$ & $\mu \mathrm{g} / \mathrm{L}$ \\
\hline Dibromochloromethane & $0 / 3$ & 1 & 0 & $1 / 1$ & $\mu \mathrm{g} / \mathrm{L}$ \\
\hline 1,1-Dichloroethane & $0 / 3$ & 1 & 0 & $1 / 1$ & $\mu \mathrm{g} / \mathrm{L}$ \\
\hline 1,2-Dichloroethane & $0 / 3$ & 1 & 0 & $1 / 1$ & $\mu \mathrm{g} / \mathrm{L}$ \\
\hline cis-1,2-Dichloroethylene & $0 / 3$ & 1 & 0 & $1 / 1$ & $\mu \mathrm{g} / \mathrm{L}$ \\
\hline trans-1,2-Dichloroethylene & $0 / 3$ & 1 & 0 & $1 / 1$ & $\mu \mathrm{g} / \mathrm{L}$ \\
\hline 1,1-Dichloroethylene & $0 / 3$ & 1 & 0 & $1 / 1$ & $\mu \mathrm{g} / \mathrm{L}$ \\
\hline $\begin{array}{l}\text { Dichloromethane (Methylene } \\
\text { chloride) }\end{array}$ & $0 / 3$ & 3.84667 & 0.404516 & $3.39 / 4.16$ & $\mu \mathrm{g} / \mathrm{L}$ \\
\hline 1,2-Dichloropropane & $0 / 3$ & 1 & 0 & $1 / 1$ & $\mu \mathrm{g} / \mathrm{L}$ \\
\hline cis-1,3-Dichloropropene & $0 / 3$ & 1 & 0 & $1 / 1$ & $\mu \mathrm{g} / \mathrm{L}$ \\
\hline trans-1,3-Dichloropropene & $0 / 3$ & 1 & 0 & $1 / 1$ & $\mu \mathrm{g} / \mathrm{L}$ \\
\hline
\end{tabular}

Table 3.25 Analytes Detected in Trip Blanks by GE (cont.)

\begin{tabular}{|l|l|l|l|l|l|}
\hline Analyte & $\begin{array}{l}\text { Frequency } \\
\text { Detected }\end{array}$ & $\begin{array}{l}\text { Mean } \\
\text { Result }\end{array}$ & Std. Dev. & $\begin{array}{l}\text { Min./Max. } \\
\text { Result }\end{array}$ & Unit \\
\hline Ethylbenzene & $0 / 3$ & 1 & 0 & $1 / 1$ & $\mu \mathrm{g} / \mathrm{L}$ \\
\hline 2-Hexanone & $0 / 3$ & 5 & 0 & $5 / 5$ & $\mu \mathrm{g} / \mathrm{L}$ \\
\hline Methyl ethyl ketone & $0 / 3$ & 10 & 0 & $10 / 10$ & $\mu \mathrm{g} / \mathrm{L}$ \\
\hline
\end{tabular}


Table 3.25 Analytes Detected in Trip Blanks by GE (cont.)

\begin{tabular}{|l|l|l|l|l|l|}
\hline Analyte & $\begin{array}{l}\text { Frequency } \\
\text { Detected }\end{array}$ & $\begin{array}{l}\text { Mean } \\
\text { Result }\end{array}$ & Std. Dev. & $\begin{array}{l}\text { Min./Max. } \\
\text { Result }\end{array}$ & Unit \\
\hline Methyl isobutyl ketone & $0 / 3$ & 5 & 0 & $5 / 5$ & $\mu \mathrm{g} / \mathrm{L}$ \\
\hline Styrene & $0 / 3$ & 1 & 0 & $1 / 1$ & $\mu \mathrm{g} / \mathrm{L}$ \\
\hline $1,1,2,2-$ Tetrachloroethane & $0 / 3$ & 1 & 0 & $1 / 1$ & $\mu \mathrm{g} / \mathrm{L}$ \\
\hline Tetrachloroethylene & $0 / 3$ & 1 & 0 & $1 / 1$ & $\mu \mathrm{g} / \mathrm{L}$ \\
\hline Toluene & $0 / 3$ & 1 & 0 & $1 / 1$ & $\mu \mathrm{g} / \mathrm{L}$ \\
\hline $1,1,1$-Trichloroethane & $0 / 3$ & 1 & 0 & $1 / 1$ & $\mu \mathrm{g} / \mathrm{L}$ \\
\hline $1,1,2$-Trichloroethane & $0 / 3$ & 1 & 0 & $1 / 1$ & $\mu \mathrm{g} / \mathrm{L}$ \\
\hline Trichloroethylene & $0 / 3$ & 1 & 0 & $1 / 1$ & $\mu \mathrm{g} / \mathrm{L}$ \\
\hline Xylenes & $0 / 3$ & 2 & 0 & $2 / 2$ & $\mu \mathrm{g} / \mathrm{L}$ \\
\hline
\end{tabular}

\section{Rinsates}

Rinsates are used to determine if sampling equipment that has been cleaned in the field is contaminated. Prior to sampling, DI water is poured over or pumped through portions of the sampling equipment that come in contact with the sample. If the rinsate is contaminated, the field cleaning procedure must be evaluated to determine the cause of the contamination. Results for all samples collected with equipment cleaned in the field must be evaluated to determine if the contamination is isolated or generalized.

This project did not use rinsates.

\subsubsection{Comparability}

The comparability of the data from the laboratories is based on results from split samples. No split samples were planned for this project. 
This page was left blank intentionally. 


\subsection{References}

DOE (U.S. Department of Energy), 1992. Environmental Measurements Laboratory Procedures Manual, HASL-300, 27th edition. Environmental Measurements Laboratory, New York, NY.

EPA (U.S. Environmental Protection Agency), 1983. Methods for Chemical Analysis of Water and Wastes, EPA-600/4-79-020. Environmental Monitoring and Support Laboratory, Cincinnati, OH.

EPA (U.S. Environmental Protection Agency), 1986. Test Methods for Evaluating Solid Waste (SW846), Volume I, including updates. Office of Solid Waste, Washington, DC.

EPA (U.S. Environmental Protection Agency), 1988a. Laboratory Data Validation Functional Guidelines for Evaluating Inorganics Analyses. Hazardous Site Evaluation Division, Washington, DC.

EPA (U.S. Environmental Protection Agency), 1988b. Laboratory Data Validation Functional Guidelines for Evaluating Organics Analyses. Hazardous Site Evaluation Division, Washington, DC.

EPA (U.S. Environmental Protection Agency), 1990. Guidance for Data Usability in Risk Assessment, EPA/540/G-90/008. Office of Emergency and Remedial Response, Washington, DC.

EPA (U.S. Environmental Protection Agency), 1991a. U.S. EPA Contract Laboratory Program, Statement of Work for Organics Analysis, Multi-Media, Multi-Concentration, OLM01.0. Washington, DC.

EPA (U.S. Environmental Protection Agency), 1991b. U.S. EPA Contract Laboratory Program, Statement of Work for Inorganics Analysis, Multi-Media, Multi-Concentration, ILM03.0. Washington, DC.

EPA (U.S. Environmental Protection Agency), 1991c. Test Method: The Determination of Inorganic Anions in Water by Ion Chromatography-Method 300.0. Environmental Monitoring and Support Laboratory, Cincinnati, $\mathrm{OH}$.

EPA (U.S. Environmental Protection Agency), 1993. Data Quality Objectives Process for Superfund, EPA 540/R-93/071. Washington, DC.

EPA (U.S. Environmental Protection Agency), 1994a. Guidelines Establishing Test Procedures for the Analysis of Pollutants, Code of Federal Regulations, Title 40, Part 136. Washington, DC.

EPA (U.S. Environmental Protection Agency), 1994b. Identification and Listing of Hazardous Waste, Code of Federal Regulations, Title 40, Part 261. Washington, DC.

WHC (Westinghouse Hanford Company), not dated. Data Validation Procedures for Radiochemical Analyses, WHC-SP-EN-SPP-001, rev. 1. Richland, WA. 
This page was left blank intentionally. 


\section{Appendix A: Analytical Methods and Method Detection Limits}

In the following tables of analytical methods and detection limits, the MDL column reports the matrixspecific method detection limit (MDL). The MDL takes into account the reagents, sample matrix, and preparation steps of a specific analytical method and is defined as the minimum concentration that can be measured and reported with $99 \%$ confidence that the analyte concentration is greater than zero.

Table A.1 Analytical Methods and Detection Limits for Liquid Matrices by GE

\begin{tabular}{|c|c|c|c|}
\hline Analyte & Method & MDL & Units \\
\hline \multicolumn{4}{|l|}{ Volatile Organic Compounds } \\
\hline Acetone & EPA8260B & 3.7 & $\mu \mathrm{g} / \mathrm{L}$ \\
\hline Benzene & EPA8260B & 0.3 & $\mu \mathrm{g} / \mathrm{L}$ \\
\hline Bromodichloromethane & EPA8260B & 0.4 & $\mu \mathrm{g} / \mathrm{L}$ \\
\hline Bromoform & EPA8260B & 0.4 & $\mu \mathrm{g} / \mathrm{L}$ \\
\hline Bromomethane (Methyl bromide) & EPA8260B & 0.3 & $\mu \mathrm{g} / \mathrm{L}$ \\
\hline Carbon disulfide & EPA8260B & 1.8 & $\mu \mathrm{g} / \mathrm{L}$ \\
\hline Carbon tetrachloride & EPA8260B & 0.2 & $\mu \mathrm{g} / \mathrm{L}$ \\
\hline Chlorobenzene & EPA8260B & 0.3 & $\mu \mathrm{g} / \mathrm{L}$ \\
\hline Chloroethane & EPA8260B & 0.3 & $\mu \mathrm{g} / \mathrm{L}$ \\
\hline Chloroethene (Vinyl chloride) & EPA8260B & 0.4 & $\mu \mathrm{g} / \mathrm{L}$ \\
\hline Chloroform & EPA8260B & 0.7 & $\mu \mathrm{g} / \mathrm{L}$ \\
\hline Chloromethane (Methyl chloride) & EPA8260B & 0.2 & $\mu \mathrm{g} / \mathrm{L}$ \\
\hline Dibromochloromethane & EPA8260B & 0.3 & $\mu \mathrm{g} / \mathrm{L}$ \\
\hline 1,1-Dichloroethane & EPA8260B & 0.4 & $\mu \mathrm{g} / \mathrm{L}$ \\
\hline 1,2-Dichloroethane & EPA8260B & 0.2 & $\mu \mathrm{g} / \mathrm{L}$ \\
\hline cis-1,2-Dichloroethylene & EPA8260B & 0.7 & $\mu \mathrm{g} / \mathrm{L}$ \\
\hline 1,1-Dichloroethylene & EPA8260B & 0.7 & $\mu \mathrm{g} / \mathrm{L}$ \\
\hline trans-1,2-Dichloroethylene & EPA8260B & 0.7 & $\mu \mathrm{g} / \mathrm{L}$ \\
\hline Dichloromethane (Methylene chloride) & EPA8260B & 1.2 & $\mu \mathrm{g} / \mathrm{L}$ \\
\hline 1,2-Dichloropropane & EPA8260B & 0.2 & $\mu \mathrm{g} / \mathrm{L}$ \\
\hline cis-1,3-Dichloropropene & EPA8260B & 0.3 & $\mu \mathrm{g} / \mathrm{L}$ \\
\hline trans-1,3-Dichloropropene & EPA8260B & 0.3 & $\mu \mathrm{g} / \mathrm{L}$ \\
\hline Ethylbenzene & EPA8260B & 0.3 & $\mu \mathrm{g} / \mathrm{L}$ \\
\hline 2-Hexanone & EPA8260B & 3.2 & $\mu \mathrm{g} / \mathrm{L}$ \\
\hline Methyl ethyl ketone & EPA8260B & 5.9 & $\mu \mathrm{g} / \mathrm{L}$ \\
\hline Methyl isobutyl ketone & EPA8260B & 1.6 & $\mu \mathrm{g} / \mathrm{L}$ \\
\hline Styrene & EPA8260B & 0.2 & $\mu \mathrm{g} / \mathrm{L}$ \\
\hline 1,1,2,2-Tetrachloroethane & EPA8260B & 0.5 & $\mu \mathrm{g} / \mathrm{L}$ \\
\hline Tetrachloroethylene & EPA8260B & 0.7 & $\mu \mathrm{g} / \mathrm{L}$ \\
\hline
\end{tabular}


Table A.1 Analytical Methods and Detection Limits for Liquid Matrices by GE (cont.)

\begin{tabular}{|l|l|l|l|}
\hline Analyte & Method & MDL & Units \\
\hline Toluene & EPA8260B & 0.5 & $\mu \mathrm{g} / \mathrm{L}$ \\
\hline $1,1,1-$ Trichloroethane & EPA8260B & 0.2 & $\mu \mathrm{g} / \mathrm{L}$ \\
\hline $1,1,2$-Trichloroethane & EPA8260B & 0.4 & $\mu \mathrm{g} / \mathrm{L}$ \\
\hline Trichloroethylene & EPA8260B & 0.6 & $\mu \mathrm{g} / \mathrm{L}$ \\
\hline Xylenes & EPA8260B & 1.1 & $\mu \mathrm{g} / \mathrm{L}$ \\
\hline
\end{tabular}

Table A.2 Analytical Methods and Detection Limits for Solid Matrices by GE

\begin{tabular}{|c|c|c|c|}
\hline Analyte & Method & MDL & Units \\
\hline \multicolumn{4}{|l|}{ Metals (total recoverable) } \\
\hline Antimony & EPA6010B & 3.82191 & $\mu \mathrm{g} / \mathrm{kg}$ \\
\hline Arsenic & EPA6010B & 4.56228 & $\mu \mathrm{g} / \mathrm{kg}$ \\
\hline Barium & EPA6010B & 0.5427 & $\mu \mathrm{g} / \mathrm{kg}$ \\
\hline Cadmium & EPA6010B & 0.3819 & $\mu \mathrm{g} / \mathrm{kg}$ \\
\hline Calcium & EPA6010B & 69.723486 & $\mu \mathrm{g} / \mathrm{kg}$ \\
\hline Chromium & EPA6010B & 0.7638 & $\mu \mathrm{g} / \mathrm{kg}$ \\
\hline Copper & EPA6010B & 1.3467 & $\mu \mathrm{g} / \mathrm{kg}$ \\
\hline Lead & EPA6010B & 1.5879 & $\mu \mathrm{g} / \mathrm{kg}$ \\
\hline Magnesium & EPA6010B & 5.08254 & $\mu \mathrm{g} / \mathrm{kg}$ \\
\hline Manganese & EPA6010B & 7.1355 & $\mu \mathrm{g} / \mathrm{kg}$ \\
\hline Mercury & EPA7471A & 0.0135 & $\mu \mathrm{g} / \mathrm{kg}$ \\
\hline Nickel & EPA6010B & 0.6432 & $\mu \mathrm{g} / \mathrm{kg}$ \\
\hline Selenium & EPA6010B & 2.7135 & $\mu \mathrm{g} / \mathrm{kg}$ \\
\hline Silver & EPA6010B & 0.6231 & $\mu \mathrm{g} / \mathrm{kg}$ \\
\hline Zinc & EPA6010B & 3.7185 & $\mu \mathrm{g} / \mathrm{kg}$ \\
\hline \multicolumn{4}{|l|}{ Volatile Organic Compounds } \\
\hline Acetone & EPA8260B & 10.3 & $\mu \mathrm{g} / \mathrm{kg}$ \\
\hline Benzene & EPA8260B & 0.5 & $\mu \mathrm{g} / \mathrm{kg}$ \\
\hline Bromodichloromethane & EPA8260B & 0.1 & $\mu \mathrm{g} / \mathrm{kg}$ \\
\hline Bromoform & EPA8260B & 0.3 & $\mu \mathrm{g} / \mathrm{kg}$ \\
\hline Bromomethane (Methyl bromide) & EPA8260B & 0.3 & $\mu \mathrm{g} / \mathrm{kg}$ \\
\hline Carbon disulfide & EPA8260B & 0.3 & $\mu \mathrm{g} / \mathrm{kg}$ \\
\hline Carbon tetrachloride & EPA8260B & 0.5 & $\mu \mathrm{g} / \mathrm{kg}$ \\
\hline Chlorobenzene & EPA8260B & 0.3 & $\mu \mathrm{g} / \mathrm{kg}$ \\
\hline Chloroethane & EPA8260B & 0.3 & $\mu \mathrm{g} / \mathrm{kg}$ \\
\hline Chloroethene (Vinyl chloride) & EPA8260B & 0.4 & $\mu \mathrm{g} / \mathrm{kg}$ \\
\hline Chloroform & EPA8260B & 0.1 & $\mu \mathrm{g} / \mathrm{kg}$ \\
\hline Chloromethane (Methyl chloride) & EPA8260B & 0.2 & $\mu \mathrm{g} / \mathrm{kg}$ \\
\hline Dibromochloromethane & EPA8260B & 0.2 & $\mu \mathrm{g} / \mathrm{kg}$ \\
\hline
\end{tabular}

Data Summary Report for Savannah River Integrator Operable Unit Fish Tissue

(WSRC-TR-99-00386) 
Table A.2 Analytical Methods and Detection Limits for Solid Matrices by GE (cont.)

\begin{tabular}{|c|c|c|c|}
\hline Analyte & Method & MDL & Units \\
\hline 1,1-Dichloroethane & EPA8260B & 0.1 & $\mu \mathrm{g} / \mathrm{kg}$ \\
\hline 1,2-Dichloroethane & EPA8260B & 0.2 & $\mu \mathrm{g} / \mathrm{kg}$ \\
\hline 1,1-Dichloroethylene & EPA8260B & 0.3 & $\mu \mathrm{g} / \mathrm{kg}$ \\
\hline 1,2-Dichloroethylene & EPA8260B & 0.25 & $\mu \mathrm{g} / \mathrm{kg}$ \\
\hline Dichloromethane (Methylene chloride) & EPA8260B & 1.4 & $\mu \mathrm{g} / \mathrm{kg}$ \\
\hline 1,2-Dichloropropane & EPA8260B & 0.2 & $\mu \mathrm{g} / \mathrm{kg}$ \\
\hline cis-1,3-Dichloropropene & EPA8260B & 0.2 & $\mu \mathrm{g} / \mathrm{kg}$ \\
\hline trans-1,3-Dichloropropene & EPA8260B & 0.3 & $\mu \mathrm{g} / \mathrm{kg}$ \\
\hline Ethylbenzene & EPA8260B & 0.3 & $\mu \mathrm{g} / \mathrm{kg}$ \\
\hline 2-Hexanone & EPA8260B & 2.8 & $\mu \mathrm{g} / \mathrm{kg}$ \\
\hline Methyl ethyl ketone & EPA8260B & 3.2 & $\mu \mathrm{g} / \mathrm{kg}$ \\
\hline Methyl isobutyl ketone & EPA8260B & 3.1 & $\mu \mathrm{g} / \mathrm{kg}$ \\
\hline Styrene & EPA8260B & 0.3 & $\mu \mathrm{g} / \mathrm{kg}$ \\
\hline 1,1,2,2-Tetrachloroethane & EPA8260B & 0.6 & $\mu \mathrm{g} / \mathrm{kg}$ \\
\hline Tetrachloroethylene & EPA8260B & 0.4 & $\mu \mathrm{g} / \mathrm{kg}$ \\
\hline Toluene & EPA8260B & 0.9 & $\mu \mathrm{g} / \mathrm{kg}$ \\
\hline 1,1,1-Trichloroethane & EPA8260B & 0.1 & $\mu \mathrm{g} / \mathrm{kg}$ \\
\hline 1,1,2-Trichloroethane & EPA8260B & 0.3 & $\mu \mathrm{g} / \mathrm{kg}$ \\
\hline Trichloroethylene & EPA8260B & 0.3 & $\mu \mathrm{g} / \mathrm{kg}$ \\
\hline Vinyl acetate & EPA8260B & 2.1 & $\mu \mathrm{g} / \mathrm{kg}$ \\
\hline Xylenes & EPA8260B & 1 & $\mu \mathrm{g} / \mathrm{kg}$ \\
\hline
\end{tabular}

In the following table of analytical methods and minimum detectable activities, the MDA column reports the mean matrix-specific minimum detectable activity. The MDA takes into account the reagents, sample matrix, and preparation steps of a specific analytical method and is defined as the smallest quantity of a radionuclide that can be detected in a sample with a $95 \%$ confidence level.

Table A.3 Analytical Methods and Minimum Detectable Activities for Solid Matrices by EP

\begin{tabular}{|c|c|c|c|}
\hline Analyte & Method & MDA & Units \\
\hline \multicolumn{4}{|l|}{ Radionuclides } \\
\hline Actinium-228 & EPIA-013B & 0.232567 & $\mathrm{pCi} / \mathrm{g}$ \\
\hline Antimony-124 & EPIA-013B & 0.0730367 & $\mathrm{pCi} / \mathrm{g}$ \\
\hline Antimony-125 & EPIA-013B & 0.127987 & $\mathrm{pCi} / \mathrm{g}$ \\
\hline Barium-133 & EPIA-013B & 0.05736 & $\overline{\mathrm{pCi} / \mathrm{g}}$ \\
\hline Cerium-144 & EPIA-013B & 0.2415 & $\mathrm{pCi} / \mathrm{g}$ \\
\hline Cesium-134 & EPIA-013B & 0.0501567 & $\mathrm{pCi} / \mathrm{g}$ \\
\hline
\end{tabular}


Table A.3 Analytical Methods and Minimum Detectable Activities for Solid Matrices by EP (cont.)

\begin{tabular}{|l|l|l|l|}
\hline Analyte & Method & MDA & Units \\
\hline Cesium-137 & EPIA-013B & 0.112288 & $\mathrm{pCi} / \mathrm{g}$ \\
\hline Cobalt-57 & EPIA-013B & 0.0303867 & $\mathrm{pCi} / \mathrm{g}$ \\
\hline Cobalt-58 & EPIA-013B & 0.0667867 & $\mathrm{pCi} / \mathrm{g}$ \\
\hline Cobalt-60 & EPIA-013B & 0.0607933 & $\mathrm{pCi} / \mathrm{g}$ \\
\hline Europium-152 & EPIA-013B & 0.133373 & $\mathrm{pCi} / \mathrm{g}$ \\
\hline Europium-154 & EPIA-013B & 0.164903 & $\mathrm{pCi} / \mathrm{g}$ \\
\hline Europium-155 & EPIA-013B & 0.114703 & $\mathrm{pCi/g}$ \\
\hline Gross alpha & EPIA-001B & 1.26465 & $\mathrm{pCi} / \mathrm{g}$ \\
\hline Lead-212 & EPIA-013B & 0.08758 & $\mathrm{pCi/g}$ \\
\hline Manganese-54 & EPIA-013B & 0.0554 & $\mathrm{pCi/g}$ \\
\hline Neptunium-239 & EPIA-013B & 0.211233 & $\mathrm{pCi/g}$ \\
\hline Nonvolatile beta & EPIA-001B & 1.54818 & $\mathrm{pCi/g}$ \\
\hline Plutonium-238 & EPIA-012B & 0.0193297 & $\mathrm{pCi/g}$ \\
\hline Plutonium-239/240 & EPIA-012B & 0.0177774 & $\mathrm{pCi/g}$ \\
\hline Potassium-40 & EPIA-013B & 0.579533 & $\mathrm{pCi/g}$ \\
\hline Promethium-144 & EPIA-013B & 0.05522 & $\mathrm{pCi/g}$ \\
\hline Promethium-146 & EPIA-013B & 0.0596267 & $\mathrm{pCi/g}$ \\
\hline Ruthenium-106 & EPIA-013B & 0.483 & $\mathrm{pCi/g}$ \\
\hline Sodium-22 & EPIA-013B & 0.0583467 & $\mathrm{pCi/g}$ \\
\hline Strontium-89/90 & EPA905 & 0.547006 & $\mathrm{pCi/g}$ \\
\hline Tin-113 & EPIA-013B & 0.0672833 & $\mathrm{pCi/g}$ \\
\hline Tritium & EPIA-002B & 0.164682 & $\mathrm{pCi/g}$ \\
\hline Uranium-233/234 & EPIA-011B & 0.0250363 & $\mathrm{pCi/g}$ \\
\hline Uranium-235 & EPIA-011B & 0.0203993 & $\mathrm{pCi/g}$ \\
\hline Uranium-238 & EPIA-011B & 0.0169079 & $\mathrm{pCi/g}$ \\
\hline Yttrium-88 & EPIA-013B & 0.0709367 & \\
\hline Zinc-65 & EPIA-013B & 0.124837 & 0.123257 \\
\hline Zirconium-95 & EPIA-013B & & \\
\hline & & \\
\hline
\end{tabular}




\section{Appendix B: Laboratory Performance Evaluation Results}

This appendix contains recent performance evaluation results for the laboratories that report data for soils and groundwater samples. Not all laboratories report data for each project.

\section{B.1 EPA Performance Evaluation Study Results}

EPA conducts the water pollution (WP) and water supply (WS) performance evaluation studies biannually to certify laboratories for specific analyses. EPA's Environmental Monitoring Systems Laboratory (EMSL) of Cincinnati, OH, distributes water samples spiked with known concentrations of constituents found in polluted waters and potable water and submits them to laboratories seeking certification to analyze wastewater and potable water. EMSL evaluates the results, using limits statistically based on the performance of approximately 100 top-rated laboratories that analyze each constituent by the same procedure as the laboratory being evaluated.

Recra reported results for WP039 (May 1998); EMAX, GE, and Recra reported results for WP040 (November 1998).

The results for WP039 are listed in table B.1. The results for Recra were all acceptable. However, the results for chemical oxygen demand and methylene chloride were close to the acceptance limits; Recra was instructed to check for error.

The results for WP040 are listed in table B.2. For EMAX, results were outside acceptance limits for antimony, fluoride, and total phenolics. Results for lead, vanadium, and chlorobenzene were close to the acceptance limits; EMAX was instructed to check for error. Results for GE were outside acceptance limits for nitrogen (Kjeldahl), chemical oxygen demand, aroclor 1016/1242, and for all volatile halocarbons reported (1,2-dichloroethane, chloroform, 1,1,1-trichloroethane, trichloroethene, carbon tetrachloride, tetrachloroethene, bromodichloromethane, dibromochloromethane, bromoform, methylene chloride, and chlorobenzene). The results for Recra were outside acceptance limits for chemical oxygen demand, total organic carbon and PCB 1016/1242 in oil. The results for PCB 1254 in oil were close to the acceptance limits; Recra was instructed to check for error.

Recra reported results for WS040 (March 1998), and EMAX and GE reported results for WS041 (September 1998).

The results for WS040 are listed in table B.3. The results for Recra were all acceptable.

The results for WS041 are listed in table B.4. The results for EMAX were outside acceptance limits for nitrate as nitrogen, dichloromethane, 1,3-dichloropropene, turbidity, and total organic carbon. For GE, results were outside acceptance limits for orthophosphate as P, 2,4-D, trans-1,2-dichloroethylene, 1,2,4trichlorobenzene, sulfate, and total organic carbon.

Throughout section B.1, D.L. means detection limit and NR means that a result was not reported by the laboratory.

The following conventions are used in the tables:

- Bold Italic indicates that the laboratory was asked to check for error.

- Bold shows that the reported values were outside acceptance limits.

Table B.1 WP039 Results for Recra 


\begin{tabular}{|c|c|c|c|}
\hline Analyte & Recra Result & True Value & Acceptance Limits \\
\hline \multicolumn{4}{|l|}{ Trace Metals $(\mu \mathrm{g} / \mathrm{L})$} \\
\hline Aluminum & 525 & 489 & $420-562$ \\
\hline Antimony & 265 & 277 & $198-325$ \\
\hline Arsenic & 880 & 864 & $739-1010$ \\
\hline Beryllium & 193 & 191 & $170-214$ \\
\hline Cadmium & 34.9 & 33.9 & $28.7-39.1$ \\
\hline Chromium & 215 & 216 & $189-244$ \\
\hline Cobalt & 81.7 & 82.3 & $71.5-92.4$ \\
\hline Copper & 76.3 & 74.3 & $68.2-84.3$ \\
\hline Iron & 193 & 191 & $165-218$ \\
\hline Lead & 1939 & 1900 & $1690-2110$ \\
\hline Manganese & 544 & 530 & $485-587$ \\
\hline Mercury & 0.524 & 0.563 & $0.307-0.782$ \\
\hline Molybdenum & 643 & 589 & $521-662$ \\
\hline Nickel & 736 & 702 & 674-780 \\
\hline Selenium & 798 & 760 & $625-862$ \\
\hline Silver & 137 & 130 & $118-150$ \\
\hline Strontium & 6.67 & 6.34 & $5.37-7.59$ \\
\hline Thallium & 148 & 141 & \begin{tabular}{|l|}
$121-167$ \\
\end{tabular} \\
\hline Titanium & 89.5 & 88.2 & $77.3-98.2$ \\
\hline Vanadium & 1417 & 1420 & $1320-1560$ \\
\hline Zinc & 139 & 131 & $113-150$ \\
\hline \multicolumn{4}{|l|}{ Minerals (mg/L, except as noted) } \\
\hline Alkalinity $\left(\right.$ as $\left.\mathrm{CaCO}_{3}\right)$, total & 39.1 & 36.8 & $32.3-44.7$ \\
\hline Calcium & 2.60 & 2.51 & $1.98-3.23$ \\
\hline Chloride & 11.1 & 10.8 & $8.30-12.7$ \\
\hline Fluoride & 3.98 & 3.80 & $3.37-4.24$ \\
\hline Hardness (as $\mathrm{CaCO}_{3}$ ), total & 14.9 & 14.8 & $11.7-18.8$ \\
\hline Magnesium & 2.18 & 2.08 & $1.84-2.37$ \\
\hline Potassium & 30.3 & 30.0 & $24.4-35.3$ \\
\hline Sodium & 32.5 & 31.8 & $28.0-36.2$ \\
\hline Specific conductance $(\mu \mathrm{S} / \mathrm{cm})$ & 286 & 296 & $271-313$ \\
\hline Sulfate & 60.6 & 58.0 & $49.0-67.3$ \\
\hline Total dissolved solids at $180^{\circ} \mathrm{C}$ & 145 & 156 & $127-200$ \\
\hline \multicolumn{4}{|l|}{ Nutrients (mg/L) } \\
\hline Ammonia nitrogen & 0.904 & 0.840 & $0.594-1.13$ \\
\hline Nitrate nitrogen & 1.06 & 1.10 & $0.868-1.31$ \\
\hline
\end{tabular}

Table B.1 WP039 Results for Recra (cont.)

\begin{tabular}{|l|l|l|l|}
\hline Analyte & Recra Result & True Value & Acceptance Limits \\
\hline Nitrogen by Kjeldahl method & 0.453 & 0.360 & D.L. -0.907 \\
\hline
\end{tabular}

Data Summary Report for Savannah River Integrator Operable Unit Fish Tissue

(WSRC-TR-99-00386) 
Table B.1 WP039 Results for Recra (cont.)

\begin{tabular}{|c|c|c|c|}
\hline Analyte & Recra Result & True Value & Acceptance Limits \\
\hline Orthophosphate & 0.250 & 0.250 & $0.203-0.296$ \\
\hline Total phosphorus & 0.207 & 0.170 & $0.150-0.252$ \\
\hline \multicolumn{4}{|l|}{ Demands (mg/L) } \\
\hline 5-day Biochemical oxygen demand & 103 & 119 & $63.5-174$ \\
\hline Carbonaceous BOD & 87.0 & 100 & $41.2-159$ \\
\hline Chemical oxygen demand & 146 & 192 & $139-232$ \\
\hline Total organic carbon & 75.4 & 76.0 & $63.6-86.4$ \\
\hline \multicolumn{4}{|l|}{ PCBs $(\mu \mathrm{g} / \mathrm{L})$} \\
\hline PCB $1016 / 1242$ & 5.70 & 6.40 & $2.55-8.54$ \\
\hline PCB 1254 & 2.75 & 3.29 & $1.45-4.34$ \\
\hline \multicolumn{4}{|l|}{ PCBs in Oil (mg/kg) } \\
\hline PCB 1016/1242 & 30.6 & 41.7 & $6.01-57.7$ \\
\hline PCB 1260 & 13.9 & 16.6 & $2.93-23.7$ \\
\hline \multicolumn{4}{|l|}{ Pesticides $(\mu \mathrm{g} / \mathrm{L})$} \\
\hline Aldrin & 1.38 & 1.48 & $0.354-2.12$ \\
\hline Chlordane & 11.6 & 12.7 & $5.86-18.9$ \\
\hline p,p'-DDD & 2.23 & 2.35 & $1.19-2.94$ \\
\hline p,p'-DDE & 1.55 & 1.47 & $0.791-2.56$ \\
\hline p,p'-DDT & 2.93 & 3.25 & $1.07-3.76$ \\
\hline Dieldrin & 1.72 & 1.72 & $0.949-2.14$ \\
\hline Heptachlor & 0.750 & 0.764 & $0.236-1.03$ \\
\hline Heptachlor epoxide & 0.480 & 0.586 & $0.317-0.693$ \\
\hline \multicolumn{4}{|l|}{ Volatile Halocarbons $(\mu \mathrm{g} / \mathrm{L})$} \\
\hline Bromodichloromethane & 18.8 & 21.6 & $15.7-25.8$ \\
\hline Bromoform & 32.2 & 36.8 & $21.7-47.5$ \\
\hline Carbon tetrachloride & 13.8 & 15.2 & $8.37-20.9$ \\
\hline Chlorobenzene & 14.3 & 16.6 & $11.8-20.6$ \\
\hline Chloroform & 23.4 & 27.2 & $18.5-33.3$ \\
\hline Dibromochloromethane & 18.1 & 20.4 & $13.7-25.0$ \\
\hline 1,2-Dichloroethane & 19.7 & 22.8 & $15.8-28.9$ \\
\hline Methylene chloride & 23.7 & 33.6 & $21.8-41.8$ \\
\hline Tetrachloroethylene & 14.2 & 17.5 & $11.1-22.3$ \\
\hline 1,1,1-Trichloroethane & 15.5 & 17.4 & $11.3-23.3$ \\
\hline Trichloroethylene & 10.5 & 12.7 & $7.88-15.3$ \\
\hline \multicolumn{4}{|l|}{ Volatile Aromatics $(\mu \mathrm{g} / \mathrm{L})$} \\
\hline Benzene & 38.3 & 46.4 & $35.0-57.9$ \\
\hline 1,2-Dichlorobenzene & 11.0 & 13.8 & $9.42-16.2$ \\
\hline 1,3-Dichlorobenzene & 18.1 & 22.4 & $15.7-26.4$ \\
\hline 1,4-Dichlorobenzene & 16.0 & 18.8 & $13.6-23.9$ \\
\hline Ethylbenzene & 22.8 & 27.3 & $19.2-34.5$ \\
\hline
\end{tabular}

Data Summary Report for Savannah River Integrator Operable Unit Fish Tissue

(WSRC-TR-99-00386) 
Table B.1 WP039 Results for Recra (cont.)

\begin{tabular}{|l|l|l|l|}
\hline Analyte & Recra Result & True Value & Acceptance Limits \\
\hline Toluene & 51.7 & 62.6 & $46.5-76.5$ \\
\hline \multicolumn{4}{|l|}{} \\
\hline Miscellaneous Parameters (mg/L, except as noted) \\
\hline Cyanide, total & 0.705 & 0.639 & $0.447-0.836$ \\
\hline Nonfilterable residue & 76.3 & 96.0 & $36.8-109$ \\
\hline Oil and grease (Freon extraction) & 41.0 & 44.0 & $31.0-49.2$ \\
\hline $\mathrm{pH}$ (pH units) & 5.01 & 5.03 & $4.93-5.14$ \\
\hline Phenolics, total & 0.942 & 0.799 & $0.443-1.15$ \\
\hline Residual chlorine, total & 0.350 & 0.280 & $0.229-0.439$ \\
\hline
\end{tabular}

Table B.2 WP040 Results for EMAX, GE, and Recra

\begin{tabular}{|c|c|c|c|c|c|}
\hline Analyte & $\begin{array}{l}\text { EMAX } \\
\text { Result }\end{array}$ & GE Result & $\begin{array}{l}\text { Recra } \\
\text { Result }\end{array}$ & True Value & $\begin{array}{l}\text { Acceptance } \\
\text { Limits }\end{array}$ \\
\hline \multicolumn{6}{|l|}{ Trace Metals $(\mu \mathrm{g} / \mathrm{L})$} \\
\hline Aluminum & 2970 & 3160 & 3192 & 3105 & $2740-3440$ \\
\hline Antimony & 364 & 512 & 485 & 499 & $381-590$ \\
\hline Arsenic & 153 & 174 & 160 & 160 & $128-190$ \\
\hline Beryllium & 37.2 & 36.8 & 37.4 & 36.9 & $33.1-42.2$ \\
\hline Cadmium & 159 & 174 & 172 & 170 & $147-193$ \\
\hline Chromium & 638 & 667 & 661 & 650 & $579-726$ \\
\hline Cobalt & 491 & 521 & 513 & 503 & $449-561$ \\
\hline Copper & 712 & 714 & 709 & 700 & $654-781$ \\
\hline Iron & 815 & 855 & 879 & 834 & $744-936$ \\
\hline Lead & 64.3 & 68.1 & 75 & 70.6 & $61.7-83.3$ \\
\hline Manganese & 229 & 242 & 246 & 240 & $216-259$ \\
\hline Mercury & 0.932 & 1.2 & 1.06 & 1.15 & $0.813-1.47$ \\
\hline Molybdenum & 16 & 17.9 & 19 & 18.2 & $14-22$ \\
\hline Nickel & 2510 & 2680 & 2627 & 2501 & $2340-2860$ \\
\hline Selenium & 231 & 264 & 246 & 260 & $189-297$ \\
\hline Silver & 801 & 834 & 708 & 851 & $736-930$ \\
\hline Strontium & 296 & 306 & 312 & 301 & $263-342$ \\
\hline Thallium & 838 & 896 & 871 & 841 & $747-970$ \\
\hline Titanium & 160 & 157 & 165 & 160 & $142-177$ \\
\hline Vanadium & 3930 & 4200 & 4405 & 4202 & $3880-4640$ \\
\hline Zinc & 633 & 646 & 644 & 631 & $563-709$ \\
\hline \multicolumn{6}{|c|}{ Minerals (mg/L, except as noted) } \\
\hline Alkalinity $\left(\right.$ as $\left.\mathrm{CaCO}_{3}\right)$, total & 43.5 & 40 & 41.1 & 42.4 & $35.5-48.1$ \\
\hline Calcium & 28.3 & 30.1 & 30.2 & 29 & $25.1-33.2$ \\
\hline
\end{tabular}


Table B.2 WP040 Results for EMAX, GE, and Recra (cont.)

\begin{tabular}{|c|c|c|c|c|c|}
\hline Analyte & $\begin{array}{l}\text { EMAX } \\
\text { Result }\end{array}$ & GE Result & $\begin{array}{l}\text { Recra } \\
\text { Result }\end{array}$ & True Value & $\begin{array}{l}\text { Acceptance } \\
\text { Limits }\end{array}$ \\
\hline Chloride & 79.9 & 74.7 & 75.4 & 74.9 & $68.1-83.2$ \\
\hline Fluoride & 4.65 & 0.784 & 0.912 & 0.860 & $0.738-1.05$ \\
\hline Hardness (as $\mathrm{CaCO}_{3}$ ), total & 102 & 108 & 106 & 105 & $93.3-119$ \\
\hline Magnesium & 7.63 & 8.03 & 8.36 & 8 & $7.06-8.86$ \\
\hline Potassium & 25.8 & 26.7 & 27 & 25 & $21.4-28.8$ \\
\hline Sodium & 38.9 & 41.8 & 39.4 & 39 & $35.7-44.1$ \\
\hline Specific conductance $(\mu \mathrm{S} / \mathrm{cm})$ & 517 & 508 & 502 & 525 & $471-544$ \\
\hline Sulfate & 66.6 & 66.9 & 71.7 & 69 & $58.3-78.4$ \\
\hline Total dissolved solids at $180^{\circ} \mathrm{C}$ & 297 & 283 & 274 & 274 & 230-337 \\
\hline \multicolumn{6}{|l|}{ Nutrients $(\mathrm{mg} / \mathrm{L})$} \\
\hline Ammonia nitrogen & 4.86 & 4.65 & 4.83 & 4.80 & $3.89-5.84$ \\
\hline Nitrate nitrogen & 12.3 & 11.8 & 12.3 & 12 & $10.1-13.9$ \\
\hline Nitrogen by Kjeldahl method & NR & 3 & 5.33 & 5.4 & $4.02-6.79$ \\
\hline Orthophosphate & 0.606 & 0.614 & 0.579 & 0.580 & $0.496-0.669$ \\
\hline Total phosphorus & NR & 4.12 & 3.85 & 4 & $3.3-4.68$ \\
\hline \multicolumn{6}{|l|}{ Demands (mg/L) } \\
\hline Chemical oxygen demand & 61.2 & 21.9 & 28.4 & 60.7 & $42.5-73.5$ \\
\hline Total organic carbon & 24.9 & 25.6 & 12 & 24 & $20.7-28.1$ \\
\hline 5-day Biological oxygen demand & NR & 44 & NR & 37.6 & $17.7-57.2$ \\
\hline $\begin{array}{l}\text { Carbonaceous biological oxygen } \\
\text { demand }\end{array}$ & NR & 43 & NR & 31.9 & $14.5-53.4$ \\
\hline \multicolumn{6}{|l|}{ PCBs $(\mu \mathrm{g} / \mathrm{L})$} \\
\hline PCB 1016/1242 & NR & 1.9 & NR & NR & Not present \\
\hline PCB 1232 & 4.89 & NR & 4.48 & 4.73 & $1.1-7.18$ \\
\hline PCB 1260 & 3.42 & 3.1 & 3.04 & 3.24 & $1.29-4.6$ \\
\hline \multicolumn{6}{|l|}{ PCBs in Oil $(\mathrm{mg} / \mathrm{kg})$} \\
\hline PCB 1016/1242 & 33.3 & 31.2 & 3.76 & 38.7 & $3.96-57.2$ \\
\hline PCB 1254 & 18.5 & 19.8 & 3.28 & 24 & D.L. -40.2 \\
\hline \multicolumn{6}{|l|}{ Pesticides $(\mu \mathrm{g} / \mathrm{L})$} \\
\hline Aldrin & 0.952 & 0.77 & 0.87 & 0.940 & $0.164-1.45$ \\
\hline Chlordane & 5.37 & 4.2 & 4.12 & 4.81 & $2.08-6.8$ \\
\hline $\mathrm{p}, \mathrm{p}^{\prime}-\mathrm{DDD}$ & 4.90 & 5.84 & 5.06 & 5.25 & $3.21-7.12$ \\
\hline $\mathrm{p}, \mathrm{p}^{\prime}-\mathrm{DDE}$ & 4.59 & 3.52 & 3.79 & 3.64 & $1.74-5.1$ \\
\hline p,p'-DDT & 6.85 & 7.76 & 7.85 & 8.42 & $2.61-12.7$ \\
\hline Dieldrin & 2.84 & 2.72 & 2.75 & 2.78 & $1.55-3.96$ \\
\hline Heptachlor & 2.29 & 2.36 & 2.46 & 2.49 & $0.837-3.41$ \\
\hline Heptachlor epoxide & 1.85 & 1.68 & 1.76 & 1.98 & $1.13-2.42$ \\
\hline \multicolumn{6}{|l|}{ Volatile Halocarbons $(\mu \mathrm{g} / \mathrm{L})$} \\
\hline Bromodichloromethane & 13.8 & 22.9 & 17 & 16.5 & $10.9-19.8$ \\
\hline
\end{tabular}


Table B.2 WP040 Results for EMAX, GE, and Recra (cont.)

\begin{tabular}{|c|c|c|c|c|c|}
\hline Analyte & $\begin{array}{l}\text { EMAX } \\
\text { Result }\end{array}$ & GE Result & $\begin{array}{l}\text { Recra } \\
\text { Result }\end{array}$ & True Value & $\begin{array}{l}\text { Acceptance } \\
\text { Limits }\end{array}$ \\
\hline Bromoform & 12 & 26.2 & 15.1 & 14.7 & $8.12-18.7$ \\
\hline Carbon tetrachloride & 25 & 33.6 & 27.5 & 26.3 & $16.3-32.9$ \\
\hline Chlorobenzene & 28.8 & 40.7 & 24.6 & 24.7 & $17.6-29.3$ \\
\hline Chloroform & 16 & 24.7 & 18.8 & 18.4 & $13.7-21.7$ \\
\hline Dibromochloromethane & 36.7 & 53.6 & 32.2 & 32.7 & $21.4-40.6$ \\
\hline 1,2-Dichloroethane & 16 & 19.2 & 17.2 & 14.6 & $9.85-18.5$ \\
\hline Methylene chloride & 41 & 75.3 & 33.6 & 44.1 & $29.7-58.8$ \\
\hline Tetrachloroethene & 36 & 51.4 & 31.6 & 32.5 & $21.6-39.5$ \\
\hline 1,1,1-Trichloroethane & 29 & 43.6 & 34.6 & 32.8 & $22.2-41.1$ \\
\hline Trichloroethene & 21.7 & 30.9 & 23.3 & 23.4 & $15.6-29.8$ \\
\hline \multicolumn{6}{|l|}{ Volatile Aromatics $(\mu \mathrm{g} / \mathrm{L})$} \\
\hline Benzene & 28.7 & 26.1 & 24.2 & 25.7 & $21.9-33.7$ \\
\hline 1,2-Dichlorobenzene & 33 & 39.4 & 34.2 & 36.3 & $27.1-45.1$ \\
\hline 1,3-Dichlorobenzene & 31 & 37.9 & 31.3 & 33.7 & $23.7-40.4$ \\
\hline 1,4-Dichlorobenzene & 40.4 & 46.5 & 39.7 & 41.6 & $30.4-52.3$ \\
\hline Ethylbenzene & 41.1 & 40 & 38.6 & 42.6 & $29.9-54$ \\
\hline Toluene & 32.6 & 31.5 & 26.9 & 32.3 & $23.9-39.8$ \\
\hline \multicolumn{6}{|c|}{ Miscellaneous Parameters (mg/L, except as noted) } \\
\hline Cyanide, total & 0.133 & 0.129 & 0.122 & 0.140 & $0.089-0.184$ \\
\hline Nonfilterable residue & $\mathrm{NR}$ & 50 & 34.9 & 64.0 & $12.4-80.7$ \\
\hline Oil and grease (hexane extraction) & 18.2 & NR & NR & 19.1 & $5.65-28.7$ \\
\hline Oil and grease (Freon extraction) & NR & 19.1 & 21.4 & 19.1 & $5.9-27.5$ \\
\hline $\mathrm{pH}$ (pH units) & 8.7 & 8.55 & 8.54 & 8.6 & $8.31-8.92$ \\
\hline Phenolics, total & 0.866 & 0.038 & 0.076 & 0.0668 & $0.0261-0.108$ \\
\hline Residual chlorine, total & 1.18 & 1.06 & 1.08 & 0.930 & $0.811-1.32$ \\
\hline
\end{tabular}

Table B.3 WS040 Results for Recra

\begin{tabular}{|l|l|l|l|}
\hline Analyte & Recra Result & True Value & Acceptance Limits \\
\hline Trace Metals, $(\boldsymbol{\mu g} / \mathbf{L})$ & \multicolumn{3}{l|}{} \\
\hline Antimony & 15.1 & 13.0 & $9.1-16.9$ \\
\hline Arsenic & 108 & 102 & $89.3-113$ \\
\hline Barium & 2724 & 2700 & $2300-3110$ \\
\hline Beryllium & 6.62 & 6.60 & $5.61-7.59$ \\
\hline Boron & 1150 & 1150 & $1050-1290$ \\
\hline Cadmium & 6.17 & 6.31 & $5.05-7.57$ \\
\hline
\end{tabular}


Table B.3 WS040 Results for Recra (cont.)

\begin{tabular}{|c|c|c|c|}
\hline Analyte & Recra Result & True Value & Acceptance Limits \\
\hline Chromium & 91.8 & 90.9 & $77.3-105$ \\
\hline Copper & 1675 & 1700 & $1530-1870$ \\
\hline Lead & 71.9 & 71.0 & 49.7-92.3 \\
\hline Manganese & 32.4 & 32.0 & $27.7-35.2$ \\
\hline Mercury & 1.35 & 1.50 & $1.05-1.95$ \\
\hline Molybdenum & 34.8 & 35.0 & $29.6-40.1$ \\
\hline Nickel & 25.7 & 25.0 & $21.3-28.8$ \\
\hline Selenium & 67.5 & 74.0 & $59.2-88.8$ \\
\hline Thallium & 11.7 & 10.0 & $7.00-13.0$ \\
\hline Zinc & 1726 & 1700 & $1620-1850$ \\
\hline \multicolumn{4}{|c|}{ Nitrate/Nitrite/Fluoride (mg/L) } \\
\hline Fluoride & 1.27 & 1.29 & $1.16-1.43$ \\
\hline Nitrate as $\mathrm{N}$ & 6.66 & 7.10 & $6.39-7.81$ \\
\hline Nitrite as $\mathrm{N}$ & 1.31 & 1.30 & $1.11-1.50$ \\
\hline Orthophosphate as $\mathrm{P}$ & 0.789 & 0.820 & $0.745-0.895$ \\
\hline \multicolumn{4}{|l|}{ Insecticides $(\mu \mathrm{g} / \mathrm{L})$} \\
\hline Alachlor & 19.4 & 17.7 & $9.74-25.7$ \\
\hline Aldrin & 1.81 & 1.87 & $0.605-2.45$ \\
\hline Atrazine & 23.3 & 24.7 & $13.6-35.8$ \\
\hline Chlordane (total) & 11.7 & 11.8 & $6.49-17.1$ \\
\hline Dieldrin & 3.12 & 2.87 & $1.86-3.71$ \\
\hline Endrin & 0.88 & 0.867 & $0.607-1.13$ \\
\hline Heptachlor & 2.32 & 2.33 & $1.28-3.38$ \\
\hline Heptachlor epoxide & 1.48 & 1.48 & $0.814-2.15$ \\
\hline Hexachlorobenzene & 3.11 & 2.90 & $1.43-3.82$ \\
\hline Hexachlorocyclopentadiene & 0.552 & 1.22 & $0.187-1.68$ \\
\hline Lindane & 1.80 & 1.77 & $0.974-2.57$ \\
\hline Methoxychlor & 49.2 & 42.8 & $23.5-62.1$ \\
\hline Propachlor & 3.34 & 3.18 & $1.9-4.29$ \\
\hline Simazine & 30.8 & 34.3 & $4.97-50.8$ \\
\hline Toxaphene & 17.1 & 16.5 & $8.08-23.9$ \\
\hline Trifluralin & 2.06 & 2.62 & $1.04-3.57$ \\
\hline \multicolumn{4}{|l|}{ Herbicides $(\mu \mathrm{g} / \mathrm{L})$} \\
\hline $2,4-\mathrm{D}$ & 43.4 & 45.0 & $22.5-67.5$ \\
\hline Dalapon & 128 & 132 & D.L. -185 \\
\hline Dicamba & 118 & 87.2 & $40.1-127$ \\
\hline Dinoseb & 11.6 & 12.7 & D.L. -20.2 \\
\hline Pentachlorophenol & 24.7 & 22.3 & $11.2-33.5$ \\
\hline Picloram & 40.9 & 44.0 & D.L. -62.9 \\
\hline 2,4,5-TP (Silvex) & 18.3 & 18.1 & $9.05-27.2$ \\
\hline
\end{tabular}


Table B.3 WS040 Results for Recra (cont.)

\begin{tabular}{|c|c|c|c|}
\hline Analyte & Recra Result & True Value & Acceptance Limits \\
\hline \multicolumn{4}{|c|}{ Polynuclear Aromatic Hydrocarbons $(\mu \mathrm{g} / \mathrm{L})$} \\
\hline Benzo $[a]$ pyrene & 1.07 & 1.48 & $0.373-1.97$ \\
\hline \multicolumn{4}{|l|}{ Adipate/Phthalates $(\mu \mathrm{g} / \mathrm{L})$} \\
\hline Di-(2-ethylhexyl) adipate & 13.6 & 15.8 & $5.85-22.7$ \\
\hline Di-(2-ethylhexyl) phthalate & 25.1 & 32.4 & $10.3-42.0$ \\
\hline \multicolumn{4}{|l|}{ Trihalomethanes $(\mu \mathrm{g} / \mathrm{L})$} \\
\hline Bromodichloromethane & 19.2 & 19.8 & $15.8-23.8$ \\
\hline Bromoform & 14.5 & 12.7 & $10.2-15.2$ \\
\hline Chlorodibromomethane & 17.1 & 15.6 & $12.5-18.7$ \\
\hline Chloroform & 27.3 & 27.4 & $21.9-32.9$ \\
\hline Total trihalomethane & 78.1 & 75.5 & $60.4-90.6$ \\
\hline \multicolumn{4}{|c|}{ Volatile Organic Compounds $(\mu \mathrm{g} / \mathrm{L})$} \\
\hline Benzene & 16.6 & 16.7 & $13.4-20.0$ \\
\hline Carbon tetrachloride & 9.44 & 8.90 & $5.34-12.5$ \\
\hline Chlorobenzene & 21.2 & 22.9 & $18.3-27.5$ \\
\hline 2-Chlorotoluene & 11.0 & 10.2 & $7.28-12.5$ \\
\hline 1,2-Dibromo-3-chloropropane & 0.437 & 0.527 & $0.316-0.738$ \\
\hline 1,2-Dichlorobenzene & 16.7 & 18.4 & $14.7-22.1$ \\
\hline 1,3-Dichlorobenzene & 21.4 & 18.3 & $13.8-22.5$ \\
\hline 1,4-Dichlorobenzene & 10.9 & 11.6 & $9.28-13.9$ \\
\hline 1,2-Dichloroethane & 18.1 & 17.2 & $13.8-20.6$ \\
\hline 1,1-Dichloroethylene & 20.2 & 18.3 & $14.6-22.0$ \\
\hline cis-1,2-Dichloroethylene & 18.1 & 18.4 & $14.7-22.1$ \\
\hline trans-1,2-Dichloroethylene & 24.6 & 26.8 & $21.4-32.2$ \\
\hline Dichloromethane & 7.11 & 6.20 & $3.72-8.68$ \\
\hline 1,2-Dichloropropane & 19.0 & 19.0 & $15.2-22.8$ \\
\hline cis-1,3-Dichloropropene & 5.92 & 7.42 & $4.15-7.52$ \\
\hline trans-1,3-Dichloropropene & 6.97 & 8.60 & $4.49-8.92$ \\
\hline Ethylbenzene & 16.5 & 17.8 & $14.2-21.4$ \\
\hline Ethylene dibromide & 0.603 & 0.638 & $0.383-0.893$ \\
\hline Hexachlorobutadiene & 16.2 & 15.3 & $11.5-19.8$ \\
\hline Styrene & 18.6 & 18.9 & $15.1-22.7$ \\
\hline 1,1,1,2-Tetrachloroethane & 13.3 & 13.3 & $9.87-15.2$ \\
\hline Tetrachloroethylene & 13.4 & 14.7 & $11.8-17.6$ \\
\hline Toluene & 13.0 & 14.6 & $11.7-17.5$ \\
\hline 1,2,4-Trichlorobenzene & 11.6 & 12.3 & $9.84-14.8$ \\
\hline 1,1,1-Trichloroethane & 7.39 & 7.20 & $4.32-10.1$ \\
\hline 1,1,2-Trichloroethane & 15.6 & 17.2 & $13.8-20.6$ \\
\hline Trichloroethylene & 6.13 & 5.80 & $3.48-8.14$ \\
\hline 1,2,3-Trichloropropane & 17.9 & 18.7 & $12.0-22.8$ \\
\hline
\end{tabular}

Data Summary Report for Savannah River Integrator Operable Unit Fish Tissue

(WSRC-TR-99-00386) 
Table B.3 WS040 Results for Recra (cont.)

\begin{tabular}{|l|l|l|l|}
\hline Analyte & Recra Result & True Value & Acceptance Limits \\
\hline Vinyl chloride & 32.3 & 27.2 & $16.3-38.1$ \\
\hline Xylenes (total) & 30.1 & 30.3 & $24.2-36.4$ \\
\hline \multicolumn{2}{|l|}{} \\
\hline Inorganic Disinfection Byproducts $(\boldsymbol{\mu g} / \mathbf{L})$ & 36.0 & $15.0-57.6$ \\
\hline Bromate & 43.0 & 379 & $310-452$ \\
\hline Bromide & 363 & 110 & $85.5-134$ \\
\hline Chlorate & 114 & 420 & $259-660$ \\
\hline Chlorite & 482 & \multicolumn{2}{l|}{} \\
\hline Miscellaneous Analytes $(\mathbf{m g} / \mathbf{L}$ except as noted) & 0.240 & $0.0199-0.460$ \\
\hline Residual free chlorine & 0.27 & 7.80 & $7.00-9.67$ \\
\hline Turbidity (NTU) & 8.49 & 232 & $147-380$ \\
\hline Total filterable residue & 215 & 95.0 & $88.0-104$ \\
\hline Calcium hardness $\left(\mathrm{mg} \mathrm{CaCO}_{3} / \mathrm{L}\right)$ & 92.2 & 9.13 & $8.93-9.33$ \\
\hline pH (units) & 9.15 & 34.4 & $32.8-39.6$ \\
\hline Alkalinity (mg CaCO $/ \mathrm{L}_{3}$ & 37.1 & 15.8 & $14.4-17.8$ \\
\hline Sodium & 16.0 & 225 & $202-247$ \\
\hline Sulfate & 222 & 0.554 & $0.416-0.693$ \\
\hline Cyanide (total) & 0.506 & 3.70 & $3.27-4.54$ \\
\hline Total organic carbon & 3.82 & \\
\hline
\end{tabular}

Table B.4 WS041 Results for EMAX and GE

\begin{tabular}{|l|l|l|l|l|}
\hline \multicolumn{2}{|l|}{ EMAX Result } & GE Result & True Value & Acceptance Limits \\
\hline \multicolumn{5}{|l|}{} \\
\hline Trace Metals $(\boldsymbol{\mu g} / \mathbf{L})$ & EMte & \multicolumn{1}{l|}{} \\
\hline Antimony & 31.6 & 33.5 & 31.4 & $22-40.8$ \\
\hline Arsenic & 60.7 & 69.4 & 65.6 & $58.2-72.9$ \\
\hline Beryllium & 2.57 & 2.74 & 2.58 & $2.19-2.97$ \\
\hline Boron & 866 & 814 & 790 & $736-874$ \\
\hline Cadmium & 18 & 20.1 & 18.2 & $14.6-21.8$ \\
\hline Chromium & 53.1 & 56.8 & 55.5 & $47.2-63.8$ \\
\hline Copper & 697 & 700 & 702 & $632-772$ \\
\hline Manganese & 177 & 189 & 183 & $167-196$ \\
\hline Mercury & 5.71 & 5.28 & 5.82 & $4.07-7.57$ \\
\hline Molybdenum & 79.1 & 75 & 76.7 & $66.2-86.4$ \\
\hline Nickel & 352 & 372 & 352 & $299-405$ \\
\hline Selenium & 41.1 & 49.9 & 46.3 & $37-55.6$ \\
\hline Thallium & 3.27 & 3.49 & 3.5 & $2.45-4.55$ \\
\hline Zinc & 390 & 423 & 402 & $359-441$ \\
\hline
\end{tabular}


Table B.4 WS041 Results for EMAX and GE (cont.)

\begin{tabular}{|c|c|c|c|c|}
\hline Analyte & EMAX Result & GE Result & True Value & Acceptance Limits \\
\hline \multicolumn{5}{|c|}{ Fluoride/Nitrate/Nitrite (mg/L) } \\
\hline Fluoride & 6.2 & 5.87 & 6.2 & $5.58-6.82$ \\
\hline Nitrate as $\mathrm{N}$ & 17.1 & 14.9 & 15.0 & $13.5-16.5$ \\
\hline Nitrite as $\mathrm{N}$ & 1.87 & 1.64 & 1.7 & $1.45-1.96$ \\
\hline Orthophosphate as $\mathrm{P}$ & 1.22 & 1.17 & 1.3 & $1.19-1.39$ \\
\hline \multicolumn{5}{|l|}{ Insecticides ( $\mu \mathrm{g} / \mathrm{L})$} \\
\hline Aldrin & 0.762 & 0.833 & 1.23 & $0.505-1.56$ \\
\hline Chlordane, total & 3.24 & 2.3 & 2.9 & $1.6-4.21$ \\
\hline Dieldrin & 0.959 & 0.79 & 0.920 & $0.622-1.14$ \\
\hline Endrin & 0.852 & 0.795 & 0.789 & $0.552-1.03$ \\
\hline Heptachlor & 0.521 & 0.567 & 0.83 & $0.457-1.2$ \\
\hline Heptachlor epoxide & 0.610 & 0.529 & 0.630 & $0.347-0.914$ \\
\hline Hexachlorocyclopentadiene & 0.635 & NR & 1.93 & $0.0861-2.58$ \\
\hline Hexachlorobenzene & 0.541 & NR & 1.03 & $0.426-1.23$ \\
\hline Lindane & 1.76 & 1.98 & 2.5 & $1.38-3.63$ \\
\hline Methoxychlor & 25.9 & 23.8 & 26.8 & $14.7-38.9$ \\
\hline Propachlor & 3.78 & NR & 5.02 & $3.14-6.8$ \\
\hline Toxaphene & 6.47 & 6.5 & 6.9 & $3.8-10$ \\
\hline Trifluralin & 2.04 & $\mathrm{NR}$ & 3.82 & $1.55-5.4$ \\
\hline \multicolumn{5}{|l|}{ Herbicides $(\mu \mathrm{g} / \mathrm{L})$} \\
\hline Acifluorfen & 50 & $\mathrm{NR}$ & 72.1 & $25.4-101$ \\
\hline $2,4-\mathrm{D}$ & 42 & 18.2 & 73.1 & $36.6-110$ \\
\hline Dalapon & 95.7 & 71.5 & 183 & D.L. -258 \\
\hline Dinoseb & 15.1 & 19.2 & 27.6 & $0.568-41.9$ \\
\hline Dicamba & 75.6 & 88 & 123 & $32.1-167$ \\
\hline Pentachlorophenol & 19.5 & $\mathrm{NR}$ & 34.6 & $17.3-51.9$ \\
\hline Picloram & 19.9 & $\mathrm{NR}$ & 62.1 & D.L. -86.9 \\
\hline 2,4,5-TP (Silvex) & 19.4 & 17.6 & 24.1 & $12.1-36.2$ \\
\hline \multicolumn{5}{|c|}{ Polynuclear Aromatic Hydrocarbons $(\mu \mathrm{g} / \mathrm{L})$} \\
\hline Benzo $[a]$ pyrene & NR & 2.23 & 2.37 & $0.502-2.87$ \\
\hline \multicolumn{5}{|l|}{ Trihalomethanes $(\mu \mathrm{g} / \mathrm{L})$} \\
\hline Bromodichloromethane & 13 & 10.5 & 12.3 & $9.84-14.8$ \\
\hline Bromoform & 19.5 & 19 & 16.6 & $13.3-19.9$ \\
\hline Chlorodibromomethane & 21.3 & 17.5 & 19.4 & $15.5-23.3$ \\
\hline Chloroform & 13.7 & 12.1 & 14.4 & $11.5-17.3$ \\
\hline Trihalomethane, total & 67.5 & 59.1 & 62.7 & $50.2-75.2$ \\
\hline \multicolumn{5}{|c|}{ Volatile Organic Compounds $(\mu \mathrm{g} / \mathrm{L})$} \\
\hline Benzene & 19.1 & 16.7 & 18.7 & $15-22.4$ \\
\hline Carbon tetrachloride & 14.4 & 12 & 14.2 & $11.4-17$ \\
\hline
\end{tabular}

Data Summary Report for Savannah River Integrator Operable Unit Fish Tissue

(WSRC-TR-99-00386) 
Table B.4 WS041 Results for EMAX and GE (cont.)

\begin{tabular}{|c|c|c|c|c|}
\hline Analyte & EMAX Result & GE Result & True Value & Acceptance Limits \\
\hline Chlorobenzene & 19.1 & 16.6 & 18.6 & $14.9-22.3$ \\
\hline 1,2-Dibromo-3-chloropropane & 0.386 & 0.49 & 0.451 & $0.271-0.631$ \\
\hline 1,2-Dichlorobenzene & 12.3 & 10.5 & 11.3 & $9.04-13.6$ \\
\hline 1,4-Dichlorobenzene & 16 & 16.7 & 15.8 & $12.6-19$ \\
\hline 1,2-Dichloroethane & 14.7 & 13.5 & 13.7 & $11-16.4$ \\
\hline 1,1-Dichloroethylene & 6.17 & 5.18 & 5.25 & $3.15-7.35$ \\
\hline cis-1,2-Dichloroethylene & 22.2 & 21 & 25.3 & $20.2-30.4$ \\
\hline trans-1,2-Dichloroethylene & 18.6 & 14 & 18.5 & $14.8-22.2$ \\
\hline Dichloromethane & 19.6 & 14.1 & 15.9 & $12.7-19.1$ \\
\hline 1,2-Dichloropropane & 15.6 & 14.5 & 15.4 & $12.3-18.5$ \\
\hline 2,2-Dichloropropane & NR & 11.7 & $12 / 7$ & $9.73-14.4$ \\
\hline cis-1,3-Dichloropropene & 17.2 & 12.9 & 15.2 & $12.1-17.4$ \\
\hline trans-1,3-Dichloropropene & 16.2 & 11.6 & 13.7 & $10-15.6$ \\
\hline Ethylbenzene & 15.5 & 13.8 & 14.7 & $11.8-17.6$ \\
\hline Ethylene dibromide (EDB) & 0.291 & 0.37 & 0.344 & $0.206-0.482$ \\
\hline Hexachlorobutadiene & 12.6 & 10.8 & 11.6 & $8.53-14.5$ \\
\hline Styrene & 13.7 & 12.2 & 12.4 & $9.92-14.9$ \\
\hline 1,1,1,2-Tetrachloroethane & 15.9 & 13.8 & 15.2 & $12-17.5$ \\
\hline Tetrachloroethylene & 11.6 & 9.64 & 11.5 & $9.2-13.8$ \\
\hline Toluene & 19.2 & 17.4 & 18.7 & $15-22.4$ \\
\hline 1,2,3-Trichlorobenzene & 20.3 & 13 & 18.4 & $12.7-21.5$ \\
\hline 1,2,4-Trichlorobenzene & 15.1 & 7.81 & 14.2 & $11.4-17$ \\
\hline 1,1,1-Trichloroethane & 12.2 & 10.9 & 12.6 & $10.1-15.1$ \\
\hline 1,1,2-Trichloroethane & 14.2 & 13.4 & 13.3 & $10.6-16$ \\
\hline Trichloroethylene & 6.84 & 6.14 & 6.87 & $4.12-9.62$ \\
\hline 1,2,3-Trichloropropane & 16.8 & 16.7 & 14.8 & $10.2-18$ \\
\hline Vinyl chloride & 26.4 & 27 & 22.3 & $13.4-31.2$ \\
\hline Xylenes, total & 31.8 & 29.8 & 30.8 & $24.6-37$ \\
\hline \multicolumn{5}{|c|}{ Miscellaneous Analytes (mg/L, except as noted) } \\
\hline Alkalinity as $\mathrm{CaCO}_{3}$ & 55.6 & 54 & 50.6 & $48-56.7$ \\
\hline Chlorine, residual free & 2.02 & 1.83 & 1.9 & $1.55-2.32$ \\
\hline Cyanide, total & 0.267 & 0.310 & 0.326 & $0.245-0.408$ \\
\hline Hardness, calcium as $\mathrm{CaCO}_{3}$ & 246 & 260 & 248 & $229-266$ \\
\hline $\mathrm{pH}$ (units) & 9.01 & 9.18 & 9.13 & $8.88-9.28$ \\
\hline Residue, total filterable & 668 & 477 & 474 & $287-826$ \\
\hline Sodium & 23.5 & 24.5 & 23.3 & $21.6-26.3$ \\
\hline Sulfate & 47.1 & 43.4 & 49 & $44.1-54.2$ \\
\hline Total organic carbon & 2.44 & 2.16 & 1.6 & $1.21-2.05$ \\
\hline Turbidity (NTU) & 2.21 & 2.71 & 2.6 & $2.37-3.31$ \\
\hline
\end{tabular}




\section{B.2 EMS Quality Control Standards Results}

During first quarter 1999, EPD/EMS conducted quality assessments of the laboratories. Each laboratory received a set of certified environmental quality control standards from Environmental Resource Associates (ERA) of Arvada, CO (lot numbers 439, 586, 3227, 3427, 8922, and 9987). Each laboratory's results were compared with the ERA-certified values and performance acceptance limits (PALs). The PALs are listed as guidelines for acceptable analytical results given the limitations of the EPA methods used to determine these parameters. The PALs closely approximate the $95 \%$ confidence interval. The laboratories' results and the certified values and limits are given in table B.5 for EMAX, table B.6 for GE, and B.7 for Recra.

All three laboratories analyzed total petroleum hydrocarbons by the infrared method.

Of 103 analyses reported by EMAX, 98 (or 95.1\%), were within the PALs. Of 103 analyses reported by GE, 102 (or $99.0 \%$ ), were within the PALs. Of 103 analyses reported by Recra, 90 (or 87.4\%) were within the PALs.

Throughout section B.2:

- NR means that a result was not reported by the laboratory.

- Bold indicates that the reported values were outside acceptance limits.

- $\mathbf{J}$ in the Functional Guidelines (FG) column means that the analytical result was an estimated quantity.

- Results reported as below detection $(<)$ do not provide sufficient information to determine whether or not the result was within performance acceptance limits.

Table B.5 Quality Control Standards for Selected Analyses for EMAX

\begin{tabular}{|l|l|l|l|l|}
\hline \multicolumn{2}{|l|}{ Analyte } & Certified Value $\begin{array}{l}\text { Performance Acceptance } \\
\text { Limits }\end{array}$ & $\begin{array}{l}\text { EMAX } \\
\text { Result }\end{array}$ & FG Code \\
\hline \multicolumn{5}{|l|}{ Acids (Lot 586) $(\boldsymbol{\mu g} / \mathbf{L})$}
\end{tabular}

Table B.5 Quality Control Standards for Selected Analyses for EMAX (cont.)

\begin{tabular}{|c|c|c|c|c|}
\hline Analyte & Certified Value & $\begin{array}{l}\text { Performance Acceptance } \\
\text { Limits }\end{array}$ & $\begin{array}{l}\text { EMAX } \\
\text { Result }\end{array}$ & FG Code \\
\hline \multicolumn{5}{|c|}{ Base/Neutrals (Lot 586) $(\mu \mathrm{g} / \mathrm{L})$} \\
\hline Anthracene & 86.3 & $40.7-101$ & 73.4 & \\
\hline Benzo $[g, h, i]$ perylene & 17.3 & $7.16-24.3$ & 14.0 & \\
\hline Bis(2-ethylhexyl) phthalate & 76.9 & $31.0-98.1$ & 70.1 & \\
\hline 4-Bromophenyl-phenylether & 185 & 91.4-221 & 128 & \\
\hline
\end{tabular}


Table B.5 Quality Control Standards for Selected Analyses for EMAX (cont.)

\begin{tabular}{|c|c|c|c|c|}
\hline Analyte & Certified Value & $\begin{array}{l}\text { Performance Acceptance } \\
\text { Limits }\end{array}$ & $\begin{array}{l}\text { EMAX } \\
\text { Result }\end{array}$ & FG Code \\
\hline Chrysene & 28.7 & $13.3-35.2$ & 26.2 & \\
\hline Dibenz $[a, h]$ anthracene & 14.7 & $5.26-19.0$ & 10.8 & $\mathrm{~J}$ \\
\hline Dibenzofuran & 118 & $56.1-131$ & 79.3 & \\
\hline 1,2-Dichlorobenzene & 113 & $26.4-129$ & 58.1 & \\
\hline Diethylphthlate & 90.9 & $21.8-117$ & 11.7 & \\
\hline Dimethylphthlate & 138 & $59.4-160$ & 4.03 & $\mathrm{~J}$ \\
\hline 2,4-Dinitrotoluene & 104 & $43.5-120$ & 74.4 & \\
\hline Naphthalene & 89.9 & $31.9-102$ & 49.6 & \\
\hline N-Nitroso-di-n-propylamine & 63.1 & $26.7-77.6$ & 30.1 & \\
\hline Phenanthrene & 55.2 & $29.9-63.5$ & 45.7 & \\
\hline Pyrene & 99.6 & $46.6-121$ & 86.5 & \\
\hline 1,2,4-Trichlorobenzene & 102 & $29.7-116$ & 53.4 & \\
\hline \multicolumn{5}{|l|}{ Cations $(\operatorname{Lot} 439)(\mu \mathrm{g} / \mathrm{L})$} \\
\hline Calcium & 101,000 & $90,900-111,000$ & 103,000 & \\
\hline Magnesium & 74,900 & $66,700-83,100$ & 72,100 & \\
\hline Potassium & 90,300 & $82,200-98,400$ & 81,700 & \\
\hline Sodium & 94,900 & $84,500-106,000$ & 87,700 & \\
\hline \multicolumn{5}{|c|}{ Cyanide and Phenol (Lot 9987) $(\mu \mathrm{g} / \mathrm{L})$} \\
\hline Cyanide, total & 82.6 & $60.3-105$ & 77.1 & \\
\hline Phenol & 106 & $80.6-131$ & 109 & \\
\hline \multicolumn{5}{|c|}{ Grease and Oil (Lot 9987) (mg/bottle) } \\
\hline Grease and oil (gravimetric) & 50.6 & $30.4-63.3$ & 43.2 & \\
\hline \multicolumn{5}{|c|}{ Inorganics (Lot 3427) ( $\mu \mathrm{g} / \mathrm{L}$, except as noted) } \\
\hline Alkalinity $\left(\right.$ as $\left.\mathrm{CaCO}_{3}\right)$ & 151,000 & $141,000-170,000$ & 166,000 & \\
\hline Chloride & 56,800 & $50,500-64,100$ & 58,800 & \\
\hline Fluoride & 4,730 & $4,260-5,200$ & 3,930 & \\
\hline Nitrate as nitrogen & 3,260 & $2,930-3,590$ & 3,160 & \\
\hline $\mathrm{pH}$ (pH units) & 9.23 & $9.03-9.43$ & 9.18 & \\
\hline Potassium & 18,800 & $16,100-21,900$ & 18,700 & \\
\hline Sodium & 144,000 & $130,000-159,000$ & 142,000 & \\
\hline Specific conductance $(\mu \mathrm{S} / \mathrm{cm})$ & 676 & 566-769 & 685 & \\
\hline Sulfate & 79,500 & $68,200-89,900$ & 80,600 & \\
\hline Total dissolved solids & 607,000 & $497,000-680,000$ & 600,000 & \\
\hline \multicolumn{5}{|l|}{ Nutrients (Lot 9987) $(\mu \mathrm{g} / \mathrm{L})$} \\
\hline Ammonia as nitrogen & 3,560 & $2,990-4,130$ & 2,910 & \\
\hline Nitrate-nitrite as nitrogen & 5,420 & $4,820-6,020$ & 5,250 & \\
\hline Total phosphates (as P) & 4,060 & $3,450-4,670$ & 4,060 & \\
\hline \multicolumn{5}{|l|}{ PCBs $($ Lot 586) $(\mu \mathrm{g} / \mathrm{L})$} \\
\hline PCB 1254 & 5.71 & $3.42-7.17$ & 4.50 & \\
\hline
\end{tabular}

Data Summary Report for Savannah River Integrator Operable Unit Fish Tissue

(WSRC-TR-99-00386) 
Table B.5 Quality Control Standards for Selected Analyses for EMAX (cont.)

\begin{tabular}{|c|c|c|c|c|}
\hline Analyte & Certified Value & $\begin{array}{l}\text { Performance Acceptance } \\
\text { Limits }\end{array}$ & $\begin{array}{l}\text { EMAX } \\
\text { Result }\end{array}$ & FG Code \\
\hline \multicolumn{5}{|l|}{ Pesticides $($ Lot 586) $(\mu \mathrm{g} / \mathrm{L})$} \\
\hline Aldrin & 1.60 & $0.830-1.97$ & 1.00 & \\
\hline beta-Benzene hexachloride & 7.12 & $3.80-9.00$ & 5.50 & \\
\hline delta-Benzene hexachloride & 5.74 & $2.53-7.37$ & 5.20 & \\
\hline gamma-Benzene hexachloride (Lindane) & 1.04 & $0.585-1.35$ & 0.64 & \\
\hline gamma-Chlordane & 8.56 & $5.96-10.2$ & 7.60 & \\
\hline $4,4^{\prime}-\mathrm{DDD}$ & 6.50 & $3.99-8.44$ & 6.90 & \\
\hline 4,4'-DDE & 0.877 & $0.511-1.10$ & 0.91 & \\
\hline 4,4'-DDT & 1.19 & $0.692-1.49$ & 1.30 & \\
\hline Dieldrin & 3.65 & $2.26-4.72$ & 3.50 & \\
\hline Endrin & 1.43 & $0.896-1.85$ & 1.40 & \\
\hline Heptachlor & 5.83 & $2.64-7.22$ & 3.50 & \\
\hline Heptachlor epoxide & 0.915 & $0.566-1.12$ & 0.77 & \\
\hline \multicolumn{5}{|l|}{ Pesticides/Herbicides (Lot 3227) $(\mu \mathrm{g} / \mathrm{L})$} \\
\hline 2-sec-Butyl-4,6-dinitrophenol & 4.24 & $1.39-5.48$ & 0.48 & \\
\hline 2,4-Dichlorophenoxyacetic acid & 5.92 & $2.96-8.88$ & 3.50 & \\
\hline 2,4,5-TP (Silvex) & 4.90 & $2.45-7.35$ & 3.72 & \\
\hline \multicolumn{5}{|c|}{ Total Petroleum Hydrocarbons (Lot 8922) (mg/bottle) } \\
\hline Total petroleum hydrocarbons, infrared & 54.6 & $34.0-70.8$ & 59.9 & \\
\hline \multicolumn{5}{|l|}{ Toxaphene (Lot 3227) ( $\mu \mathrm{g} / \mathrm{L})$} \\
\hline Toxaphene & 3.02 & $1.66-4.38$ & 3.60 & \\
\hline \multicolumn{5}{|l|}{ Trace Metals $(\operatorname{Lot} 9987)(\mu \mathrm{g} / \mathrm{L})$} \\
\hline Aluminum & 233 & $191-275$ & 249 & \\
\hline Antimony & 192 & $144-240$ & 220 & \\
\hline Arsenic & 117 & $87.8-138$ & 118 & \\
\hline Barium & 617 & $506-728$ & 636 & \\
\hline Beryllium & 105 & $86.1-124$ & 106 & \\
\hline Boron & 641 & $526-801$ & 709 & \\
\hline Cadmium & 175 & 144-207 & 162 & \\
\hline Chromium & 242 & $198-285$ & 249 & \\
\hline Cobalt & 517 & $424-610$ & 547 & \\
\hline Copper & 275 & $226-325$ & 265 & \\
\hline Iron & 467 & $383-551$ & 494 & \\
\hline Lead & 433 & $355-511$ & 430 & \\
\hline Manganese & 558 & $458-658$ & 572 & \\
\hline Mercury & 6.67 & $5.00-8.34$ & 5.92 & \\
\hline Molybdenum & 142 & $116-168$ & 155 & \\
\hline Nickel & 342 & 280-404 & 338 & \\
\hline Selenium & 139 & $104-164$ & 139 & \\
\hline
\end{tabular}

Data Summary Report for Savannah River Integrator Operable Unit Fish Tissue 
Table B.5 Quality Control Standards for Selected Analyses for EMAX (cont.)

\begin{tabular}{|c|c|c|c|c|}
\hline Analyte & Certified Value & $\begin{array}{l}\text { Performance Acceptance } \\
\text { Limits }\end{array}$ & $\begin{array}{l}\text { EMAX } \\
\text { Result }\end{array}$ & FG Code \\
\hline Silver & 183 & $150-216$ & 173 & \\
\hline Strontium & 533 & $437-629$ & 539 & \\
\hline Thallium & 108 & $81.0-135$ & 112 & \\
\hline Vanadium & 458 & $376-540$ & 451 & \\
\hline Zinc & 117 & 95.9-138 & 109 & \\
\hline \multicolumn{5}{|l|}{ Turbidity (Lot 3427) (NTU) } \\
\hline Turbidity & 3.36 & $2.86-3.93$ & 3.35 & \\
\hline \multicolumn{5}{|l|}{ Volatiles $($ Lot 586) $(\mu \mathrm{g} / \mathrm{L})$} \\
\hline Benzene & 110 & $85.4-137$ & 120 & \\
\hline Bromodichloromethane & 61.3 & $47.1-76.3$ & 72.0 & \\
\hline Bromoform & 86.8 & $63.5-112$ & 110 & \\
\hline Carbon tetrachloride & 175 & 129-218 & 190 & \\
\hline Chlorobenzene & 78.1 & $61.1-93.7$ & 81.0 & \\
\hline Chloroform & 96.1 & $73.7-117$ & 100 & \\
\hline Dibromochloromethane & 15.9 & $12.4-19.6$ & 16.0 & \\
\hline 1,2-Dichlorobenzene & 62.6 & $47.5-76.9$ & 66.0 & \\
\hline 1,3-Dichlorobenzene & 127 & $97.0-153$ & 120 & \\
\hline 1,4-Dichlorobenzene & 57.6 & $43.3-70.2$ & 53.0 & \\
\hline 1,2-Dichloroethane & 60.1 & $46.9-75.7$ & 67.0 & \\
\hline Dichloromethane (methylene chloride) & 60.8 & $43.0-79.2$ & 62.0 & \\
\hline Ethylbenzene & 49.3 & $36.9-57.6$ & 54.0 & \\
\hline 4-Methyl-2-pentanone (MIBK) & 63.5 & $36.7-86.7$ & 62.0 & \\
\hline 1,1,2,2-Tetrachloroethane & 81.0 & $59.0-102$ & 80.0 & \\
\hline Tetrachloroethylene & 64.3 & $47.4-77.6$ & 60.0 & \\
\hline Toluene & 84.4 & $65.1-102$ & 88.0 & \\
\hline 1,1,1-Trichloroethane & 59.0 & $42.6-70.4$ & 60.0 & \\
\hline 1,1,2-Trichloroethane & 36.4 & $27.7-45.7$ & 37.0 & \\
\hline Trichloroethylene & 37.6 & $27.9-45.5$ & 39.0 & \\
\hline $\mathrm{m} / \mathrm{p}$-Xylene & 41.2 & $26.6-51.8$ & 42.0 & \\
\hline
\end{tabular}

Table B.6 Quality Control Standards for Selected Analyses for GE

\begin{tabular}{|c|c|c|c|c|}
\hline Analyte & $\begin{array}{l}\text { Certified } \\
\text { Value }\end{array}$ & $\begin{array}{l}\text { Performance } \\
\text { Acceptance Limits }\end{array}$ & GE Result & FG Code \\
\hline \multicolumn{5}{|l|}{ Acids $($ Lot 586) $(\mu \mathrm{g} / \mathrm{L})$} \\
\hline 2,6-Dichlorophenol & 132 & $72.9-153$ & 97.2 & \\
\hline 2-Methylphenol & 59.9 & $19.0-69.3$ & 40.5 & \\
\hline 4-Methylphenol & 82.8 & 25.4-95.9 & 47.6 & \\
\hline Pentachlorophenol & 129 & $40.2-162$ & 110 & \\
\hline 2,4,5-Trichlorophenol & 60.5 & $23.8-69.9$ & 39.9 & \\
\hline
\end{tabular}

Data Summary Report for Savannah River Integrator Operable Unit Fish Tissue

(WSRC-TR-99-00386) 


\begin{tabular}{|c|c|c|c|c|}
\hline Analyte & $\begin{array}{l}\text { Certified } \\
\text { Value }\end{array}$ & $\begin{array}{l}\text { Performance } \\
\text { Acceptance Limits }\end{array}$ & GE Result & FG Code \\
\hline 2,4,6-Trichlorophenol & 55.7 & $24.0-63.8$ & 35.5 & \\
\hline \multicolumn{5}{|l|}{ Base/Neutrals $($ Lot 586) $(\mu \mathrm{g} / \mathrm{L})$} \\
\hline Anthracene & 86.3 & $40.7-101$ & 73.2 & \\
\hline Benzo $[g, h, i]$ perylene & 17.3 & $7.16-24.3$ & 12.9 & \\
\hline Bis(2-ethylhexyl) phthalate & 76.9 & $31.0-98.1$ & 71.9 & \\
\hline 4-Bromophenyl-phenylether & 185 & 91.4-221 & 144 & \\
\hline Chrysene & 28.7 & $13.3-35.2$ & 25.1 & \\
\hline Dibenz $[a, h]$ anthracene & 14.7 & $5.26-19.0$ & 10.4 & \\
\hline Dibenzofuran & 118 & $56.1-131$ & 91.4 & \\
\hline 1,2-Dichlorobenzene & 113 & $26.4-129$ & 64.3 & \\
\hline Diethylphthlate & 90.9 & $21.8-117$ & 58.1 & \\
\hline Dimethylphthlate & 138 & $59.4-160$ & 53.3 & \\
\hline 2,4-Dinitrotoluene & 104 & $43.5-120$ & 75.4 & \\
\hline Naphthalene & 89.9 & $31.9-102$ & 64.3 & \\
\hline N-Nitroso-di-n-propylamine & 63.1 & $26.7-77.6$ & 50.3 & \\
\hline Phenanthrene & 55.2 & $29.9-63.5$ & 44.8 & \\
\hline Pyrene & 99.6 & $46.6-121$ & 84.6 & \\
\hline 1,2,4-Trichlorobenzene & 102 & $29.7-116$ & 58.7 & \\
\hline \multicolumn{5}{|l|}{ Cations $(\operatorname{Lot} 439)(\mu \mathrm{g} / \mathrm{L})$} \\
\hline Calcium & 101,000 & $90,900-111,000$ & 104,000 & \\
\hline Magnesium & 74,900 & $66,700-83,100$ & 75,400 & \\
\hline Potassium & 90,300 & $82,200-98,400$ & 82,500 & \\
\hline Sodium & 94,900 & $84,500-106,000$ & 98,300 & \\
\hline \multicolumn{5}{|c|}{ Cyanide and Phenol (Lot 9987) $(\mu \mathrm{g} / \mathrm{L})$} \\
\hline Cyanide, total & 82.6 & $60.3-105$ & 82.6 & \\
\hline Phenol & 106 & $80.6-131$ & 89.7 & \\
\hline \multicolumn{5}{|c|}{ Grease and Oil (Lot 9987) (mg/bottle) } \\
\hline Grease and oil (gravimetric) & 50.6 & $30.4-63.3$ & 41.4 & \\
\hline \multicolumn{5}{|c|}{ Inorganics (Lot 3427) ( $\mu \mathrm{g} / \mathrm{L}$, except as noted) } \\
\hline Alkalinity (as $\left.\mathrm{CaCO}_{3}\right)$ & 151,000 & $141,000-170,000$ & 145,000 & \\
\hline Chloride & 56,800 & $50,500-64,100$ & 54,300 & \\
\hline Fluoride & 4,730 & $4,260-5,200$ & 4,530 & \\
\hline
\end{tabular}

Table B.6 Quality Control Standards for Selected Analyses for GE (cont.)

\begin{tabular}{|l|l|l|l|l|}
\hline Analyte & $\begin{array}{l}\text { Certified } \\
\text { Value }\end{array}$ & $\begin{array}{l}\text { Performance } \\
\text { Acceptance Limits }\end{array}$ & GE Result & FG Code \\
\hline Nitrate as nitrogen & 3,260 & $2,930-3,590$ & 3,010 & \\
\hline $\mathrm{pH}$ (pH units) & 9.23 & $9.03-9.43$ & 9.07 & \\
\hline Potassium & 18,800 & $16,100-21,900$ & 19,100 & \\
\hline Sodium & 144,000 & $130,000-159,000$ & 151,000 & \\
\hline
\end{tabular}

Data Summary Report for Savannah River Integrator Operable Unit Fish Tissue

(WSRC-TR-99-00386) 
Table B.6 Quality Control Standards for Selected Analyses for GE (cont.)

\begin{tabular}{|c|c|c|c|c|}
\hline Analyte & $\begin{array}{l}\text { Certified } \\
\text { Value }\end{array}$ & $\begin{array}{l}\text { Performance } \\
\text { Acceptance Limits }\end{array}$ & GE Result & FG Code \\
\hline Specific conductance $(\mu \mathrm{S} / \mathrm{cm})$ & 676 & $566-769$ & 718 & \\
\hline Sulfate & 79,500 & $68,200-89,900$ & 75,700 & \\
\hline Total dissolved solids & 607,000 & $497,000-680,000$ & 580,000 & \\
\hline \multicolumn{5}{|l|}{ Nutrients (Lot 9987) $(\mu \mathrm{g} / \mathrm{L})$} \\
\hline Ammonia as nitrogen & 3,560 & $2,990-4,130$ & 3,350 & \\
\hline Nitrate-nitrite as nitrogen & 5,420 & $4,820-6,020$ & 5,450 & \\
\hline Total phosphates (as P) & 4,060 & $3,450-4,670$ & 3,630 & \\
\hline \multicolumn{5}{|l|}{ PCBs (Lot 586) } \\
\hline PCB 1254 & 5.71 & $3.42-7.17$ & 5.30 & $\mathrm{~J}$ \\
\hline \multicolumn{5}{|l|}{ Pesticides (Lot 586) $(\mu \mathrm{g} / \mathrm{L})$} \\
\hline Aldrin & 1.60 & $0.830-1.97$ & 1.51 & $\mathrm{~J}$ \\
\hline beta-Benzene hexachloride & 7.12 & $3.80-9.00$ & 6.69 & $\mathrm{~J}$ \\
\hline delta-Benzene hexachloride & 5.74 & $2.53-7.37$ & 5.78 & $\mathrm{~J}$ \\
\hline gamma-Benzene hexachloride (Lindane) & 1.04 & $0.585-1.35$ & 0.87 & $\mathrm{~J}$ \\
\hline gamma-Chlordane & 8.56 & $5.96-10.2$ & 8.0 & $\mathrm{~J}$ \\
\hline 4,4 '-DDD & 6.50 & $3.99-8.44$ & 5.52 & $\mathrm{~J}$ \\
\hline 4,4'-DDE & 0.877 & $0.511-1.10$ & 0.76 & $\mathrm{~J}$ \\
\hline 4,4'-DDT & 1.19 & $0.692-1.49$ & 1.15 & $\mathrm{~J}$ \\
\hline Dieldrin & 3.65 & $2.26-4.72$ & 3.22 & $\mathrm{~J}$ \\
\hline Endrin & 1.43 & $0.896-1.85$ & 1.36 & $\mathrm{~J}$ \\
\hline Heptachlor & 5.83 & $2.64-7.22$ & 5.20 & $\mathrm{~J}$ \\
\hline Heptachlor epoxide & 0.915 & $0.566-1.12$ & 0.79 & $\mathrm{~J}$ \\
\hline \multicolumn{5}{|l|}{ Pesticides/Herbicides (Lot 3227) $(\mu \mathrm{g} / \mathrm{L})$} \\
\hline 2-sec-Butyl-4,6-dinitrophenol & 4.24 & $1.39-5.48$ & 3.92 & \\
\hline 2,4-Dichlorophenoxyacetic acid & 5.92 & $2.96-8.88$ & 5.66 & \\
\hline 2,4,5-TP (Silvex) & 4.90 & $2.45-7.35$ & 4.33 & \\
\hline \multicolumn{5}{|c|}{ Total Petroleum Hydrocarbons (Lot 8922) (mg/bottle) } \\
\hline Total petroleum hydrocarbons, infrared & 54.6 & $34.0-70.8$ & 49.5 & \\
\hline \multicolumn{5}{|l|}{ Toxaphene $($ Lot 3227) $(\mu \mathrm{g} / \mathrm{L})$} \\
\hline Toxaphene & 3.02 & $1.66-4.38$ & NR & \\
\hline \multicolumn{5}{|l|}{ Trace Metals $($ Lot 9987) $(\mu \mathrm{g} / \mathrm{L})$} \\
\hline Aluminum & 233 & $191-275$ & 253 & \\
\hline Antimony & 192 & $144-240$ & 194 & \\
\hline Arsenic & 117 & $87.8-138$ & 118 & \\
\hline Barium & 617 & $506-728$ & 604 & \\
\hline Beryllium & 105 & $86.1-124$ & 103 & \\
\hline Boron & 641 & $526-801$ & 706 & \\
\hline Cadmium & 175 & \begin{tabular}{|l|}
$144-207$ \\
\end{tabular} & 176 & \\
\hline Chromium & 242 & $198-285$ & 240 & \\
\hline
\end{tabular}

Data Summary Report for Savannah River Integrator Operable Unit Fish Tissue

(WSRC-TR-99-00386) 
Table B.6 Quality Control Standards for Selected Analyses for GE (cont.)

\begin{tabular}{|c|c|c|c|c|}
\hline Analyte & $\begin{array}{l}\text { Certified } \\
\text { Value }\end{array}$ & $\begin{array}{l}\text { Performance } \\
\text { Acceptance Limits }\end{array}$ & GE Result & FG Code \\
\hline Cobalt & 517 & $424-610$ & 528 & \\
\hline Copper & 275 & $226-325$ & 267 & \\
\hline Iron & 467 & $383-551$ & 461 & \\
\hline Lead & 433 & $355-511$ & 414 & \\
\hline Manganese & 558 & $458-658$ & 556 & \\
\hline Mercury & 6.67 & $5.00-8.34$ & 6.08 & \\
\hline Molybdenum & 142 & $116-168$ & 132 & \\
\hline Nickel & 342 & $280-404$ & 352 & \\
\hline Selenium & 139 & \begin{tabular}{|l|}
$104-164$ \\
\end{tabular} & 140 & \\
\hline Silver & 183 & $150-216$ & 175 & \\
\hline Strontium & 533 & $437-629$ & 521 & \\
\hline Thallium & 108 & $81.0-135$ & 110 & \\
\hline Vanadium & 458 & $376-540$ & 448 & \\
\hline Zinc & 117 & 95.9-138 & 118 & \\
\hline \multicolumn{5}{|l|}{ Turbidity (Lot 3427) (NTU) } \\
\hline Turbidity & 3.36 & $2.86-3.93$ & 3.22 & \\
\hline \multicolumn{5}{|l|}{ Volatiles $($ Lot 586) $(\mu \mathrm{g} / \mathrm{L})$} \\
\hline Benzene & 110 & $85.4-137$ & 104 & \\
\hline Bromodichloromethane & 61.3 & $47.1-76.3$ & 59.0 & \\
\hline Bromoform & 86.8 & $63.5-112$ & 93.2 & \\
\hline Carbon tetrachloride & 175 & $129-218$ & 188 & \\
\hline Chlorobenzene & 78.1 & $61.1-93.7$ & 79.2 & \\
\hline Chloroform & 96.1 & $73.7-117$ & 95.2 & \\
\hline Dibromochloromethane & 15.9 & $12.4-19.6$ & 16.3 & \\
\hline 1,2-Dichlorobenzene & 62.6 & $47.5-76.9$ & 63.3 & \\
\hline 1,3-Dichlorobenzene & 127 & $97.0-153$ & 134 & \\
\hline 1,4-Dichlorobenzene & 57.6 & $43.3-70.2$ & 65.4 & \\
\hline 1,2-Dichloroethane & 60.1 & $46.9-75.7$ & 61.9 & \\
\hline Dichloromethane (methylene chloride) & 60.8 & $43.0-79.2$ & 61.8 & \\
\hline Ethylbenzene & 49.3 & $36.9-57.6$ & 49.5 & \\
\hline 4-Methyl-2-pentanone (MIBK) & 63.5 & $36.7-86.7$ & 60.0 & \\
\hline 1,1,2,2-Tetrachloroethane & 81.0 & $59.0-102$ & 72.4 & \\
\hline Tetrachloroethylene & 64.3 & $47.4-77.6$ & 71.7 & \\
\hline Toluene & 84.4 & $65.1-102$ & 80.7 & \\
\hline 1,1,1-Trichloroethane & 59.0 & $42.6-70.4$ & 62.5 & \\
\hline 1,1,2-Trichloroethane & 36.4 & $27.7-45.7$ & 34.7 & \\
\hline Trichloroethylene & 37.6 & 27.9-45.5 & 38.4 & \\
\hline $\mathrm{m} / \mathrm{p}$-Xylene & 41.2 & $26.6-51.8$ & 43.3 & \\
\hline
\end{tabular}


Table B.7 Quality Control Standards for Selected Analyses for Recra

\begin{tabular}{|c|c|c|c|c|}
\hline Analyte & $\begin{array}{l}\text { Certified } \\
\text { Value }\end{array}$ & $\begin{array}{l}\text { Performance } \\
\text { Acceptance Limits }\end{array}$ & Recra Result & FG Code \\
\hline \multicolumn{5}{|l|}{ Acids $($ Lot 586) $(\mu \mathrm{g} / \mathrm{L})$} \\
\hline 2,6-Dichlorophenol & 132 & $72.9-153$ & 106 & \\
\hline 2-Methylphenol & 59.9 & 19.0-69.3 & 42.7 & \\
\hline 4-Methylphenol & 82.8 & $25.4-95.9$ & 59.8 & \\
\hline Pentachlorophenol & 129 & $40.2-162$ & 108 & \\
\hline 2,4,5-Trichlorophenol & 60.5 & $23.8-69.9$ & 52.2 & \\
\hline 2,4,6-Trichlorophenol & 55.7 & $24.0-63.8$ & 49.0 & \\
\hline \multicolumn{5}{|l|}{ Base/Neutrals (Lot 586) $(\mu \mathrm{g} / \mathrm{L})$} \\
\hline Anthracene & 86.3 & $40.7-101$ & 64.1 & \\
\hline Benzo $[g, h, i]$ perylene & 17.3 & $7.16-24.3$ & 17.3 & \\
\hline Bis(2-ethylhexyl) phthalate & 76.9 & 31.0-98.1 & 61.4 & \\
\hline 4-Bromophenyl-phenylether & 185 & 91.4-221 & 165 & \\
\hline Chrysene & 28.7 & $13.3-35.2$ & 24.4 & \\
\hline Dibenz $[a, h]$ anthracene & 14.7 & $5.26-19.0$ & 16.0 & \\
\hline Dibenzofuran & 118 & 56.1-131 & 106 & \\
\hline 1,2-Dichlorobenzene & 113 & 26.4-129 & 85.0 & \\
\hline Diethylphthlate & 90.9 & \begin{tabular}{|l|}
$21.8-117$ \\
\end{tabular} & 68.0 & \\
\hline Dimethylphthlate & 138 & $59.4-160$ & 123 & \\
\hline 2,4-Dinitrotoluene & 104 & $43.5-120$ & 85.1 & \\
\hline Naphthalene & 89.9 & 31.9-102 & 62.3 & \\
\hline N-Nitroso-di-n-propylamine & 63.1 & $26.7-77.6$ & 50.2 & \\
\hline Phenanthrene & 55.2 & $29.9-63.5$ & 48.4 & \\
\hline Pyrene & 99.6 & $46.6-121$ & 74.9 & \\
\hline 1,2,4-Trichlorobenzene & 102 & $29.7-116$ & 68.8 & \\
\hline \multicolumn{5}{|l|}{ Cations $(\operatorname{Lot} 439)(\mu \mathrm{g} / \mathrm{L})$} \\
\hline Calcium & 101,000 & $90,900-111,000$ & 102,000 & \\
\hline Magnesium & 74,900 & $66,700-83,100$ & 71,100 & \\
\hline
\end{tabular}

Table B.7 Quality Control Standards for Selected Analyses for Recra (cont.)

\begin{tabular}{|c|c|c|c|c|}
\hline Analyte & $\begin{array}{l}\text { Certified } \\
\text { Value }\end{array}$ & $\begin{array}{l}\text { Performance } \\
\text { Acceptance Limits }\end{array}$ & Recra Result & FG Code \\
\hline Potassium & 90,300 & $82,200-98,400$ & 90,800 & \\
\hline Sodium & 94,900 & $84,500-106,000$ & 91,100 & \\
\hline \multicolumn{5}{|c|}{ Cyanide and Phenol $(\operatorname{Lot} 9987)(\mu \mathrm{g} / \mathrm{L})$} \\
\hline Cyanide, total & 82.6 & $60.3-105$ & 83.1 & \\
\hline Phenol & 106 & $80.6-131$ & 102 & \\
\hline \multicolumn{5}{|c|}{ Grease and Oil (Lot 9987) (mg/bottle) } \\
\hline Grease and oil (gravimetric) & 50.6 & $30.4-63.3$ & 36.1 & $\mathrm{~J}$ \\
\hline
\end{tabular}

Data Summary Report for Savannah River Integrator Operable Unit Fish Tissue

(WSRC-TR-99-00386) 
Table B.7 Quality Control Standards for Selected Analyses for Recra (cont.)

\begin{tabular}{|c|c|c|c|c|}
\hline Analyte & $\begin{array}{l}\text { Certified } \\
\text { Value }\end{array}$ & $\begin{array}{l}\text { Performance } \\
\text { Acceptance Limits }\end{array}$ & Recra Result & FG Code \\
\hline \multicolumn{5}{|c|}{ Inorganics (Lot 3427) $(\mu \mathrm{g} / \mathrm{L}$, except as noted) } \\
\hline Alkalinity $\left(\right.$ as $\left.\mathrm{CaCO}_{3}\right)$ & 151,000 & $141,000-170,000$ & 151,000 & \\
\hline Chloride & 56,800 & $50,500-64,100$ & 63,000 & \\
\hline Fluoride & 4,730 & $4,260-5,200$ & 4,740 & \\
\hline Nitrate as nitrogen & 3,260 & $2,930-3,590$ & 3,230 & \\
\hline $\mathrm{pH}$ (pH units) & 9.23 & $9.03-9.43$ & 9.17 & \\
\hline Potassium & 18,800 & $16,100-21,900$ & 19,200 & \\
\hline Sodium & 144,000 & $130,000-159,000$ & 134,000 & \\
\hline Specific conductance $(\mu \mathrm{S} / \mathrm{cm})$ & 676 & $566-769$ & 559 & \\
\hline Sulfate & 79,500 & $68,200-89,900$ & 81,600 & \\
\hline Total dissolved solids & 607,000 & $497,000-680,000$ & 581,000 & \\
\hline \multicolumn{5}{|l|}{ Nutrients $($ Lot 9987) $(\mu \mathrm{g} / \mathrm{L})$} \\
\hline Ammonia as nitrogen & 3,560 & $2,990-4,130$ & 3,500 & \\
\hline Nitrate-nitrite as nitrogen & 5,420 & $4,820-6,020$ & 5,410 & \\
\hline Total phosphates (as P) & 4,060 & $3,450-4,670$ & 4,080 & \\
\hline \multicolumn{5}{|l|}{ PCBs $($ Lot 586) $(\mu \mathrm{g} / \mathrm{L})$} \\
\hline PCB 1254 & 5.71 & $3.42-7.17$ & 5.28 & \\
\hline \multicolumn{5}{|l|}{ Pesticides $($ Lot 586) $(\mu \mathrm{g} / \mathrm{L})$} \\
\hline Aldrin & 1.60 & $0.830-1.97$ & 1.82 & \\
\hline beta-Benzene hexachloride & 7.12 & $3.80-9.00$ & 8.01 & \\
\hline delta-Benzene hexachloride & 5.74 & $2.53-7.37$ & 5.92 & \\
\hline gamma-Benzene hexachloride (Lindane) & 1.04 & $0.585-1.35$ & 1.03 & \\
\hline gamma-Chlordane & 8.56 & $5.96-10.2$ & 8.48 & \\
\hline 4,4'-DDD & 6.50 & $3.99-8.44$ & 5.93 & \\
\hline 4,4'-DDE & 0.877 & $0.511-1.10$ & 0.76 & \\
\hline 4,4'-DDT & 1.19 & $0.692-1.49$ & 1.23 & \\
\hline Dieldrin & 3.65 & $2.26-4.72$ & 3.72 & \\
\hline Endrin & 1.43 & $0.896-1.85$ & 1.69 & \\
\hline Heptachlor & 5.83 & $2.64-7.22$ & 6.01 & \\
\hline Heptachlor epoxide & 0.915 & $0.566-1.12$ & 0.84 & \\
\hline \multicolumn{5}{|l|}{ Pesticides/Herbicides (Lot 3227) ( $\mu \mathrm{g} / \mathrm{L})$} \\
\hline 2-sec-Butyl-4,6-dinitrophenol & 4.24 & $1.39-5.48$ & 4.19 & \\
\hline 2,4-Dichlorophenoxyacetic acid & 5.92 & $2.96-8.88$ & 6.24 & \\
\hline $2,4,5$-TP (Silvex) & 4.90 & $2.45-7.35$ & 4.56 & \\
\hline \multicolumn{5}{|c|}{ Total Petroleum Hydrocarbons (Lot 8922) (mg/bottle) } \\
\hline Total petroleum hydrocarbons, infrared & 54.6 & $34.0-70.8$ & $<1,000$ & \\
\hline \multicolumn{5}{|l|}{ Toxaphene (Lot 3227) $(\mu \mathrm{g} / \mathrm{L})$} \\
\hline Toxaphene & 3.02 & $1.66-4.38$ & NR & \\
\hline
\end{tabular}


Table B.7 Quality Control Standards for Selected Analyses for Recra (cont.)

\begin{tabular}{|c|c|c|c|c|}
\hline Analyte & $\begin{array}{l}\text { Certified } \\
\text { Value }\end{array}$ & $\begin{array}{l}\text { Performance } \\
\text { Acceptance Limits }\end{array}$ & Recra Result & FG Code \\
\hline \multicolumn{5}{|l|}{ Trace Metals $($ Lot 9987) $(\mu \mathrm{g} / \mathrm{L})$} \\
\hline Aluminum & 233 & $191-275$ & 308 & \\
\hline Antimony & 192 & $144-240$ & 200 & \\
\hline Arsenic & 117 & $87.8-138$ & 118 & \\
\hline Barium & 617 & $506-728$ & 623 & \\
\hline Beryllium & 105 & $86.1-124$ & 101 & \\
\hline Boron & 641 & $526-801$ & 660 & \\
\hline Cadmium & 175 & $144-207$ & 176 & \\
\hline Chromium & 242 & $198-285$ & 241 & \\
\hline Cobalt & 517 & $424-610$ & 521 & \\
\hline Copper & 275 & $226-325$ & 269 & \\
\hline Iron & 467 & $383-551$ & 461 & \\
\hline Lead & 433 & $355-511$ & 441 & \\
\hline Manganese & 558 & $458-658$ & 576 & \\
\hline Mercury & 6.67 & $5.00-8.34$ & 6.58 & \\
\hline Molybdenum & 142 & $116-168$ & 144 & \\
\hline Nickel & 342 & $280-404$ & 350 & \\
\hline Selenium & 139 & $104-164$ & 148 & \\
\hline Silver & 183 & $150-216$ & 183 & \\
\hline Strontium & 533 & $437-629$ & 531 & \\
\hline Thallium & 108 & $81.0-135$ & 110 & \\
\hline Vanadium & 458 & $376-540$ & 454 & \\
\hline Zinc & 117 & 95.9-138 & 119 & \\
\hline \multicolumn{5}{|l|}{ Turbidity (Lot 3427) (NTU) } \\
\hline Turbidity & 3.36 & $2.86-3.93$ & 3.30 & \\
\hline \multicolumn{5}{|l|}{ Volatiles $($ Lot 586) $(\mu \mathrm{g} / \mathrm{L})$} \\
\hline Benzene & 110 & \begin{tabular}{|l|}
$85.4-137$ \\
\end{tabular} & 134 & \\
\hline Bromodichloromethane & 61.3 & $47.1-76.3$ & 75.6 & \\
\hline Bromoform & 86.8 & $63.5-112$ & 123 & \\
\hline Carbon tetrachloride & 175 & $129-218$ & 194 & \\
\hline Chlorobenzene & 78.1 & $61.1-93.7$ & 99.2 & \\
\hline Chloroform & 96.1 & $73.7-117$ & 116 & \\
\hline Dibromochloromethane & 15.9 & $12.4-19.6$ & 20.7 & \\
\hline 1,2-Dichlorobenzene & 62.6 & $47.5-76.9$ & 69.8 & \\
\hline 1,3-Dichlorobenzene & 127 & $97.0-153$ & 167 & \\
\hline 1,4-Dichlorobenzene & 57.6 & $43.3-70.2$ & 78.0 & \\
\hline 1,2-Dichloroethane & 60.1 & $46.9-75.7$ & 77.3 & \\
\hline Dichloromethane (methylene chloride) & 60.8 & $43.0-79.2$ & 64.8 & \\
\hline Ethylbenzene & 49.3 & $36.9-57.6$ & 63.2 & \\
\hline 4-Methyl-2-pentanone (MIBK) & 63.5 & $36.7-86.7$ & 76.2 & \\
\hline
\end{tabular}

Data Summary Report for Savannah River Integrator Operable Unit Fish Tissue 
Table B.7 Quality Control Standards for Selected Analyses for Recra (cont.)

\begin{tabular}{|l|l|l|l|l|}
\hline Analyte & $\begin{array}{l}\text { Certified } \\
\text { Value }\end{array}$ & $\begin{array}{l}\text { Performance } \\
\text { Acceptance Limits }\end{array}$ & Recra Result & FG Code \\
\hline $1,1,2,2$-Tetrachloroethane & 81.0 & $59.0-102$ & $\mathbf{1 1 1}$ & \\
\hline Tetrachloroethylene & 64.3 & $47.4-77.6$ & 74.5 & \\
\hline Toluene & 84.4 & $65.1-102$ & $\mathbf{1 0 4}$ & \\
\hline $1,1,1$-Trichloroethane & 59.0 & $42.6-70.4$ & 66.1 & \\
\hline $1,1,2$-Trichloroethane & 36.4 & $27.7-45.7$ & $\mathbf{4 7 . 4}$ & \\
\hline Trichloroethylene & 37.6 & $27.9-45.5$ & 42.7 & \\
\hline m/p-Xylene & 41.2 & $26.6-51.8$ & $\mathbf{5 2 . 2}$ & \\
\hline
\end{tabular}

\section{B.3 Mixed Analyte Performance Evaluation Program}

The Department of Energy mixed analyte performance evaluation program provides proficiency samples containing metals, pesticides, and radionuclides. The results from the June 1996 MAPEP-96-S3 study were reported in December 1996. For GE, copper was not acceptable and nickel was reported at 23.4 $\mathrm{mg} / \mathrm{kg}$ although it was not included in the original sample. Five of the eight pesticides had a negative bias and were not acceptable; 4,4'-DDE and methoxychlor were not reported. For Recra, all the metals were within the acceptance limits. Four of the eight pesticides were not acceptable, three were acceptable, and methoxychlor was not reported. For Environmental Physics, Inc. (EP), all eight radionuclides were acceptable, but plutonium-238 was close to the upper acceptance limit and given a warning flag. For Thermo NUtech (TNU), seven of eight radionuclides were within the acceptance limits; however the result for strontium-90 was not acceptable.

Methoxychlor was correctly identified by only approximately $50 \%$ of the laboratories and was reported at levels significantly below the reference value.

The results for GE and Recra are reported in table B.8 and for EP and TNU in table B.9.

The results for MAPEP-96-W4 were reported in April 1997. For QST, the results for 4-methylphenol and phenol were outside the acceptance limits. The results are reported in table B.10.

Throughout section B.3, NR means that a result was not reported by the laboratory. The following indicators appear in the tables:

- Bold indicates that the reported values were outside acceptance limits.

- Bold italic indicates that a warning was given to the laboratory. 
Table B.8 Mixed Analyte Performance Evaluation Program Results for MAPEP-96-S3 for GE and Recra

\begin{tabular}{|c|c|c|c|c|}
\hline Analyte & GE Result & Recra Result & $\begin{array}{l}\text { Reference } \\
\text { Value }\end{array}$ & Acceptance Limits \\
\hline \multicolumn{5}{|l|}{ Metals (mg/kg) } \\
\hline Aluminum & 15300 & 15000 & 19100 & 13370-24830 \\
\hline Arsenic & 39.1 & 40 & 35.6 & $24.92-46.28$ \\
\hline Barium & 260 & 256 & 282 & $197.4-366.6$ \\
\hline Cadmium & 7.34 & 6.41 & 7.93 & $5.55-10.31$ \\
\hline Calcium & 64700 & 63400 & 68500 & $47950-89050$ \\
\hline Chromium & 47.8 & 46.5 & 48.9 & $34.23-63.57$ \\
\hline Copper & 26.0 & 18.0 & 15.9 & $11.13-20.67$ \\
\hline Iron & 18300 & 17700 & 18300 & $12810-23790$ \\
\hline Lead & 51.8 & 50.3 & 49.4 & $34.58-64.22$ \\
\hline Magnesium & 8890 & 8970 & 9650 & $6755-12545$ \\
\hline Manganese & 277 & 277 & 286 & $200.2-371.8$ \\
\hline Nickel & 23.4 & Not found & 0.00 & $0.00-0.00$ \\
\hline Potassium & 3340 & 3470 & 4050 & $2835-5265$ \\
\hline Selenium & 7.49 & 8.14 & 6.90 & $4.83-8.97$ \\
\hline Silver & 36.4 & 36.6 & 32.5 & $22.75-42.25$ \\
\hline Sodium & 458 & 491 & 514 & $359.8-668.2$ \\
\hline Vanadium & 79.8 & 76.3 & 87.3 & $61.11-113.49$ \\
\hline Zinc & 66.0 & 67.5 & 69.5 & $48.65-90.35$ \\
\hline \multicolumn{5}{|l|}{ Pesticides $(\mu \mathrm{g} / \mathrm{kg})$} \\
\hline Aldrin & 32.4 & 84 & 137.5 & $96.25-178.75$ \\
\hline alpha-Benzene hexachloride & 48.6 & 120 & 179.6 & $125.72-233.48$ \\
\hline delta-Benzene hexachloride & 18.4 & 52 & 79.0 & $55.3-102.7$ \\
\hline $4,4^{\prime}-\mathrm{DDE}$ & NR & 32 & 40.0 & $28.0-52.0$ \\
\hline Dieldrin & 49.6 & 71 & 97.5 & $68.25-126.75$ \\
\hline Endosulfan II & 37.3 & 31 & 42.9 & $30.03-55.77$ \\
\hline Endrin aldehyde & 34.1 & 49 & 89.7 & $62.79-116.61$ \\
\hline Methoxychlor & NR & NR & 42.1 & $29.47-54.73$ \\
\hline
\end{tabular}

Table B.9 Mixed Analyte Performance Evaluation Program Results for MAPEP-96-S3 for EP and TNU

\begin{tabular}{|c|c|c|c|c|}
\hline Analyte & EP Result & TNU Result & $\begin{array}{l}\text { Reference } \\
\text { Value }\end{array}$ & $\begin{array}{l}\text { Acceptance } \\
\text { Limits }\end{array}$ \\
\hline \multicolumn{5}{|c|}{ Radionuclides (Bq/kg) } \\
\hline Americium-241 & 29.0 & 28.7 & 28.7 & $20.09-37.31$ \\
\hline Cesium-137 & 1770 & 1359 & 1531 & $1071.7-1990.3$ \\
\hline Cobalt-60 & 931 & 727 & 812 & $568.4-1055.6$ \\
\hline Plutonium-238 & 20.5 & 17.3 & 15.9 & $11.13-20.67$ \\
\hline
\end{tabular}

Data Summary Report for Savannah River Integrator Operable Unit Fish Tissue

(WSRC-TR-99-00386) 


\begin{tabular}{|l|l|l|l|l|}
\hline Analyte & EP Result & TNU Result & $\begin{array}{l}\text { Reference } \\
\text { Value }\end{array}$ & $\begin{array}{l}\text { Acceptance } \\
\text { Limits }\end{array}$ \\
\hline Plutonium-239/240 & 22.6 & 18.9 & 19.7 & $13.79-25.61$ \\
\hline Strontium-90 & 540 & $\mathbf{3 4 7}$ & 536.0 & $375.2-696.8$ \\
\hline Uranium-233/234 & 56.6 & 59.7 & 63.9 & $44.73-83.07$ \\
\hline Uranium-238 & 52.9 & 59.7 & 64.0 & $44.80-83.20$ \\
\hline
\end{tabular}

Table B.10 Mixed Analyte Performance Evaluation Program Results for MAPEP-96-W4 for QST

\begin{tabular}{|l|l|l|l|}
\hline Analyte & QST Result & Reference Value & Acceptance Limits \\
\hline \multicolumn{4}{|l|}{} \\
\hline Metals (mg/L) & 1.03 & 1.00 & $0.70-1.30$ \\
\hline Antimony & 1.01 & 1.00 & $0.70-1.30$ \\
\hline Arsenic & 10.8 & 10.2 & $7.14-13.26$ \\
\hline Barium & 1.05 & 1.00 & $0.70-1.30$ \\
\hline Beryllium & 0.251 & 0.260 & $0.18-0.34$ \\
\hline Cadmium & 1.01 & 1.00 & $0.70-1.30$ \\
\hline Chromium & 0.732 & 0.720 & $0.50-0.94$ \\
\hline Lead & 2.93 & 3.10 & $2.17-4.03$ \\
\hline Nickel & 0.271 & 0.260 & $0.18-0.34$ \\
\hline Selenium & 0.512 & 0.510 & $0.36-0.66$ \\
\hline Silver & 1.05 & 1.00 & $0.70-1.30$ \\
\hline Thallium & 2.09 & 2.00 & $1.40-2.60$ \\
\hline Vanadium & 2.88 & 3.10 & $2.17-4.03$ \\
\hline Zinc & & & \\
\hline Radionuclides $(\mathbf{B q} / \mathbf{L})$ & 0.79 & 0.90 & $0.63-1.17$ \\
\hline Americium-241 & 212 & 201 & $140.7-261.3$ \\
\hline Cesium-137 & 217 & 222 & $155.4-288.6$ \\
\hline Cobalt-57 & 269 & 267 & $186.9-347.1$ \\
\hline Cobalt-60 & 1.12 & 1.219 & $0.85-1.59$ \\
\hline Plutonium-238 & 1.35 & 1.495 & $1.05-1.95$ \\
\hline Plutonium-239/240 & 31.06 & 26.38 & $18.47-34.29$ \\
\hline Strontium-90 & 0.40 & 0.402 & $0.28-0.52$ \\
\hline Uranium-233/234 & 0.42 & 0.417 & $0.29-0.55$ \\
\hline Uranium-238 & \multicolumn{2}{|l|}{} \\
\hline
\end{tabular}

Table B.10 Mixed Analyte Performance Evaluation Program Results for MAPEP-96-W4 for QST (cont.)

\begin{tabular}{|l|l|l|l|}
\hline Analyte & QST Result & Reference Value & Acceptance Limits \\
\hline Zinc-65 & 425 & 403 & $282.1-523.9$ \\
\hline Semivolatile Organics $(\boldsymbol{\mu g} / \mathbf{L})$ & \multicolumn{3}{|l|}{} \\
\hline Anthracene & 250 & 295.1 & $206.57-383.63$ \\
\hline Benzo $[a]$ anthracene & 540 & 566.4 & $396.48-736.32$ \\
\hline Benzo $[a]$ pyrene & 430 & 463.8 & $324.66-602.94$ \\
\hline \hline
\end{tabular}

Data Summary Report for Savannah River Integrator Operable Unit Fish Tissue

(WSRC-TR-99-00386) 
Table B.10 Mixed Analyte Performance Evaluation Program Results for MAPEP-96-W4 for QST (cont.)

\begin{tabular}{|l|l|l|l|}
\hline Analyte & QST Result & Reference Value & Acceptance Limits \\
\hline Butylbenzyl phthalate & 540 & 489.6 & $342.72-636.48$ \\
\hline 2-Chlorophenol & 930 & 797.4 & $558.18-1036.62$ \\
\hline Di-n-butyl phthalate & 510 & 496.6 & $347.62-645.58$ \\
\hline 1,3-Dichlorobenzene & 460 & 400.5 & $280.35-520.65$ \\
\hline Diethyl phthalate & 510 & 458.8 & $321.16-596.44$ \\
\hline 2,4-Dimethylphenol & 660 & 582.1 & $407.47-756.73$ \\
\hline Di-n-octyl phthalate & 460 & 477 & $333.9-620.1$ \\
\hline Hexachlorobenzene & 210 & 254.5 & $178.15-330.85$ \\
\hline 2-Methylphenol & 21 & 17.6 & $12.32-22.88$ \\
\hline 3-Methylphenol & $\mathrm{NR}$ & 768.5 & $537.95-999.05$ \\
\hline 4-Methylphenol & $\mathbf{1 1 0 0}$ & 768.5 & $537.95-999.05$ \\
\hline Naphthalene & 330 & 305.2 & $213.64-396.76$ \\
\hline Phenanthrene & 260 & 281.9 & $197.33-366.47$ \\
\hline Phenol & $\mathbf{7 3 0}$ & 509.6 & $356.72-662.48$ \\
\hline Pyrene & 450 & 514.8 & $360.36-669.24$ \\
\hline 2,4,5-Trichlorophenol & 450 & & $287.77-534.43$ \\
\hline
\end{tabular}

\section{B.4 EPA Radiological Performance Evaluation Studies}

EP and QR participated in the EPA radiological performance evaluation studies administered by the Characterization Research Division-Las Vegas. The studies included in this order are the following:

- $\quad$ Tritium in Water, August 9, 1996

- Blind-A, October 15, 1996

- Blind B, October 15, 1996

- Gamma in Water, November 8, 1996

- Strontium in Water, January 17, 1997

- Gross Alpha-Beta in Water, January 31, 1997

- $\quad$ Iodine in Water, February 7, 1997

- Uranium-Radium in Water, February 14, 1997

For tritium in water (8/9/96), the results were $9,613 \mathrm{pCi} / \mathrm{L}$ for $\mathrm{EP}$ and $10,707 \mathrm{pCi} / \mathrm{L}$ for $\mathrm{QR}$. The EPA reference value was $10,879 \mathrm{pCi} / \mathrm{L}$ and the acceptance limits were $8,991-12,767 \mathrm{pCi} / \mathrm{L}$.

In the following tables, all results are reported in $\mathrm{pCi} / \mathrm{L}$. Bold indicates that the reported values were out of range. 
Table B.11 Blind-A Results for EP and QR (10/15/96)

\begin{tabular}{|l|l|l|l|l|}
\hline Analyte & EP Result & QR Result & $\begin{array}{l}\text { Reference } \\
\text { Value }\end{array}$ & $\begin{array}{l}\text { Acceptance } \\
\text { Limits }\end{array}$ \\
\hline Gross Alpha & 65.27 & 48.87 & 59.1 & $33.4-84.8$ \\
\hline Radium-226 & 9.40 & 9.60 & 9.9 & $7.3-12.5$ \\
\hline Radium-228 & 4.67 & 4.57 & 5.1 & $2.8-7.4$ \\
\hline Uranium (natural) & 37.33 & 40.10 & 40.9 & $33.8-48.0$ \\
\hline
\end{tabular}

Table B.12 Blind-B Results for EP and QR (10/15/96)

\begin{tabular}{|l|l|l|l|l|}
\hline Analyte & EP Result & QR Result & $\begin{array}{l}\text { Reference } \\
\text { Value }\end{array}$ & $\begin{array}{l}\text { Acceptance } \\
\text { Limits }\end{array}$ \\
\hline Cesium-134 & 18.00 & 18.00 & 20.0 & $11.3-28.7$ \\
\hline Cesium-137 & 31.33 & 30.67 & 30.0 & $21.3-38.7$ \\
\hline Cobalt-60 & 15.33 & 15.33 & 15.0 & $6.3-23.7$ \\
\hline Gross Beta & $\mathbf{4 3 . 4 3}$ & 119.67 & 111.8 & $82.7-140.9$ \\
\hline Strontium-89 & 11.67 & 16.67 & 10.0 & $1.3-18.7$ \\
\hline Strontium-90 & 24.67 & 21.33 & 25.0 & $16.3-33.7$ \\
\hline
\end{tabular}

Table B.13 Gamma in Water Results for EP and QR (11/8/96)

\begin{tabular}{|l|l|l|l|l|}
\hline Analyte & EP Result & QR Result & $\begin{array}{l}\text { Reference } \\
\text { Value }\end{array}$ & $\begin{array}{l}\text { Acceptance } \\
\text { Limits }\end{array}$ \\
\hline Cobalt-60 & 45.67 & 43.33 & 44.0 & $35.3-52.7$ \\
\hline Zinc-65 & 39.33 & 36.67 & 35.0 & $26.3-43.7$ \\
\hline Cesium-134 & 10.67 & 9.33 & 11.0 & $2.3-19.7$ \\
\hline Cesium-137 & 22.67 & 20.33 & 19.0 & $10.3-27.7$ \\
\hline Barium-133 & 64.67 & 61.00 & 64.0 & $53.6-74.4$ \\
\hline
\end{tabular}

Table B.14 Strontium in Water Results for EP and QR (1/17/97)

\begin{tabular}{|l|l|l|l|l|}
\hline Analyte & EP Result & QR Result & $\begin{array}{l}\text { Reference } \\
\text { Value }\end{array}$ & $\begin{array}{l}\text { Acceptance } \\
\text { Limits }\end{array}$ \\
\hline Strontium-89 & 8.67 & 11.00 & 12.0 & $3.3-20.7$ \\
\hline Strontium-90 & 26.33 & 24.33 & 25.0 & $16.3-33.7$ \\
\hline
\end{tabular}

Table B.15 Gross Alpha-Beta in Water Results for EP and QR (1/31/97)

\begin{tabular}{|l|l|l|l|l|}
\hline Analyte & EP Result & QR Result & $\begin{array}{l}\text { Reference } \\
\text { Value }\end{array}$ & $\begin{array}{l}\text { Acceptance } \\
\text { Limits }\end{array}$ \\
\hline Gross alpha & 4.83 & 4.60 & 5.2 & $0.0-13.9$ \\
\hline Gross beta & 14.23 & 17.40 & 14.7 & $6.0-23.4$ \\
\hline
\end{tabular}

For iodine in water (2/7/97), both EP and QR reported results of $88.00 \mathrm{pCi} / \mathrm{L}$. The EPA reference value was $86.0 \mathrm{pCi} / \mathrm{L}$ and the acceptance limits were $70.4-101.6 \mathrm{pCi} / \mathrm{L}$.

Data Summary Report for Savannah River Integrator Operable Unit Fish Tissue

(WSRC-TR-99-00386) 
Table B.16 Uranium-Radium in Water Results for EP and QR (2/14/97)

\begin{tabular}{|l|l|l|l|l|}
\hline Analyte & EP Result & QR Result & $\begin{array}{l}\text { Reference } \\
\text { Value }\end{array}$ & Acceptance Limits \\
\hline Radium-226 & 5.17 & 5.90 & 5.9 & $4.3-7.5$ \\
\hline Radium-228 & 7.30 & 8.33 & 8.2 & $4.6-11.8$ \\
\hline Uranium (natural) & 32.07 & 27.8 & 27.0 & $21.8-32.2$ \\
\hline
\end{tabular}


This page was left blank intentionally. 


\section{Appendix C: Formats of the Field and Laboratory QC Codes}

The formats presented in this appendix have been established by WSRC-EPD/EMS to identify field and laboratory QC samples.

Table C. 1 contains the codes for laboratory QC samples.

Table C.1 AN98 Laboratory QC Samples Codes

\begin{tabular}{|l|l|}
\hline Code & Definition \\
\hline (blank) & Data not remarked. The result is a routine analysis of a sample. \\
\hline 1 & Laboratory duplicate \\
\hline 2A & Matrix spike \\
\hline 2B & Matrix spike duplicate \\
\hline 2C & Surrogate spike \\
\hline 2D & Laboratory control sample \\
\hline 2E & Laboratory control sample duplicate \\
\hline 2F & Radiochemical tracer \\
\hline 2G & Radiochemical carrier \\
\hline 3 & Laboratory blank \\
\hline 4 & Reanalysis of a sample at the request of EPD/EMS \\
\hline CCV & Continuing calibration verification standard \\
\hline CCB & Continuing calibration blank \\
\hline ICS & Interference check sample \\
\hline ICV & Initial calibration verification standard \\
\hline ICB & Initial calibration blank \\
\hline ICSA & Interference Check Standard A \\
\hline ICSB & Interference Check Standard AB \\
\hline
\end{tabular}

Table C.2 contains the AN98 field QC codes.

\section{Table C.2 AN98 Field QC Codes}

\begin{tabular}{|l|l|}
\hline Code & Description \\
\hline A & Field duplicate \\
\hline B & Trip blank \\
\hline C & Field blank \\
\hline D & Split sample \\
\hline E & Rinsate blank \\
\hline F & Matrix spike performance evaluation standard \\
\hline G & Blank spike performance evaluation standard \\
\hline R & Resampled \\
\hline
\end{tabular}


This page was left blank intentionally. 


\section{Appendix D: Data Tables}

\section{D.1 Qualifiers}

The following qualifiers may have been applied to the analytical results during data validation.

\section{Table D.1 AN98 EPA Functional Guideline Codes}

\begin{tabular}{|c|c|}
\hline Qualifier & Description \\
\hline (Blank) & Data not remarked. The analytical result is acceptable for use as reported. \\
\hline $\mathrm{J}$ & $\begin{array}{l}\text { The analyte was positively identified; the associated numerical value is an estimated } \\
\text { concentration of the analyte in the sample. }\end{array}$ \\
\hline $\mathrm{N}$ & $\begin{array}{l}\text { The analysis indicates the presence of an analyte for which there is presumptive evidence to } \\
\text { make a tentative identification. Used for all TIC results. }\end{array}$ \\
\hline $\mathrm{R}$ & $\begin{array}{l}\text { The sample results are rejected due to serious deficiencies in the ability to analyze the } \\
\text { sample and meet quality control criteria. The presence or absence of the analyte cannot be } \\
\text { verified. Assignment of } R \text { requires approval by the appropriate WSRC data validation } \\
\text { coordinator. }\end{array}$ \\
\hline $\mathrm{U}$ & Material analyzed for, but not detected. The analyte concentration is $<$ ssEQL. \\
\hline NJ & $\begin{array}{l}\text { The analysis indicates the presence of an analyte that has been tentatively identified and the } \\
\text { associated numerical value represents its approximate concentration. }\end{array}$ \\
\hline UJ & $\begin{array}{l}\text { The analyte was not detected above the reported sample quantitation limit. The reported } \\
\text { quantitation limit is approximate, and may not represent the actual limit of quantitation } \\
\text { necessary to accurately and precisely measure the analyte in the sample. }\end{array}$ \\
\hline
\end{tabular}

Only EPA STORET qualifiers in table D.2 are to appear in the appropriate AN98 field.

Table D.2 AN98 EPA STORET Codes Used by EMS

\begin{tabular}{|l|l|}
\hline Qualifier & Description \\
\hline (Blank) & Data not remarked \\
\hline A & The result is the mean of two or more results. \\
\hline C & The result is calculated. \\
\hline D & Field measurement \\
\hline G & The result reported is the maximum of two or more results. \\
\hline H & The result is from a field kit determination and may not be accurate. \\
\hline
\end{tabular}

Table D2 AN98 EPA STORET Codes Used by EMS (cont.)

\begin{tabular}{|l|l|}
\hline Qualifier & Description \\
\hline I & The result is less than the ssEQL, but equal to or greater than the MDL. \\
\hline K & The actual concentration is known to be less than the reported result. \\
\hline L & The actual concentration is known to be greater than the reported result. \\
\hline M & Indicates male of the species \\
\hline
\end{tabular}


Table D2 AN98 EPA STORET Codes Used by EMS (cont.)

\begin{tabular}{|l|l|}
\hline Qualifier & Description \\
\hline O & Sample received by laboratory, but the analysis was lost or not performed. \\
\hline Q & The sample was held beyond the normal holding time prior to analysis. \\
\hline S & Laboratory test \\
\hline U & Undetermined sex of the species \\
\hline V & The analyte was detected in both the method blank and the sample. \\
\hline Y & $\begin{array}{l}\text { The result is from an unpreserved or incorrectly preserved sample; the data may not be } \\
\text { accurate. }\end{array}$ \\
\hline
\end{tabular}

Table D.3 shows the AN98 EMS codes, which are defined by EMS/EGG and are used to aid the validators in qualifying the data.

\section{Table D.3 AN98 EMS Codes}

\begin{tabular}{|c|c|}
\hline Code & Description \\
\hline (Blank) & Data not remarked. The analytical result is acceptable for use as reported. \\
\hline A & Compound identification criteria were not met. \\
\hline $\mathrm{B}$ & Not currently used \\
\hline $\mathrm{C}$ & LCS or BS criteria were not met. \\
\hline $\mathrm{D}$ & ICP serial dilution criteria were not met. \\
\hline $\mathrm{E}$ & Not currently used \\
\hline $\mathrm{F}$ & Not currently used \\
\hline G & Not currently used \\
\hline $\mathrm{H}$ & Internal standard criteria were not met when the IS was used for quantitation. \\
\hline $\mathrm{I}$ & Matrix spike recovery was not within the control limits. \\
\hline $\mathrm{K}$ & A tentatively identified compound is a suspected aldol-condensation product. \\
\hline $\mathrm{L}$ & Initial or continuing calibration criteria were not met. \\
\hline $\mathrm{M}$ & Not currently used \\
\hline $\mathrm{N}$ & Not currently used \\
\hline $\mathrm{O}$ & Surrogate or tracer spike recovery is out of specification. \\
\hline $\bar{P}$ & $\begin{array}{l}\text { Graphite furnace atomic absorption QC } \\
\text { a. Duplicate injection criteria were not met. } \\
\text { b. Post-digestion spike recovery was not within control limits and the sample absorbance is } \\
>50 \% \text { of the post-digestion spike absorbance. }\end{array}$ \\
\hline Q & Not currently used \\
\hline $\mathrm{R}$ & Not currently used \\
\hline $\mathrm{S}$ & The sample was analyzed by the method of standard additions. \\
\hline $\mathrm{T}$ & Not currently used \\
\hline
\end{tabular}

Table D.3 AN98 EMS Codes (cont.)

\begin{tabular}{|l|l|}
\hline Code & Description \\
\hline $\mathrm{U}$ & Not currently used \\
\hline
\end{tabular}

Data Summary Report for Savannah River Integrator Operable Unit Fish Tissue (WSRC-TR-99-00386) 


\begin{tabular}{|l|l|}
\hline Code & Description \\
\hline V & Not currently used \\
\hline W & $\begin{array}{l}\text { Graphite furnace atomic absorption QC: the post-digestion spike recovery is not within } \\
\text { control limits and the sample absorbance is < 50\% of the post-digestion spike absorbance. }\end{array}$ \\
\hline X & The laboratory duplicate RPD or MS/MSD RPD was not within control limits. \\
\hline Y & Not currently used \\
\hline Z & Not currently used \\
\hline 4 & Matrix interference is present. \\
\hline 5 & $\begin{array}{l}\text { Matrix spike concentration was }<0.25 \times \text { the sample concentration, and the percent recovery } \\
\text { cannot be determined. }\end{array}$ \\
\hline 6 & The analyte was detected in both the sample and associated field blank. \\
\hline 7 & The analyte was detected in both the sample and associated rinsate. \\
\hline 8 & The analyte was detected in both the sample and associated trip blank. \\
\hline 9 & The field duplicate RPD was not within control limits. \\
\hline
\end{tabular}

\section{D.2 Data Summaries}

The remainder of this appendix contains data tables.

Table D.4 Number of Analytical Records by Method and Type

Table D.5 Analytes Not Detected in Fish Samples

Table D.6 Analytical Results Sorted by Sample

Table D.7 Results for Blanks Sorted by Sample

Table D.8 Analytical Results Sorted by Analyte

Table D.9 Results for Blanks Sorted by Analyte 
Table D.4 Number of Analytical Records by Method and Type

\begin{tabular}{|c|c|c|c|c|c|c|}
\hline $\begin{array}{l}\text { Analysis } \\
\text { Method }\end{array}$ & $\begin{array}{l}\text { Sample } \\
\text { Records }\end{array}$ & $\begin{array}{l}\text { Laboratory } \\
\text { Duplicates }\end{array}$ & Laboratory Blanks & $\begin{array}{l}\text { Matrix Spikes/ } \\
\text { Duplicates }\end{array}$ & $\begin{array}{l}\text { Calibration } \\
\text { Blanks }\end{array}$ & Totals \\
\hline \multicolumn{7}{|c|}{ Solids (Fauna and Method Blanks) } \\
\hline EPA6010B & 364 & 0 & 24 & 48 & 0 & 436 \\
\hline EPA7471A & 26 & 0 & 3 & 6 & 0 & 35 \\
\hline EPA8260B & 884 & 0 & 136 & 20 & 0 & 1,040 \\
\hline EPA905 & 26 & 2 & 2 & 2 & 0 & 32 \\
\hline EPIA-001B & 52 & 4 & 4 & 4 & 0 & 64 \\
\hline EPIA-002B & 26 & 2 & 2 & 2 & 0 & 32 \\
\hline EPIA-011B & 78 & 6 & 6 & 2 & 0 & 92 \\
\hline EPIA-012B & 52 & 4 & 4 & 2 & 0 & 62 \\
\hline EPIA-013B & 650 & 50 & 50 & 0 & 0 & 750 \\
\hline \multicolumn{7}{|c|}{ Water (Trip Blanks) } \\
\hline EPA8260B & 102 & 0 & 68 & 10 & 0 & 180 \\
\hline
\end{tabular}


Table D.5 Analytes Not Detected in Fish Samples

\begin{tabular}{|c|c|c|c|}
\hline Analyte & Minimum ssMDL & Maximum ssMDL & Unit \\
\hline Antimony-124 & 0.042 & 0.107 & $\mathrm{pCi} / \mathrm{g}$ \\
\hline Antimony-125 & 0.075 & 0.179 & $\mathrm{pCi} / \mathrm{g}$ \\
\hline Antimony & 0.079 & 0.191 & $\mathrm{mg} / \mathrm{kg}$ \\
\hline Barium-133 & 0.034 & 0.078 & $\mathrm{pCi} / \mathrm{g}$ \\
\hline Bromodichloromethane & 0.500 & 0.500 & $\mu \mathrm{g} / \mathrm{kg}$ \\
\hline Bromoform & 1.350 & 1.350 & $\mu \mathrm{g} / \mathrm{kg}$ \\
\hline Bromomethane (Methyl bromide) & 1.500 & 1.500 & $\mu \mathrm{g} / \mathrm{kg}$ \\
\hline Carbon tetrachloride & 2.500 & 2.500 & $\mu \mathrm{g} / \mathrm{kg}$ \\
\hline Cesium-134 & 0.032 & 0.071 & $\mathrm{pCi} / \mathrm{g}$ \\
\hline Chlorobenzene & 1.500 & 1.500 & $\mu \mathrm{g} / \mathrm{kg}$ \\
\hline Chloroethane & 1.500 & 1.500 & $\mu \mathrm{g} / \mathrm{kg}$ \\
\hline Chloroethene (Vinyl chloride) & 2.000 & 2.000 & $\mu \mathrm{g} / \mathrm{kg}$ \\
\hline Chloroform & 0.500 & 0.500 & $\mu \mathrm{g} / \mathrm{kg}$ \\
\hline Chloromethane (Methyl chloride) & 1.000 & 1.000 & $\mu \mathrm{g} / \mathrm{kg}$ \\
\hline Cobalt-57 & 0.021 & 0.041 & $\mathrm{pCi} / \mathrm{g}$ \\
\hline Cobalt-58 & 0.040 & 0.106 & $\mathrm{pCi} / \mathrm{g}$ \\
\hline Cobalt-60 & 0.035 & 0.088 & $\mathrm{pCi} / \mathrm{g}$ \\
\hline Dibromochloromethane & 1.050 & 1.050 & $\mu \mathrm{g} / \mathrm{kg}$ \\
\hline 1,1-Dichloroethane & 0.500 & 0.500 & $\mu \mathrm{g} / \mathrm{kg}$ \\
\hline 1,2-Dichloroethane & 1.150 & 1.150 & $\mu \mathrm{g} / \mathrm{kg}$ \\
\hline 1,1-Dichloroethylene & 1.500 & 1.500 & $\mu \mathrm{g} / \mathrm{kg}$ \\
\hline 1,2-Dichloroethylene & 1.250 & 1.250 & $\mu \mathrm{g} / \mathrm{kg}$ \\
\hline 1,2-Dichloropropane & 1.150 & 1.150 & $\mu \mathrm{g} / \mathrm{kg}$ \\
\hline cis-1,3-Dichloropropene & 1.000 & 1.000 & $\mu \mathrm{g} / \mathrm{kg}$ \\
\hline trans-1,3-Dichloropropene & 1.500 & 1.500 & $\mu \mathrm{g} / \mathrm{kg}$ \\
\hline Europium-152 & 0.082 & 0.191 & $\mathrm{pCi} / \mathrm{g}$ \\
\hline Europium-154 & 0.090 & 0.235 & $\mathrm{pCi} / \mathrm{g}$ \\
\hline Europium-155 & 0.074 & 0.159 & $\mathrm{pCi} / \mathrm{g}$ \\
\hline Gross alpha & 0.377 & 2.740 & $\mathrm{pCi} / \mathrm{g}$ \\
\hline 2-Hexanone & 14.000 & 14.000 & $\mu \mathrm{g} / \mathrm{kg}$ \\
\hline Manganese-54 & 0.030 & 0.087 & $\mathrm{pCi} / \mathrm{g}$ \\
\hline Methyl isobutyl ketone & 14.600 & 14.600 & $\mu \mathrm{g} / \mathrm{kg}$ \\
\hline Neptunium-239 & 0.141 & 0.304 & $\mathrm{pCi} / \mathrm{g}$ \\
\hline Plutonium-238 & 0.007 & 0.059 & $\mathrm{pCi} / \mathrm{g}$ \\
\hline Plutonium-239/240 & 0.007 & 0.045 & $\mathrm{pCi} / \mathrm{g}$ \\
\hline Promethium-144 & 0.031 & 0.078 & $\mathrm{pCi} / \mathrm{g}$ \\
\hline Promethium-146 & 0.036 & 0.082 & $\mathrm{pCi} / \mathrm{g}$ \\
\hline Ruthenium-106 & 0.277 & 0.751 & $\mathrm{pCi} / \mathrm{g}$ \\
\hline Sodium-22 & 0.032 & 0.085 & $\mathrm{pCi} / \mathrm{g}$ \\
\hline
\end{tabular}


Table D.5 Analytes Not Detected in Fish Samples(cont.)

\begin{tabular}{|l|l|l|l|}
\hline Analyte & Minimum ssMDL & Maximum ssMDL & Unit \\
\hline Styrene & 1.500 & 1.500 & $\mu \mathrm{g} / \mathrm{kg}$ \\
\hline $1,1,2,2-$-Tetrachloroethane & 3.000 & 3.000 & $\mu \mathrm{g} / \mathrm{kg}$ \\
\hline Tetrachloroethylene & 2.000 & 2.000 & $\mu \mathrm{g} / \mathrm{kg}$ \\
\hline Tin-113 & 0.037 & 0.101 & $\mathrm{pCi} / \mathrm{g}$ \\
\hline $1,1,1-$ Trichloroethane & 0.500 & 0.500 & $\mu \mathrm{g} / \mathrm{kg}$ \\
\hline $1,1,2$-Trichloroethane & 1.200 & 1.200 & $\mu \mathrm{g} / \mathrm{kg}$ \\
\hline Trichloroethylene & 1.350 & 1.350 & $\mu \mathrm{g} / \mathrm{kg}$ \\
\hline Uranium-235 & 0.006 & 0.045 & $\mathrm{pCi} / \mathrm{g}$ \\
\hline Vinyl acetate & 10.500 & 10.500 & $\mu \mathrm{g} / \mathrm{kg}$ \\
\hline Yttrium-88 & 0.044 & 0.114 & $\mathrm{pCi} / \mathrm{g}$ \\
\hline Zinc-65 & 0.081 & 0.201 & $\mathrm{pCi} / \mathrm{g}$ \\
\hline Zirconium-95 & 0.068 & 0.188 & $\mathrm{pCi} / \mathrm{g}$ \\
\hline
\end{tabular}


Table D.6 Analytical Results Sorted by Sample

SURVEY ID: SRF-01-01

Sample type: Normal

\section{Constituent}

Metals (total recoverable)
Arsenic

Barium

Cadmium

Calcium

Chromium

Copper

Lead

Magnesium

Manganese

Mercury

Nickel

Selenium

Silver

Zinc

Radionuclides

Actinium-228

Antimony-124

Antimony-125

Barium-133

Cerium-144

Cesium-134

Cesium-137

Cobalt-57

Cobalt-58

Cobalt-60

Europium-152

Europium-154

Europium-155

Gross alpha

Lead-212

Manganese-54

Neptunium-239

Nonvolatile beta

Plutonium-238

Plutonium-239/240

Potassium-40

Promethium-144

Promethium-146

Ruthenium-106

Sodium-22

Strontium-89/90

Tin-1 13

Tritium
Sample ID: 117018

Sample matrix: Fauna

Percent solids: NA
U

FG SC E Result Unit ssMDL

$\mathrm{mg} / \mathrm{kg} \quad 0.174 \quad$ EPA6010B

$\mathrm{mg} / \mathrm{kg} \quad 0.207 \quad$ EPA6010B

$\mathrm{mg} / \mathrm{kg} \quad 0.025 \quad$ EPA6010B

$\mathrm{mg} / \mathrm{kg} \quad 0.017 \quad$ EPA6010B

$\mathrm{mg} / \mathrm{kg} 3.172 \quad$ EPA6010B

$\mathrm{mg} / \mathrm{kg} \quad 0.0331 \quad$ EPA6010B

$\mathrm{mg} / \mathrm{kg} \quad 0.06 \quad$ EPA6010B

$\mathrm{mg} / \mathrm{kg} \quad 0.072 \quad$ EPA6010B

$\mathrm{mg} / \mathrm{kg} \quad 0.231 \quad$ EPA6010B

$\mathrm{mg} / \mathrm{kg} \quad 0.323 \quad$ EPA6010B

$\mathrm{mg} / \mathrm{kg} \quad 0.00225 \quad$ EPA7471A

$\mathrm{mg} / \mathrm{kg} \quad 0.029 \quad$ EPA6010B

$\mathrm{mg} / \mathrm{kg} \quad 0.123 \quad$ EPA6010B

$\mathrm{mg} / \mathrm{kg} \quad 0.0282 \quad$ EPA6010B

$\mathrm{mg} / \mathrm{kg} \quad 0.168$

EPA6010B

$\begin{array}{llll}0.0883 & \mathrm{pCi} / \mathrm{g} & 0.261 & \text { EPIA-013B } \\ -0.0184 & \mathrm{pCi} / \mathrm{g} & 0.072 & \text { EPIA-013B } \\ -0.0158 & \mathrm{pCi} / \mathrm{g} & 0.142 & \text { EPIA-013B } \\ -0.0241 & \mathrm{pCi} / \mathrm{g} & 0.059 & \text { EPIA-013B } \\ 0.0737 & \mathrm{pCi} / \mathrm{g} & 0.275 & \text { EPIA-013B } \\ -0.0232 & \mathrm{pCi} / \mathrm{g} & 0.0487 & \text { EPIA-013B } \\ 0.0207 & \mathrm{pCi} / \mathrm{g} & 0.0583 & \text { EPIA-013B } \\ 0.0125 & \mathrm{pCi} / \mathrm{g} & 0.0334 & \text { EPIA-013B } \\ 0.00701 & \mathrm{pCi} / \mathrm{g} & 0.0673 & \text { EPIA-013B } \\ -0.00754 & \mathrm{pCi} / \mathrm{g} & 0.061 & \text { EPIA-013B } \\ -0.0452 & \mathrm{pCi} / \mathrm{g} & 0.143 & \text { EPIA-013B } \\ -0.014 & \mathrm{pCi} / \mathrm{g} & 0.152 & \text { EPIA-013B } \\ -0.0109 & \mathrm{pCi} / \mathrm{g} & 0.126 & \text { EPIA-013B } \\ -0.0306 & \mathrm{pCi} / \mathrm{g} & 0.733 & \text { EPIA-001B } \\ 0.0379 & \mathrm{pCi} / \mathrm{g} & 0.0783 & \text { EPIA-013B } \\ -0.0118 & \mathrm{pCi} / \mathrm{g} & 0.0484 & \text { EPIA-013B } \\ -0.00946 & \mathrm{pCi} / \mathrm{g} & 0.222 & \text { EPIA-013B } \\ 5.12 & \mathrm{pCi} / \mathrm{g} & 0.817 & \text { EPIA-001B } \\ 0.00334 & \mathrm{pCi} / \mathrm{g} & 0.01 & \text { EPIA-012B } \\ -0.0048 & \mathrm{pCi} / \mathrm{g} & 0.0286 & \text { EPIA-012B } \\ 3.97 & \mathrm{pCi} / \mathrm{g} & 0.611 & \text { EPIA-013B } \\ 0.0151 & \mathrm{pCi} / \mathrm{g} & 0.0622 & \text { EPIA-013B } \\ -0.00415 & \mathrm{pCi} / \mathrm{g} & 0.0672 & \text { EPIA-013B } \\ -0.103 & \mathrm{pCi} / \mathrm{g} & 0.457 & \text { EPIA-013B } \\ -0.00507 & \mathrm{pCi} / \mathrm{g} & 0.0549 & \text { EPIA-013B } \\ 0.428 & \mathrm{pCi} / \mathrm{g} & 0.631 & \text { EPA905 } \\ -0.0116 & \mathrm{pCi} / \mathrm{g} & 0.0698 & \text { EPIA-013B } \\ 0 & \mathrm{pCi} / \mathrm{g} & 0.157 & \text { EPIA-002B }\end{array}$


SURVEY ID: SRF-01-01 (cont.)

Constituent

Radionuclides

Uranium-233/234

Uranium-235

Uranium-238

Yttrium-88

Zinc-65

Zirconium-95

Volatile Organic Compounds

\begin{tabular}{|c|c|c|c|c|}
\hline Acetone & $\mathrm{J}$ & $\mathrm{K}$ & $\mathrm{O}$ & 925 \\
\hline Benzene & $\mathrm{J}$ & $\mathrm{K}$ & $\mathrm{O}$ & 10.3 \\
\hline Bromodichloromethane & $\mathrm{U}$ & & & 5 \\
\hline Bromoform & $\mathrm{U}$ & & & 5 \\
\hline Bromomethane (Methyl bromide) & $\mathrm{U}$ & & & 5 \\
\hline Carbon disulfide & $\mathrm{J}$ & IK & $\mathrm{O}$ & 20.8 \\
\hline Carbon tetrachloride & $\mathrm{U}$ & & & 5 \\
\hline Chlorobenzene & $\mathrm{U}$ & & & 5 \\
\hline Chloroethane & $\mathrm{U}$ & & & 5 \\
\hline Chloroethene (Vinyl chloride) & $\mathrm{U}$ & & & 5 \\
\hline Chloroform & $\mathrm{U}$ & & & 5 \\
\hline Chloromethane (Methyl chloride) & $\mathrm{U}$ & & & 5 \\
\hline Dibromochloromethane & $\mathrm{U}$ & & & 5 \\
\hline 1,1-Dichloroethane & $\mathrm{U}$ & & & 5 \\
\hline 1,2-Dichloroethane & $\mathrm{U}$ & & & 5 \\
\hline 1,1-Dichloroethylene & $\mathrm{U}$ & & & 5 \\
\hline 1,2-Dichloroethylene & $\mathrm{U}$ & & & 50 \\
\hline Dichloromethane (Methylene chloride) & $\mathrm{J}$ & $\mathrm{K}$ & $\mathrm{O}$ & 209 \\
\hline 1,2-Dichloropropane & $\mathrm{U}$ & & & 5 \\
\hline cis-1,3-Dichloropropene & $\mathrm{U}$ & & & 5 \\
\hline trans-1,3-Dichloropropene & $\mathrm{U}$ & & & 5 \\
\hline Ethylbenzene & $\mathrm{J}$ & $\mathrm{K}$ & $\mathrm{O}$ & 8.23 \\
\hline 2-Hexanone & $\mathrm{U}$ & & & 25 \\
\hline Methyl ethyl ketone & $\mathrm{U}$ & & & 25 \\
\hline Methyl isobutyl ketone & $\mathrm{U}$ & & & 25 \\
\hline Styrene & $\mathrm{U}$ & & & 5 \\
\hline $1,1,2,2$-Tetrachloroethane & $\mathrm{U}$ & & & 5 \\
\hline Tetrachloroethylene & $\mathrm{U}$ & & & 5 \\
\hline Toluene & $\mathrm{J}$ & $\mathrm{K}$ & $\mathrm{O}$ & 11.3 \\
\hline 1,1,1-Trichloroethane & $\mathrm{U}$ & & & 5 \\
\hline $1,1,2$-Trichloroethane & $\mathrm{U}$ & & & 5 \\
\hline Trichloroethylene & $\mathrm{U}$ & & & 5 \\
\hline Vinyl acetate & $\mathrm{U}$ & & & 75 \\
\hline Xylenes & $\mathrm{J}$ & $\mathrm{K}$ & $\mathrm{O}$ & 25.8 \\
\hline
\end{tabular}

0.00283
-0.000545
0.00685
0.00144
-0.0125
0.0173

$\begin{array}{ll}\mathrm{pCi} / \mathrm{g} & 0.0205 \\ \mathrm{pCi} / \mathrm{g} & 0.0221 \\ \mathrm{pCi} / \mathrm{g} & 0.0169 \\ \mathrm{pCi} / \mathrm{g} & 0.0803 \\ \mathrm{pCi} / \mathrm{g} & 0.139 \\ \mathrm{pCi} / \mathrm{g} & 0.13\end{array}$

EPIA-011B EPIA-011B EPIA-011B EPIA-013B EPIA-013B EPIA-013B

EPA8260B EPA8260B EPA8260B EPA8260B EPA8260B EPA8260B EPA8260B

EPA8260B EPA8260B EPA8260B EPA8260B EPA8260B EPA8260B EPA8260B EPA8260B EPA8260B EPA8260B

EPA8260B EPA8260B EPA8260B EPA8260B EPA8260B EPA8260B EPA8260B EPA8260B EPA8260B EPA8260B EPA8260B EPA8260B EPA8260B EPA8260B EPA8260B EPA8260B EPA8260B 
SURVEY ID: SRF-01-02

Sample type: Normal

Constituent

Metals (total recoverable)

Antimony
Arsenic
Barium
Cadmium
Calcium
Chromium
Copper
Lead
Magnesium
Manganese
Mercury
Nickel
Selenium
Silver
Zinc

Radionuclides

Actinium-228

Antimony-124

Antimony-125

Barium-133

Cerium-144

Cesium-134

Cesium-137

Cobalt-57

Cobalt-58

Cobalt-60

Europium-152

Europium-154

Europium-155

Gross alpha

Lead-212

Manganese-54

Neptunium-239

Nonvolatile beta

Plutonium-238

Plutonium-239/240

Potassium-40

Promethium-144

Promethium-146

Ruthenium-106

Sodium-22

Strontium-89/90

Tin-1 13

Tritium
Sample ID: 117019

Sample matrix: Fauna

Percent solids: NA

\begin{tabular}{|c|c|c|c|c|}
\hline \multirow[t]{3}{*}{ JU } & 0.205 & $\mathrm{mg} / \mathrm{kg}$ & 0.182 & EPA6010B \\
\hline & 0.621 & $\mathrm{mg} / \mathrm{kg}$ & 0.217 & EPA6010B \\
\hline & 3.93 & $\mathrm{mg} / \mathrm{kg}$ & 0.026 & EPA6010B \\
\hline \multirow[t]{2}{*}{$\mathrm{J}$} & 0.0376 & $\mathrm{mg} / \mathrm{kg}$ & 0.018 & EPA6010B \\
\hline & 11200 & $\mathrm{mg} / \mathrm{kg}$ & 3.319 & EPA6010B \\
\hline \multirow[t]{2}{*}{$\mathrm{J}$} & 0.214 & $\mathrm{mg} / \mathrm{kg}$ & 0.0347 & EPA6010B \\
\hline & 0.731 & $\mathrm{mg} / \mathrm{kg}$ & 0.0629 & EPA6010B \\
\hline \multirow[t]{3}{*}{$\mathrm{J}$} & 0.173 & $\mathrm{mg} / \mathrm{kg}$ & 0.075 & EPA6010B \\
\hline & 357 & $\mathrm{mg} / \mathrm{kg}$ & 0.242 & EPA6010B \\
\hline & 66.6 & $\mathrm{mg} / \mathrm{kg}$ & 0.338 & EPA6010B \\
\hline \multirow[t]{3}{*}{$\mathrm{J}$} & 0.0285 & $\mathrm{mg} / \mathrm{kg}$ & 0.00196 & EPA7471A \\
\hline & 0.276 & $\mathrm{mg} / \mathrm{kg}$ & 0.03 & EPA6010B \\
\hline & 1 & $\mathrm{mg} / \mathrm{kg}$ & 0.129 & EPA6010B \\
\hline \multirow[t]{2}{*}{$\mathrm{J}$} & 0.105 & $\mathrm{mg} / \mathrm{kg}$ & 0.0295 & EPA6010B \\
\hline & 15.2 & $\mathrm{mg} / \mathrm{kg}$ & 0.176 & EPA6010B \\
\hline
\end{tabular}

U

U

U

$\mathrm{U}$

U

$\mathrm{U}$

$\mathrm{R}$

U

U

U

U

$\mathrm{U}$

U

$\mathrm{U}$

$\mathrm{R}$

U

$\mathrm{U}$

$\mathrm{U}$

$\mathrm{U}$

U

U

$\mathrm{U}$

$\mathrm{U}$

U

$\mathrm{U}$

$\mathrm{U}$
Uni

SSMDL

Method

EPA6010B

$\begin{array}{lll}\mathrm{pCi} / \mathrm{g} & 0.196 & \text { EPIA-013B } \\ \mathrm{pCi} / \mathrm{g} & 0.0586 & \text { EPIA-013B }\end{array}$

$\mathrm{pCi} / \mathrm{g} \quad 0.117 \quad$ EPIA-013B

$\begin{array}{lll}\mathrm{pCi} / \mathrm{g} & 0.0481 & \text { EPIA-013B }\end{array}$

$\mathrm{pCi} / \mathrm{g} \quad 0.192 \quad$ EPIA-013B

$\mathrm{pCi} / \mathrm{g} \quad 0.0435 \quad$ EPIA-013B

$\mathrm{pCi} / \mathrm{g} \quad 0.0794 \quad$ EPIA-013B

$\mathrm{pCi} / \mathrm{g} \quad 0.0236 \quad$ EPIA-013B

$\mathrm{pCi} / \mathrm{g} \quad 0.0641 \quad$ EPIA-013B

$\mathrm{pCi} / \mathrm{g} \quad 0.0484 \quad$ EPIA-013B

$\mathrm{pCi} / \mathrm{g} \quad 0.113 \quad$ EPIA-013B

$\mathrm{pCi} / \mathrm{g} \quad 0.18 \quad$ EPIA-013B

$\mathrm{pCi} / \mathrm{g} \quad 0.377 \quad$ EPIA-001B

$\mathrm{pCi} / \mathrm{g} \quad 0.0751 \quad$ EPIA-013B

$\mathrm{pCi} / \mathrm{g} \quad 0.0516 \quad$ EPIA-013B

$\mathrm{pCi} / \mathrm{g} \quad 0.174 \quad$ EPIA-013B

$\mathrm{pCi} / \mathrm{g} \quad 0.0231 \quad$ EPIA-012B

$\mathrm{pCi} / \mathrm{g} \quad 0.0131 \quad$ EPIA-012B

$\mathrm{pCi} / \mathrm{g} \quad 0.487 \quad$ EPIA-013B

$\mathrm{pCi} / \mathrm{g} \quad 0.0449 \quad$ EPIA-013B

$\mathrm{pCi} / \mathrm{g} \quad 0.058 \quad$ EPIA-013B

$\mathrm{pCi} / \mathrm{g} \quad 0.383 \quad$ EPIA-013B

$\mathrm{pCi} / \mathrm{g} \quad 0.0649 \quad$ EPIA-013B

$\mathrm{pCi} / \mathrm{g} \quad 0.826 \quad$ EPA905

$\mathrm{pCi} / \mathrm{g} \quad 0.06 \quad$ EPIA-013B

$\mathrm{pCi} / \mathrm{g} \quad 0.171 \quad$ EPIA-002B
$\mathrm{pCi} / \mathrm{g} \quad 0.0891 \quad$ EPIA-013B

$\mathrm{pCi} / \mathrm{g} \quad 0.579 \quad$ EPIA-001B 
SURVEY ID: SRF-01-02 (cont.)

Constituent

Radionuclides

Uranium-233/234

Uranium-235

Uranium-238

Yttrium-88

Zinc-65

Zirconium-95

Volatile Organic Compounds

\begin{tabular}{|c|c|c|c|c|}
\hline Acetone & $\mathrm{J}$ & $\mathrm{K}$ & $\mathrm{O}$ & 402 \\
\hline Benzene & $\mathrm{J}$ & $\mathrm{K}$ & $\mathrm{O}$ & 5.63 \\
\hline Bromodichloromethane & $\mathrm{U}$ & & & 5 \\
\hline Bromoform & $\mathrm{U}$ & & & 5 \\
\hline Bromomethane (Methyl bromide) & $\mathrm{U}$ & & & 5 \\
\hline Carbon disulfide & $\mathrm{U}$ & & & 25 \\
\hline Carbon tetrachloride & $\mathrm{U}$ & & & 5 \\
\hline Chlorobenzene & $\mathrm{U}$ & & & 5 \\
\hline Chloroethane & $\mathrm{U}$ & & & 5 \\
\hline Chloroethene (Vinyl chloride) & $\mathrm{U}$ & & & 5 \\
\hline Chloroform & $\mathrm{U}$ & & & 5 \\
\hline Chloromethane (Methyl chloride) & $\mathrm{U}$ & & & 5 \\
\hline Dibromochloromethane & $\mathrm{U}$ & & & 5 \\
\hline 1,1-Dichloroethane & $\mathrm{U}$ & & & 5 \\
\hline 1,2-Dichloroethane & $\mathrm{U}$ & & & 5 \\
\hline 1,1-Dichloroethylene & $\mathrm{U}$ & & & 5 \\
\hline 1,2-Dichloroethylene & $\mathrm{U}$ & & & 50 \\
\hline Dichloromethane (Methylene chloride) & $\mathrm{J}$ & $\mathrm{K}$ & $\mathrm{O}$ & 66.7 \\
\hline 1,2-Dichloropropane & $\mathrm{U}$ & & & 5 \\
\hline cis-1,3-Dichloropropene & $\mathrm{U}$ & & & 5 \\
\hline trans-1,3-Dichloropropene & $\mathrm{U}$ & & & 5 \\
\hline Ethylbenzene & $\mathrm{J}$ & $\mathrm{K}$ & $\mathrm{O}$ & 7.6 \\
\hline 2-Hexanone & $\mathrm{U}$ & & & 25 \\
\hline Methyl ethyl ketone & $\mathrm{J}$ & $\mathrm{K}$ & $\mathrm{O}$ & 58.2 \\
\hline Methyl isobutyl ketone & $\mathrm{U}$ & & & 25 \\
\hline Styrene & $\mathrm{U}$ & & & 5 \\
\hline $1,1,2,2$-Tetrachloroethane & $\mathrm{U}$ & & & 5 \\
\hline Tetrachloroethylene & $\mathrm{U}$ & & & 5 \\
\hline Toluene & $\mathrm{J}$ & K & $\mathrm{O}$ & 7.11 \\
\hline 1,1,1-Trichloroethane & $\mathrm{U}$ & & & 5 \\
\hline 1,1,2-Trichloroethane & $\mathrm{U}$ & & & 5 \\
\hline Trichloroethylene & $\mathrm{U}$ & & & 5 \\
\hline Vinyl acetate & $\mathrm{U}$ & & & 75 \\
\hline Xylenes & $\mathrm{J}$ & $\mathrm{K}$ & $\mathrm{O}$ & 33.6 \\
\hline
\end{tabular}

Sample ID: 117019

FG SC E Result

Unit ssMDL

Method

$\begin{array}{lll}\mathrm{pCi} / \mathrm{g} & 0.0256 & \text { EPIA-011B } \\ \mathrm{pCi} / \mathrm{g} & 0.0195 & \text { EPIA-011B } \\ \mathrm{pCi} / \mathrm{g} & 0.0229 & \text { EPIA-011B } \\ \mathrm{pCi} / \mathrm{g} & 0.0656 & \text { EPIA-013B } \\ \mathrm{pCi} / \mathrm{g} & 0.116 & \text { EPIA-013B } \\ \mathrm{pCi} / \mathrm{g} & 0.112 & \text { EPIA-013B }\end{array}$

$\mu \mathrm{g} / \mathrm{kg} \quad 51.5 \quad$ EPA8260B

$\mu \mathrm{g} / \mathrm{kg} \quad 2.5 \quad$ EPA8260B

$\mu \mathrm{g} / \mathrm{kg} \quad 0.5 \quad$ EPA8260B

$\mu \mathrm{g} / \mathrm{kg} \quad 1.35 \quad$ EPA8260B

$\mu \mathrm{g} / \mathrm{kg} \quad 1.5 \quad$ EPA8260B

$\mu \mathrm{g} / \mathrm{kg} \quad 1.5 \quad$ EPA8260B

$\mu \mathrm{g} / \mathrm{kg} \quad 2.5 \quad$ EPA8260B

$\mu \mathrm{g} / \mathrm{kg} \quad 1.5 \quad$ EPA8260B

$\mu \mathrm{g} / \mathrm{kg} \quad 1.5 \quad$ EPA8260B

$\mu \mathrm{g} / \mathrm{kg} \quad 2 \quad$ EPA8260B

$\mu \mathrm{g} / \mathrm{kg} \quad 0.5 \quad$ EPA8260B

$\mu \mathrm{g} / \mathrm{kg} \quad 1 \quad$ EPA8260B

$\mu \mathrm{g} / \mathrm{kg} \quad 1.05 \quad$ EPA8260B

$\mu \mathrm{g} / \mathrm{kg} \quad 0.5 \quad$ EPA8260B

$\mu \mathrm{g} / \mathrm{kg} \quad 1.15 \quad$ EPA8260B

$\mu \mathrm{g} / \mathrm{kg} \quad 1.5 \quad$ EPA8260B

$\mu \mathrm{g} / \mathrm{kg} \quad 1.25 \quad$ EPA8260B

$\mu \mathrm{g} / \mathrm{kg} 7$ EPA8260B

$\mu \mathrm{g} / \mathrm{kg} \quad 1.15 \quad$ EPA8260B

$\mu \mathrm{g} / \mathrm{kg} \quad 1 \quad$ EPA8260B

$\mu \mathrm{g} / \mathrm{kg} \quad 1.5 \quad$ EPA8260B

$\mu \mathrm{g} / \mathrm{kg} \quad 1.5 \quad$ EPA8260B

$\mu \mathrm{g} / \mathrm{kg} \quad 14 \quad$ EPA8260B

$\mu \mathrm{g} / \mathrm{kg} \quad 16 \quad$ EPA8260B

$\mu \mathrm{g} / \mathrm{kg} \quad 14.6 \quad$ EPA8260B

$\mu \mathrm{g} / \mathrm{kg} \quad 1.5 \quad$ EPA8260B

$\mu \mathrm{g} / \mathrm{kg} \quad 3 \quad$ EPA8260B

$\mu \mathrm{g} / \mathrm{kg} \quad 2 \quad$ EPA8260B

$\mu \mathrm{g} / \mathrm{kg} \quad 4.5 \quad$ EPA8260B

$\mu \mathrm{g} / \mathrm{kg} \quad 0.5 \quad$ EPA8260B

$\mu \mathrm{g} / \mathrm{kg} \quad 1.2 \quad$ EPA8260B

$\mu \mathrm{g} / \mathrm{kg} \quad 1.35 \quad$ EPA8260B

$\mu \mathrm{g} / \mathrm{kg} \quad 10.5 \quad$ EPA8260B

$\mu \mathrm{g} / \mathrm{kg} \quad 5 \quad$ EPA8260B 
SURVEY ID: SRF-01-03

Sample type: Normal

Constituent

Metals (total recoverable)

Antimony
Arsenic
Barium
Cadmium
Calcium
Chromium
Copper
Lead
Magnesium
Manganese
Mercury
Nickel
Selenium
Silver
Zinc

$\mathrm{U}$

0.841

3.18

U $\quad 0.227$

$\begin{array}{lll}\text { J } & \text { I } & 0.104\end{array}$

$\begin{array}{ll} & 1.28 \\ \mathrm{U} & 0.227\end{array}$

336

20.1

0.0706

$\mathrm{U}$

0.227

0.925

U $\quad 0.227$

6.96

Radionuclides

Actinium-228

Antimony-124

Antimony-125

Barium-133

Cerium-144

Cesium-134

Cesium-137

Cobalt-57

Cobalt-58

Cobalt-60

Europium-152

Europium-154

Europium-155

Gross alpha

Lead-212

Manganese-54

Neptunium-239

Nonvolatile beta

Plutonium-238

Plutonium-239/240

Potassium-40

Promethium-144

Promethium-146

Ruthenium-106

Sodium-22

Strontium-89/90

Tin-1 13

Tritium

Sample ID: 117020
Percent solids: NA

Method

Unit ssMDL

$\mathrm{mg} / \mathrm{kg} \quad 0.174 \quad$ EPA6010B

$\mathrm{mg} / \mathrm{kg} \quad 0.207 \quad$ EPA6010B

$\mathrm{mg} / \mathrm{kg} \quad 0.025 \quad$ EPA6010B

$\mathrm{mg} / \mathrm{kg} \quad 0.017 \quad$ EPA6010B

$\mathrm{mg} / \mathrm{kg} \quad 3.172 \quad$ EPA6010B

$\mathrm{mg} / \mathrm{kg} \quad 0.0331 \quad$ EPA6010B

$\mathrm{mg} / \mathrm{kg} \quad 0.06 \quad$ EPA6010B

$\mathrm{mg} / \mathrm{kg} \quad 0.072 \quad$ EPA6010B

$\mathrm{mg} / \mathrm{kg} \quad 0.231 \quad$ EPA6010B

$\mathrm{mg} / \mathrm{kg} \quad 0.323 \quad$ EPA6010B

$\mathrm{mg} / \mathrm{kg} \quad 0.002 \quad$ EPA7471A

$\mathrm{mg} / \mathrm{kg} \quad 0.029 \quad$ EPA6010B

$\mathrm{mg} / \mathrm{kg} \quad 0.123 \quad$ EPA6010B

$\mathrm{mg} / \mathrm{kg} \quad 0.0282 \quad$ EPA6010B

$\mathrm{mg} / \mathrm{kg} \quad 0.168 \quad$ EPA6010B

$\begin{array}{lllll}\mathrm{U} & 0.186 & \mathrm{pCi} / \mathrm{g} & 0.233 & \text { EPIA-013B } \\ \mathrm{U} & -0.00905 & \mathrm{pCi} / \mathrm{g} & 0.0638 & \text { EPIA-013B } \\ \mathrm{U} & -0.0509 & \mathrm{pCi} / \mathrm{g} & 0.114 & \text { EPIA-013B } \\ \mathrm{U} & -0.00135 & \mathrm{pCi} / \mathrm{g} & 0.0522 & \text { EPIA-013B } \\ \mathrm{U} & -0.0966 & \mathrm{pCi} / \mathrm{g} & 0.243 & \text { EPIA-013B } \\ \mathrm{U} & -0.000852 & \mathrm{pCi} / \mathrm{g} & 0.044 & \text { EPIA-013B } \\ \mathrm{R} & 0.114 & \mathrm{pCi} / \mathrm{g} & 0.0809 & \text { EPIA-013B } \\ \mathrm{U} & 0.00995 & \mathrm{pCi} / \mathrm{g} & 0.033 & \text { EPIA-013B } \\ \mathrm{U} & 0.0134 & \mathrm{pCi} / \mathrm{g} & 0.062 & \text { EPIA-013B } \\ \mathrm{U} & 0.00821 & \mathrm{pCi} / \mathrm{g} & 0.0672 & \text { EPIA-013B } \\ \mathrm{U} & -0.00701 & \mathrm{pCi} / \mathrm{g} & 0.126 & \text { EPIA-013B } \\ \mathrm{U} & 0.0512 & \mathrm{pCi} / \mathrm{g} & 0.149 & \text { EPIA-013B } \\ \mathrm{U} & 0.0223 & \mathrm{pCi} / \mathrm{g} & 0.114 & \text { EPIA-013B } \\ \mathrm{U} & -0.142 & \mathrm{pCi} / \mathrm{g} & 0.801 & \text { EPIA-001B } \\ \mathrm{U} & 0.0704 & \mathrm{pCi} / \mathrm{g} & 0.0872 & \text { EPIA-013B } \\ \mathrm{U} & -0.00517 & \mathrm{pCi} / \mathrm{g} & 0.0517 & \text { EPIA-013B } \\ \mathrm{U} & -0.0623 & \mathrm{pCi} / \mathrm{g} & 0.208 & \text { EPIA-013B } \\ & 7.32 & \mathrm{pCi} / \mathrm{g} & 1.26 & \text { EPIA-001B } \\ \mathrm{U} & -0.00917 & \mathrm{pCi} / \mathrm{g} & 0.0406 & \text { EPIA-012B } \\ \mathrm{U} & -0.00217 & \mathrm{pCi} / \mathrm{g} & 0.0209 & \text { EPIA-012B } \\ & 4.41 & \mathrm{pCi} / \mathrm{g} & 0.459 & \text { EPIA-013B } \\ \mathrm{U} & -0.0104 & \mathrm{pCi} / \mathrm{g} & 0.0443 & \text { EPIA-013B } \\ \mathrm{U} & -0.0185 & \mathrm{pCi} / \mathrm{g} & 0.0575 & \text { EPIA-013B } \\ \mathrm{U} & -0.0132 & \mathrm{pCi} / \mathrm{g} & 0.467 & \text { EPIA-013B } \\ \mathrm{U} & 0.0184 & \mathrm{pCi} / \mathrm{g} & 0.0538 & \text { EPIA-013B } \\ \mathrm{U} & 0.543 & \mathrm{pCi} / \mathrm{g} & 0.545 & \text { EPA905 } \\ \mathrm{U} & 0.0118 & \mathrm{pCi} / \mathrm{g} & 0.0694 & \text { EPIA-013B } \\ \mathrm{U} & 0.0205 & \mathrm{pCi} / \mathrm{g} & 0.132 & \text { EPIA-002B }\end{array}$


SURVEY ID: SRF-01-03 (cont.)

Constituent

Radionuclides

Uranium-233/234

Uranium-235

Uranium-238

Yttrium-88

Zinc-65

Zirconium-95

Volatile Organic Compounds

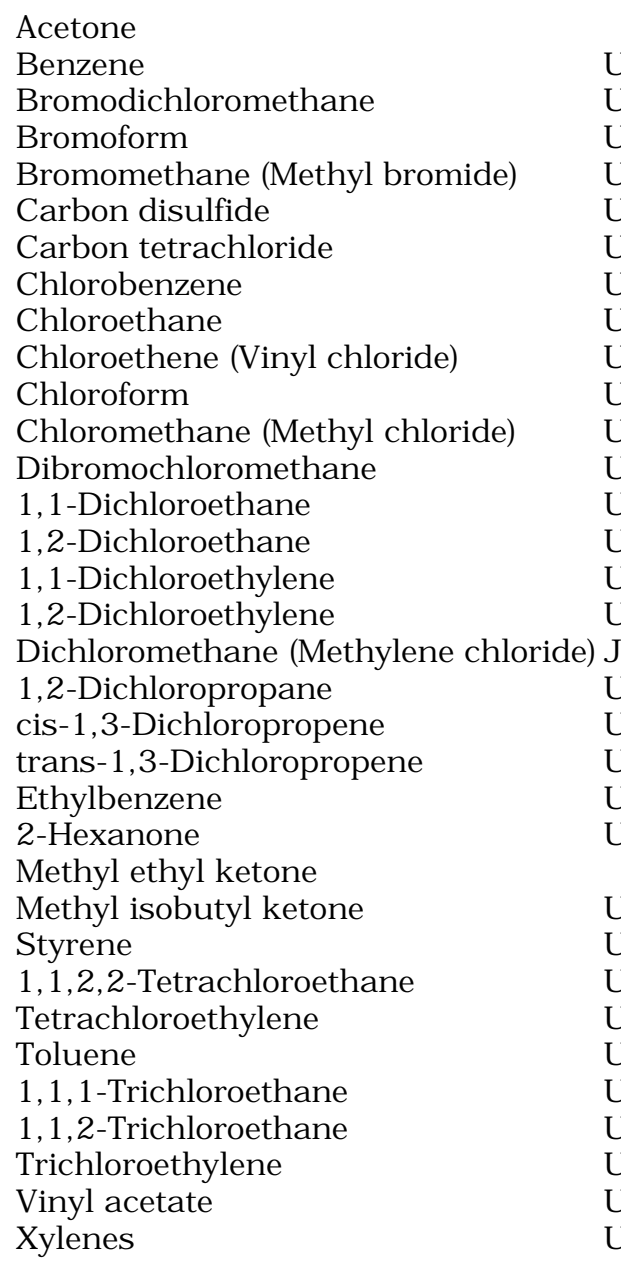

Sample ID: 117020

FG SC E Result

Unit ssMDL

Method

$\begin{array}{lll}\mathrm{pCi} / \mathrm{g} & 0.023 & \text { EPIA-011B } \\ \mathrm{pCi} / \mathrm{g} & 0.0203 & \text { EPIA-011B } \\ \mathrm{pCi} / \mathrm{g} & 0.023 & \text { EPIA-011B } \\ \mathrm{pCi} / \mathrm{g} & 0.0775 & \text { EPIA-013B } \\ \mathrm{pCi} / \mathrm{g} & 0.114 & \text { EPIA-013B } \\ \mathrm{pCi} / \mathrm{g} & 0.112 & \text { EPIA-013B }\end{array}$

0.00685
-0.00258
-0.00118
0.0122
-0.0219
-0.0247

16

5

5

5

25

5

5

5

5

5

5

5

5

5

50

I

$\begin{array}{ll}\mu g / \mathrm{kg} & 51.5 \\ \mu \mathrm{g} / \mathrm{kg} & 2.5 \\ \mu \mathrm{g} / \mathrm{kg} & 0.5 \\ \mu \mathrm{g} / \mathrm{kg} & 1.35 \\ \mu \mathrm{g} / \mathrm{kg} & 1.5 \\ \mu \mathrm{g} / \mathrm{kg} & 1.5 \\ \mu \mathrm{g} / \mathrm{kg} & 2.5 \\ \mu \mathrm{g} / \mathrm{kg} & 1.5 \\ \mu \mathrm{g} / \mathrm{kg} & 1.5 \\ \mu \mathrm{g} / \mathrm{kg} & 2 \\ \mu \mathrm{g} / \mathrm{kg} & 0.5 \\ \mu \mathrm{g} / \mathrm{kg} & 1 \\ \mu \mathrm{g} / \mathrm{kg} & 1.05 \\ \mu \mathrm{g} / \mathrm{kg} & 0.5 \\ \mu \mathrm{g} / \mathrm{kg} & 1.15 \\ \mu \mathrm{g} / \mathrm{kg} & 1.5 \\ \mu \mathrm{g} / \mathrm{kg} & 1.25 \\ \mu \mathrm{g} / \mathrm{kg} & 7 \\ \mu \mathrm{g} / \mathrm{kg} & 1.15 \\ \mu \mathrm{g} / \mathrm{kg} & 1 \\ \mu \mathrm{g} / \mathrm{kg} & 1.5 \\ \mu \mathrm{g} / \mathrm{kg} & 1.5 \\ \mu \mathrm{g} / \mathrm{kg} & 14 \\ \mu \mathrm{g} / \mathrm{kg} & 16 \\ \mu \mathrm{g} / \mathrm{kg} & 14.6 \\ \mu \mathrm{g} / \mathrm{kg} & 1.5 \\ \mu \mathrm{g} / \mathrm{kg} & 3 \\ \mu \mathrm{g} / \mathrm{kg} & 2 \\ \mu \mathrm{g} / \mathrm{kg} & 4.5 \\ \mu \mathrm{g} / \mathrm{kg} & 0.5 \\ \mu \mathrm{g} / \mathrm{kg} & 1.2 \\ \mu \mathrm{g} / \mathrm{kg} & 1.35 \\ \mu \mathrm{g} / \mathrm{kg} & 10.5 \\ \mu \mathrm{g} / \mathrm{kg} & 5 \\ & \end{array}$

EPA8260B

EPA8260B

EPA8260B

EPA8260B

EPA8260B

EPA8260B

EPA8260B

EPA8260B

EPA8260B

EPA8260B

EPA8260B

EPA8260B

EPA8260B

EPA8260B

EPA8260B

EPA8260B

EPA8260B

EPA8260B

EPA8260B

EPA8260B

EPA8260B

EPA8260B

EPA8260B

EPA8260B

EPA8260B

EPA8260B

EPA8260B

EPA8260B

EPA8260B

EPA8260B

EPA8260B

EPA8260B

EPA8260B

EPA8260B 
SURVEY ID: SRF-01-04

Sample type: Normal

Constituent

Metals (total recoverable)

Antimony
Arsenic
Barium
Cadmium
Calcium
Chromium
Copper
Lead
Magnesium
Manganese
Mercury
Nickel
Selenium
Silver
Zinc

Radionuclides

Actinium-228

Antimony-124

Antimony-125

Barium-133

Cerium-144

Cesium-134

Cesium-137

Cobalt-57

Cobalt-58

Cobalt-60

Europium-152

Europium-154

Europium-155

Gross alpha

Lead-212

Manganese-54

Neptunium-239

Nonvolatile beta

Plutonium-238

Plutonium-239/240

Potassium-40

Promethium-144

Promethium-146

Ruthenium-106

Sodium-22

Strontium-89/90

Tin-1 13

Tritium
Sample ID: 117021

Sample matrix: Fauna

Percent solids: NA

\begin{tabular}{|c|c|c|c|c|c|c|}
\hline \multirow[t]{3}{*}{ JU } & & 4 & 0.259 & $\mathrm{mg} / \mathrm{kg}$ & 0.177 & EPA6010B \\
\hline & & & 0.355 & $\mathrm{mg} / \mathrm{kg}$ & 0.211 & EPA6010B \\
\hline & & & 3.92 & $\mathrm{mg} / \mathrm{kg}$ & 0.025 & EPA6010B \\
\hline \multirow[t]{4}{*}{ JU } & & 4 & 0.0252 & $\mathrm{mg} / \mathrm{kg}$ & 0.018 & EPA6010B \\
\hline & & & 17800 & $\mathrm{mg} / \mathrm{kg}$ & 3.228 & EPA6010B \\
\hline & & & 0.251 & $\mathrm{mg} / \mathrm{kg}$ & 0.0338 & EPA6010B \\
\hline & & & 0.316 & $\mathrm{mg} / \mathrm{kg}$ & 0.0611 & EPA6010B \\
\hline \multirow[t]{3}{*}{ J } & I & & 0.146 & $\mathrm{mg} / \mathrm{kg}$ & 0.073 & EPA6010B \\
\hline & & & 475 & $\mathrm{mg} / \mathrm{kg}$ & 0.235 & EPA6010B \\
\hline & & & 37 & $\mathrm{mg} / \mathrm{kg}$ & 0.329 & EPA6010B \\
\hline$J$ & I & & 0.0306 & $\mathrm{mg} / \mathrm{kg}$ & 0.00222 & EPA7471A \\
\hline \multirow[t]{2}{*}{$\mathrm{U}$} & & & 0.231 & $\mathrm{mg} / \mathrm{kg}$ & 0.03 & EPA6010B \\
\hline & & & 0.938 & $\mathrm{mg} / \mathrm{kg}$ & 0.125 & EPA6010F \\
\hline \multirow[t]{2}{*}{$J$} & I & & 0.0501 & $\mathrm{mg} / \mathrm{kg}$ & 0.0287 & EPA6010B \\
\hline & & & 19.5 & $\mathrm{mg} / \mathrm{kg}$ & 0.171 & EPA6010B \\
\hline
\end{tabular}

U

U

U

U

U

U

U

$\mathrm{U}$

$\mathrm{U}$

U

$\mathrm{U}$

U

U

U

U

$\mathrm{U}$

$\mathrm{U}$

U

$\mathrm{U}$

U

U

U

U

U
0.0867

0.0173

0.0124

0.00889

0.0362

$-0.00304$

$-0.00711$

0.00987

$-0.00533$

0.0187

0.0114

0.0174

0.0404

0.122

0.014

0.0061

0.028

7.7

0

$-0.000901$

3.39

$-0.00147$

0.00492

$-0.0714$

0.00639

0.532

$-0.0225$

0.0632

$\begin{array}{ll} & \\ \mathrm{pCi} / \mathrm{g} & 0.174 \\ \mathrm{pCi} / \mathrm{g} & 0.0584 \\ \mathrm{pCi} / \mathrm{g} & 0.101 \\ \mathrm{pCi} / \mathrm{g} & 0.0478 \\ \mathrm{pCi} / \mathrm{g} & 0.211 \\ \mathrm{pCi} / \mathrm{g} & 0.0408 \\ \mathrm{pCi} / \mathrm{g} & 0.0348 \\ \mathrm{pCi} / \mathrm{g} & 0.0237 \\ \mathrm{pCi} / \mathrm{g} & 0.0518 \\ \mathrm{pCi} / \mathrm{g} & 0.0547 \\ \mathrm{pCi} / \mathrm{g} & 0.112 \\ \mathrm{pCi} / \mathrm{g} & 0.132 \\ \mathrm{pCi} / \mathrm{g} & 0.111 \\ \mathrm{pCi} / \mathrm{g} & 0.428 \\ \mathrm{pCi} / \mathrm{g} & 0.11 \\ \mathrm{pCi} / \mathrm{g} & 0.0453 \\ \mathrm{pCi} / \mathrm{g} & 0.184 \\ \mathrm{pCi} / \mathrm{g} & 0.568 \\ \mathrm{pCi} / \mathrm{g} & 0.0113 \\ \mathrm{pCi} / \mathrm{g} & 0.0198 \\ \mathrm{pCi} / \mathrm{g} & 0.456 \\ \mathrm{pCi} / \mathrm{g} & 0.0413 \\ \mathrm{pCi} / \mathrm{g} & 0.0499 \\ \mathrm{pCi} / \mathrm{g} & 0.364 \\ \mathrm{pCi} / \mathrm{g} & 0.0476 \\ \mathrm{pCi} / \mathrm{g} & 0.765 \\ \mathrm{pCi} / \mathrm{g} & 0.0512 \\ \mathrm{pCi} / \mathrm{g} & 0.144 \\ & \end{array}$

EPIA-013B

EPIA-013B

EPIA-013B

EPIA-013B

EPIA-013B

EPIA-013B

EPIA-013B

EPIA-013B

EPIA-013B

EPIA-013B

EPIA-013B

EPIA-013B

EPIA-013B

EPIA-001B

EPIA-013B

EPIA-013B

EPIA-013B

EPIA-001B

EPIA-012B

EPIA-012B

EPIA-013B

EPIA-013B

EPIA-013B

EPIA-013B

EPIA-013B

EPA905

EPIA-013B

EPIA-002B 
SURVEY ID: SRF-01-04 (cont.)

Constituent

Radionuclides

Tritium

Uranium-233/234

Uranium-235

Uranium-238

Yttrium-88

Zinc-65

Zirconium-95

Volatile Organic Compounds

Acetone

Benzene

Bromodichloromethane

Bromoform

Bromomethane (Methyl bromide)

Carbon disulfide

Carbon tetrachloride

Chlorobenzene

Chloroethane

Chloroethene (Vinyl chloride)

Chloroform

Chloromethane (Methyl chloride)

Dibromochloromethane

1,1-Dichloroethane

1,2-Dichloroethane

1,1-Dichloroethylene

1,2-Dichloroethylene

Dichloromethane (Methylene chloride) J

1,2-Dichloropropane cis-1,3-Dichloropropene

trans-1,3-Dichloropropene

Ethylbenzene

2-Hexanone

Methyl ethyl ketone

Methyl isobutyl ketone

Styrene

1,1,2,2-Tetrachloroethane

Tetrachloroethylene

Toluene

1,1,1-Trichloroethane

1,1,2-Trichloroethane

Trichloroethylene

Vinyl acetate

Xylenes
Sample ID: 117021

FG SC E Result

Unit ssMDL

Method

EPIA-002B

EPIA-011B

EPIA-011B

EPIA-011B

EPIA-013B

EPIA-013B

EPIA-013B

EPA8260B

$\mu g / \mathrm{kg} \quad 51.5$

EPA8260B

EPA8260B

EPA8260B

EPA8260B

EPA8260B

EPA8260B

EPA8260B

EPA8260B

EPA8260B

EPA8260B

EPA8260B

EPA8260B

EPA8260B

EPA8260B

EPA8260B

EPA8260B

EPA8260B

EPA8260B

EPA8260B

EPA8260B

EPA8260B

EPA8260B

EPA8260B

EPA8260B

EPA8260B

EPA8260B

EPA8260B

EPA8260B

EPA8260B

EPA8260B

EPA8260B

EPA8260B

EPA8260B 
SURVEY ID: SRF-01-05

Sample type: Normal

Constituent

Metals (total recoverable)

Antimony
Arsenic
Barium
Cadmium
Calcium
Chromium
Copper
Lead
Magnesium
Manganese
Mercury
Nickel
Selenium
Silver
Zinc

Radionuclides

Actinium-228

Actinium-228

Antimony-124

Antimony-124

Antimony-125

Antimony-125

Barium-133

Barium-133

Cerium-144

Cerium-144

Cesium-134

Cesium-134

Cesium-137

Cesium-137

Cobalt-57

Cobalt-57

Cobalt-58

Cobalt-58

Cobalt-60

Cobalt-60

Europium-152

Europium-152

Europium-154

Europium-154

Europium-155

Europium-155

Gross alpha

Gross alpha
Sample ID: 117022

Sample matrix: Fauna

Percent solids: NA

\begin{tabular}{|c|c|c|c|c|c|c|}
\hline \multirow[t]{3}{*}{ U } & & & 0.459 & $\mathrm{mg} / \mathrm{kg}$ & 0.175 & EPA6010B \\
\hline & & & 0.543 & $\mathrm{mg} / \mathrm{kg}$ & 0.209 & EPA6010B \\
\hline & & & 3.33 & $\mathrm{mg} / \mathrm{kg}$ & 0.024 & EPA6010B \\
\hline \multirow[t]{2}{*}{ JU } & & 4 & 0.0224 & $\mathrm{mg} / \mathrm{kg}$ & 0.017 & EPA6010B \\
\hline & & & 10400 & $\mathrm{mg} / \mathrm{kg}$ & 3.2 & EPA6010B \\
\hline \multirow[t]{2}{*}{$\mathrm{J}$} & I & & 0.166 & $\mathrm{mg} / \mathrm{kg}$ & 0.0334 & EPA6010B \\
\hline & & & 0.413 & $\mathrm{mg} / \mathrm{kg}$ & 0.0606 & EPA6010B \\
\hline \multirow[t]{2}{*}{$J$} & I & & 0.119 & $\mathrm{mg} / \mathrm{kg}$ & 0.072 & EPA6010B \\
\hline & & & 375 & $\mathrm{mg} / \mathrm{kg}$ & 0.233 & EPA6010B \\
\hline $\mathrm{J}$ & $\mathrm{K}$ & I & 18.5 & $\mathrm{mg} / \mathrm{kg}$ & 0.326 & EPA6010B \\
\hline$J$ & $\mathrm{~L}$ & I & 0.0992 & $\mathrm{mg} / \mathrm{kg}$ & 0.00221 & EPA7471A \\
\hline \multirow{2}{*}{$\mathrm{U}$} & & & 0.229 & $\mathrm{mg} / \mathrm{kg}$ & 0.029 & EPA6010B \\
\hline & & & 1.02 & $\mathrm{mg} / \mathrm{kg}$ & 0.124 & EPA6010B \\
\hline \multirow[t]{2}{*}{$\mathrm{U}$} & & & 0.229 & $\mathrm{mg} / \mathrm{kg}$ & 0.0284 & EPA6010B \\
\hline & & & 17.9 & $\mathrm{mg} / \mathrm{kg}$ & 0.17 & EPA6010B \\
\hline
\end{tabular}

$\begin{array}{lllll}\mathrm{U} & 0.145 & \mathrm{pCi} / \mathrm{g} & 0.203 & \text { EPIA-013B } \\ \mathrm{U} & 0.179 & \mathrm{pCi} / \mathrm{g} & 0.201 & \text { EPIA-013B } \\ \mathrm{U} & -0.000566 & \mathrm{pCi} / \mathrm{g} & 0.0663 & \text { EPIA-013B } \\ \mathrm{U} & -0.00867 & \mathrm{pCi} / \mathrm{g} & 0.0604 & \text { EPIA-013B } \\ \mathrm{U} & 0.00376 & \mathrm{pCi} / \mathrm{g} & 0.114 & \text { EPIA-013B } \\ \mathrm{U} & 0.0298 & \mathrm{pCi} / \mathrm{g} & 0.0955 & \text { EPIA-013B } \\ \mathrm{U} & 0.00386 & \mathrm{pCi} / \mathrm{g} & 0.0515 & \text { EPIA-013B } \\ \mathrm{U} & 0.0286 & \mathrm{pCi} / \mathrm{g} & 0.0523 & \text { EPIA-013B } \\ \mathrm{U} & -0.00121 & \mathrm{pCi} / \mathrm{g} & 0.225 & \text { EPIA-013B } \\ \mathrm{U} & 0.00574 & \mathrm{pCi} / \mathrm{g} & 0.209 & \text { EPIA-013B } \\ \mathrm{U} & -0.00193 & \mathrm{pCi} / \mathrm{g} & 0.0419 & \text { EPIA-013B } \\ \mathrm{U} & 0.0152 & \mathrm{pCi} / \mathrm{g} & 0.0465 & \text { EPIA-013B } \\ \mathrm{U} & 0.00304 & \mathrm{pCi} / \mathrm{g} & 0.0437 & \text { EPIA-013B } \\ \mathrm{U} & 0.0119 & \mathrm{pCi} / \mathrm{g} & 0.0449 & \text { EPIA-013B } \\ \mathrm{U} & 0.00534 & \mathrm{pCi} / \mathrm{g} & 0.0261 & \text { EPIA-013B } \\ \mathrm{U} & 0.00733 & \mathrm{pCi} / \mathrm{g} & 0.0262 & \text { EPIA-013B } \\ \mathrm{U} & 0.00134 & \mathrm{pCi} / \mathrm{g} & 0.0559 & \text { EPIA-013B } \\ \mathrm{U} & -0.0156 & \mathrm{pCi} / \mathrm{g} & 0.05 & \text { EPIA-013B } \\ \mathrm{U} & 0.0128 & \mathrm{pCi} / \mathrm{g} & 0.0552 & \text { EPIA-013B } \\ \mathrm{U} & 0.0145 & \mathrm{pCi} / \mathrm{g} & 0.0543 & \text { EPIA-013B } \\ \mathrm{U} & 0.000658 & \mathrm{pCi} / \mathrm{g} & 0.114 & \text { EPIA-013B } \\ \mathrm{U} & -0.00988 & \mathrm{pCi} / \mathrm{g} & 0.108 & \text { EPIA-013B } \\ \mathrm{U} & -0.00085 & \mathrm{pCi} / \mathrm{g} & 0.149 & \text { EPIA-013B } \\ \mathrm{U} & 0.0158 & \mathrm{pCi} / \mathrm{g} & 0.14 & \text { EPIA-013B } \\ \mathrm{U} & -0.0104 & \mathrm{pCi} / \mathrm{g} & 0.0947 & \text { EPIA-013B } \\ \mathrm{U} & 0.0355 & \mathrm{pCi} / \mathrm{g} & 0.114 & \text { EPIA-013B } \\ \mathrm{U} & 0.502 & \mathrm{pCi} / \mathrm{g} & 1.58 & \text { EPIA-001B } \\ \mathrm{U} & 0.653 & \mathrm{pCi} / \mathrm{g} & 1.29 & \text { EPIA-001B } \\ & & & & \\ & & & & \end{array}$


SURVEY ID: SRF-01-05 (cont.)

Constituent

Radionuclides

Lead-212

Lead-212

Manganese-54

Manganese-54

Neptunium-239

Neptunium-239

Nonvolatile beta

Nonvolatile beta

Plutonium-238

Plutonium-239/240

Potassium-40

Potassium-40

Promethium-144

Promethium-144

Promethium-146

Promethium-146

Ruthenium-106

Ruthenium-106

Sodium-22

Sodium-22

Strontium-89/90

Tin-1 13

Tin-1 13

Tritium

Uranium-233/234

Uranium-235

Uranium-238

Yttrium-88

Yttrium-88

Zinc-65

Zinc-65

Zirconium-95

Zirconium-95

Volatile Organic Compounds

Acetone

Benzene

Bromodichloromethane

Bromoform

Bromomethane (Methyl bromide)

Carbon disulfide

Carbon tetrachloride

Chlorobenzene

Chloroethane

Chloroethene (Vinyl chloride)

Chloroform

Chloromethane (Methyl chloride)

Dibromochloromethane

1,1-Dichloroethane

1,2-Dichloroethane

1,1-Dichloroethylene

1,2-Dichloroethylene

Sample ID: 117022

FG SC E Result

Unit ssMDL

Method

$\mathrm{U}$
$\mathrm{R}$
$\mathrm{U}$

$\mathrm{R}$

$\mathrm{U}$

U

$\mathrm{U}$

U

$\mathrm{U}$

U

$\mathrm{U}$

U

U

$\mathrm{U}$

U

U

$\mathrm{U}$

U

U

$\mathrm{U}$

$\mathrm{U}$

$\mathrm{U}$

$\mathrm{U}$

$\mathrm{U}$

$\begin{array}{ll} & 0.0725 \\ 4 & 0.0923 \\ -0.00379 \\ 0.0103 \\ -0.0297 \\ -0.0625 \\ 8.14 \\ 8.22 \\ 0 \\ 0.0168 \\ 2.88 \\ 3.64 \\ -0.0155 \\ 0.0193 \\ -0.00327 \\ -0.0233 \\ -0.0232 \\ 0.138 \\ 0.00542 \\ -0.000404 \\ 0.733 \\ -0.0245 \\ -0.00129 \\ -0.0433 \\ -0.00837 \\ -0.00575 \\ -0.0043 \\ 0.0119 \\ -0.00547 \\ 0.0308 \\ 0.00905 \\ 0.00184 \\ 0.00348 \\ \end{array}$

0.00348

$\begin{array}{ll}\mathrm{pCi} / \mathrm{g} & 0.0797 \\ \mathrm{pCi} / \mathrm{g} & 0.0779 \\ \mathrm{pCi} / \mathrm{g} & 0.0436 \\ \mathrm{pCi} / \mathrm{g} & 0.0434 \\ \mathrm{pCi} / \mathrm{g} & 0.183 \\ \mathrm{pCi} / \mathrm{g} & 0.168 \\ \mathrm{pCi} / \mathrm{g} & 2.57 \\ \mathrm{pCi} / \mathrm{g} & 2.48 \\ \mathrm{pCi} / \mathrm{g} & 0.0183 \\ \mathrm{pCi} / \mathrm{g} & 0.0321 \\ \mathrm{pCi} / \mathrm{g} & 1.37 \\ \mathrm{pCi} / \mathrm{g} & 0.498 \\ \mathrm{pCi} / \mathrm{g} & 0.0451 \\ \mathrm{pCi} / \mathrm{g} & 0.0495 \\ \mathrm{pCi} / \mathrm{g} & 0.0528 \\ \mathrm{pCi} / \mathrm{g} & 0.0475 \\ \mathrm{pCi} / \mathrm{g} & 0.396 \\ \mathrm{pCi} / \mathrm{g} & 0.415 \\ \mathrm{pCi} / \mathrm{g} & 0.0503 \\ \mathrm{pCi} / \mathrm{g} & 0.0538 \\ \mathrm{pCi} / \mathrm{g} & 1.15 \\ \mathrm{pCi} / \mathrm{g} & 0.0483 \\ \mathrm{pCi} / \mathrm{g} & 0.0504 \\ \mathrm{pCi} / \mathrm{g} & 0.214 \\ \mathrm{pCi} / \mathrm{g} & 0.061 \\ \mathrm{pCi} / \mathrm{g} & 0.0453 \\ \mathrm{pCi} / \mathrm{g} & 0.0415 \\ \mathrm{pCi} / \mathrm{g} & 0.0574 \\ \mathrm{pCi} / \mathrm{g} & 0.0586 \\ \mathrm{pCi} / \mathrm{g} & 0.111 \\ \mathrm{pCi} / \mathrm{g} & 0.0994 \\ \mathrm{pCi} / \mathrm{g} & 0.0993 \\ \mathrm{pCi} / \mathrm{g} & 0.101 \\ & \end{array}$

EPIA-013B EPIA-013B EPIA-013B

EPIA-013B

EPIA-013B

EPIA-013B

EPIA-001B

EPIA-001B

EPIA-012B

EPIA-012B

EPIA-013B

EPIA-013B

EPIA-013B

EPIA-013B

EPIA-013B

EPIA-013B

EPIA-013B

EPIA-013B

EPIA-013B

EPIA-013B

EPA905

EPIA-013B

EPIA-013B

EPIA-002B

EPIA-011B

EPIA-011B

EPIA-011B

EPIA-013B

EPIA-013B

EPIA-013B

EPIA-013B

EPIA-013B

EPIA-013B
EPA8260B EPA8260B EPA8260B

EPA8260B

EPA8260B

EPA8260B

EPA8260B

EPA8260B

EPA8260B

EPA8260B

EPA8260B

EPA8260B

EPA8260B

EPA8260B

EPA8260B

EPA8260B

EPA8260B 
SURVEY ID: SRF-01-05 (cont.)

Constituent

Volatile Organic Compounds

1,2-Dichloropropane cis-1,3-Dichloropropene

trans-1,3-Dichloropropene

Ethylbenzene

2-Hexanone

Methyl ethyl ketone

Methyl isobutyl ketone

Styrene

1,1,2,2-Tetrachloroethane

Tetrachloroethylene

Toluene

1,1,1-Trichloroethane

1,1,2-Trichloroethane

Trichloroethylene

Vinyl acetate

Xylenes

SURVEY ID: SRF-02-01

Sample type: Normal

\section{Constituent}

Metals (total recoverable)

Antimony
Arsenic
Barium
Cadmium
Calcium
Chromium
Copper
Lead
Magnesium
Manganese
Mercury
Nickel
Selenium
Silver
Zinc

Radionuclides

Actinium-228

Antimony-124

Antimony-125

Barium-133
Sample ID: 117022

FG SC E Result

Unit ssMDL

Method

$\begin{array}{lll}\mu g / \mathrm{kg} & 7 & \text { EPA8260B } \\ \mu g / \mathrm{kg} & 1.15 & \text { EPA8260B } \\ \mu \mathrm{g} / \mathrm{kg} & 1 & \text { EPA8260B } \\ \mu \mathrm{g} / \mathrm{kg} & 1.5 & \text { EPA8260B } \\ \mu \mathrm{g} / \mathrm{kg} & 1.5 & \text { EPA8260B } \\ \mu \mathrm{g} / \mathrm{kg} & 14 & \text { EPA8260B } \\ \mu \mathrm{g} / \mathrm{kg} & 16 & \text { EPA8260B } \\ \mu \mathrm{g} / \mathrm{kg} & 14.6 & \text { EPA8260B } \\ \mu \mathrm{gg} & 1.5 & \text { EPA8260B } \\ \mu g / \mathrm{kg} & 3 & \text { EPA8260B } \\ \mu g / \mathrm{kg} & 2 & \text { EPA8260B } \\ \mu g / \mathrm{kg} & 4.5 & \text { EPA8260B } \\ \mu \mathrm{g} / \mathrm{kg} & 0.5 & \text { EPA8260B } \\ \mu g / \mathrm{kg} & 1.2 & \text { EPA8260B } \\ \mu g / \mathrm{kg} & 1.35 & \text { EPA8260B } \\ \mu g / \mathrm{kg} & 10.5 & \end{array}$

Sample ID: 117023

Sample matrix: Fauna Percent solids: NA
FG SC E Result Unit ssMDL

\begin{tabular}{|c|c|c|c|c|c|}
\hline \multirow[t]{3}{*}{$\mathrm{U}$} & & 0.467 & $\mathrm{mg} / \mathrm{kg}$ & 0.178 & EPA6010B \\
\hline & & 0.534 & $\mathrm{mg} / \mathrm{kg}$ & 0.213 & EPA6010B \\
\hline & & 6.5 & $\mathrm{mg} / \mathrm{kg}$ & 0.025 & EPA6010B \\
\hline \multirow[t]{8}{*}{$\mathrm{U}$} & & 0.234 & $\mathrm{mg} / \mathrm{kg}$ & 0.018 & EPA6010B \\
\hline & & 34500 & $\mathrm{mg} / \mathrm{kg}$ & 6.519 & EPA6010B \\
\hline & & 0.682 & $\mathrm{mg} / \mathrm{kg}$ & 0.0341 & EPA6010B \\
\hline & & 0.826 & $\mathrm{mg} / \mathrm{kg}$ & 0.0617 & EPA6010B \\
\hline & & 0.299 & $\mathrm{mg} / \mathrm{kg}$ & 0.074 & EPA6010B \\
\hline & & 750 & $\mathrm{mg} / \mathrm{kg}$ & 0.237 & EPA6010B \\
\hline & & 38 & $\mathrm{mg} / \mathrm{kg}$ & 0.332 & EPA6010B \\
\hline & & 0.119 & $\mathrm{mg} / \mathrm{kg}$ & 0.00207 & EPA7471A \\
\hline & V & 0.373 & $\mathrm{mg} / \mathrm{kg}$ & 0.03 & EPA6010B \\
\hline & & 0.859 & $\mathrm{mg} / \mathrm{kg}$ & 0.126 & EPA6010F \\
\hline \multirow[t]{2}{*}{$\mathrm{U}$} & & 0.234 & $\mathrm{mg} / \mathrm{kg}$ & 0.029 & EPA6010B \\
\hline & & 23.6 & $\mathrm{mg} / \mathrm{kg}$ & 0.173 & EPA6010F \\
\hline
\end{tabular}

$\begin{array}{lllll}\mathrm{U} & -0.0298 & \mathrm{pCi} / \mathrm{g} & 0.21 & \text { EPIA-013B } \\ \mathrm{U} & 0.0344 & \mathrm{pCi} / \mathrm{g} & 0.0824 & \text { EPIA-013B } \\ \mathrm{U} & -0.0292 & \mathrm{pCi} / \mathrm{g} & 0.142 & \text { EPIA-013B } \\ \mathrm{U} & -0.00508 & \mathrm{pCi} / \mathrm{g} & 0.0604 & \text { EPIA-013B }\end{array}$


SURVEY ID: SRF-02-01 (cont.)

Constituent

Radionuclides

Cerium-144

Cesium-134

Cesium-137

Cobalt-57

Cobalt-58

Cobalt-60

Europium-152

Europium-154

Europium-155

Gross alpha

Lead-212

Manganese-54

Neptunium-239

Nonvolatile beta

Plutonium-238

Plutonium-239/240

Potassium-40

Promethium-144

Promethium-146

Ruthenium-106

Sodium-22

Strontium-89/90

Tin-113

Tritium

Uranium-233/234

Uranium-235

Uranium-238

Yttrium-88

Zinc-65

Zirconium-95

Volatile Organic Compounds

Acetone

Benzene

Bromodichloromethane

Bromoform

Bromomethane (Methyl bromide)

Carbon disulfide

Carbon tetrachloride

Chlorobenzene

Chloroethane

Chloroethene (Vinyl chloride)

Chloroform

Chloromethane (Methyl chloride)

Dibromochloromethane

1,1-Dichloroethane

1,2-Dichloroethane

1,1-Dichloroethylene

1,2-Dichloroethylene

Dichloromethane (Methylene chloride) J

1,2-Dichloropropane

cis-1,3-Dichloropropene
Sample ID: 117023

FG SC E Result

Unit ssMDL

Method

$\begin{array}{lll}\mathrm{pCi} / \mathrm{g} & 0.272 & \text { EPIA-013B } \\ \mathrm{pCi} / \mathrm{g} & 0.0559 & \text { EPIA-013B } \\ \mathrm{pCi} / \mathrm{g} & 0.0631 & \text { EPIA-013B } \\ \mathrm{pCi} / \mathrm{g} & 0.0329 & \text { EPIA-013B } \\ \mathrm{pCi} / \mathrm{g} & 0.0551 & \text { EPIA-013B } \\ \mathrm{pCi} / \mathrm{g} & 0.0637 & \text { EPIA-013B } \\ \mathrm{pCi} / \mathrm{g} & 0.149 & \text { EPIA-013B } \\ \mathrm{pCi} / \mathrm{g} & 0.149 & \text { EPIA-013B } \\ \mathrm{pCi} / \mathrm{g} & 0.122 & \text { EPIA-013B } \\ \mathrm{pCi} / \mathrm{g} & 1.5 & \text { EPIA-001B } \\ \mathrm{pCi} / \mathrm{g} & 0.0955 & \text { EPIA-013B } \\ \mathrm{pCi} / \mathrm{g} & 0.0584 & \text { EPIA-013B } \\ \mathrm{pCi} / \mathrm{g} & 0.234 & \text { EPIA-013B } \\ \mathrm{pCi} / \mathrm{g} & 1.7 & \text { EPIA-001B } \\ \mathrm{pCi} / \mathrm{g} & 0.0264 & \text { EPIA-012B } \\ \mathrm{pCi} / \mathrm{g} & 0.0264 & \text { EPIA-012B } \\ \mathrm{pCi} / \mathrm{g} & 0.726 & \text { EPIA-013B } \\ \mathrm{pCi} / \mathrm{g} & 0.0659 & \text { EPIA-013B } \\ \mathrm{pCi} / \mathrm{g} & 0.0655 & \text { EPIA-013B } \\ \mathrm{pCi} / \mathrm{g} & 0.547 & \text { EPIA-013B } \\ \mathrm{pCi} / \mathrm{g} & 0.0539 & \text { EPIA-013B } \\ \mathrm{pCi} / \mathrm{g} & 0.752 & \text { EPA905 } \\ \mathrm{pCi} / \mathrm{g} & 0.0764 & \text { EPIA-013B } \\ \mathrm{pCi} / \mathrm{g} & 0.0984 & \text { EPIA-002B } \\ \mathrm{pCi} / \mathrm{g} & 0.0277 & \text { EPIA-011B } \\ \mathrm{pCi} / \mathrm{g} & 0.0239 & \text { EPIA-011B } \\ \mathrm{pCi} / \mathrm{g} & 0.0238 & \text { EPIA-011B } \\ \mathrm{pCi} / \mathrm{g} & 0.0764 & \text { EPIA-013B } \\ \mathrm{pCi} / \mathrm{g} & 0.122 & \text { EPIA-013B } \\ \mathrm{pCi} / \mathrm{g} & 0.138 & \text { EPIA-013B }\end{array}$

0.0335

0.0141

0.0295

0.0318

$-0.0171$

$-0.0251$

0.000223

$-0.19$

0.0869

0.0257

0.0165

7.76

0.00645

$-0.00204$

4.23

0.0247

$-0.044$

0.245

$-0.00917$

0.037

0.0263

$-0.0229$

0.0164

0.000961

0.000959

0.0152

0.109

0.0612

$\mathrm{pCi} / \mathrm{g}$

EPIA-013B

$\begin{array}{ll}\mu g / \mathrm{kg} & 51.5 \\ \mu g / \mathrm{kg} & 2.5 \\ \mu g / \mathrm{kg} & 0.5 \\ \mu g / \mathrm{kg} & 1.35 \\ \mu g / \mathrm{kg} & 1.5 \\ \mu g / \mathrm{kg} & 1.5 \\ \mu g / \mathrm{kg} & 2.5 \\ \mu g / \mathrm{kg} & 1.5 \\ \mu g / \mathrm{kg} & 1.5 \\ \mu g / \mathrm{kg} & 2 \\ \mu g / \mathrm{kg} & 0.5 \\ \mu g / \mathrm{kg} & 1 \\ \mu g / \mathrm{kg} & 1.05 \\ \mu g / \mathrm{kg} & 0.5 \\ \mu g / \mathrm{kg} & 1.15 \\ \mu g / \mathrm{kg} & 1.5 \\ \mu g / \mathrm{kg} & 1.25 \\ \mu g / \mathrm{kg} & 7 \\ \mu g / \mathrm{kg} & 1.15 \\ \mu g / \mathrm{kg} & 1\end{array}$

EPA8260B

EPA8260B

EPA8260B

EPA8260B

EPA8260B

EPA8260B

EPA8260B

EPA8260B

EPA8260B

EPA8260B

EPA8260B

EPA8260B

EPA8260B

EPA8260B

EPA8260B

EPA8260B

EPA8260B

EPA8260B

EPA8260B

EPA8260B 
SURVEY ID: SRF-02-01 (cont.)

Constituent

Volatile Organic Compounds

trans-1,3-Dichloropropene

Ethylbenzene

2-Hexanone

Methyl ethyl ketone

Methyl isobutyl ketone

Styrene

1,1,2,2-Tetrachloroethane

Tetrachloroethylene

Toluene

1,1,1-Trichloroethane

1,1,2-Trichloroethane

Trichloroethylene

Vinyl acetate

Xylenes

SURVEY ID: SRF-02-02

Sample type: Normal

\section{Constituent}

Metals (total recoverable)

Antimony

Arsenic

Barium

Cadmium

Calcium

Chromium

Copper

Lead

Magnesium

Manganese

Mercury

Nickel

Selenium

Silver

Zinc

Radionuclides

Actinium-228

Antimony-124

Antimony-125

Barium-133

Cerium-144

Cesium-134

Cesium-137
Sample ID: 117023

FG SC E Result

Unit ssMDL

Method

$\begin{array}{ll}\mu g / \mathrm{kg} & 1.5 \\ \mu g / \mathrm{kg} & 1.5 \\ \mu g / \mathrm{kg} & 14 \\ \mu g / \mathrm{kg} & 16 \\ \mu g / \mathrm{kg} & 14.6 \\ \mu g / \mathrm{kg} & 1.5 \\ \mu g / \mathrm{kg} & 3 \\ \mu g / \mathrm{kg} & 2 \\ \mu g / \mathrm{kg} & 4.5 \\ \mu g / \mathrm{kg} & 0.5 \\ \mu g / \mathrm{kg} & 1.2 \\ \mu g / \mathrm{kg} & 1.35 \\ \mu g / \mathrm{kg} & 10.5 \\ \mu g / \mathrm{kg} & 5\end{array}$

EPA8260B

EPA8260B

EPA8260B

EPA8260B

EPA8260B

EPA8260B

EPA8260B

EPA8260B

EPA8260B

EPA8260B

EPA8260B

EPA8260B

EPA8260B

EPA8260B
Sample ID: 117024

Sample matrix: Fauna

Percent solids: NA
FG SC E Result Unit ssMDL

\begin{tabular}{|c|c|c|c|c|c|}
\hline \multirow[t]{3}{*}{$\mathrm{U}$} & & 0.481 & $\mathrm{mg} / \mathrm{kg}$ & 0.184 & EPA6010B \\
\hline & & 0.55 & $\mathrm{mg} / \mathrm{kg}$ & 0.219 & EPA6010B \\
\hline & & 3.84 & $\mathrm{mg} / \mathrm{kg}$ & 0.026 & EPA6010B \\
\hline \multirow[t]{3}{*}{ JU } & 4 & 0.0225 & $\mathrm{mg} / \mathrm{kg}$ & 0.018 & EPA6010B \\
\hline & & 32400 & $\mathrm{mg} / \mathrm{kg}$ & 6.707 & EPA6010B \\
\hline & & 0.43 & $\mathrm{mg} / \mathrm{kg}$ & 0.035 & EPA6010B \\
\hline \multirow[t]{5}{*}{$\mathrm{J}$} & I & 0.217 & $\mathrm{mg} / \mathrm{kg}$ & 0.0635 & EPA6010B \\
\hline & & 0.245 & $\mathrm{mg} / \mathrm{kg}$ & 0.076 & EPA6010B \\
\hline & & 677 & $\mathrm{mg} / \mathrm{kg}$ & 0.244 & EPA6010B \\
\hline & & 8.99 & $\mathrm{mg} / \mathrm{kg}$ & 0.342 & EPA6010B \\
\hline & & 0.0532 & $\mathrm{mg} / \mathrm{kg}$ & 0.00212 & EPA7471A \\
\hline \multirow[t]{2}{*}{$\mathrm{U}$} & & 0.24 & $\mathrm{mg} / \mathrm{kg}$ & 0.031 & EPA6010B \\
\hline & & 1.07 & $\mathrm{mg} / \mathrm{kg}$ & 0.13 & EPA6010B \\
\hline \multirow[t]{2}{*}{$\mathrm{U}$} & & 0.24 & $\mathrm{mg} / \mathrm{kg}$ & 0.0298 & EPA6010B \\
\hline & & 18.6 & $\mathrm{mg} / \mathrm{kg}$ & 0.178 & EPA6010B \\
\hline
\end{tabular}

$\begin{array}{lllll}\mathrm{U} & 0.085 & \mathrm{pCi} / \mathrm{g} & 0.18 & \text { EPIA-013B } \\ \mathrm{U} & -0.0146 & \mathrm{pCi} / \mathrm{g} & 0.0545 & \text { EPIA-013B } \\ \mathrm{U} & -0.0076 & \mathrm{pCi} / \mathrm{g} & 0.102 & \text { EPIA-013B } \\ \mathrm{U} & -0.0242 & \mathrm{pCi} / \mathrm{g} & 0.0383 & \text { EPIA-013B } \\ \mathrm{U} & 0.0858 & \mathrm{pCi} / \mathrm{g} & 0.189 & \text { EPIA-013B } \\ \mathrm{U} & -0.00547 & \mathrm{pCi} / \mathrm{g} & 0.0373 & \text { EPIA-013B } \\ \mathrm{U} & 0.0144 & \mathrm{pCi} / \mathrm{g} & 0.05 & \text { EPIA-013B }\end{array}$


SURVEY ID: SRF-02-02 (cont.)

Constituent

Radionuclides

Cobalt-57

Cobalt-58

Cobalt-60

Europium-152

Europium-154

Europium-155

Gross alpha

Lead-212

Manganese-54

Neptunium-239

Nonvolatile beta

Plutonium-238

Plutonium-239/240

Potassium-40

Promethium-144

Promethium-146

Ruthenium-106

Sodium-22

Strontium-89/90

Tin-1 13

Tritium

Uranium-233/234

Uranium-235

Uranium-238

Yttrium-88

Zinc-65

Zirconium-95

Volatile Organic Compounds

Acetone

Benzene

Bromodichloromethane

Bromoform

Bromomethane (Methyl bromide)

Carbon disulfide

Carbon tetrachloride

Chlorobenzene

Chloroethane

Chloroethene (Vinyl chloride)

Chloroform

Chloromethane (Methyl chloride)

Dibromochloromethane

1,1-Dichloroethane

1,2-Dichloroethane

1,1-Dichloroethylene

1,2-Dichloroethylene

Dichloromethane (Methylene chloride) J

1,2-Dichloropropane

cis-1,3-Dichloropropene

trans-1,3-Dichloropropene

Ethylbenzene

2-Hexanone
Sample ID: 117024

FG SC E Result

Unit ssMDL

Method

EPIA-013B

$\begin{array}{llll}0.0119 & \mathrm{pCi} / \mathrm{g} & 0.0573 & \text { EPIA-013B } \\ 0.0421 & \mathrm{pCi} / \mathrm{g} & 0.0582 & \text { EPIA-013B }\end{array}$

$0.0431 \quad \mathrm{pCi} / \mathrm{g} \quad 0.111 \quad$ EPIA-013B

$\begin{array}{llll}0.019 & \mathrm{pCi} / \mathrm{g} & 0.139 & \text { EPIA-013B }\end{array}$

$\begin{array}{llll}-0.00745 & \mathrm{pCi} / \mathrm{g} & 0.0899 \quad \text { EPIA-013B }\end{array}$

$\begin{array}{lll}-0.214 & \mathrm{pCi} / \mathrm{g} & 1.22\end{array}$

$\begin{array}{lll}0.0586 & \mathrm{pCi} / \mathrm{g} & 0.0773\end{array}$

$\begin{array}{lll}-0.00243 & \mathrm{pCi} / \mathrm{g} & 0.0452\end{array}$

$\begin{array}{lll}-0.0467 & \mathrm{pCi} / \mathrm{g} & 0.174\end{array}$

5.87

0.00398

$-0.00116$

$\mathrm{pCi} / \mathrm{g} \quad 1.06$

$\mathrm{pCi} / \mathrm{g} \quad 0.028$

$\mathrm{pCi} / \mathrm{g} \quad 0.0151$

$\mathrm{pCi} / \mathrm{g} \quad 0.459$

$\mathrm{pCi} / \mathrm{g} \quad 0.0489$

0.0223

0.00314

0.0177

0.00668

I

0.49

$-0.00762$

$-0.0264$

0.00679

$-0.000914$

$\mathrm{pCi} / \mathrm{g} \quad 0.047$

$\mathrm{pCi} / \mathrm{g} \quad 0.41$

$\mathrm{pCi} / \mathrm{g} \quad 0.05$

$\mathrm{pCi} / \mathrm{g} \quad 0.48$

pCi/g 0.0537

$\mathrm{pCi} / \mathrm{g} \quad 0.107$

$\mathrm{pCi} / \mathrm{g} \quad 0.0144$

$\mathrm{pCi} / \mathrm{g} \quad 0.0178$

$\mathrm{pCi} / \mathrm{g} \quad 0.0109$

$\mathrm{pCi} / \mathrm{g} \quad 0.0576$

0.00638

$-0.0305$

0.0125

$\mathrm{pCi} / \mathrm{g} \quad 0.104$

$\mathrm{pCi} / \mathrm{g} \quad 0.0955$

EPIA-001B

EPIA-013B

EPIA-013B

EPIA-013B

EPIA-001B

EPIA-012B

EPIA-012B

EPIA-013B

EPIA-013B

EPIA-013B

EPIA-013B

EPIA-013B

EPA905

EPIA-013B

EPIA-002B

EPIA-011B

EPIA-011B

EPIA-011B

EPIA-013B

EPIA-013B

EPIA-013B

EPA8260B

EPA8260B

EPA8260B

EPA8260B

EPA8260B

EPA8260B

EPA8260B

EPA8260B

EPA8260B

EPA8260B

EPA8260B

EPA8260B

EPA8260B

EPA8260B

EPA8260B

EPA8260B

EPA8260B

EPA8260B

EPA8260B

EPA8260B

EPA8260B

EPA8260B

EPA8260B 
SURVEY ID: SRF-02-02 (cont.)

Constituent

Volatile Organic Compounds

Methyl ethyl ketone

Methyl isobutyl ketone

Styrene

1,1,2,2-Tetrachloroethane

Tetrachloroethylene

Toluene

1,1,1-Trichloroethane

1,1,2-Trichloroethane

Trichloroethylene

Vinyl acetate

Xylenes

SURVEY ID: SRF-02-03

Sample type: Normal

Constituent

Metals (total recoverable)

Antimony

Arsenic

Barium

Cadmium

Calcium

Chromium

Copper

Lead

Magnesium

Manganese

Mercury

Nickel

Selenium

Silver

Zinc

Radionuclides

Actinium-228

Antimony-124

Antimony-125

Barium-133

Cerium-144

Cesium-134

Cesium-137

Cobalt-57

Cobalt-58

Cobalt-60
Sample ID: 117024

FG SC E Result

Unit ssMDL

Method

$\begin{array}{llll}\mathrm{U} & & & 25 \\ \mathrm{U} & & & 25 \\ \mathrm{U} & & & 5 \\ \mathrm{U} & & & 5 \\ \mathrm{U} & & & 5 \\ \mathrm{~J} & \mathrm{~K} & \mathrm{O} & 5.4 \\ \mathrm{U} & & & 5 \\ \mathrm{U} & & & 5 \\ \mathrm{U} & & & 5 \\ \mathrm{U} & & & 75 \\ \mathrm{~J} & \mathrm{~K} & \mathrm{O} & 34.3\end{array}$

$\begin{array}{ll}\mu g / \mathrm{kg} & 16 \\ \mu g / \mathrm{kg} & 14.6 \\ \mu g / \mathrm{kg} & 1.5 \\ \mu g / \mathrm{kg} & 3 \\ \mu g / \mathrm{kg} & 2 \\ \mu g / \mathrm{kg} & 4.5 \\ \mu g / \mathrm{kg} & 0.5 \\ \mu g / \mathrm{kg} & 1.2 \\ \mu g / \mathrm{kg} & 1.35 \\ \mu g / \mathrm{kg} & 10.5 \\ \mu g / \mathrm{kg} & 5\end{array}$

EPA8260B EPA8260B EPA8260B EPA8260B EPA8260B EPA8260B EPA8260B EPA8260B EPA8260B EPA8260B EPA8260B
Sample ID: 117025

Sample matrix: Fauna
Percent solids: NA
FG SC E Result Unit ssMDL

$\begin{array}{lllll}\mathrm{U} & 0.5 & \mathrm{mg} / \mathrm{kg} & 0.191 & \text { EPA6010B }\end{array}$

$0.535 \quad \mathrm{mg} / \mathrm{kg} \quad 0.228 \quad$ EPA6010B

$2.96 \mathrm{mg} / \mathrm{kg} \quad 0.027 \quad$ EPA6010B

$0.25 \mathrm{mg} / \mathrm{kg} \quad 0.019 \quad$ EPA6010B

$14200 \quad \mathrm{mg} / \mathrm{kg} 3.486 \quad$ EPA6010B

$0.509 \quad \mathrm{mg} / \mathrm{kg} \quad 0.0365 \quad$ EPA6010B

$1.48 \quad \mathrm{mg} / \mathrm{kg} \quad 0.066 \quad$ EPA6010B

1.46

537

7.11

$\begin{array}{llll}\mathrm{J} & \mathrm{L} & \mathrm{I} & 0.618\end{array}$

$\mathrm{U} \quad \mathrm{V} \quad 0.366$

1.27

0.0969

12.6

$\mathrm{mg} / \mathrm{kg} \quad 0.079$

$\mathrm{mg} / \mathrm{kg} \quad 0.254$

$\mathrm{mg} / \mathrm{kg} \quad 0.355$

$\mathrm{mg} / \mathrm{kg} \quad 0.00205$

$\mathrm{mg} / \mathrm{kg} \quad 0.032$

$\mathrm{mg} / \mathrm{kg} \quad 0.135$

$\mathrm{mg} / \mathrm{kg} \quad 0.031$

$\mathrm{mg} / \mathrm{kg} \quad 0.185$

EPA6010B

EPA6010B

EPA6010B

EPA7471A

EPA6010B

EPA6010B

EPA6010B

EPA6010B

$\begin{array}{lllll}\mathrm{U} & 0.0133 & \mathrm{pCi} / \mathrm{g} & 0.27 & \text { EPIA-013B } \\ \mathrm{U} & 0.0434 & \mathrm{pCi} / \mathrm{g} & 0.105 & \text { EPIA-013B } \\ \mathrm{U} & -0.106 & \mathrm{pCi} / \mathrm{g} & 0.16 & \text { EPIA-013B } \\ \mathrm{U} & 0.0178 & \mathrm{pCi} / \mathrm{g} & 0.0759 & \text { EPIA-013B } \\ \mathrm{U} & 0.000742 & \mathrm{pCi} / \mathrm{g} & 0.326 & \text { EPIA-013B } \\ \mathrm{U} & -0.00891 & \mathrm{pCi} / \mathrm{g} & 0.0637 & \text { EPIA-013B } \\ & 0.621 & \mathrm{pCi} / \mathrm{g} & 0.0656 & \text { EPIA-013B } \\ \mathrm{U} & 0.0289 & \mathrm{pCi} / \mathrm{g} & 0.0411 & \text { EPIA-013B } \\ \mathrm{U} & -0.00466 & \mathrm{pCi} / \mathrm{g} & 0.0946 & \text { EPIA-013B } \\ \mathrm{U} & 0.0198 & \mathrm{pCi} / \mathrm{g} & 0.0882 & \text { EPIA-013B }\end{array}$


SURVEY ID: SRF-02-03 (cont.)

Constituent

Radionuclides

Europium-152

Europium-154

Europium-155

Gross alpha

Lead-212

Manganese-54

Neptunium-239

Nonvolatile beta

Plutonium-238

Plutonium-239/240

Potassium-40

Promethium-144

Promethium-146

Ruthenium-106

Sodium-22

Strontium-89/90

Tin-1 13

Tritium

Uranium-233/234

Uranium-235

Uranium-238

Yttrium-88

Zinc-65

Zirconium-95

Volatile Organic Compounds

Acetone

Benzene

Bromodichloromethane

Bromoform

Bromomethane (Methyl bromide)

Carbon disulfide

Carbon tetrachloride

Chlorobenzene

Chloroethane

Chloroethene (Vinyl chloride)

Chloroform

Chloromethane (Methyl chloride)

Dibromochloromethane

1,1-Dichloroethane

1,2-Dichloroethane

1,1-Dichloroethylene

1,2-Dichloroethylene

Dichloromethane (Methylene chloride) J

1,2-Dichloropropane

cis-1,3-Dichloropropene

trans-1,3-Dichloropropene

Ethylbenzene

2-Hexanone

Methyl ethyl ketone

Methyl isobutyl ketone

Styrene
Sample ID: 117025

FG SC E Result

Unit ssMDL

Method

$\begin{array}{lll}\mathrm{pCi} / \mathrm{g} & 0.191 & \text { EPIA-013B } \\ \mathrm{pCi} / \mathrm{g} & 0.207 & \text { EPIA-013B } \\ \mathrm{pCi} / \mathrm{g} & 0.146 & \text { EPIA-013B } \\ \mathrm{pCi} / \mathrm{g} & 0.995 & \text { EPIA-001B } \\ \mathrm{pCi} / \mathrm{g} & 0.11 & \text { EPIA-013B } \\ \mathrm{pCi} / \mathrm{g} & 0.0872 & \text { EPIA-013B } \\ \mathrm{pCi} / \mathrm{g} & 0.269 & \text { EPIA-013B } \\ \mathrm{pCi} / \mathrm{g} & 1.04 & \text { EPIA-001B } \\ \mathrm{pCi} / \mathrm{g} & 0.0303 & \text { EPIA-012B } \\ \mathrm{pCi} / \mathrm{g} & 0.0149 & \text { EPIA-012B } \\ \mathrm{pCi} / \mathrm{g} & 0.695 & \text { EPIA-013B } \\ \mathrm{pCi} / \mathrm{g} & 0.075 & \text { EPIA-013B } \\ \mathrm{pCi} / \mathrm{g} & 0.0788 & \text { EPIA-013B } \\ \mathrm{pCi} / \mathrm{g} & 0.699 & \text { EPIA-013B } \\ \mathrm{pCi} / \mathrm{g} & 0.0467 & \text { EPIA-013B } \\ \mathrm{pCi} / \mathrm{g} & 0.505 & \text { EPA905 } \\ \mathrm{pCi} / \mathrm{g} & 0.097 & \text { EPIA-013B } \\ \mathrm{pCi} / \mathrm{g} & 0.162 & \text { EPIA-002B } \\ \mathrm{pCi} / \mathrm{g} & 0.0203 & \text { EPIA-011B } \\ \mathrm{pCi} / \mathrm{g} & 0.0142 & \text { EPIA-011B } \\ \mathrm{pCi} / \mathrm{g} & 0.0167 & \text { EPIA-011B } \\ \mathrm{pCi} / \mathrm{g} & 0.0872 & \text { EPIA-013B } \\ \mathrm{pCi} / \mathrm{g} & 0.151 & \text { EPIA-013B } \\ \mathrm{pCi} / \mathrm{g} & 0.164 & \text { EPIA-013B }\end{array}$

0.0139
0.0376
0.0159
0.111
0.0564
0.0811
-0.0876
8.12
0.00402
-0.00115
3.58
0.015
-0.00799
0.0415
0.0136
0.427
0.0399
-0.0238
0.00548
0.00205
0.0014
-0.0388
0.0773
0.00407

$\mathrm{pCi} / \mathrm{g}$

0.164
EPA8260B

EPA8260B

EPA8260B

EPA8260B

EPA8260B

EPA8260B

EPA8260B

EPA8260B

EPA8260B

EPA8260B

EPA8260B

EPA8260B

EPA8260B

EPA8260B

EPA8260B

EPA8260B

EPA8260B

EPA8260B

EPA8260B

EPA8260B

EPA8260B

EPA8260B

EPA8260B

EPA8260B

EPA8260B

EPA8260B 
SURVEY ID: SRF-02-03 (cont.)

Constituent

Volatile Organic Compounds

1,1,2,2-Tetrachloroethane

Tetrachloroethylene

Toluene

1,1,1-Trichloroethane

1,1,2-Trichloroethane

Trichloroethylene

Vinyl acetate

Xylenes

SURVEY ID: SRF-02-03-A

Sample type: Field Duplicate

\section{Constituent}

Metals (total recoverable)

Antimony
Arsenic
Barium
Cadmium
Calcium
Chromium
Copper
Lead
Magnesium
Manganese
Mercury
Nickel
Selenium
Silver
Zinc

Radionuclides

Actinium-228

Antimony-124

Antimony-125

Barium-133

Cerium-144

Cesium-134

Cesium-137

Cobalt-57

Cobalt-58

Cobalt-60

Europium-152

Europium-154

Europium-155
Sample ID: 117025

FG SC E Result

Unit ssMDL

Method

$\begin{array}{ll}\mu g / \mathrm{kg} & 3 \\ \mu \mathrm{g} / \mathrm{kg} & 2 \\ \mu \mathrm{g} / \mathrm{kg} & 4.5 \\ \mu \mathrm{g} / \mathrm{kg} & 0.5 \\ \mu \mathrm{g} / \mathrm{kg} & 1.2 \\ \mu \mathrm{g} / \mathrm{kg} & 1.35 \\ \mu \mathrm{g} / \mathrm{kg} & 10.5 \\ \mu \mathrm{g} / \mathrm{kg} & 5\end{array}$

EPA8260B

EPA8260B

EPA8260B

EPA8260B

EPA8260B

EPA8260B

EPA8260B

EPA8260B
Sample ID: 117026

Sample matrix: Fauna

Percent solids: NA
FG SC E Result Unit ssMDL

\begin{tabular}{|c|c|c|c|c|c|}
\hline $\mathrm{U}$ & & 0.472 & $\mathrm{mg} / \mathrm{kg}$ & 0.18 & EPA6010B \\
\hline & & 0.666 & $\mathrm{mg} / \mathrm{kg}$ & 0.215 & EPA6010B \\
\hline & & 5.99 & $\mathrm{mg} / \mathrm{kg}$ & 0.025 & EPA6010B \\
\hline JU & 4 & 0.0574 & $\mathrm{mg} / \mathrm{kg}$ & 0.018 & EPA6010B \\
\hline & & 29600 & $\mathrm{mg} / \mathrm{kg}$ & 6.575 & EPA6010B \\
\hline & & 0.461 & $\mathrm{mg} / \mathrm{kg}$ & 0.0344 & EPA6010B \\
\hline & & 7.3 & $\mathrm{mg} / \mathrm{kg}$ & 0.0623 & EPA6010B \\
\hline J & I & 0.189 & $\mathrm{mg} / \mathrm{kg}$ & 0.075 & EPA6010B \\
\hline & & 1400 & $\mathrm{mg} / \mathrm{kg}$ & 0.24 & EPA6010B \\
\hline & & 14.3 & $\mathrm{mg} / \mathrm{kg}$ & 0.335 & EPA6010B \\
\hline & & 0.396 & $\mathrm{mg} / \mathrm{kg}$ & 0.00197 & EPA7471A \\
\hline $\mathrm{U}$ & & 0.236 & $\mathrm{mg} / \mathrm{kg}$ & 0.03 & EPA6010B \\
\hline & & 2.85 & $\mathrm{mg} / \mathrm{kg}$ & 0.127 & EPA6010B \\
\hline & & 0.712 & $\mathrm{mg} / \mathrm{kg}$ & 0.0292 & EPA6010B \\
\hline & & 21.5 & $\mathrm{mg} / \mathrm{kg}$ & 0.175 & EPA6010B \\
\hline
\end{tabular}

$-0.0295$

0.035

0.00484

0.121

$-0.0383$

0.751

$-0.00317$

$-0.0398$

$-0.0107$

0.0379

$-0.0501$

0.0157

$\begin{array}{ll}\mathrm{pCi} / \mathrm{g} & 0.296 \\ \mathrm{pCi} / \mathrm{g} & 0.0869 \\ \mathrm{pCi} / \mathrm{g} & 0.168 \\ \mathrm{pCi} / \mathrm{g} & 0.0702 \\ \mathrm{pCi} / \mathrm{g} & 0.309 \\ \mathrm{pCi} / \mathrm{g} & 0.061 \\ \mathrm{pCi} / \mathrm{g} & 0.0596 \\ \mathrm{pCi} / \mathrm{g} & 0.0378 \\ \mathrm{pCi} / \mathrm{g} & 0.0747 \\ \mathrm{pCi} / \mathrm{g} & 0.0717 \\ \mathrm{pCi} / \mathrm{g} & 0.171 \\ \mathrm{pCi} / \mathrm{g} & 0.199 \\ \mathrm{pCi} / \mathrm{g} & 0.141\end{array}$

EPIA-013B

EPIA-013B

EPIA-013B

EPIA-013B

EPIA-013B

EPIA-013B

EPIA-013B

EPIA-013B

EPIA-013B

EPIA-013B

EPIA-013B

EPIA-013B

EPIA-013B 
SURVEY ID: SRF-02-03-A (cont.)

Constituent

Radionuclides

Gross alpha

Lead-212

Manganese-54

Neptunium-239

Nonvolatile beta

Plutonium-238

Plutonium-239/240

Potassium-40

Promethium-144

Promethium-146

Ruthenium-106

Sodium-22

Strontium-89/90

Tin-1 13

Tritium

Uranium-233/234

Uranium-235

Uranium-238

Yttrium-88

Zinc-65

Zirconium-95

Volatile Organic Compounds

Acetone

Benzene

Bromodichloromethane

Bromoform

Bromomethane (Methyl bromide)

Carbon disulfide

Carbon tetrachloride

Chlorobenzene

Chloroethane

Chloroethene (Vinyl chloride)

Chloroform

Chloromethane (Methyl chloride)

Dibromochloromethane

1,1-Dichloroethane

1,2-Dichloroethane

1,1-Dichloroethylene

1,2-Dichloroethylene

Dichloromethane (Methylene chloride)

1,2-Dichloropropane

cis-1,3-Dichloropropene

trans-1,3-Dichloropropene

Ethylbenzene

2-Hexanone

Methyl ethyl ketone

Methyl isobutyl ketone

Styrene

1,1,2,2-Tetrachloroethane

Tetrachloroethylene

Toluene

Sample ID: 117026

FG SC E Result

Unit ssMDL

Method

$\begin{array}{lll}\mathrm{pCi} / \mathrm{g} & 1.07 & \text { EPIA-001B } \\ \mathrm{pCi} / \mathrm{g} & 0.107 & \text { EPIA-013B } \\ \mathrm{pCi} / \mathrm{g} & 0.0693 & \text { EPIA-013B } \\ \mathrm{pCi} / \mathrm{g} & 0.277 & \text { EPIA-013B } \\ \mathrm{pCi} / \mathrm{g} & 1.03 & \text { EPIA-001B } \\ \mathrm{pCi} / \mathrm{g} & 0.0438 & \text { EPIA-012B } \\ \mathrm{pCi} / \mathrm{g} & 0.0242 & \text { EPIA-012B } \\ \mathrm{pCi} / \mathrm{g} & 0.739 & \text { EPIA-013B } \\ \mathrm{pCi} / \mathrm{g} & 0.0621 & \text { EPIA-013B } \\ \mathrm{pCi} / \mathrm{g} & 0.0706 & \text { EPIA-013B } \\ \mathrm{pCi} / \mathrm{g} & 0.575 & \text { EPIA-013B } \\ \mathrm{pCi} / \mathrm{g} & 0.0722 & \text { EPIA-013B } \\ \mathrm{pCi} / \mathrm{g} & 0.603 & \text { EPA905 } \\ \mathrm{pCi} / \mathrm{g} & 0.0845 & \text { EPIA-013B } \\ \mathrm{pCi} / \mathrm{g} & 0.13 & \text { EPIA-002B } \\ \mathrm{pCi} / \mathrm{g} & 0.0265 & \text { EPIA-011B } \\ \mathrm{pCi} / \mathrm{g} & 0.0181 & \text { EPIA-011B } \\ \mathrm{pCi} / \mathrm{g} & 0.0078 & \text { EPIA-011B } \\ \mathrm{pCi} / \mathrm{g} & 0.0977 & \text { EPIA-013B } \\ \mathrm{pCi} / \mathrm{g} & 0.138 & \text { EPIA-013B } \\ \mathrm{pCi} / \mathrm{g} & 0.143 & \text { EPIA-013B }\end{array}$

0.162

0.0862

0.0215

0.114

6.29

$-0.0137$

$-0.00307$

3.53

$-0.0191$

$-0.0416$

0.0977

$-0.0179$

0.391

0.00248

$-0.0247$

$-0.00104$

$-0.00188$

0.0078

0.0237

$-0.0228$

0.0143

79.2

5

5

5

25

5

5

5

5

5

5

5

5

5

5

50

36.1

5

5

5

5

25

25

25

5

5

5

5

$\begin{array}{ll}\mu g / \mathrm{kg} & 51.5 \\ \mu g / \mathrm{kg} & 2.5 \\ \mu g / \mathrm{kg} & 0.5 \\ \mu \mathrm{g} / \mathrm{kg} & 1.35 \\ \mu g / \mathrm{kg} & 1.5 \\ \mu \mathrm{g} / \mathrm{kg} & 1.5 \\ \mu \mathrm{g} / \mathrm{kg} & 2.5 \\ \mu \mathrm{g} / \mathrm{kg} & 1.5 \\ \mu \mathrm{g} / \mathrm{kg} & 1.5 \\ \mu g / \mathrm{kg} & 2 \\ \mu g / \mathrm{kg} & 0.5 \\ \mu g / \mathrm{kg} & 1 \\ \mu \mathrm{g} / \mathrm{kg} & 1.05 \\ \mu \mathrm{g} / \mathrm{kg} & 0.5 \\ \mu g / \mathrm{kg} & 1.15 \\ \mu g / \mathrm{kg} & 1.5 \\ \mu g / \mathrm{kg} & 1.25 \\ \mu g / \mathrm{kg} & 7 \\ \mu g / \mathrm{kg} & 1.15 \\ \mu g / \mathrm{kg} & 1 \\ \mu g / \mathrm{kg} & 1.5 \\ \mu g / \mathrm{kg} & 1.5 \\ \mu g / \mathrm{kg} & 14 \\ \mu g / \mathrm{kg} & 16 \\ \mu g / \mathrm{kg} & 14.6 \\ \mu g / \mathrm{kg} & 1.5 \\ \mu g / \mathrm{kg} & 3 \\ \mu g / \mathrm{kg} & 2 \\ \mu g / \mathrm{kg} & 4.5\end{array}$

EPA8260B

EPA8260B

EPA8260B

EPA8260B

EPA8260B

EPA8260B

EPA8260B

EPA8260B

EPA8260B

EPA8260B

EPA8260B

EPA8260B

EPA8260B

EPA8260B

EPA8260B

EPA8260B

EPA8260B

EPA8260B

EPA8260B

EPA8260B

EPA8260B

EPA8260B

EPA8260B

EPA8260B

EPA8260B

EPA8260B

EPA8260B

EPA8260B

EPA8260B 
SURVEY ID: SRF-02-03-A (cont.)

Constituent

Volatile Organic Compounds

1,1,1-Trichloroethane

1,1,2-Trichloroethane

Trichloroethylene

Vinyl acetate

Xylenes

SURVEY ID: SRF-03-01

Sample type: Normal

\section{Constituent}

Metals (total recoverable)
Sample ID: 117026

FG SC E Result
Unit ssMDL

Method

$\begin{array}{ll}\mu g / \mathrm{kg} & 0.5 \\ \mu g / \mathrm{kg} & 1.2 \\ \mu g / \mathrm{kg} & 1.35 \\ \mu g / \mathrm{kg} & 10.5 \\ \mu g / \mathrm{kg} & 5\end{array}$

EPA8260B

EPA8260B

EPA8260B

EPA8260B

EPA8260B
Sample ID: 117027

Sample matrix: Fauna
Percent solids: NA
FG SC E Result Unit ssMDL $\quad$ Uethod

\begin{tabular}{|c|c|c|c|c|c|}
\hline \multirow[t]{3}{*}{$\mathrm{U}$} & & 0.495 & $\mathrm{mg} / \mathrm{kg}$ & 0.189 & EPA6010B \\
\hline & & 0.465 & $\mathrm{mg} / \mathrm{kg}$ & 0.226 & EPA6010B \\
\hline & & 1.74 & $\mathrm{mg} / \mathrm{kg}$ & 0.027 & EPA6010B \\
\hline \multirow[t]{2}{*}{ J } & I & 0.0308 & $\mathrm{mg} / \mathrm{kg}$ & 0.019 & EPA6010B \\
\hline & & 9240 & $\mathrm{mg} / \mathrm{kg}$ & 3.451 & EPA6010B \\
\hline \multirow[t]{2}{*}{ J } & I & 0.152 & $\mathrm{mg} / \mathrm{kg}$ & 0.0361 & EPA6010B \\
\hline & & 0.363 & $\mathrm{mg} / \mathrm{kg}$ & 0.0653 & EPA6010B \\
\hline \multirow[t]{4}{*}{$J$} & I & 0.0998 & $\mathrm{mg} / \mathrm{kg}$ & 0.078 & EPA6010B \\
\hline & & 375 & $\mathrm{mg} / \mathrm{kg}$ & 0.251 & EPA6010B \\
\hline & & 19.2 & $\mathrm{mg} / \mathrm{kg}$ & 0.351 & EPA6010B \\
\hline & & 0.268 & $\mathrm{mg} / \mathrm{kg}$ & 0.00205 & EPA7471A \\
\hline \multirow[t]{2}{*}{$\mathrm{U}$} & & 0.248 & $\mathrm{mg} / \mathrm{kg}$ & 0.032 & EPA6010B \\
\hline & & 0.905 & $\mathrm{mg} / \mathrm{kg}$ & 0.134 & EPA6010B \\
\hline \multirow[t]{2}{*}{$\mathrm{U}$} & & 0.248 & $\mathrm{mg} / \mathrm{kg}$ & 0.0307 & EPA6010B \\
\hline & & 12.4 & $\mathrm{mg} / \mathrm{kg}$ & 0.184 & EPA6010I \\
\hline
\end{tabular}

Radionuclides

Actinium-228

Antimony-124

Antimony-125

Barium-133

Cerium-144

Cesium-134

Cesium-137

Cobalt-57

Cobalt-58

Cobalt-60

Europium-152

Europium-154

Europium-155

Gross alpha

Lead-212

Manganese-54
0.126
0.0153
0.0385
$-0.0676$
$-0.0603$
$-0.0117$
0.032
0.00686
0.0495
0.0444
0.0307
$-0.0319$
$-0.0712$
$-0.0176$
0.0128
0.0347

$\begin{array}{ll}\mathrm{pCi} / \mathrm{g} & 0.249 \\ \mathrm{pCi} / \mathrm{g} & 0.0876 \\ \mathrm{pCi} / \mathrm{g} & 0.153 \\ \mathrm{pCi} / \mathrm{g} & 0.0591 \\ \mathrm{pCi} / \mathrm{g} & 0.283 \\ \mathrm{pCi} / \mathrm{g} & 0.0599 \\ \mathrm{pCi} / \mathrm{g} & 0.0616 \\ \mathrm{pCi} / \mathrm{g} & 0.0375 \\ \mathrm{pCi} / \mathrm{g} & 0.0667 \\ \mathrm{pCi} / \mathrm{g} & 0.0685 \\ \mathrm{pCi} / \mathrm{g} & 0.172 \\ \mathrm{pCi} / \mathrm{g} & 0.186 \\ \mathrm{pCi} / \mathrm{g} & 0.134 \\ \mathrm{pCi} / \mathrm{g} & 1.75 \\ \mathrm{pCi} / \mathrm{g} & 0.107 \\ \mathrm{pCi} / \mathrm{g} & 0.0649\end{array}$

EPIA-013B EPIA-013B EPIA-013B EPIA-013B EPIA-013B EPIA-013B EPIA-013B EPIA-013B EPIA-013B EPIA-013B EPIA-013B EPIA-013B EPIA-013B EPIA-001B EPIA-013B EPIA-013B 
SURVEY ID: SRF-03-01 (cont.)

Constituent

Radionuclides

Neptunium-239

Nonvolatile beta

Plutonium-238

Plutonium-239/240

Potassium-40

Promethium-144

Promethium-146

Ruthenium-106

Sodium-22

Strontium-89/90

Tin-1 13

Tritium

Tritium

Uranium-233/234

Uranium-235

Uranium-238

Yttrium-88

Zinc-65

Zirconium-95

Volatile Organic Compounds

$\begin{array}{ll}\text { Acetone } & \\ \text { Benzene } & \\ \text { Bromodichloromethane } & \\ \text { Bromoform } & \\ \text { Bromomethane (Methyl bromide) } & \\ \text { Carbon disulfide } & \\ \text { Carbon tetrachloride } & \\ \text { Chlorobenzene } & \mathrm{U} \\ \text { Chloroethane } & \mathrm{U} \\ \text { Chloroethene (Vinyl chloride) } & \mathrm{U} \\ \text { Chloroform } & \mathrm{U} \\ \text { Chloromethane (Methyl chloride) } & \mathrm{U} \\ \text { Dibromochloromethane } & \mathrm{U} \\ \text { 1,1-Dichloroethane } & \mathrm{U} \\ \text { 1,2-Dichloroethane } & \mathrm{U} \\ \text { 1,1-Dichloroethylene } & \mathrm{U} \\ \text { 1,2-Dichloroethylene } & \mathrm{U} \\ \text { Dichloromethane (Methylene chloride) } \\ \text { 1,2-Dichloropropane } & \mathrm{U} \\ \text { cis-1,3-Dichloropropene } & \mathrm{U} \\ \text { trans-1,3-Dichloropropene } & \mathrm{U} \\ \text { Ethylbenzene } & \mathrm{U} \\ \text { 2-Hexanone } & \mathrm{U} \\ \text { Methyl ethyl ketone } & \mathrm{U} \\ \text { Methyl isobutyl ketone } & \mathrm{U} \\ \text { Styrene } & \\ \text { 1,1,2,2-Tetrachloroethane } & \\ \text { Tetrachloroethylene } & \\ \text { Toluene } & \\ \text { 1,1,1-Trichloroethane } & \end{array}$

Sample ID: 117027

FG SC E Result

Unit ssMDL

Method

$\begin{array}{lll}\mathrm{pCi} / \mathrm{g} & 0.277 & \text { EPIA-013B } \\ \mathrm{pCi} / \mathrm{g} & 1.75 & \text { EPIA-001B } \\ \mathrm{pCi} / \mathrm{g} & 0.0594 & \text { EPIA-012B } \\ \mathrm{pCi} / \mathrm{g} & 0.0261 & \text { EPIA-012B } \\ \mathrm{pCi} / \mathrm{g} & 0.6 & \text { EPIA-013B } \\ \mathrm{pCi} / \mathrm{g} & 0.0621 & \text { EPIA-013B } \\ \mathrm{pCi} / \mathrm{g} & 0.0695 & \text { EPIA-013B } \\ \mathrm{pCi} / \mathrm{g} & 0.514 & \text { EPIA-013B } \\ \mathrm{pCi} / \mathrm{g} & 0.0672 & \text { EPIA-013B } \\ \mathrm{pCi} / \mathrm{g} & 0.868 & \text { EPA905 } \\ \mathrm{pCi} / \mathrm{g} & 0.0729 & \text { EPIA-013B } \\ \mathrm{pCi} / \mathrm{g} & 0.144 & \text { EPIA-002B } \\ \mathrm{pCi} / \mathrm{g} & 0.137 & \text { EPIA-002B } \\ \mathrm{pCi} / \mathrm{g} & 0.047 & \text { EPIA-011B } \\ \mathrm{pCi} / \mathrm{g} & 0.0327 & \text { EPIA-011B } \\ \mathrm{pCi} / \mathrm{g} & 0.0228 & \text { EPIA-011B } \\ \mathrm{pCi} / \mathrm{g} & 0.0679 & \text { EPIA-013B } \\ \mathrm{pCi} / \mathrm{g} & 0.128 & \text { EPIA-013B } \\ \mathrm{pCi} / \mathrm{g} & 0.136 & \text { EPIA-013B }\end{array}$

0.0519

7.32

$-0.00319$

0.00218

2.47

0.03

0.0264

$-0.0495$

$-0.00353$

0.675

$-0.0209$

0

$-0.01$

0.000518

0.0045

$4 \quad 0.0248$

$-0.00252$

$-0.0121$

0.0463

U

U

U

U

U

U

U

U

U

U

U

$\mathrm{U}$

$\mathrm{J}$

U

$\mathrm{U}$

U

$\mathrm{U}$

U

U

U

U

U

U

U

U

347
5
5
5
5
25
5
5
5
5
5
5
5
5
5
5
50
17.8
5
5
5
5
25
25
25
5
5
5
5
5
5

5
5
5
5
5
5
5
5
5
5
5
5
5
5
5
5
5
5
5
5
5
5
5
5
5

\begin{tabular}{|c|c|}
\hline$\mu g / \mathrm{kg}$ & 51.5 \\
\hline$\mu \mathrm{g} / \mathrm{kg}$ & 2.5 \\
\hline$\mu \mathrm{g} / \mathrm{kg}$ & 0.5 \\
\hline$\mu \mathrm{g} / \mathrm{kg}$ & 1.35 \\
\hline$\mu \mathrm{g} / \mathrm{kg}$ & 1.5 \\
\hline$\mu \mathrm{g} / \mathrm{kg}$ & 1.5 \\
\hline$\mu \mathrm{g} / \mathrm{kg}$ & 2.5 \\
\hline$\mu \mathrm{g} / \mathrm{kg}$ & 1.5 \\
\hline$\mu \mathrm{g} / \mathrm{kg}$ & 1.5 \\
\hline$\mu \mathrm{g} / \mathrm{kg}$ & 2 \\
\hline$\mu \mathrm{g} / \mathrm{kg}$ & 0.5 \\
\hline$\mu \mathrm{g} / \mathrm{kg}$ & 1 \\
\hline$\mu \mathrm{g} / \mathrm{kg}$ & 1.05 \\
\hline$\mu \mathrm{g} / \mathrm{kg}$ & 0.5 \\
\hline$\mu \mathrm{g} / \mathrm{kg}$ & 1.15 \\
\hline$\mu \mathrm{g} / \mathrm{kg}$ & 1.5 \\
\hline$\mu \mathrm{g} / \mathrm{kg}$ & 1.25 \\
\hline$\mu g / \mathrm{kg}$ & 7 \\
\hline$\mu \mathrm{g} / \mathrm{kg}$ & 1.15 \\
\hline$\mu g / \mathrm{kg}$ & 1 \\
\hline$\mu \mathrm{g} / \mathrm{kg}$ & 1.5 \\
\hline$\mu \mathrm{g} / \mathrm{kg}$ & 1.5 \\
\hline$\mu \mathrm{g} / \mathrm{kg}$ & 14 \\
\hline$\mu \mathrm{g} / \mathrm{kg}$ & 16 \\
\hline$\mu \mathrm{g} / \mathrm{kg}$ & 14.6 \\
\hline$\mu \mathrm{g} / \mathrm{kg}$ & 1.5 \\
\hline$\mu \mathrm{g} / \mathrm{kg}$ & 3 \\
\hline$\mu \mathrm{g} / \mathrm{kg}$ & 2 \\
\hline$\mu \mathrm{g} / \mathrm{kg}$ & 4.5 \\
\hline$\mu g / \mathrm{kg}$ & 0.5 \\
\hline$\mu \mathrm{g} / \mathrm{kg}$ & 1.2 \\
\hline
\end{tabular}

EPA8260B

EPA8260B

EPA8260B

EPA8260B

EPA8260B

EPA8260B

EPA8260B

EPA8260B

EPA8260B

EPA8260B

EPA8260B

EPA8260B

EPA8260B

EPA8260B

EPA8260B

EPA8260B

EPA8260B

EPA8260B

EPA8260B

EPA8260B

EPA8260B

EPA8260B

EPA8260B

EPA8260B

EPA8260B

EPA8260B

EPA8260B

EPA8260B

EPA8260B

EPA8260B

EPA8260B 
SURVEY ID: SRF-03-01 (cont.)

Constituent

Volatile Organic Compounds

Trichloroethylene

Vinyl acetate

Xylenes

SURVEY ID: SRF-03-02

Sample type: Normal

Constituent

Metals (total recoverable)

Antimony

Arsenic

Barium

Cadmium

Calcium

Chromium

Copper

Lead

Magnesium

Manganese

Mercury

Nickel

Selenium

Silver

Zinc

Radionuclides

Actinium-228

Antimony-124

Antimony-125

Barium-133

Cerium-144

Cesium-134

Cesium-137

Cobalt-57

Cobalt-58

Cobalt-60

Europium-152

Europium-154

Europium-155

Gross alpha

Lead-212

Manganese-54

Neptunium-239

Nonvolatile beta
Sample ID: 117027

FG SC E Result

Unit ssMDL

Method

\begin{tabular}{|c|c|c|c|c|}
\hline $\mathrm{U}$ & 5 & $\mu g / \mathrm{kg}$ & 1.35 & EPA8260B \\
\hline $\mathrm{U}$ & 75 & $\mu \mathrm{g} / \mathrm{kg}$ & 10.5 & EPA8260B \\
\hline $\mathrm{J} \quad \mathrm{I}$ & 5.89 & $\mu \mathrm{g} / \mathrm{kg}$ & 5 & EPA82601 \\
\hline
\end{tabular}

Sample ID: 117028

Sample matrix: Fauna Percent solids: NA

\section{FG SC E Result Unit ssMDL}

U $\quad 0.485 \quad \mathrm{mg} / \mathrm{kg} \quad 0.185 \quad$ EPA6010B

$0.546 \quad \mathrm{mg} / \mathrm{kg} \quad 0.221 \quad$ EPA6010B

$6.63 \mathrm{mg} / \mathrm{kg} \quad 0.026 \quad$ EPA6010B

$0.022 \mathrm{mg} / \mathrm{kg} \quad 0.018 \quad$ EPA6010B

$4970 \quad \mathrm{mg} / \mathrm{kg} 3.381 \quad$ EPA6010B

$0.974 \mathrm{mg} / \mathrm{kg} \quad 0.0354 \quad$ EPA6010B

$2.55 \mathrm{mg} / \mathrm{kg} \quad 0.0641 \quad$ EPA6010B

$0.588 \quad \mathrm{mg} / \mathrm{kg} \quad 0.077 \quad$ EPA6010B

$278 \quad \mathrm{mg} / \mathrm{kg} \quad 0.246 \quad$ EPA6010B

$76.2 \quad \mathrm{mg} / \mathrm{kg} \quad 0.344 \quad$ EPA6010B

$0.0767 \quad \mathrm{mg} / \mathrm{kg} \quad 0.00223 \quad$ EPA7471A

$0.517 \quad \mathrm{mg} / \mathrm{kg} \quad 0.031 \quad$ EPA6010B

$0.883 \quad \mathrm{mg} / \mathrm{kg} \quad 0.131 \quad$ EPA6010B

$0.243 \quad \mathrm{mg} / \mathrm{kg} \quad 0.0301 \quad$ EPA6010B

$16.3 \quad \mathrm{mg} / \mathrm{kg} \quad 0.18 \quad$ EPA6010B

\begin{tabular}{|c|c|c|c|c|}
\hline & 0.883 & $\mathrm{mg} / \mathrm{kg}$ & 0.131 & EPA6010B \\
\hline 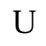 & 0.243 & $\mathrm{mg} / \mathrm{kg}$ & 0.0301 & EPA6010B \\
\hline & 16.3 & $\mathrm{mg} / \mathrm{kg}$ & 0.18 & EPA6010B \\
\hline
\end{tabular}

$\begin{array}{lllll}\mathrm{U} & 0.185 & \mathrm{pCi} / \mathrm{g} & 0.202 & \text { EPIA-013B } \\ \mathrm{U} & -0.0296 & \mathrm{pCi} / \mathrm{g} & 0.0597 & \text { EPIA-013B } \\ \mathrm{U} & 0.0238 & \mathrm{pCi} / \mathrm{g} & 0.105 & \text { EPIA-013B } \\ \mathrm{U} & 0.0213 & \mathrm{pCi} / \mathrm{g} & 0.0522 & \text { EPIA-013B } \\ \mathrm{U} & -0.00921 & \mathrm{pCi} / \mathrm{g} & 0.232 & \text { EPIA-013B } \\ \mathrm{U} & -0.0552 & \mathrm{pCi} / \mathrm{g} & 0.0419 & \text { EPIA-013B } \\ \mathrm{U} & 0.009 & \mathrm{pCi} / \mathrm{g} & 0.0501 & \text { EPIA-013B } \\ \mathrm{U} & 0.0121 & \mathrm{pCi} / \mathrm{g} & 0.0305 & \text { EPIA-013B } \\ \mathrm{U} & -0.0258 & \mathrm{pCi} / \mathrm{g} & 0.0533 & \text { EPIA-013B } \\ \mathrm{U} & -0.00223 & \mathrm{pCi} / \mathrm{g} & 0.0532 & \text { EPIA-013B } \\ \mathrm{U} & -0.0325 & \mathrm{pCi} / \mathrm{g} & 0.112 & \text { EPIA-013B } \\ \mathrm{U} & -0.0526 & \mathrm{pCi} / \mathrm{g} & 0.127 & \text { EPIA-013B } \\ \mathrm{U} & 0.0502 & \mathrm{pCi} / \mathrm{g} & 0.11 & \text { EPIA-013B } \\ \mathrm{U} & 0.0683 & \mathrm{pCi} / \mathrm{g} & 1.69 & \text { EPIA-001B } \\ \mathrm{R} & 0.0493 & \mathrm{pCi} / \mathrm{g} & 0.0825 & \text { EPIA-013B } \\ \mathrm{U} & -0.000495 & \mathrm{pCi} / \mathrm{g} & 0.0498 & \text { EPIA-013B } \\ \mathrm{U} & 0.0502 & \mathrm{pCi} / \mathrm{g} & 0.207 & \text { EPIA-013B } \\ & 7.88 & \mathrm{pCi} / \mathrm{g} & 1.49 & \text { EPIA-001B }\end{array}$


SURVEY ID: SRF-03-02 (cont.)

Constituent

Radionuclides

Plutonium-238

Plutonium-238

Plutonium-239/240

Plutonium-239/240

Potassium-40

Promethium-144

Promethium-146

Ruthenium-106

Sodium-22

Strontium-89/90

Tin-1 13

Tritium

Uranium-233/234

Uranium-233/234

Uranium-235

Uranium-235

Uranium-238

Uranium-238

Yttrium-88

Zinc-65

Zirconium-95

Volatile Organic Compounds

Acetone

Benzene

Bromodichloromethane

Bromoform

Bromomethane (Methyl bromide)

Carbon disulfide

Carbon tetrachloride

Chlorobenzene

Chloroethane

Chloroethene (Vinyl chloride)

Chloroform

Chloromethane (Methyl chloride)

Dibromochloromethane

1,1-Dichloroethane

1,2-Dichloroethane

1,1-Dichloroethylene

1,2-Dichloroethylene

Dichloromethane (Methylene chloride)

1,2-Dichloropropane cis-1,3-Dichloropropene

trans-1,3-Dichloropropene

Ethylbenzene

2-Hexanone

Methyl ethyl ketone

Methyl isobutyl ketone

Styrene

1,1,2,2-Tetrachloroethane

Tetrachloroethylene

Toluene
Sample ID: 117028

FG SC E Result

Unit ssMDL

Method

$\begin{array}{lll}\mathrm{pCi} / \mathrm{g} & 0.0136 & \text { EPIA-012B } \\ \mathrm{pCi} / \mathrm{g} & 0.0129 & \text { EPIA-012B } \\ \mathrm{pCi} / \mathrm{g} & 0.0238 & \text { EPIA-012B } \\ \mathrm{pCi} / \mathrm{g} & 0.0227 & \text { EPIA-012B } \\ \mathrm{pCi} / \mathrm{g} & 0.423 & \text { EPIA-013B } \\ \mathrm{pCi} / \mathrm{g} & 0.0471 & \text { EPIA-013B } \\ \mathrm{pCi} / \mathrm{g} & 0.05 & \text { EPIA-013B } \\ \mathrm{pCi} / \mathrm{g} & 0.411 & \text { EPIA-013B } \\ \mathrm{pCi} / \mathrm{g} & 0.0458 & \text { EPIA-013B } \\ \mathrm{pCi} / \mathrm{g} & 0.221 & \text { EPA905 } \\ \mathrm{pCi} / \mathrm{g} & 0.0579 & \text { EPIA-013B } \\ \mathrm{pCi} / \mathrm{g} & 0.145 & \text { EPIA-002B } \\ \mathrm{pCi} / \mathrm{g} & 0.0314 & \text { EPIA-011B } \\ \mathrm{pCi} / \mathrm{g} & 0.0256 & \text { EPIA-011B } \\ \mathrm{pCi} / \mathrm{g} & 0.024 & \text { EPIA-011B } \\ \mathrm{pCi} / \mathrm{g} & 0.0257 & \text { EPIA-011B } \\ \mathrm{pCi} / \mathrm{g} & 0.0239 & \text { EPIA-011B } \\ \mathrm{pCi} / \mathrm{g} & 0.0217 & \text { EPIA-011B } \\ \mathrm{pCi} / \mathrm{g} & 0.0548 & \text { EPIA-013B } \\ \mathrm{pCi} / \mathrm{g} & 0.125 & \text { EPIA-013B } \\ \mathrm{pCi} / \mathrm{g} & 0.109 & \text { EPIA-013B }\end{array}$

$\mu \mathrm{g} / \mathrm{kg} \quad 51.5$

EPA8260B

$\mu \mathrm{g} / \mathrm{kg} \quad 2.5$

$\mu g / \mathrm{kg} \quad 0.5$

$\mu \mathrm{g} / \mathrm{kg} \quad 1.35$

$\mu \mathrm{g} / \mathrm{kg} \quad 1.5$

$\mu \mathrm{g} / \mathrm{kg} \quad 1.5$

$\mu \mathrm{g} / \mathrm{kg} \quad 2.5$

$\mu g / \mathrm{kg} \quad 1.5$

$\mu \mathrm{g} / \mathrm{kg} \quad 1.5$

$\mu \mathrm{g} / \mathrm{kg} \quad 2$

$\mu g / k g \quad 0.5$

$\mu \mathrm{g} / \mathrm{kg} \quad 1$

$\mu \mathrm{g} / \mathrm{kg} \quad 1.05$

$\mu \mathrm{g} / \mathrm{kg} \quad 0.5$

$\mu \mathrm{g} / \mathrm{kg} \quad 1.15$

$\mu \mathrm{g} / \mathrm{kg} \quad 1.5$

$\mu \mathrm{g} / \mathrm{kg} \quad 1.25$

$\mu g / \mathrm{kg} \quad 7$

$\mu \mathrm{g} / \mathrm{kg} \quad 1.15$

$\mu \mathrm{g} / \mathrm{kg} \quad 1$

$\mu g / \mathrm{kg} \quad 1.5$

$\mu \mathrm{g} / \mathrm{kg} \quad 1.5$

$\mu \mathrm{g} / \mathrm{kg} \quad 14$

$\mu \mathrm{g} / \mathrm{kg} \quad 16$

$\mu \mathrm{g} / \mathrm{kg} \quad 14.6$

$\mu \mathrm{g} / \mathrm{kg} \quad 1.5$

$\mu \mathrm{g} / \mathrm{kg} \quad 3$

$\mu \mathrm{g} / \mathrm{kg} \quad 2$

$\mu \mathrm{g} / \mathrm{kg} \quad 4.5$
EPA8260B

EPA8260B

EPA8260B

EPA8260B

EPA8260B

EPA8260B

EPA8260B

EPA8260B

EPA8260B

EPA8260B

EPA8260B

EPA8260B

EPA8260B

EPA8260B

EPA8260B

EPA8260B

EPA8260B

EPA8260B

EPA8260B

EPA8260B

EPA8260B

EPA8260B

EPA8260B

EPA8260B

EPA8260B

EPA8260B

EPA8260B

EPA8260B 
SURVEY ID: SRF-03-02 (cont.)

Constituent

Volatile Organic Compounds

1,1,1-Trichloroethane

1,1,2-Trichloroethane

Trichloroethylene

Vinyl acetate

Xylenes

SURVEY ID: SRF-03-03

Sample type: Normal

\section{Constituent}

Metals (total recoverable)

Antimony
Arsenic
Barium
Cadmium
Calcium
Chromium
Copper
Lead
Magnesium
Manganese
Mercury
Nickel
Selenium
Silver
Zinc
Radionuclides
Actinium-228
Antimony-124
Antimony-125
Barium-133
Cerium-144
Cesium-134
Cesium-137
Cobalt-57
Cobalt-58
Cobalt-60
Europium-152
Europium-154
Europium-155
Gross alpha
Lead-212
Manganese-54

Sample ID: 117028

FG SC E Result

Unit ssMDL

Method

$\begin{array}{lllll}\mathrm{U} & 5 & \mu g / \mathrm{kg} & 0.5 & \text { EPA8260B } \\ \mathrm{U} & 5 & \mu \mathrm{gg} & 1.2 & \text { EPA8260B } \\ \mathrm{U} & 5 & \mu \mathrm{kg} / \mathrm{kg} & 1.35 & \text { EPA8260B } \\ \mathrm{U} & 75 & \mu \mathrm{kg} & 10.5 & \text { EPA8260B } \\ & 25.1 & \mu \mathrm{kg} / \mathrm{kg} & 5 & \text { EPA8260B }\end{array}$

Sample ID: 117029

Sample matrix: Fauna Percent solids: NA

\begin{tabular}{|c|c|c|c|c|c|}
\hline \multirow[t]{3}{*}{$\mathrm{U}$} & & 0.467 & $\mathrm{mg} / \mathrm{kg}$ & 0.178 & EPA6010B \\
\hline & & 0.713 & $\mathrm{mg} / \mathrm{kg}$ & 0.213 & EPA6010B \\
\hline & & 4.43 & $\mathrm{mg} / \mathrm{kg}$ & 0.025 & EPA6010B \\
\hline \multirow[t]{4}{*}{ JU } & 4 & 0.0273 & $\mathrm{mg} / \mathrm{kg}$ & 0.018 & EPA6010B \\
\hline & & 20500 & $\mathrm{mg} / \mathrm{kg}$ & 3.256 & EPA6010B \\
\hline & & 0.332 & $\mathrm{mg} / \mathrm{kg}$ & 0.0341 & EPA6010B \\
\hline & & 1.16 & $\mathrm{mg} / \mathrm{kg}$ & 0.0617 & EPA6010B \\
\hline \multirow[t]{4}{*}{$\mathrm{J}$} & I & 0.174 & $\mathrm{mg} / \mathrm{kg}$ & 0.074 & EPA6010B \\
\hline & & 825 & $\mathrm{mg} / \mathrm{kg}$ & 0.237 & EPA6010B \\
\hline & & 9.96 & $\mathrm{mg} / \mathrm{kg}$ & 0.332 & EPA6010B \\
\hline & & 0.261 & $\mathrm{mg} / \mathrm{kg}$ & 0.0141 & EPA7471A \\
\hline \multirow[t]{2}{*}{$\mathrm{U}$} & & 0.234 & $\mathrm{mg} / \mathrm{kg}$ & 0.03 & EPA6010B \\
\hline & & 1.13 & $\mathrm{mg} / \mathrm{kg}$ & 0.126 & EPA6010B \\
\hline \multirow[t]{2}{*}{$\mathrm{J}$} & I & 0.067 & $\mathrm{mg} / \mathrm{kg}$ & 0.029 & EPA6010B \\
\hline & & 23.8 & $\mathrm{mg} / \mathrm{kg}$ & 0.173 & EPA6010B \\
\hline
\end{tabular}

$\begin{array}{lllll}\mathrm{U} & 0.0927 & \mathrm{pCi} / \mathrm{g} & 0.235 & \text { EPIA-013B } \\ \mathrm{U} & -0.00361 & \mathrm{pCi} / \mathrm{g} & 0.0698 & \text { EPIA-013B } \\ \mathrm{U} & 0.0233 & \mathrm{pCi} / \mathrm{g} & 0.135 & \text { EPIA-013B } \\ \mathrm{U} & 0.00717 & \mathrm{pCi} / \mathrm{g} & 0.0562 & \text { EPIA-013B } \\ \mathrm{U} & 0.056 & \mathrm{pCi} / \mathrm{g} & 0.254 & \text { EPIA-013B } \\ \mathrm{U} & -0.0272 & \mathrm{pCi} / \mathrm{g} & 0.0463 & \text { EPIA-013B } \\ \mathrm{U} & 0.049 & \mathrm{pCi} / \mathrm{g} & 0.0619 & \text { EPIA-013B } \\ \mathrm{U} & 0.000747 & \mathrm{pCi} / \mathrm{g} & 0.0308 & \text { EPIA-013B } \\ \mathrm{U} & 0.0331 & \mathrm{pCi} / \mathrm{g} & 0.0736 & \text { EPIA-013B } \\ \mathrm{U} & 0.0219 & \mathrm{pCi} / \mathrm{g} & 0.0666 & \text { EPIA-013B } \\ \mathrm{U} & 0.0149 & \mathrm{pCi} / \mathrm{g} & 0.129 & \text { EPIA-013B } \\ \mathrm{U} & -0.014 & \mathrm{pCi} / \mathrm{g} & 0.157 & \text { EPIA-013B } \\ \mathrm{U} & 0.0129 & \mathrm{pCi} / \mathrm{g} & 0.12 & \text { EPIA-013B } \\ \mathrm{U} & -0.266 & \mathrm{pCi} / \mathrm{g} & 0.978 & \text { EPIA-001B } \\ \mathrm{U} & 0.0493 & \mathrm{pCi} / \mathrm{g} & 0.0823 & \text { EPIA-013B } \\ \mathrm{U} & 0 & \mathrm{pCi} / \mathrm{g} & 0.0569 & \text { EPIA-013B }\end{array}$


SURVEY ID: SRF-03-03 (cont.)

Constituent

Radionuclides

Neptunium-239

Nonvolatile beta

Plutonium-238

Plutonium-239/240

Potassium-40

Promethium-144

Promethium-146

Ruthenium-106

Sodium-22

Strontium-89/90

Tin-1 13

Tritium

Uranium-233/234

Uranium-235

Uranium-238

Yttrium-88

Zinc-65

Zirconium-95

Volatile Organic Compounds

Acetone

Benzene

Bromodichloromethane

Bromoform

Bromomethane (Methyl bromide)

Carbon disulfide

Carbon tetrachloride

Chlorobenzene

Chloroethane

Chloroethene (Vinyl chloride)

Chloroform

Chloromethane (Methyl chloride)

Dibromochloromethane

1,1-Dichloroethane

1,2-Dichloroethane

1,1-Dichloroethylene

1,2-Dichloroethylene

Dichloromethane (Methylene chloride)

1,2-Dichloropropane

cis-1,3-Dichloropropene

trans-1,3-Dichloropropene

Ethylbenzene

2-Hexanone

Methyl ethyl ketone

Methyl isobutyl ketone

Styrene

1,1,2,2-Tetrachloroethane

Tetrachloroethylene

Toluene

1,1,1-Trichloroethane

1,1,2-Trichloroethane

Trichloroethylene
Sample ID: 117029

FG SC E Result

Unit ssMDL

Method

$\begin{array}{lll}\mathrm{pCi} / \mathrm{g} & 0.196 & \text { EPIA-013B } \\ \mathrm{pCi} / \mathrm{g} & 1.18 & \text { EPIA-001B } \\ \mathrm{pCi} / \mathrm{g} & 0.024 & \text { EPIA-012B } \\ \mathrm{pCi} / \mathrm{g} & 0.0198 & \text { EPIA-012B } \\ \mathrm{pCi} / \mathrm{g} & 0.556 & \text { EPIA-013B } \\ \mathrm{pCi} / \mathrm{g} & 0.0529 & \text { EPIA-013B } \\ \mathrm{pCi} / \mathrm{g} & 0.0622 & \text { EPIA-013B } \\ \mathrm{pCi} / \mathrm{g} & 0.521 & \text { EPIA-013B } \\ \mathrm{pCi} / \mathrm{g} & 0.0567 & \text { EPIA-013B } \\ \mathrm{pCi} / \mathrm{g} & 0.198 & \text { EPA905 } \\ \mathrm{pCi} / \mathrm{g} & 0.0637 & \text { EPIA-013B } \\ \mathrm{pCi} / \mathrm{g} & 0.179 & \text { EPIA-002B } \\ \mathrm{pCi} / \mathrm{g} & 0.0224 & \text { EPIA-011B } \\ \mathrm{pCi} / \mathrm{g} & 0.0281 & \text { EPIA-011B } \\ \mathrm{pCi} / \mathrm{g} & 0.0157 & \text { EPIA-011B } \\ \mathrm{pCi} / \mathrm{g} & 0.0693 & \text { EPIA-013B } \\ \mathrm{pCi} / \mathrm{g} & 0.106 & \text { EPIA-013B } \\ \mathrm{pCi} / \mathrm{g} & 0.109 & \text { EPIA-013B }\end{array}$

-0.117
6.13
-0.00305
-0.00153
2.97
-0.00607
0.0157
0.127
-0.00506
0.177
0.0191
-0.0231
0.000119
0.00322
0.00226
-0.00236
-0.0198
-0.079

$\mathrm{pCi} / \mathrm{g}$

$\begin{array}{ll}\mu g / \mathrm{kg} & 51.5 \\ \mu g / \mathrm{kg} & 2.5 \\ \mu g / \mathrm{kg} & 0.5 \\ \mu g / \mathrm{kg} & 1.35 \\ \mu g / \mathrm{kg} & 1.5 \\ \mu g / \mathrm{kg} & 1.5 \\ \mu g / \mathrm{kg} & 2.5 \\ \mu g / \mathrm{kg} & 1.5 \\ \mu g / \mathrm{kg} & 1.5 \\ \mu g / \mathrm{kg} & 2 \\ \mu g / \mathrm{kg} & 0.5 \\ \mu g / \mathrm{kg} & 1 \\ \mu g / \mathrm{kg} & 1.05 \\ \mu g / \mathrm{kg} & 0.5 \\ \mu g / \mathrm{kg} & 1.15 \\ \mu g / \mathrm{kg} & 1.5 \\ \mu g / \mathrm{kg} & 1.25 \\ \mu g / \mathrm{kg} & 7 \\ \mu g / \mathrm{kg} & 1.15 \\ \mu g / \mathrm{kg} & 1 \\ \mu g / \mathrm{kg} & 1.5 \\ \mu g / \mathrm{kg} & 1.5 \\ \mu g / \mathrm{kg} & 14 \\ \mu g / \mathrm{kg} & 16 \\ \mu g / \mathrm{kg} & 14.6 \\ \mu g / \mathrm{kg} & 1.5 \\ \mu g / \mathrm{kg} & 3 \\ \mu g / \mathrm{kg} & 2 \\ \mu g / \mathrm{kg} & 4.5 \\ \mu g / \mathrm{kg} & 0.5 \\ \mu g / \mathrm{kg} & 1.2 \\ \mu g / \mathrm{kg} & 1.35\end{array}$

EPA8260B

EPA8260B

EPA8260B

EPA8260B

EPA8260B

EPA8260B

EPA8260B

EPA8260B

EPA8260B

EPA8260B

EPA8260B

EPA8260B

EPA8260B

EPA8260B

EPA8260B

EPA8260B

EPA8260B

EPA8260B

EPA8260B

EPA8260B

EPA8260B

EPA8260B

EPA8260B

EPA8260B

EPA8260B

EPA8260B

EPA8260B

EPA8260B

EPA8260B

EPA8260B

EPA8260B

EPA8260B 
SURVEY ID: SRF-03-03 (cont.)

Constituent

Volatile Organic Compounds

Vinyl acetate

Xylenes

SURVEY ID: SRF-03-04

Sample type: Normal

\section{Constituent}

Metals (total recoverable)
Sample ID: 117029

FG SC E Result

75

10.9

$\mu g / k g \quad 10.5$

$\mu g / \mathrm{kg} \quad 5$
Method

Sample ID: 117030

Sample matrix: Fauna

Percent solids: NA
FG SC E Result $\quad$ Unit ssMDL $\quad$ Method

\begin{tabular}{|c|c|c|c|c|c|c|}
\hline Antimony & JU & 4 & 0.196 & $\mathrm{mg} / \mathrm{kg}$ & 0.187 & EPA6010B \\
\hline Arsenic & & & 0.638 & $\mathrm{mg} / \mathrm{kg}$ & 0.223 & EPA6010B \\
\hline Barium & & & 7.83 & $\mathrm{mg} / \mathrm{kg}$ & 0.026 & EPA6010B \\
\hline Cadmium & $\mathrm{U}$ & & 0.245 & $\mathrm{mg} / \mathrm{kg}$ & 0.019 & EPA6010B \\
\hline Calcium & & & 23200 & $\mathrm{mg} / \mathrm{kg}$ & 3.416 & EPA6010B \\
\hline Chromium & & & 0.335 & $\mathrm{mg} / \mathrm{kg}$ & 0.0357 & EPA6010B \\
\hline Copper & & & 0.455 & $\mathrm{mg} / \mathrm{kg}$ & 0.0647 & EPA6010B \\
\hline Lead & $\mathrm{J}$ & I & 0.146 & $\mathrm{mg} / \mathrm{kg}$ & 0.077 & EPA6010B \\
\hline Magnesium & & & 533 & $\mathrm{mg} / \mathrm{kg}$ & 0.249 & EPA6010B \\
\hline Manganese & & & 62.6 & $\mathrm{mg} / \mathrm{kg}$ & 0.348 & EPA6010B \\
\hline Mercury & & & 0.0569 & $\mathrm{mg} / \mathrm{kg}$ & 0.00225 & EPA7471A \\
\hline Nickel & $\mathrm{U}$ & & 0.245 & $\mathrm{mg} / \mathrm{kg}$ & 0.031 & EPA6010B \\
\hline Selenium & & & 1.05 & $\mathrm{mg} / \mathrm{kg}$ & 0.132 & EPA6010B \\
\hline Silver & $\mathrm{U}$ & & 0.245 & $\mathrm{mg} / \mathrm{kg}$ & 0.0304 & EPA6010B \\
\hline Zinc & & & 36.2 & $\mathrm{mg} / \mathrm{kg}$ & 0.181 & EPA6010B \\
\hline \multicolumn{7}{|l|}{ Radionuclides } \\
\hline Actinium-228 & $\mathrm{R}$ & 4 & 0.17 & $\mathrm{pCi} / \mathrm{g}$ & 0.141 & EPIA-013B \\
\hline Antimony-124 & $\mathrm{U}$ & & -0.0277 & $\mathrm{pCi} / \mathrm{g}$ & 0.0419 & EPIA-013B \\
\hline Antimony-125 & $\mathrm{U}$ & & 0.0121 & $\mathrm{pCi} / \mathrm{g}$ & 0.0746 & EPIA-013B \\
\hline Barium-133 & $\mathrm{U}$ & & -0.00926 & $\mathrm{pCi} / \mathrm{g}$ & 0.0344 & EPIA-013B \\
\hline Cerium-144 & $\mathrm{U}$ & & 0.0405 & $\mathrm{pCi} / \mathrm{g}$ & 0.162 & EPIA-013B \\
\hline Cesium-134 & $\mathrm{U}$ & & 0.0194 & $\mathrm{pCi} / \mathrm{g}$ & 0.0321 & EPIA-013B \\
\hline Cesium-137 & & & 0.0582 & $\mathrm{pCi} / \mathrm{g}$ & 0.028 & EPIA-013B \\
\hline Cobalt-57 & $\mathrm{U}$ & & 0.00322 & $\mathrm{pCi} / \mathrm{g}$ & 0.0205 & EPIA-013B \\
\hline Cobalt-58 & $\mathrm{U}$ & & 0.0134 & $\mathrm{pCi} / \mathrm{g}$ & 0.04 & EPIA-013B \\
\hline Cobalt-60 & $\mathrm{U}$ & & 0.00656 & $\mathrm{pCi} / \mathrm{g}$ & 0.0352 & EPIA-013B \\
\hline Europium-152 & $\mathrm{U}$ & & 0.0205 & $\mathrm{pCi} / \mathrm{g}$ & 0.0822 & EPIA-013B \\
\hline Europium-154 & $\mathrm{U}$ & & -0.0228 & $\mathrm{pCi} / \mathrm{g}$ & 0.0897 & EPIA-013B \\
\hline Europium-155 & $\mathrm{U}$ & & 0.00112 & $\mathrm{pCi} / \mathrm{g}$ & 0.0741 & EPIA-013B \\
\hline Gross alpha & $\mathrm{U}$ & & 0.17 & $\mathrm{pCi} / \mathrm{g}$ & 2.74 & EPIA-001B \\
\hline Lead-212 & & & 0.0683 & $\mathrm{pCi} / \mathrm{g}$ & 0.0443 & EPIA-013B \\
\hline Manganese-54 & $\mathrm{U}$ & & -0.00853 & $\mathrm{pCi} / \mathrm{g}$ & 0.0303 & EPIA-013B \\
\hline Neptunium-239 & $\mathrm{U}$ & & 0.00155 & $\mathrm{pCi} / \mathrm{g}$ & 0.141 & EPIA-013B \\
\hline Nonvolatile beta & & & 7.07 & $\mathrm{pCi} / \mathrm{g}$ & 1.98 & EPIA-001B \\
\hline Plutonium-238 & $\mathrm{U}$ & & 0 & $\mathrm{pCi} / \mathrm{g}$ & 0.0157 & EPIA-012B \\
\hline
\end{tabular}

Radionuclides

Actinium-228

Antimony-124

Antimony-125

Barium-133

Cerium-144

Cesium-134

Cesium-137

Cobalt-57

Cobalt-58

Cobalt-60

Europium-152

Europium-154

Europium-155

Gross alpha

Lead-212

Manganese-54

Neptunium-239

Nonvolatile beta

Plutonium-238

\begin{tabular}{|c|c|c|c|c|c|c|}
\hline Antimony & JU & 4 & 0.196 & $\mathrm{mg} / \mathrm{kg}$ & 0.187 & EPA6010B \\
\hline Arsenic & & & 0.638 & $\mathrm{mg} / \mathrm{kg}$ & 0.223 & EPA6010B \\
\hline Barium & & & 7.83 & $\mathrm{mg} / \mathrm{kg}$ & 0.026 & EPA6010B \\
\hline Cadmium & $\mathrm{U}$ & & 0.245 & $\mathrm{mg} / \mathrm{kg}$ & 0.019 & EPA6010B \\
\hline Calcium & & & 23200 & $\mathrm{mg} / \mathrm{kg}$ & 3.416 & EPA6010B \\
\hline Chromium & & & 0.335 & $\mathrm{mg} / \mathrm{kg}$ & 0.0357 & EPA6010B \\
\hline Copper & & & 0.455 & $\mathrm{mg} / \mathrm{kg}$ & 0.0647 & EPA6010B \\
\hline Lead & $\mathrm{J}$ & I & 0.146 & $\mathrm{mg} / \mathrm{kg}$ & 0.077 & EPA6010B \\
\hline Magnesium & & & 533 & $\mathrm{mg} / \mathrm{kg}$ & 0.249 & EPA6010B \\
\hline Manganese & & & 62.6 & $\mathrm{mg} / \mathrm{kg}$ & 0.348 & EPA6010B \\
\hline Mercury & & & 0.0569 & $\mathrm{mg} / \mathrm{kg}$ & 0.00225 & EPA7471A \\
\hline Nickel & $\mathrm{U}$ & & 0.245 & $\mathrm{mg} / \mathrm{kg}$ & 0.031 & EPA6010B \\
\hline Selenium & & & 1.05 & $\mathrm{mg} / \mathrm{kg}$ & 0.132 & EPA6010B \\
\hline Silver & $\mathrm{U}$ & & 0.245 & $\mathrm{mg} / \mathrm{kg}$ & 0.0304 & EPA6010B \\
\hline Zinc & & & 36.2 & $\mathrm{mg} / \mathrm{kg}$ & 0.181 & EPA6010B \\
\hline \multicolumn{7}{|l|}{ Radionuclides } \\
\hline Actinium-228 & $\mathrm{R}$ & 4 & 0.17 & $\mathrm{pCi} / \mathrm{g}$ & 0.141 & EPIA-013B \\
\hline Antimony-124 & $\mathrm{U}$ & & -0.0277 & $\mathrm{pCi} / \mathrm{g}$ & 0.0419 & EPIA-013B \\
\hline Antimony-125 & $\mathrm{U}$ & & 0.0121 & $\mathrm{pCi} / \mathrm{g}$ & 0.0746 & EPIA-013B \\
\hline Barium-133 & $\mathrm{U}$ & & -0.00926 & $\mathrm{pCi} / \mathrm{g}$ & 0.0344 & EPIA-013B \\
\hline Cerium-144 & $\mathrm{U}$ & & 0.0405 & $\mathrm{pCi} / \mathrm{g}$ & 0.162 & EPIA-013B \\
\hline Cesium-134 & $\mathrm{U}$ & & 0.0194 & $\mathrm{pCi} / \mathrm{g}$ & 0.0321 & EPIA-013B \\
\hline Cesium-137 & & & 0.0582 & $\mathrm{pCi} / \mathrm{g}$ & 0.028 & EPIA-013B \\
\hline Cobalt-57 & $\mathrm{U}$ & & 0.00322 & $\mathrm{pCi} / \mathrm{g}$ & 0.0205 & EPIA-013B \\
\hline Cobalt-58 & $\mathrm{U}$ & & 0.0134 & $\mathrm{pCi} / \mathrm{g}$ & 0.04 & EPIA-013B \\
\hline Cobalt-60 & $\mathrm{U}$ & & 0.00656 & $\mathrm{pCi} / \mathrm{g}$ & 0.0352 & EPIA-013B \\
\hline Europium-152 & $\mathrm{U}$ & & 0.0205 & $\mathrm{pCi} / \mathrm{g}$ & 0.0822 & EPIA-013B \\
\hline Europium-154 & $\mathrm{U}$ & & -0.0228 & $\mathrm{pCi} / \mathrm{g}$ & 0.0897 & EPIA-013B \\
\hline Europium-155 & $\mathrm{U}$ & & 0.00112 & $\mathrm{pCi} / \mathrm{g}$ & 0.0741 & EPIA-013B \\
\hline Gross alpha & $\mathrm{U}$ & & 0.17 & $\mathrm{pCi} / \mathrm{g}$ & 2.74 & EPIA-001B \\
\hline Lead-212 & & & 0.0683 & $\mathrm{pCi} / \mathrm{g}$ & 0.0443 & EPIA-013B \\
\hline Manganese-54 & $\mathrm{U}$ & & -0.00853 & $\mathrm{pCi} / \mathrm{g}$ & 0.0303 & EPIA-013B \\
\hline Neptunium-239 & $\mathrm{U}$ & & 0.00155 & $\mathrm{pCi} / \mathrm{g}$ & 0.141 & EPIA-013B \\
\hline Nonvolatile beta & & & 7.07 & $\mathrm{pCi} / \mathrm{g}$ & 1.98 & EPIA-001B \\
\hline Plutonium-238 & $\mathrm{U}$ & & 0 & $\mathrm{pCi} / \mathrm{g}$ & 0.0157 & EPIA-012B \\
\hline
\end{tabular}

\begin{tabular}{|c|c|c|c|c|c|c|}
\hline Antimony & JU & 4 & 0.196 & $\mathrm{mg} / \mathrm{kg}$ & 0.187 & EPA6010B \\
\hline Arsenic & & & 0.638 & $\mathrm{mg} / \mathrm{kg}$ & 0.223 & EPA6010B \\
\hline Barium & & & 7.83 & $\mathrm{mg} / \mathrm{kg}$ & 0.026 & EPA6010B \\
\hline Cadmium & $\mathrm{U}$ & & 0.245 & $\mathrm{mg} / \mathrm{kg}$ & 0.019 & EPA6010B \\
\hline Calcium & & & 23200 & $\mathrm{mg} / \mathrm{kg}$ & 3.416 & EPA6010B \\
\hline Chromium & & & 0.335 & $\mathrm{mg} / \mathrm{kg}$ & 0.0357 & EPA6010B \\
\hline Copper & & & 0.455 & $\mathrm{mg} / \mathrm{kg}$ & 0.0647 & EPA6010B \\
\hline Lead & $\mathrm{J}$ & I & 0.146 & $\mathrm{mg} / \mathrm{kg}$ & 0.077 & EPA6010B \\
\hline Magnesium & & & 533 & $\mathrm{mg} / \mathrm{kg}$ & 0.249 & EPA6010B \\
\hline Manganese & & & 62.6 & $\mathrm{mg} / \mathrm{kg}$ & 0.348 & EPA6010B \\
\hline Mercury & & & 0.0569 & $\mathrm{mg} / \mathrm{kg}$ & 0.00225 & EPA7471A \\
\hline Nickel & $\mathrm{U}$ & & 0.245 & $\mathrm{mg} / \mathrm{kg}$ & 0.031 & EPA6010B \\
\hline Selenium & & & 1.05 & $\mathrm{mg} / \mathrm{kg}$ & 0.132 & EPA6010B \\
\hline Silver & $\mathrm{U}$ & & 0.245 & $\mathrm{mg} / \mathrm{kg}$ & 0.0304 & EPA6010B \\
\hline Zinc & & & 36.2 & $\mathrm{mg} / \mathrm{kg}$ & 0.181 & EPA6010B \\
\hline \multicolumn{7}{|l|}{ Radionuclides } \\
\hline Actinium-228 & $\mathrm{R}$ & 4 & 0.17 & $\mathrm{pCi} / \mathrm{g}$ & 0.141 & EPIA-013B \\
\hline Antimony-124 & $\mathrm{U}$ & & -0.0277 & $\mathrm{pCi} / \mathrm{g}$ & 0.0419 & EPIA-013B \\
\hline Antimony-125 & $\mathrm{U}$ & & 0.0121 & $\mathrm{pCi} / \mathrm{g}$ & 0.0746 & EPIA-013B \\
\hline Barium-133 & $\mathrm{U}$ & & -0.00926 & $\mathrm{pCi} / \mathrm{g}$ & 0.0344 & EPIA-013B \\
\hline Cerium-144 & $\mathrm{U}$ & & 0.0405 & $\mathrm{pCi} / \mathrm{g}$ & 0.162 & EPIA-013B \\
\hline Cesium-134 & $\mathrm{U}$ & & 0.0194 & $\mathrm{pCi} / \mathrm{g}$ & 0.0321 & EPIA-013B \\
\hline Cesium-137 & & & 0.0582 & $\mathrm{pCi} / \mathrm{g}$ & 0.028 & EPIA-013B \\
\hline Cobalt-57 & $\mathrm{U}$ & & 0.00322 & $\mathrm{pCi} / \mathrm{g}$ & 0.0205 & EPIA-013B \\
\hline Cobalt-58 & $\mathrm{U}$ & & 0.0134 & $\mathrm{pCi} / \mathrm{g}$ & 0.04 & EPIA-013B \\
\hline Cobalt-60 & $\mathrm{U}$ & & 0.00656 & $\mathrm{pCi} / \mathrm{g}$ & 0.0352 & EPIA-013B \\
\hline Europium-152 & $\mathrm{U}$ & & 0.0205 & $\mathrm{pCi} / \mathrm{g}$ & 0.0822 & EPIA-013B \\
\hline Europium-154 & $\mathrm{U}$ & & -0.0228 & $\mathrm{pCi} / \mathrm{g}$ & 0.0897 & EPIA-013B \\
\hline Europium-155 & $\mathrm{U}$ & & 0.00112 & $\mathrm{pCi} / \mathrm{g}$ & 0.0741 & EPIA-013B \\
\hline Gross alpha & $\mathrm{U}$ & & 0.17 & $\mathrm{pCi} / \mathrm{g}$ & 2.74 & EPIA-001B \\
\hline Lead-212 & & & 0.0683 & $\mathrm{pCi} / \mathrm{g}$ & 0.0443 & EPIA-013B \\
\hline Manganese-54 & $\mathrm{U}$ & & -0.00853 & $\mathrm{pCi} / \mathrm{g}$ & 0.0303 & EPIA-013B \\
\hline Neptunium-239 & $\mathrm{U}$ & & 0.00155 & $\mathrm{pCi} / \mathrm{g}$ & 0.141 & EPIA-013B \\
\hline Nonvolatile beta & & & 7.07 & $\mathrm{pCi} / \mathrm{g}$ & 1.98 & EPIA-001B \\
\hline Plutonium-238 & $\mathrm{U}$ & & 0 & $\mathrm{pCi} / \mathrm{g}$ & 0.0157 & EPIA-012B \\
\hline
\end{tabular}

EPA8260B

EPA8260B 
SURVEY ID: SRF-03-04 (cont.)

Constituent

Radionuclides

Plutonium-239/240

Potassium-40

Promethium-144

Promethium-146

Ruthenium-106

Sodium-22

Strontium-89/90

Tin-1 13

Tritium

Uranium-233/234

Uranium-235

Uranium-238

Yttrium-88

Zinc-65

Zirconium-95

Volatile Organic Compounds

Acetone

Benzene

Bromodichloromethane

Bromoform

Bromomethane (Methyl bromide)

Carbon disulfide

Carbon tetrachloride

Chlorobenzene

Chloroethane

Chloroethene (Vinyl chloride)

Chloroform

Chloromethane (Methyl chloride)

Dibromochloromethane

1,1-Dichloroethane

1,2-Dichloroethane

1,1-Dichloroethylene

1,2-Dichloroethylene

Dichloromethane (Methylene chloride) U

1,2-Dichloropropane

cis-1,3-Dichloropropene

trans-1,3-Dichloropropene

Ethylbenzene

2-Hexanone

Methyl ethyl ketone

Methyl isobutyl ketone

Styrene

1,1,2,2-Tetrachloroethane

Tetrachloroethylene

Toluene

1,1,1-Trichloroethane

1,1,2-Trichloroethane

Trichloroethylene

Vinyl acetate

Xylenes
Sample ID: 117030

FG SC E Result

Unit ssMDL

Method

$\begin{array}{lllll}\mathrm{U} & -0.00753 & \mathrm{pCi} / \mathrm{g} & 0.0448 & \text { EPIA-012B } \\ \mathrm{U} & 3.84 & \mathrm{pCi} / \mathrm{g} & 0.309 & \text { EPIA-013B } \\ \mathrm{U} & -0.00494 & \mathrm{pCi} / \mathrm{g} & 0.0308 & \text { EPIA-013B } \\ \mathrm{U} & 0.0111 & \mathrm{pCi} / \mathrm{g} & 0.0362 & \text { EPIA-013B } \\ \mathrm{U} & -0.0324 & \mathrm{pCi} / \mathrm{g} & 0.277 & \text { EPIA-013B } \\ & -0.00829 & \mathrm{pCi} / \mathrm{g} & 0.0324 & \text { EPIA-013B } \\ \mathrm{U} & 0.361 & \mathrm{pCi} / \mathrm{g} & 0.16 & \text { EPA905 } \\ \mathrm{U} & -0.0143 & \mathrm{pCi} / \mathrm{g} & 0.0372 & \text { EPIA-013B } \\ \mathrm{U} & 0.151 & \mathrm{pCi} / \mathrm{g} & 0.175 & \text { EPIA-002B } \\ \mathrm{U} & 0.00156 & \mathrm{pCi} / \mathrm{g} & 0.0457 & \text { EPIA-011B } \\ \mathrm{U} & 0.000174 & \mathrm{pCi} / \mathrm{g} & 0.0328 & \text { EPIA-011B } \\ \mathrm{U} & 0.0109 & \mathrm{pCi} / \mathrm{g} & 0.0269 & \text { EPIA-011B } \\ \mathrm{U} & 0.00828 & \mathrm{pCi} / \mathrm{g} & 0.0436 & \text { EPIA-013B } \\ \mathrm{U} & 0.0138 & \mathrm{pCi} / \mathrm{g} & 0.0811 & \text { EPIA-013B } \\ & 0.00882 & \mathrm{pCi} / \mathrm{g} & 0.0676 & \text { EPIA-013B }\end{array}$

\begin{tabular}{|c|c|c|c|}
\hline 164 & $\mu \mathrm{g} / \mathrm{kg}$ & 51.5 & EPA8260B \\
\hline 5 & $\mu \mathrm{g} / \mathrm{kg}$ & 2.5 & EPA8260B \\
\hline 5 & $\mathrm{ug} / \mathrm{kg}$ & 0.5 & EPA8260B \\
\hline 5 & $\mu \mathrm{g} / \mathrm{kg}$ & 1.35 & EPA8260B \\
\hline 5 & $\mu g / \mathrm{kg}$ & 1.5 & EPA8260B \\
\hline 25 & $\mu \mathrm{g} / \mathrm{kg}$ & 1.5 & EPA8260B \\
\hline 5 & $\mu \mathrm{g} / \mathrm{kg}$ & 2.5 & EPA8260B \\
\hline 5 & $\mu \mathrm{g} / \mathrm{kg}$ & 1.5 & EPA8260B \\
\hline 5 & $\mu \mathrm{g} / \mathrm{kg}$ & 1.5 & EPA8260B \\
\hline 5 & $\mathrm{\mu g} / \mathrm{kg}$ & 2 & EPA8260B \\
\hline 5 & $\mu \mathrm{g} / \mathrm{kg}$ & 0.5 & EPA8260B \\
\hline 5 & $\mu \mathrm{g} / \mathrm{kg}$ & 1 & EPA8260B \\
\hline 5 & $\mu \mathrm{g} / \mathrm{kg}$ & 1.05 & EPA8260B \\
\hline 5 & $\mu \mathrm{g} / \mathrm{kg}$ & 0.5 & EPA8260B \\
\hline 5 & $\mu \mathrm{g} / \mathrm{kg}$ & 1.15 & EPA8260B \\
\hline 5 & $\mu \mathrm{g} / \mathrm{kg}$ & 1.5 & EPA8260B \\
\hline 50 & $\mu \mathrm{g} / \mathrm{kg}$ & 1.25 & EPA8260B \\
\hline 25 & $\mu \mathrm{g} / \mathrm{kg}$ & 7 & EPA8260B \\
\hline 5 & $\mu \mathrm{g} / \mathrm{kg}$ & 1.15 & EPA8260B \\
\hline 5 & $\mu \mathrm{g} / \mathrm{kg}$ & 1 & EPA8260B \\
\hline 5 & $\mu \mathrm{g} / \mathrm{kg}$ & 1.5 & EPA8260B \\
\hline 11.4 & $\mu \mathrm{g} / \mathrm{kg}$ & 1.5 & EPA8260B \\
\hline 25 & $\mu \mathrm{g} / \mathrm{kg}$ & 14 & EPA8260B \\
\hline 25 & $\mu \mathrm{g} / \mathrm{kg}$ & 16 & EPA8260B \\
\hline 25 & $\mu \mathrm{g} / \mathrm{kg}$ & 14.6 & EPA8260B \\
\hline 5 & $\mu \mathrm{g} / \mathrm{kg}$ & 1.5 & EPA8260B \\
\hline 5 & $\mu \mathrm{g} / \mathrm{kg}$ & 3 & EPA8260B \\
\hline 5 & $\mu \mathrm{g} / \mathrm{kg}$ & 2 & EPA8260B \\
\hline 9.57 & $\mu \mathrm{g} / \mathrm{kg}$ & 4.5 & EPA8260B \\
\hline 5 & $\mu \mathrm{g} / \mathrm{kg}$ & 0.5 & EPA8260B \\
\hline 5 & $\mu \mathrm{g} / \mathrm{kg}$ & 1.2 & EPA8260B \\
\hline 5 & $\mu \mathrm{g} / \mathrm{kg}$ & 1.35 & EPA8260B \\
\hline 75 & $\mu \mathrm{g} / \mathrm{kg}$ & 10.5 & EPA8260B \\
\hline 50 & $\mathrm{ug} / \mathrm{kg}$ & 5 & EPA8260B \\
\hline
\end{tabular}


SURVEY ID: SRF-04-01

Sample type: Normal

Constituent

Metals (total recoverable)

Antimony
Arsenic
Barium
Cadmium
Calcium
Chromium
Copper
Lead
Magnesium
Manganese
Mercury
Nickel
Selenium
Silver
Zinc

Radionuclides

Actinium-228

Antimony-124

Antimony-125

Barium-133

Cerium-144

Cesium-134

Cesium-137

Cobalt-57

Cobalt-58

Cobalt-60

Europium-152

Europium-154

Europium-155

Gross alpha

Lead-212

Manganese-54

Neptunium-239

Nonvolatile beta

Plutonium-238

Plutonium-239/240

Potassium-40

Promethium-144

Promethium-146

Ruthenium-106

Sodium-22

Strontium-89/90

Tin-1 13

Tritium
Sample ID: 117031

Sample matrix: Fauna

Percent solids: NA

\begin{tabular}{|c|c|c|c|c|c|}
\hline \multirow[t]{3}{*}{ JU } & 4 & 0.221 & $\mathrm{mg} / \mathrm{kg}$ & 0.184 & EPA6010B \\
\hline & & 0.528 & $\mathrm{mg} / \mathrm{kg}$ & 0.219 & EPA6010B \\
\hline & & 5.82 & $\mathrm{mg} / \mathrm{kg}$ & 0.026 & EPA6010B \\
\hline \multirow[t]{8}{*}{$J$} & I & 0.059 & $\mathrm{mg} / \mathrm{kg}$ & 0.018 & EPA6010B \\
\hline & & 20100 & $\mathrm{mg} / \mathrm{kg}$ & 3.354 & EPA6010B \\
\hline & & 0.403 & $\mathrm{mg} / \mathrm{kg}$ & 0.035 & EPA6010B \\
\hline & & 0.746 & $\mathrm{mg} / \mathrm{kg}$ & 0.0635 & EPA6010B \\
\hline & & 0.264 & $\mathrm{mg} / \mathrm{kg}$ & 0.076 & EPA6010B \\
\hline & & 558 & $\mathrm{mg} / \mathrm{kg}$ & 0.244 & EPA6010B \\
\hline & & 56.9 & $\mathrm{mg} / \mathrm{kg}$ & 0.342 & EPA6010B \\
\hline & & 0.0655 & $\mathrm{mg} / \mathrm{kg}$ & 0.00214 & EPA7471A \\
\hline \multirow[t]{2}{*}{ JU } & IV & 0.133 & $\mathrm{mg} / \mathrm{kg}$ & 0.031 & EPA6010B \\
\hline & & 1.01 & $\mathrm{mg} / \mathrm{kg}$ & 0.13 & EPA6010B \\
\hline \multirow[t]{2}{*}{$\mathrm{U}$} & & 0.24 & $\mathrm{mg} / \mathrm{kg}$ & 0.0298 & EPA6010B \\
\hline & & 22.2 & $\mathrm{mg} / \mathrm{kg}$ & 0.178 & EPA6010B \\
\hline
\end{tabular}

U

U

U

U

U

U

U

$\mathrm{U}$

U

$\mathrm{U}$

U

U

U

U

$\mathrm{U}$

$\mathrm{U}$

U

U

U

U

U

U

U

0.162
-0.00379
0.00765
-0.0183
0.279
0.00467
0.0791
-0.0119
0.0453
-0.0147
0.0204
-0.0187
0.0387
0.0186
0.00678
-0.000791
0.0669
9.76
-0.00057
0
3.01
0.0023
0.00421
-0.27
-0.0064
1.81
0.016
4.07

Unit ssMDL

Method

EPA6010B

$\begin{array}{lll}\mathrm{pCi} / \mathrm{g} & 0.21 & \text { EPIA-013B } \\ \mathrm{pCi} / \mathrm{g} & 0.0688 & \text { EPIA-013B } \\ \mathrm{pCi} / \mathrm{g} & 0.122 & \text { EPIA-013B } \\ \mathrm{pCi} / \mathrm{g} & 0.0525 & \text { EPIA-013B } \\ \mathrm{pCi} / \mathrm{g} & 0.324 & \text { EPIA-013B } \\ \mathrm{pCi} / \mathrm{g} & 0.0498 & \text { EPIA-013B } \\ \mathrm{pCi} / \mathrm{g} & 0.0438 & \text { EPIA-013B } \\ \mathrm{pCi} / \mathrm{g} & 0.0357 & \text { EPIA-013B } \\ \mathrm{pCi} / \mathrm{g} & 0.0754 & \text { EPIA-013B } \\ \mathrm{pCi} / \mathrm{g} & 0.0491 & \text { EPIA-013B } \\ \mathrm{pCi} / \mathrm{g} & 0.128 & \text { EPIA-013B } \\ \mathrm{pCi} / \mathrm{g} & 0.141 & \text { EPIA-013B } \\ \mathrm{pCi} / \mathrm{g} & 0.14 & \text { EPIA-013B } \\ \mathrm{pCi} / \mathrm{g} & 1.12 & \text { EPIA-001B } \\ \mathrm{pCi} / \mathrm{g} & 0.0927 & \text { EPIA-013B } \\ \mathrm{pCi} / \mathrm{g} & 0.0488 & \text { EPIA-013B } \\ \mathrm{pCi} / \mathrm{g} & 0.253 & \text { EPIA-013B } \\ \mathrm{pCi} / \mathrm{g} & 1.06 & \text { EPIA-001B } \\ \mathrm{pCi} / \mathrm{g} & 0.0125 & \text { EPIA-012B } \\ \mathrm{pCi} / \mathrm{g} & 0.00712 & \text { EPIA-012B } \\ \mathrm{pCi} / \mathrm{g} & 0.561 & \text { EPIA-013B } \\ \mathrm{pCi} / \mathrm{g} & 0.054 & \text { EPIA-013B } \\ \mathrm{pCi} / \mathrm{g} & 0.0553 & \text { EPIA-013B } \\ \mathrm{pCi} / \mathrm{g} & 0.433 & \text { EPIA-013B } \\ \mathrm{pCi} / \mathrm{g} & 0.0511 & \text { EPIA-013B } \\ \mathrm{pCi} / \mathrm{g} & 0.0918 & \text { EPA905 } \\ \mathrm{pCi} / \mathrm{g} & 0.0645 & \text { EPIA-013B } \\ \mathrm{pCi} / \mathrm{g} & 0.127 & \text { EPIA-002B }\end{array}$


SURVEY ID: SRF-04-01 (cont.)

Constituent

Radionuclides

Uranium-233/234

Uranium-235

Uranium-238

Yttrium-88

Zinc-65

Zirconium-95

Volatile Organic Compounds

\begin{tabular}{|c|c|c|c|c|}
\hline Acetone & $\mathrm{J}$ & IK & $\mathrm{O}$ & 122 \\
\hline Benzene & $\mathrm{U}$ & & & 5 \\
\hline Bromodichloromethane & $\mathrm{U}$ & & & 5 \\
\hline Bromoform & $\mathrm{U}$ & & & 5 \\
\hline Bromomethane (Methyl bromide) & $\mathrm{U}$ & & & 5 \\
\hline Carbon disulfide & $\mathrm{J}$ & IK & $\mathrm{O}$ & 16. \\
\hline Carbon tetrachloride & $\mathrm{U}$ & & & 5 \\
\hline Chlorobenzene & $\mathrm{U}$ & & & 5 \\
\hline Chloroethane & $\mathrm{U}$ & & & 5 \\
\hline Chloroethene (Vinyl chloride) & $\mathrm{U}$ & & & 5 \\
\hline Chloroform & $\mathrm{U}$ & & & 5 \\
\hline Chloromethane (Methyl chloride) & $\mathrm{U}$ & & & 5 \\
\hline Dibromochloromethane & $\mathrm{U}$ & & & 5 \\
\hline 1,1-Dichloroethane & $\mathrm{U}$ & & & 5 \\
\hline 1,2-Dichloroethane & $\mathrm{U}$ & & & 5 \\
\hline 1,1-Dichloroethylene & $\mathrm{U}$ & & & 5 \\
\hline 1,2-Dichloroethylene & $\mathrm{U}$ & & & 50 \\
\hline Dichloromethane (Methylene chloride) & $\mathrm{J}$ & $\mathrm{K}$ & $\mathrm{O}$ & 34.1 \\
\hline 1,2-Dichloropropane & $\mathrm{U}$ & & & 5 \\
\hline cis-1,3-Dichloropropene & $\mathrm{U}$ & & & 5 \\
\hline trans-1,3-Dichloropropene & $\mathrm{U}$ & & & 5 \\
\hline Ethylbenzene & $\mathrm{J}$ & IK & $\mathrm{O}$ & 4.49 \\
\hline 2-Hexanone & $\mathrm{U}$ & & & 25 \\
\hline Methyl ethyl ketone & $\mathrm{U}$ & & & 25 \\
\hline Methyl isobutyl ketone & $\mathrm{U}$ & & & 25 \\
\hline Styrene & $\mathrm{U}$ & & & 5 \\
\hline $1,1,2,2$-Tetrachloroethane & $\mathrm{U}$ & & & 5 \\
\hline Tetrachloroethylene & $\mathrm{U}$ & & & 5 \\
\hline Toluene & $\mathrm{J}$ & $\mathrm{K}$ & $\mathrm{O}$ & 5.6 \\
\hline 1,1,1-Trichloroethane & $\mathrm{U}$ & & & 5 \\
\hline 1,1,2-Trichloroethane & $\mathrm{U}$ & & & 5 \\
\hline Trichloroethylene & $\mathrm{U}$ & & & 5 \\
\hline Vinyl acetate & $\mathrm{U}$ & & & 75 \\
\hline Xylenes & $\mathrm{J}$ & $\mathrm{K}$ & $\mathrm{O}$ & $2 b$ \\
\hline
\end{tabular}

Sample ID: 117031

FG SC E Result

Unit ssMDL

Method

0.0154
0.00399
0.000723
0.0136
-0.0117
0.0125

$\begin{array}{ll}\mathrm{pCi} / \mathrm{g} & 0.0222 \\ \mathrm{pCi} / \mathrm{g} & 0.012 \\ \mathrm{pCi} / \mathrm{g} & 0.0204 \\ \mathrm{pCi} / \mathrm{g} & 0.0622 \\ \mathrm{pCi} / \mathrm{g} & 0.115 \\ \mathrm{pCi} / \mathrm{g} & 0.117\end{array}$

EPIA-011B EPIA-011B EPIA-011B EPIA-013B EPIA-013B EPIA-013B

EPA8260B EPA8260B EPA8260B EPA8260B EPA8260B EPA8260B EPA8260B

EPA8260B EPA8260B EPA8260B EPA8260B EPA8260B EPA8260B EPA8260B EPA8260B EPA8260B EPA8260B

EPA8260B EPA8260B EPA8260B

EPA8260B EPA8260B EPA8260B EPA8260B EPA8260B EPA8260B EPA8260B EPA8260B EPA8260B EPA8260B EPA8260B EPA8260B EPA8260B EPA8260B 
SURVEY ID: SRF-04-02

Sample type: Normal

Constituent

Metals (total recoverable)

Antimony
Arsenic
Barium
Cadmium
Calcium
Chromium
Copper
Lead
Magnesium
Manganese
Mercury
Nickel
Selenium
Silver
Zinc

Radionuclides

Actinium-228

Antimony-124

Antimony-125

Barium-133

Cerium-144

Cesium-134

Cesium-137

Cobalt-57

Cobalt-58

Cobalt-60

Europium-152

Europium-154

Europium-155

Gross alpha

Lead-212

Manganese-54

Neptunium-239

Nonvolatile beta

Plutonium-238

Plutonium-239/240

Potassium-40

Promethium-144

Promethium-146

Ruthenium-106

Sodium-22

Strontium-89/90

Tin-1 13

Tritium
Sample ID: 117032

Sample matrix: Fauna

Percent solids: NA

\begin{tabular}{|c|c|c|c|c|c|c|}
\hline \multirow[t]{3}{*}{$\mathrm{U}$} & & & 0.459 & $\mathrm{mg} / \mathrm{kg}$ & 0.175 & EPA6010B \\
\hline & & & 0.544 & $\mathrm{mg} / \mathrm{kg}$ & 0.209 & EPA6010B \\
\hline & & & 3.25 & $\mathrm{mg} / \mathrm{kg}$ & 0.024 & EPA6010B \\
\hline \multirow[t]{4}{*}{ JU } & & 4 & 0.0199 & $\mathrm{mg} / \mathrm{kg}$ & 0.017 & EPA6010B \\
\hline & & & 20000 & $\mathrm{mg} / \mathrm{kg}$ & 3.2 & EPA6010B \\
\hline & & & 0.293 & $\mathrm{mg} / \mathrm{kg}$ & 0.0334 & EPA6010B \\
\hline & & & 0.305 & $\mathrm{mg} / \mathrm{kg}$ & 0.0606 & EPA6010B \\
\hline \multirow[t]{4}{*}{$\mathrm{J}$} & I & & 0.177 & $\mathrm{mg} / \mathrm{kg}$ & 0.072 & EPA6010B \\
\hline & & & 524 & $\mathrm{mg} / \mathrm{kg}$ & 0.233 & EPA6010B \\
\hline & & & 31 & $\mathrm{mg} / \mathrm{kg}$ & 0.326 & EPA6010B \\
\hline & & & 0.151 & $\mathrm{mg} / \mathrm{kg}$ & 0.002 & EPA7471 \\
\hline \multirow[t]{2}{*}{ JU } & IV & & 0.145 & $\mathrm{mg} / \mathrm{kg}$ & 0.029 & EPA6010B \\
\hline & & & 1.15 & mg/kg & 0.124 & EPA6010I \\
\hline \multirow[t]{2}{*}{$\mathrm{U}$} & & & 0.229 & $\mathrm{mg} / \mathrm{kg}$ & 0.0284 & EPA6010B \\
\hline & & & 16.9 & $\mathrm{mg} / \mathrm{kg}$ & 0.17 & EPA6010H \\
\hline
\end{tabular}

U

U

U

U

U

U

U

U

U

$\mathrm{U}$

U

U

U

U

$\mathrm{U}$

$\mathrm{U}$

$\mathrm{U}$

U

U

U

U

U

U
Result

16.9

\begin{tabular}{l}
0.0839 \\
-0.0292 \\
0.0531 \\
0.0114 \\
-0.0704 \\
0.0251 \\
0.0882 \\
-0.00906 \\
0.00544 \\
0.0218 \\
0.046 \\
-0.0562 \\
-0.0614 \\
-0.471 \\
0.0877 \\
0.00577 \\
0.0289 \\
6.65 \\
0 \\
-0.00112 \\
2.25 \\
-0.00452 \\
-0.0232 \\
0.261 \\
-0.0205 \\
0.643 \\
0.0729 \\
1.31 \\
\hline
\end{tabular}

Unit ssMDL

Method

EPA6010B

$\begin{array}{lll}\mathrm{pCi} / \mathrm{g} & 0.265 & \text { EPIA-013B } \\ \mathrm{pCi} / \mathrm{g} & 0.0935 & \text { EPIA-013B } \\ \mathrm{pCi} / \mathrm{g} & 0.166 & \text { EPIA-013B } \\ \mathrm{pCi} / \mathrm{g} & 0.0703 & \text { EPIA-013B } \\ \mathrm{pCi} / \mathrm{g} & 0.282 & \text { EPIA-013B } \\ \mathrm{pCi} / \mathrm{g} & 0.0705 & \text { EPIA-013B } \\ \mathrm{pCi} / \mathrm{g} & 0.0617 & \text { EPIA-013B } \\ \mathrm{pCi} / \mathrm{g} & 0.0381 & \text { EPIA-013B } \\ \mathrm{pCi} / \mathrm{g} & 0.0911 & \text { EPIA-013B } \\ \mathrm{pCi} / \mathrm{g} & 0.0758 & \text { EPIA-013B } \\ \mathrm{pCi} / \mathrm{g} & 0.167 & \text { EPIA-013B } \\ \mathrm{pCi} / \mathrm{g} & 0.215 & \text { EPIA-013B } \\ \mathrm{pCi} / \mathrm{g} & 0.14 & \text { EPIA-013B } \\ \mathrm{pCi} / \mathrm{g} & 1.71 & \text { EPIA-001B } \\ \mathrm{pCi} / \mathrm{g} & 0.106 & \text { EPIA-013B } \\ \mathrm{pCi} / \mathrm{g} & 0.0586 & \text { EPIA-013B } \\ \mathrm{pCi} / \mathrm{g} & 0.264 & \text { EPIA-013B } \\ \mathrm{pCi} / \mathrm{g} & 1.75 & \text { EPIA-001B } \\ \mathrm{pCi} / \mathrm{g} & 0.014 & \text { EPIA-012B } \\ \mathrm{pCi} / \mathrm{g} & 0.0247 & \text { EPIA-012B } \\ \mathrm{pCi} / \mathrm{g} & 0.646 & \text { EPIA-013B } \\ \mathrm{pCi} / \mathrm{g} & 0.0713 & \text { EPIA-013B } \\ \mathrm{pCi} / \mathrm{g} & 0.068 & \text { EPIA-013B } \\ \mathrm{pCi} / \mathrm{g} & 0.633 & \text { EPIA-013B } \\ \mathrm{pCi} / \mathrm{g} & 0.0777 & \text { EPIA-013B } \\ \mathrm{pCi} / \mathrm{g} & 0.15 & \text { EPA905 } \\ \mathrm{pCi} / \mathrm{g} & 0.0979 & \text { EPIA-013B } \\ \mathrm{pCi} / \mathrm{g} & 0.155 & \text { EPIA-002B }\end{array}$


SURVEY ID: SRF-04-02 (cont.)

Constituent

Radionuclides

Uranium-233/234

Uranium-235

Uranium-238

Yttrium-88

Zinc-65

Zirconium-95

Volatile Organic Compounds
Sample ID: 117032

FG SC E Result
Unit ssMDL Method

$\begin{array}{lllll}\mathrm{U} & 0.00409 & \mathrm{pCi} / \mathrm{g} & 0.0297 & \text { EPIA-011B } \\ \mathrm{U} & -0.00473 & \mathrm{pCi} / \mathrm{g} & 0.0319 & \text { EPIA-011B } \\ \mathrm{U} & -0.00189 & \mathrm{pCi} / \mathrm{g} & 0.0244 & \text { EPIA-011B } \\ \mathrm{U} & -0.0251 & \mathrm{pCi} / \mathrm{g} & 0.0715 & \text { EPIA-013B } \\ \mathrm{U} & -0.0767 & \mathrm{pCi} / \mathrm{g} & 0.158 & \text { EPIA-013B } \\ \mathrm{U} & 0.0651 & \mathrm{pCi} / \mathrm{g} & 0.173 & \text { EPIA-013B }\end{array}$

Acetone

Benzene

Bromodichloromethane

Bromoform

129
5
5
5
5
25
5
5
5
5
5
5
5
5
5
5
50
25.5
5
5
5
4.09
25
17
25
5
5
5
5
5
5
5
75
20

5
5
5
5
5
5
5
5
5
5

EPA8260B

EPA8260B

EPA8260B

EPA8260B

EPA8260B

EPA8260B

EPA8260B

EPA8260B

EPA8260B

EPA8260B

EPA8260B

EPA8260B

EPA8260B

EPA8260B

EPA8260B

EPA8260B

EPA8260B

EPA8260B

EPA8260B

EPA8260B

EPA8260B

EPA8260B

EPA8260B

EPA8260B

EPA8260B

EPA8260B

EPA8260B

EPA8260B

EPA8260B

EPA8260B

EPA8260B

EPA8260B

EPA8260B

EPA8260B 
SURVEY ID: SRF-04-03

Sample type: Normal

\section{Constituent}

Metals (total recoverable)

Antimony
Arsenic
Barium
Cadmium
Calcium
Chromium
Copper
Lead
Magnesium
Manganese
Mercury
Nickel
Selenium
Silver
Zinc

Radionuclides

Actinium-228

Antimony-124

Antimony-125

Barium-133

Cerium-144

Cesium-134

Cesium-137

Cobalt-57

Cobalt-58

Cobalt-60

Europium-152

Europium-154

Europium-155

Gross alpha

Lead-212

Manganese-54

Neptunium-239

Nonvolatile beta

Plutonium-238

Plutonium-239/240

Potassium-40

Promethium-144

Promethium-146

Ruthenium-106

Sodium-22

Strontium-89/90

Tin-1 13

Tritium
Sample ID: 117033

Sample matrix: Fauna

Percent solids: NA

\begin{tabular}{|c|c|c|c|c|c|}
\hline \multirow{3}{*}{\multicolumn{2}{|c|}{$\mathrm{U}$}} & 0.495 & $\mathrm{mg} / \mathrm{kg}$ & 0.189 & EPA6010B \\
\hline & & 0.586 & $\mathrm{mg} / \mathrm{kg}$ & 0.226 & EPA6010B \\
\hline & & 0.427 & $\mathrm{mg} / \mathrm{kg}$ & 0.027 & EPA6010B \\
\hline \multirow[t]{2}{*}{$\mathrm{U}$} & & 0.248 & $\mathrm{mg} / \mathrm{kg}$ & 0.019 & EPA6010B \\
\hline & & 2670 & $\mathrm{mg} / \mathrm{kg}$ & 3.451 & EPA6010B \\
\hline \multirow[t]{2}{*}{$J$} & I & 0.0858 & $\mathrm{mg} / \mathrm{kg}$ & 0.0361 & EPA6010B \\
\hline & & 0.26 & $\mathrm{mg} / \mathrm{kg}$ & 0.0653 & EPA6010F \\
\hline \multirow[t]{4}{*}{$\mathrm{U}$} & & 0.248 & $\mathrm{mg} / \mathrm{kg}$ & 0.078 & EPA6010B \\
\hline & & 294 & $\mathrm{mg} / \mathrm{kg}$ & 0.251 & EPA6010B \\
\hline & & 1.66 & $\mathrm{mg} / \mathrm{kg}$ & 0.351 & EPA6010B \\
\hline & & 0.222 & $\mathrm{mg} / \mathrm{kg}$ & 0.00213 & EPA7471 \\
\hline \multirow[t]{2}{*}{ JU } & IV & 0.15 & $\mathrm{mg} / \mathrm{kg}$ & 0.032 & EPA6010B \\
\hline & & 0.764 & $\mathrm{mg} / \mathrm{kg}$ & 0.134 & EPA6010E \\
\hline \multirow[t]{2}{*}{$\mathrm{U}$} & & 0.248 & $\mathrm{mg} / \mathrm{kg}$ & 0.0307 & EPA6010I \\
\hline & & 5.98 & $\mathrm{mg} / \mathrm{kg}$ & 0.184 & EPA60101 \\
\hline
\end{tabular}

\begin{tabular}{|c|c|c|c|c|}
\hline $\mathrm{U}$ & 0.0281 & $\mathrm{pCi} / \mathrm{g}$ & 0.291 & EPIA-013B \\
\hline $\mathrm{U}$ & -0.0103 & $\mathrm{pCi} / \mathrm{g}$ & 0.0725 & EPIA-013B \\
\hline $\mathrm{U}$ & 0.0995 & $\mathrm{pCi} / \mathrm{g}$ & 0.163 & EPIA-013B \\
\hline $\mathrm{U}$ & 0.0484 & $\mathrm{pCi} / \mathrm{g}$ & 0.0728 & EPIA-013B \\
\hline $\mathrm{U}$ & 0.0639 & $\mathrm{pCi} / \mathrm{g}$ & 0.28 & EPIA-013B \\
\hline $\mathrm{U}$ & 0.0395 & $\mathrm{pCi} / \mathrm{g}$ & 0.0604 & EPIA-013B \\
\hline $\mathrm{U}$ & 0.0535 & $\mathrm{pCi} / \mathrm{g}$ & 0.0538 & EPIA-013B \\
\hline $\mathrm{U}$ & -0.0245 & $\mathrm{pCi} / \mathrm{g}$ & 0.0338 & EPIA-013B \\
\hline $\mathrm{U}$ & -0.00279 & $\mathrm{pCi} / \mathrm{g}$ & 0.0806 & EPIA-013B \\
\hline $\mathrm{U}$ & 0.0048 & $\mathrm{pCi} / \mathrm{g}$ & 0.0816 & EPIA-013I \\
\hline $\mathrm{U}$ & 0.0512 & $\mathrm{pCi} / \mathrm{g}$ & 0.162 & EPIA-013B \\
\hline $\mathrm{U}$ & 0.0607 & $\mathrm{pCi} / \mathrm{g}$ & 0.203 & EPIA-013B \\
\hline $\mathrm{U}$ & -0.00634 & $\mathrm{pCi} / \mathrm{g}$ & 0.14 & EPIA-0135 \\
\hline $\mathrm{U}$ & 0.611 & $\mathrm{pCi} / \mathrm{g}$ & 0.885 & EPIA-001B \\
\hline $\mathrm{U}$ & 0.089 & $\mathrm{pCi} / \mathrm{g}$ & 0.103 & EPIA-013B \\
\hline $\mathrm{U}$ & -0.0036 & $\mathrm{pCi} / \mathrm{g}$ & 0.0714 & EPIA-013B \\
\hline \multirow[t]{2}{*}{$\mathrm{U}$} & 0.0212 & $\mathrm{pCi} / \mathrm{g}$ & 0.24 & EPIA-013I \\
\hline & 7.74 & $\mathrm{pCi} / \mathrm{g}$ & 1.04 & EPIA-001B \\
\hline $\mathrm{U}$ & 0 & $\mathrm{pCi} / \mathrm{g}$ & 0.00802 & EPIA-012E \\
\hline \multirow[t]{2}{*}{$\mathrm{U}$} & -0.000641 & $\mathrm{pCi} / \mathrm{g}$ & 0.0141 & EPIA-012B \\
\hline & 4.01 & $\mathrm{pCi} / \mathrm{g}$ & 0.778 & EPIA-013B \\
\hline $\mathrm{U}$ & 0.044 & $\mathrm{pCi} / \mathrm{g}$ & 0.0744 & EPIA-013B \\
\hline $\mathrm{U}$ & 0.0343 & $\mathrm{pCi} / \mathrm{g}$ & 0.0681 & EPIA-013E \\
\hline $\mathrm{U}$ & -0.101 & $\mathrm{pCi} / \mathrm{g}$ & 0.484 & EPIA-013F \\
\hline \multirow[t]{2}{*}{$\mathrm{U}$} & 0.0218 & $\mathrm{pCi} / \mathrm{g}$ & 0.0732 & EPIA-013B \\
\hline & 0.859 & $\mathrm{pCi} / \mathrm{g}$ & 0.0874 & EPA905 \\
\hline $\mathrm{U}$ & -0.0179 & $\mathrm{pCi} / \mathrm{g}$ & 0.0713 & EPIA-013I \\
\hline $\mathrm{U}$ & 0.105 & $\mathrm{pCi} / \mathrm{g}$ & 0.116 & EPIA-002] \\
\hline
\end{tabular}


SURVEY ID: SRF-04-03 (cont.)

Constituent

Radionuclides

Uranium-233/234

Uranium-235

Uranium-238

Yttrium-88

Zinc-65

Zirconium-95

Volatile Organic Compounds

\begin{tabular}{|c|c|c|c|c|}
\hline Acetone & $\mathrm{J}$ & IK & $\mathrm{O}$ & 94.4 \\
\hline Benzene & $\mathrm{U}$ & & & 5 \\
\hline Bromodichloromethane & $\mathrm{U}$ & & & 5 \\
\hline Bromoform & $\mathrm{U}$ & & & 5 \\
\hline Bromomethane (Methyl bromide) & $\mathrm{U}$ & & & 5 \\
\hline Carbon disulfide & $\mathrm{U}$ & & & 25 \\
\hline Carbon tetrachloride & $\mathrm{U}$ & & & 5 \\
\hline Chlorobenzene & $\mathrm{U}$ & & & 5 \\
\hline Chloroethane & $\mathrm{U}$ & & & 5 \\
\hline Chloroethene (Vinyl chloride) & $\mathrm{U}$ & & & 5 \\
\hline Chloroform & $\mathrm{U}$ & & & 5 \\
\hline Chloromethane (Methyl chloride) & $\mathrm{U}$ & & & 5 \\
\hline Dibromochloromethane & $\mathrm{U}$ & & & 5 \\
\hline 1,1-Dichloroethane & $\mathrm{U}$ & & & 5 \\
\hline 1,2-Dichloroethane & $\mathrm{U}$ & & & 5 \\
\hline 1,1-Dichloroethylene & $\mathrm{U}$ & & & 5 \\
\hline 1,2-Dichloroethylene & $\mathrm{U}$ & & & 50 \\
\hline Dichloromethane (Methylene chloride) & $\mathrm{J}$ & $\mathrm{K}$ & $\mathrm{O}$ & 103 \\
\hline 1,2-Dichloropropane & $\mathrm{U}$ & & & 5 \\
\hline cis-1,3-Dichloropropene & $\mathrm{U}$ & & & 5 \\
\hline trans-1,3-Dichloropropene & $\mathrm{U}$ & & & 5 \\
\hline Ethylbenzene & $\mathrm{J}$ & IK & $\mathrm{O}$ & 2.73 \\
\hline 2-Hexanone & $\mathrm{U}$ & & & 25 \\
\hline Methyl ethyl ketone & $\mathrm{U}$ & & & 25 \\
\hline Methyl isobutyl ketone & $\mathrm{U}$ & & & 25 \\
\hline Styrene & $\mathrm{U}$ & & & 5 \\
\hline $1,1,2,2$-Tetrachloroethane & $\mathrm{U}$ & & & 5 \\
\hline Tetrachloroethylene & $\mathrm{U}$ & & & 5 \\
\hline Toluene & $\mathrm{U}$ & & & 5 \\
\hline $1,1,1$-Trichloroethane & $\mathrm{U}$ & & & 5 \\
\hline 1,1,2-Trichloroethane & $\mathrm{U}$ & & & 5 \\
\hline Trichloroethylene & $\mathrm{U}$ & & & 5 \\
\hline Vinyl acetate & $\mathrm{U}$ & & & 75 \\
\hline Xylenes & $\mathrm{J}$ & IK & $\mathrm{O}$ & 8.61 \\
\hline
\end{tabular}

$$
\begin{aligned}
& 0.00548 \\
& 0.00183 \\
& 0.00605 \\
& 0.0328 \\
& -0.0511 \\
& -0.0114
\end{aligned}
$$

Unit

SSMDL

Method

$\begin{array}{lll}\mathrm{pCi} / \mathrm{g} & 0.0167 & \text { EPIA-011B } \\ \mathrm{pCi} / \mathrm{g} & 0.0127 & \text { EPIA-011B } \\ \mathrm{pCi} / \mathrm{g} & 0.0149 & \text { EPIA-011B } \\ \mathrm{pCi} / \mathrm{g} & 0.103 & \text { EPIA-013B } \\ \mathrm{pCi} / \mathrm{g} & 0.138 & \text { EPIA-013B } \\ \mathrm{pCi} / \mathrm{g} & 0.139 & \text { EPIA-013B }\end{array}$

$\mu \mathrm{g} / \mathrm{kg} \quad 51.5 \quad$ EPA8260B

$\mu \mathrm{g} / \mathrm{kg} \quad 2.5 \quad$ EPA8260B

$\mu \mathrm{g} / \mathrm{kg} \quad 0.5 \quad$ EPA8260B

$\mu \mathrm{g} / \mathrm{kg} \quad 1.35 \quad$ EPA8260B

$\mu \mathrm{g} / \mathrm{kg} \quad 1.5 \quad$ EPA8260B

$\mu \mathrm{g} / \mathrm{kg} \quad 1.5 \quad$ EPA8260B

$\mu \mathrm{g} / \mathrm{kg} \quad 2.5 \quad$ EPA8260B

$\mu \mathrm{g} / \mathrm{kg} \quad 1.5 \quad$ EPA8260B

$\mu \mathrm{g} / \mathrm{kg} \quad 1.5 \quad$ EPA8260B

$\mu \mathrm{g} / \mathrm{kg} \quad 2 \quad$ EPA8260B

$\mu \mathrm{g} / \mathrm{kg} \quad 0.5 \quad$ EPA8260B

$\mu \mathrm{g} / \mathrm{kg} \quad 1 \quad$ EPA8260B

$\mu \mathrm{g} / \mathrm{kg} \quad 1.05 \quad$ EPA8260B

$\mu \mathrm{g} / \mathrm{kg} \quad 0.5 \quad$ EPA8260B

$\mu \mathrm{g} / \mathrm{kg} \quad 1.15 \quad$ EPA8260B

$\mu \mathrm{g} / \mathrm{kg} \quad 1.5 \quad$ EPA8260B

$\mu \mathrm{g} / \mathrm{kg} \quad 1.25 \quad$ EPA8260B

$\mu \mathrm{g} / \mathrm{kg} \quad 7 \quad$ EPA8260B

$\mu \mathrm{g} / \mathrm{kg} \quad 1.15 \quad$ EPA8260B

$\mu \mathrm{g} / \mathrm{kg} \quad 1 \quad$ EPA8260B

$\mu \mathrm{g} / \mathrm{kg} \quad 1.5 \quad$ EPA8260B

$\mu \mathrm{g} / \mathrm{kg} \quad 1.5 \quad$ EPA8260B

$\mu \mathrm{g} / \mathrm{kg} \quad 14 \quad$ EPA8260B

$\mu \mathrm{g} / \mathrm{kg} \quad 16 \quad$ EPA8260B

$\mu \mathrm{g} / \mathrm{kg} \quad 14.6 \quad$ EPA8260B

$\mu \mathrm{g} / \mathrm{kg} \quad 1.5 \quad$ EPA8260B

$\mu \mathrm{g} / \mathrm{kg} \quad 3 \quad$ EPA8260B

$\mu \mathrm{g} / \mathrm{kg} \quad 2 \quad$ EPA8260B

$\mu g / \mathrm{kg} \quad 4.5 \quad$ EPA8260B

$\mu \mathrm{g} / \mathrm{kg} \quad 0.5 \quad$ EPA8260B

$\mu \mathrm{g} / \mathrm{kg} \quad 1.2 \quad$ EPA8260B

$\mu \mathrm{g} / \mathrm{kg} \quad 1.35 \quad$ EPA8260B

$\mu \mathrm{g} / \mathrm{kg} \quad 10.5 \quad$ EPA8260B

$\mu \mathrm{g} / \mathrm{kg} \quad 5 \quad$ EPA8260B 
SURVEY ID: SRF-05-01

Sample type: Normal

Constituent

Metals (total recoverable)

Antimony
Arsenic
Barium
Cadmium
Calcium
Chromium
Copper
Lead
Magnesium
Manganese
Mercury
Nickel
Selenium
Silver
Zinc

Radionuclides

Actinium-228

Antimony-124

Antimony-125

Barium-133

Cerium-144

Cesium-134

Cesium-137

Cobalt-57

Cobalt-58

Cobalt-60

Europium-152

Europium-154

Europium-155

Gross alpha

Lead-212

Manganese-54

Neptunium-239

Nonvolatile beta

Plutonium-238

Plutonium-239/240

Potassium-40

Promethium-144

Promethium-146

Ruthenium-106

Sodium-22

Strontium-89/90

Tin-1 13

Tritium
Sample ID: 117034

Sample matrix: Fauna

Percent solids: NA

\begin{tabular}{|c|c|c|c|c|c|}
\hline \multirow[t]{3}{*}{$\mathrm{U}$} & & 0.485 & $\mathrm{mg} / \mathrm{kg}$ & 0.185 & EPA6010B \\
\hline & & 0.41 & $\mathrm{mg} / \mathrm{kg}$ & 0.221 & EPA6010B \\
\hline & & 5.5 & $\mathrm{mg} / \mathrm{kg}$ & 0.026 & EPA6010B \\
\hline \multirow[t]{2}{*}{$\mathrm{J}$} & I & 0.139 & $\mathrm{mg} / \mathrm{kg}$ & 0.018 & EPA6010B \\
\hline & & 8270 & $\mathrm{mg} / \mathrm{kg}$ & 3.381 & EPA6010B \\
\hline \multirow[t]{2}{*}{$J$} & I & 0.19 & $\mathrm{mg} / \mathrm{kg}$ & 0.0354 & EPA6010B \\
\hline & & 0.778 & $\mathrm{mg} / \mathrm{kg}$ & 0.0641 & EPA6010F \\
\hline \multirow[t]{4}{*}{$\mathrm{J}$} & I & 0.109 & $\mathrm{mg} / \mathrm{kg}$ & 0.077 & EPA6010B \\
\hline & & 381 & $\mathrm{mg} / \mathrm{kg}$ & 0.246 & EPA6010B \\
\hline & & 34.4 & $\mathrm{mg} / \mathrm{kg}$ & 0.344 & EPA6010B \\
\hline & & 0.165 & $\mathrm{mg} / \mathrm{kg}$ & 0.00218 & EPA7471 \\
\hline \multirow[t]{2}{*}{ U } & V & 0.372 & $\mathrm{mg} / \mathrm{kg}$ & 0.031 & EPA6010B \\
\hline & & 1.04 & $\mathrm{mg} / \mathrm{kg}$ & 0.131 & EPA6010 \\
\hline \multirow[t]{2}{*}{$\mathrm{U}$} & & 0.243 & $\mathrm{mg} / \mathrm{kg}$ & 0.0301 & EPA60101 \\
\hline & & 15.7 & $\mathrm{mg} / \mathrm{kg}$ & 0.18 & EPA6010 \\
\hline
\end{tabular}

$\begin{array}{lllll}\mathrm{U} & 0.101 & \mathrm{pCi} / \mathrm{g} & 0.214 & \text { EPIA-013B } \\ \mathrm{U} & 0.00332 & \mathrm{pCi} / \mathrm{g} & 0.0612 & \text { EPIA-013B } \\ \mathrm{U} & -0.0328 & \mathrm{pCi} / \mathrm{g} & 0.1 & \text { EPIA-013B } \\ \mathrm{U} & 0.00224 & \mathrm{pCi} / \mathrm{g} & 0.0488 & \text { EPIA-013B } \\ \mathrm{U} & -0.00918 & \mathrm{pCi} / \mathrm{g} & 0.205 & \text { EPIA-013B } \\ \mathrm{U} & -0.0159 & \mathrm{pCi} / \mathrm{g} & 0.0412 & \text { EPIA-013B } \\ & 0.243 & \mathrm{pCi} / \mathrm{g} & 0.0454 & \text { EPIA-013B } \\ \mathrm{U} & 0.000975 & \mathrm{pCi} / \mathrm{g} & 0.0254 & \text { EPIA-013B } \\ \mathrm{U} & -0.00482 & \mathrm{pCi} / \mathrm{g} & 0.06 & \text { EPIA-013B } \\ \mathrm{U} & 0.0086 & \mathrm{pCi} / \mathrm{g} & 0.0529 & \text { EPIA-013B } \\ \mathrm{U} & -0.0127 & \mathrm{pCi} / \mathrm{g} & 0.111 & \text { EPIA-013B } \\ \mathrm{U} & -0.0189 & \mathrm{pCi} / \mathrm{g} & 0.15 & \text { EPIA-013B } \\ \mathrm{U} & 0.058 & \mathrm{pCi} / \mathrm{g} & 0.102 & \text { EPIA-013B } \\ \mathrm{U} & 0.24 & \mathrm{pCi} / \mathrm{g} & 0.948 & \text { EPIA-001B } \\ \mathrm{U} & 0.00228 & \mathrm{pCi} / \mathrm{g} & 0.0718 & \text { EPIA-013B } \\ \mathrm{U} & -0.0143 & \mathrm{pCi} / \mathrm{g} & 0.0467 & \text { EPIA-013B } \\ \mathrm{U} & -0.00259 & \mathrm{pCi} / \mathrm{g} & 0.179 & \text { EPIA-013B } \\ & 6.39 & \mathrm{pCi} / \mathrm{g} & 1.33 & \text { EPIA-001B } \\ \mathrm{U} & -0.000653 & \mathrm{pCi} / \mathrm{g} & 0.0144 & \text { EPIA-012B } \\ \mathrm{U} & 0 & \mathrm{pCi} / \mathrm{g} & 0.00816 & \text { EPIA-012B } \\ & 3.16 & \mathrm{pCi} / \mathrm{g} & 0.372 & \text { EPIA-013B } \\ \mathrm{U} & 0.00753 & \mathrm{pCi} / \mathrm{g} & 0.0472 & \text { EPIA-013B } \\ \mathrm{U} & -0.0345 & \mathrm{pCi} / \mathrm{g} & 0.0463 & \text { EPIA-013B } \\ \mathrm{U} & 0.0452 & \mathrm{pCi} / \mathrm{g} & 0.449 & \text { EPIA-013B } \\ \mathrm{U} & -0.00672 & \mathrm{pCi} / \mathrm{g} & 0.0542 & \text { EPIA-013B } \\ \mathrm{U} & 0.403 & \mathrm{pCi} / \mathrm{g} & 0.107 & \text { EPA905 } \\ & 0.0147 & \mathrm{pCi} / \mathrm{g} & 0.0605 & \text { EPIA-013B } \\ & 3.32 & \mathrm{pCi} / \mathrm{g} & 0.127 & \text { EPIA-002B }\end{array}$


SURVEY ID: SRF-05-01 (cont.)

Constituent

Radionuclides

Uranium-233/234

Uranium-235

Uranium-238

Yttrium-88

Zinc-65

Zirconium-95

Volatile Organic Compounds

\begin{tabular}{|c|c|c|c|c|}
\hline Acetone & $\mathrm{J}$ & IK & $\mathrm{O}$ & 77.1 \\
\hline Benzene & $\mathrm{U}$ & & & 5 \\
\hline Bromodichloromethane & $\mathrm{U}$ & & & 5 \\
\hline Bromoform & $\mathrm{U}$ & & & 5 \\
\hline Bromomethane (Methyl bromide) & $\mathrm{U}$ & & & 5 \\
\hline Carbon disulfide & $\mathrm{J}$ & IK & $\mathrm{O}$ & 16.6 \\
\hline Carbon tetrachloride & $\mathrm{U}$ & & & 5 \\
\hline Chlorobenzene & $\mathrm{U}$ & & & 5 \\
\hline Chloroethane & $\mathrm{U}$ & & & 5 \\
\hline Chloroethene (Vinyl chloride) & $\mathrm{U}$ & & & 5 \\
\hline Chloroform & $\mathrm{U}$ & & & 5 \\
\hline Chloromethane (Methyl chloride) & $\mathrm{U}$ & & & 5 \\
\hline Dibromochloromethane & $\mathrm{U}$ & & & 5 \\
\hline 1,1-Dichloroethane & $\mathrm{U}$ & & & 5 \\
\hline 1,2-Dichloroethane & $\mathrm{U}$ & & & 5 \\
\hline 1,1-Dichloroethylene & $\mathrm{U}$ & & & 5 \\
\hline 1,2-Dichloroethylene & $\mathrm{U}$ & & & 50 \\
\hline Dichloromethane (Methylene chloride) & $\mathrm{J}$ & $\mathrm{K}$ & $\mathrm{O}$ & 32 \\
\hline 1,2-Dichloropropane & $\mathrm{U}$ & & & 5 \\
\hline cis-1,3-Dichloropropene & $\mathrm{U}$ & & & 5 \\
\hline trans-1,3-Dichloropropene & $\mathrm{U}$ & & & 5 \\
\hline Ethylbenzene & $\mathrm{U}$ & & & 5 \\
\hline 2-Hexanone & $\mathrm{U}$ & & & 25 \\
\hline Methyl ethyl ketone & $\mathrm{U}$ & & & 25 \\
\hline Methyl isobutyl ketone & $\mathrm{U}$ & & & 25 \\
\hline Styrene & $\mathrm{U}$ & & & 5 \\
\hline $1,1,2,2$-Tetrachloroethane & $\mathrm{U}$ & & & 5 \\
\hline Tetrachloroethylene & $\mathrm{U}$ & & & 5 \\
\hline Toluene & $\mathrm{U}$ & & & 5 \\
\hline $1,1,1$-Trichloroethane & $\mathrm{U}$ & & & 5 \\
\hline 1,1,2-Trichloroethane & $\mathrm{U}$ & & & 5 \\
\hline Trichloroethylene & $\mathrm{U}$ & & & 5 \\
\hline Vinyl acetate & $\mathrm{U}$ & & & 75 \\
\hline Xylenes & $\mathrm{J}$ & IK & $\mathrm{O}$ & 5.99 \\
\hline
\end{tabular}

Sample ID: 117034
FG SC E Result

Unit ssMDL

Method

$\begin{array}{llll}0.0156 & \mathrm{pCi} / \mathrm{g} & 0.0195 & \text { EPIA-011B } \\ -0.00187 & \mathrm{pCi} / \mathrm{g} & 0.018 & \text { EPIA-011B } \\ 0.00775 & \mathrm{pCi} / \mathrm{g} & 0.00775 & \text { EPIA-011B } \\ 0.0016 & \mathrm{pCi} / \mathrm{g} & 0.0479 & \text { EPIA-013B } \\ -0.0392 & \mathrm{pCi} / \mathrm{g} & 0.108 & \text { EPIA-013B } \\ -0.00215 & \mathrm{pCi} / \mathrm{g} & 0.109 & \text { EPIA-013B }\end{array}$

$\mu \mathrm{g} / \mathrm{kg} \quad 51.5 \quad$ EPA8260B

$\mu \mathrm{g} / \mathrm{kg} \quad 2.5 \quad$ EPA8260B

$\mu \mathrm{g} / \mathrm{kg} \quad 0.5 \quad$ EPA8260B

$\mu \mathrm{g} / \mathrm{kg} \quad 1.35 \quad$ EPA8260B

$\mu \mathrm{g} / \mathrm{kg} \quad 1.5 \quad$ EPA8260B

$\mu \mathrm{g} / \mathrm{kg} \quad 1.5 \quad$ EPA8260B

$\mu \mathrm{g} / \mathrm{kg} \quad 2.5 \quad$ EPA8260B

$\mu \mathrm{g} / \mathrm{kg} \quad 1.5 \quad$ EPA8260B

$\mu \mathrm{g} / \mathrm{kg} \quad 1.5 \quad$ EPA8260B

$\mu \mathrm{g} / \mathrm{kg} \quad 2 \quad$ EPA8260B

$\mu \mathrm{g} / \mathrm{kg} \quad 0.5 \quad$ EPA8260B

$\mu \mathrm{g} / \mathrm{kg} \quad 1 \quad$ EPA8260B

$\mu g / \mathrm{kg} \quad 1.05 \quad$ EPA8260B

$\mu \mathrm{g} / \mathrm{kg} \quad 0.5 \quad$ EPA8260B

$\mu \mathrm{g} / \mathrm{kg} \quad 1.15 \quad$ EPA8260B

$\mu \mathrm{g} / \mathrm{kg} \quad 1.5 \quad$ EPA8260B

$\mu \mathrm{g} / \mathrm{kg} \quad 1.25 \quad$ EPA8260B

$\mu \mathrm{g} / \mathrm{kg} \quad 7 \quad$ EPA8260B

$\mu \mathrm{g} / \mathrm{kg} \quad 1.15 \quad$ EPA8260B

$\mu \mathrm{g} / \mathrm{kg} \quad 1 \quad$ EPA8260B

$\mu \mathrm{g} / \mathrm{kg} \quad 1.5 \quad$ EPA8260B

$\mu \mathrm{g} / \mathrm{kg} \quad 1.5 \quad$ EPA8260B

$\mu \mathrm{g} / \mathrm{kg} \quad 14 \quad$ EPA8260B

$\mu \mathrm{g} / \mathrm{kg} \quad 16 \quad$ EPA8260B

$\mu \mathrm{g} / \mathrm{kg} \quad 14.6 \quad$ EPA8260B

$\mu g / \mathrm{kg} \quad 1.5 \quad$ EPA8260B

$\mu \mathrm{g} / \mathrm{kg} \quad 3 \quad$ EPA8260B

$\mu \mathrm{g} / \mathrm{kg} \quad 2 \quad$ EPA8260B

$\mu \mathrm{g} / \mathrm{kg} \quad 4.5 \quad$ EPA8260B

$\mu g / \mathrm{kg} \quad 0.5 \quad$ EPA8260B

$\mu \mathrm{g} / \mathrm{kg} \quad 1.2 \quad$ EPA8260B

$\mu \mathrm{g} / \mathrm{kg} \quad 1.35 \quad$ EPA8260B

$\mu \mathrm{g} / \mathrm{kg} \quad 10.5 \quad$ EPA8260B

$\mu \mathrm{g} / \mathrm{kg} \quad 5 \quad$ EPA8260B 
SURVEY ID: SRF-05-02

Sample type: Normal

\section{Constituent}

Metals (total recoverable)

Antimony
Arsenic
Barium
Cadmium
Calcium
Chromium
Copper
Lead
Magnesium
Manganese
Mercury
Nickel
Selenium
Silver
Zinc

Radionuclides

Actinium-228

Antimony-124

Antimony-125

Barium-133

Cerium-144

Cesium-134

Cesium-137

Cobalt-57

Cobalt-58

Cobalt-60

Europium-152

Europium-154

Europium-155

Gross alpha

Lead-212

Manganese-54

Neptunium-239

Nonvolatile beta

Plutonium-238

Plutonium-239/240

Potassium-40

Promethium-144

Promethium-146

Ruthenium-106

Sodium-22

Strontium-89/90

Strontium-89/90

Tin-1 13
Sample ID: 117035

Sample matrix: Fauna

Percent solids: NA

$\begin{array}{ll}\mathrm{U} & 0.476 \\ & 0.496 \\ \mathrm{U} & 3.96 \\ & 0.238 \\ & 28500 \\ & 0.359 \\ & 0.251 \\ & 0.306 \\ & 617 \\ & 10.1 \\ \mathrm{U} & 0.134 \\ & 0.238 \\ \mathrm{U} & 0.88 \\ & 0.238 \\ & 24\end{array}$

24
Unit ssMDL

$\mathrm{mg} / \mathrm{kg} \quad 0.182$

$\mathrm{mg} / \mathrm{kg} \quad 0.217$

$\mathrm{mg} / \mathrm{kg} \quad 0.026$

$\mathrm{mg} / \mathrm{kg} \quad 0.018$

$\mathrm{mg} / \mathrm{kg} \quad 6.637$

$\mathrm{mg} / \mathrm{kg} \quad 0.0347$

$\mathrm{mg} / \mathrm{kg} \quad 0.0629$

$\mathrm{mg} / \mathrm{kg} \quad 0.075$

$\mathrm{mg} / \mathrm{kg} \quad 0.242$

$\mathrm{mg} / \mathrm{kg} \quad 0.338$

$\mathrm{mg} / \mathrm{kg} \quad 0.00225$

$\mathrm{mg} / \mathrm{kg} \quad 0.03$

$\mathrm{mg} / \mathrm{kg} \quad 0.129$

$\mathrm{mg} / \mathrm{kg} \quad 0.0295$

$\mathrm{mg} / \mathrm{kg} \quad 0.176$
EPA6010B

EPA6010B

EPA6010B

EPA6010B

EPA6010B

EPA6010B

EPA6010B

EPA6010B

EPA6010B

EPA6010B

EPA7471A

EPA6010B

EPA6010B

EPA6010B

EPA6010B
$\mathrm{U}$

U

U

U

U

U

U

U

U

U

U

U

U

$\mathrm{R}$

U

$\mathrm{U}$

$\mathrm{U}$

$\mathrm{U}$

U

U

U

U

U
0.144

0.0113

$-0.00353$

0.0256

0.0354

$-0.0413$

0.103

0.0139

0.0175

0.0272

$-0.0167$

0.0971

0.0187

0.224

$4 \quad 0.0924$

0.0105

$-0.0482$

5.47

$-0.000944$

$-0.00113$

3.28

0.012

0.00102

0.209

0.0351

0.559

0.553

0.013

$\begin{array}{ll}\mathrm{pCi} / \mathrm{g} & 0.244 \\ \mathrm{pCi} / \mathrm{g} & 0.0711 \\ \mathrm{pCi} / \mathrm{g} & 0.109 \\ \mathrm{pCi} / \mathrm{g} & 0.0524 \\ \mathrm{pCi} / \mathrm{g} & 0.199 \\ \mathrm{pCi} / \mathrm{g} & 0.042 \\ \mathrm{pCi} / \mathrm{g} & 0.0474 \\ \mathrm{pCi} / \mathrm{g} & 0.0252 \\ \mathrm{pCi} / \mathrm{g} & 0.0727 \\ \mathrm{pCi} / \mathrm{g} & 0.0615 \\ \mathrm{pCi} / \mathrm{g} & 0.112 \\ \mathrm{pCi} / \mathrm{g} & 0.185 \\ \mathrm{pCi} / \mathrm{g} & 0.0934 \\ \mathrm{pCi} / \mathrm{g} & 1.29 \\ \mathrm{pCi} / \mathrm{g} & 0.0767 \\ \mathrm{pCi} / \mathrm{g} & 0.0599 \\ \mathrm{pCi} / \mathrm{g} & 0.162 \\ \mathrm{pCi} / \mathrm{g} & 1.8 \\ \mathrm{pCi} / \mathrm{g} & 0.0382 \\ \mathrm{pCi} / \mathrm{g} & 0.0249 \\ \mathrm{pCi} / \mathrm{g} & 0.44 \\ \mathrm{pCi} / \mathrm{g} & 0.0552 \\ \mathrm{pCi} / \mathrm{g} & 0.0612 \\ \mathrm{pCi} / \mathrm{g} & 0.506 \\ \mathrm{pCi} / \mathrm{g} & 0.0614 \\ \mathrm{pCi} / \mathrm{g} & 0.14 \\ \mathrm{pCi} / \mathrm{g} & 0.148 \\ \mathrm{pCi} / \mathrm{g} & 0.0605 \\ & \end{array}$

EPIA-013B

EPIA-013B

EPIA-013B

EPIA-013B

EPIA-013B

EPIA-013B

EPIA-013B

EPIA-013B

EPIA-013B

EPIA-013B

EPIA-013B

EPIA-013B

EPIA-013B

EPIA-001B

EPIA-013B

EPIA-013B

EPIA-013B

EPIA-001B

EPIA-012B

EPIA-012B

EPIA-013B

EPIA-013B

EPIA-013B

EPIA-013B

EPIA-013B

EPA905

EPA905

EPIA-013B 
SURVEY ID: SRF-05-02 (cont.)

Constituent

Radionuclides

Tritium

Uranium-233/234

Uranium-235

Uranium-238

Yttrium-88

Zinc-65

Zirconium-95

Volatile Organic Compounds

Acetone

Benzene

Bromodichloromethane

Bromoform

Bromomethane (Methyl bromide)

Carbon disulfide

Carbon tetrachloride

Chlorobenzene

Chloroethane

Chloroethene (Vinyl chloride)

Chloroform

Chloromethane (Methyl chloride)

Dibromochloromethane

1,1-Dichloroethane

1,2-Dichloroethane

1,1-Dichloroethylene

1,2-Dichloroethylene

Dichloromethane (Methylene chloride)

1,2-Dichloropropane

cis-1,3-Dichloropropene

trans-1,3-Dichloropropene

Ethylbenzene

2-Hexanone

Methyl ethyl ketone

Methyl isobutyl ketone

Styrene

1,1,2,2-Tetrachloroethane

Tetrachloroethylene

Toluene

1,1,1-Trichloroethane

1,1,2-Trichloroethane

Trichloroethylene

Vinyl acetate

Xylenes
Sample ID: 117035

FG SC E Result

Unit ssMDL

Method

0.425
0.0104
0.00417
0.0131
0.0083
0.0243
-0.00859

$\begin{array}{ll}\mathrm{pCi} / \mathrm{g} & 0.108 \\ \mathrm{pCi} / \mathrm{g} & 0.0408 \\ \mathrm{pCi} / \mathrm{g} & 0.0303 \\ \mathrm{pCi} / \mathrm{g} & 0.0277 \\ \mathrm{pCi} / \mathrm{g} & 0.0739 \\ \mathrm{pCi} / \mathrm{g} & 0.138 \\ \mathrm{pCi} / \mathrm{g} & 0.127\end{array}$

EPIA-002B EPIA-011B EPIA-011B

EPIA-011B

EPIA-013B

EPIA-013B

EPIA-013B

EPA8260B

EPA8260B

EPA8260B

EPA8260B

EPA8260B

EPA8260B

EPA8260B

EPA8260B

EPA8260B

EPA8260B

EPA8260B

EPA8260B

EPA8260B

EPA8260B

EPA8260B

EPA8260B

EPA8260B

EPA8260B

EPA8260B

EPA8260B

EPA8260B

EPA8260B

EPA8260B

EPA8260B

EPA8260B

EPA8260B

EPA8260B

EPA8260B

EPA8260B

EPA8260B

EPA8260B

EPA8260B

EPA8260B

EPA8260B 
SURVEY ID: SRF-05-03

Sample type: Normal

Constituent

Metals (total recoverable)

\begin{tabular}{|c|c|c|c|}
\hline Antimony & \multicolumn{2}{|l|}{$\mathrm{U}$} & 0.467 \\
\hline Arsenic & & & 0.758 \\
\hline Barium & \multirow{3}{*}{\multicolumn{2}{|c|}{$\mathrm{U}$}} & 1.46 \\
\hline Cadmium & & & 0.234 \\
\hline Calcium & & & 8790 \\
\hline Chromium & \multirow[t]{2}{*}{$\mathrm{J}$} & I & 0.148 \\
\hline Copper & & & 0.519 \\
\hline Lead & \multirow[t]{4}{*}{$\mathrm{U}$} & & 0.234 \\
\hline Magnesium & & & 419 \\
\hline Manganese & & & 4.56 \\
\hline Mercury & & & 1.25 \\
\hline Nickel & \multirow[t]{2}{*}{$\mathrm{U}$} & & 0.234 \\
\hline Selenium & & & 1.08 \\
\hline Silver & \multirow[t]{2}{*}{$\mathrm{U}$} & & 0.234 \\
\hline Zinc & & & 10.6 \\
\hline
\end{tabular}

Radionuclides

Actinium-228

Antimony-124

Antimony-125

Barium-133

Cerium-144

Cesium-134

Cesium-137

Cobalt-57

Cobalt-58

Cobalt-60

Europium-152

Europium-154

Europium-155

Gross alpha

Lead-212

Manganese-54

Neptunium-239

Nonvolatile beta

Plutonium-238

Plutonium-239/240

Potassium-40

Promethium-144

Promethium-146

Ruthenium-106

Sodium-22

Strontium-89/90

Tin-1 13

Tritium
Sample ID: 117036

Sample matrix: Fauna

Percent solids: NA
Method

Unit ssMDL

$\mathrm{mg} / \mathrm{kg} \quad 0.178$

$\mathrm{mg} / \mathrm{kg} \quad 0.213$

$\mathrm{mg} / \mathrm{kg} \quad 0.025$

$\mathrm{mg} / \mathrm{kg} \quad 0.018$

$\mathrm{mg} / \mathrm{kg} 3.256$

$\mathrm{mg} / \mathrm{kg} \quad 0.0341$

$\mathrm{mg} / \mathrm{kg} \quad 0.0617$

$\mathrm{mg} / \mathrm{kg} \quad 0.074$

$\mathrm{mg} / \mathrm{kg} \quad 0.237$

$\mathrm{mg} / \mathrm{kg} \quad 0.332$

$\mathrm{mg} / \mathrm{kg} \quad 0.00214$

$\mathrm{mg} / \mathrm{kg} \quad 0.03$

$\mathrm{mg} / \mathrm{kg} \quad 0.126$

$\mathrm{mg} / \mathrm{kg} \quad 0.029$

$\mathrm{mg} / \mathrm{kg} \quad 0.173$

$\begin{array}{ll}\mathrm{pCi} / \mathrm{g} & 0.21 \\ \mathrm{pCi} / \mathrm{g} & 0.0747 \\ \mathrm{pCi} / \mathrm{g} & 0.139 \\ \mathrm{pCi} / \mathrm{g} & 0.0558 \\ \mathrm{pCi} / \mathrm{g} & 0.223 \\ \mathrm{pCi} / \mathrm{g} & 0.0446 \\ \mathrm{pCi} / \mathrm{g} & 0.0504 \\ \mathrm{pCi} / \mathrm{g} & 0.026 \\ \mathrm{pCi} / \mathrm{g} & 0.0723 \\ \mathrm{pCi} / \mathrm{g} & 0.061 \\ \mathrm{pCi} / \mathrm{g} & 0.122 \\ \mathrm{pCi} / \mathrm{g} & 0.135 \\ \mathrm{pCi} / \mathrm{g} & 0.0986 \\ \mathrm{pCi} / \mathrm{g} & 1.02 \\ \mathrm{pCi} / \mathrm{g} & 0.0849 \\ \mathrm{pCi} / \mathrm{g} & 0.0543 \\ \mathrm{pCi} / \mathrm{g} & 0.189 \\ \mathrm{pCi} / \mathrm{g} & 1.06 \\ \mathrm{pCi} / \mathrm{g} & 0.0159 \\ \mathrm{pCi} / \mathrm{g} & 0.0135 \\ \mathrm{pCi} / \mathrm{g} & 0.406 \\ \mathrm{pCi} / \mathrm{g} & 0.056 \\ \mathrm{pCi} / \mathrm{g} & 0.0603 \\ \mathrm{pCi} / \mathrm{g} & 0.444 \\ \mathrm{pCi} / \mathrm{g} & 0.0491 \\ \mathrm{pCi} / \mathrm{g} & 0.119 \\ \mathrm{pCi} / \mathrm{g} & 0.0637 \\ \mathrm{pCi} / \mathrm{g} & 0.112 \\ & \end{array}$

$\mathrm{pCi} / \mathrm{g} \quad 0.21$

0.021

0.0032

0.00258

$-0.00206$

$-0.0215$

0.832

$-0.00767$

0.0109

0.0189

$-0.0418$

$-0.016$

0.0327

$-0.0606$

0.00465

0.00248

0.042

5.95

$-0.00123$

0.00451

3.56

0.0193

$-0.0232$

$-0.0522$

$-0.00555$

0.315

$-0.00556$

1.28
$\mathrm{pCi} / \mathrm{g}$
EPA6010B

EPA6010B

EPA6010B

EPA6010B

EPA6010B

EPA6010B

EPA6010B

EPA6010B

EPA6010B

EPA6010B

EPA7471A

EPA6010B

EPA6010B

EPA6010B

EPA6010B

EPIA-013B

EPIA-013B

EPIA-013B

EPIA-013B

EPIA-013B

EPIA-013B

EPIA-013B

EPIA-013B

EPIA-013B

EPIA-013B

EPIA-013B

EPIA-013B

EPIA-013B

EPIA-001B

EPIA-013B

EPIA-013B

EPIA-013B

EPIA-001B

EPIA-012B

EPIA-012B

EPIA-013B

EPIA-013B

EPIA-013B

EPIA-013B

EPIA-013B

EPA905

EPIA-013B

EPIA-002B 
SURVEY ID: SRF-05-03 (cont.)

Constituent

Radionuclides

Uranium-233/234

Uranium-235

Uranium-238

Yttrium-88

Zinc-65

Zirconium-95

Volatile Organic Compounds

\begin{tabular}{|c|c|c|c|c|}
\hline Acetone & $\mathrm{J}$ & IK & $\mathrm{O}$ & 106 \\
\hline Benzene & $\mathrm{U}$ & & & 5 \\
\hline Bromodichloromethane & $\mathrm{U}$ & & & 5 \\
\hline Bromoform & $\mathrm{U}$ & & & 5 \\
\hline Bromomethane (Methyl bromide) & $\mathrm{U}$ & & & 5 \\
\hline Carbon disulfide & $\mathrm{J}$ & $\mathrm{K}$ & $\mathrm{O}$ & 32.6 \\
\hline Carbon tetrachloride & $\mathrm{U}$ & & & 5 \\
\hline Chlorobenzene & $\mathrm{U}$ & & & 5 \\
\hline Chloroethane & $\mathrm{U}$ & & & 5 \\
\hline Chloroethene (Vinyl chloride) & $\mathrm{U}$ & & & 5 \\
\hline Chloroform & $\mathrm{U}$ & & & 5 \\
\hline Chloromethane (Methyl chloride) & $\mathrm{U}$ & & & 5 \\
\hline Dibromochloromethane & $\mathrm{U}$ & & & 5 \\
\hline 1,1-Dichloroethane & $\mathrm{U}$ & & & 5 \\
\hline 1,2-Dichloroethane & $\mathrm{U}$ & & & 5 \\
\hline 1,1-Dichloroethylene & $\mathrm{U}$ & & & 5 \\
\hline 1,2-Dichloroethylene & $\mathrm{U}$ & & & 50 \\
\hline Dichloromethane (Methylene chloride) & $\mathrm{J}$ & $\mathrm{K}$ & $\mathrm{O}$ & 78.4 \\
\hline 1,2-Dichloropropane & $\mathrm{U}$ & & & 5 \\
\hline cis-1,3-Dichloropropene & $\mathrm{U}$ & & & 5 \\
\hline trans-1,3-Dichloropropene & $\mathrm{U}$ & & & 5 \\
\hline Ethylbenzene & $\mathrm{U}$ & & & 5 \\
\hline 2-Hexanone & $\mathrm{U}$ & & & 25 \\
\hline Methyl ethyl ketone & $\mathrm{U}$ & & & 25 \\
\hline Methyl isobutyl ketone & $\mathrm{U}$ & & & 25 \\
\hline Styrene & $\mathrm{U}$ & & & 5 \\
\hline $1,1,2,2$-Tetrachloroethane & $\mathrm{U}$ & & & 5 \\
\hline Tetrachloroethylene & $\mathrm{U}$ & & & 5 \\
\hline Toluene & $\mathrm{U}$ & & & 5 \\
\hline 1,1,1-Trichloroethane & $\mathrm{U}$ & & & 5 \\
\hline $1,1,2$-Trichloroethane & $\mathrm{U}$ & & & 5 \\
\hline Trichloroethylene & $\mathrm{U}$ & & & 5 \\
\hline Vinyl acetate & $\mathrm{U}$ & & & 75 \\
\hline Xylenes & $\mathrm{J}$ & IK & $\mathrm{O}$ & 8.05 \\
\hline
\end{tabular}
0.000186
0.00177
0.00408
0.00973
$-0.0397$
0.00296

$\begin{array}{ll}\mathrm{U} & 0.000186 \\ \mathrm{U} & 0.00177 \\ \mathrm{U} & 0.00408 \\ \mathrm{U} & 0.00973 \\ \mathrm{U} & -0.0397 \\ \mathrm{U} & 0.00296\end{array}$

EPIA-011B EPIA-011B EPIA-011B EPIA-013B EPIA-013B EPIA-013B
EPA8260B

EPA8260B

EPA8260B

EPA8260B

EPA8260B

EPA8260B

EPA8260B

EPA8260B

EPA8260B

EPA8260B

EPA8260B

EPA8260B

EPA8260B

EPA8260B

EPA8260B

EPA8260B

EPA8260B

EPA8260B

EPA8260B

EPA8260B

EPA8260B

EPA8260B

EPA8260B

EPA8260B

EPA8260B

EPA8260B

EPA8260B

EPA8260B

EPA8260B

EPA8260B

EPA8260B

EPA8260B

EPA8260B

EPA8260B 
SURVEY ID: SRF-06-01

Sample type: Normal

Constituent

Metals (total recoverable)

Antimony
Arsenic
Barium
Cadmium
Calcium
Chromium
Copper
Lead
Magnesium
Manganese
Mercury
Nickel
Selenium
Silver
Zinc

Radionuclides

Actinium-228

Antimony-124

Antimony-125

Barium-133

Cerium-144

Cesium-134

Cesium-137

Cobalt-57

Cobalt-58

Cobalt-60

Europium-152

Europium-154

Europium-155

Gross alpha

Lead-212

Manganese-54

Neptunium-239

Nonvolatile beta

Plutonium-238

Plutonium-239/240

Potassium-40

Promethium-144

Promethium-146

Ruthenium-106

Sodium-22

Strontium-89/90

Tin-1 13

Tritium
Sample ID: 117037

Sample matrix: Fauna

Percent solids: NA

\begin{tabular}{|c|c|c|c|c|c|c|}
\hline \multirow[t]{3}{*}{ JU } & & 4 & 0.185 & $\mathrm{mg} / \mathrm{kg}$ & 0.182 & EPA6010B \\
\hline & & & 0.604 & $\mathrm{mg} / \mathrm{kg}$ & 0.217 & EPA6010B \\
\hline & & & 6.58 & $\mathrm{mg} / \mathrm{kg}$ & 0.026 & EPA6010B \\
\hline \multirow[t]{4}{*}{ JU } & & 4 & 0.0473 & $\mathrm{mg} / \mathrm{kg}$ & 0.018 & EPA6010B \\
\hline & & & 51200 & $\mathrm{mg} / \mathrm{kg}$ & 33.187 & EPA6010B \\
\hline & & & 0.657 & $\mathrm{mg} / \mathrm{kg}$ & 0.0347 & EPA6010B \\
\hline & & & 2.91 & $\mathrm{mg} / \mathrm{kg}$ & 0.0629 & EPA6010B \\
\hline \multirow[t]{4}{*}{$\mathrm{J}$} & I & & 0.227 & $\mathrm{mg} / \mathrm{kg}$ & 0.075 & EPA6010B \\
\hline & & & 1010 & $\mathrm{mg} / \mathrm{kg}$ & 0.242 & EPA6010B \\
\hline & & & 93.4 & $\mathrm{mg} / \mathrm{kg}$ & 0.338 & EPA6010B \\
\hline & & & 0.0959 & $\mathrm{mg} / \mathrm{kg}$ & 0.00195 & EPA7471A \\
\hline \multirow[t]{2}{*}{ JU } & IV & & 0.181 & $\mathrm{mg} / \mathrm{kg}$ & 0.03 & EPA6010B \\
\hline & & & 1.34 & $\mathrm{mg} / \mathrm{kg}$ & 0.129 & EPA6010B \\
\hline \multirow[t]{2}{*}{$\mathrm{U}$} & & & 0.238 & $\mathrm{mg} / \mathrm{kg}$ & 0.0295 & EPA6010B \\
\hline & & & 28 & $\mathrm{mg} / \mathrm{kg}$ & 0.176 & EPA6010B \\
\hline
\end{tabular}

U $\quad 0.0734$

0.0109

0.0596

0.0181

$-0.104$

0.0125

0.0418

0.00931

0.0246

0.00582

$-0.00621$

$-0.0336$

0.0123

$-0.0717$

0.0237

$-0.00667$

0.0376

7.33

$-0.00168$

$-0.00168$

2.95

$-0.00499$

$-0.0152$

0.071

$-0.0122$

0.546

0.00509

0.151
Unit ssMDL

Method

EPA6010B

EPIA-013B

$\mathrm{pCi} / \mathrm{g} \quad 0.261$

$\mathrm{pCi} / \mathrm{g} \quad 0.0841$

$\mathrm{pCi} / \mathrm{g} \quad 0.143$

$\mathrm{pCi} / \mathrm{g} \quad 0.0613$

EPIA-013B

EPIA-013B

EPIA-013B

$\mathrm{pCi} / \mathrm{g} \quad 0.224$

$\mathrm{pCi} / \mathrm{g} \quad 0.0588$

$\mathrm{pCi} / \mathrm{g} \quad 0.069$

$\mathrm{pCi} / \mathrm{g} \quad 0.0898$

$\mathrm{pCi} / \mathrm{g} \quad 0.0686$

$\mathrm{pCi} / \mathrm{g} \quad 0.137$

$\mathrm{pCi} / \mathrm{g} \quad 0.171$

$\mathrm{pCi} / \mathrm{g} \quad 0.105$

$\mathrm{pCi} / \mathrm{g} \quad 0.861$

$\mathrm{pCi} / \mathrm{g} \quad 0.0919$

$\mathrm{pCi} / \mathrm{g} \quad 0.0644$

$\mathrm{pCi} / \mathrm{g} \quad 0.2$

$\mathrm{pCi} / \mathrm{g} \quad 1.42$

$\mathrm{pCi} / \mathrm{g} \quad 0.0218$

$\mathrm{pCi} / \mathrm{g} \quad 0.0218$

$\mathrm{pCi} / \mathrm{g} \quad 0.58$

$\mathrm{pCi} / \mathrm{g} \quad 0.0576$

$\mathrm{pCi} / \mathrm{g} \quad 0.0653$

$\mathrm{pCi} / \mathrm{g} \quad 0.614$

$\mathrm{pCi} / \mathrm{g} \quad 0.0617$

$\begin{array}{ll}\mathrm{pCi} / \mathrm{g} & 0.17\end{array}$

$\mathrm{pCi} / \mathrm{g} \quad 0.0772$

EPIA-013B

EPIA-013B

EPIA-013B

EPIA-013B

EPIA-013B

EPIA-013B

EPIA-013B

EPIA-013B

EPIA-001B

EPIA-013B

EPIA-013B

EPIA-013B

EPIA-001B

EPIA-012B

EPIA-012B

EPIA-013B

EPIA-013B

EPIA-013B

EPIA-013B

EPIA-013B

EPA905

EPIA-013B

EPIA-002B
$\mathrm{pCi} / \mathrm{g} \quad 0.0286$

EPIA-013B 
SURVEY ID: SRF-06-01 (cont.)

Constituent

Radionuclides

Uranium-233/234

Uranium-235

Uranium-238

Yttrium-88

Zinc-65

Zirconium-95

Volatile Organic Compounds

\begin{tabular}{|c|c|c|c|c|}
\hline Acetone & $\mathrm{J}$ & $\mathrm{K}$ & $\mathrm{O}$ & 156 \\
\hline Benzene & $\mathrm{U}$ & & & 5 \\
\hline Bromodichloromethane & $\mathrm{U}$ & & & 5 \\
\hline Bromoform & $\mathrm{U}$ & & & 5 \\
\hline Bromomethane (Methyl bromide) & $\mathrm{U}$ & & & 5 \\
\hline Carbon disulfide & $\mathrm{J}$ & $\mathrm{K}$ & $\mathrm{O}$ & 26.7 \\
\hline Carbon tetrachloride & $\mathrm{U}$ & & & 5 \\
\hline Chlorobenzene & $\mathrm{U}$ & & & 5 \\
\hline Chloroethane & $\mathrm{U}$ & & & 5 \\
\hline Chloroethene (Vinyl chloride) & $\mathrm{U}$ & & & 5 \\
\hline Chloroform & $\mathrm{U}$ & & & 5 \\
\hline Chloromethane (Methyl chloride) & $\mathrm{U}$ & & & 5 \\
\hline Dibromochloromethane & $\mathrm{U}$ & & & 5 \\
\hline 1,1-Dichloroethane & $\mathrm{U}$ & & & 5 \\
\hline 1,2-Dichloroethane & $\mathrm{U}$ & & & 5 \\
\hline 1,1-Dichloroethylene & $\mathrm{U}$ & & & 5 \\
\hline 1,2-Dichloroethylene & $\mathrm{U}$ & & & 50 \\
\hline Dichloromethane (Methylene chloride) & $\mathrm{J}$ & $\mathrm{K}$ & $\mathrm{O}$ & 50 \\
\hline 1,2-Dichloropropane & $\mathrm{U}$ & & & 5 \\
\hline cis-1,3-Dichloropropene & $\mathrm{U}$ & & & 5 \\
\hline trans-1,3-Dichloropropene & $\mathrm{U}$ & & & 5 \\
\hline Ethylbenzene & $\mathrm{J}$ & $\mathrm{K}$ & $\mathrm{O}$ & 10.3 \\
\hline 2-Hexanone & $\mathrm{U}$ & & & 25 \\
\hline Methyl ethyl ketone & $\mathrm{U}$ & & & 25 \\
\hline Methyl isobutyl ketone & $\mathrm{U}$ & & & 25 \\
\hline Styrene & $\mathrm{U}$ & & & 5 \\
\hline $1,1,2,2$-Tetrachloroethane & $\mathrm{U}$ & & & 5 \\
\hline Tetrachloroethylene & $\mathrm{U}$ & & & 5 \\
\hline Toluene & $\mathrm{J}$ & $\mathrm{K}$ & $\mathrm{O}$ & 17.4 \\
\hline 1,1,1-Trichloroethane & $\mathrm{U}$ & & & 5 \\
\hline 1,1,2-Trichloroethane & $\mathrm{U}$ & & & 5 \\
\hline Trichloroethylene & $\mathrm{U}$ & & & 5 \\
\hline Vinyl acetate & $\mathrm{U}$ & & & 75 \\
\hline Xylenes & $\mathrm{J}$ & $\mathrm{K}$ & $\mathrm{O}$ & 51.3 \\
\hline
\end{tabular}

Sample ID: 117037

FG SC E Result

Unit ssMDL

Method

\begin{tabular}{|c|c|c|c|c|c|}
\hline $\mathrm{R}$ & 4 & 0.019 & $\mathrm{pCi} / \mathrm{g}$ & 0.00949 & EPIA-011B \\
\hline $\mathrm{U}$ & & 0 & $\mathrm{pCi} / \mathrm{g}$ & 0.00952 & EPIA-011B \\
\hline $\mathrm{U}$ & & 0.00949 & $\mathrm{pCi} / \mathrm{g}$ & 0.00949 & EPIA-011B \\
\hline $\mathrm{U}$ & & -0.00179 & $\mathrm{pCi} / \mathrm{g}$ & 0.0683 & EPIA-013H \\
\hline $\mathrm{U}$ & & 0.0543 & $\mathrm{pCi} / \mathrm{g}$ & 0.157 & EPIA-013B \\
\hline $\mathrm{U}$ & & 0.0643 & $\mathrm{pCi} / \mathrm{g}$ & 0.154 & EPIA-013I \\
\hline
\end{tabular}

$\begin{array}{ll}\mu g / \mathrm{kg} & 51.5 \\ \mu g / \mathrm{kg} & 2.5 \\ \mu g / \mathrm{kg} & 0.5 \\ \mu g / \mathrm{kg} & 1.35 \\ \mu g / \mathrm{kg} & 1.5 \\ \mu g / \mathrm{kg} & 1.5 \\ \mu g / \mathrm{kg} & 2.5 \\ \mu g / \mathrm{kg} & 1.5 \\ \mu g / \mathrm{kg} & 1.5 \\ \mu g / \mathrm{kg} & 2 \\ \mu g / \mathrm{kg} & 0.5 \\ \mu g / \mathrm{kg} & 1 \\ \mu g / \mathrm{kg} & 1.05 \\ \mu g / \mathrm{kg} & 0.5 \\ \mu g / \mathrm{kg} & 1.15 \\ \mu g / \mathrm{kg} & 1.5 \\ \mu g / \mathrm{kg} & 1.25 \\ \mu g / \mathrm{kg} & 7 \\ \mu g / \mathrm{kg} & 1.15 \\ \mu g / \mathrm{kg} & 1 \\ \mu g / \mathrm{kg} & 1.5 \\ \mu g / \mathrm{kg} & 1.5 \\ \mu g / \mathrm{kg} & 14 \\ \mu g / \mathrm{kg} & 16 \\ \mu g / \mathrm{kg} & 14.6 \\ \mu g / \mathrm{kg} & 1.5 \\ \mu g / \mathrm{kg} & 3 \\ \mu g / \mathrm{kg} & 2 \\ \mu g / \mathrm{kg} & 4.5 \\ \mu g / \mathrm{kg} & 0.5 \\ \mu g / \mathrm{kg} & 1.2 \\ \mu g / \mathrm{kg} & 1.35 \\ \mu g / \mathrm{kg} & 10.5 \\ \mu g / \mathrm{kg} & 5 \\ & \end{array}$

EPA8260B

EPA8260B

EPA8260B

EPA8260B

EPA8260B

EPA8260B

EPA8260B

EPA8260B

EPA8260B

EPA8260B

EPA8260B

EPA8260B

EPA8260B

EPA8260B

EPA8260B

EPA8260B

EPA8260B

EPA8260B

EPA8260B

EPA8260B

EPA8260B

EPA8260B

EPA8260B

EPA8260B

EPA8260B

EPA8260B

EPA8260B

EPA8260B

EPA8260B

EPA8260B

EPA8260B

EPA8260B

EPA8260B

EPA8260B 
SURVEY ID: SRF-06-02

Sample type: Normal

Constituent

Metals (total recoverable)

Antimony
Arsenic
Barium
Cadmium
Calcium
Chromium
Copper
Lead
Magnesium
Manganese
Mercury
Nickel
Selenium
Silver
Zinc

Radionuclides

Actinium-228

Antimony-124

Antimony-125

Barium-133

Cerium-144

Cesium-134

Cesium-137

Cobalt-57

Cobalt-58

Cobalt-60

Europium-152

Europium-154

Europium-155

Gross alpha

Lead-212

Manganese-54

Neptunium-239

Nonvolatile beta

Plutonium-238

Plutonium-239/240

Potassium-40

Promethium-144

Promethium-146

Ruthenium-106

Sodium-22

Strontium-89/90

Tin-1 13

Tritium
Sample ID: 117038

Sample matrix: Fauna

Percent solids: NA

FG SC E Result Unit ssMDL

\begin{tabular}{|c|c|c|c|c|c|}
\hline \multirow[t]{3}{*}{$\mathrm{U}$} & & 0.463 & $\mathrm{mg} / \mathrm{kg}$ & 0.177 & EPA6010B \\
\hline & & 0.489 & $\mathrm{mg} / \mathrm{kg}$ & 0.211 & EPA6010B \\
\hline & & 3.82 & $\mathrm{mg} / \mathrm{kg}$ & 0.025 & EPA6010B \\
\hline \multirow[t]{4}{*}{ JU } & 4 & 0.0216 & $\mathrm{mg} / \mathrm{kg}$ & 0.018 & EPA6010B \\
\hline & & 12800 & $\mathrm{mg} / \mathrm{kg}$ & 3.228 & EPA6010B \\
\hline & & 0.354 & $\mathrm{mg} / \mathrm{kg}$ & 0.0338 & EPA6010B \\
\hline & & 1.19 & $\mathrm{mg} / \mathrm{kg}$ & 0.0611 & EPA6010B \\
\hline \multirow[t]{4}{*}{$\mathrm{J}$} & I & 0.161 & $\mathrm{mg} / \mathrm{kg}$ & 0.073 & EPA6010B \\
\hline & & 411 & $\mathrm{mg} / \mathrm{kg}$ & 0.235 & EPA6010B \\
\hline & & 28.5 & $\mathrm{mg} / \mathrm{kg}$ & 0.329 & EPA6010B \\
\hline & & 0.134 & $\mathrm{mg} / \mathrm{kg}$ & 0.00219 & EPA7471A \\
\hline \multirow[t]{2}{*}{$\mathrm{U}$} & V & 0.796 & $\mathrm{mg} / \mathrm{kg}$ & 0.03 & EPA6010B \\
\hline & & 0.941 & $\mathrm{mg} / \mathrm{kg}$ & 0.125 & EPA6010B \\
\hline \multirow[t]{2}{*}{$\mathrm{U}$} & & 0.231 & $\mathrm{mg} / \mathrm{kg}$ & 0.0287 & EPA6010B \\
\hline & & 17.9 & $\mathrm{mg} / \mathrm{kg}$ & 0.171 & EPA6010B \\
\hline
\end{tabular}

$\mathrm{U}$

0.16

U $\quad 0.0331$

U $\quad-0.019$

U $\quad 0.00842$

U $\quad 0.0146$

0.0693

0.00479

$-0.0297$

$-0.0125$

0.0397

0.0818

$-0.028$

$-0.521$

0.0159

$-0.00517$

0.0176

8.49

$\begin{array}{ll}\mathrm{U} & 0 \\ \mathrm{U} & -0.00158\end{array}$

2.94

0.00184

$-0.0244$

$-0.00601$

0.0294

0.553

$-0.00714$

$\begin{array}{ll}\mathrm{pCi} / \mathrm{g} & 0.189 \\ \mathrm{pCi} / \mathrm{g} & 0.0617 \\ \mathrm{pCi} / \mathrm{g} & 0.106 \\ \mathrm{pCi} / \mathrm{g} & 0.0468 \\ \mathrm{pCi} / \mathrm{g} & 0.201 \\ \mathrm{pCi} / \mathrm{g} & 0.0433 \\ \mathrm{pCi} / \mathrm{g} & 0.0443 \\ \mathrm{pCi} / \mathrm{g} & 0.027 \\ \mathrm{pCi} / \mathrm{g} & 0.0496 \\ \mathrm{pCi} / \mathrm{g} & 0.0463 \\ \mathrm{pCi} / \mathrm{g} & 0.105 \\ \mathrm{pCi} / \mathrm{g} & 0.155 \\ \mathrm{pCi} / \mathrm{g} & 0.0867 \\ \mathrm{pCi} / \mathrm{g} & 1.42 \\ \mathrm{pCi} / \mathrm{g} & 0.0658 \\ \mathrm{pCi} / \mathrm{g} & 0.0365 \\ \mathrm{pCi} / \mathrm{g} & 0.175 \\ \mathrm{pCi} / \mathrm{g} & 1.31 \\ \mathrm{pCi} / \mathrm{g} & 0.00987 \\ \mathrm{pCi} / \mathrm{g} & 0.0204 \\ \mathrm{pCi} / \mathrm{g} & 0.389 \\ \mathrm{pCi} / \mathrm{g} & 0.0391 \\ \mathrm{pCi} / \mathrm{g} & 0.0398 \\ \mathrm{pCi} / \mathrm{g} & 0.349 \\ \mathrm{pCi} / \mathrm{g} & 0.056 \\ \mathrm{pCi} / \mathrm{g} & 0.203 \\ \mathrm{pCi} / \mathrm{g} & 0.0529 \\ \mathrm{pCi} / \mathrm{g} & 0.146\end{array}$

EPIA-013B

EPIA-013B

EPIA-013B

EPIA-013B

EPIA-013B

EPIA-013B

EPIA-013B

EPIA-013B

EPIA-013B

EPIA-013B

EPIA-013B

EPIA-013B

EPIA-013B

EPIA-001B

EPIA-013B

EPIA-013B

EPIA-013B

EPIA-001B

EPIA-012B

EPIA-012B

EPIA-013B

EPIA-013B

EPIA-013B

EPIA-013B

EPIA-013B

EPA905

EPIA-013B

EPIA-002B 
SURVEY ID: SRF-06-02 (cont.)

Constituent

Radionuclides

Uranium-233/234

Uranium-235

Uranium-238

Yttrium-88

Zinc-65

Zirconium-95

Volatile Organic Compounds

$\begin{array}{lll}\text { Acetone } & \mathrm{J} & \mathrm{I} \\ \text { Benzene } & \mathrm{U} & 117 \\ \text { Bromodichloromethane } & \mathrm{U} & 5 \\ \text { Bromoform } & \mathrm{U} & 5 \\ \text { Bromomethane (Methyl bromide) } & \mathrm{U} & 5 \\ \text { Carbon disulfide } & \mathrm{U} & 5 \\ \text { Carbon tetrachloride } & \mathrm{U} & 25 \\ \text { Chlorobenzene } & \mathrm{U} & 5 \\ \text { Chloroethane } & \mathrm{U} & 5 \\ \text { Chloroethene (Vinyl chloride) } & \mathrm{U} & 5 \\ \text { Chloroform } & \mathrm{U} & 5 \\ \text { Chloromethane (Methyl chloride) } & \mathrm{U} & 5 \\ \text { Dibromochloromethane } & \mathrm{U} & 5 \\ \text { 1,1-Dichloroethane } & \mathrm{U} & 5 \\ \text { 1,2-Dichloroethane } & \mathrm{U} & 5 \\ \text { 1,1-Dichloroethylene } & \mathrm{U} & 5 \\ \text { 1,2-Dichloroethylene } & \mathrm{U} & 5 \\ \text { Dichloromethane (Methylene chloride) J } & \mathrm{I} & 50 \\ \text { 1,2-Dichloropropane } & \mathrm{U} & 19.8 \\ \text { cis-1,3-Dichloropropene } & \mathrm{U} & 5 \\ \text { trans-1,3-Dichloropropene } & \mathrm{U} & 5 \\ \text { Ethylbenzene } & \mathrm{U} & 5 \\ \text { 2-Hexanone } & \mathrm{U} & 5 \\ \text { Methyl ethyl ketone } & \mathrm{U} & 25 \\ \text { Methyl isobutyl ketone } & \mathrm{U} & 25 \\ \text { Styrene } & \mathrm{U} & 25 \\ \text { 1,1,2,2-Tetrachloroethane } & \mathrm{U} & 5 \\ \text { Tetrachloroethylene } & \mathrm{U} & 5 \\ \text { Toluene } & \mathrm{U} & 5 \\ \text { 1,1,1-Trichloroethane } & \mathrm{U} & 5 \\ \text { 1,1,2-Trichloroethane } & \mathrm{U} & 5 \\ \text { Trichloroethylene } & \mathrm{U} & 5 \\ \text { Vinyl acetate } & \mathrm{U} & 75 \\ \text { Xylenes } & & 25\end{array}$

Sample ID: 117038

FG SC E Result

Unit ssMDL

Method

EPIA-011B

$\mathrm{pCi} / \mathrm{g} \quad 0.0362 \quad$ EPIA-011B

$\mathrm{pCi} / \mathrm{g} \quad 0.0288 \quad$ EPIA-011B

$\mathrm{pCi} / \mathrm{g} \quad 0.0551 \quad$ EPIA-013B

$\begin{array}{llll}-0.0389 & \mathrm{pCi} / \mathrm{g} & 0.0855 & \text { EPIA-013B } \\ 0.0417 & \mathrm{pCi} / \mathrm{g} & 0.0947 & \text { EPIA-013B }\end{array}$

$\begin{array}{llll}-0.0389 & \mathrm{pCi} / \mathrm{g} & 0.0855 & \text { EPIA-013B } \\ 0.0417 & \mathrm{pCi} / \mathrm{g} & 0.0947 & \text { EPIA-013B }\end{array}$

\begin{tabular}{|c|c|c|}
\hline $\mathrm{lg} / \mathrm{kg}$ & 51.5 & EPA8260B \\
\hline $\mathrm{ug} / \mathrm{kg}$ & 2.5 & EPA8260B \\
\hline ug/ $/ \mathrm{kg}$ & 0.5 & EPA8260B \\
\hline $\mathrm{ug} / \mathrm{kg}$ & 1.35 & EPA8260B \\
\hline $\mathrm{ug} / \mathrm{kg}$ & 1.5 & EPA8260F \\
\hline $\mathrm{ug} / \mathrm{kg}$ & 1.5 & EPA8260H \\
\hline ug/kg & 2.5 & EPA8260B \\
\hline $\mathrm{ug} / \mathrm{kg}$ & 1.5 & EPA8260B \\
\hline $\mathrm{ug} / \mathrm{kg}$ & 1.5 & EPA82601 \\
\hline $\mathrm{ug} / \mathrm{kg}$ & 2 & EPA8260F \\
\hline ug/ $/ \mathrm{kg}$ & 0.5 & EPA8260E \\
\hline $\mathrm{ug} / \mathrm{kg}$ & 1 & EPA8260F \\
\hline $\mathrm{ug} / \mathrm{kg}$ & 1.05 & EPA8260F \\
\hline ug/ $/ \mathrm{kg}$ & 0.5 & EPA8260E \\
\hline $\mathrm{ug} / \mathrm{kg}$ & 1.15 & EPA8260F \\
\hline ug/kg & 1.5 & EPA8260F \\
\hline $\mathrm{ug} / \mathrm{kg}$ & 1.25 & EPA82601 \\
\hline ug/ $/ \mathrm{kg}$ & 7 & EPA8260B \\
\hline $\mathrm{ug} / \mathrm{kg}$ & 1.15 & EPA8260B \\
\hline $\mathrm{lg} / \mathrm{kg}$ & 1 & EPA8260F \\
\hline $\mathrm{ug} / \mathrm{kg}$ & 1.5 & EPA8260B \\
\hline ug/ $/ \mathrm{kg}$ & 1.5 & EPA8260B \\
\hline $\mathrm{ug} / \mathrm{kg}$ & 14 & EPA8260B \\
\hline $\mathrm{lg} / \mathrm{kg}$ & 16 & EPA82601 \\
\hline ug/kg & 14.6 & EPA8260E \\
\hline $\mathrm{ug} / \mathrm{kg}$ & 1.5 & EPA8260E \\
\hline $\mathrm{gg} / \mathrm{kg}$ & 3 & EPA82601 \\
\hline $\mathrm{ug} / \mathrm{kg}$ & 2 & EPA82601 \\
\hline $\mathrm{ug} / \mathrm{kg}$ & 4.5 & EPA8260F \\
\hline ug/kg & 0.5 & EPA8260B \\
\hline $\mathrm{lg} / \mathrm{kg}$ & 1.2 & EPA82601 \\
\hline$/ \mathrm{kg}$ & 1.35 & EPA8260 \\
\hline ;/kg & 10.5 & EPA82601 \\
\hline / Kg & 5 & EPA8260B \\
\hline
\end{tabular}


SURVEY ID: SRF-06-03

Sample type: Normal

Constituent

Metals (total recoverable)

Antimony
Arsenic
Barium
Cadmium
Calcium
Chromium
Copper
Lead
Magnesium
Manganese
Mercury
Nickel
Selenium
Silver
Zinc

Radionuclides

Actinium-228

Antimony-124

Antimony-125

Barium-133

Cerium-144

Cesium-134

Cesium-137

Cobalt-57

Cobalt-58

Cobalt-60

Europium-152

Europium-154

Europium-155

Gross alpha

Lead-212

Manganese-54

Neptunium-239

Nonvolatile beta

Plutonium-238

Plutonium-239/240

Potassium-40

Promethium-144

Promethium-146

Ruthenium-106

Sodium-22

Strontium-89/90

Tin-1 13

Tritium
Sample ID: 117039

Sample matrix: Fauna

Percent solids: NA

\begin{tabular}{|c|c|c|c|c|c|}
\hline \multirow[t]{3}{*}{ JU } & 4 & 0.186 & $\mathrm{mg} / \mathrm{kg}$ & 0.182 & EPA6010B \\
\hline & & 0.873 & $\mathrm{mg} / \mathrm{kg}$ & 0.217 & EPA6010B \\
\hline & & 2.02 & $\mathrm{mg} / \mathrm{kg}$ & 0.026 & EPA6010B \\
\hline \multirow[t]{8}{*}{ JU } & 4 & 0.0364 & $\mathrm{mg} / \mathrm{kg}$ & 0.018 & EPA6010B \\
\hline & & 13300 & $\mathrm{mg} / \mathrm{kg}$ & 3.319 & EPA6010B \\
\hline & & 0.303 & $\mathrm{mg} / \mathrm{kg}$ & 0.0347 & EPA6010B \\
\hline & & 0.444 & $\mathrm{mg} / \mathrm{kg}$ & 0.0629 & EPA6010B \\
\hline & & 0.443 & $\mathrm{mg} / \mathrm{kg}$ & 0.075 & EPA6010B \\
\hline & & 463 & $\mathrm{mg} / \mathrm{kg}$ & 0.242 & EPA6010B \\
\hline & & 5.89 & $\mathrm{mg} / \mathrm{kg}$ & 0.338 & EPA6010B \\
\hline & & 0.456 & $\mathrm{mg} / \mathrm{kg}$ & 0.0021 & EPA7471A \\
\hline \multirow[t]{2}{*}{ JU } & & 0.233 & $\mathrm{mg} / \mathrm{kg}$ & 0.03 & EPA6010B \\
\hline & & 0.962 & $\mathrm{mg} / \mathrm{kg}$ & 0.129 & EPA6010B \\
\hline \multirow[t]{2}{*}{$J$} & & 0.0421 & $\mathrm{mg} / \mathrm{kg}$ & 0.0295 & EPA6010B \\
\hline & & 11.5 & $\mathrm{mg} / \mathrm{kg}$ & 0.176 & EPA6010B \\
\hline
\end{tabular}

U

U

U

U

U

U

U

U

U

U

U

U

U

$\mathrm{U}$

U

$\mathrm{U}$

U

$\mathrm{U}$

$\mathrm{U}$

U

U

$\mathrm{U}$

U $\quad \begin{array}{ll}0.31 \\ \text { U }\end{array}$

J I $\quad 0.13$
0.226

0.00128

$-0.0504$

$-0.0279$

0.0102

$-0.0285$

1.25

$-0.00711$

$-0.0105$

0.0023

0.0213

0.00836

$-0.0854$

0.0231

0.0565

0.0255

0.046

6.71

0

2.45

0.0261

$-0.0424$

$-0.371$

0.00276
Unit ssMDL

Method

PA6010B

EPA6010B

EPA6010B

EPA6010B

EPA6010B

EPA747

EPA6010B

EPA6010B

EPA6010B

$\begin{array}{lll}\mathrm{pCi} / \mathrm{g} & 0.287 & \text { EPIA-013B } \\ \mathrm{pCi} / \mathrm{g} & 0.0913 & \text { EPIA-013B }\end{array}$

$\mathrm{pCi} / \mathrm{g} \quad 0.169 \quad$ EPIA-013B

$\mathrm{pCi} / \mathrm{g} \quad 0.0755 \quad$ EPIA-013B

$\mathrm{pCi} / \mathrm{g} \quad 0.293 \quad$ EPIA-013B

$\mathrm{pCi} / \mathrm{g} \quad 0.0549 \quad$ EPIA-013B

$\mathrm{pCi} / \mathrm{g} \quad 0.0637 \quad$ EPIA-013B

$\mathrm{pCi} / \mathrm{g} \quad 0.037 \quad$ EPIA-013B

$\mathrm{pCi} / \mathrm{g} \quad 0.069 \quad$ EPIA-013B

$\mathrm{pCi} / \mathrm{g} \quad 0.067 \quad$ EPIA-013B

$\mathrm{pCi} / \mathrm{g} \quad 0.165 \quad$ EPIA-013B

$\mathrm{pCi} / \mathrm{g} \quad 0.176 \quad$ EPIA-013B

$\mathrm{pCi} / \mathrm{g} \quad 0.138 \quad$ EPIA-013B

$\mathrm{pCi} / \mathrm{g} \quad 0.558 \quad$ EPIA-001B

$\mathrm{pCi} / \mathrm{g} \quad 0.0904 \quad$ EPIA-013B

$\mathrm{pCi} / \mathrm{g} \quad 0.0711 \quad$ EPIA-013B

$\mathrm{pCi} / \mathrm{g} \quad 0.257 \quad$ EPIA-013B

$\mathrm{pCi} / \mathrm{g} \quad 0.857 \quad$ EPIA-001B

$\mathrm{pCi} / \mathrm{g} \quad 0.00704 \quad$ EPIA-012B

$\mathrm{pCi} / \mathrm{g} \quad 0.00703 \quad$ EPIA-012B

$\mathrm{pCi} / \mathrm{g} \quad 0.605 \quad$ EPIA-013B

$\mathrm{pCi} / \mathrm{g} \quad 0.0582 \quad$ EPIA-013B

$\mathrm{pCi} / \mathrm{g} \quad 0.0751 \quad$ EPIA-013B

$\mathrm{pCi} / \mathrm{g} \quad 0.492 \quad$ EPIA-013B

$\mathrm{pCi} / \mathrm{g} \quad 0.0633 \quad$ EPIA-013B

$\mathrm{pCi} / \mathrm{g} \quad 0.114 \quad$ EPA905

$\mathrm{pCi} / \mathrm{g} \quad 0.0903 \quad$ EPIA-013B

$\mathrm{pCi} / \mathrm{g} \quad 0.0974 \quad$ EPIA-002B
EPA6010B 
SURVEY ID: SRF-06-03 (cont.)

Constituent

Radionuclides

Uranium-233/234

Uranium-235

Uranium-238

Yttrium-88

Zinc-65

Zirconium-95

Volatile Organic Compounds

\begin{tabular}{|c|c|c|c|c|}
\hline Acetone & $\mathrm{U}$ & & & 125 \\
\hline Benzene & $\mathrm{U}$ & & & 5 \\
\hline Bromodichloromethane & $\mathrm{U}$ & & & 5 \\
\hline Bromoform & $\mathrm{U}$ & & & 5 \\
\hline Bromomethane (Methyl bromide) & $\mathrm{U}$ & & & 5 \\
\hline Carbon disulfide & $\mathrm{U}$ & & & 25 \\
\hline Carbon tetrachloride & $\mathrm{U}$ & & & 5 \\
\hline Chlorobenzene & $\mathrm{U}$ & & & 5 \\
\hline Chloroethane & $\mathrm{U}$ & & & 5 \\
\hline Chloroethene (Vinyl chloride) & $\mathrm{U}$ & & & 5 \\
\hline Chloroform & $\mathrm{U}$ & & & 5 \\
\hline Chloromethane (Methyl chloride) & $\mathrm{U}$ & & & 5 \\
\hline Dibromochloromethane & $\mathrm{U}$ & & & 5 \\
\hline 1,1-Dichloroethane & $\mathrm{U}$ & & & 5 \\
\hline 1,2-Dichloroethane & $\mathrm{U}$ & & & 5 \\
\hline 1,1-Dichloroethylene & $\mathrm{U}$ & & & 5 \\
\hline 1,2-Dichloroethylene & $\mathrm{U}$ & & & 50 \\
\hline Dichloromethane (Methylene chloride) & $\mathrm{J}$ & $\mathrm{K}$ & $\mathrm{O}$ & 54.5 \\
\hline 1,2-Dichloropropane & $\mathrm{U}$ & & & 5 \\
\hline cis-1,3-Dichloropropene & $\mathrm{U}$ & & & 5 \\
\hline trans-1,3-Dichloropropene & $\mathrm{U}$ & & & 5 \\
\hline Ethylbenzene & $\mathrm{U}$ & & & 5 \\
\hline 2-Hexanone & $\mathrm{U}$ & & & 25 \\
\hline Methyl ethyl ketone & $\mathrm{U}$ & & & 25 \\
\hline Methyl isobutyl ketone & $\mathrm{U}$ & & & 25 \\
\hline Styrene & $\mathrm{U}$ & & & 5 \\
\hline $1,1,2,2$-Tetrachloroethane & $\mathrm{U}$ & & & 5 \\
\hline Tetrachloroethylene & $\mathrm{U}$ & & & 5 \\
\hline Toluene & $\mathrm{U}$ & & & 5 \\
\hline 1,1,1-Trichloroethane & $\mathrm{U}$ & & & 5 \\
\hline $1,1,2$-Trichloroethane & $\mathrm{U}$ & & & 5 \\
\hline Trichloroethylene & $\mathrm{U}$ & & & 5 \\
\hline Vinyl acetate & $\mathrm{U}$ & & & 75 \\
\hline Xylenes & $\mathrm{J}$ & IK & $\mathrm{O}$ & 5.67 \\
\hline
\end{tabular}

0.00849
0.00398
0.00198
-0.0151
-0.0666
0.0122

$\mathrm{pCi} / \mathrm{g} \quad 0.0138$

$\mathrm{pCi} / \mathrm{g} \quad 0.00597$

$\mathrm{pCi} / \mathrm{g} \quad 0.00595$

$\mathrm{pCi} / \mathrm{g} \quad 0.0678$

$\mathrm{pCi} / \mathrm{g} \quad 0.12$

$\mathrm{pCi} / \mathrm{g} \quad 0.136$

$\begin{array}{ll}\mu g / \mathrm{kg} & 51.5 \\ \mu g / \mathrm{kg} & 2.5 \\ \mu g / \mathrm{kg} & 0.5 \\ \mu g / \mathrm{kg} & 1.35 \\ \mu g / \mathrm{kg} & 1.5 \\ \mu g / \mathrm{kg} & 1.5 \\ \mu g / \mathrm{kg} & 2.5 \\ \mu g / \mathrm{kg} & 1.5 \\ \mu g / \mathrm{kg} & 1.5 \\ \mu g / \mathrm{kg} & 2 \\ \mu g / \mathrm{kg} & 0.5 \\ \mu g / \mathrm{kg} & 1 \\ \mu g / \mathrm{kg} & 1.05 \\ \mu g / \mathrm{kg} & 0.5 \\ \mu g / \mathrm{kg} & 1.15 \\ \mu g / \mathrm{kg} & 1.5 \\ \mu g / \mathrm{kg} & 1.25 \\ \mu g / \mathrm{kg} & 7 \\ \mu g / \mathrm{kg} & 1.15 \\ \mu g / \mathrm{kg} & 1 \\ \mu g / \mathrm{kg} & 1.5 \\ \mu g / \mathrm{kg} & 1.5 \\ \mu g / \mathrm{kg} & 14 \\ \mu g / \mathrm{kg} & 16 \\ \mu g / \mathrm{kg} & 14.6 \\ \mu g / \mathrm{kg} & 1.5 \\ \mu g / \mathrm{kg} & 3 \\ \mu g / \mathrm{kg} & 2 \\ \mu g / \mathrm{kg} & 4.5 \\ \mu g / \mathrm{kg} & 0.5 \\ \mu g / \mathrm{kg} & 1.2 \\ \mu g / \mathrm{kg} & 1.35 \\ \mu g / \mathrm{kg} & 10.5 \\ \mu g / \mathrm{kg} & 5 \\ & 1.5\end{array}$

EPIA-011B EPIA-011B EPIA-011B EPIA-013B EPIA-013B EPIA-013B

EPA8260B EPA8260B EPA8260B EPA8260B

EPA8260B

EPA8260B

EPA8260B

EPA8260B

EPA8260B

EPA8260B

EPA8260B

EPA8260B

EPA8260B

EPA8260B

EPA8260B

EPA8260B

EPA8260B

EPA8260B

EPA8260B

EPA8260B

EPA8260B

EPA8260B

EPA8260B

EPA8260B

EPA8260B

EPA8260B

EPA8260B

EPA8260B

EPA8260B

EPA8260B

EPA8260B

EPA8260B

EPA8260B

EPA8260B 
SURVEY ID: SRF-06-03-A

Sample type: Field Duplicate

Constituent

Metals (total recoverable)

Antimony
Arsenic
Barium
Cadmium
Calcium
Chromium
Copper
Lead
Magnesium
Manganese
Mercury
Nickel
Selenium
Silver
Zinc

Radionuclides

Actinium-228

Antimony-124

Antimony-125

Barium-133

Cerium-144

Cesium-134

Cesium-137

Cobalt-57

Cobalt-58

Cobalt-60

Europium-152

Europium-154

Europium-155

Gross alpha

Lead-212

Manganese-54

Neptunium-239

Nonvolatile beta

Plutonium-238

Plutonium-239/240

Potassium-40

Promethium-144

Promethium-146

Ruthenium-106

Sodium-22

Strontium-89/90

Tin-1 13

Tritium
Sample ID: 117040

Sample matrix: Fauna

Percent solids: NA

\begin{tabular}{|c|c|c|c|c|c|}
\hline \multirow[t]{3}{*}{$\mathrm{U}$} & & 0.495 & $\mathrm{mg} / \mathrm{kg}$ & 0.189 & EPA6010B \\
\hline & & 0.647 & $\mathrm{mg} / \mathrm{kg}$ & 0.226 & EPA6010B \\
\hline & & 3.06 & $\mathrm{mg} / \mathrm{kg}$ & 0.027 & EPA6010B \\
\hline \multirow[t]{8}{*}{ JU } & 4 & 0.0248 & $\mathrm{mg} / \mathrm{kg}$ & 0.019 & EPA6010B \\
\hline & & 13500 & $\mathrm{mg} / \mathrm{kg}$ & 3.451 & EPA6010B \\
\hline & & 0.313 & $\mathrm{mg} / \mathrm{kg}$ & 0.0361 & EPA6010B \\
\hline & & 0.6 & $\mathrm{mg} / \mathrm{kg}$ & 0.0653 & EPA6010B \\
\hline & & 0.498 & $\mathrm{mg} / \mathrm{kg}$ & 0.078 & EPA6010B \\
\hline & & 550 & $\mathrm{mg} / \mathrm{kg}$ & 0.251 & EPA6010B \\
\hline & & 6.7 & $\mathrm{mg} / \mathrm{kg}$ & 0.351 & EPA6010B \\
\hline & & 0.474 & $\mathrm{mg} / \mathrm{kg}$ & 0.00194 & EPA7471A \\
\hline \multirow[t]{2}{*}{$\mathrm{U}$} & $\mathrm{V}$ & 0.284 & $\mathrm{mg} / \mathrm{kg}$ & 0.032 & EPA6010B \\
\hline & & 1.04 & $\mathrm{mg} / \mathrm{kg}$ & 0.134 & EPA6010F \\
\hline \multirow[t]{2}{*}{$\mathrm{U}$} & & 0.248 & $\mathrm{mg} / \mathrm{kg}$ & 0.0307 & EPA6010B \\
\hline & & 18 & $\mathrm{mg} / \mathrm{kg}$ & 0.184 & EPA60101 \\
\hline
\end{tabular}

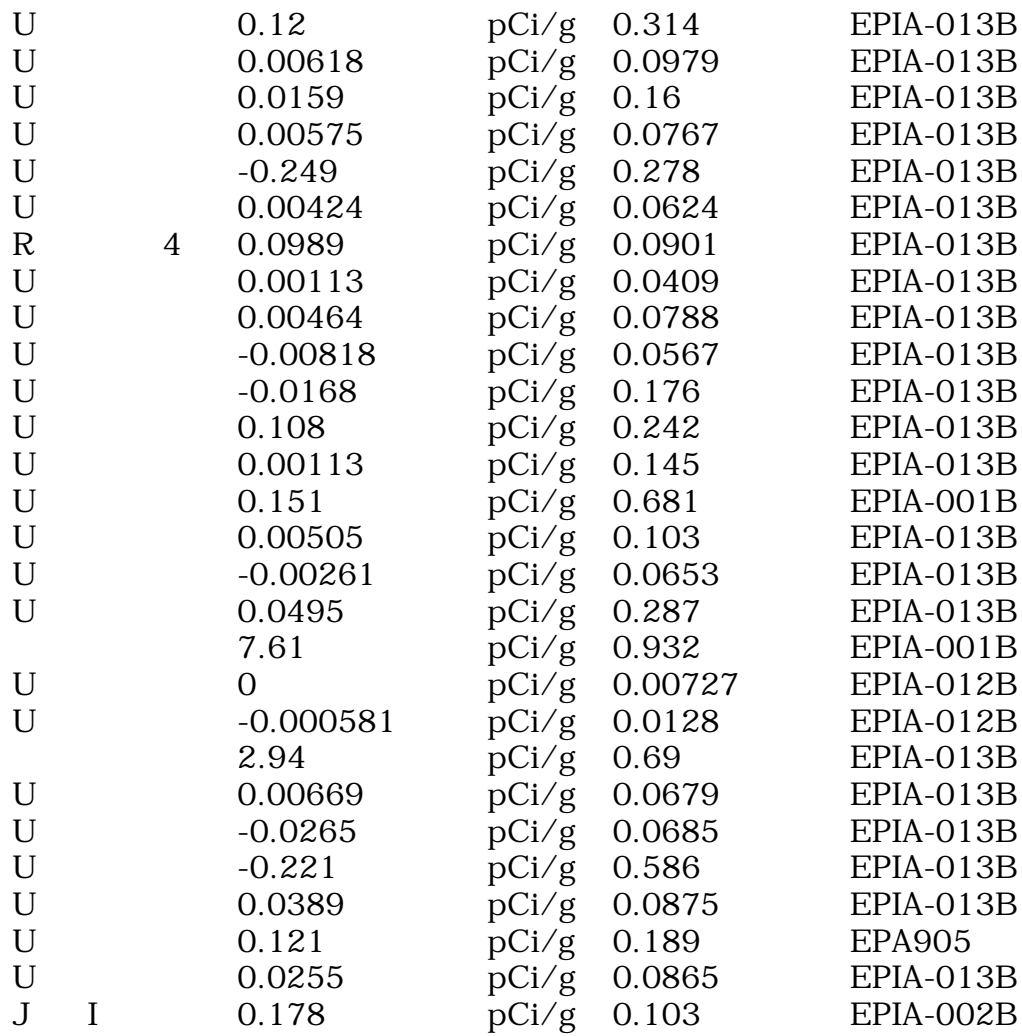


SURVEY ID: SRF-06-03-A (cont.)

Constituent

Radionuclides

Uranium-233/234

Uranium-235

Uranium-238

Yttrium-88

Zinc-65

Zirconium-95

Volatile Organic Compounds

Acetone
Benzene
Bromodichloromethane
Bromoform
Bromomethane (Methyl bromide)
Carbon disulfide
Carbon tetrachloride
Chlorobenzene

Sample ID: 117040

FG SC E Result

Unit ssMDL

Method

EPIA-011B

pCi/g $0.00691 \quad$ EPIA-011B

$\mathrm{pCi} / \mathrm{g} \quad 0.0121 \quad$ EPIA-011B

$\mathrm{pCi} / \mathrm{g} \quad 0.0956 \quad$ EPIA-013B

$\mathrm{pCi} / \mathrm{g} \quad 0.168 \quad$ EPIA-013B

$\mathrm{pCi} / \mathrm{g} \quad 0.147 \quad$ EPIA-013B

$\begin{array}{llll}0.00294 & \mathrm{pCi} / \mathrm{g} & 0.016 & \text { EPIA-011B } \\ 0.0023 & \mathrm{pCi} / \mathrm{g} & 0.00691 & \text { EPIA-011B } \\ 0.00404 & \mathrm{pCi} / \mathrm{g} & 0.0121 & \text { EPIA-011B } \\ -0.00322 & \mathrm{pCi} / \mathrm{g} & 0.0956 & \text { EPIA-013B } \\ 0.00423 & \mathrm{pCi} / \mathrm{g} & 0.168 & \text { EPIA-013B } \\ 0.0199 & \mathrm{pCi} / \mathrm{g} & 0.147 & \text { EPIA-013B }\end{array}$

125

5

5

5

5

25

5

5

5

5

5

5

5

5

5

5

50

282

5

5

5

6.42

25

25

25

5

5

5

18.3

5

5

5

75

32.4

$\begin{array}{ll}\mu g / \mathrm{kg} & 51.5 \\ \mu \mathrm{g} / \mathrm{kg} & 2.5 \\ \mu \mathrm{g} / \mathrm{kg} & 0.5 \\ \mu \mathrm{g} / \mathrm{kg} & 1.35 \\ \mu \mathrm{g} / \mathrm{kg} & 1.5 \\ \mu \mathrm{g} / \mathrm{kg} & 1.5 \\ \mu \mathrm{g} / \mathrm{kg} & 2.5 \\ \mu \mathrm{g} / \mathrm{kg} & 1.5 \\ \mu \mathrm{g} / \mathrm{kg} & 1.5 \\ \mu \mathrm{g} / \mathrm{kg} & 2 \\ \mu \mathrm{g} / \mathrm{kg} & 0.5 \\ \mu \mathrm{g} / \mathrm{kg} & 1 \\ \mu \mathrm{g} / \mathrm{kg} & 1.05 \\ \mu \mathrm{g} / \mathrm{kg} & 0.5 \\ \mu \mathrm{g} / \mathrm{kg} & 1.15 \\ \mu \mathrm{g} / \mathrm{kg} & 1.5 \\ \mu \mathrm{g} / \mathrm{kg} & 1.25 \\ \mu \mathrm{g} / \mathrm{kg} & 7 \\ \mu \mathrm{g} / \mathrm{kg} & 1.15 \\ \mu \mathrm{g} / \mathrm{kg} & 1 \\ \mu \mathrm{g} / \mathrm{kg} & 1.5 \\ \mu \mathrm{g} / \mathrm{kg} & 1.5 \\ \mu \mathrm{g} / \mathrm{kg} & 14 \\ \mu \mathrm{g} / \mathrm{kg} & 16 \\ \mu \mathrm{g} / \mathrm{kg} & 14.6 \\ \mu \mathrm{g} / \mathrm{kg} & 1.5 \\ \mu \mathrm{g} / \mathrm{kg} & 3 \\ \mu \mathrm{g} / \mathrm{kg} & 2 \\ \mu \mathrm{g} / \mathrm{kg} & 4.5 \\ \mu \mathrm{g} / \mathrm{kg} & 0.5 \\ \mu \mathrm{g} / \mathrm{kg} & 1.2 \\ \mu \mathrm{g} / \mathrm{kg} & 1.35 \\ \mu \mathrm{g} / \mathrm{kg} & 10.5 \\ \mu \mathrm{g} / \mathrm{kg} & 5 \\ & \end{array}$

EPA8260B

EPA8260B

EPA8260B

EPA8260B

EPA8260B

EPA8260B

EPA8260B

EPA8260B

EPA8260B

EPA8260B

EPA8260B

EPA8260B

EPA8260B

EPA8260B

EPA8260B

EPA8260B

EPA8260B

EPA8260B

EPA8260B

EPA8260B

EPA8260B

EPA8260B

EPA8260B

EPA8260B

EPA8260B

EPA8260B

EPA8260B

EPA8260B

EPA8260B

EPA8260B

EPA8260B

EPA8260B

EPA8260B

EPA8260B 
SURVEY ID: SRF-07-01

Sample type: Normal

Constituent

Metals (total recoverable)

Antimony
Arsenic
Barium
Cadmium
Calcium
Chromium
Copper
Lead
Magnesium
Manganese
Mercury
Nickel
Selenium
Silver
Zinc

Radionuclides

Actinium-228

Antimony-124

Antimony-125

Barium-133

Cerium-144

Cesium-134

Cesium-137

Cobalt-57

Cobalt-58

Cobalt-60

Europium-152

Europium-154

Europium-155

Gross alpha

Lead-212

Manganese-54

Neptunium-239

Nonvolatile beta

Plutonium-238

Plutonium-239/240

Potassium-40

Promethium-144

Promethium-146

Ruthenium-106

Sodium-22

Strontium-89/90

Tin-1 13

Tritium
Sample ID: 117041

Sample matrix: Fauna

Percent solids: NA

\begin{tabular}{|c|c|c|c|c|c|}
\hline \multirow[t]{3}{*}{ JU } & 4 & 0.233 & $\mathrm{mg} / \mathrm{kg}$ & 0.184 & EPA6010B \\
\hline & & 0.336 & $\mathrm{mg} / \mathrm{kg}$ & 0.219 & EPA6010B \\
\hline & & 1.8 & $\mathrm{mg} / \mathrm{kg}$ & 0.026 & EPA6010B \\
\hline \multirow[t]{2}{*}{ JU } & 4 & 0.0205 & $\mathrm{mg} / \mathrm{kg}$ & 0.018 & EPA6010B \\
\hline & & 9960 & $\mathrm{mg} / \mathrm{kg}$ & 3.354 & EPA6010B \\
\hline \multirow[t]{2}{*}{$\mathrm{J}$} & & 0.184 & $\mathrm{mg} / \mathrm{kg}$ & 0.035 & EPA6010B \\
\hline & & 0.377 & $\mathrm{mg} / \mathrm{kg}$ & 0.0635 & EPA6010B \\
\hline \multirow[t]{4}{*}{$J$} & & 0.0933 & $\mathrm{mg} / \mathrm{kg}$ & 0.076 & EPA6010B \\
\hline & & 382 & $\mathrm{mg} / \mathrm{kg}$ & 0.244 & EPA6010B \\
\hline & & 18.6 & $\mathrm{mg} / \mathrm{kg}$ & 0.342 & EPA6010B \\
\hline & & 0.194 & $\mathrm{mg} / \mathrm{kg}$ & 0.00209 & EPA7471A \\
\hline \multirow[t]{2}{*}{$J$} & & 0.115 & $\mathrm{mg} / \mathrm{kg}$ & 0.031 & EPA6010B \\
\hline & & 0.874 & $\mathrm{mg} / \mathrm{kg}$ & 0.13 & EPA6010B \\
\hline \multirow[t]{2}{*}{$\mathrm{J}$} & & 0.0896 & $\mathrm{mg} / \mathrm{kg}$ & 0.0298 & EPA6010B \\
\hline & & 20.8 & $\mathrm{mg} / \mathrm{kg}$ & 0.178 & EPA6010B \\
\hline
\end{tabular}

U

U

U

U

U

U

U

U

U

U

$\mathrm{U}$

$\mathrm{U}$

U

$\mathrm{U}$

$\mathrm{R}$

U

U

U

U

U

U

U

U

U

U

U

Uni

SSMDL

Method

G SC E Result Result

$\mathrm{pCi} / \mathrm{g} \quad 0.318$

$\mathrm{pCi} / \mathrm{g} \quad 0.0912$

$\mathrm{pCi} / \mathrm{g} \quad 0.171$

$\mathrm{pCi} / \mathrm{g} \quad 0.0669$

$\mathrm{pCi} / \mathrm{g} \quad 0.244$

$\mathrm{pCi} / \mathrm{g} \quad 0.065$

$\mathrm{pCi} / \mathrm{g} \quad 0.0792$

$\mathrm{pCi} / \mathrm{g} \quad 0.032$

$\mathrm{pCi} / \mathrm{g} \quad 0.0682$

$\mathrm{pCi} / \mathrm{g} \quad 0.077$

$\mathrm{pCi} / \mathrm{g} \quad 0.142$

$\mathrm{pCi} / \mathrm{g} \quad 0.199$

$\mathrm{pCi} / \mathrm{g} \quad 0.119$

$\mathrm{pCi} / \mathrm{g} \quad 0.556$

$\mathrm{pCi} / \mathrm{g} \quad 0.106$

$\mathrm{pCi} / \mathrm{g} \quad 0.0702$

$\mathrm{pCi} / \mathrm{g} \quad 0.213$

$\mathrm{pCi} / \mathrm{g} \quad 0.897$

$\mathrm{pCi} / \mathrm{g} \quad 0.0224$

$\mathrm{pCi} / \mathrm{g} \quad 0.0184$

$\mathrm{pCi} / \mathrm{g} \quad 0.743$

$\mathrm{pCi} / \mathrm{g} \quad 0.0707$

$\mathrm{pCi} / \mathrm{g} \quad 0.0764$

$\mathrm{pCi} / \mathrm{g} \quad 0.583$

$\mathrm{pCi} / \mathrm{g} \quad 0.0719$

$\mathrm{pCi} / \mathrm{g} \quad 0.537$

$\mathrm{pCi} / \mathrm{g} \quad 0.0777$

$\mathrm{pCi} / \mathrm{g} \quad 0.17$
EPIA-013B

EPIA-013B

EPIA-013B

EPIA-013B

EPIA-013B

EPIA-013B

EPIA-013B

EPIA-013B

EPIA-013B

EPIA-013B

EPIA-013B

EPIA-013B

EPIA-013B

EPIA-001B

EPIA-013B

EPIA-013B

EPIA-013B

EPIA-001B

EPIA-012B

EPIA-012B

EPIA-013B

EPIA-013B

EPIA-013B

EPIA-013B

EPIA-013B

EPA905

EPIA-013B

EPIA-002B 
SURVEY ID: SRF-07-01 (cont.)

Constituent

Radionuclides

Uranium-233/234

Uranium-235

Uranium-238

Yttrium-88

Zinc-65

Zirconium-95

Volatile Organic Compounds

\begin{tabular}{|c|c|c|c|c|}
\hline Acetone & $\mathrm{J}$ & $\mathrm{K}$ & $\mathrm{O}$ & 423 \\
\hline Benzene & $\mathrm{J}$ & IK & $\mathrm{O}$ & 2.6 \\
\hline Bromodichloromethane & $\mathrm{U}$ & & & 5 \\
\hline Bromoform & $\mathrm{U}$ & & & 5 \\
\hline Bromomethane (Methyl bromide) & $\mathrm{U}$ & & & 5 \\
\hline Carbon disulfide & $\mathrm{U}$ & & & 25 \\
\hline Carbon tetrachloride & $\mathrm{U}$ & & & 5 \\
\hline Chlorobenzene & $\mathrm{U}$ & & & 5 \\
\hline Chloroethane & $\mathrm{U}$ & & & 5 \\
\hline Chloroethene (Vinyl chloride) & $\mathrm{U}$ & & & 5 \\
\hline Chloroform & $\mathrm{U}$ & & & 5 \\
\hline Chloromethane (Methyl chloride) & $\mathrm{U}$ & & & 5 \\
\hline Dibromochloromethane & $\mathrm{U}$ & & & 5 \\
\hline 1,1-Dichloroethane & $\mathrm{U}$ & & & 5 \\
\hline 1,2-Dichloroethane & $\mathrm{U}$ & & & 5 \\
\hline 1,1-Dichloroethylene & $\mathrm{U}$ & & & 5 \\
\hline 1,2-Dichloroethylene & $\mathrm{U}$ & & & 50 \\
\hline Dichloromethane (Methylene chloride) & $\mathrm{J}$ & $\mathrm{K}$ & $\mathrm{O}$ & 75.3 \\
\hline 1,2-Dichloropropane & $\mathrm{U}$ & & & 5 \\
\hline cis-1,3-Dichloropropene & $\mathrm{U}$ & & & 5 \\
\hline trans-1,3-Dichloropropene & $\mathrm{U}$ & & & 5 \\
\hline Ethylbenzene & $\mathrm{U}$ & & & 5 \\
\hline 2-Hexanone & $\mathrm{U}$ & & & 25 \\
\hline Methyl ethyl ketone & $\mathrm{U}$ & & & 25 \\
\hline Methyl isobutyl ketone & $\mathrm{U}$ & & & 25 \\
\hline Styrene & $\mathrm{U}$ & & & 5 \\
\hline 1,1,2,2-Tetrachloroethane & $\mathrm{U}$ & & & 5 \\
\hline Tetrachloroethylene & $\mathrm{U}$ & & & 5 \\
\hline Toluene & $\mathrm{U}$ & & & 5 \\
\hline 1,1,1-Trichloroethane & $\mathrm{U}$ & & & 5 \\
\hline $1,1,2$-Trichloroethane & $\mathrm{U}$ & & & 5 \\
\hline Trichloroethylene & $\mathrm{U}$ & & & 5 \\
\hline Vinyl acetate & $\mathrm{U}$ & & & 75 \\
\hline Xylenes & $\mathrm{J}$ & IK & $\mathrm{O}$ & 5.55 \\
\hline
\end{tabular}

\author{
0.00216 \\ 0.00196 \\ 0.000821 \\ $-0.0196$ \\ $-0.00653$ \\ 0.054
}

Unit ssMDL

Method

$\begin{array}{lll}\mathrm{pCi} / \mathrm{g} & 0.0252 & \text { EPIA-011B } \\ \mathrm{pCi} / \mathrm{g} & 0.0136 & \text { EPIA-011B } \\ \mathrm{pCi} / \mathrm{g} & 0.0232 & \text { EPIA-011B } \\ \mathrm{pCi} / \mathrm{g} & 0.098 & \text { EPIA-013B } \\ \mathrm{pCi} / \mathrm{g} & 0.168 & \text { EPIA-013B } \\ \mathrm{pCi} / \mathrm{g} & 0.184 & \text { EPIA-013B }\end{array}$

$\mu \mathrm{g} / \mathrm{kg} \quad 51.5$

EPA8260B

$\mu g / \mathrm{kg} \quad 2.5$

$\mu g / \mathrm{kg} \quad 0.5$

$\mu g / k g \quad 1.35$

$\mu \mathrm{g} / \mathrm{kg} \quad 1.5$

$\mu \mathrm{g} / \mathrm{kg} \quad 1.5$

$\mu \mathrm{g} / \mathrm{kg} \quad 2.5$

$\mu g / \mathrm{kg} \quad 1.5$

$\mu \mathrm{g} / \mathrm{kg} \quad 1.5$

$\mu \mathrm{g} / \mathrm{kg} \quad 2$

$\mu g / \mathrm{kg} \quad 0.5$

$\mu \mathrm{g} / \mathrm{kg} \quad 1$

$\mu g / \mathrm{kg} \quad 1.05$

$\mu g / \mathrm{kg} \quad 0.5$

$\mu g / \mathrm{kg} \quad 1.15$

$\mu \mathrm{g} / \mathrm{kg} \quad 1.5$

$\mu g / \mathrm{kg} \quad 1.25$

$\mu \mathrm{g} / \mathrm{kg} \quad 7$

$\mu \mathrm{g} / \mathrm{kg} \quad 1.15$

$\mu \mathrm{g} / \mathrm{kg} \quad 1$

$\mu g / \mathrm{kg} \quad 1.5$

$\mu \mathrm{g} / \mathrm{kg} \quad 1.5$

$\mu g / \mathrm{kg} \quad 14$

$\mu \mathrm{g} / \mathrm{kg} \quad 16$

$\mu g / \mathrm{kg} \quad 14.6$

$\mu g / \mathrm{kg} \quad 1.5$

$\mu \mathrm{g} / \mathrm{kg} \quad 3$

$\mu \mathrm{g} / \mathrm{kg} \quad 2$

$\mu \mathrm{g} / \mathrm{kg} \quad 4.5$

$\mu g / k g \quad 0.5$

$\mu \mathrm{g} / \mathrm{kg} \quad 1.2$

$\mu \mathrm{g} / \mathrm{kg} \quad 1.35$

$\mu g / k g \quad 10.5$

$\mu \mathrm{g} / \mathrm{kg} \quad 5$
EPA8260B

EPA8260B

EPA8260B

EPA8260B

EPA8260B

EPA8260B

EPA8260B

EPA8260B

EPA8260B

EPA8260B

EPA8260B

EPA8260B

EPA8260B

EPA8260B

EPA8260B

EPA8260B

EPA8260B

EPA8260B

EPA8260B

EPA8260B

EPA8260B

EPA8260B

EPA8260B

EPA8260B

EPA8260B

EPA8260B

EPA8260B

EPA8260B

EPA8260B

EPA8260B

EPA8260B
EPA8260B

EPA8260B 
SURVEY ID: SRF-07-02

Sample type: Normal

Constituent

Metals (total recoverable)

Antimony
Arsenic
Barium
Cadmium
Calcium
Chromium
Copper
Lead
Magnesium
Manganese
Mercury
Nickel
Selenium
Silver
Zinc

Radionuclides

Actinium-228

Antimony-124

Antimony-125

Barium-133

Cerium-144

Cesium-134

Cesium-137

Cobalt-57

Cobalt-58

Cobalt-60

Europium-152

Europium-154

Europium-155

Gross alpha

Lead-212

Manganese-54

Neptunium-239

Nonvolatile beta

Plutonium-238

Plutonium-239/240

Potassium-40

Promethium-144

Promethium-146

Ruthenium-106

Sodium-22

Strontium-89/90

Tin-1 13

Tritium
Sample ID: 117042

Sample matrix: Fauna

Percent solids: NA

\begin{tabular}{|c|c|c|c|c|c|}
\hline \multirow[t]{3}{*}{ JU } & 4 & 0.177 & $\mathrm{mg} / \mathrm{kg}$ & 0.0788 & EPA6010B \\
\hline & & 0.354 & $\mathrm{mg} / \mathrm{kg}$ & 0.219 & EPA6010B \\
\hline & & 2.69 & $\mathrm{mg} / \mathrm{kg}$ & 0.026 & EPA6010B \\
\hline \multirow[t]{4}{*}{$\mathrm{U}$} & & 0.24 & $\mathrm{mg} / \mathrm{kg}$ & 0.018 & EPA6010B \\
\hline & & 18100 & $\mathrm{mg} / \mathrm{kg}$ & 3.354 & EPA6010B \\
\hline & & 0.305 & $\mathrm{mg} / \mathrm{kg}$ & 0.035 & EPA6010B \\
\hline & & 0.414 & $\mathrm{mg} / \mathrm{kg}$ & 0.0635 & EPA6010B \\
\hline \multirow[t]{4}{*}{$\mathrm{J}$} & I & 0.154 & $\mathrm{mg} / \mathrm{kg}$ & 0.076 & EPA6010B \\
\hline & & 437 & $\mathrm{mg} / \mathrm{kg}$ & 0.244 & EPA6010B \\
\hline & & 10.1 & $\mathrm{mg} / \mathrm{kg}$ & 0.342 & EPA6010B \\
\hline & & 0.204 & $\mathrm{mg} / \mathrm{kg}$ & 0.0022 & EPA7471A \\
\hline \multirow[t]{2}{*}{$\mathrm{J}$} & I & 0.115 & $\mathrm{mg} / \mathrm{kg}$ & 0.031 & EPA6010B \\
\hline & & 0.845 & $\mathrm{mg} / \mathrm{kg}$ & 0.13 & EPA6010B \\
\hline \multirow[t]{2}{*}{$\mathrm{J}$} & I & 0.082 & $\mathrm{mg} / \mathrm{kg}$ & 0.0298 & EPA6010B \\
\hline & & 24.6 & $\mathrm{mg} / \mathrm{kg}$ & 0.178 & EPA6010B \\
\hline
\end{tabular}

\begin{tabular}{|c|c|c|c|c|}
\hline $\mathrm{U}$ & 0.107 & $\mathrm{pCi} / \mathrm{g}$ & 0.341 & EPIA-013B \\
\hline $\mathrm{U}$ & 0.0325 & $\mathrm{pCi} / \mathrm{g}$ & 0.107 & EPIA-013B \\
\hline $\mathrm{U}$ & -0.0796 & $\mathrm{pCi} / \mathrm{g}$ & 0.152 & EPIA-013E \\
\hline $\mathrm{U}$ & -0.0363 & $\mathrm{pCi} / \mathrm{g}$ & 0.0776 & EPIA-013B \\
\hline $\mathrm{U}$ & -0.124 & $\mathrm{pCi} / \mathrm{g}$ & 0.319 & EPIA-013B \\
\hline $\mathrm{U}$ & -0.0101 & $\mathrm{pCi} / \mathrm{g}$ & 0.0667 & EPIA-013E \\
\hline $\mathrm{U}$ & 0.0291 & $\mathrm{pCi} / \mathrm{g}$ & 0.0804 & EPIA-013E \\
\hline $\mathrm{U}$ & 0.0241 & $\mathrm{pCi} / \mathrm{g}$ & 0.0406 & EPIA-013B \\
\hline $\mathrm{U}$ & 0.00927 & $\mathrm{pCi} / \mathrm{g}$ & 0.0992 & EPIA-013E \\
\hline $\mathrm{U}$ & 0.00603 & $\mathrm{pCi} / \mathrm{g}$ & 0.0763 & EPIA-013I \\
\hline $\mathrm{U}$ & 0.00832 & $\mathrm{pCi} / \mathrm{g}$ & 0.189 & EPIA-013. \\
\hline $\mathrm{U}$ & -0.0648 & $\mathrm{pCi} / \mathrm{g}$ & 0.216 & EPIA-013B \\
\hline $\mathrm{U}$ & 0.0292 & $\mathrm{pCi} / \mathrm{g}$ & 0.159 & EPIA-013B \\
\hline $\mathrm{U}$ & -0.539 & $\mathrm{pCi} / \mathrm{g}$ & 1.34 & EPIA-0011 \\
\hline U & 0.0868 & $\mathrm{pCi} / \mathrm{g}$ & 0.123 & EPIA-013B \\
\hline $\mathrm{U}$ & -0.0262 & $\mathrm{pCi} / \mathrm{g}$ & 0.0714 & EPIA-013E \\
\hline \multirow[t]{2}{*}{$\mathrm{U}$} & 0.0014 & $\mathrm{pCi} / \mathrm{g}$ & 0.304 & EPIA-013B \\
\hline & 6.33 & $\mathrm{pCi} / \mathrm{g}$ & 1.33 & EPIA-0011 \\
\hline $\mathrm{U}$ & 0.00451 & $\mathrm{pCi} / \mathrm{g}$ & 0.0135 & EPIA-012B \\
\hline \multirow[t]{2}{*}{$\mathrm{U}$} & -0.00108 & $\mathrm{pCi} / \mathrm{g}$ & 0.0238 & EPIA-012E \\
\hline & 2.8 & $\mathrm{pCi} / \mathrm{g}$ & 0.881 & EPIA-013I \\
\hline $\mathrm{U}$ & -0.00318 & $\mathrm{pCi} / \mathrm{g}$ & 0.0755 & EPIA-013B \\
\hline $\mathrm{U}$ & -0.031 & $\mathrm{pCi} / \mathrm{g}$ & 0.0755 & EPIA-013E \\
\hline $\mathrm{U}$ & 0.36 & $\mathrm{pCi} / \mathrm{g}$ & 0.751 & EPIA-013I \\
\hline $\mathrm{U}$ & -0.0236 & $\mathrm{pCi} / \mathrm{g}$ & 0.0782 & EPIA-013 \\
\hline $\mathrm{U}$ & 0.364 & $\mathrm{pCi} / \mathrm{g}$ & 0.948 & EPA905 \\
\hline $\mathrm{U}$ & 0.0498 & $\mathrm{pCi} / \mathrm{g}$ & 0.101 & EPIA-013I \\
\hline $\mathrm{J}$ & 0.229 & $\mathrm{pCi} / \mathrm{g}$ & 0.19 & EPIA-002] \\
\hline
\end{tabular}


SURVEY ID: SRF-07-02 (cont.)

Constituent

Radionuclides

Uranium-233/234

Uranium-235

Uranium-238

Yttrium-88

Zinc-65

Zirconium-95

Volatile Organic Compounds

Acetone

Benzene

Bromodichloromethane

Bromoform

Bromomethane (Methyl bromide)

Carbon disulfide

Carbon tetrachloride

Chlorobenzene

Chloroethane

Chloroethene (Vinyl chloride)

Chloroform

Chloromethane (Methyl chloride)

Dibromochloromethane

1,1-Dichloroethane

1,2-Dichloroethane

1,1-Dichloroethylene

1,2-Dichloroethylene

Dichloromethane (Methylene chloride)

1,2-Dichloropropane

cis-1,3-Dichloropropene

trans-1,3-Dichloropropene

Ethylbenzene

2-Hexanone

Methyl ethyl ketone

Methyl isobutyl ketone

Styrene

1,1,2,2-Tetrachloroethane

Tetrachloroethylene

Toluene

1,1,1-Trichloroethane

1,1,2-Trichloroethane

Trichloroethylene

Vinyl acetate

Xylenes
Sample ID: 117042

FG SC E Result

Unit ssMDL

Method

EPIA-011B

$0.000171 \quad \mathrm{pCi} / \mathrm{g} \quad 0.0322 \quad$ EPIA-011B

$0.00221 \quad \mathrm{pCi} / \mathrm{g} \quad 0.0265 \quad$ EPIA-011B

$\begin{array}{llll}-0.00231 & \mathrm{pCi} / \mathrm{g} & 0.114 & \text { EPIA-013B }\end{array}$

$\begin{array}{llll}0.0628 & \mathrm{pCi} / \mathrm{g} & 0.201 & \text { EPIA-013B }\end{array}$

$0.13 \quad \mathrm{pCi} / \mathrm{g} \quad 0.188 \quad$ EPIA-013B

$\mu g / \mathrm{kg} \quad 51.5 \quad$ EPA8260B

$\mu \mathrm{g} / \mathrm{kg} \quad 2.5 \quad$ EPA8260B

$\mu \mathrm{g} / \mathrm{kg} \quad 0.5 \quad$ EPA8260B

$\mu \mathrm{g} / \mathrm{kg} \quad 1.35 \quad$ EPA8260B

$\mu \mathrm{g} / \mathrm{kg} \quad 1.5 \quad$ EPA8260B

$\mu \mathrm{g} / \mathrm{kg} \quad 1.5 \quad$ EPA8260B

$\mu \mathrm{g} / \mathrm{kg} \quad 2.5 \quad$ EPA8260B

$\mu \mathrm{g} / \mathrm{kg} \quad 1.5 \quad$ EPA8260B

$\mu \mathrm{g} / \mathrm{kg} \quad 1.5 \quad$ EPA8260B

$\mu \mathrm{g} / \mathrm{kg} \quad 2 \quad$ EPA8260B

$\mu \mathrm{g} / \mathrm{kg} \quad 0.5 \quad$ EPA8260B

$\mu \mathrm{g} / \mathrm{kg} \quad 1 \quad$ EPA8260B

$\mu \mathrm{g} / \mathrm{kg} \quad 1.05 \quad$ EPA8260B

$\mu \mathrm{g} / \mathrm{kg} \quad 0.5 \quad$ EPA8260B

$\mu \mathrm{g} / \mathrm{kg} \quad 1.15 \quad$ EPA8260B

$\mu \mathrm{g} / \mathrm{kg} \quad 1.5 \quad$ EPA8260B

$\mu \mathrm{g} / \mathrm{kg} \quad 1.25 \quad$ EPA8260B

$\mu \mathrm{g} / \mathrm{kg} \quad 7 \quad$ EPA8260B

$\mu \mathrm{g} / \mathrm{kg} \quad 1.15 \quad$ EPA8260B

$\mu \mathrm{g} / \mathrm{kg} \quad 1 \quad$ EPA8260B

$\mu \mathrm{g} / \mathrm{kg} \quad 1.5 \quad$ EPA8260B

$\mu \mathrm{g} / \mathrm{kg} \quad 1.5 \quad$ EPA8260B

$\mu \mathrm{g} / \mathrm{kg} \quad 14 \quad$ EPA8260B

$\mu \mathrm{g} / \mathrm{kg} \quad 16 \quad$ EPA8260B

$\mu \mathrm{g} / \mathrm{kg} \quad 14.6 \quad$ EPA8260B

$\mu \mathrm{g} / \mathrm{kg} \quad 1.5 \quad$ EPA8260B

$\mu \mathrm{g} / \mathrm{kg} \quad 3 \quad$ EPA8260B

$\mu \mathrm{g} / \mathrm{kg} \quad 2 \quad$ EPA8260B

$\mu \mathrm{g} / \mathrm{kg} \quad 4.5 \quad$ EPA8260B

$\mu \mathrm{g} / \mathrm{kg} \quad 0.5 \quad$ EPA8260B

$\mu \mathrm{g} / \mathrm{kg} \quad 1.2 \quad$ EPA8260B

$\mu \mathrm{g} / \mathrm{kg} \quad 1.35 \quad$ EPA8260B

$\mu \mathrm{g} / \mathrm{kg} \quad 10.5 \quad$ EPA8260B

$\mu \mathrm{g} / \mathrm{kg} \quad 5 \quad$ EPA8260B 
SURVEY ID: SRF-07-03

Sample type: Normal

Constituent

Metals (total recoverable)

Antimony
Arsenic
Barium
Cadmium
Calcium
Chromium
Copper
Lead
Magnesium
Manganese
Mercury
Nickel
Selenium
Silver
Zinc

Radionuclides

Actinium-228

Actinium-228

Antimony-124

Antimony-124

Antimony-125

Antimony-125

Barium-133

Barium-133

Cerium-144

Cerium-144

Cesium-134

Cesium-134

Cesium-137

Cesium-137

Cobalt-57

Cobalt-57

Cobalt-58

Cobalt-58

Cobalt-60

Cobalt-60

Europium-152

Europium-152

Europium-154

Europium-154

Europium-155

Europium-155

Gross alpha

Gross alpha
Sample ID: 117043

Sample matrix: Fauna

Percent solids: NA

\begin{tabular}{|c|c|c|c|c|c|}
\hline \multirow[t]{3}{*}{ JU } & 4 & 0.263 & $\mathrm{mg} / \mathrm{kg}$ & 0.175 & EPA6010B \\
\hline & & 0.426 & $\mathrm{mg} / \mathrm{kg}$ & 0.209 & EPA6010B \\
\hline & & 2.68 & $\mathrm{mg} / \mathrm{kg}$ & 0.024 & EPA6010B \\
\hline \multirow[t]{8}{*}{$\mathrm{U}$} & & 0.229 & $\mathrm{mg} / \mathrm{kg}$ & 0.017 & EPA6010B \\
\hline & & 14200 & $\mathrm{mg} / \mathrm{kg}$ & 3.2 & EPA6010B \\
\hline & & 0.47 & $\mathrm{mg} / \mathrm{kg}$ & 0.0334 & EPA6010B \\
\hline & & 1.2 & $\mathrm{mg} / \mathrm{kg}$ & 0.0606 & EPA6010B \\
\hline & & 0.68 & $\mathrm{mg} / \mathrm{kg}$ & 0.072 & EPA6010B \\
\hline & & 490 & $\mathrm{mg} / \mathrm{kg}$ & 0.233 & EPA6010B \\
\hline & & 6.96 & $\mathrm{mg} / \mathrm{kg}$ & 0.326 & EPA6010B \\
\hline & & 0.543 & $\mathrm{mg} / \mathrm{kg}$ & 0.00224 & EPA7471A \\
\hline $\mathrm{J}$ & & 0.12 & $\mathrm{mg} / \mathrm{kg}$ & 0.029 & EPA6010B \\
\hline & & 1.07 & $\mathrm{mg} / \mathrm{kg}$ & 0.124 & EPA6010B \\
\hline IL & I & 0.135 & $\mathrm{mg} / \mathrm{kg}$ & 0.0284 & EPA6010B \\
\hline & & 15.1 & $\mathrm{mg} / \mathrm{kg}$ & 0.17 & EPA6010B \\
\hline
\end{tabular}

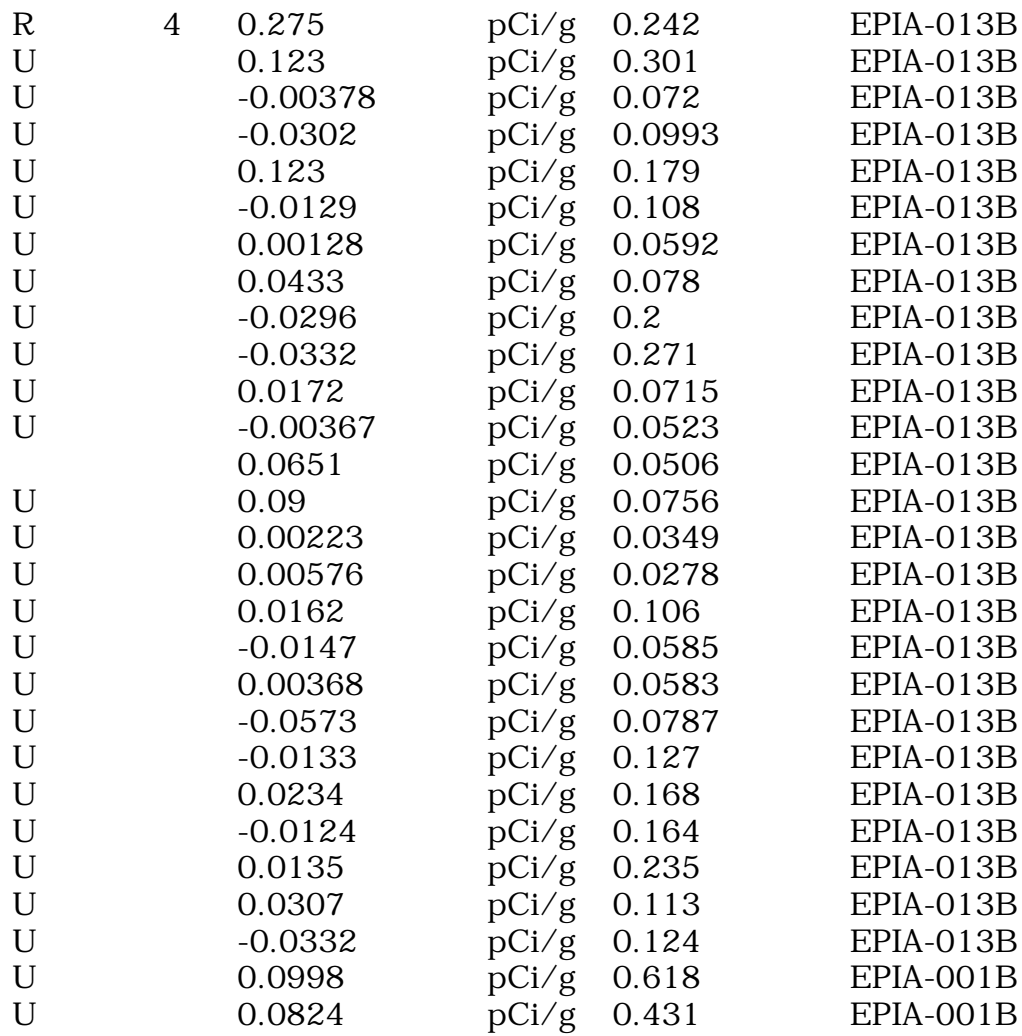


SURVEY ID: SRF-07-03 (cont.)

Constituent

Radionuclides

Lead-212

Lead-212

Manganese-54

Manganese-54

Neptunium-239

Neptunium-239

Nonvolatile beta

Nonvolatile beta

Plutonium-238

Plutonium-238

Plutonium-239/240

Plutonium-239/240

Potassium-40

Potassium-40

Promethium-144

Promethium-144

Promethium-146

Promethium-146

Ruthenium-106

Ruthenium-106

Sodium-22

Sodium-22

Strontium-89/90

Strontium-89/90

Tin-113

Tin-1 13

Tritium

Uranium-233/234

Uranium-233/234

Uranium-235

Uranium-235

Uranium-238

Uranium-238

Yttrium-88

Yttrium-88

Zinc-65

Zinc-65

Zirconium-95

Zirconium-95

Volatile Organic Compounds

Acetone

Benzene

Bromodichloromethane

Bromoform

Bromomethane (Methyl bromide)

Carbon disulfide

Carbon tetrachloride

Chlorobenzene

Chloroethane

Chloroethene (Vinyl chloride)

Chloroform

Sample ID: 117043

FG SC E Result

Unit ssMDL

Method

$\mathrm{U}$
$\mathrm{R}$
$\mathrm{U}$

U

U

U

$\mathrm{U}$

U

$\mathrm{U}$

U

U

U

U

U

U

U

U

U

$\mathrm{U}$

U

U

U

$\mathrm{U}$

U

U

U

$\mathrm{U}$

U

U

U

U

\begin{tabular}{|c|c|c|}
\hline 0.0271 & $\mathrm{pCi} / \mathrm{g}$ & 0.0842 \\
\hline 0.134 & $\mathrm{pCi} / \mathrm{g}$ & 0.109 \\
\hline-0.000359 & $\mathrm{pCi} / \mathrm{g}$ & 0.0806 \\
\hline 0.0134 & $\mathrm{pCi} / \mathrm{g}$ & 0.0586 \\
\hline 0.105 & $\mathrm{pCi} / \mathrm{g}$ & 0.211 \\
\hline-0.162 & $\mathrm{pCi} / \mathrm{g}$ & 0.224 \\
\hline 5.31 & $\mathrm{pCi} / \mathrm{g}$ & 0.586 \\
\hline 5.4 & $\mathrm{pCi} / \mathrm{g}$ & 0.642 \\
\hline-0.000479 & $\mathrm{pCi} / \mathrm{g}$ & 0.0105 \\
\hline 0.00317 & $\mathrm{pCi} / \mathrm{g}$ & 0.013 \\
\hline-0.000501 & $\mathrm{pCi} / \mathrm{g}$ & 0.011 \\
\hline 0.00199 & $\mathrm{pCi} / \mathrm{g}$ & 0.00598 \\
\hline 2.86 & $\mathrm{pCi} / \mathrm{g}$ & 0.561 \\
\hline 2.76 & $\mathrm{pCi} / \mathrm{g}$ & 0.818 \\
\hline 0.0118 & $\mathrm{pCi} / \mathrm{g}$ & 0.0779 \\
\hline 0.0127 & $\mathrm{pCi} / \mathrm{g}$ & 0.0525 \\
\hline 0.0137 & $\mathrm{pCi} / \mathrm{g}$ & 0.0605 \\
\hline 0.000611 & $\mathrm{pCi} / \mathrm{g}$ & 0.0823 \\
\hline 0.252 & $\mathrm{pCi} / \mathrm{g}$ & 0.497 \\
\hline 0.0762 & $\mathrm{pCi} / \mathrm{g}$ & 0.702 \\
\hline-0.00447 & $\mathrm{pCi} / \mathrm{g}$ & 0.0592 \\
\hline 0.00507 & $\mathrm{pCi} / \mathrm{g}$ & 0.0852 \\
\hline 0.365 & $\mathrm{pCi} / \mathrm{g}$ & 0.433 \\
\hline 0.336 & $\mathrm{pCi} / \mathrm{g}$ & 0.466 \\
\hline-0.0125 & $\mathrm{pCi} / \mathrm{g}$ & 0.0884 \\
\hline 0.0115 & $\mathrm{pCi} / \mathrm{g}$ & 0.0639 \\
\hline 0 & $\mathrm{pCi} / \mathrm{g}$ & 0.0844 \\
\hline 7.1E-05 & $\mathrm{pCi} / \mathrm{g}$ & 0.0134 \\
\hline 0.00995 & $\mathrm{pCi} / \mathrm{g}$ & 0.0131 \\
\hline 0.000142 & $\mathrm{pCi} / \mathrm{g}$ & 0.0168 \\
\hline 0.00521 & $\mathrm{pCi} / \mathrm{g}$ & 0.00997 \\
\hline 0.00135 & $\mathrm{pCi} / \mathrm{g}$ & 0.00937 \\
\hline 0.00143 & $\mathrm{pCi} / \mathrm{g}$ & 0.00994 \\
\hline-0.00343 & $\mathrm{pCi} / \mathrm{g}$ & 0.0863 \\
\hline 0.00984 & $\mathrm{pCi} / \mathrm{g}$ & 0.0743 \\
\hline-0.0264 & $\mathrm{pCi} / \mathrm{g}$ & 0.123 \\
\hline-0.00123 & $\mathrm{pCi} / \mathrm{g}$ & 0.187 \\
\hline-0.0093 & $\mathrm{pCi} / \mathrm{g}$ & 0.176 \\
\hline-0.0442 & $\mathrm{pCi} / \mathrm{g}$ & 0.101 \\
\hline
\end{tabular}

EPIA-013B

EPIA-013B

EPIA-013B

EPIA-013B

EPIA-013B

EPIA-013B

EPIA-001B

EPIA-001B

EPIA-012B

EPIA-012B

EPIA-012B

EPIA-012B

EPIA-013B

EPIA-013B

EPIA-013B

EPIA-013B

EPIA-013B

EPIA-013B

EPIA-013B

EPIA-013B

EPIA-013B

EPIA-013B

EPA905

EPA905

EPIA-013B

EPIA-013B

EPIA-002B

EPIA-011B

EPIA-011B

EPIA-011B

EPIA-011B

EPIA-011B

EPIA-011B

EPIA-013B

EPIA-013B

EPIA-013B

EPIA-013B

EPIA-013B

EPIA-013B
EPA8260B EPA8260B EPA8260B EPA8260B EPA8260B EPA8260B EPA8260B EPA8260B EPA8260B EPA8260B EPA8260B

$\begin{array}{ll}\mu g / \mathrm{kg} & 51.5 \\ \mu g / \mathrm{kg} & 2.5 \\ \mu g / \mathrm{kg} & 0.5 \\ \mu g / \mathrm{kg} & 1.35 \\ \mu g / \mathrm{kg} & 1.5 \\ \mu g / \mathrm{kg} & 1.5 \\ \mu g / \mathrm{kg} & 2.5 \\ \mu g / \mathrm{kg} & 1.5 \\ \mu g / \mathrm{kg} & 1.5 \\ \mu g / \mathrm{kg} & 2 \\ \mu g / \mathrm{kg} & 0.5\end{array}$


SURVEY ID: SRF-07-03 (cont.)

Constituent

Volatile Organic Compounds

Chloromethane (Methyl chloride) U

Dibromochloromethane

1,1-Dichloroethane

1,2-Dichloroethane

1,1-Dichloroethylene

1,2-Dichloroethylene

Dichloromethane (Methylene chloride) J

1,2-Dichloropropane

cis-1,3-Dichloropropene

trans-1,3-Dichloropropene

Ethylbenzene

2-Hexanone

Methyl ethyl ketone

Methyl isobutyl ketone

Styrene

1,1,2,2-Tetrachloroethane

Tetrachloroethylene

Toluene

1,1,1-Trichloroethane

1,1,2-Trichloroethane

Trichloroethylene

Vinyl acetate

Xylenes
Sample ID: 117043

FG SC E Result

Unit ssMDL Method

$\begin{array}{lll}\mu \mathrm{g} / \mathrm{kg} & 1 & \text { EPA8260B } \\ \mu \mathrm{g} / \mathrm{kg} & 1.05 & \text { EPA8260B } \\ \mu \mathrm{g} / \mathrm{kg} & 0.5 & \text { EPA8260B } \\ \mu \mathrm{g} / \mathrm{kg} & 1.15 & \text { EPA8260B } \\ \mu \mathrm{g} / \mathrm{kg} & 1.5 & \text { EPA8260B } \\ \mu \mathrm{g} / \mathrm{kg} & 1.25 & \text { EPA8260B } \\ \mu \mathrm{g} / \mathrm{kg} & 7 & \text { EPA8260B } \\ \mu \mathrm{g} / \mathrm{kg} & 1.15 & \text { EPA8260B } \\ \mu \mathrm{g} / \mathrm{kg} & 1 & \text { EPA8260B } \\ \mu \mathrm{g} / \mathrm{kg} & 1.5 & \text { EPA8260B } \\ \mu \mathrm{g} / \mathrm{kg} & 1.5 & \text { EPA8260B } \\ \mu \mathrm{g} / \mathrm{kg} & 14 & \text { EPA8260B } \\ \mu \mathrm{g} / \mathrm{kg} & 16 & \text { EPA8260B } \\ \mu \mathrm{g} / \mathrm{kg} & 14.6 & \text { EPA8260B } \\ \mu \mathrm{g} / \mathrm{kg} & 1.5 & \text { EPA8260B } \\ \mu \mathrm{g} / \mathrm{kg} & 3 & \text { EPA8260B } \\ \mu \mathrm{g} / \mathrm{kg} & 2 & \text { EPA8260B } \\ \mu \mathrm{g} / \mathrm{kg} & 4.5 & \text { EPA8260B } \\ \mu \mathrm{g} / \mathrm{kg} & 0.5 & \text { EPA8260B } \\ \mu \mathrm{g} / \mathrm{kg} & 1.2 & \text { EPA8260B } \\ \mu \mathrm{g} / \mathrm{kg} & 1.35 & \text { EPA8260B } \\ \mu \mathrm{g} / \mathrm{kg} & 10.5 & \text { EPA8260B } \\ \mu \mathrm{g} / \mathrm{kg} & 5 & \text { EPA8260B }\end{array}$




\section{Table D.7 Results for Blanks Sorted by Sample}

SURVEY ID: SRF-TB-01-B

Sample type: Trip Blank

\section{Constituent}

Volatile Organic Compounds

Acetone U

Benzene

Bromodichloromethane

Bromoform

Bromomethane (Methyl bromide)

Carbon disulfide

Carbon tetrachloride

Chlorobenzene

Chloroethane

Chloroethene (Vinyl chloride)

Chloroform

Chloromethane (Methyl chloride)

Dibromochloromethane

1,1-Dichloroethane

1,2-Dichloroethane

cis-1,2-Dichloroethylene

1,1-Dichloroethylene trans-1,2-Dichloroethylene

Dichloromethane (Methylene chloride) JU

1,2-Dichloropropane

cis-1,3-Dichloropropene

trans-1,3-Dichloropropene

Ethylbenzene

2-Hexanone

Methyl ethyl ketone

Methyl isobutyl ketone

Styrene

1,1,2,2-Tetrachloroethane

Tetrachloroethylene

Toluene

1,1,1-Trichloroethane

1,1,2-Trichloroethane

Trichloroethylene

Xylenes
Sample ID: 117044

Sample matrix: Water

Percent solids: 0
Method

ug/L $\quad 3.7$

$\mu \mathrm{g} / \mathrm{L} \quad 0.3$

$\mu \mathrm{g} / \mathrm{L} \quad 0.4$

$\mu \mathrm{g} / \mathrm{L} \quad 0.4$

$\mu \mathrm{g} / \mathrm{L} \quad 0.3$

$\mu \mathrm{g} / \mathrm{L} \quad 1.8$

$\mu \mathrm{g} / \mathrm{L} \quad 0.2$

$\mu \mathrm{g} / \mathrm{L} \quad 0.3$

$\mu \mathrm{g} / \mathrm{L} \quad 0.3$

$\mu \mathrm{g} / \mathrm{L} \quad 0.4$

$\mu \mathrm{g} / \mathrm{L} \quad 0.7$

$\mu \mathrm{g} / \mathrm{L} \quad 0.2$

$\mu \mathrm{g} / \mathrm{L} \quad 0.3$

$\mu \mathrm{g} / \mathrm{L} \quad 0.4$

$\mu \mathrm{g} / \mathrm{L} \quad 0.2$

$\mu \mathrm{g} / \mathrm{L} \quad 0.7$

$\mu \mathrm{g} / \mathrm{L} \quad 0.7$

$\mu \mathrm{g} / \mathrm{L} \quad 0.7$

$\mu \mathrm{g} / \mathrm{L} \quad 1.2$

$\mu \mathrm{g} / \mathrm{L} \quad 0.2$

$\mu g / L \quad 0.3$

$\mu \mathrm{g} / \mathrm{L} \quad 0.3$

$\mu g / L \quad 0.3$

$\mu \mathrm{g} / \mathrm{L} \quad 3.2$

$\mu g / L \quad 5.9$

$\mu \mathrm{g} / \mathrm{L} \quad 1.6$

$\mu g / L \quad 0.2$

$\mu \mathrm{g} / \mathrm{L} \quad 0.5$

$\mu \mathrm{g} / \mathrm{L} \quad 0.7$

$\mu g / L \quad 0.5$

$\mu \mathrm{g} / \mathrm{L} \quad 0.2$

$\mu g / L \quad 0.4$

$\mu \mathrm{g} / \mathrm{L} \quad 0.6$

$\mu \mathrm{g} / \mathrm{L} \quad 1.1$
EPA8260B

EPA8260B

EPA8260B

EPA8260B

EPA8260B

EPA8260B

EPA8260B

EPA8260B

EPA8260B

EPA8260B

EPA8260B

EPA8260B

EPA8260B

EPA8260B

EPA8260B

EPA8260B

EPA8260B

EPA8260B

EPA8260B

EPA8260B

EPA8260B

EPA8260B

EPA8260B

EPA8260B

EPA8260B

EPA8260B

EPA8260B

EPA8260B

EPA8260B

EPA8260B

EPA8260B

EPA8260B

EPA8260B

EPA8260B 
SURVEY ID: SRF-TB-02-B

Sample type: Trip Blank

Constituent

Volatile Organic Compounds

\begin{tabular}{|c|c|c|}
\hline Acetone & $\mathrm{U}$ & 5 \\
\hline Benzene & $\mathrm{U}$ & 1 \\
\hline Bromodichloromethane & $\mathrm{U}$ & 1 \\
\hline Bromoform & $\mathrm{U}$ & 1 \\
\hline Bromomethane (Methyl bromide) & $\mathrm{U}$ & 1 \\
\hline Carbon disulfide & $\mathrm{U}$ & 5 \\
\hline Carbon tetrachloride & $\mathrm{U}$ & 1 \\
\hline Chlorobenzene & $\mathrm{U}$ & 1 \\
\hline Chloroethane & $\mathrm{U}$ & 1 \\
\hline Chloroethene (Vinyl chloride) & $\mathrm{U}$ & 1 \\
\hline Chloroform & $\mathrm{U}$ & 1 \\
\hline Chloromethane (Methyl chloride) & $\mathrm{U}$ & 1 \\
\hline Dibromochloromethane & $\mathrm{U}$ & 1 \\
\hline 1,1-Dichloroethane & $\mathrm{U}$ & 1 \\
\hline 1,2-Dichloroethane & $\mathrm{U}$ & 1 \\
\hline cis-1,2-Dichloroethylene & $\mathrm{U}$ & 1 \\
\hline 1,1-Dichloroethylene & $\mathrm{U}$ & 1 \\
\hline trans-1,2-Dichloroethylene & $\mathrm{U}$ & 1 \\
\hline Dichloromethane (Methylene chloride) & $\mathrm{JU}$ & 3.99 \\
\hline 1,2-Dichloropropane & $\mathrm{U}$ & 1 \\
\hline cis-1,3-Dichloropropene & $\mathrm{U}$ & 1 \\
\hline trans-1,3-Dichloropropene & $\mathrm{U}$ & 1 \\
\hline Ethylbenzene & $\mathrm{U}$ & 1 \\
\hline 2-Hexanone & $\mathrm{U}$ & 5 \\
\hline Methyl ethyl ketone & $\mathrm{U}$ & 10 \\
\hline Methyl isobutyl ketone & $\mathrm{U}$ & 5 \\
\hline Styrene & $\mathrm{U}$ & 1 \\
\hline $1,1,2,2$-Tetrachloroethane & $\mathrm{U}$ & 1 \\
\hline Tetrachloroethylene & $\mathrm{U}$ & 1 \\
\hline Toluene & $\mathrm{U}$ & 1 \\
\hline $1,1,1$-Trichloroethane & $\mathrm{U}$ & 1 \\
\hline $1,1,2$-Trichloroethane & $\mathrm{U}$ & 1 \\
\hline Trichloroethylene & $\mathrm{U}$ & 1 \\
\hline Xylenes & $\mathrm{U}$ & 2 \\
\hline
\end{tabular}

Sample ID: 117045

Sample matrix: Water

Percent solids: 0

FG SC E Result Unit ssMDL

\begin{tabular}{|c|c|c|}
\hline$\mu g / L$ & 3.7 & EPA8260B \\
\hline$\mu g / L$ & 0.3 & EPA8260B \\
\hline$\mu g / L$ & 0.4 & EPA8260B \\
\hline$\mu g / L$ & 0.4 & EPA8260B \\
\hline$\mu \mathrm{g} / \mathrm{L}$ & 0.3 & EPA8260B \\
\hline$\mu g / L$ & 1.8 & EPA8260B \\
\hline$\mu g / L$ & 0.2 & EPA8260B \\
\hline$\mu g / L$ & 0.3 & EPA8260B \\
\hline$\mu g / L$ & 0.3 & EPA8260B \\
\hline$\mu g / L$ & 0.4 & EPA8260B \\
\hline$\mu g / L$ & 0.7 & EPA8260B \\
\hline$\mu g / L$ & 0.2 & EPA8260B \\
\hline$\mu g / L$ & 0.3 & EPA8260B \\
\hline$\mu g / L$ & 0.4 & EPA8260B \\
\hline$\mu g / L$ & 0.2 & EPA8260B \\
\hline$\mu g / L$ & 0.7 & EPA8260B \\
\hline$\mu g / L$ & 0.7 & EPA8260B \\
\hline$\mu g / L$ & 0.7 & EPA8260B \\
\hline$\mu g / L$ & 1.2 & EPA8260B \\
\hline$\mu g / L$ & 0.2 & EPA8260B \\
\hline$\mu g / L$ & 0.3 & EPA8260B \\
\hline$\mu g / L$ & 0.3 & EPA8260B \\
\hline$\mu \mathrm{g} / \mathrm{L}$ & 0.3 & EPA8260B \\
\hline$\mu g / L$ & 3.2 & EPA8260B \\
\hline$\mu g / L$ & 5.9 & EPA8260B \\
\hline$\mu g / L$ & 1.6 & EPA8260B \\
\hline$\mu g / L$ & 0.2 & EPA8260B \\
\hline$\mu g / L$ & 0.5 & EPA8260B \\
\hline$\mu g / L$ & 0.7 & EPA8260B \\
\hline$\mu g / L$ & 0.5 & EPA8260B \\
\hline$\mu g / L$ & 0.2 & EPA8260B \\
\hline$\mu g / L$ & 0.4 & EPA8260B \\
\hline$\mu g / L$ & 0.6 & EPA8260B \\
\hline$\mu g / L$ & 1.1 & EPA8260B \\
\hline
\end{tabular}


SURVEY ID: SRF-TB-03-B

Sample type: Trip Blank

Constituent

Volatile Organic Compounds

\begin{tabular}{|c|c|c|}
\hline Acetone & $\mathrm{U}$ & 5 \\
\hline Benzene & $\mathrm{U}$ & 1 \\
\hline Bromodichloromethane & $\mathrm{U}$ & 1 \\
\hline Bromoform & $\mathrm{U}$ & 1 \\
\hline Bromomethane (Methyl bromide) & $\mathrm{U}$ & 1 \\
\hline Carbon disulfide & $\mathrm{U}$ & 5 \\
\hline Carbon tetrachloride & $\mathrm{U}$ & 1 \\
\hline Chlorobenzene & $\mathrm{U}$ & 1 \\
\hline Chloroethane & $\mathrm{U}$ & 1 \\
\hline Chloroethene (Vinyl chloride) & $\mathrm{U}$ & 1 \\
\hline Chloroform & $\mathrm{U}$ & 1 \\
\hline Chloromethane (Methyl chloride) & $\mathrm{U}$ & 1 \\
\hline Dibromochloromethane & $\mathrm{U}$ & 1 \\
\hline 1,1-Dichloroethane & $\mathrm{U}$ & 1 \\
\hline 1,2-Dichloroethane & $\mathrm{U}$ & 1 \\
\hline cis-1,2-Dichloroethylene & $\mathrm{U}$ & 1 \\
\hline 1,1-Dichloroethylene & $\mathrm{U}$ & 1 \\
\hline trans-1,2-Dichloroethylene & $\mathrm{U}$ & 1 \\
\hline Dichloromethane (Methylene chloride) & $\mathrm{JU}$ & 3.39 \\
\hline 1,2-Dichloropropane & $\mathrm{U}$ & 1 \\
\hline cis-1,3-Dichloropropene & $\mathrm{U}$ & 1 \\
\hline trans-1,3-Dichloropropene & $\mathrm{U}$ & 1 \\
\hline Ethylbenzene & $\mathrm{U}$ & 1 \\
\hline 2-Hexanone & $\mathrm{U}$ & 5 \\
\hline Methyl ethyl ketone & $\mathrm{U}$ & 10 \\
\hline Methyl isobutyl ketone & $\mathrm{U}$ & 5 \\
\hline Styrene & $\mathrm{U}$ & 1 \\
\hline $1,1,2,2$-Tetrachloroethane & $\mathrm{U}$ & 1 \\
\hline Tetrachloroethylene & $\mathrm{U}$ & 1 \\
\hline Toluene & $\mathrm{U}$ & 1 \\
\hline $1,1,1$-Trichloroethane & $\mathrm{U}$ & 1 \\
\hline $1,1,2$-Trichloroethane & $\mathrm{U}$ & 1 \\
\hline Trichloroethylene & $\mathrm{U}$ & 1 \\
\hline Xylenes & $\mathrm{U}$ & 2 \\
\hline
\end{tabular}

Sample ID: 117046

Sample matrix: Water

Percent solids: 0

FG SC E Result

Unit ssMDL Method

\begin{tabular}{|c|c|c|}
\hline$\mu \mathrm{g} / \mathrm{L}$ & 3.7 & EPA8260B \\
\hline$\mu g / L$ & 0.3 & EPA8260B \\
\hline$\mu g / L$ & 0.4 & EPA8260B \\
\hline$\mu g / L$ & 0.4 & EPA8260B \\
\hline$\mu g / L$ & 0.3 & EPA8260B \\
\hline$\mu g / L$ & 1.8 & EPA8260B \\
\hline$\mu g / L$ & 0.2 & EPA8260B \\
\hline$\mu g / L$ & 0.3 & EPA8260B \\
\hline$\mu g / L$ & 0.3 & EPA8260B \\
\hline$\mu g / L$ & 0.4 & EPA8260B \\
\hline$\mu g / L$ & 0.7 & EPA8260B \\
\hline$\mu g / L$ & 0.2 & EPA8260B \\
\hline$\mu g / L$ & 0.3 & EPA8260B \\
\hline$\mu g / L$ & 0.4 & EPA8260B \\
\hline$\mu g / L$ & 0.2 & EPA8260B \\
\hline$\mu g / L$ & 0.7 & EPA8260B \\
\hline$\mu g / L$ & 0.7 & EPA8260B \\
\hline$\mu g / L$ & 0.7 & EPA8260B \\
\hline$\mu g / L$ & 1.2 & EPA8260B \\
\hline$\mu g / L$ & 0.2 & EPA8260B \\
\hline$\mu g / L$ & 0.3 & EPA8260B \\
\hline$\mu g / L$ & 0.3 & EPA8260B \\
\hline$\mu g / L$ & 0.3 & EPA8260B \\
\hline$\mu g / L$ & 3.2 & EPA8260B \\
\hline$\mu g / L$ & 5.9 & EPA8260B \\
\hline$\mu g / L$ & 1.6 & EPA8260B \\
\hline$\mu g / L$ & 0.2 & EPA8260B \\
\hline$\mu g / L$ & 0.5 & EPA8260B \\
\hline$\mu g / L$ & 0.7 & EPA8260B \\
\hline$\mu g / L$ & 0.5 & EPA8260B \\
\hline$\mu g / L$ & 0.2 & EPA8260B \\
\hline$\mu g / L$ & 0.4 & EPA8260B \\
\hline$\mu g / L$ & 0.6 & EPA8260B \\
\hline$\mu g / L$ & 1.1 & EPA8260B \\
\hline
\end{tabular}


Table D.8 Analytical Results Sorted by Analyte

ANALYTE: Acetone

Analytical Group: Volatile Organic Compounds

$\begin{array}{lllrl}\text { Survey ID } & \text { Sample ID } & \text { FG } & \text { Result } & \text { Unit } \\ \text { SRF-01-01 } & 117018 & \mathrm{~J} & 925 & \mu \mathrm{g} / \mathrm{kg} \\ \text { SRF-01-02 } & 117019 & \mathrm{~J} & 402 & \mu \mathrm{g} / \mathrm{kg} \\ \text { SRF-01-03 } & 117020 & & 161 & \mu \mathrm{g} / \mathrm{kg} \\ \text { SRF-01-04 } & 117021 & \mathrm{~J} & 593 & \mu \mathrm{g} / \mathrm{kg} \\ \text { SRF-01-05 } & 117022 & \mathrm{~J} & 2490 & \mu \mathrm{g} / \mathrm{kg} \\ \text { SRF-02-01 } & 117023 & \mathrm{~J} & 180 & \mu \mathrm{g} / \mathrm{kg} \\ \text { SRF-02-02 } & 117024 & \mathrm{~J} & 104 & \mu \mathrm{g} / \mathrm{kg} \\ \text { SRF-02-03 } & 117025 & \mathrm{~J} & 173 & \mu \mathrm{g} / \mathrm{kg} \\ \text { SRF-02-03-A } & 117026 & \mathrm{~J} & 79.2 & \mu \mathrm{g} / \mathrm{kg} \\ \text { SRF-03-01 } & 117027 & & 347 & \mu \mathrm{g} / \mathrm{kg} \\ \text { SRF-03-02 } & 117028 & & 127 & \mu \mathrm{g} / \mathrm{kg} \\ \text { SRF-03-03 } & 117029 & \mathrm{~J} & 80 & \mu \mathrm{g} / \mathrm{kg} \\ \text { SRF-03-04 } & 117030 & & 164 & \mu \mathrm{g} / \mathrm{kg} \\ \text { SRF-04-01 } & 117031 & \mathrm{~J} & 122 & \mu \mathrm{g} / \mathrm{kg} \\ \text { SRF-04-02 } & 117032 & & 129 & \mu \mathrm{g} / \mathrm{kg} \\ \text { SRF-04-03 } & 117033 & \mathrm{~J} & 94.4 & \mu \mathrm{g} / \mathrm{kg} \\ \text { SRF-05-01 } & 117034 & \mathrm{~J} & 77.1 & \mu \mathrm{g} / \mathrm{kg} \\ \text { SRF-05-02 } & 117035 & & 223 & \mu \mathrm{g} / \mathrm{kg} \\ \text { SRF-05-03 } & 117036 & \mathrm{~J} & 106 & \mu \mathrm{g} / \mathrm{kg} \\ \text { SRF-06-01 } & 117037 & \mathrm{~J} & 156 & \mu \mathrm{g} / \mathrm{kg} \\ \text { SRF-06-02 } & 117038 & \mathrm{~J} & 117 & \mu \mathrm{g} / \mathrm{kg} \\ \text { SRF-06-03 } & 117039 & \mathrm{U} & 125 & \mu \mathrm{g} / \mathrm{kg} \\ \text { SRF-06-03-A } & 117040 & \mathrm{U} & 125 & \mu \mathrm{g} / \mathrm{kg} \\ \text { SRF-07-01 } & 117041 & \mathrm{~J} & 423 & \mu \mathrm{g} / \mathrm{kg} \\ \text { SRF-07-02 } & 117042 & \mathrm{U} & 125 & \mu \mathrm{g} / \mathrm{kg} \\ \text { SRF-07-03 } & 117043 & \mathrm{~J} & 132 & \mu \mathrm{g} / \mathrm{kg} \\ & & & & \end{array}$

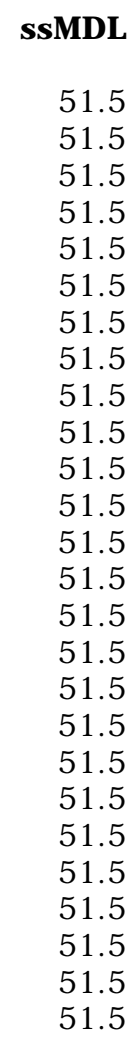

51.5

51.5

51.5

51.5

51.5

51.5

51.5

51.5

51.5

51.5

51.5

51.5

51.5

51.5

51.5

51.5

51.5

51.5

51.5

51.5

51.5

51.5

Method
EPA8260B
EPA8260B
EPA8260B
EPA8260B
EPA8260B
EPA8260B
EPA8260B
EPA8260B
EPA8260B
EPA8260B
EPA8260B
EPA8260B
EPA8260B
EPA8260B
EPA8260B
EPA8260B
EPA8260B
EPA8260B
EPA8260B
EPA8260B
EPA8260B
EPA8260B
EPA8260B
EPA8260B
EPA8260B
EPA8260B

SC E

K O

K O

K O

$\mathrm{K} \quad \mathrm{O}$

$\mathrm{K} \quad \mathrm{O}$

IK O

$\mathrm{K}$

I

I

IK O

IK O

IK O

IK O

$\mathrm{K} \quad \mathrm{O}$

K O

K O

ANALYTE: Actinium-228

Analytical Group: Radionuclides

\begin{tabular}{|c|c|c|c|c|c|c|c|}
\hline Survey ID & Sample ID & FG & Result & Unit & SSMDL & Method & SC \\
\hline SRF-01-01 & 117018 & $\mathrm{U}$ & 0.0883 & $\mathrm{pCi} / \mathrm{g}$ & 0.261 & EPIA-013B & \\
\hline SRF-01-02 & 117019 & $\mathrm{U}$ & 0.0375 & $\mathrm{pCi} / \mathrm{g}$ & 0.196 & EPIA-013B & \\
\hline SRF-01-03 & 117020 & $\mathrm{U}$ & 0.186 & $\mathrm{pCi} / \mathrm{g}$ & 0.233 & EPIA-013B & \\
\hline SRF-01-04 & 117021 & $\mathrm{U}$ & 0.0867 & $\mathrm{pCi} / \mathrm{g}$ & 0.174 & EPIA-013B & \\
\hline SRF-01-05 & 117022 & $\mathrm{U}$ & 0.145 & $\mathrm{pCi} / \mathrm{g}$ & 0.203 & EPIA-013B & \\
\hline SRF-01-05 & 117022 & $\mathrm{U}$ & 0.179 & $\mathrm{pCi} / \mathrm{g}$ & 0.201 & EPIA-013B & \\
\hline SRF-02-01 & 117023 & $\mathrm{U}$ & -0.0298 & $\mathrm{pCi} / \mathrm{g}$ & 0.21 & EPIA-013B & \\
\hline SRF-02-02 & 117024 & $\mathrm{U}$ & 0.085 & $\mathrm{pCi} / \mathrm{g}$ & 0.18 & EPIA-013B & \\
\hline SRF-02-03 & 117025 & $\mathrm{U}$ & 0.0133 & $\mathrm{pCi} / \mathrm{g}$ & 0.27 & EPIA-013B & \\
\hline SRF-02-03-A & 117026 & $\mathrm{U}$ & 0.283 & $\mathrm{pCi} / \mathrm{g}$ & 0.296 & EPIA-013B & \\
\hline SRF-03-01 & 117027 & $\mathrm{U}$ & 0.126 & $\mathrm{pCi} / \mathrm{g}$ & 0.249 & EPIA-013B & \\
\hline SRF-03-02 & 117028 & $\mathrm{U}$ & 0.185 & $\mathrm{pCi} / \mathrm{g}$ & 0.202 & EPIA-013B & \\
\hline SRF-03-03 & 117029 & $\mathrm{U}$ & 0.0927 & $\mathrm{pCi} / \mathrm{g}$ & 0.235 & EPIA-013B & \\
\hline SRF-03-04 & 117030 & $\mathrm{R}$ & 0.17 & $\mathrm{pCi} / \mathrm{g}$ & 0.141 & EPIA-013B & \\
\hline SRF-04-01 & 117031 & $\mathrm{U}$ & 0.162 & $\mathrm{pCi} / \mathrm{g}$ & 0.21 & EPIA-013B & \\
\hline SRF-04-02 & 117032 & $\mathrm{U}$ & 0.0839 & $\mathrm{pCi} / \mathrm{g}$ & 0.265 & EPIA-013B & \\
\hline SRF-04-03 & 117033 & $\mathrm{U}$ & 0.0281 & $\mathrm{pCi} / \mathrm{g}$ & 0.291 & EPIA-013B & \\
\hline SRF-05-01 & 117034 & $\mathrm{U}$ & 0.101 & $\mathrm{pCi} / \mathrm{g}$ & 0.214 & EPIA-013B & \\
\hline SRF-05-02 & 117035 & $\mathrm{U}$ & 0.144 & $\mathrm{pCi} / \mathrm{g}$ & 0.244 & EPIA-013B & \\
\hline SRF-05-03 & 117036 & $\mathrm{U}$ & 0.122 & $\mathrm{pCi} / \mathrm{g}$ & 0.21 & EPIA-013B & \\
\hline
\end{tabular}


ANALYTE: Actinium-228 (cont.)

$\begin{array}{lllrllll}\text { Survey ID } & \text { Sample ID } & \text { FG } & \text { Result } & \text { Unit } & \text { ssMDL } & \text { Method } & \text { SC } \\ \text { SRF-06-01 } & 117037 & \mathrm{U} & 0.0734 & \mathrm{pCi} / \mathrm{g} & 0.261 & \text { EPIA-013B } & \text { EPIA-013B } \\ \text { SRF-06-02 } & 117038 & \mathrm{U} & 0.16 & \mathrm{pCi} / \mathrm{g} & 0.189 & \text { EPIA-013B } \\ \text { SRF-06-03 } & 117039 & \mathrm{U} & 0.226 & \mathrm{pCi} / \mathrm{g} & 0.287 & \text { EPIA-013B } \\ \text { SRF-06-03-A } & 117040 & \mathrm{U} & 0.12 & \mathrm{pCi} / \mathrm{g} & 0.314 & \text { EPIA-013B } \\ \text { SRF-07-01 } & 117041 & \mathrm{U} & 0.189 & \mathrm{pCi} / \mathrm{g} & 0.318 & \text { EPIA-013B } \\ \text { SRF-07-02 } & 117042 & \mathrm{U} & 0.107 & \mathrm{pCi} / \mathrm{g} & 0.341 & \text { EPIA-013B } \\ \text { SRF-07-03 } & 117043 & \mathrm{R} & 0.275 & \mathrm{pCi} / \mathrm{g} & 0.242 & \text { EPIA-013B } & \end{array}$

ANALYTE: Antimony-124

Analytical Group: Radionuclides

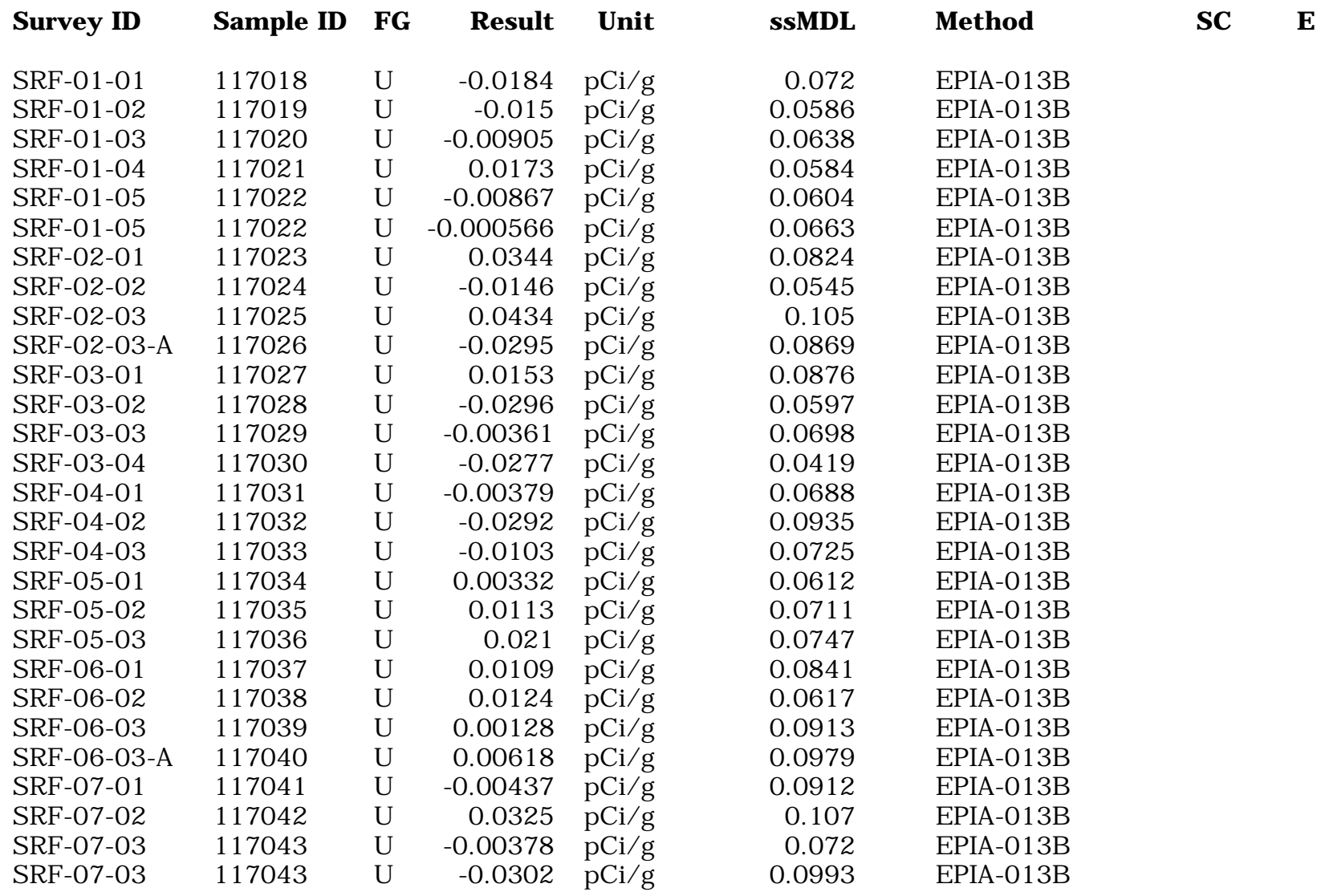

ANALYTE: Antimony-125

Analytical Group: Radionuclides

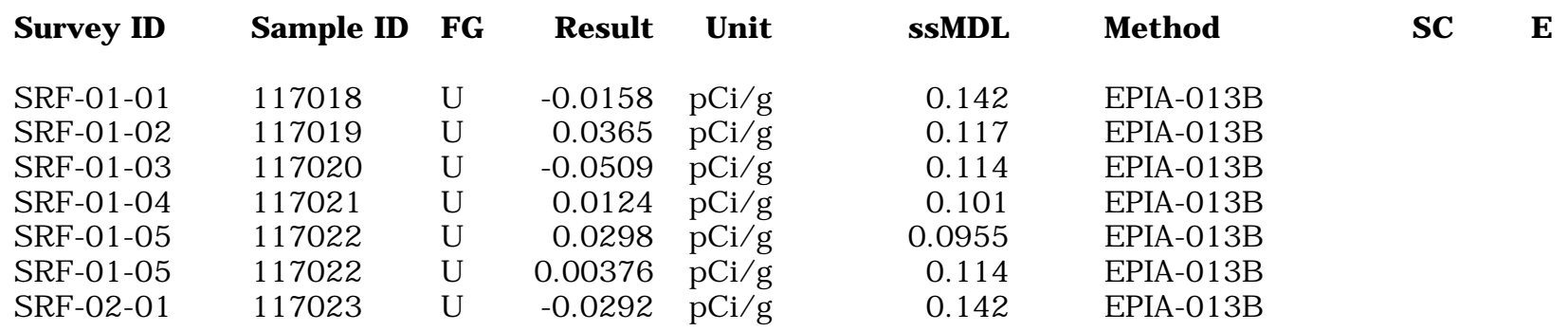


ANALYTE: Antimony-125 (cont.)

$\begin{array}{lllrlrl}\text { Survey ID } & \text { Sample ID } & \text { FG } & \text { Result } & \text { Unit } & \text { ssMDL } & \text { Method } \\ \text { SRF-02-02 } & 117024 & \mathrm{U} & -0.0076 & \mathrm{pCi} / \mathrm{g} & 0.102 & \text { EPIA-013B } \\ \text { SRF-02-03 } & 117025 & \mathrm{U} & -0.106 & \mathrm{pCi} / \mathrm{g} & 0.16 & \text { EPIA-013B } \\ \text { SRF-02-03-A } & 117026 & \mathrm{U} & 0.035 & \mathrm{pCi} / \mathrm{g} & 0.168 & \text { EPIA-013B } \\ \text { SRF-03-01 } & 117027 & \mathrm{U} & 0.0385 & \mathrm{pCi} / \mathrm{g} & 0.153 & \text { EPIA-013B } \\ \text { SRF-03-02 } & 117028 & \mathrm{U} & 0.0238 & \mathrm{pCi} / \mathrm{g} & 0.105 & \text { EPIA-013B } \\ \text { SRF-03-03 } & 117029 & \mathrm{U} & 0.0233 & \mathrm{pCi} / \mathrm{g} & 0.135 & \text { EPIA-013B } \\ \text { SRF-03-04 } & 117030 & \mathrm{U} & 0.0121 & \mathrm{pCi} / \mathrm{g} & 0.0746 & \text { EPIA-013B } \\ \text { SRF-04-01 } & 117031 & \mathrm{U} & 0.00765 & \mathrm{pCi} / \mathrm{g} & 0.122 & \text { EPIA-013B } \\ \text { SRF-04-02 } & 117032 & \mathrm{U} & 0.0531 & \mathrm{pCi} / \mathrm{g} & 0.166 & \text { EPIA-013B } \\ \text { SRF-04-03 } & 117033 & \mathrm{U} & 0.0995 & \mathrm{pCi} / \mathrm{g} & 0.163 & \text { EPIA-013B } \\ \text { SRF-05-01 } & 117034 & \mathrm{U} & -0.0328 & \mathrm{pCi} / \mathrm{g} & 0.1 & \text { EPIA-013B } \\ \text { SRF-05-02 } & 117035 & \mathrm{U} & -0.00353 & \mathrm{pCi} / \mathrm{g} & 0.109 & \text { EPIA-013B } \\ \text { SRF-05-03 } & 117036 & \mathrm{U} & 0.0032 & \mathrm{pCi} / \mathrm{g} & 0.139 & \text { EPIA-013B } \\ \text { SRF-06-01 } & 117037 & \mathrm{U} & 0.0596 & \mathrm{pCi} / \mathrm{g} & 0.143 & \text { EPIA-013B } \\ \text { SRF-06-02 } & 117038 & \mathrm{U} & 0.0331 & \mathrm{pCi} / \mathrm{g} & 0.106 & \text { EPIA-013B } \\ \text { SRF-06-03 } & 117039 & \mathrm{U} & -0.0504 & \mathrm{pCi} / \mathrm{g} & 0.169 & \text { EPIA-013B } \\ \text { SRF-06-03-A } & 117040 & \mathrm{U} & 0.0159 & \mathrm{pCi/g} & 0.16 & \text { EPIA-013B } \\ \text { SRF-07-01 } & 117041 & \mathrm{U} & 0.0414 & \mathrm{pCi} / \mathrm{g} & 0.171 & \text { EPIA-013B } \\ \text { SRF-07-02 } & 117042 & \mathrm{U} & -0.0796 & \mathrm{pCi} / \mathrm{g} & 0.152 & \text { EPIA-013B } \\ \text { SRF-07-03 } & 117043 & \mathrm{U} & -0.0129 & \mathrm{pCi} / \mathrm{g} & 0.108 & \text { EPIA-013B } \\ \text { SRF-07-03 } & 117043 & \mathrm{U} & 0.123 & \mathrm{pCi} / \mathrm{g} & 0.179 & \text { EPIA-013B }\end{array}$

ANALYTE: Antimony

Analytical Group: Metals (total recoverable)

\begin{tabular}{|c|c|c|c|c|c|c|c|}
\hline Survey ID & Sample ID & $\mathbf{F G}$ & Result & Unit & SSMDL & Method & SC \\
\hline SRF-01-01 & 117018 & $\mathrm{U}$ & 0.455 & $\mathrm{mg} / \mathrm{kg}$ & 0.174 & EPA6010B & \\
\hline SRF-01-02 & 117019 & JU & 0.205 & $\mathrm{mg} / \mathrm{kg}$ & 0.182 & EPA6010B & \\
\hline SRF-01-03 & 117020 & $\mathrm{U}$ & 0.455 & $\mathrm{mg} / \mathrm{kg}$ & 0.174 & EPA6010B & \\
\hline SRF-01-04 & 117021 & JU & 0.259 & $\mathrm{mg} / \mathrm{kg}$ & 0.177 & EPA6010B & \\
\hline SRF-01-05 & 117022 & $\mathrm{U}$ & 0.459 & $\mathrm{mg} / \mathrm{kg}$ & 0.175 & EPA6010B & \\
\hline SRF-02-01 & 117023 & $\mathrm{U}$ & 0.467 & $\mathrm{mg} / \mathrm{kg}$ & 0.178 & EPA6010B & \\
\hline SRF-02-02 & 117024 & $\mathrm{U}$ & 0.481 & $\mathrm{mg} / \mathrm{kg}$ & 0.184 & EPA6010B & \\
\hline SRF-02-03 & 117025 & $\mathrm{U}$ & 0.5 & $\mathrm{mg} / \mathrm{kg}$ & 0.191 & EPA6010B & \\
\hline SRF-02-03-A & 117026 & $\mathrm{U}$ & 0.472 & $\mathrm{mg} / \mathrm{kg}$ & 0.18 & EPA6010B & \\
\hline SRF-03-01 & 117027 & $\mathrm{U}$ & 0.495 & $\mathrm{mg} / \mathrm{kg}$ & 0.189 & EPA6010B & \\
\hline SRF-03-02 & 117028 & $\mathrm{U}$ & 0.485 & $\mathrm{mg} / \mathrm{kg}$ & 0.185 & EPA6010B & \\
\hline SRF-03-03 & 117029 & $\mathrm{U}$ & 0.467 & $\mathrm{mg} / \mathrm{kg}$ & 0.178 & EPA6010B & \\
\hline SRF-03-04 & 117030 & JU & 0.196 & $\mathrm{mg} / \mathrm{kg}$ & 0.187 & EPA6010B & \\
\hline SRF-04-01 & 117031 & JU & 0.221 & $\mathrm{mg} / \mathrm{kg}$ & 0.184 & EPA6010B & \\
\hline SRF-04-02 & 117032 & $\mathrm{U}$ & 0.459 & $\mathrm{mg} / \mathrm{kg}$ & 0.175 & EPA6010B & \\
\hline SRF-04-03 & 117033 & $\mathrm{U}$ & 0.495 & $\mathrm{mg} / \mathrm{kg}$ & 0.189 & EPA6010B & \\
\hline SRF-05-01 & 117034 & $\mathrm{U}$ & 0.485 & $\mathrm{mg} / \mathrm{kg}$ & 0.185 & EPA6010B & \\
\hline SRF-05-02 & 117035 & $\mathrm{U}$ & 0.476 & $\mathrm{mg} / \mathrm{kg}$ & 0.182 & EPA6010B & \\
\hline SRF-05-03 & 117036 & $\mathrm{U}$ & 0.467 & $\mathrm{mg} / \mathrm{kg}$ & 0.178 & EPA6010B & \\
\hline SRF-06-01 & 117037 & JU & 0.185 & $\mathrm{mg} / \mathrm{kg}$ & 0.182 & EPA6010B & \\
\hline SRF-06-02 & 117038 & $\mathrm{U}$ & 0.463 & $\mathrm{mg} / \mathrm{kg}$ & 0.177 & EPA6010B & \\
\hline SRF-06-03 & 117039 & JU & 0.186 & $\mathrm{mg} / \mathrm{kg}$ & 0.182 & EPA6010B & \\
\hline SRF-06-03-A & 117040 & $\mathrm{U}$ & 0.495 & $\mathrm{mg} / \mathrm{kg}$ & 0.189 & EPA6010B & \\
\hline SRF-07-01 & 117041 & JU & 0.233 & $\mathrm{mg} / \mathrm{kg}$ & 0.184 & EPA6010B & \\
\hline SRF-07-02 & 117042 & JU & 0.177 & $\mathrm{mg} / \mathrm{kg}$ & 0.0788 & EPA6010B & \\
\hline SRF-07-03 & 117043 & JU & 0.263 & $\mathrm{mg} / \mathrm{kg}$ & 0.175 & EPA6010B & \\
\hline
\end{tabular}


ANALYTE: Arsenic

Analytical Group: Metals (total recoverable)

$\begin{array}{ll}\text { Survey ID } & \text { Sample } \\ \text { SRF-01-01 } & 117018 \\ \text { SRF-01-02 } & 117019 \\ \text { SRF-01-03 } & 117020 \\ \text { SRF-01-04 } & 117021 \\ \text { SRF-01-05 } & 117022 \\ \text { SRF-02-01 } & 117023 \\ \text { SRF-02-02 } & 117024 \\ \text { SRF-02-03 } & 117025 \\ \text { SRF-02-03-A } & 117026 \\ \text { SRF-03-01 } & 117027 \\ \text { SRF-03-02 } & 117028 \\ \text { SRF-03-03 } & 117029 \\ \text { SRF-03-04 } & 117030 \\ \text { SRF-04-01 } & 117031 \\ \text { SRF-04-02 } & 117032 \\ \text { SRF-04-03 } & 117033 \\ \text { SRF-05-01 } & 117034 \\ \text { SRF-05-02 } & 117035 \\ \text { SRF-05-03 } & 117036 \\ \text { SRF-06-01 } & 117037 \\ \text { SRF-06-02 } & 117038 \\ \text { SRF-06-03 } & 117039 \\ \text { SRF-06-03-A } & 117040 \\ \text { SRF-07-01 } & 117041 \\ \text { SRF-07-02 } & 117042 \\ \text { SRF-07-03 } & 117043\end{array}$

\begin{tabular}{|c|c|}
\hline Result & Unit \\
\hline 0.279 & $\mathrm{mg} / \mathrm{kg}$ \\
\hline 0.621 & $\mathrm{mg} / \mathrm{kg}$ \\
\hline 0.841 & $\mathrm{mg} / \mathrm{kg}$ \\
\hline 0.355 & $\mathrm{mg} / \mathrm{kg}$ \\
\hline 0.543 & $\mathrm{mg} / \mathrm{kg}$ \\
\hline 0.534 & $\mathrm{mg} / \mathrm{kg}$ \\
\hline 0.55 & $\mathrm{mg} / \mathrm{kg}$ \\
\hline 0.535 & $\mathrm{mg} / \mathrm{kg}$ \\
\hline 0.666 & $\mathrm{mg} / \mathrm{kg}$ \\
\hline 0.465 & $\mathrm{mg} / \mathrm{kg}$ \\
\hline 0.546 & $\mathrm{mg} / \mathrm{kg}$ \\
\hline 0.713 & $\mathrm{mg} / \mathrm{kg}$ \\
\hline 0.638 & $\mathrm{mg} / \mathrm{kg}$ \\
\hline 0.528 & $\mathrm{mg} / \mathrm{kg}$ \\
\hline 0.544 & $\mathrm{mg} / \mathrm{kg}$ \\
\hline 0.586 & $\mathrm{mg} / \mathrm{kg}$ \\
\hline 0.41 & $\mathrm{mg} / \mathrm{kg}$ \\
\hline 0.496 & $\mathrm{mg} / \mathrm{kg}$ \\
\hline 0.758 & $\mathrm{mg} / \mathrm{kg}$ \\
\hline 0.604 & $\mathrm{mg} / \mathrm{kg}$ \\
\hline 0.489 & $\mathrm{mg} / \mathrm{k}$ \\
\hline 0.873 & $\mathrm{mg} / \mathrm{kg}$ \\
\hline 0.647 & $\mathrm{mg} / \mathrm{kg}$ \\
\hline 0.336 & $\mathrm{mg} / \mathrm{k}$ \\
\hline 0.354 & $\mathrm{mg} / \mathrm{k}$ \\
\hline 0.426 & $\mathrm{mg} / \mathrm{k}$ \\
\hline
\end{tabular}

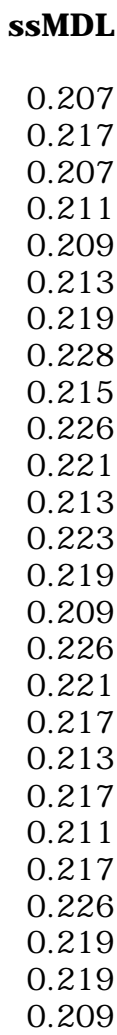

Method

SC

EPA6010B

EPA6010B

EPA6010B

EPA6010B

EPA6010B

EPA6010B

EPA6010B

EPA6010B

EPA6010B

EPA6010B

EPA6010B

EPA6010B

EPA6010B

EPA6010B

EPA6010B

EPA6010B

EPA6010B

EPA6010B

EPA6010B

EPA6010B

EPA6010B

EPA6010B

EPA6010B

EPA6010B

EPA6010B

EPA6010B

ANALYTE: Barium-133

Analytical Group: Radionuclides

\begin{tabular}{|c|c|c|c|c|c|c|}
\hline Survey ID & Sample ID & $\mathbf{F G}$ & Result & Unit & SSMDL & Method \\
\hline SRF-01-01 & 117018 & $\mathrm{U}$ & -0.0241 & $\mathrm{pCi} / \mathrm{g}$ & 0.059 & EPIA-013B \\
\hline SRF-01-02 & 117019 & $\mathrm{U}$ & 0.00484 & $\mathrm{pCi} / \mathrm{g}$ & 0.0481 & EPIA-013B \\
\hline SRF-01-03 & 117020 & $\mathrm{U}$ & -0.00135 & $\mathrm{pCi} / \mathrm{g}$ & 0.0522 & EPIA-013B \\
\hline SRF-01-04 & 117021 & $\mathrm{U}$ & 0.00889 & $\mathrm{pCi} / \mathrm{g}$ & 0.0478 & EPIA-013B \\
\hline SRF-01-05 & 117022 & $\mathrm{U}$ & 0.0286 & $\mathrm{pCi} / \mathrm{g}$ & 0.0523 & EPIA-013B \\
\hline SRF-01-05 & 117022 & $\mathrm{U}$ & 0.00386 & $\mathrm{pCi} / \mathrm{g}$ & 0.0515 & EPIA-013B \\
\hline SRF-02-01 & 117023 & $\mathrm{U}$ & -0.00508 & $\mathrm{pCi} / \mathrm{g}$ & 0.0604 & EPIA-013B \\
\hline SRF-02-02 & 117024 & $\mathrm{U}$ & -0.0242 & $\mathrm{pCi} / \mathrm{g}$ & 0.0383 & EPIA-013B \\
\hline SRF-02-03 & 117025 & $\mathrm{U}$ & 0.0178 & $\mathrm{pCi} / \mathrm{g}$ & 0.0759 & EPIA-013B \\
\hline SRF-02-03-A & 117026 & $\mathrm{U}$ & 0.00484 & $\mathrm{pCi} / \mathrm{g}$ & 0.0702 & EPIA-013B \\
\hline SRF-03-01 & 117027 & $\mathrm{U}$ & -0.0676 & $\mathrm{pCi} / \mathrm{g}$ & 0.0591 & EPIA-013B \\
\hline SRF-03-02 & 117028 & $\mathrm{U}$ & 0.0213 & $\mathrm{pCi} / \mathrm{g}$ & 0.0522 & EPIA-013B \\
\hline SRF-03-03 & 117029 & $\mathrm{U}$ & 0.00717 & $\mathrm{pCi} / \mathrm{g}$ & 0.0562 & EPIA-013B \\
\hline SRF-03-04 & 117030 & $\mathrm{U}$ & -0.00926 & $\mathrm{pCi} / \mathrm{g}$ & 0.0344 & EPIA-013B \\
\hline SRF-04-01 & 117031 & $\mathrm{U}$ & -0.0183 & $\mathrm{pCi} / \mathrm{g}$ & 0.0525 & EPIA-013B \\
\hline SRF-04-02 & 117032 & $\mathrm{U}$ & 0.0114 & $\mathrm{pCi} / \mathrm{g}$ & 0.0703 & EPIA-013B \\
\hline SRF-04-03 & 117033 & $\mathrm{U}$ & 0.0484 & $\mathrm{pCi} / \mathrm{g}$ & 0.0728 & EPIA-013B \\
\hline SRF-05-01 & 117034 & $\mathrm{U}$ & 0.00224 & $\mathrm{pCi} / \mathrm{g}$ & 0.0488 & EPIA-013B \\
\hline SRF-05-02 & 117035 & $\mathrm{U}$ & 0.0256 & $\mathrm{pCi} / \mathrm{g}$ & 0.0524 & EPIA-013B \\
\hline SRF-05-03 & 117036 & $\mathrm{U}$ & 0.00258 & $\mathrm{pCi} / \mathrm{g}$ & 0.0558 & EPIA-013B \\
\hline SRF-06-01 & 117037 & $\mathrm{U}$ & 0.0181 & $\mathrm{pCi} / \mathrm{g}$ & 0.0613 & EPIA-013B \\
\hline SRF-06-02 & 117038 & $\mathrm{U}$ & -0.019 & $\mathrm{pCi} / \mathrm{g}$ & 0.0468 & EPIA-013B \\
\hline SRF-06-03 & 117039 & $\mathrm{U}$ & -0.0279 & $\mathrm{pCi} / \mathrm{g}$ & 0.0755 & EPIA-013B \\
\hline
\end{tabular}


ANALYTE: Barium-133 (cont.)

$\begin{array}{lllrlrl}\text { Survey ID } & \text { Sample ID } & \text { FG } & \text { Result } & \text { Unit } & \text { ssMDL } & \text { Method } \\ \text { SRF-06-03-A } & 117040 & \mathrm{U} & 0.00575 & \mathrm{pCi} / \mathrm{g} & 0.0767 & \text { EPIA-013B } \\ \text { SRF-07-01 } & 117041 & \mathrm{U} & 0.0439 & \mathrm{pCi} / \mathrm{g} & 0.0669 & \text { EPIA-013B } \\ \text { SRF-07-02 } & 117042 & \mathrm{U} & -0.0363 & \mathrm{pCi} / \mathrm{g} & 0.0776 & \text { EPIA-013B } \\ \text { SRF-07-03 } & 117043 & \mathrm{U} & 0.0433 & \mathrm{pCi} / \mathrm{g} & 0.078 & \text { EPIA-013B } \\ \text { SRF-07-03 } & 117043 & \mathrm{U} & 0.00128 & \mathrm{pCi} / \mathrm{g} & 0.0592 & \text { EPIA-013B }\end{array}$

ANALYTE: Barium

Analytical Group: Metals (total recoverable)

\begin{tabular}{|c|c|c|c|c|c|c|c|}
\hline Survey ID & Sample ID & $\mathbf{F G}$ & Result & Unit & SSMDL & Method & SC \\
\hline SRF-01-01 & 117018 & & 3.9 & $\mathrm{mg} / \mathrm{kg}$ & 0.025 & EPA6010B & \\
\hline SRF-01-02 & 117019 & & 3.93 & $\mathrm{mg} / \mathrm{kg}$ & 0.026 & EPA6010B & \\
\hline SRF-01-03 & 117020 & & 3.18 & $\mathrm{mg} / \mathrm{kg}$ & 0.025 & EPA6010B & \\
\hline SRF-01-04 & 117021 & & 3.92 & $\mathrm{mg} / \mathrm{kg}$ & 0.025 & EPA6010B & \\
\hline SRF-01-05 & 117022 & & 3.33 & $\mathrm{mg} / \mathrm{kg}$ & 0.024 & EPA6010B & \\
\hline SRF-02-01 & 117023 & & 6.5 & $\mathrm{mg} / \mathrm{kg}$ & 0.025 & EPA6010B & \\
\hline SRF-02-02 & 117024 & & 3.84 & $\mathrm{mg} / \mathrm{kg}$ & 0.026 & EPA6010B & \\
\hline SRF-02-03 & 117025 & & 2.96 & $\mathrm{mg} / \mathrm{kg}$ & 0.027 & EPA6010B & \\
\hline SRF-02-03-A & 117026 & & 5.99 & $\mathrm{mg} / \mathrm{kg}$ & 0.025 & EPA6010B & \\
\hline SRF-03-01 & 117027 & & 1.74 & $\mathrm{mg} / \mathrm{kg}$ & 0.027 & EPA6010B & \\
\hline SRF-03-02 & 117028 & & 6.63 & $\mathrm{mg} / \mathrm{kg}$ & 0.026 & EPA6010B & \\
\hline SRF-03-03 & 117029 & & 4.43 & $\mathrm{mg} / \mathrm{kg}$ & 0.025 & EPA6010B & \\
\hline SRF-03-04 & 117030 & & 7.83 & $\mathrm{mg} / \mathrm{kg}$ & 0.026 & EPA6010B & \\
\hline SRF-04-01 & 117031 & & 5.82 & $\mathrm{mg} / \mathrm{kg}$ & 0.026 & EPA6010B & \\
\hline SRF-04-02 & 117032 & & 3.25 & $\mathrm{mg} / \mathrm{kg}$ & 0.024 & EPA6010B & \\
\hline SRF-04-03 & 117033 & & 0.427 & $\mathrm{mg} / \mathrm{kg}$ & 0.027 & EPA6010B & \\
\hline SRF-05-01 & 117034 & & 5.5 & $\mathrm{mg} / \mathrm{kg}$ & 0.026 & EPA6010B & \\
\hline SRF-05-02 & 117035 & & 3.96 & $\mathrm{mg} / \mathrm{kg}$ & 0.026 & EPA6010B & \\
\hline SRF-05-03 & 117036 & & 1.46 & $\mathrm{mg} / \mathrm{kg}$ & 0.025 & EPA6010B & \\
\hline SRF-06-01 & 117037 & & 6.58 & $\mathrm{mg} / \mathrm{kg}$ & 0.026 & EPA6010B & \\
\hline SRF-06-02 & 117038 & & 3.82 & $\mathrm{mg} / \mathrm{kg}$ & 0.025 & EPA6010B & \\
\hline SRF-06-03 & 117039 & & 2.02 & $\mathrm{mg} / \mathrm{kg}$ & 0.026 & EPA6010B & \\
\hline SRF-06-03-A & 117040 & & 3.06 & $\mathrm{mg} / \mathrm{kg}$ & 0.027 & EPA6010B & \\
\hline SRF-07-01 & 117041 & & 1.8 & $\mathrm{mg} / \mathrm{kg}$ & 0.026 & EPA6010B & \\
\hline SRF-07-02 & 117042 & & 2.69 & $\mathrm{mg} / \mathrm{kg}$ & 0.026 & EPA6010B & \\
\hline SRF-07-03 & 117043 & & 2.68 & $\mathrm{mg} / \mathrm{kg}$ & 0.024 & EPA6010B & \\
\hline
\end{tabular}

ANALYTE: Benzene

Analytical Group: Volatile Organic Compounds

\begin{tabular}{|c|c|c|c|c|c|c|c|}
\hline Survey ID & Sample ID & $\mathbf{F G}$ & Result & Unit & SSMDL & Method & SC \\
\hline SRF-01-01 & 117018 & $\mathrm{~J}$ & 10.3 & $\mu g / \mathrm{kg}$ & 2.5 & EPA8260B & $\mathrm{K}$ \\
\hline SRF-01-02 & 117019 & $\mathrm{~J}$ & 5.63 & $\mu \mathrm{g} / \mathrm{kg}$ & 2.5 & EPA8260B & $\mathrm{K}$ \\
\hline SRF-01-03 & 117020 & $\mathrm{U}$ & 5 & $\mu \mathrm{g} / \mathrm{kg}$ & 2.5 & EPA8260B & \\
\hline SRF-01-04 & 117021 & $\mathrm{~J}$ & 2.64 & $\mu g / \mathrm{kg}$ & 2.5 & EPA8260B & IK \\
\hline SRF-01-05 & 117022 & $\mathrm{~J}$ & 3.44 & $\mu g / \mathrm{kg}$ & 2.5 & EPA8260B & IK \\
\hline SRF-02-01 & 117023 & $\mathrm{U}$ & 5 & $\mu g / k g$ & 2.5 & EPA8260B & \\
\hline SRF-02-02 & 117024 & $\mathrm{U}$ & 5 & $\mu \mathrm{g} / \mathrm{kg}$ & 2.5 & EPA8260B & \\
\hline SRF-02-03 & 117025 & $\mathrm{U}$ & 5 & $\mu \mathrm{g} / \mathrm{kg}$ & 2.5 & EPA8260B & \\
\hline SRF-02-03-A & 117026 & $\mathrm{U}$ & 5 & $\mu g / k g$ & 2.5 & EPA8260B & \\
\hline SRF-03-01 & 117027 & $\mathrm{U}$ & 5 & $\mu \mathrm{g} / \mathrm{kg}$ & 2.5 & EPA8260B & \\
\hline SRF-03-02 & 117028 & $\mathrm{U}$ & 5 & $\mu g / \mathrm{kg}$ & 2.5 & EPA8260B & \\
\hline SRF-03-03 & 117029 & $\mathrm{U}$ & 5 & $\mu \mathrm{g} / \mathrm{kg}$ & 2.5 & EPA8260B & \\
\hline
\end{tabular}


ANALYTE: Benzene (cont.)

\begin{tabular}{|c|c|c|c|c|c|c|c|}
\hline Survey ID & Sample ID & FG & Result & Unit & SsMDL & Method & SC \\
\hline SRF-03-04 & 117030 & $\mathrm{U}$ & 5 & $\mu g / \mathrm{kg}$ & 2.5 & EPA8260B & \\
\hline SRF-04-01 & 117031 & $\mathrm{U}$ & 5 & $\mu \mathrm{g} / \mathrm{kg}$ & 2.5 & EPA8260B & \\
\hline SRF-04-02 & 117032 & $\mathrm{U}$ & 5 & $\mu g / k g$ & 2.5 & EPA8260B & \\
\hline SRF-04-03 & 117033 & $\mathrm{U}$ & 5 & $\mu \mathrm{g} / \mathrm{kg}$ & 2.5 & EPA8260B & \\
\hline SRF-05-01 & 117034 & $\mathrm{U}$ & 5 & $\mu g / \mathrm{kg}$ & 2.5 & EPA8260B & \\
\hline SRF-05-02 & 117035 & $\mathrm{U}$ & 5 & $\mu g / \mathrm{kg}$ & 2.5 & EPA8260B & \\
\hline SRF-05-03 & 117036 & $\mathrm{U}$ & 5 & $\mu g / k g$ & 2.5 & EPA8260B & \\
\hline SRF-06-01 & 117037 & $\mathrm{U}$ & 5 & $\mu g / \mathrm{kg}$ & 2.5 & EPA8260B & \\
\hline SRF-06-02 & 117038 & $\mathrm{U}$ & 5 & $\mu \mathrm{g} / \mathrm{kg}$ & 2.5 & EPA8260B & \\
\hline SRF-06-03 & 117039 & $\mathrm{U}$ & 5 & $\mu \mathrm{g} / \mathrm{kg}$ & 2.5 & EPA8260B & \\
\hline SRF-06-03-A & 117040 & $\mathrm{U}$ & 5 & $\mu \mathrm{g} / \mathrm{kg}$ & 2.5 & EPA8260B & \\
\hline SRF-07-01 & 117041 & $\mathrm{~J}$ & 2.6 & $\mu g / \mathrm{kg}$ & 2.5 & EPA8260B & IK \\
\hline SRF-07-02 & 117042 & $\mathrm{~J}$ & 4.84 & $\mu \mathrm{g} / \mathrm{kg}$ & 2.5 & EPA8260B & IK \\
\hline SRF-07-03 & 117043 & $\mathrm{U}$ & 5 & $\mu g / \mathrm{kg}$ & 2.5 & EPA8260B & \\
\hline
\end{tabular}

ANALYTE: Bromodichloromethane

Analytical Group: Volatile Organic Compounds

\begin{tabular}{|c|c|c|c|c|c|c|c|}
\hline Survey ID & Sample ID & $\mathbf{F G}$ & Result & Unit & SSMDL & Method & SC \\
\hline SRF-01-01 & 117018 & $\mathrm{U}$ & 5 & $\mu g / \mathrm{kg}$ & 0.5 & EPA8260B & \\
\hline SRF-01-02 & 117019 & $\mathrm{U}$ & 5 & $\mu g / \mathrm{kg}$ & 0.5 & EPA8260B & \\
\hline SRF-01-03 & 117020 & $\mathrm{U}$ & 5 & $\mu g / k g$ & 0.5 & EPA8260B & \\
\hline SRF-01-04 & 117021 & $\mathrm{U}$ & 5 & $\mu g / \mathrm{kg}$ & 0.5 & EPA8260B & \\
\hline SRF-01-05 & 117022 & $\mathrm{U}$ & 5 & $\mu \mathrm{g} / \mathrm{kg}$ & 0.5 & EPA8260B & \\
\hline SRF-02-01 & 117023 & $\mathrm{U}$ & 5 & $\mu \mathrm{g} / \mathrm{kg}$ & 0.5 & EPA8260B & \\
\hline SRF-02-02 & 117024 & $\mathrm{U}$ & 5 & $\mu \mathrm{g} / \mathrm{kg}$ & 0.5 & EPA8260B & \\
\hline SRF-02-03 & 117025 & $\mathrm{U}$ & 5 & $\mu g / k g$ & 0.5 & EPA8260B & \\
\hline SRF-02-03-A & 117026 & $\mathrm{U}$ & 5 & $\mu \mathrm{g} / \mathrm{kg}$ & 0.5 & EPA8260B & \\
\hline SRF-03-01 & 117027 & $\mathrm{U}$ & 5 & $\mu g / \mathrm{kg}$ & 0.5 & EPA8260B & \\
\hline SRF-03-02 & 117028 & $\mathrm{U}$ & 5 & $\mu g / \mathrm{kg}$ & 0.5 & EPA8260B & \\
\hline SRF-03-03 & 117029 & $\mathrm{U}$ & 5 & $\mu g / \mathrm{kg}$ & 0.5 & EPA8260B & \\
\hline SRF-03-04 & 117030 & $\mathrm{U}$ & 5 & $\mu \mathrm{g} / \mathrm{kg}$ & 0.5 & EPA8260B & \\
\hline SRF-04-01 & 117031 & $\mathrm{U}$ & 5 & $\mu g / k g$ & 0.5 & EPA8260B & \\
\hline SRF-04-02 & 117032 & $\mathrm{U}$ & 5 & $\mu g / \mathrm{kg}$ & 0.5 & EPA8260B & \\
\hline SRF-04-03 & 117033 & $\mathrm{U}$ & 5 & $\mu g / \mathrm{kg}$ & 0.5 & EPA8260B & \\
\hline SRF-05-01 & 117034 & $\mathrm{U}$ & 5 & $\mu \mathrm{g} / \mathrm{kg}$ & 0.5 & EPA8260B & \\
\hline SRF-05-02 & 117035 & $\mathrm{U}$ & 5 & $\mu g / k g$ & 0.5 & EPA8260B & \\
\hline SRF-05-03 & 117036 & $\mathrm{U}$ & 5 & $\mu g / \mathrm{kg}$ & 0.5 & EPA8260B & \\
\hline SRF-06-01 & 117037 & $\mathrm{U}$ & 5 & $\mu \mathrm{g} / \mathrm{kg}$ & 0.5 & EPA8260B & \\
\hline SRF-06-02 & 117038 & $\mathrm{U}$ & 5 & $\mu g / \mathrm{kg}$ & 0.5 & EPA8260B & \\
\hline SRF-06-03 & 117039 & $\mathrm{U}$ & 5 & $\mu \mathrm{g} / \mathrm{kg}$ & 0.5 & EPA8260B & \\
\hline SRF-06-03-A & 117040 & $\mathrm{U}$ & 5 & $\mu g / \mathrm{kg}$ & 0.5 & EPA8260B & \\
\hline SRF-07-01 & 117041 & $\mathrm{U}$ & 5 & $\mu \mathrm{g} / \mathrm{kg}$ & 0.5 & EPA8260B & \\
\hline SRF-07-02 & 117042 & $\mathrm{U}$ & 5 & $\mu g / \mathrm{kg}$ & 0.5 & EPA8260B & \\
\hline SRF-07-03 & 117043 & $\mathrm{U}$ & 5 & $\mu g / k g$ & 0.5 & EPA8260B & \\
\hline
\end{tabular}

ANALYTE: Bromoform

Analytical Group: Volatile Organic Compounds

\begin{tabular}{|c|c|c|c|c|c|c|c|}
\hline Survey ID & Sample ID & $\mathbf{F G}$ & Result & Unit & SSMDL & Method & SC \\
\hline SRF-01-01 & 117018 & $\mathrm{U}$ & 5 & $\mu \mathrm{g} / \mathrm{kg}$ & 1.35 & EPA8260B & \\
\hline SRF-01-02 & 117019 & $\mathrm{U}$ & 5 & $\mu g / k g$ & 1.35 & EPA8260B & \\
\hline SRF-01-03 & 117020 & $\mathrm{U}$ & 5 & $\mu \mathrm{g} / \mathrm{kg}$ & 1.35 & EPA8260B & \\
\hline
\end{tabular}


ANALYTE: Bromoform (cont.)

\begin{tabular}{|c|c|c|c|c|c|c|c|}
\hline Survey ID & Sample ID & $\mathbf{F G}$ & Result & Unit & SSMDL & Method & SC \\
\hline SRF-01-04 & 117021 & $\mathrm{U}$ & 5 & $\mu g / \mathrm{kg}$ & 1.35 & EPA8260B & \\
\hline SRF-01-05 & 117022 & $\mathrm{U}$ & 5 & $\mu \mathrm{g} / \mathrm{kg}$ & 1.35 & EPA8260B & \\
\hline SRF-02-01 & 117023 & $\mathrm{U}$ & 5 & $\mu g / \mathrm{kg}$ & 1.35 & EPA8260B & \\
\hline SRF-02-02 & 117024 & $\mathrm{U}$ & 5 & $\mu \mathrm{g} / \mathrm{kg}$ & 1.35 & EPA8260B & \\
\hline SRF-02-03 & 117025 & $\mathrm{U}$ & 5 & $\mu g / k g$ & 1.35 & EPA8260B & \\
\hline SRF-02-03-A & 117026 & $\mathrm{U}$ & 5 & $\mu \mathrm{g} / \mathrm{kg}$ & 1.35 & EPA8260B & \\
\hline SRF-03-01 & 117027 & $\mathrm{U}$ & 5 & $\mu g / \mathrm{kg}$ & 1.35 & EPA8260B & \\
\hline SRF-03-02 & 117028 & $\mathrm{U}$ & 5 & $\mu g / \mathrm{kg}$ & 1.35 & EPA8260B & \\
\hline SRF-03-03 & 117029 & $\mathrm{U}$ & 5 & $\mu g / k g$ & 1.35 & EPA8260B & \\
\hline SRF-03-04 & 117030 & $\mathrm{U}$ & 5 & $\mu g / \mathrm{kg}$ & 1.35 & EPA8260B & \\
\hline SRF-04-01 & 117031 & $\mathrm{U}$ & 5 & $\mu \mathrm{g} / \mathrm{kg}$ & 1.35 & EPA8260B & \\
\hline SRF-04-02 & 117032 & $\mathrm{U}$ & 5 & $\mu g / \mathrm{kg}$ & 1.35 & EPA8260B & \\
\hline SRF-04-03 & 117033 & $\mathrm{U}$ & 5 & $\mu \mathrm{g} / \mathrm{kg}$ & 1.35 & EPA8260B & \\
\hline SRF-05-01 & 117034 & $\mathrm{U}$ & 5 & $\mu \mathrm{g} / \mathrm{kg}$ & 1.35 & EPA8260B & \\
\hline SRF-05-02 & 117035 & $\mathrm{U}$ & 5 & $\mu \mathrm{g} / \mathrm{kg}$ & 1.35 & EPA8260B & \\
\hline SRF-05-03 & 117036 & $\mathrm{U}$ & 5 & $\mu g / \mathrm{kg}$ & 1.35 & EPA8260B & \\
\hline SRF-06-01 & 117037 & $\mathrm{U}$ & 5 & $\mu \mathrm{g} / \mathrm{kg}$ & 1.35 & EPA8260B & \\
\hline SRF-06-02 & 117038 & $\mathrm{U}$ & 5 & $\mu g / \mathrm{kg}$ & 1.35 & EPA8260B & \\
\hline SRF-06-03 & 117039 & $\mathrm{U}$ & 5 & $\mu \mathrm{g} / \mathrm{kg}$ & 1.35 & EPA8260B & \\
\hline SRF-06-03-A & 117040 & $\mathrm{U}$ & 5 & $\mu g / k g$ & 1.35 & EPA8260B & \\
\hline SRF-07-01 & 117041 & $\mathrm{U}$ & 5 & $\mu \mathrm{g} / \mathrm{kg}$ & 1.35 & EPA8260B & \\
\hline SRF-07-02 & 117042 & $\mathrm{U}$ & 5 & $\mu \mathrm{g} / \mathrm{kg}$ & 1.35 & EPA8260B & \\
\hline SRF-07-03 & 117043 & $\mathrm{U}$ & 5 & $\mu \mathrm{g} / \mathrm{kg}$ & 1.35 & EPA8260B & \\
\hline
\end{tabular}

ANALYTE: Bromomethane (Methyl bromide)

Analytical Group: Volatile Organic Compounds

\begin{tabular}{|c|c|c|c|c|c|c|c|}
\hline Survey ID & Sample ID & $\mathbf{F G}$ & Result & Unit & ssMDL & Method & SC \\
\hline SRF-01-01 & 117018 & $\mathrm{U}$ & 5 & $\mu \mathrm{g} / \mathrm{kg}$ & 1.5 & EPA8260B & \\
\hline SRF-01-02 & 117019 & $\mathrm{U}$ & 5 & $\mu g / \mathrm{kg}$ & 1.5 & EPA8260B & \\
\hline SRF-01-03 & 117020 & $\mathrm{U}$ & 5 & $\mu \mathrm{g} / \mathrm{kg}$ & 1.5 & EPA8260B & \\
\hline SRF-01-04 & 117021 & $\mathrm{U}$ & 5 & $\mu g / k g$ & 1.5 & EPA8260B & \\
\hline SRF-01-05 & 117022 & $\mathrm{U}$ & 5 & $\mu \mathrm{g} / \mathrm{kg}$ & 1.5 & EPA8260B & \\
\hline SRF-02-01 & 117023 & $\mathrm{U}$ & 5 & $\mu g / k g$ & 1.5 & EPA8260B & \\
\hline SRF-02-02 & 117024 & $\mathrm{U}$ & 5 & $\mu g / \mathrm{kg}$ & 1.5 & EPA8260B & \\
\hline SRF-02-03 & 117025 & $\mathrm{U}$ & 5 & $\mu \mathrm{g} / \mathrm{kg}$ & 1.5 & EPA8260B & \\
\hline SRF-02-03-A & 117026 & $\mathrm{U}$ & 5 & $\mu g / \mathrm{kg}$ & 1.5 & EPA8260B & \\
\hline SRF-03-01 & 117027 & $\mathrm{U}$ & 5 & $\mu \mathrm{g} / \mathrm{kg}$ & 1.5 & EPA8260B & \\
\hline SRF-03-02 & 117028 & $\mathrm{U}$ & 5 & $\mu g / \mathrm{kg}$ & 1.5 & EPA8260B & \\
\hline SRF-03-03 & 117029 & $\mathrm{U}$ & 5 & $\mu \mathrm{g} / \mathrm{kg}$ & 1.5 & EPA8260B & \\
\hline SRF-03-04 & 117030 & $\mathrm{U}$ & 5 & $\mu \mathrm{g} / \mathrm{kg}$ & 1.5 & EPA8260B & \\
\hline SRF-04-01 & 117031 & $\mathrm{U}$ & 5 & $\mu \mathrm{g} / \mathrm{kg}$ & 1.5 & EPA8260B & \\
\hline SRF-04-02 & 117032 & $\mathrm{U}$ & 5 & $\mu g / k g$ & 1.5 & EPA8260B & \\
\hline SRF-04-03 & 117033 & $\mathrm{U}$ & 5 & $\mu \mathrm{g} / \mathrm{kg}$ & 1.5 & EPA8260B & \\
\hline SRF-05-01 & 117034 & $\mathrm{U}$ & 5 & $\mu \mathrm{g} / \mathrm{kg}$ & 1.5 & EPA8260B & \\
\hline SRF-05-02 & 117035 & $\mathrm{U}$ & 5 & $\mu g / k g$ & 1.5 & EPA8260B & \\
\hline SRF-05-03 & 117036 & $\mathrm{U}$ & 5 & $\mu g / k g$ & 1.5 & EPA8260B & \\
\hline SRF-06-01 & 117037 & $\mathrm{U}$ & 5 & $\mu g / \mathrm{kg}$ & 1.5 & EPA8260B & \\
\hline SRF-06-02 & 117038 & $\mathrm{U}$ & 5 & $\mu \mathrm{g} / \mathrm{kg}$ & 1.5 & EPA8260B & \\
\hline SRF-06-03 & 117039 & $\mathrm{U}$ & 5 & $\mu g / \mathrm{kg}$ & 1.5 & EPA8260B & \\
\hline SRF-06-03-A & 117040 & $\mathrm{U}$ & 5 & $\mu \mathrm{g} / \mathrm{kg}$ & 1.5 & EPA8260B & \\
\hline SRF-07-01 & 117041 & $\mathrm{U}$ & 5 & $\mu \mathrm{g} / \mathrm{kg}$ & 1.5 & EPA8260B & \\
\hline SRF-07-02 & 117042 & $\mathrm{U}$ & 5 & $\mu \mathrm{g} / \mathrm{kg}$ & 1.5 & EPA8260B & \\
\hline SRF-07-03 & 117043 & $\mathrm{U}$ & 5 & $\mu g / \mathrm{kg}$ & 1.5 & EPA8260B & \\
\hline
\end{tabular}


ANALYTE: Cadmium

Analytical Group: Metals (total recoverable)

\begin{tabular}{|c|c|c|c|c|c|c|c|}
\hline Survey ID & Sample ID & FG & Result & Unit & SsMDL & Method & SC \\
\hline SRF-01-01 & 117018 & $\mathrm{~J}$ & 0.0385 & $\mathrm{mg} / \mathrm{kg}$ & 0.017 & EPA6010B & I \\
\hline SRF-01-02 & 117019 & $\mathrm{~J}$ & 0.0376 & $\mathrm{mg} / \mathrm{kg}$ & 0.018 & EPA6010B & I \\
\hline SRF-01-03 & 117020 & $\mathrm{U}$ & 0.227 & $\mathrm{mg} / \mathrm{kg}$ & 0.017 & EPA6010B & \\
\hline SRF-01-04 & 117021 & JU & 0.0252 & $\mathrm{mg} / \mathrm{kg}$ & 0.018 & EPA6010B & \\
\hline SRF-01-05 & 117022 & JU & 0.0224 & $\mathrm{mg} / \mathrm{kg}$ & 0.017 & EPA6010B & \\
\hline SRF-02-01 & 117023 & $\mathrm{U}$ & 0.234 & $\mathrm{mg} / \mathrm{kg}$ & 0.018 & EPA6010B & \\
\hline SRF-02-02 & 117024 & JU & 0.0225 & $\mathrm{mg} / \mathrm{kg}$ & 0.018 & EPA6010B & \\
\hline SRF-02-03 & 117025 & $\mathrm{U}$ & 0.25 & $\mathrm{mg} / \mathrm{kg}$ & 0.019 & EPA6010B & \\
\hline SRF-02-03-A & 117026 & JU & 0.0574 & $\mathrm{mg} / \mathrm{kg}$ & 0.018 & EPA6010B & \\
\hline SRF-03-01 & 117027 & $\mathrm{~J}$ & 0.0308 & $\mathrm{mg} / \mathrm{kg}$ & 0.019 & EPA6010B & I \\
\hline SRF-03-02 & 117028 & $\mathrm{~J}$ & 0.022 & $\mathrm{mg} / \mathrm{kg}$ & 0.018 & EPA6010B & I \\
\hline SRF-03-03 & 117029 & JU & 0.0273 & $\mathrm{mg} / \mathrm{kg}$ & 0.018 & EPA6010B & \\
\hline SRF-03-04 & 117030 & $\mathrm{U}$ & 0.245 & $\mathrm{mg} / \mathrm{kg}$ & 0.019 & EPA6010B & \\
\hline SRF-04-01 & 117031 & $\mathrm{~J}$ & 0.059 & $\mathrm{mg} / \mathrm{kg}$ & 0.018 & EPA6010B & I \\
\hline SRF-04-02 & 117032 & JU & 0.0199 & $\mathrm{mg} / \mathrm{kg}$ & 0.017 & EPA6010B & \\
\hline SRF-04-03 & 117033 & $\mathrm{U}$ & 0.248 & $\mathrm{mg} / \mathrm{kg}$ & 0.019 & EPA6010B & \\
\hline SRF-05-01 & 117034 & $\mathrm{~J}$ & 0.139 & $\mathrm{mg} / \mathrm{kg}$ & 0.018 & EPA6010B & I \\
\hline SRF-05-02 & 117035 & $\mathrm{U}$ & 0.238 & $\mathrm{mg} / \mathrm{kg}$ & 0.018 & EPA6010B & \\
\hline SRF-05-03 & 117036 & $\mathrm{U}$ & 0.234 & $\mathrm{mg} / \mathrm{kg}$ & 0.018 & EPA6010B & \\
\hline SRF-06-01 & 117037 & JU & 0.0473 & $\mathrm{mg} / \mathrm{kg}$ & 0.018 & EPA6010B & \\
\hline SRF-06-02 & 117038 & JU & 0.0216 & $\mathrm{mg} / \mathrm{kg}$ & 0.018 & EPA6010B & \\
\hline SRF-06-03 & 117039 & JU & 0.0364 & $\mathrm{mg} / \mathrm{kg}$ & 0.018 & EPA6010B & \\
\hline SRF-06-03-A & 117040 & JU & 0.0248 & $\mathrm{mg} / \mathrm{kg}$ & 0.019 & EPA6010B & \\
\hline SRF-07-01 & 117041 & JU & 0.0205 & $\mathrm{mg} / \mathrm{kg}$ & 0.018 & EPA6010B & \\
\hline SRF-07-02 & 117042 & $\mathrm{U}$ & 0.24 & $\mathrm{mg} / \mathrm{kg}$ & 0.018 & EPA6010B & \\
\hline SRF-07-03 & 117043 & $\mathrm{U}$ & 0.229 & $\mathrm{mg} / \mathrm{kg}$ & 0.017 & EPA6010B & \\
\hline
\end{tabular}

ANALYTE: Calcium

Analytical Group: Metals (total recoverable)

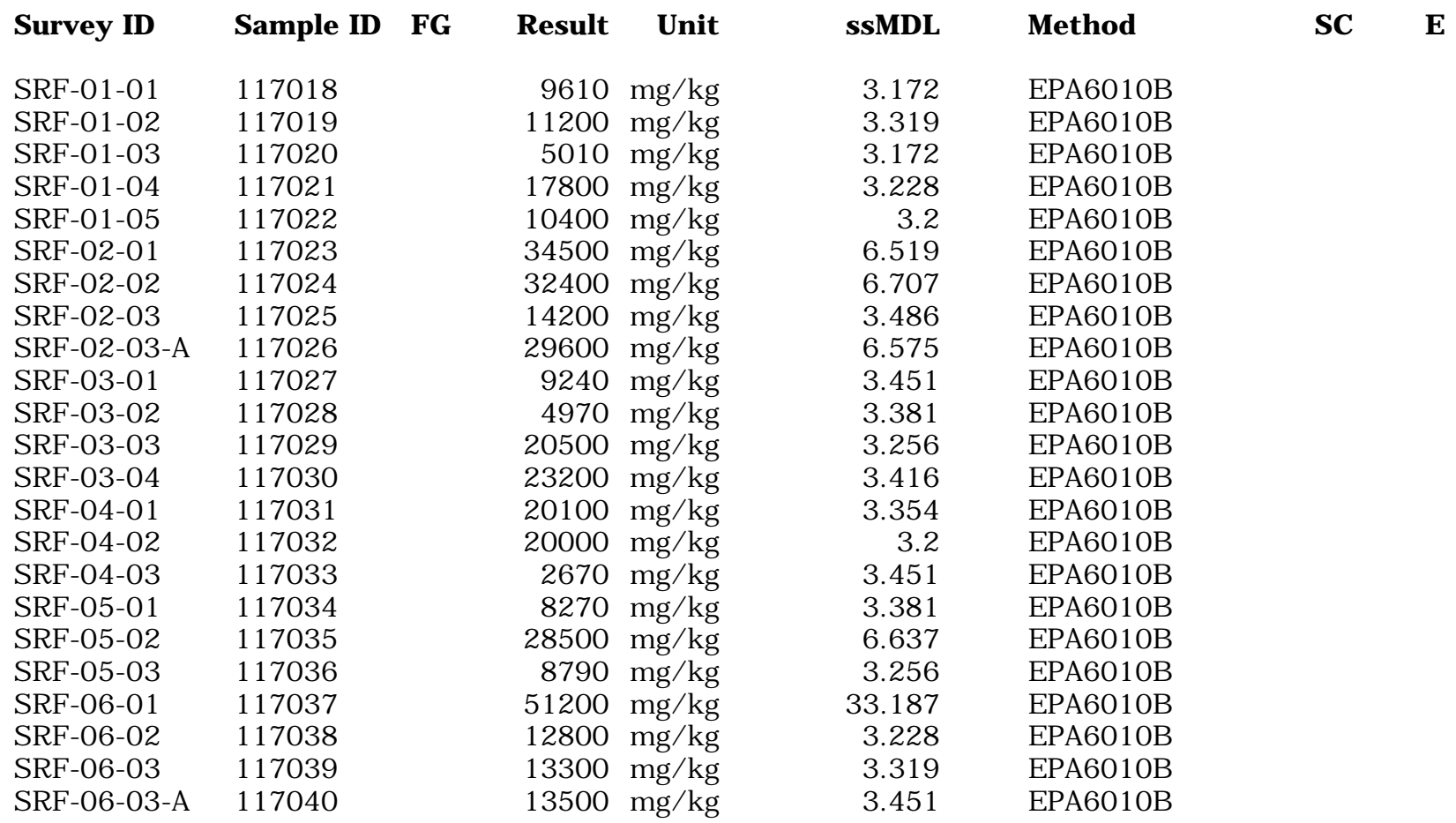


ANALYTE: Calcium (cont.)

\begin{tabular}{|c|c|c|c|c|c|c|c|}
\hline Survey ID & Sample ID & FG & Result & Unit & SsMDL & Method & SC \\
\hline SRF-07-01 & 117041 & & 9960 & $\mathrm{mg} / \mathrm{kg}$ & 3.354 & EPA6010B & \\
\hline SRF-07-02 & 117042 & & 18100 & $\mathrm{mg} / \mathrm{kg}$ & 3.354 & EPA6010B & \\
\hline SRF-07-03 & 117043 & & 14200 & $\mathrm{mg} / \mathrm{kg}$ & 3.2 & EPA6010B & \\
\hline
\end{tabular}

ANALYTE: Carbon disulfide

Analytical Group: Volatile Organic Compounds

\begin{tabular}{|c|c|c|c|c|c|c|c|}
\hline Survey ID & Sample ID & FG & Result & Unit & SsMDL & Method & SC \\
\hline SRF-01-01 & 117018 & $\mathrm{~J}$ & 20.8 & $\mu g / \mathrm{kg}$ & 1.5 & EPA8260B & IK \\
\hline SRF-01-02 & 117019 & $\mathrm{U}$ & 25 & $\mu \mathrm{g} / \mathrm{kg}$ & 1.5 & EPA8260B & \\
\hline SRF-01-03 & 117020 & $\mathrm{U}$ & 25 & $\mu \mathrm{g} / \mathrm{kg}$ & 1.5 & EPA8260B & \\
\hline SRF-01-04 & 117021 & $\mathrm{U}$ & 25 & $\mu \mathrm{g} / \mathrm{kg}$ & 1.5 & EPA8260B & \\
\hline SRF-01-05 & 117022 & $\mathrm{U}$ & 25 & $\mu \mathrm{g} / \mathrm{kg}$ & 1.5 & EPA8260B & \\
\hline SRF-02-01 & 117023 & $\mathrm{U}$ & 25 & $\mu \mathrm{g} / \mathrm{kg}$ & 1.5 & EPA8260B & \\
\hline SRF-02-02 & 117024 & $\mathrm{U}$ & 25 & $\mu \mathrm{g} / \mathrm{kg}$ & 1.5 & EPA8260B & \\
\hline SRF-02-03 & 117025 & $\mathrm{U}$ & 25 & $\mu g / \mathrm{kg}$ & 1.5 & EPA8260B & \\
\hline SRF-02-03-A & 117026 & $\mathrm{U}$ & 25 & $\mu \mathrm{g} / \mathrm{kg}$ & 1.5 & EPA8260B & \\
\hline SRF-03-01 & 117027 & $\mathrm{U}$ & 25 & $\mu \mathrm{g} / \mathrm{kg}$ & 1.5 & EPA8260B & \\
\hline SRF-03-02 & 117028 & $\mathrm{U}$ & 25 & $\mu \mathrm{g} / \mathrm{kg}$ & 1.5 & EPA8260B & \\
\hline SRF-03-03 & 117029 & $\mathrm{U}$ & 25 & $\mu \mathrm{g} / \mathrm{kg}$ & 1.5 & EPA8260B & \\
\hline SRF-03-04 & 117030 & $\mathrm{U}$ & 25 & $\mu \mathrm{g} / \mathrm{kg}$ & 1.5 & EPA8260B & \\
\hline SRF-04-01 & 117031 & $\mathrm{~J}$ & 16.2 & $\mu \mathrm{g} / \mathrm{kg}$ & 1.5 & EPA8260B & IK \\
\hline SRF-04-02 & 117032 & $\mathrm{U}$ & 25 & $\mu \mathrm{g} / \mathrm{kg}$ & 1.5 & EPA8260B & \\
\hline SRF-04-03 & 117033 & $\mathrm{U}$ & 25 & $\mu \mathrm{g} / \mathrm{kg}$ & 1.5 & EPA8260B & \\
\hline SRF-05-01 & 117034 & $\mathrm{~J}$ & 16.6 & $\mu \mathrm{g} / \mathrm{kg}$ & 1.5 & EPA8260B & IK \\
\hline SRF-05-02 & 117035 & $\mathrm{U}$ & 25 & $\mu \mathrm{g} / \mathrm{kg}$ & 1.5 & EPA8260B & \\
\hline SRF-05-03 & 117036 & $\mathrm{~J}$ & 32.6 & $\mu \mathrm{g} / \mathrm{kg}$ & 1.5 & EPA8260B & $\mathrm{K}$ \\
\hline SRF-06-01 & 117037 & $\mathrm{~J}$ & 26.7 & $\mu \mathrm{g} / \mathrm{kg}$ & 1.5 & EPA8260B & $\mathrm{K}$ \\
\hline SRF-06-02 & 117038 & $\mathrm{U}$ & 25 & $\mu \mathrm{g} / \mathrm{kg}$ & 1.5 & EPA8260B & \\
\hline SRF-06-03 & 117039 & $\mathrm{U}$ & 25 & $\mu \mathrm{g} / \mathrm{kg}$ & 1.5 & EPA8260B & \\
\hline SRF-06-03-A & 117040 & $\mathrm{U}$ & 25 & $\mu \mathrm{g} / \mathrm{kg}$ & 1.5 & EPA8260B & \\
\hline SRF-07-01 & 117041 & $\mathrm{U}$ & 25 & $\mu \mathrm{g} / \mathrm{kg}$ & 1.5 & EPA8260B & \\
\hline SRF-07-02 & 117042 & $\mathrm{U}$ & 25 & $\mu \mathrm{g} / \mathrm{kg}$ & 1.5 & EPA8260B & \\
\hline SRF-07-03 & 117043 & $\mathrm{U}$ & 25 & $\mu \mathrm{g} / \mathrm{kg}$ & 1.5 & EPA8260B & \\
\hline
\end{tabular}

ANALYTE: Carbon tetrachloride

Analytical Group: Volatile Organic Compounds

\begin{tabular}{|c|c|c|c|c|c|c|c|}
\hline Survey ID & Sample ID & FG & Result & Unit & SsMDL & Method & SC \\
\hline SRF-01-01 & 117018 & $\mathrm{U}$ & 5 & $\mu g / \mathrm{kg}$ & 2.5 & EPA8260B & \\
\hline SRF-01-02 & 117019 & $\mathrm{U}$ & 5 & $\mu g / k g$ & 2.5 & EPA8260B & \\
\hline SRF-01-03 & 117020 & $\mathrm{U}$ & 5 & $\mu g / \mathrm{kg}$ & 2.5 & EPA8260B & \\
\hline SRF-01-04 & 117021 & $\mathrm{U}$ & 5 & $\mu \mathrm{g} / \mathrm{kg}$ & 2.5 & EPA8260B & \\
\hline SRF-01-05 & 117022 & $\mathrm{U}$ & 5 & $\mu \mathrm{g} / \mathrm{kg}$ & 2.5 & EPA8260B & \\
\hline SRF-02-01 & 117023 & $\mathrm{U}$ & 5 & $\mu \mathrm{g} / \mathrm{kg}$ & 2.5 & EPA8260B & \\
\hline SRF-02-02 & 117024 & $\mathrm{U}$ & 5 & $\mu g / \mathrm{kg}$ & 2.5 & EPA8260B & \\
\hline SRF-02-03 & 117025 & $\mathrm{U}$ & 5 & $\mu g / \mathrm{kg}$ & 2.5 & EPA8260B & \\
\hline SRF-02-03-A & 117026 & $\mathrm{U}$ & 5 & $\mu g / \mathrm{kg}$ & 2.5 & EPA8260B & \\
\hline SRF-03-01 & 117027 & $\mathrm{U}$ & 5 & $\mu g / \mathrm{kg}$ & 2.5 & EPA8260B & \\
\hline SRF-03-02 & 117028 & $\mathrm{U}$ & 5 & $\mu \mathrm{g} / \mathrm{kg}$ & 2.5 & EPA8260B & \\
\hline SRF-03-03 & 117029 & $\mathrm{U}$ & 5 & $\mu \mathrm{g} / \mathrm{kg}$ & 2.5 & EPA8260B & \\
\hline SRF-03-04 & 117030 & $\mathrm{U}$ & 5 & $\mu \mathrm{g} / \mathrm{kg}$ & 2.5 & EPA8260B & \\
\hline SRF-04-01 & 117031 & $\mathrm{U}$ & 5 & $\mu \mathrm{g} / \mathrm{kg}$ & 2.5 & EPA8260B & \\
\hline
\end{tabular}


ANALYTE: Carbon tetrachloride (cont.)

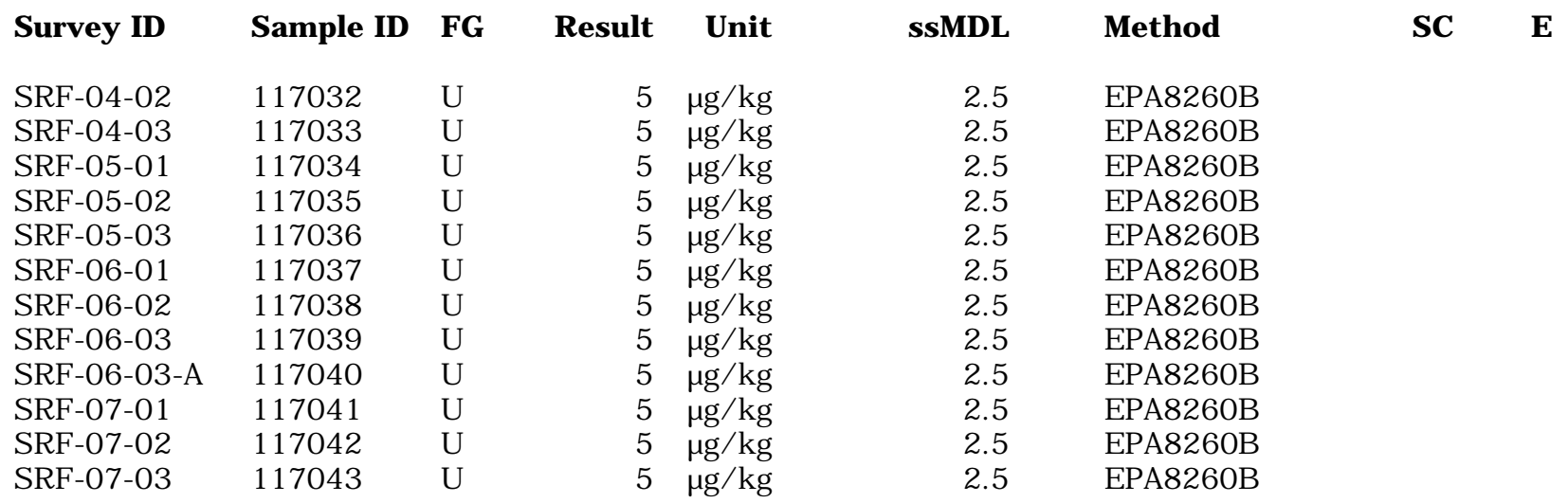

ANALYTE: Cerium-144

Analytical Group: Radionuclides

\begin{tabular}{|c|c|c|c|c|c|c|c|}
\hline Survey ID & Sample ID & $\mathbf{F G}$ & Result & Unit & SSMDL & Method & SC \\
\hline SRF-01-01 & 117018 & $\mathrm{U}$ & 0.0737 & $\mathrm{pCi} / \mathrm{g}$ & 0.275 & EPIA-013B & \\
\hline SRF-01-02 & 117019 & $\mathrm{U}$ & -0.0298 & $\mathrm{pCi} / \mathrm{g}$ & 0.192 & EPIA-013B & \\
\hline SRF-01-03 & 117020 & $\mathrm{U}$ & -0.0966 & $\mathrm{pCi} / \mathrm{g}$ & 0.243 & EPIA-013B & \\
\hline SRF-01-04 & 117021 & $\mathrm{U}$ & 0.0362 & $\mathrm{pCi} / \mathrm{g}$ & 0.211 & EPIA-013B & \\
\hline SRF-01-05 & 117022 & $\mathrm{U}$ & -0.00121 & $\mathrm{pCi} / \mathrm{g}$ & 0.225 & EPIA-013B & \\
\hline SRF-01-05 & 117022 & $\mathrm{U}$ & 0.00574 & $\mathrm{pCi} / \mathrm{g}$ & 0.209 & EPIA-013B & \\
\hline SRF-02-01 & 117023 & $\mathrm{R}$ & 0.294 & $\mathrm{pCi} / \mathrm{g}$ & 0.272 & EPIA-013B & \\
\hline SRF-02-02 & 117024 & $\mathrm{U}$ & 0.0858 & $\mathrm{pCi} / \mathrm{g}$ & 0.189 & EPIA-013B & \\
\hline SRF-02-03 & 117025 & $\mathrm{U}$ & 0.000742 & $\mathrm{pCi} / \mathrm{g}$ & 0.326 & EPIA-013B & \\
\hline SRF-02-03-A & 117026 & $\mathrm{U}$ & 0.121 & $\mathrm{pCi} / \mathrm{g}$ & 0.309 & EPIA-013B & \\
\hline SRF-03-01 & 117027 & $\mathrm{U}$ & -0.0603 & $\mathrm{pCi} / \mathrm{g}$ & 0.283 & EPIA-013B & \\
\hline SRF-03-02 & 117028 & $\mathrm{U}$ & -0.00921 & $\mathrm{pCi} / \mathrm{g}$ & 0.232 & EPIA-013B & \\
\hline SRF-03-03 & 117029 & $\mathrm{U}$ & 0.056 & $\mathrm{pCi} / \mathrm{g}$ & 0.254 & EPIA-013B & \\
\hline SRF-03-04 & 117030 & $\mathrm{U}$ & 0.0405 & $\mathrm{pCi} / \mathrm{g}$ & 0.162 & EPIA-013B & \\
\hline SRF-04-01 & 117031 & $\mathrm{U}$ & 0.279 & $\mathrm{pCi} / \mathrm{g}$ & 0.324 & EPIA-013B & \\
\hline SRF-04-02 & 117032 & $\mathrm{U}$ & -0.0704 & $\mathrm{pCi} / \mathrm{g}$ & 0.282 & EPIA-013B & \\
\hline SRF-04-03 & 117033 & $\mathrm{U}$ & 0.0639 & $\mathrm{pCi} / \mathrm{g}$ & 0.28 & EPIA-013B & \\
\hline SRF-05-01 & 117034 & $\mathrm{U}$ & -0.00918 & $\mathrm{pCi} / \mathrm{g}$ & 0.205 & EPIA-013B & \\
\hline SRF-05-02 & 117035 & $\mathrm{U}$ & 0.0354 & $\mathrm{pCi} / \mathrm{g}$ & 0.199 & EPIA-013B & \\
\hline SRF-05-03 & 117036 & $\mathrm{U}$ & -0.00206 & $\mathrm{pCi} / \mathrm{g}$ & 0.223 & EPIA-013B & \\
\hline SRF-06-01 & 117037 & $\mathrm{U}$ & -0.104 & $\mathrm{pCi} / \mathrm{g}$ & 0.224 & EPIA-013B & \\
\hline SRF-06-02 & 117038 & $\mathrm{U}$ & 0.00842 & $\mathrm{pCi} / \mathrm{g}$ & 0.201 & EPIA-013B & \\
\hline SRF-06-03 & 117039 & $\mathrm{U}$ & 0.0102 & $\mathrm{pCi} / \mathrm{g}$ & 0.293 & EPIA-013B & \\
\hline SRF-06-03-A & 117040 & $\mathrm{U}$ & -0.249 & $\mathrm{pCi} / \mathrm{g}$ & 0.278 & EPIA-013B & \\
\hline SRF-07-01 & 117041 & $\mathrm{U}$ & -0.0536 & $\mathrm{pCi} / \mathrm{g}$ & 0.244 & EPIA-013B & \\
\hline SRF-07-02 & 117042 & $\mathrm{U}$ & -0.124 & $\mathrm{pCi} / \mathrm{g}$ & 0.319 & EPIA-013B & \\
\hline SRF-07-03 & 117043 & $\mathrm{U}$ & -0.0332 & $\mathrm{pCi} / \mathrm{g}$ & 0.271 & EPIA-013B & \\
\hline SRF-07-03 & 117043 & $\mathrm{U}$ & -0.0296 & $\mathrm{pCi} / \mathrm{g}$ & 0.2 & EPIA-013B & \\
\hline \multicolumn{8}{|c|}{$\begin{array}{l}\text { ANALYTE: Cesium-134 } \\
\text { Analytical Group: Radionuclides }\end{array}$} \\
\hline Survey ID & Sample ID & $\mathbf{F G}$ & Result & Unit & SsMDL & Method & SC \\
\hline SRF-01-01 & 117018 & $\mathrm{U}$ & -0.0232 & $\mathrm{pCi} / \mathrm{g}$ & 0.0487 & EPIA-013B & \\
\hline SRF-01-02 & 117019 & $\mathrm{U}$ & 0.00854 & $\mathrm{pCi} / \mathrm{g}$ & 0.0435 & EPIA-013B & \\
\hline SRF-01-03 & 117020 & $\mathrm{U}$ & -0.000852 & $\mathrm{pCi} / \mathrm{g}$ & 0.044 & EPIA-013B & \\
\hline
\end{tabular}


ANALYTE: Cesium-134 (cont.)

\begin{tabular}{|c|c|c|c|c|c|c|c|}
\hline Survey ID & Sample ID & FG & Result & Unit & SSMDL & Method & SC \\
\hline SRF-01-04 & 117021 & $\mathrm{U}$ & -0.00304 & $\mathrm{pCi} / \mathrm{g}$ & 0.0408 & EPIA-013B & \\
\hline SRF-01-05 & 117022 & $\mathrm{U}$ & 0.0152 & $\mathrm{pCi} / \mathrm{g}$ & 0.0465 & EPIA-013B & \\
\hline SRF-01-05 & 117022 & $\mathrm{U}$ & -0.00193 & $\mathrm{pCi} / \mathrm{g}$ & 0.0419 & EPIA-013B & \\
\hline SRF-02-01 & 117023 & $\mathrm{U}$ & 0.0239 & $\mathrm{pCi} / \mathrm{g}$ & 0.0559 & EPIA-013B & \\
\hline SRF-02-02 & 117024 & $\mathrm{U}$ & -0.00547 & $\mathrm{pCi} / \mathrm{g}$ & 0.0373 & EPIA-013B & \\
\hline SRF-02-03 & 117025 & $\mathrm{U}$ & -0.00891 & $\mathrm{pCi} / \mathrm{g}$ & 0.0637 & EPIA-013B & \\
\hline SRF-02-03-A & 117026 & $\mathrm{U}$ & -0.0383 & $\mathrm{pCi} / \mathrm{g}$ & 0.061 & EPIA-013B & \\
\hline SRF-03-01 & 117027 & $\mathrm{U}$ & -0.0117 & $\mathrm{pCi} / \mathrm{g}$ & 0.0599 & EPIA-013B & \\
\hline SRF-03-02 & 117028 & $\mathrm{U}$ & -0.0552 & $\mathrm{pCi} / \mathrm{g}$ & 0.0419 & EPIA-013B & \\
\hline SRF-03-03 & 117029 & $\mathrm{U}$ & -0.0272 & $\mathrm{pCi} / \mathrm{g}$ & 0.0463 & EPIA-013B & \\
\hline SRF-03-04 & 117030 & $\mathrm{U}$ & 0.0194 & $\mathrm{pCi} / \mathrm{g}$ & 0.0321 & EPIA-013B & \\
\hline SRF-04-01 & 117031 & $\mathrm{U}$ & 0.00467 & $\mathrm{pCi} / \mathrm{g}$ & 0.0498 & EPIA-013B & \\
\hline SRF-04-02 & 117032 & $\mathrm{U}$ & 0.0251 & $\mathrm{pCi} / \mathrm{g}$ & 0.0705 & EPIA-013B & \\
\hline SRF-04-03 & 117033 & $\mathrm{U}$ & 0.0395 & $\mathrm{pCi} / \mathrm{g}$ & 0.0604 & EPIA-013B & \\
\hline SRF-05-01 & 117034 & $\mathrm{U}$ & -0.0159 & $\mathrm{pCi} / \mathrm{g}$ & 0.0412 & EPIA-013B & \\
\hline SRF-05-02 & 117035 & $\mathrm{U}$ & -0.0413 & $\mathrm{pCi} / \mathrm{g}$ & 0.042 & EPIA-013B & \\
\hline SRF-05-03 & 117036 & $\mathrm{U}$ & -0.0215 & $\mathrm{pCi} / \mathrm{g}$ & 0.0446 & EPIA-013B & \\
\hline SRF-06-01 & 117037 & $\mathrm{U}$ & 0.0125 & $\mathrm{pCi} / \mathrm{g}$ & 0.0588 & EPIA-013B & \\
\hline SRF-06-02 & 117038 & $\mathrm{U}$ & 0.0146 & $\mathrm{pCi} / \mathrm{g}$ & 0.0433 & EPIA-013B & \\
\hline SRF-06-03 & 117039 & $\mathrm{U}$ & -0.0285 & $\mathrm{pCi} / \mathrm{g}$ & 0.0549 & EPIA-013B & \\
\hline SRF-06-03-A & 117040 & $\mathrm{U}$ & 0.00424 & $\mathrm{pCi} / \mathrm{g}$ & 0.0624 & EPIA-013B & \\
\hline SRF-07-01 & 117041 & $\mathrm{U}$ & 0.0293 & $\mathrm{pCi} / \mathrm{g}$ & 0.065 & EPIA-013B & \\
\hline SRF-07-02 & 117042 & $\mathrm{U}$ & -0.0101 & $\mathrm{pCi} / \mathrm{g}$ & 0.0667 & EPIA-013B & \\
\hline SRF-07-03 & 117043 & $\mathrm{U}$ & -0.00367 & $\mathrm{pCi} / \mathrm{g}$ & 0.0523 & EPIA-013B & \\
\hline SRF-07-03 & 117043 & $\mathrm{U}$ & 0.0172 & $\mathrm{pCi} / \mathrm{g}$ & 0.0715 & EPIA-013B & \\
\hline
\end{tabular}

ANALYTE: Cesium-137

Analytical Group: Radionuclides

\begin{tabular}{|c|c|c|c|c|c|c|c|}
\hline Survey ID & Sample ID & $\mathbf{F G}$ & Result & Unit & SSMDL & Method & SC \\
\hline SRF-01-01 & 117018 & $\mathrm{U}$ & 0.0207 & $\mathrm{pCi} / \mathrm{g}$ & 0.0583 & EPIA-013B & \\
\hline SRF-01-02 & 117019 & $\mathrm{R}$ & 0.108 & $\mathrm{pCi} / \mathrm{g}$ & 0.0794 & EPIA-013B & \\
\hline SRF-01-03 & 117020 & $\mathrm{R}$ & 0.114 & $\mathrm{pCi} / \mathrm{g}$ & 0.0809 & EPIA-013B & \\
\hline SRF-01-04 & 117021 & $\mathrm{U}$ & -0.00711 & $\mathrm{pCi} / \mathrm{g}$ & 0.0348 & EPIA-013B & \\
\hline SRF-01-05 & 117022 & $\mathrm{U}$ & 0.0119 & $\mathrm{pCi} / \mathrm{g}$ & 0.0449 & EPIA-013B & \\
\hline SRF-01-05 & 117022 & $\mathrm{U}$ & 0.00304 & $\mathrm{pCi} / \mathrm{g}$ & 0.0437 & EPIA-013B & \\
\hline SRF-02-01 & 117023 & $\mathrm{U}$ & 0.0335 & $\mathrm{pCi} / \mathrm{g}$ & 0.0631 & EPIA-013B & \\
\hline SRF-02-02 & 117024 & $\mathrm{U}$ & 0.0144 & $\mathrm{pCi} / \mathrm{g}$ & 0.05 & EPIA-013B & \\
\hline SRF-02-03 & 117025 & & 0.621 & $\mathrm{pCi} / \mathrm{g}$ & 0.0656 & EPIA-013B & \\
\hline SRF-02-03-A & 117026 & & 0.751 & $\mathrm{pCi} / \mathrm{g}$ & 0.0596 & EPIA-013B & \\
\hline SRF-03-01 & 117027 & $\mathrm{U}$ & 0.032 & $\mathrm{pCi} / \mathrm{g}$ & 0.0616 & EPIA-013B & \\
\hline SRF-03-02 & 117028 & $\mathrm{U}$ & 0.009 & $\mathrm{pCi} / \mathrm{g}$ & 0.0501 & EPIA-013B & \\
\hline SRF-03-03 & 117029 & $\mathrm{U}$ & 0.049 & $\mathrm{pCi} / \mathrm{g}$ & 0.0619 & EPIA-013B & \\
\hline SRF-03-04 & 117030 & & 0.0582 & $\mathrm{pCi} / \mathrm{g}$ & 0.028 & EPIA-013B & \\
\hline SRF-04-01 & 117031 & & 0.0791 & $\mathrm{pCi} / \mathrm{g}$ & 0.0438 & EPIA-013B & \\
\hline SRF-04-02 & 117032 & & 0.0882 & $\mathrm{pCi} / \mathrm{g}$ & 0.0617 & EPIA-013B & \\
\hline SRF-04-03 & 117033 & $\mathrm{U}$ & 0.0535 & $\mathrm{pCi} / \mathrm{g}$ & 0.0538 & EPIA-013B & \\
\hline SRF-05-01 & 117034 & & 0.243 & $\mathrm{pCi} / \mathrm{g}$ & 0.0454 & EPIA-013B & \\
\hline SRF-05-02 & 117035 & & 0.103 & $\mathrm{pCi} / \mathrm{g}$ & 0.0474 & EPIA-013B & \\
\hline SRF-05-03 & 117036 & & 0.832 & $\mathrm{pCi} / \mathrm{g}$ & 0.0504 & EPIA-013B & \\
\hline SRF-06-01 & 117037 & $\mathrm{U}$ & 0.0418 & $\mathrm{pCi} / \mathrm{g}$ & 0.069 & EPIA-013B & \\
\hline SRF-06-02 & 117038 & & 0.0693 & $\mathrm{pCi} / \mathrm{g}$ & 0.0443 & EPIA-013B & \\
\hline SRF-06-03 & 117039 & & 1.25 & $\mathrm{pCi} / \mathrm{g}$ & 0.0637 & EPIA-013B & \\
\hline SRF-06-03-A & 117040 & $\mathrm{R}$ & 0.0989 & $\mathrm{pCi} / \mathrm{g}$ & 0.0901 & EPIA-013B & \\
\hline SRF-07-01 & 117041 & $\mathrm{U}$ & 0.0363 & $\mathrm{pCi} / \mathrm{g}$ & 0.0792 & EPIA-013B & \\
\hline
\end{tabular}


ANALYTE: Cesium-137 (cont.)

$\begin{array}{llllllll}\text { Survey ID } & \text { Sample ID } & \text { FG } & \text { Result } & \text { Unit } & \text { ssMDL } & \text { Method } & \text { SC } \\ \text { SRF-07-02 } & 117042 & \text { U } & 0.0291 & \mathrm{pCi} / \mathrm{g} & 0.0804 & \text { EPIA-013B } & \\ \text { SRF-07-03 } & 117043 & & 0.0651 & \mathrm{pCi} / \mathrm{g} & 0.0506 & \text { EPIA-013B } & \\ \text { SRF-07-03 } & 117043 & \mathrm{U} & 0.09 & \mathrm{pCi} / \mathrm{g} & 0.0756 & \text { EPIA-013B }\end{array}$

ANALYTE: Chlorobenzene

Analytical Group: Volatile Organic Compounds

\begin{tabular}{|c|c|c|c|c|c|c|c|}
\hline Survey ID & Sample ID & $\mathbf{F G}$ & Result & Unit & SsMDL & Method & SC \\
\hline SRF-01-01 & 117018 & $\mathrm{U}$ & 5 & $\mu \mathrm{g} / \mathrm{kg}$ & 1.5 & EPA8260B & \\
\hline SRF-01-02 & 117019 & $\mathrm{U}$ & 5 & $\mu g / \mathrm{kg}$ & 1.5 & EPA8260B & \\
\hline SRF-01-03 & 117020 & $\mathrm{U}$ & 5 & $\mu \mathrm{g} / \mathrm{kg}$ & 1.5 & EPA8260B & \\
\hline SRF-01-04 & 117021 & $\mathrm{U}$ & 5 & $\mu g / \mathrm{kg}$ & 1.5 & EPA8260B & \\
\hline SRF-01-05 & 117022 & $\mathrm{U}$ & 5 & $\mu \mathrm{g} / \mathrm{kg}$ & 1.5 & EPA8260B & \\
\hline SRF-02-01 & 117023 & $\mathrm{U}$ & 5 & $\mu g / k g$ & 1.5 & EPA8260B & \\
\hline SRF-02-02 & 117024 & $\mathrm{U}$ & 5 & $\mu \mathrm{g} / \mathrm{kg}$ & 1.5 & EPA8260B & \\
\hline SRF-02-03 & 117025 & $\mathrm{U}$ & 5 & $\mu g / k g$ & 1.5 & EPA8260B & \\
\hline SRF-02-03-A & 117026 & $\mathrm{U}$ & 5 & $\mu g / \mathrm{kg}$ & 1.5 & EPA8260B & \\
\hline SRF-03-01 & 117027 & $\mathrm{U}$ & 5 & $\mu \mathrm{g} / \mathrm{kg}$ & 1.5 & EPA8260B & \\
\hline SRF-03-02 & 117028 & $\mathrm{U}$ & 5 & $\mu g / \mathrm{kg}$ & 1.5 & EPA8260B & \\
\hline SRF-03-03 & 117029 & $\mathrm{U}$ & 5 & $\mu g / k g$ & 1.5 & EPA8260B & \\
\hline SRF-03-04 & 117030 & $\mathrm{U}$ & 5 & $\mu \mathrm{g} / \mathrm{kg}$ & 1.5 & EPA8260B & \\
\hline SRF-04-01 & 117031 & $\mathrm{U}$ & 5 & $\mu g / \mathrm{kg}$ & 1.5 & EPA8260B & \\
\hline SRF-04-02 & 117032 & $\mathrm{U}$ & 5 & $\mu g / k g$ & 1.5 & EPA8260B & \\
\hline SRF-04-03 & 117033 & $\mathrm{U}$ & 5 & $\mu g / \mathrm{kg}$ & 1.5 & EPA8260B & \\
\hline SRF-05-01 & 117034 & $\mathrm{U}$ & 5 & $\mu g / k g$ & 1.5 & EPA8260B & \\
\hline SRF-05-02 & 117035 & $\mathrm{U}$ & 5 & $\mu g / \mathrm{kg}$ & 1.5 & EPA8260B & \\
\hline SRF-05-03 & 117036 & $\mathrm{U}$ & 5 & $\mu \mathrm{g} / \mathrm{kg}$ & 1.5 & EPA8260B & \\
\hline SRF-06-01 & 117037 & $\mathrm{U}$ & 5 & $\mu g / k g$ & 1.5 & EPA8260B & \\
\hline SRF-06-02 & 117038 & $\mathrm{U}$ & 5 & $\mu \mathrm{g} / \mathrm{kg}$ & 1.5 & EPA8260B & \\
\hline SRF-06-03 & 117039 & $\mathrm{U}$ & 5 & $\mu g / \mathrm{kg}$ & 1.5 & EPA8260B & \\
\hline SRF-06-03-A & 117040 & $\mathrm{U}$ & 5 & $\mu \mathrm{g} / \mathrm{kg}$ & 1.5 & EPA8260B & \\
\hline SRF-07-01 & 117041 & $\mathrm{U}$ & 5 & $\mu g / \mathrm{kg}$ & 1.5 & EPA8260B & \\
\hline SRF-07-02 & 117042 & $\mathrm{U}$ & 5 & $\mu \mathrm{g} / \mathrm{kg}$ & 1.5 & EPA8260B & \\
\hline SRF-07-03 & 117043 & $\mathrm{U}$ & 5 & $\mu \mathrm{g} / \mathrm{kg}$ & 1.5 & EPA8260B & \\
\hline
\end{tabular}

ANALYTE: Chloroethane

Analytical Group: Volatile Organic Compounds

\begin{tabular}{|c|c|c|c|c|c|c|c|}
\hline Survey ID & Sample ID & FG & Result & Unit & SsMDL & Method & SC \\
\hline SRF-01-01 & 117018 & $\mathrm{U}$ & 5 & $\mu g / \mathrm{kg}$ & 1.5 & EPA8260B & \\
\hline SRF-01-02 & 117019 & $\mathrm{U}$ & 5 & $\mu g / k g$ & 1.5 & EPA8260B & \\
\hline SRF-01-03 & 117020 & $\mathrm{U}$ & 5 & $\mu g / \mathrm{kg}$ & 1.5 & EPA8260B & \\
\hline SRF-01-04 & 117021 & $\mathrm{U}$ & 5 & $\mu \mathrm{g} / \mathrm{kg}$ & 1.5 & EPA8260B & \\
\hline SRF-01-05 & 117022 & $\mathrm{U}$ & 5 & $\mu g / \mathrm{kg}$ & 1.5 & EPA8260B & \\
\hline SRF-02-01 & 117023 & $\mathrm{U}$ & 5 & $\mu \mathrm{g} / \mathrm{kg}$ & 1.5 & EPA8260B & \\
\hline SRF-02-02 & 117024 & $\mathrm{U}$ & 5 & $\mu g / \mathrm{kg}$ & 1.5 & EPA8260B & \\
\hline SRF-02-03 & 117025 & $\mathrm{U}$ & 5 & $\mu g / \mathrm{kg}$ & 1.5 & EPA8260B & \\
\hline SRF-02-03-A & 117026 & $\mathrm{U}$ & 5 & $\mu g / \mathrm{kg}$ & 1.5 & EPA8260B & \\
\hline SRF-03-01 & 117027 & $\mathrm{U}$ & 5 & $\mu g / \mathrm{kg}$ & 1.5 & EPA8260B & \\
\hline SRF-03-02 & 117028 & $\mathrm{U}$ & 5 & $\mu \mathrm{g} / \mathrm{kg}$ & 1.5 & EPA8260B & \\
\hline SRF-03-03 & 117029 & $\mathrm{U}$ & 5 & $\mu \mathrm{g} / \mathrm{kg}$ & 1.5 & EPA8260B & \\
\hline SRF-03-04 & 117030 & $\mathrm{U}$ & 5 & $\mu \mathrm{g} / \mathrm{kg}$ & 1.5 & EPA8260B & \\
\hline SRF-04-01 & 117031 & $\mathrm{U}$ & 5 & $\mu \mathrm{g} / \mathrm{kg}$ & 1.5 & EPA8260B & \\
\hline
\end{tabular}


ANALYTE: Chloroethane (cont.)

$\begin{array}{lllrlrl}\text { Survey ID } & \text { Sample ID } & \text { FG } & \text { Result } & \text { Unit } & \text { ssMDL } & \text { Method } \\ \text { SRF-04-02 } & 117032 & \mathrm{U} & 5 & \mu \mathrm{g} / \mathrm{kg} & 1.5 & \text { EPA8260B } \\ \text { SRF-04-03 } & 117033 & \mathrm{U} & 5 & \mu \mathrm{g} / \mathrm{kg} & 1.5 & \text { EPA8260B } \\ \text { SRF-05-01 } & 117034 & \mathrm{U} & 5 & \mu \mathrm{g} / \mathrm{kg} & 1.5 & \text { EPA8260B } \\ \text { SRF-05-02 } & 117035 & \mathrm{U} & 5 & \mu \mathrm{g} / \mathrm{kg} & 1.5 & \text { EPA8260B } \\ \text { SRF-05-03 } & 117036 & \mathrm{U} & 5 & \mu \mathrm{g} / \mathrm{kg} & 1.5 & \text { EPA8260B } \\ \text { SRF-06-01 } & 117037 & \mathrm{U} & 5 & \mu \mathrm{g} / \mathrm{kg} & 1.5 & \text { EPA8260B } \\ \text { SRF-06-02 } & 117038 & \mathrm{U} & 5 & \mu \mathrm{g} / \mathrm{kg} & 1.5 & \text { EPA8260B } \\ \text { SRF-06-03 } & 117039 & \mathrm{U} & 5 & \mu \mathrm{g} / \mathrm{kg} & 1.5 & \text { EPA8260B } \\ \text { SRF-06-03-A } & 117040 & \mathrm{U} & 5 & \mu \mathrm{g} / \mathrm{kg} & 1.5 & \text { EPA8260B } \\ \text { SRF-07-01 } & 117041 & \mathrm{U} & 5 & \mu \mathrm{g} / \mathrm{kg} & 1.5 & \text { EPA8260B } \\ \text { SRF-07-02 } & 117042 & \mathrm{U} & 5 & \mu \mathrm{g} / \mathrm{kg} & 1.5 & \text { EPA8260B } \\ \text { SRF-07-03 } & 117043 & \mathrm{U} & 5 & \mu \mathrm{g} / \mathrm{kg} & 1.5 & \text { EPA8260B }\end{array}$

ANALYTE: Chloroethene (Vinyl chloride)

Analytical Group: Volatile Organic Compounds

\begin{tabular}{|c|c|c|c|c|c|c|c|}
\hline Survey ID & Sample ID & $\mathbf{F G}$ & Result & Unit & SsMDL & Method & SC \\
\hline SRF-01-01 & 117018 & $\mathrm{U}$ & 5 & $\mu g / \mathrm{kg}$ & 2 & EPA8260B & \\
\hline SRF-01-02 & 117019 & $\mathrm{U}$ & 5 & $\mu \mathrm{g} / \mathrm{kg}$ & 2 & EPA8260B & \\
\hline SRF-01-03 & 117020 & $\mathrm{U}$ & 5 & $\mu g / k g$ & 2 & EPA8260B & \\
\hline SRF-01-04 & 117021 & $\mathrm{U}$ & 5 & $\mu \mathrm{g} / \mathrm{kg}$ & 2 & EPA8260B & \\
\hline SRF-01-05 & 117022 & $\mathrm{U}$ & 5 & $\mu g / k g$ & 2 & EPA8260B & \\
\hline SRF-02-01 & 117023 & $\mathrm{U}$ & 5 & $\mu g / \mathrm{kg}$ & 2 & EPA8260B & \\
\hline SRF-02-02 & 117024 & $\mathrm{U}$ & 5 & $\mu \mathrm{g} / \mathrm{kg}$ & 2 & EPA8260B & \\
\hline SRF-02-03 & 117025 & $\mathrm{U}$ & 5 & $\mu g / \mathrm{kg}$ & 2 & EPA8260B & \\
\hline SRF-02-03-A & 117026 & $\mathrm{U}$ & 5 & $\mu \mathrm{g} / \mathrm{kg}$ & 2 & EPA8260B & \\
\hline SRF-03-01 & 117027 & $\mathrm{U}$ & 5 & $\mu \mathrm{g} / \mathrm{kg}$ & 2 & EPA8260B & \\
\hline SRF-03-02 & 117028 & $\mathrm{U}$ & 5 & $\mu \mathrm{g} / \mathrm{kg}$ & 2 & EPA8260B & \\
\hline SRF-03-03 & 117029 & $\mathrm{U}$ & 5 & $\mu g / \mathrm{kg}$ & 2 & EPA8260B & \\
\hline SRF-03-04 & 117030 & $\mathrm{U}$ & 5 & $\mu \mathrm{g} / \mathrm{kg}$ & 2 & EPA8260B & \\
\hline SRF-04-01 & 117031 & $\mathrm{U}$ & 5 & $\mu g / k g$ & 2 & EPA8260B & \\
\hline SRF-04-02 & 117032 & $\mathrm{U}$ & 5 & $\mu \mathrm{g} / \mathrm{kg}$ & 2 & EPA8260B & \\
\hline SRF-04-03 & 117033 & $\mathrm{U}$ & 5 & $\mu \mathrm{g} / \mathrm{kg}$ & 2 & EPA8260B & \\
\hline SRF-05-01 & 117034 & $\mathrm{U}$ & 5 & $\mu g / k g$ & 2 & EPA8260B & \\
\hline SRF-05-02 & 117035 & $\mathrm{U}$ & 5 & $\mu g / k g$ & 2 & EPA8260B & \\
\hline SRF-05-03 & 117036 & $\mathrm{U}$ & 5 & $\mu \mathrm{g} / \mathrm{kg}$ & 2 & EPA8260B & \\
\hline SRF-06-01 & 117037 & $\mathrm{U}$ & 5 & $\mu \mathrm{g} / \mathrm{kg}$ & 2 & EPA8260B & \\
\hline SRF-06-02 & 117038 & $\mathrm{U}$ & 5 & $\mu \mathrm{g} / \mathrm{kg}$ & 2 & EPA8260B & \\
\hline SRF-06-03 & 117039 & $\mathrm{U}$ & 5 & $\mu \mathrm{g} / \mathrm{kg}$ & 2 & EPA8260B & \\
\hline SRF-06-03-A & 117040 & $\mathrm{U}$ & 5 & $\mu \mathrm{g} / \mathrm{kg}$ & 2 & EPA8260B & \\
\hline SRF-07-01 & 117041 & $\mathrm{U}$ & 5 & $\mu \mathrm{g} / \mathrm{kg}$ & 2 & EPA8260B & \\
\hline SRF-07-02 & 117042 & $\mathrm{U}$ & 5 & $\mu g / \mathrm{kg}$ & 2 & EPA8260B & \\
\hline SRF-07-03 & 117043 & $\mathrm{U}$ & 5 & $\mu \mathrm{g} / \mathrm{kg}$ & 2 & EPA8260B & \\
\hline
\end{tabular}

ANALYTE: Chloroform

Analytical Group: Volatile Organic Compounds

$\begin{array}{lllrllll}\text { Survey ID } & \text { Sample ID } & \text { FG } & \text { Result } & \text { Unit } & \text { ssMDL } & \text { Method } & \text { SC } \\ \text { SRF-01-01 } & 117018 & \mathrm{U} & 5 & \mu \mathrm{g} / \mathrm{kg} & 0.5 & \text { EPA8260B } & \text { EPA8260B } \\ \text { SRF-01-02 } & 117019 & \mathrm{U} & 5 & \mu \mathrm{g} / \mathrm{kg} & 0.5 & 0.5 & \text { EPA8260B } \\ \text { SRF-01-03 } & 117020 & \mathrm{U} & 5 & \mu \mathrm{g} / \mathrm{kg} & 0.5 & \text { EPA8260B } \\ \text { SRF-01-04 } & 117021 & \mathrm{U} & 5 \mathrm{\mu g} / \mathrm{kg} & \text { EPA8260B } & \end{array}$


ANALYTE: Chloroform (cont.)

$\begin{array}{lllrlll}\text { Survey ID } & \text { Sample ID } & \text { FG } & \text { Result } & \text { Unit } & \text { ssMDL } & \text { Method } \\ \text { SRF-02-01 } & 117023 & \mathrm{U} & 5 & \mu \mathrm{g} / \mathrm{kg} & 0.5 & \text { EPA8260B } \\ \text { SRF-02-02 } & 117024 & \mathrm{U} & 5 & \mu \mathrm{g} / \mathrm{kg} & 0.5 & \text { EPA8260B } \\ \text { SRF-02-03 } & 117025 & \mathrm{U} & 5 & \mu \mathrm{g} / \mathrm{kg} & 0.5 & \text { EPA8260B } \\ \text { SRF-02-03-A } & 117026 & \mathrm{U} & 5 & \mu \mathrm{g} / \mathrm{kg} & 0.5 & \text { EPA8260B } \\ \text { SRF-03-01 } & 117027 & \mathrm{U} & 5 & \mu \mathrm{g} / \mathrm{kg} & 0.5 & \text { EPA8260B } \\ \text { SRF-03-02 } & 117028 & \mathrm{U} & 5 & \mu \mathrm{g} / \mathrm{kg} & 0.5 & \text { EPA8260B } \\ \text { SRF-03-03 } & 117029 & \mathrm{U} & 5 & \mu \mathrm{g} / \mathrm{kg} & 0.5 & \text { EPA8260B } \\ \text { SRF-03-04 } & 117030 & \mathrm{U} & 5 & \mu \mathrm{g} / \mathrm{kg} & 0.5 & \text { EPA8260B } \\ \text { SRF-04-01 } & 117031 & \mathrm{U} & 5 & \mu \mathrm{g} / \mathrm{kg} & 0.5 & \text { EPA8260B } \\ \text { SRF-04-02 } & 117032 & \mathrm{U} & 5 & \mu \mathrm{g} / \mathrm{kg} & 0.5 & \text { EPA8260B } \\ \text { SRF-04-03 } & 117033 & \mathrm{U} & 5 & \mu \mathrm{g} / \mathrm{kg} & 0.5 & \text { EPA8260B } \\ \text { SRF-05-01 } & 117034 & \mathrm{U} & 5 & \mu \mathrm{g} / \mathrm{kg} & 0.5 & \text { EPA8260B } \\ \text { SRF-05-02 } & 117035 & \mathrm{U} & 5 & \mu \mathrm{g} / \mathrm{kg} & 0.5 & \text { EPA8260B } \\ \text { SRF-05-03 } & 117036 & \mathrm{U} & 5 & \mu \mathrm{g} / \mathrm{kg} & 0.5 & \text { EPA8260B } \\ \text { SRF-06-01 } & 117037 & \mathrm{U} & 5 & \mu \mathrm{g} / \mathrm{kg} & 0.5 & \text { EPA8260B } \\ \text { SRF-06-02 } & 117038 & \mathrm{U} & 5 & \mu \mathrm{g} / \mathrm{kg} & 0.5 & \text { EPA8260B } \\ \text { SRF-06-03 } & 117039 & \mathrm{U} & 5 & \mu \mathrm{g} / \mathrm{kg} & 0.5 & \text { EPA8260B } \\ \text { SRF-06-03-A } & 117040 & \mathrm{U} & 5 & \mu \mathrm{g} / \mathrm{kg} & 0.5 & \text { EPA8260B } \\ \text { SRF-07-01 } & 117041 & \mathrm{U} & 5 & \mu \mathrm{g} / \mathrm{kg} & 0.5 & \text { EPA8260B } \\ \text { SRF-07-02 } & 117042 & \mathrm{U} & 5 & \mu \mathrm{g} / \mathrm{kg} & 0.5 & \text { EPA8260B } \\ \text { SRF-07-03 } & 117043 & \mathrm{U} & 5 & \mu \mathrm{g} / \mathrm{kg} & 0.5 & \text { EPA8260B }\end{array}$

ANALYTE: Chloromethane (Methyl chloride)

Analytical Group: Volatile Organic Compounds

\begin{tabular}{|c|c|c|c|c|c|c|c|}
\hline Survey ID & Sample ID & $\mathbf{F G}$ & Result & Unit & SSMDL & Method & SC \\
\hline SRF-01-01 & 117018 & $\mathrm{U}$ & 5 & $\mu g / \mathrm{kg}$ & 1 & EPA8260B & \\
\hline SRF-01-02 & 117019 & $\mathrm{U}$ & 5 & $\mu \mathrm{g} / \mathrm{kg}$ & 1 & EPA8260B & \\
\hline SRF-01-03 & 117020 & $\mathrm{U}$ & 5 & $\mu g / \mathrm{kg}$ & 1 & EPA8260B & \\
\hline SRF-01-04 & 117021 & $\mathrm{U}$ & 5 & $\mu \mathrm{g} / \mathrm{kg}$ & 1 & EPA8260B & \\
\hline SRF-01-05 & 117022 & $\mathrm{U}$ & 5 & $\mu g / k g$ & 1 & EPA8260B & \\
\hline SRF-02-01 & 117023 & $\mathrm{U}$ & 5 & $\mu g / k g$ & 1 & EPA8260B & \\
\hline SRF-02-02 & 117024 & $\mathrm{U}$ & 5 & $\mu g / k g$ & 1 & EPA8260B & \\
\hline SRF-02-03 & 117025 & $\mathrm{U}$ & 5 & $\mu g / \mathrm{kg}$ & 1 & EPA8260B & \\
\hline SRF-02-03-A & 117026 & $\mathrm{U}$ & 5 & $\mu \mathrm{g} / \mathrm{kg}$ & 1 & EPA8260B & \\
\hline SRF-03-01 & 117027 & $\mathrm{U}$ & 5 & $\mu \mathrm{g} / \mathrm{kg}$ & 1 & EPA8260B & \\
\hline SRF-03-02 & 117028 & $\mathrm{U}$ & 5 & $\mu g / k g$ & 1 & EPA8260B & \\
\hline SRF-03-03 & 117029 & $\mathrm{U}$ & 5 & $\mu g / \mathrm{kg}$ & 1 & EPA8260B & \\
\hline SRF-03-04 & 117030 & $\mathrm{U}$ & 5 & $\mu \mathrm{g} / \mathrm{kg}$ & 1 & EPA8260B & \\
\hline SRF-04-01 & 117031 & $\mathrm{U}$ & 5 & $\mu \mathrm{g} / \mathrm{kg}$ & 1 & EPA8260B & \\
\hline SRF-04-02 & 117032 & $\mathrm{U}$ & 5 & $\mu \mathrm{g} / \mathrm{kg}$ & 1 & EPA8260B & \\
\hline SRF-04-03 & 117033 & $\mathrm{U}$ & 5 & $\mu g / \mathrm{kg}$ & 1 & EPA8260B & \\
\hline SRF-05-01 & 117034 & $\mathrm{U}$ & 5 & $\mu \mathrm{g} / \mathrm{kg}$ & 1 & EPA8260B & \\
\hline SRF-05-02 & 117035 & $\mathrm{U}$ & 5 & $\mu \mathrm{g} / \mathrm{kg}$ & 1 & EPA8260B & \\
\hline SRF-05-03 & 117036 & $\mathrm{U}$ & 5 & $\mu \mathrm{g} / \mathrm{kg}$ & 1 & EPA8260B & \\
\hline SRF-06-01 & 117037 & $\mathrm{U}$ & 5 & $\mu g / k g$ & 1 & EPA8260B & \\
\hline SRF-06-02 & 117038 & $\mathrm{U}$ & 5 & $\mu \mathrm{g} / \mathrm{kg}$ & 1 & EPA8260B & \\
\hline SRF-06-03 & 117039 & $\mathrm{U}$ & 5 & $\mu g / k g$ & 1 & EPA8260B & \\
\hline SRF-06-03-A & 117040 & $\mathrm{U}$ & 5 & $\mu g / k g$ & 1 & EPA8260B & \\
\hline SRF-07-01 & 117041 & $\mathrm{U}$ & 5 & $\mu g / k g$ & 1 & EPA8260B & \\
\hline SRF-07-02 & 117042 & $\mathrm{U}$ & 5 & $\mu g / \mathrm{kg}$ & 1 & EPA8260B & \\
\hline SRF-07-03 & 117043 & $\mathrm{U}$ & 5 & $\mu \mathrm{g} / \mathrm{kg}$ & 1 & EPA8260B & \\
\hline
\end{tabular}


ANALYTE: Chromium

Analytical Group: Metals (total recoverable)

\begin{tabular}{ll} 
Survey ID & Sample ID \\
SRF-01-01 & 117018 \\
SRF-01-02 & 117019 \\
SRF-01-03 & 117020 \\
SRF-01-04 & 117021 \\
SRF-01-05 & 117022 \\
SRF-02-01 & 117023 \\
SRF-02-02 & 117024 \\
SRF-02-03 & 117025 \\
SRF-02-03-A & 117026 \\
SRF-03-01 & 117027 \\
SRF-03-02 & 117028 \\
SRF-03-03 & 117029 \\
SRF-03-04 & 117030 \\
SRF-04-01 & 117031 \\
SRF-04-02 & 117032 \\
SRF-04-03 & 117033 \\
SRF-05-01 & 117034 \\
SRF-05-02 & 117035 \\
SRF-05-03 & 117036 \\
SRF-06-01 & 117037 \\
SRF-06-02 & 117038 \\
SRF-06-03 & 117039 \\
SRF-06-03-A & 117040 \\
SRF-07-01 & 117041 \\
SRF-07-02 & 117042 \\
SRF-07-03 & 117043 \\
\hline
\end{tabular}

$\begin{array}{crc}\text { FG } & \text { Result } & \text { Unit } \\ \mathrm{J} & 0.174 \mathrm{mg} / \mathrm{kg} \\ \mathrm{J} & 0.214 \mathrm{mg} / \mathrm{kg} \\ \mathrm{J} & 0.104 \mathrm{mg} / \mathrm{kg} \\ & 0.251 \mathrm{mg} / \mathrm{kg} \\ \mathrm{J} & 0.166 \mathrm{mg} / \mathrm{kg} \\ & 0.682 \mathrm{mg} / \mathrm{kg} \\ & 0.43 \mathrm{mg} / \mathrm{kg} \\ & 0.509 \mathrm{mg} / \mathrm{kg} \\ & 0.461 & \mathrm{mg} / \mathrm{kg} \\ \mathrm{J} & 0.152 & \mathrm{mg} / \mathrm{kg} \\ & 0.974 & \mathrm{mg} / \mathrm{kg} \\ & 0.332 \mathrm{mg} / \mathrm{kg} \\ & 0.335 \mathrm{mg} / \mathrm{kg} \\ & 0.403 \mathrm{mg} / \mathrm{kg} \\ \mathrm{J} & 0.293 \mathrm{mg} / \mathrm{kg} \\ \mathrm{J} & 0.0858 \mathrm{mg} / \mathrm{kg} \\ & 0.19 \mathrm{mg} / \mathrm{kg} \\ \mathrm{J} & 0.359 \mathrm{mg} / \mathrm{kg} \\ & 0.148 \mathrm{mg} / \mathrm{kg} \\ & 0.657 \mathrm{mg} / \mathrm{kg} \\ & 0.354 \mathrm{mg} / \mathrm{kg} \\ \mathrm{J} & 0.303 \mathrm{mg} / \mathrm{kg} \\ & 0.313 \mathrm{mg} / \mathrm{kg} \\ & 0.184 \mathrm{mg} / \mathrm{kg} \\ & 0.305 \mathrm{mg} / \mathrm{kg} \\ & 0.47 \mathrm{mg} / \mathrm{kg}\end{array}$

SSMDL
0.0331
0.0347
0.0331
0.0338
0.0334
0.0341
0.035
0.0365
0.0344
0.0361
0.0354
0.0341
0.0357
0.035
0.0334
0.0361
0.0354
0.0347
0.0341
0.0347
0.0338
0.0347
0.0361
0.035
0.035
0.0334

Method

SC $\quad \mathbf{E}$

EPA6010B

EPA6010B

EPA6010B

EPA6010B

EPA6010B

EPA6010B

EPA6010B

EPA6010B

EPA6010B

EPA6010B

EPA6010B

EPA6010B

EPA6010B

EPA6010B

EPA6010B

EPA6010B

EPA6010B

EPA6010B

EPA6010B

EPA6010B

EPA6010B

EPA6010B

EPA6010B

EPA6010B

EPA6010B

EPA6010B

ANALYTE: Cobalt-57

Analytical Group: Radionuclides

\begin{tabular}{|c|c|c|c|c|c|c|}
\hline Survey ID & Sample ID & $\mathbf{F G}$ & Result & Unit & SsMDL & Method \\
\hline SRF-01-01 & 117018 & $\mathrm{U}$ & 0.0125 & $\mathrm{pCi} / \mathrm{g}$ & 0.0334 & EPIA-013B \\
\hline SRF-01-02 & 117019 & $\mathrm{U}$ & -0.00188 & $\mathrm{pCi} / \mathrm{g}$ & 0.0236 & EPIA-013B \\
\hline SRF-01-03 & 117020 & $\mathrm{U}$ & 0.00995 & $\mathrm{pCi} / \mathrm{g}$ & 0.033 & EPIA-013B \\
\hline SRF-01-04 & 117021 & $\mathrm{U}$ & 0.00987 & $\mathrm{pCi} / \mathrm{g}$ & 0.0237 & EPIA-013B \\
\hline SRF-01-05 & 117022 & $\mathrm{U}$ & 0.00534 & $\mathrm{pCi} / \mathrm{g}$ & 0.0261 & EPIA-013B \\
\hline SRF-01-05 & 117022 & $\mathrm{U}$ & 0.00733 & $\mathrm{pCi} / \mathrm{g}$ & 0.0262 & EPIA-013B \\
\hline SRF-02-01 & 117023 & $\mathrm{U}$ & 0.0141 & $\mathrm{pCi} / \mathrm{g}$ & 0.0329 & EPIA-013B \\
\hline SRF-02-02 & 117024 & $\mathrm{U}$ & -0.00706 & $\mathrm{pCi} / \mathrm{g}$ & 0.0228 & EPIA-013B \\
\hline SRF-02-03 & 117025 & $\mathrm{U}$ & 0.0289 & $\mathrm{pCi} / \mathrm{g}$ & 0.0411 & EPIA-013B \\
\hline SRF-02-03-A & 117026 & $\mathrm{U}$ & -0.00317 & $\mathrm{pCi} / \mathrm{g}$ & 0.0378 & EPIA-013B \\
\hline SRF-03-01 & 117027 & $\mathrm{U}$ & 0.00686 & $\mathrm{pCi} / \mathrm{g}$ & 0.0375 & EPIA-013B \\
\hline SRF-03-02 & 117028 & $\mathrm{U}$ & 0.0121 & $\mathrm{pCi} / \mathrm{g}$ & 0.0305 & EPIA-013B \\
\hline SRF-03-03 & 117029 & $\mathrm{U}$ & 0.000747 & $\mathrm{pCi} / \mathrm{g}$ & 0.0308 & EPIA-013B \\
\hline SRF-03-04 & 117030 & $\mathrm{U}$ & 0.00322 & $\mathrm{pCi} / \mathrm{g}$ & 0.0205 & EPIA-013B \\
\hline SRF-04-01 & 117031 & $\mathrm{U}$ & -0.0119 & $\mathrm{pCi} / \mathrm{g}$ & 0.0357 & EPIA-013B \\
\hline SRF-04-02 & 117032 & $\mathrm{U}$ & -0.00906 & $\mathrm{pCi} / \mathrm{g}$ & 0.0381 & EPIA-013B \\
\hline SRF-04-03 & 117033 & $\mathrm{U}$ & -0.0245 & $\mathrm{pCi} / \mathrm{g}$ & 0.0338 & EPIA-013B \\
\hline SRF-05-01 & 117034 & $\mathrm{U}$ & 0.000975 & $\mathrm{pCi} / \mathrm{g}$ & 0.0254 & EPIA-013B \\
\hline SRF-05-02 & 117035 & $\mathrm{U}$ & 0.0139 & $\mathrm{pCi} / \mathrm{g}$ & 0.0252 & EPIA-013B \\
\hline SRF-05-03 & 117036 & $\mathrm{U}$ & -0.00767 & $\mathrm{pCi} / \mathrm{g}$ & 0.026 & EPIA-013B \\
\hline SRF-06-01 & 117037 & $\mathrm{U}$ & 0.00931 & $\mathrm{pCi} / \mathrm{g}$ & 0.0286 & EPIA-013B \\
\hline SRF-06-02 & 117038 & $\mathrm{U}$ & 0.00479 & $\mathrm{pCi} / \mathrm{g}$ & 0.027 & EPIA-013B \\
\hline SRF-06-03 & 117039 & $\mathrm{U}$ & -0.00711 & $\mathrm{pCi} / \mathrm{g}$ & 0.037 & EPIA-013B \\
\hline
\end{tabular}


ANALYTE: Cobalt-57 (cont.)

$\begin{array}{lllrlrrr}\text { Survey ID } & \text { Sample ID } & \text { FG } & \text { Result } & \text { Unit } & \text { ssMDL } & \text { Method } & \text { SC } \\ \text { SRF-06-03-A } & 117040 & \mathrm{U} & 0.00113 & \mathrm{pCi} / \mathrm{g} & 0.0409 & \text { EPIA-013B } & \text { EPIA-013B } \\ \text { SRF-07-01 } & 117041 & \mathrm{U} & -0.00321 & \mathrm{pCi} / \mathrm{g} & 0.032 & \text { EPIA-013B } \\ \text { SRF-07-02 } & 117042 & \mathrm{U} & 0.0241 & \mathrm{pCi} / \mathrm{g} & 0.0406 & \text { EPIA-013B } \\ \text { SRF-07-03 } & 117043 & \mathrm{U} & 0.00576 & \mathrm{pCi} / \mathrm{g} & 0.0278 & \text { EPIA-013B } \\ \text { SRF-07-03 } & 117043 & \mathrm{U} & 0.00223 & \mathrm{pCi} / \mathrm{g} & 0.0349 & \end{array}$

ANALYTE: Cobalt-58

Analytical Group: Radionuclides

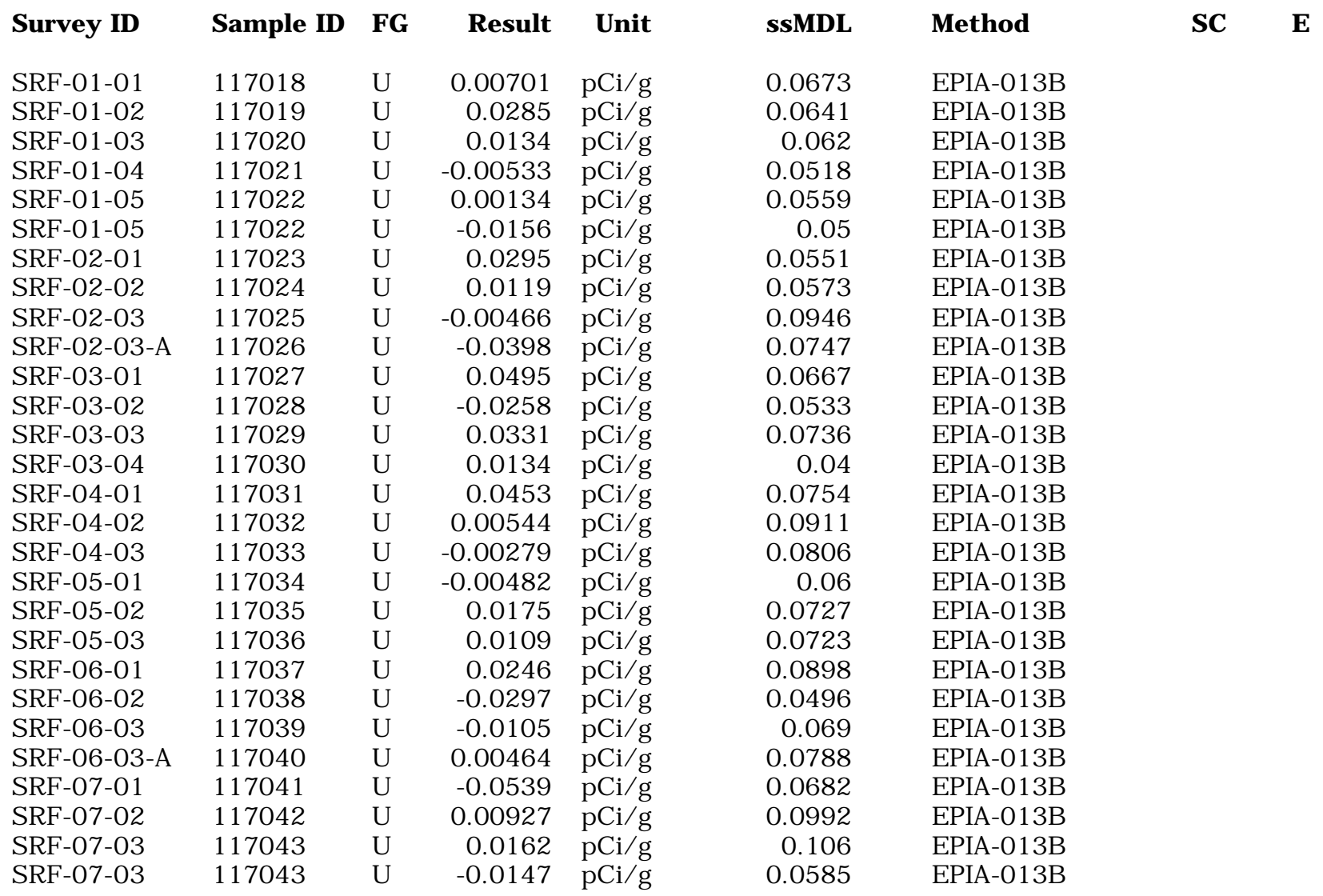

ANALYTE: Cobalt-60

Analytical Group: Radionuclides

$\begin{array}{lllrlrlr}\text { Survey ID } & \text { Sample ID } & \text { FG } & \text { Result } & \text { Unit } & \text { ssMDL } & \text { Method } & \text { SC } \\ \text { SRF-01-01 } & 117018 & \mathrm{U} & -0.00754 & \mathrm{pCi} / \mathrm{g} & 0.061 & \text { EPIA-013B } \\ \text { SRF-01-02 } & 117019 & \mathrm{U} & -0.00673 & \mathrm{pCi} / \mathrm{g} & 0.0484 & \text { EPIA-013B } \\ \text { SRF-01-03 } & 117020 & \mathrm{U} & 0.00821 & \mathrm{pCi} / \mathrm{g} & 0.0672 & \text { EPIA-013B } \\ \text { SRF-01-04 } & 117021 & \mathrm{U} & 0.0187 & \mathrm{pCi} / \mathrm{g} & 0.0547 & \text { EPIA-013B } \\ \text { SRF-01-05 } & 117022 & \mathrm{U} & 0.0145 & \mathrm{pCi} / \mathrm{g} & 0.0543 & \text { EPIA-013B } \\ \text { SRF-01-05 } & 117022 & \mathrm{U} & 0.0128 & \mathrm{pCi} / \mathrm{g} & 0.0552 & \text { EPIA-013B } \\ \text { SRF-02-01 } & 117023 & \mathrm{U} & 0.0318 & \mathrm{pCi} / \mathrm{g} & 0.0637 & \text { EPIA-013B } \\ \text { SRF-02-02 } & 117024 & \mathrm{U} & 0.0421 & \mathrm{pCi} / \mathrm{g} & 0.0582 & \text { EPIA-013B } \\ \text { SRF-02-03 } & 117025 & \mathrm{U} & 0.0198 & \mathrm{pCi} / \mathrm{g} & 0.0882 & \text { EPIA-013B } \\ \text { SRF-02-03-A } & 117026 & \mathrm{U} & -0.0107 & \mathrm{pCi} / \mathrm{g} & 0.0717 & \text { EPIA-013B }\end{array}$


ANALYTE: Cobalt-60 (cont.)

$\begin{array}{lllrlrl}\text { Survey ID } & \text { Sample ID } & \text { FG } & \text { Result } & \text { Unit } & \text { ssMDL } & \text { Method } \\ \text { SRF-03-01 } & 117027 & \mathrm{U} & 0.0444 & \mathrm{pCi} / \mathrm{g} & 0.0685 & \text { EPIA-013B } \\ \text { SRF-03-02 } & 117028 & \mathrm{U} & -0.00223 & \mathrm{pCi} / \mathrm{g} & 0.0532 & \text { EPIA-013B } \\ \mathrm{SRF}-03-03 & 117029 & \mathrm{U} & 0.0219 & \mathrm{pCi} / \mathrm{g} & 0.0666 & \text { EPIA-013B } \\ \mathrm{SRF}-03-04 & 117030 & \mathrm{U} & 0.00656 & \mathrm{pCi} / \mathrm{g} & 0.0352 & \text { EPIA-013B } \\ \text { SRF-04-01 } & 117031 & \mathrm{U} & -0.0147 & \mathrm{pCi} / \mathrm{g} & 0.0491 & \text { EPIA-013B } \\ \text { SRF-04-02 } & 117032 & \mathrm{U} & 0.0218 & \mathrm{pCi} / \mathrm{g} & 0.0758 & \text { EPIA-013B } \\ \text { SRF-04-03 } & 117033 & \mathrm{U} & 0.0048 & \mathrm{pCi} / \mathrm{g} & 0.0816 & \text { EPIA-013B } \\ \text { SRF-05-01 } & 117034 & \mathrm{U} & 0.0086 & \mathrm{pCi} / \mathrm{g} & 0.0529 & \text { EPIA-013B } \\ \text { SRF-05-02 } & 117035 & \mathrm{U} & 0.0272 & \mathrm{pCi} / \mathrm{g} & 0.0615 & \text { EPIA-013B } \\ \text { SRF-05-03 } & 117036 & \mathrm{U} & 0.0189 & \mathrm{pCi} / \mathrm{g} & 0.061 & \text { EPIA-013B } \\ \text { SRF-06-01 } & 117037 & \mathrm{U} & 0.00582 & \mathrm{pCi} / \mathrm{g} & 0.0686 & \text { EPIA-013B } \\ \text { SRF-06-02 } & 117038 & \mathrm{U} & -0.0125 & \mathrm{pCi} / \mathrm{g} & 0.0463 & \text { EPIA-013B } \\ \text { SRF-06-03 } & 117039 & \mathrm{U} & 0.0023 & \mathrm{pCi} / \mathrm{g} & 0.067 & \text { EPIA-013B } \\ \text { SRF-06-03-A } & 117040 & \mathrm{U} & -0.00818 & \mathrm{pCi} / \mathrm{g} & 0.0567 & \text { EPIA-013B } \\ \text { SRF-07-01 } & 117041 & \mathrm{U} & -0.00956 & \mathrm{pCi} / \mathrm{g} & 0.077 & \text { EPIA-013B } \\ \text { SRF-07-02 } & 117042 & \mathrm{U} & 0.00603 & \mathrm{pCi} / \mathrm{g} & 0.0763 & \text { EPIA-013B } \\ \text { SRF-07-03 } & 117043 & \mathrm{U} & 0.00368 & \mathrm{pCi} / \mathrm{g} & 0.0583 & \text { EPIA-013B } \\ \text { SRF-07-03 } & 117043 & \mathrm{U} & -0.0573 & \mathrm{pCi} / \mathrm{g} & 0.0787 & \text { EPIA-013B }\end{array}$

ANALYTE: Copper

Analytical Group: Metals (total recoverable)

\begin{tabular}{|c|c|c|c|c|c|c|c|}
\hline Survey ID & Sample ID & $\mathbf{F G}$ & Result & Unit & SSMDL & Method & SC \\
\hline SRF-01-01 & 117018 & & 0.702 & $\mathrm{mg} / \mathrm{kg}$ & 0.06 & EPA6010B & \\
\hline SRF-01-02 & 117019 & & 0.731 & $\mathrm{mg} / \mathrm{kg}$ & 0.0629 & EPA6010B & \\
\hline SRF-01-03 & 117020 & & 1.28 & $\mathrm{mg} / \mathrm{kg}$ & 0.06 & EPA6010B & \\
\hline SRF-01-04 & 117021 & & 0.316 & $\mathrm{mg} / \mathrm{kg}$ & 0.0611 & EPA6010B & \\
\hline SRF-01-05 & 117022 & & 0.413 & $\mathrm{mg} / \mathrm{kg}$ & 0.0606 & EPA6010B & \\
\hline SRF-02-01 & 117023 & & 0.826 & $\mathrm{mg} / \mathrm{kg}$ & 0.0617 & EPA6010B & \\
\hline SRF-02-02 & 117024 & $\mathrm{~J}$ & 0.217 & $\mathrm{mg} / \mathrm{kg}$ & 0.0635 & EPA6010B & I \\
\hline SRF-02-03 & 117025 & & 1.48 & $\mathrm{mg} / \mathrm{kg}$ & 0.066 & EPA6010B & \\
\hline SRF-02-03-A & 117026 & & 7.3 & $\mathrm{mg} / \mathrm{kg}$ & 0.0623 & EPA6010B & \\
\hline SRF-03-01 & 117027 & & 0.363 & $\mathrm{mg} / \mathrm{kg}$ & 0.0653 & EPA6010B & \\
\hline SRF-03-02 & 117028 & & 2.55 & $\mathrm{mg} / \mathrm{kg}$ & 0.0641 & EPA6010B & \\
\hline SRF-03-03 & 117029 & & 1.16 & $\mathrm{mg} / \mathrm{kg}$ & 0.0617 & EPA6010B & \\
\hline SRF-03-04 & 117030 & & 0.455 & $\mathrm{mg} / \mathrm{kg}$ & 0.0647 & EPA6010B & \\
\hline SRF-04-01 & 117031 & & 0.746 & $\mathrm{mg} / \mathrm{kg}$ & 0.0635 & EPA6010B & \\
\hline SRF-04-02 & 117032 & & 0.305 & $\mathrm{mg} / \mathrm{kg}$ & 0.0606 & EPA6010B & \\
\hline SRF-04-03 & 117033 & & 0.26 & $\mathrm{mg} / \mathrm{kg}$ & 0.0653 & EPA6010B & \\
\hline SRF-05-01 & 117034 & & 0.778 & $\mathrm{mg} / \mathrm{kg}$ & 0.0641 & EPA6010B & \\
\hline SRF-05-02 & 117035 & & 0.251 & $\mathrm{mg} / \mathrm{kg}$ & 0.0629 & EPA6010B & \\
\hline SRF-05-03 & 117036 & & 0.519 & $\mathrm{mg} / \mathrm{kg}$ & 0.0617 & EPA6010B & \\
\hline SRF-06-01 & 117037 & & 2.91 & $\mathrm{mg} / \mathrm{kg}$ & 0.0629 & EPA6010B & \\
\hline SRF-06-02 & 117038 & & 1.19 & $\mathrm{mg} / \mathrm{kg}$ & 0.0611 & EPA6010B & \\
\hline SRF-06-03 & 117039 & & 0.444 & $\mathrm{mg} / \mathrm{kg}$ & 0.0629 & EPA6010B & \\
\hline SRF-06-03-A & 117040 & & 0.6 & $\mathrm{mg} / \mathrm{kg}$ & 0.0653 & EPA6010B & \\
\hline SRF-07-01 & 117041 & & 0.377 & $\mathrm{mg} / \mathrm{kg}$ & 0.0635 & EPA6010B & \\
\hline SRF-07-02 & 117042 & & 0.414 & $\mathrm{mg} / \mathrm{kg}$ & 0.0635 & EPA6010B & \\
\hline SRF-07-03 & 117043 & & 1.2 & $\mathrm{mg} / \mathrm{kg}$ & 0.0606 & EPA6010B & \\
\hline
\end{tabular}

ANALYTE: Dibromochloromethane

Analytical Group: Volatile Organic Compounds

$\begin{array}{lllrrrrr}\text { Survey ID } & \text { Sample ID } & \text { FG } & \text { Result } & \text { Unit } & \text { ssMDL } & \text { Method } & \text { SC } \\ \text { SRF-01-01 } & 117018 & \text { U } & 5 & \mu g / k g & 1.05 & \text { EPA8260B } & \\ \text { SRF-01-02 } & 117019 & \text { U } & 5 & \mu g / k g & 1.05 & \text { EPA8260B } & \end{array}$




\begin{tabular}{|c|c|c|c|c|c|c|}
\hline SRF-01-03 & 117020 & $\mathrm{U}$ & 5 & $\mu g / \mathrm{kg}$ & 1.05 & EPA8260B \\
\hline SRF-01-04 & 117021 & $\mathrm{U}$ & 5 & $\mu g / k g$ & 1.05 & EPA8260B \\
\hline SRF-01-05 & 117022 & $\mathrm{U}$ & 5 & $\mu g / k g$ & 1.05 & EPA8260B \\
\hline SRF-02-01 & 117023 & $\mathrm{U}$ & 5 & $\mu \mathrm{g} / \mathrm{kg}$ & 1.05 & EPA8260B \\
\hline SRF-02-02 & 117024 & $\mathrm{U}$ & 5 & $\mu \mathrm{g} / \mathrm{kg}$ & 1.05 & EPA8260B \\
\hline SRF-02-03 & 117025 & $\mathrm{U}$ & 5 & $\mu \mathrm{g} / \mathrm{kg}$ & 1.05 & EPA8260B \\
\hline SRF-02-03-A & 117026 & $\mathrm{U}$ & 5 & $\mu \mathrm{g} / \mathrm{kg}$ & 1.05 & EPA8260B \\
\hline SRF-03-01 & 117027 & $\mathrm{U}$ & 5 & $\mu \mathrm{g} / \mathrm{kg}$ & 1.05 & EPA8260B \\
\hline SRF-03-02 & 117028 & $\mathrm{U}$ & 5 & $\mu g / k g$ & 1.05 & EPA8260B \\
\hline SRF-03-03 & 117029 & $\mathrm{U}$ & 5 & $\mu g / k g$ & 1.05 & EPA8260B \\
\hline SRF-03-04 & 117030 & $\mathrm{U}$ & 5 & $\mu \mathrm{g} / \mathrm{kg}$ & 1.05 & EPA8260B \\
\hline SRF-04-01 & 117031 & $\mathrm{U}$ & 5 & $\mu \mathrm{g} / \mathrm{kg}$ & 1.05 & EPA8260B \\
\hline SRF-04-02 & 117032 & $\mathrm{U}$ & 5 & $\mu \mathrm{g} / \mathrm{kg}$ & 1.05 & EPA8260B \\
\hline SRF-04-03 & 117033 & $\mathrm{U}$ & 5 & $\mu \mathrm{g} / \mathrm{kg}$ & 1.05 & EPA8260B \\
\hline SRF-05-01 & 117034 & $\mathrm{U}$ & 5 & $\mu \mathrm{g} / \mathrm{kg}$ & 1.05 & EPA8260B \\
\hline SRF-05-02 & 117035 & $\mathrm{U}$ & 5 & $\mu \mathrm{g} / \mathrm{kg}$ & 1.05 & EPA8260B \\
\hline SRF-05-03 & 117036 & $\mathrm{U}$ & 5 & $\mu \mathrm{g} / \mathrm{kg}$ & 1.05 & EPA8260B \\
\hline SRF-06-01 & 117037 & $\mathrm{U}$ & 5 & $\mu \mathrm{g} / \mathrm{kg}$ & 1.05 & EPA8260B \\
\hline SRF-06-02 & 117038 & $\mathrm{U}$ & 5 & $\mu \mathrm{g} / \mathrm{kg}$ & 1.05 & EPA8260B \\
\hline SRF-06-03 & 117039 & $\mathrm{U}$ & 5 & $\mu \mathrm{g} / \mathrm{kg}$ & 1.05 & EPA8260B \\
\hline SRF-06-03-A & 117040 & $\mathrm{U}$ & 5 & $\mu g / k g$ & 1.05 & EPA8260B \\
\hline SRF-07-01 & 117041 & $\mathrm{U}$ & 5 & $\mu \mathrm{g} / \mathrm{kg}$ & 1.05 & EPA8260B \\
\hline SRF-07-02 & 117042 & $\mathrm{U}$ & 5 & $\mu \mathrm{g} / \mathrm{kg}$ & 1.05 & EPA8260B \\
\hline SRF-07-03 & 117043 & $\mathrm{U}$ & 5 & $\mu \mathrm{g} / \mathrm{kg}$ & 1.05 & EPA8260B \\
\hline
\end{tabular}

ANALYTE: 1,1-Dichloroethane

Analytical Group: Volatile Organic Compounds

\begin{tabular}{|c|c|c|c|c|c|c|c|}
\hline Survey ID & Sample ID & FG & Result & Unit & ssMDL & Method & SC \\
\hline SRF-01-01 & 117018 & $\mathrm{U}$ & 5 & $\mu g / \mathrm{kg}$ & 0.5 & EPA8260B & \\
\hline SRF-01-02 & 117019 & $\mathrm{U}$ & 5 & $\mu g / k g$ & 0.5 & EPA8260B & \\
\hline SRF-01-03 & 117020 & $\mathrm{U}$ & 5 & $\mu \mathrm{g} / \mathrm{kg}$ & 0.5 & EPA8260B & \\
\hline SRF-01-04 & 117021 & $\mathrm{U}$ & 5 & $\mu \mathrm{g} / \mathrm{kg}$ & 0.5 & EPA8260B & \\
\hline SRF-01-05 & 117022 & $\mathrm{U}$ & 5 & $\mu g / \mathrm{kg}$ & 0.5 & EPA8260B & \\
\hline SRF-02-01 & 117023 & $\mathrm{U}$ & 5 & $\mu \mathrm{g} / \mathrm{kg}$ & 0.5 & EPA8260B & \\
\hline SRF-02-02 & 117024 & $\mathrm{U}$ & 5 & $\mu g / \mathrm{kg}$ & 0.5 & EPA8260B & \\
\hline SRF-02-03 & 117025 & $\mathrm{U}$ & 5 & $\mu \mathrm{g} / \mathrm{kg}$ & 0.5 & EPA8260B & \\
\hline SRF-02-03-A & 117026 & $\mathrm{U}$ & 5 & $\mu g / \mathrm{kg}$ & 0.5 & EPA8260B & \\
\hline SRF-03-01 & 117027 & $\mathrm{U}$ & 5 & $\mu \mathrm{g} / \mathrm{kg}$ & 0.5 & EPA8260B & \\
\hline SRF-03-02 & 117028 & $\mathrm{U}$ & 5 & $\mu g / \mathrm{kg}$ & 0.5 & EPA8260B & \\
\hline SRF-03-03 & 117029 & $\mathrm{U}$ & 5 & $\mu g / \mathrm{kg}$ & 0.5 & EPA8260B & \\
\hline SRF-03-04 & 117030 & $\mathrm{U}$ & 5 & $\mu g / k g$ & 0.5 & EPA8260B & \\
\hline SRF-04-01 & 117031 & $\mathrm{U}$ & 5 & $\mu g / \mathrm{kg}$ & 0.5 & EPA8260B & \\
\hline SRF-04-02 & 117032 & $\mathrm{U}$ & 5 & $\mu \mathrm{g} / \mathrm{kg}$ & 0.5 & EPA8260B & \\
\hline SRF-04-03 & 117033 & $\mathrm{U}$ & 5 & $\mu \mathrm{g} / \mathrm{kg}$ & 0.5 & EPA8260B & \\
\hline SRF-05-01 & 117034 & $\mathrm{U}$ & 5 & $\mu \mathrm{g} / \mathrm{kg}$ & 0.5 & EPA8260B & \\
\hline SRF-05-02 & 117035 & $\mathrm{U}$ & 5 & $\mu g / \mathrm{kg}$ & 0.5 & EPA8260B & \\
\hline SRF-05-03 & 117036 & $\mathrm{U}$ & 5 & $\mu \mathrm{g} / \mathrm{kg}$ & 0.5 & EPA8260B & \\
\hline SRF-06-01 & 117037 & $\mathrm{U}$ & 5 & $\mu g / k g$ & 0.5 & EPA8260B & \\
\hline SRF-06-02 & 117038 & $\mathrm{U}$ & 5 & $\mu \mathrm{g} / \mathrm{kg}$ & 0.5 & EPA8260B & \\
\hline SRF-06-03 & 117039 & $\mathrm{U}$ & 5 & $\mu \mathrm{g} / \mathrm{kg}$ & 0.5 & EPA8260B & \\
\hline SRF-06-03-A & 117040 & $\mathrm{U}$ & 5 & $\mu g / \mathrm{kg}$ & 0.5 & EPA8260B & \\
\hline \multicolumn{8}{|c|}{ ANALYTE: 1,1 -Dichloroethane (cont.) } \\
\hline Survey ID & Sample ID & $\mathbf{F G}$ & Result & Unit & ssMDL & Method & SC \\
\hline SRF-07-01 & 117041 & $\mathrm{U}$ & 5 & $\mu g / \mathrm{kg}$ & 0.5 & EPA8260B & \\
\hline SRF-07-02 & 117042 & $\mathrm{U}$ & 5 & $\mu \mathrm{g} / \mathrm{kg}$ & 0.5 & EPA8260B & \\
\hline SRF-07-03 & 117043 & $\mathrm{U}$ & 5 & $\mu \mathrm{g} / \mathrm{kg}$ & 0.5 & EPA8260B & \\
\hline
\end{tabular}


ANALYTE: 1,2 -Dichloroethane

Analytical Group: Volatile Organic Compounds

\begin{tabular}{|c|c|c|c|c|c|c|c|}
\hline Survey ID & Sample ID & $\mathbf{F G}$ & Result & Unit & ssMDL & Method & SC \\
\hline SRF-01-01 & 117018 & $\mathrm{U}$ & 5 & $\mu g / \mathrm{kg}$ & 1.15 & EPA8260B & \\
\hline SRF-01-02 & 117019 & $\mathrm{U}$ & 5 & $\mu \mathrm{g} / \mathrm{kg}$ & 1.15 & EPA8260B & \\
\hline SRF-01-03 & 117020 & $\mathrm{U}$ & 5 & $\mu g / \mathrm{kg}$ & 1.15 & EPA8260B & \\
\hline SRF-01-04 & 117021 & $\mathrm{U}$ & 5 & $\mu \mathrm{g} / \mathrm{kg}$ & 1.15 & EPA8260B & \\
\hline SRF-01-05 & 117022 & $\mathrm{U}$ & 5 & $\mu g / \mathrm{kg}$ & 1.15 & EPA8260B & \\
\hline SRF-02-01 & 117023 & $\mathrm{U}$ & 5 & $\mu g / \mathrm{kg}$ & 1.15 & EPA8260B & \\
\hline SRF-02-02 & 117024 & $\mathrm{U}$ & 5 & $\mu g / k g$ & 1.15 & EPA8260B & \\
\hline SRF-02-03 & 117025 & $\mathrm{U}$ & 5 & $\mu \mathrm{g} / \mathrm{kg}$ & 1.15 & EPA8260B & \\
\hline SRF-02-03-A & 117026 & $\mathrm{U}$ & 5 & $\mu g / k g$ & 1.15 & EPA8260B & \\
\hline SRF-03-01 & 117027 & $\mathrm{U}$ & 5 & $\mu g / \mathrm{kg}$ & 1.15 & EPA8260B & \\
\hline SRF-03-02 & 117028 & $\mathrm{U}$ & 5 & $\mu \mathrm{g} / \mathrm{kg}$ & 1.15 & EPA8260B & \\
\hline SRF-03-03 & 117029 & $\mathrm{U}$ & 5 & $\mu g / \mathrm{kg}$ & 1.15 & EPA8260B & \\
\hline SRF-03-04 & 117030 & $\mathrm{U}$ & 5 & $\mu \mathrm{g} / \mathrm{kg}$ & 1.15 & EPA8260B & \\
\hline SRF-04-01 & 117031 & $\mathrm{U}$ & 5 & $\mu g / \mathrm{kg}$ & 1.15 & EPA8260B & \\
\hline SRF-04-02 & 117032 & $\mathrm{U}$ & 5 & $\mu \mathrm{g} / \mathrm{kg}$ & 1.15 & EPA8260B & \\
\hline SRF-04-03 & 117033 & $\mathrm{U}$ & 5 & $\mu g / \mathrm{kg}$ & 1.15 & EPA8260B & \\
\hline SRF-05-01 & 117034 & $\mathrm{U}$ & 5 & $\mu g / \mathrm{kg}$ & 1.15 & EPA8260B & \\
\hline SRF-05-02 & 117035 & $\mathrm{U}$ & 5 & $\mu g / \mathrm{kg}$ & 1.15 & EPA8260B & \\
\hline SRF-05-03 & 117036 & $\mathrm{U}$ & 5 & $\mu \mathrm{g} / \mathrm{kg}$ & 1.15 & EPA8260B & \\
\hline SRF-06-01 & 117037 & $\mathrm{U}$ & 5 & $\mu g / \mathrm{kg}$ & 1.15 & EPA8260B & \\
\hline SRF-06-02 & 117038 & $\mathrm{U}$ & 5 & $\mu g / \mathrm{kg}$ & 1.15 & EPA8260B & \\
\hline SRF-06-03 & 117039 & $\mathrm{U}$ & 5 & $\mu \mathrm{g} / \mathrm{kg}$ & 1.15 & EPA8260B & \\
\hline SRF-06-03-A & 117040 & $\mathrm{U}$ & 5 & $\mu g / \mathrm{kg}$ & 1.15 & EPA8260B & \\
\hline SRF-07-01 & 117041 & $\mathrm{U}$ & 5 & $\mu \mathrm{g} / \mathrm{kg}$ & 1.15 & EPA8260B & \\
\hline SRF-07-02 & 117042 & $\mathrm{U}$ & 5 & $\mu g / \mathrm{kg}$ & 1.15 & EPA8260B & \\
\hline SRF-07-03 & 117043 & $\mathrm{U}$ & 5 & $\mu g / \mathrm{kg}$ & 1.15 & EPA8260B & \\
\hline
\end{tabular}

ANALYTE: 1,1-Dichloroethylene

Analytical Group: Volatile Organic Compounds

\begin{tabular}{|c|c|c|c|c|c|c|c|c|}
\hline Survey ID & Sample ID & FG & Result & Unit & SSMDL & Method & SC & $\mathbf{E}$ \\
\hline SRF-01-01 & 117018 & $\mathrm{U}$ & 5 & $\mu g / \mathrm{kg}$ & 1.5 & EPA8260B & & \\
\hline SRF-01-02 & 117019 & $\mathrm{U}$ & 5 & $\mu \mathrm{g} / \mathrm{kg}$ & 1.5 & EPA8260B & & \\
\hline SRF-01-03 & 117020 & $\mathrm{U}$ & 5 & $\mu \mathrm{g} / \mathrm{kg}$ & 1.5 & EPA8260B & & \\
\hline SRF-01-04 & 117021 & JU & 5 & $\mu \mathrm{g} / \mathrm{kg}$ & 1.5 & EPA8260B & $\mathrm{L}$ & IX \\
\hline SRF-01-05 & 117022 & $\mathrm{U}$ & 5 & $\mu g / k g$ & 1.5 & EPA8260B & & \\
\hline SRF-02-01 & 117023 & $\mathrm{U}$ & 5 & $\mu \mathrm{g} / \mathrm{kg}$ & 1.5 & EPA8260B & & \\
\hline SRF-02-02 & 117024 & $\mathrm{U}$ & 5 & $\mu g / k g$ & 1.5 & EPA8260B & & \\
\hline SRF-02-03 & 117025 & $\mathrm{U}$ & 5 & $\mu \mathrm{g} / \mathrm{kg}$ & 1.5 & EPA8260B & & \\
\hline SRF-02-03-A & 117026 & $\mathrm{U}$ & 5 & $\mu g / k g$ & 1.5 & EPA8260B & & \\
\hline SRF-03-01 & 117027 & $\mathrm{U}$ & 5 & $\mu \mathrm{g} / \mathrm{kg}$ & 1.5 & EPA8260B & & \\
\hline SRF-03-02 & 117028 & $\mathrm{U}$ & 5 & $\mu g / k g$ & 1.5 & EPA8260B & & \\
\hline SRF-03-03 & 117029 & $\mathrm{U}$ & 5 & $\mu \mathrm{g} / \mathrm{kg}$ & 1.5 & EPA8260B & & \\
\hline SRF-03-04 & 117030 & $\mathrm{U}$ & 5 & $\mu \mathrm{g} / \mathrm{kg}$ & 1.5 & EPA8260B & & \\
\hline SRF-04-01 & 117031 & $\mathrm{U}$ & 5 & $\mu \mathrm{g} / \mathrm{kg}$ & 1.5 & EPA8260B & & \\
\hline \multicolumn{9}{|c|}{ ANALYTE: 1,1-Dichloroethylene (cont.) } \\
\hline Survey ID & Sample ID & FG & Result & Unit & SsMDL & Method & SC & $\mathbf{E}$ \\
\hline SRF-04-02 & 117032 & $\mathrm{U}$ & 5 & $\mu g / \mathrm{kg}$ & 1.5 & EPA8260B & & \\
\hline SRF-04-03 & 117033 & $\mathrm{U}$ & 5 & $\mu g / k g$ & 1.5 & EPA8260B & & \\
\hline SRF-05-01 & 117034 & $\mathrm{U}$ & 5 & $\mu g / \mathrm{kg}$ & 1.5 & EPA8260B & & \\
\hline SRF-05-02 & 117035 & $\mathrm{U}$ & 5 & $\mu g / k g$ & 1.5 & EPA8260B & & \\
\hline SRF-05-03 & 117036 & $\mathrm{U}$ & 5 & $\mu \mathrm{g} / \mathrm{kg}$ & 1.5 & EPA8260B & & \\
\hline SRF-06-01 & 117037 & $\mathrm{U}$ & 5 & $\mu g / k g$ & 1.5 & EPA8260B & & \\
\hline SRF-06-02 & 117038 & $\mathrm{U}$ & 5 & $\mu g / k g$ & 1.5 & EPA8260B & & \\
\hline
\end{tabular}




$\begin{array}{lllllll}\text { SRF-06-03 } & 117039 & \mathrm{U} & 5 & \mu \mathrm{g} / \mathrm{kg} & 1.5 & \text { EPA8260B } \\ \text { SRF-06-03-A } & 117040 & \mathrm{U} & 5 & \mu \mathrm{g} / \mathrm{kg} & 1.5 & \text { EPA8260B } \\ \text { SRF-07-01 } & 117041 & \mathrm{U} & 5 & \mu \mathrm{g} / \mathrm{kg} & 1.5 & \text { EPA8260B } \\ \text { SRF-07-02 } & 117042 & \mathrm{U} & 5 & \mu \mathrm{g} / \mathrm{kg} & 1.5 & \text { EPA8260B } \\ \text { SRF-07-03 } & 117043 & \mathrm{U} & 5 \mu \mathrm{gg} & 1.5 & \text { EPA8260B }\end{array}$

ANALYTE: 1,2 -Dichloroethylene

Analytical Group: Volatile Organic Compounds

$\begin{array}{lllrlrl}\text { Survey ID } & \text { Sample ID } & \text { FG } & \text { Result } & \text { Unit } & \text { ssMDL } & \text { Method } \\ \text { SRF-01-01 } & 117018 & \mathrm{U} & 50 & \mu \mathrm{g} / \mathrm{kg} & 1.25 & \text { EPA8260B } \\ \text { SRF-01-02 } & 117019 & \mathrm{U} & 50 & \mu \mathrm{g} / \mathrm{kg} & 1.25 & \text { EPA8260B } \\ \text { SRF-01-03 } & 117020 & \mathrm{U} & 50 & \mu \mathrm{g} / \mathrm{kg} & 1.25 & \text { EPA8260B } \\ \text { SRF-01-04 } & 117021 & \mathrm{U} & 50 & \mu \mathrm{g} / \mathrm{kg} & 1.25 & \text { EPA8260B } \\ \text { SRF-01-05 } & 117022 & \mathrm{U} & 50 & \mu \mathrm{g} / \mathrm{kg} & 1.25 & \text { EPA8260B } \\ \text { SRF-02-01 } & 117023 & \mathrm{U} & 50 & \mu \mathrm{g} / \mathrm{kg} & 1.25 & \text { EPA8260B } \\ \text { SRF-02-02 } & 117024 & \mathrm{U} & 50 & \mu \mathrm{g} / \mathrm{kg} & 1.25 & \text { EPA8260B } \\ \text { SRF-02-03 } & 117025 & \mathrm{U} & 50 & \mu \mathrm{g} / \mathrm{kg} & 1.25 & \text { EPA8260B } \\ \text { SRF-02-03-A } & 117026 & \mathrm{U} & 50 & \mu \mathrm{g} / \mathrm{kg} & 1.25 & \text { EPA8260B } \\ \text { SRF-03-01 } & 117027 & \mathrm{U} & 50 & \mu \mathrm{g} / \mathrm{kg} & 1.25 & \text { EPA8260B } \\ \text { SRF-03-02 } & 117028 & \mathrm{U} & 50 & \mu \mathrm{g} / \mathrm{kg} & 1.25 & \text { EPA8260B } \\ \text { SRF-03-03 } & 117029 & \mathrm{U} & 50 & \mu \mathrm{g} / \mathrm{kg} & 1.25 & \text { EPA8260B } \\ \text { SRF-03-04 } & 117030 & \mathrm{U} & 50 & \mu \mathrm{g} / \mathrm{kg} & 1.25 & \text { EPA8260B } \\ \text { SRF-04-01 } & 117031 & \mathrm{U} & 50 & \mu \mathrm{g} / \mathrm{kg} & 1.25 & \text { EPA8260B } \\ \text { SRF-04-02 } & 117032 & \mathrm{U} & 50 & \mu \mathrm{g} / \mathrm{kg} & 1.25 & \text { EPA8260B } \\ \text { SRF-04-03 } & 117033 & \mathrm{U} & 50 & \mu \mathrm{g} / \mathrm{kg} & 1.25 & \text { EPA8260B } \\ \text { SRF-05-01 } & 117034 & \mathrm{U} & 50 & \mu \mathrm{g} / \mathrm{kg} & 1.25 & \text { EPA8260B } \\ \text { SRF-05-02 } & 117035 & \mathrm{U} & 50 & \mu \mathrm{g} / \mathrm{kg} & 1.25 & \text { EPA8260B } \\ \text { SRF-05-03 } & 117036 & \mathrm{U} & 50 & \mu \mathrm{g} / \mathrm{kg} & 1.25 & \text { EPA8260B } \\ \text { SRF-06-01 } & 117037 & \mathrm{U} & 50 & \mu \mathrm{g} / \mathrm{kg} & 1.25 & \text { EPA8260B } \\ \text { SRF-06-02 } & 117038 & \mathrm{U} & 50 & \mu \mathrm{g} / \mathrm{kg} & 1.25 & \text { EPA8260B } \\ \text { SRF-06-03 } & 117039 & \mathrm{U} & 50 & \mu \mathrm{g} / \mathrm{kg} & 1.25 & \text { EPA8260B } \\ \text { SRF-06-03-A } & 117040 & \mathrm{U} & 50 & \mu \mathrm{g} / \mathrm{kg} & 1.25 & \text { EPA8260B } \\ \text { SRF-07-01 } & 117041 & \mathrm{U} & 50 & \mu \mathrm{g} / \mathrm{kg} & 1.25 & \text { EPA8260B } \\ \text { SRF-07-02 } & 117042 & \mathrm{U} & 50 & \mu \mathrm{g} / \mathrm{kg} & 1.25 & \text { EPA8260B } \\ \text { SRF-07-03 } & 117043 & \mathrm{U} & 50 & \mu \mathrm{g} / \mathrm{kg} & 1.25 & \text { EPA8260B } \\ & & & & & \end{array}$

ANALYTE: Dichloromethane (Methylene chloride)

Analytical Group: Volatile Organic Compounds

\begin{tabular}{|c|c|c|c|c|c|c|c|c|}
\hline Survey ID & Sample ID & FG & Result & Unit & SSMDL & Method & $\mathbf{S C}$ & $\mathbf{E}$ \\
\hline SRF-01-01 & 117018 & $\mathrm{~J}$ & 209 & $\mu g / \mathrm{kg}$ & 7 & EPA8260B & $\mathrm{K}$ & $\mathrm{O}$ \\
\hline SRF-01-02 & 117019 & $\mathrm{~J}$ & 66.7 & $\mu g / \mathrm{kg}$ & 7 & EPA8260B & $\mathrm{K}$ & $\mathrm{O}$ \\
\hline SRF-01-03 & 117020 & $\mathrm{~J}$ & 19.1 & $\mu \mathrm{g} / \mathrm{kg}$ & 7 & EPA8260B & I & \\
\hline SRF-01-04 & 117021 & $\mathrm{~J}$ & 39.6 & $\mu g / \mathrm{kg}$ & 7 & EPA8260B & $\mathrm{K}$ & $\mathrm{O}$ \\
\hline SRF-01-05 & 117022 & $\mathrm{~J}$ & 91.8 & $\mu g / \mathrm{kg}$ & 7 & EPA8260B & $\mathrm{K}$ & $\mathrm{O}$ \\
\hline \multicolumn{9}{|c|}{ ANALYTE: Dichloromethane (Methylene chloride) (cont.) } \\
\hline Survey ID & Sample ID & FG & Result & Unit & SSMDL & Method & SC & $\mathbf{E}$ \\
\hline SRF-02-01 & 117023 & $\mathrm{~J}$ & 62.6 & $\mu g / \mathrm{kg}$ & 7 & EPA8260B & $\mathrm{K}$ & $\mathrm{O}$ \\
\hline SRF-02-02 & 117024 & $J$ & 45.7 & $\mu g / \mathrm{kg}$ & 7 & EPA8260B & $\mathrm{K}$ & $\mathrm{O}$ \\
\hline SRF-02-03 & 117025 & $J$ & 35.8 & $\mu g / \mathrm{kg}$ & 7 & EPA8260B & $\mathrm{K}$ & $\mathrm{O}$ \\
\hline SRF-02-03-A & 117026 & & 36.1 & $\mu g / \mathrm{kg}$ & 7 & EPA8260B & & \\
\hline SRF-03-01 & 117027 & $J$ & 17.8 & $\mu g / \mathrm{kg}$ & 7 & EPA8260B & I & \\
\hline SRF-03-02 & 117028 & & 29.2 & $\mu g / \mathrm{kg}$ & 7 & EPA8260B & & \\
\hline SRF-03-03 & 117029 & & 112 & $\mu g / \mathrm{kg}$ & 7 & EPA8260B & & \\
\hline SRF-03-04 & 117030 & $\mathrm{U}$ & 25 & $\mu g / \mathrm{kg}$ & 7 & EPA8260B & & \\
\hline SRF-04-01 & 117031 & $\mathrm{~J}$ & 34.1 & $\mu g / \mathrm{kg}$ & 7 & EPA8260B & $\mathrm{K}$ & $\mathrm{O}$ \\
\hline
\end{tabular}




\begin{tabular}{lllrlllll}
\hline SRF-04-02 & 117032 & & 25.5 & $\mu \mathrm{g} / \mathrm{kg}$ & 7 & EPA8260B & & \\
SRF-04-03 & 117033 & $\mathrm{~J}$ & 103 & $\mu \mathrm{g} / \mathrm{kg}$ & 7 & EPA8260B & $\mathrm{K}$ & $\mathrm{O}$ \\
SRF-05-01 & 117034 & $\mathrm{~J}$ & 32 & $\mu \mathrm{g} / \mathrm{kg}$ & 7 & EPA8260B & $\mathrm{K}$ & $\mathrm{O}$ \\
SRF-05-02 & 117035 & & 48.9 & $\mu \mathrm{g} / \mathrm{kg}$ & 7 & EPA8260B & & \\
SRF-05-03 & 117036 & $\mathrm{~J}$ & 78.4 & $\mu \mathrm{g} / \mathrm{kg}$ & 7 & EPA8260B & $\mathrm{K}$ & $\mathrm{O}$ \\
SRF-06-01 & 117037 & $\mathrm{~J}$ & 50 & $\mu \mathrm{g} / \mathrm{kg}$ & 7 & EPA8260B & $\mathrm{K}$ & $\mathrm{O}$ \\
SRF-06-02 & 117038 & $\mathrm{~J}$ & 19.8 & $\mu \mathrm{g} / \mathrm{kg}$ & 7 & EPA8260B & $\mathrm{I}$ & \\
SRF-06-03 & 117039 & $\mathrm{~J}$ & 54.5 & $\mu \mathrm{g} / \mathrm{kg}$ & 7 & EPA8260B & $\mathrm{K}$ & $\mathrm{O}$ \\
SRF-06-03-A & 117040 & & 282 & $\mu \mathrm{g} / \mathrm{kg}$ & 7 & EPA8260B & & \\
SRF-07-01 & 117041 & $\mathrm{~J}$ & 75.3 & $\mu \mathrm{g} / \mathrm{kg}$ & 7 & EPA8260B & $\mathrm{K}$ & $\mathrm{O}$ \\
SRF-07-02 & 117042 & $\mathrm{~J}$ & 61.4 & $\mu \mathrm{g} / \mathrm{kg}$ & 7 & EPA8260B & $\mathrm{K}$ & $\mathrm{O}$ \\
SRF-07-03 & 117043 & $\mathrm{~J}$ & 103 & $\mu \mathrm{g} / \mathrm{kg}$ & 7 & EPA8260B & $\mathrm{K}$ & $\mathrm{O}$
\end{tabular}

ANALYTE: 1,2-Dichloropropane

Analytical Group: Volatile Organic Compounds

$\begin{array}{lllrlrll}\text { Survey ID } & \text { Sample ID } & \text { FG } & \text { Result } & \text { Unit } & \text { ssMDL } & \text { Method } & \text { SC } \\ \text { SRF-01-01 } & 117018 & \mathrm{U} & 5 & \mu \mathrm{g} / \mathrm{kg} & 1.15 & \text { EPA8260B } \\ \text { SRF-01-02 } & 117019 & \mathrm{U} & 5 & \mu \mathrm{g} / \mathrm{kg} & 1.15 & \text { EPA8260B } \\ \text { SRF-01-03 } & 117020 & \mathrm{U} & 5 & \mu \mathrm{g} / \mathrm{kg} & 1.15 & \text { EPA8260B } \\ \text { SRF-01-04 } & 117021 & \mathrm{U} & 5 & \mu \mathrm{g} / \mathrm{kg} & 1.15 & \text { EPA8260B } \\ \text { SRF-01-05 } & 117022 & \mathrm{U} & 5 & \mu \mathrm{g} / \mathrm{kg} & 1.15 & \text { EPA8260B } \\ \text { SRF-02-01 } & 117023 & \mathrm{U} & 5 & \mu \mathrm{g} / \mathrm{kg} & 1.15 & \text { EPA8260B } \\ \text { SRF-02-02 } & 117024 & \mathrm{U} & 5 & \mu \mathrm{g} / \mathrm{kg} & 1.15 & \text { EPA8260B } \\ \text { SRF-02-03 } & 117025 & \mathrm{U} & 5 & \mu \mathrm{g} / \mathrm{kg} & 1.15 & \text { EPA8260B } \\ \text { SRF-02-03-A } & 117026 & \mathrm{U} & 5 & \mu \mathrm{g} / \mathrm{kg} & 1.15 & \text { EPA8260B } \\ \text { SRF-03-01 } & 117027 & \mathrm{U} & 5 & \mu \mathrm{g} / \mathrm{kg} & 1.15 & \text { EPA8260B } \\ \text { SRF-03-02 } & 117028 & \mathrm{U} & 5 & \mu \mathrm{g} / \mathrm{kg} & 1.15 & \text { EPA8260B } \\ \text { SRF-03-03 } & 117029 & \mathrm{U} & 5 & \mu \mathrm{g} / \mathrm{kg} & 1.15 & \text { EPA8260B } \\ \text { SRF-03-04 } & 117030 & \mathrm{U} & 5 & \mu \mathrm{g} / \mathrm{kg} & 1.15 & \text { EPA8260B } \\ \text { SRF-04-01 } & 117031 & \mathrm{U} & 5 & \mu \mathrm{g} / \mathrm{kg} & 1.15 & \text { EPA8260B } \\ \text { SRF-04-02 } & 117032 & \mathrm{U} & 5 & \mu \mathrm{g} / \mathrm{kg} & 1.15 & \text { EPA8260B } \\ \text { SRF-04-03 } & 117033 & \mathrm{U} & 5 & \mu \mathrm{g} / \mathrm{kg} & 1.15 & \text { EPA8260B } \\ \text { SRF-05-01 } & 117034 & \mathrm{U} & 5 & \mu \mathrm{g} / \mathrm{kg} & 1.15 & \text { EPA8260B } \\ \text { SRF-05-02 } & 117035 & \mathrm{U} & 5 & \mu \mathrm{g} / \mathrm{kg} & 1.15 & \text { EPA8260B } \\ \text { SRF-05-03 } & 117036 & \mathrm{U} & 5 & \mu \mathrm{g} / \mathrm{kg} & 1.15 & \text { EPA8260B } \\ \text { SRF-06-01 } & 117037 & \mathrm{U} & 5 & \mu \mathrm{g} / \mathrm{kg} & 1.15 & \text { EPA8260B } \\ \text { SRF-06-02 } & 117038 & \mathrm{U} & 5 & \mu \mathrm{g} / \mathrm{kg} & 1.15 & \text { EPA8260B } \\ \text { SRF-06-03 } & 117039 & \mathrm{U} & 5 & \mu \mathrm{g} / \mathrm{kg} & 1.15 & \text { EPA8260B } \\ \text { SRF-06-03-A } & 117040 & \mathrm{U} & 5 & \mu \mathrm{g} / \mathrm{kg} & 1.15 & \text { EPA8260B } \\ \text { SRF-07-01 } & 117041 & \mathrm{U} & 5 & \mu \mathrm{g} / \mathrm{kg} & 1.15 & \text { EPA8260B } \\ \text { SRF-07-02 } & 117042 & \mathrm{U} & 5 & \mu \mathrm{g} / \mathrm{kg} & 1.15 & \text { EPA8260B } \\ \text { SRF-07-03 } & 117043 & \mathrm{U} & 5 & \mu \mathrm{g} / \mathrm{kg} & 1.15 & \text { EPA8260B } \\ & & & & & & \end{array}$

ANALYTE: cis-1,3-Dichloropropene

Analytical Group: Volatile Organic Compounds

\begin{tabular}{|c|c|c|c|c|c|c|c|}
\hline Survey ID & Sample ID & FG & Result & Unit & SsMDL & Method & $\mathbf{S C}$ \\
\hline SRF-01-01 & 117018 & $\mathrm{U}$ & 5 & $\mu \mathrm{g} / \mathrm{kg}$ & 1 & EPA8260B & \\
\hline SRF-01-02 & 117019 & $\mathrm{U}$ & 5 & $\mu g / \mathrm{kg}$ & 1 & EPA8260B & \\
\hline SRF-01-03 & 117020 & $\mathrm{U}$ & 5 & $\mu \mathrm{g} / \mathrm{kg}$ & 1 & EPA8260B & \\
\hline SRF-01-04 & 117021 & U & 5 & $\mu g / \mathrm{kg}$ & 1 & EPA8260B & \\
\hline SRF-01-05 & 117022 & $\mathrm{U}$ & 5 & $\mu \mathrm{g} / \mathrm{kg}$ & 1 & EPA8260B & \\
\hline SRF-02-01 & 117023 & $\mathrm{U}$ & 5 & $\mu \mathrm{g} / \mathrm{kg}$ & 1 & EPA8260B & \\
\hline SRF-02-02 & 117024 & $\mathrm{U}$ & 5 & $\mu g / \mathrm{kg}$ & 1 & EPA8260B & \\
\hline SRF-02-03 & 117025 & U & 5 & $\mu \mathrm{g} / \mathrm{kg}$ & 1 & EPA8260B & \\
\hline SRF-02-03-A & 117026 & $\mathrm{U}$ & 5 & $\mu g / \mathrm{kg}$ & 1 & EPA8260B & \\
\hline SRF-03-01 & 117027 & $\mathrm{U}$ & 5 & $\mu \mathrm{g} / \mathrm{kg}$ & 1 & EPA8260B & \\
\hline SRF-03-02 & 117028 & $\mathrm{U}$ & 5 & $\mu g / \mathrm{kg}$ & 1 & EPA8260B & \\
\hline SRF-03-03 & 117029 & $\mathrm{U}$ & 5 & $\mu \mathrm{g} / \mathrm{kg}$ & 1 & EPA8260B & \\
\hline
\end{tabular}




$\begin{array}{lllllll}\text { SRF-03-04 } & 117030 & \mathrm{U} & 5 & \mu \mathrm{g} / \mathrm{kg} & 1 & \text { EPA8260B } \\ \text { SRF-04-01 } & 117031 & \mathrm{U} & 5 & \mu \mathrm{g} / \mathrm{kg} & 1 & \text { EPA8260B } \\ \text { SRF-04-02 } & 117032 & \mathrm{U} & 5 & \mu \mathrm{g} / \mathrm{kg} & 1 & \text { EPA8260B } \\ \text { SRF-04-03 } & 117033 & \mathrm{U} & 5 & \mu \mathrm{g} / \mathrm{kg} & 1 & \text { EPA8260B } \\ \text { SRF-05-01 } & 117034 & \mathrm{U} & 5 & \mu \mathrm{g} / \mathrm{kg} & 1 & \text { EPA8260B } \\ \text { SRF-05-02 } & 117035 & \mathrm{U} & 5 & \mu \mathrm{g} / \mathrm{kg} & 1 & \text { EPA8260B } \\ \text { SRF-05-03 } & 117036 & \mathrm{U} & 5 & \mu \mathrm{g} / \mathrm{kg} & 1 & \text { EPA8260B } \\ \text { SRF-06-01 } & 117037 & \mathrm{U} & 5 & \mu \mathrm{g} / \mathrm{kg} & 1 & \text { EPA8260B } \\ \text { SRF-06-02 } & 117038 & \mathrm{U} & 5 & \mu \mathrm{g} / \mathrm{kg} & 1 & \text { EPA8260B } \\ \text { SRF-06-03 } & 117039 & \mathrm{U} & 5 & \mu \mathrm{g} / \mathrm{kg} & 1 & \text { EPA8260B } \\ \text { SRF-06-03-A } & 117040 & \mathrm{U} & 5 & \mu \mathrm{g} / \mathrm{kg} & 1 & \text { EPA8260B } \\ \text { SRF-07-01 } & 117041 & \mathrm{U} & 5 & \mu \mathrm{g} / \mathrm{kg} & 1 & \text { EPA8260B } \\ \text { SRF-07-02 } & 117042 & \mathrm{U} & 5 & \mu \mathrm{g} / \mathrm{kg} & 1 & \text { EPA8260B } \\ \text { SRF-07-03 } & 117043 & \mathrm{U} & 5 & \mu \mathrm{g} / \mathrm{kg} & 1 & \text { EPA8260B }\end{array}$

ANALYTE: trans-1,3-Dichloropropene

Analytical Group: Volatile Organic Compounds

\begin{tabular}{|c|c|c|c|c|c|c|c|}
\hline Survey ID & Sample ID & FG & Result & Unit & ssMDL & Method & SC \\
\hline SRF-01-01 & 117018 & $\mathrm{U}$ & 5 & $\mu g / \mathrm{kg}$ & 1.5 & EPA8260B & \\
\hline SRF-01-02 & 117019 & $\mathrm{U}$ & 5 & $\mu g / \mathrm{kg}$ & 1.5 & EPA8260B & \\
\hline SRF-01-03 & 117020 & $\mathrm{U}$ & 5 & $\mu \mathrm{g} / \mathrm{kg}$ & 1.5 & EPA8260B & \\
\hline SRF-01-04 & 117021 & $\mathrm{U}$ & 5 & $\mu \mathrm{g} / \mathrm{kg}$ & 1.5 & EPA8260B & \\
\hline SRF-01-05 & 117022 & $\mathrm{U}$ & 5 & $\mu g / \mathrm{kg}$ & 1.5 & EPA8260B & \\
\hline SRF-02-01 & 117023 & $\mathrm{U}$ & 5 & $\mu \mathrm{g} / \mathrm{kg}$ & 1.5 & EPA8260B & \\
\hline SRF-02-02 & 117024 & $\mathrm{U}$ & 5 & $\mu \mathrm{g} / \mathrm{kg}$ & 1.5 & EPA8260B & \\
\hline SRF-02-03 & 117025 & $\mathrm{U}$ & 5 & $\mu g / \mathrm{kg}$ & 1.5 & EPA8260B & \\
\hline SRF-02-03-A & 117026 & $\mathrm{U}$ & 5 & $\mu g / \mathrm{kg}$ & 1.5 & EPA8260B & \\
\hline SRF-03-01 & 117027 & $\mathrm{U}$ & 5 & $\mu \mathrm{g} / \mathrm{kg}$ & 1.5 & EPA8260B & \\
\hline SRF-03-02 & 117028 & $\mathrm{U}$ & 5 & $\mu g / \mathrm{kg}$ & 1.5 & EPA8260B & \\
\hline SRF-03-03 & 117029 & $\mathrm{U}$ & 5 & $\mu g / \mathrm{kg}$ & 1.5 & EPA8260B & \\
\hline SRF-03-04 & 117030 & $\mathrm{U}$ & 5 & $\mu \mathrm{g} / \mathrm{kg}$ & 1.5 & EPA8260B & \\
\hline SRF-04-01 & 117031 & $\mathrm{U}$ & 5 & $\mu \mathrm{g} / \mathrm{kg}$ & 1.5 & EPA8260B & \\
\hline SRF-04-02 & 117032 & $\mathrm{U}$ & 5 & $\mu \mathrm{g} / \mathrm{kg}$ & 1.5 & EPA8260B & \\
\hline SRF-04-03 & 117033 & $\mathrm{U}$ & 5 & $\mu \mathrm{g} / \mathrm{kg}$ & 1.5 & EPA8260B & \\
\hline SRF-05-01 & 117034 & $\mathrm{U}$ & 5 & $\mu \mathrm{g} / \mathrm{kg}$ & 1.5 & EPA8260B & \\
\hline SRF-05-02 & 117035 & $\mathrm{U}$ & 5 & $\mu g / \mathrm{kg}$ & 1.5 & EPA8260B & \\
\hline SRF-05-03 & 117036 & $\mathrm{U}$ & 5 & $\mu g / \mathrm{kg}$ & 1.5 & EPA8260B & \\
\hline SRF-06-01 & 117037 & $\mathrm{U}$ & 5 & $\mu g / \mathrm{kg}$ & 1.5 & EPA8260B & \\
\hline SRF-06-02 & 117038 & $\mathrm{U}$ & 5 & $\mu \mathrm{g} / \mathrm{kg}$ & 1.5 & EPA8260B & \\
\hline SRF-06-03 & 117039 & $\mathrm{U}$ & 5 & $\mu \mathrm{g} / \mathrm{kg}$ & 1.5 & EPA8260B & \\
\hline SRF-06-03-A & 117040 & $\mathrm{U}$ & 5 & $\mu g / \mathrm{kg}$ & 1.5 & EPA8260B & \\
\hline \multicolumn{8}{|c|}{ ANALYTE: trans-1,3-Dichloropropene (cont.) } \\
\hline Survey ID & Sample ID & FG & Result & Unit & SSMDL & Method & SC \\
\hline SRF-07-01 & 117041 & $\mathrm{U}$ & 5 & $\mu \mathrm{g} / \mathrm{kg}$ & 1.5 & EPA8260B & \\
\hline SRF-07-02 & 117042 & $\mathrm{U}$ & 5 & $\mu g / \mathrm{kg}$ & 1.5 & EPA8260B & \\
\hline SRF-07-03 & 117043 & $\mathrm{U}$ & 5 & $\mu \mathrm{g} / \mathrm{kg}$ & 1.5 & EPA8260B & \\
\hline
\end{tabular}

ANALYTE: Ethylbenzene

Analytical Group: Volatile Organic Compounds

$\begin{array}{lllrlrlll}\text { Survey ID } & \text { Sample ID } & \text { FG } & \text { Result } & \text { Unit } & \text { ssMDL } & \text { Method } & \text { SC } & \text { E } \\ \text { SRF-01-01 } & 117018 & \mathrm{~J} & 8.23 & \mu \mathrm{g} / \mathrm{kg} & 1.5 & \text { EPA8260B } & \mathrm{K} & \mathrm{O} \\ \text { SRF-01-02 } & 117019 & \mathrm{~J} & 7.6 & \mu \mathrm{g} / \mathrm{kg} & 1.5 & \text { EPA8260B } & \mathrm{K} & \mathrm{O} \\ \text { SRF-01-03 } & 117020 & \mathrm{U} & 5 & \mu \mathrm{g} / \mathrm{kg} & 1.5 & \text { EPA8260B } & & \\ \text { SRF-01-04 } & 117021 & \mathrm{~J} & 4.23 & \mu \mathrm{g} / \mathrm{kg} & 1.5 & \text { EPA8260B } & \mathrm{IK} & \mathrm{O} \\ \text { SRF-01-05 } & 117022 & \mathrm{~J} & 66.4 & \mu \mathrm{g} / \mathrm{kg} & 1.5 & \text { EPA8260B } & \mathrm{K} & \mathrm{O}\end{array}$




\begin{tabular}{|c|c|c|c|c|c|c|c|c|}
\hline SRF-02-01 & 117023 & $\mathrm{~J}$ & 3.18 & $\mu g / \mathrm{kg}$ & 1.5 & EPA8260B & IK & $\mathrm{O}$ \\
\hline SRF-02-02 & 117024 & $\mathrm{~J}$ & 6.88 & $\mu g / \mathrm{kg}$ & 1.5 & EPA8260B & $\mathrm{K}$ & $\mathrm{O}$ \\
\hline SRF-02-03 & 117025 & $\mathrm{U}$ & 5 & $\mu \mathrm{g} / \mathrm{kg}$ & 1.5 & EPA8260B & & \\
\hline SRF-02-03-A & 117026 & $\mathrm{U}$ & 5 & $\mu \mathrm{g} / \mathrm{kg}$ & 1.5 & EPA8260B & & \\
\hline SRF-03-01 & 117027 & $\mathrm{U}$ & 5 & $\mu \mathrm{g} / \mathrm{kg}$ & 1.5 & EPA8260B & & \\
\hline SRF-03-02 & 117028 & & 6.1 & $\mu g / \mathrm{kg}$ & 1.5 & EPA8260B & & \\
\hline SRF-03-03 & 117029 & U & 5 & $\mu g / \mathrm{kg}$ & 1.5 & EPA8260B & & \\
\hline SRF-03-04 & 117030 & & 11.4 & $\mu g / \mathrm{kg}$ & 1.5 & EPA8260B & & \\
\hline SRF-04-01 & 117031 & $\mathrm{~J}$ & 4.49 & $\mu g / \mathrm{kg}$ & 1.5 & EPA8260B & IK & $\mathrm{O}$ \\
\hline SRF-04-02 & 117032 & $\mathrm{~J}$ & 4.09 & $\mu g / \mathrm{kg}$ & 1.5 & EPA8260B & I & \\
\hline SRF-04-03 & 117033 & $\mathrm{~J}$ & 2.73 & $\mu g / \mathrm{kg}$ & 1.5 & EPA8260B & IK & $\mathrm{O}$ \\
\hline SRF-05-01 & 117034 & $\mathrm{U}$ & 5 & $\mu g / \mathrm{kg}$ & 1.5 & EPA8260B & & \\
\hline SRF-05-02 & 117035 & $\mathrm{U}$ & 5 & $\mu g / \mathrm{kg}$ & 1.5 & EPA8260B & & \\
\hline SRF-05-03 & 117036 & $\mathrm{U}$ & 5 & $\mu g / \mathrm{kg}$ & 1.5 & EPA8260B & & \\
\hline SRF-06-01 & 117037 & $\mathrm{~J}$ & 10.3 & $\mu g / \mathrm{kg}$ & 1.5 & EPA8260B & $\mathrm{K}$ & $\mathrm{O}$ \\
\hline SRF-06-02 & 117038 & $\mathrm{U}$ & 5 & $\mu g / \mathrm{kg}$ & 1.5 & EPA8260B & & \\
\hline SRF-06-03 & 117039 & U & 5 & $\mu g / \mathrm{kg}$ & 1.5 & EPA8260B & & \\
\hline SRF-06-03-A & 117040 & & 6.42 & $\mu \mathrm{g} / \mathrm{kg}$ & 1.5 & EPA8260B & & \\
\hline SRF-07-01 & 117041 & U & 5 & $\mu g / \mathrm{kg}$ & 1.5 & EPA8260B & & \\
\hline SRF-07-02 & 117042 & $J$ & 3.45 & $\mu g / \mathrm{kg}$ & 1.5 & EPA8260B & IK & $\mathrm{O}$ \\
\hline SRF-07-03 & 117043 & $\mathrm{U}$ & 5 & $\mu g / \mathrm{kg}$ & 1.5 & EPA8260B & & \\
\hline
\end{tabular}

ANALYTE: Europium-152

Analytical Group: Radionuclides

\begin{tabular}{|c|c|c|c|c|c|c|c|}
\hline Survey ID & Sample ID & FG & Result & Unit & ssMDL & Method & SC \\
\hline SRF-01-01 & 117018 & $\mathrm{U}$ & -0.0452 & $\mathrm{pCi} / \mathrm{g}$ & 0.143 & EPIA-013B & \\
\hline SRF-01-02 & 117019 & $\mathrm{U}$ & 0.0249 & $\mathrm{pCi} / \mathrm{g}$ & 0.113 & EPIA-013B & \\
\hline SRF-01-03 & 117020 & U & -0.00701 & $\mathrm{pCi} / \mathrm{g}$ & 0.126 & EPIA-013B & \\
\hline SRF-01-04 & 117021 & $\mathrm{U}$ & 0.0114 & $\mathrm{pCi} / \mathrm{g}$ & 0.112 & EPIA-013B & \\
\hline SRF-01-05 & 117022 & $\mathrm{U}$ & -0.00988 & $\mathrm{pCi} / \mathrm{g}$ & 0.108 & EPIA-013B & \\
\hline SRF-01-05 & 117022 & $\mathrm{U}$ & 0.000658 & $\mathrm{pCi} / \mathrm{g}$ & 0.114 & EPIA-013B & \\
\hline SRF-02-01 & 117023 & $\mathrm{U}$ & -0.0171 & $\mathrm{pCi} / \mathrm{g}$ & 0.149 & EPIA-013B & \\
\hline SRF-02-02 & 117024 & $\mathrm{U}$ & 0.0431 & $\mathrm{pCi} / \mathrm{g}$ & 0.111 & EPIA-013B & \\
\hline SRF-02-03 & 117025 & U & 0.0139 & $\mathrm{pCi} / \mathrm{g}$ & 0.191 & EPIA-013B & \\
\hline SRF-02-03-A & 117026 & $\mathrm{U}$ & 0.0379 & $\mathrm{pCi} / \mathrm{g}$ & 0.171 & EPIA-013B & \\
\hline SRF-03-01 & 117027 & U & 0.0307 & $\mathrm{pCi} / \mathrm{g}$ & 0.172 & EPIA-013B & \\
\hline SRF-03-02 & 117028 & $\mathrm{U}$ & -0.0325 & $\mathrm{pCi} / \mathrm{g}$ & 0.112 & EPIA-013B & \\
\hline SRF-03-03 & 117029 & $\mathrm{U}$ & 0.0149 & $\mathrm{pCi} / \mathrm{g}$ & 0.129 & EPIA-013B & \\
\hline SRF-03-04 & 117030 & $\mathrm{U}$ & 0.0205 & $\mathrm{pCi} / \mathrm{g}$ & 0.0822 & EPIA-013B & \\
\hline \multicolumn{8}{|c|}{ ANALYTE: Europium-152 (cont.) } \\
\hline Survey ID & Sample ID & $\mathbf{F G}$ & Result & Unit & ssMDL & Method & SC \\
\hline SRF-04-01 & 117031 & $\mathrm{U}$ & 0.0204 & $\mathrm{pCi} / \mathrm{g}$ & 0.128 & EPIA-013B & \\
\hline SRF-04-02 & 117032 & U & 0.046 & $\mathrm{pCi} / \mathrm{g}$ & 0.167 & EPIA-013B & \\
\hline SRF-04-03 & 117033 & $\mathrm{U}$ & 0.0512 & $\mathrm{pCi} / \mathrm{g}$ & 0.162 & EPIA-013B & \\
\hline SRF-05-01 & 117034 & $\mathrm{U}$ & -0.0127 & $\mathrm{pCi} / \mathrm{g}$ & 0.111 & EPIA-013B & \\
\hline SRF-05-02 & 117035 & $\mathrm{U}$ & -0.0167 & $\mathrm{pCi} / \mathrm{g}$ & 0.112 & EPIA-013B & \\
\hline SRF-05-03 & 117036 & U & -0.0418 & $\mathrm{pCi} / \mathrm{g}$ & 0.122 & EPIA-013B & \\
\hline SRF-06-01 & 117037 & $\mathrm{U}$ & -0.00621 & $\mathrm{pCi} / \mathrm{g}$ & 0.137 & EPIA-013B & \\
\hline SRF-06-02 & 117038 & U & 0.0397 & $\mathrm{pCi} / \mathrm{g}$ & 0.105 & EPIA-013B & \\
\hline SRF-06-03 & 117039 & $\mathrm{U}$ & 0.0213 & $\mathrm{pCi} / \mathrm{g}$ & 0.165 & EPIA-013B & \\
\hline SRF-06-03-A & 117040 & U & -0.0168 & $\mathrm{pCi} / \mathrm{g}$ & 0.176 & EPIA-013B & \\
\hline SRF-07-01 & 117041 & $\mathrm{U}$ & 0.0838 & $\mathrm{pCi} / \mathrm{g}$ & 0.142 & EPIA-013B & \\
\hline SRF-07-02 & 117042 & U & 0.00832 & $\mathrm{pCi} / \mathrm{g}$ & 0.189 & EPIA-013B & \\
\hline SRF-07-03 & 117043 & $\mathrm{U}$ & -0.0133 & $\mathrm{pCi} / \mathrm{g}$ & 0.127 & EPIA-013B & \\
\hline SRF-07-03 & 117043 & $\mathrm{U}$ & 0.0234 & $\mathrm{pCi} / \mathrm{g}$ & 0.168 & EPIA-013B & \\
\hline
\end{tabular}


ANALYTE: Europium-154

Analytical Group: Radionuclides

\begin{tabular}{|c|c|c|c|c|c|c|c|c|}
\hline Survey ID & Sample ID & $\mathbf{F G}$ & Result & Unit & ssMDL & Method & SC & $\mathbf{E}$ \\
\hline SRF-01-01 & 117018 & $\mathrm{U}$ & -0.014 & $\mathrm{pCi} / \mathrm{g}$ & 0.152 & EPIA-013B & & \\
\hline SRF-01-02 & 117019 & $\mathrm{U}$ & 0.0534 & $\mathrm{pCi} / \mathrm{g}$ & 0.18 & EPIA-013B & & \\
\hline SRF-01-03 & 117020 & $\mathrm{U}$ & 0.0512 & $\mathrm{pCi} / \mathrm{g}$ & 0.149 & EPIA-013B & & \\
\hline SRF-01-04 & 117021 & $\mathrm{U}$ & 0.0174 & $\mathrm{pCi} / \mathrm{g}$ & 0.132 & EPIA-013B & & \\
\hline SRF-01-05 & 117022 & $\mathrm{U}$ & -0.00085 & $\mathrm{pCi} / \mathrm{g}$ & 0.149 & EPIA-013B & & \\
\hline SRF-01-05 & 117022 & $\mathrm{U}$ & 0.0158 & $\mathrm{pCi} / \mathrm{g}$ & 0.14 & EPIA-013B & & \\
\hline SRF-02-01 & 117023 & $\mathrm{U}$ & -0.0251 & $\mathrm{pCi} / \mathrm{g}$ & 0.149 & EPIA-013B & & \\
\hline SRF-02-02 & 117024 & $\mathrm{U}$ & 0.019 & $\mathrm{pCi} / \mathrm{g}$ & 0.139 & EPIA-013B & & \\
\hline SRF-02-03 & 117025 & $\mathrm{U}$ & 0.0376 & $\mathrm{pCi} / \mathrm{g}$ & 0.207 & EPIA-013B & & \\
\hline SRF-02-03-A & 117026 & $\mathrm{U}$ & -0.0501 & $\mathrm{pCi} / \mathrm{g}$ & 0.199 & EPIA-013B & & \\
\hline SRF-03-01 & 117027 & $\mathrm{U}$ & -0.0319 & $\mathrm{pCi} / \mathrm{g}$ & 0.186 & EPIA-013B & & \\
\hline SRF-03-02 & 117028 & $\mathrm{U}$ & -0.0526 & $\mathrm{pCi} / \mathrm{g}$ & 0.127 & EPIA-013B & & \\
\hline SRF-03-03 & 117029 & $\mathrm{U}$ & -0.014 & $\mathrm{pCi} / \mathrm{g}$ & 0.157 & EPIA-013B & & \\
\hline SRF-03-04 & 117030 & $\mathrm{U}$ & -0.0228 & $\mathrm{pCi} / \mathrm{g}$ & 0.0897 & EPIA-013B & & \\
\hline SRF-04-01 & 117031 & $\mathrm{U}$ & -0.0187 & $\mathrm{pCi} / \mathrm{g}$ & 0.141 & EPIA-013B & & \\
\hline SRF-04-02 & 117032 & $\mathrm{U}$ & -0.0562 & $\mathrm{pCi} / \mathrm{g}$ & 0.215 & EPIA-013B & & \\
\hline SRF-04-03 & 117033 & $\mathrm{U}$ & 0.0607 & $\mathrm{pCi} / \mathrm{g}$ & 0.203 & EPIA-013B & & \\
\hline SRF-05-01 & 117034 & $\mathrm{U}$ & -0.0189 & $\mathrm{pCi} / \mathrm{g}$ & 0.15 & EPIA-013B & & \\
\hline SRF-05-02 & 117035 & $\mathrm{U}$ & 0.0971 & $\mathrm{pCi} / \mathrm{g}$ & 0.185 & EPIA-013B & & \\
\hline SRF-05-03 & 117036 & $\mathrm{U}$ & -0.016 & $\mathrm{pCi} / \mathrm{g}$ & 0.135 & EPIA-013B & & \\
\hline SRF-06-01 & 117037 & $\mathrm{U}$ & -0.0336 & $\mathrm{pCi} / \mathrm{g}$ & 0.171 & EPIA-013B & & \\
\hline SRF-06-02 & 117038 & $\mathrm{U}$ & 0.0818 & $\mathrm{pCi} / \mathrm{g}$ & 0.155 & EPIA-013B & & \\
\hline SRF-06-03 & 117039 & $\mathrm{U}$ & 0.00836 & $\mathrm{pCi} / \mathrm{g}$ & 0.176 & EPIA-013B & & \\
\hline SRF-06-03-A & 117040 & $\mathrm{U}$ & 0.108 & $\mathrm{pCi} / \mathrm{g}$ & 0.242 & EPIA-013B & & \\
\hline SRF-07-01 & 117041 & $\mathrm{U}$ & -0.0287 & $\mathrm{pCi} / \mathrm{g}$ & 0.199 & EPIA-013B & & \\
\hline SRF-07-02 & 117042 & $\mathrm{U}$ & -0.0648 & $\mathrm{pCi} / \mathrm{g}$ & 0.216 & EPIA-013B & & \\
\hline SRF-07-03 & 117043 & $\mathrm{U}$ & 0.0135 & $\mathrm{pCi} / \mathrm{g}$ & 0.235 & EPIA-013B & & \\
\hline SRF-07-03 & 117043 & $\mathrm{U}$ & -0.0124 & $\mathrm{pCi} / \mathrm{g}$ & 0.164 & EPIA-013B & & \\
\hline \multicolumn{9}{|c|}{$\begin{array}{l}\text { ANALYTE: Europium-155 } \\
\text { Analytical Group: Radionuclides }\end{array}$} \\
\hline Survey ID & Sample ID & FG & Result & Unit & SSMDL & Method & SC & $\mathbf{E}$ \\
\hline SRF-01-01 & 117018 & $\mathrm{U}$ & -0.0109 & $\mathrm{pCi} / \mathrm{g}$ & 0.126 & EPIA-013B & & \\
\hline SRF-01-02 & 117019 & $\mathrm{U}$ & 0.0169 & $\mathrm{pCi} / \mathrm{g}$ & 0.0891 & EPIA-013B & & \\
\hline SRF-01-03 & 117020 & $\mathrm{U}$ & 0.0223 & $\mathrm{pCi} / \mathrm{g}$ & 0.114 & EPIA-013B & & \\
\hline SRF-01-04 & 117021 & $\mathrm{U}$ & 0.0404 & $\mathrm{pCi} / \mathrm{g}$ & 0.111 & EPIA-013B & & \\
\hline SRF-01-05 & 117022 & $\mathrm{U}$ & -0.0104 & $\mathrm{pCi} / \mathrm{g}$ & 0.0947 & EPIA-013B & & \\
\hline SRF-01-05 & 117022 & $\mathrm{U}$ & 0.0355 & $\mathrm{pCi} / \mathrm{g}$ & 0.114 & EPIA-013B & & \\
\hline SRF-02-01 & 117023 & $\mathrm{U}$ & 0.000223 & $\mathrm{pCi} / \mathrm{g}$ & 0.122 & EPIA-013B & & \\
\hline SRF-02-02 & 117024 & $\mathrm{U}$ & -0.00745 & $\mathrm{pCi} / \mathrm{g}$ & 0.0899 & EPIA-013B & & \\
\hline SRF-02-03 & 117025 & $\mathrm{U}$ & 0.0159 & $\mathrm{pCi} / \mathrm{g}$ & 0.146 & EPIA-013B & & \\
\hline SRF-02-03-A & 117026 & $\mathrm{U}$ & 0.0157 & $\mathrm{pCi} / \mathrm{g}$ & 0.141 & EPIA-013B & & \\
\hline SRF-03-01 & 117027 & $\mathrm{U}$ & -0.0712 & $\mathrm{pCi} / \mathrm{g}$ & 0.134 & EPIA-013B & & \\
\hline SRF-03-02 & 117028 & $\mathrm{U}$ & 0.0502 & $\mathrm{pCi} / \mathrm{g}$ & 0.11 & EPIA-013B & & \\
\hline SRF-03-03 & 117029 & $\mathrm{U}$ & 0.0129 & $\mathrm{pCi} / \mathrm{g}$ & 0.12 & EPIA-013B & & \\
\hline SRF-03-04 & 117030 & $\mathrm{U}$ & 0.00112 & $\mathrm{pCi} / \mathrm{g}$ & 0.0741 & EPIA-013B & & \\
\hline SRF-04-01 & 117031 & $\mathrm{U}$ & 0.0387 & $\mathrm{pCi} / \mathrm{g}$ & 0.14 & EPIA-013B & & \\
\hline SRF-04-02 & 117032 & $\mathrm{U}$ & -0.0614 & $\mathrm{pCi} / \mathrm{g}$ & 0.14 & EPIA-013B & & \\
\hline SRF-04-03 & 117033 & $\mathrm{U}$ & -0.00634 & $\mathrm{pCi} / \mathrm{g}$ & 0.14 & EPIA-013B & & \\
\hline SRF-05-01 & 117034 & $\mathrm{U}$ & 0.058 & $\mathrm{pCi} / \mathrm{g}$ & 0.102 & EPIA-013B & & \\
\hline SRF-05-02 & 117035 & $\mathrm{U}$ & 0.0187 & $\mathrm{pCi} / \mathrm{g}$ & 0.0934 & EPIA-013B & & \\
\hline SRF-05-03 & 117036 & $\mathrm{U}$ & 0.0327 & $\mathrm{pCi} / \mathrm{g}$ & 0.0986 & EPIA-013B & & \\
\hline SRF-06-01 & 117037 & $\mathrm{U}$ & 0.0123 & $\mathrm{pCi} / \mathrm{g}$ & 0.105 & EPIA-013B & & \\
\hline SRF-06-02 & 117038 & $\mathrm{U}$ & -0.028 & $\mathrm{pCi} / \mathrm{g}$ & 0.0867 & EPIA-013B & & \\
\hline SRF-06-03 & 117039 & $\mathrm{U}$ & -0.0854 & $\mathrm{pCi} / \mathrm{g}$ & 0.138 & EPIA-013B & & \\
\hline SRF-06-03-A & 117040 & $\mathrm{U}$ & 0.00113 & $\mathrm{pCi} / \mathrm{g}$ & 0.145 & EPIA-013B & & \\
\hline
\end{tabular}




\begin{tabular}{lllrlll}
\hline SRF-07-01 & 117041 & $\mathrm{U}$ & 0.00657 & $\mathrm{pCi} / \mathrm{g}$ & 0.119 & EPIA-013B \\
$\mathrm{SRF}-07-02$ & 117042 & $\mathrm{U}$ & 0.0292 & $\mathrm{pCi} / \mathrm{g}$ & 0.159 & EPIA-013B \\
$\mathrm{SRF}-07-03$ & 117043 & $\mathrm{U}$ & -0.0332 & $\mathrm{pCi} / \mathrm{g}$ & 0.124 & EPIA-013B \\
$\mathrm{SRF}-07-03$ & 117043 & $\mathrm{U}$ & 0.0307 & $\mathrm{pCi} / \mathrm{g}$ & 0.113 & EPIA-013B
\end{tabular}

ANALYTE: Gross alpha

Analytical Group: Radionuclides

\begin{tabular}{|c|c|c|c|c|c|c|c|}
\hline Survey ID & Sample ID & $\mathbf{F G}$ & Result & Unit & SsMDL & Method & SC \\
\hline SRF-01-01 & 117018 & $\mathrm{U}$ & -0.0306 & $\mathrm{pCi} / \mathrm{g}$ & 0.733 & EPIA-001B & \\
\hline SRF-01-02 & 117019 & $\mathrm{U}$ & 0.1 & $\mathrm{pCi} / \mathrm{g}$ & 0.377 & EPIA-001B & \\
\hline SRF-01-03 & 117020 & $\mathrm{U}$ & -0.142 & $\mathrm{pCi} / \mathrm{g}$ & 0.801 & EPIA-001B & \\
\hline SRF-01-04 & 117021 & $\mathrm{U}$ & 0.122 & $\mathrm{pCi} / \mathrm{g}$ & 0.428 & EPIA-001B & \\
\hline SRF-01-05 & 117022 & $\mathrm{U}$ & 0.502 & $\mathrm{pCi} / \mathrm{g}$ & 1.58 & EPIA-001B & \\
\hline SRF-01-05 & 117022 & $\mathrm{U}$ & 0.653 & $\mathrm{pCi} / \mathrm{g}$ & 1.29 & EPIA-001B & \\
\hline SRF-02-01 & 117023 & $\mathrm{U}$ & -0.19 & $\mathrm{pCi} / \mathrm{g}$ & 1.5 & EPIA-001B & \\
\hline SRF-02-02 & 117024 & $\mathrm{U}$ & -0.214 & $\mathrm{pCi} / \mathrm{g}$ & 1.22 & EPIA-001B & \\
\hline SRF-02-03 & 117025 & $\mathrm{U}$ & 0.111 & $\mathrm{pCi} / \mathrm{g}$ & 0.995 & EPIA-001B & \\
\hline SRF-02-03-A & 117026 & $\mathrm{U}$ & 0.162 & $\mathrm{pCi} / \mathrm{g}$ & 1.07 & EPIA-001B & \\
\hline SRF-03-01 & 117027 & $\mathrm{U}$ & -0.0176 & $\mathrm{pCi} / \mathrm{g}$ & 1.75 & EPIA-001B & \\
\hline SRF-03-02 & 117028 & $\mathrm{U}$ & 0.0683 & $\mathrm{pCi} / \mathrm{g}$ & 1.69 & EPIA-001B & \\
\hline SRF-03-03 & 117029 & $\mathrm{U}$ & -0.266 & $\mathrm{pCi} / \mathrm{g}$ & 0.978 & EPIA-001B & \\
\hline SRF-03-04 & 117030 & $\mathrm{U}$ & 0.17 & $\mathrm{pCi} / \mathrm{g}$ & 2.74 & EPIA-001B & \\
\hline SRF-04-01 & 117031 & $\mathrm{U}$ & 0.0186 & $\mathrm{pCi} / \mathrm{g}$ & 1.12 & EPIA-001B & \\
\hline SRF-04-02 & 117032 & $\mathrm{U}$ & -0.471 & $\mathrm{pCi} / \mathrm{g}$ & 1.71 & EPIA-001B & \\
\hline SRF-04-03 & 117033 & $\mathrm{U}$ & 0.611 & $\mathrm{pCi} / \mathrm{g}$ & 0.885 & EPIA-001B & \\
\hline SRF-05-01 & 117034 & $\mathrm{U}$ & 0.24 & $\mathrm{pCi} / \mathrm{g}$ & 0.948 & EPIA-001B & \\
\hline SRF-05-02 & 117035 & $\mathrm{U}$ & 0.224 & $\mathrm{pCi} / \mathrm{g}$ & 1.29 & EPIA-001B & \\
\hline SRF-05-03 & 117036 & $\mathrm{U}$ & -0.0606 & $\mathrm{pCi} / \mathrm{g}$ & 1.02 & EPIA-001B & \\
\hline SRF-06-01 & 117037 & $\mathrm{U}$ & -0.0717 & $\mathrm{pCi} / \mathrm{g}$ & 0.861 & EPIA-001B & \\
\hline \multicolumn{8}{|c|}{ ANALYTE: Gross alpha (cont.) } \\
\hline Survey ID & Sample ID & $\mathbf{F G}$ & Result & Unit & SsMDL & Method & SC \\
\hline SRF-06-02 & 117038 & $\mathrm{U}$ & -0.521 & $\mathrm{pCi} / \mathrm{g}$ & 1.42 & EPIA-001B & \\
\hline SRF-06-03 & 117039 & $\mathrm{U}$ & 0.0231 & $\mathrm{pCi} / \mathrm{g}$ & 0.558 & EPIA-001B & \\
\hline SRF-06-03-A & 117040 & $\mathrm{U}$ & 0.151 & $\mathrm{pCi} / \mathrm{g}$ & 0.681 & EPIA-001B & \\
\hline SRF-07-01 & 117041 & $\mathrm{U}$ & 0.289 & $\mathrm{pCi} / \mathrm{g}$ & 0.556 & EPIA-001B & \\
\hline SRF-07-02 & 117042 & $\mathrm{U}$ & -0.539 & $\mathrm{pCi} / \mathrm{g}$ & 1.34 & EPIA-001B & \\
\hline SRF-07-03 & 117043 & $\mathrm{U}$ & 0.0824 & $\mathrm{pCi} / \mathrm{g}$ & 0.431 & EPIA-001B & \\
\hline SRF-07-03 & 117043 & $\mathrm{U}$ & 0.0998 & $\mathrm{pCi} / \mathrm{g}$ & 0.618 & EPIA-001B & \\
\hline
\end{tabular}

ANALYTE: 2-Hexanone

Analytical Group: Volatile Organic Compounds

\begin{tabular}{lllrllll} 
Survey ID & Sample ID & FG & Result & Unit & ssMDL & Method & SC \\
SRF-01-01 & 117018 & $\mathrm{U}$ & 25 & $\mu \mathrm{g} / \mathrm{kg}$ & 14 & EPA8260B \\
SRF-01-02 & 117019 & $\mathrm{U}$ & 25 & $\mu \mathrm{g} / \mathrm{kg}$ & 14 & EPA8260B \\
SRF-01-03 & 117020 & $\mathrm{U}$ & 25 & $\mu \mathrm{g} / \mathrm{kg}$ & 14 & EPA8260B \\
SRF-01-04 & 117021 & $\mathrm{U}$ & 25 & $\mu \mathrm{g} / \mathrm{kg}$ & 14 & EPA8260B \\
SRF-01-05 & 117022 & $\mathrm{U}$ & 25 & $\mu \mathrm{g} / \mathrm{kg}$ & 14 & EPA8260B \\
SRF-02-01 & 117023 & $\mathrm{U}$ & 25 & $\mu \mathrm{g} / \mathrm{kg}$ & 14 & EPA8260B \\
SRF-02-02 & 117024 & $\mathrm{U}$ & 25 & $\mu \mathrm{g} / \mathrm{kg}$ & 14 & EPA8260B \\
SRF-02-03 & 117025 & $\mathrm{U}$ & 25 & $\mu \mathrm{g} / \mathrm{kg}$ & 14 & EPA8260B \\
SRF-02-03-A & 117026 & $\mathrm{U}$ & 25 & $\mu \mathrm{g} / \mathrm{kg}$ & 14 & EPA8260B \\
SRF-03-01 & 117027 & $\mathrm{U}$ & 25 & $\mu \mathrm{g} / \mathrm{kg}$ & 14 & EPA8260B \\
SRF-03-02 & 117028 & $\mathrm{U}$ & 25 & $\mu \mathrm{g} / \mathrm{kg}$ & 14 & EPA8260B \\
SRF-03-03 & 117029 & $\mathrm{U}$ & 25 & $\mu \mathrm{g} / \mathrm{kg}$ & 14 & EPA8260B \\
SRF-03-04 & 117030 & $\mathrm{U}$ & 25 & $\mu \mathrm{g} / \mathrm{kg}$ & 14 & EPA8260B \\
\hline
\end{tabular}




\begin{tabular}{lllllll}
\hline SRF-04-01 & 117031 & $\mathrm{U}$ & 25 & $\mu \mathrm{g} / \mathrm{kg}$ & 14 & EPA8260B \\
SRF-04-02 & 117032 & $\mathrm{U}$ & 25 & $\mu \mathrm{g} / \mathrm{kg}$ & 14 & EPA8260B \\
SRF-04-03 & 117033 & $\mathrm{U}$ & 25 & $\mu \mathrm{g} / \mathrm{kg}$ & 14 & EPA8260B \\
SRF-05-01 & 117034 & $\mathrm{U}$ & 25 & $\mu \mathrm{g} / \mathrm{kg}$ & 14 & EPA8260B \\
SRF-05-02 & 117035 & $\mathrm{U}$ & 25 & $\mu \mathrm{g} / \mathrm{kg}$ & 14 & EPA8260B \\
SRF-05-03 & 117036 & $\mathrm{U}$ & 25 & $\mu \mathrm{g} / \mathrm{kg}$ & 14 & EPA8260B \\
SRF-06-01 & 117037 & $\mathrm{U}$ & 25 & $\mu \mathrm{g} / \mathrm{kg}$ & 14 & EPA8260B \\
SRF-06-02 & 117038 & $\mathrm{U}$ & 25 & $\mu \mathrm{g} / \mathrm{kg}$ & 14 & EPA8260B \\
SRF-06-03 & 117039 & $\mathrm{U}$ & 25 & $\mu \mathrm{g} / \mathrm{kg}$ & 14 & EPA8260B \\
SRF-06-03-A & 117040 & $\mathrm{U}$ & 25 & $\mu \mathrm{g} / \mathrm{kg}$ & 14 & EPA8260B \\
SRF-07-01 & 117041 & $\mathrm{U}$ & 25 & $\mu \mathrm{g} / \mathrm{kg}$ & 14 & EPA8260B \\
SRF-07-02 & 117042 & $\mathrm{U}$ & 25 & $\mu \mathrm{g} / \mathrm{kg}$ & 14 & EPA8260B \\
SRF-07-03 & 117043 & $\mathrm{U}$ & 25 & $\mu \mathrm{g} / \mathrm{kg}$ & 14 & EPA8260B
\end{tabular}

ANALYTE: Lead-212

Analytical Group: Radionuclides

\begin{tabular}{|c|c|c|c|c|c|c|c|c|}
\hline Survey ID & Sample ID & FG & Result & Unit & ssMDL & Method & SC & $\mathbf{E}$ \\
\hline SRF-01-01 & 117018 & $\mathrm{U}$ & 0.0379 & $\mathrm{pCi} / \mathrm{g}$ & 0.0783 & EPIA-013B & & \\
\hline SRF-01-02 & 117019 & $\mathrm{R}$ & 0.874 & $\mathrm{pCi} / \mathrm{g}$ & 0.0751 & EPIA-013B & & 4 \\
\hline SRF-01-03 & 117020 & U & 0.0704 & $\mathrm{pCi} / \mathrm{g}$ & 0.0872 & EPIA-013B & & \\
\hline SRF-01-04 & 117021 & $\mathrm{U}$ & 0.014 & $\mathrm{pCi} / \mathrm{g}$ & 0.11 & EPIA-013B & & \\
\hline SRF-01-05 & 117022 & $\mathrm{U}$ & 0.0725 & $\mathrm{pCi} / \mathrm{g}$ & 0.0797 & EPIA-013B & & \\
\hline SRF-01-05 & 117022 & $\mathrm{R}$ & 0.0923 & $\mathrm{pCi} / \mathrm{g}$ & 0.0779 & EPIA-013B & & 4 \\
\hline SRF-02-01 & 117023 & $\mathrm{U}$ & 0.0869 & $\mathrm{pCi} / \mathrm{g}$ & 0.0955 & EPIA-013B & & \\
\hline SRF-02-02 & 117024 & $\mathrm{U}$ & 0.0586 & $\mathrm{pCi} / \mathrm{g}$ & 0.0773 & EPIA-013B & & \\
\hline SRF-02-03 & 117025 & $\mathrm{U}$ & 0.0564 & $\mathrm{pCi} / \mathrm{g}$ & 0.11 & EPIA-013B & & \\
\hline SRF-02-03-A & 117026 & $\mathrm{U}$ & 0.0862 & $\mathrm{pCi} / \mathrm{g}$ & 0.107 & EPIA-013B & & \\
\hline \multicolumn{9}{|c|}{ ANALYTE: Lead-212 (cont.) } \\
\hline Survey ID & Sample ID & FG & Result & Unit & SSMDL & Method & SC & $\mathbf{E}$ \\
\hline SRF-03-01 & 117027 & U & 0.0128 & $\mathrm{pCi} / \mathrm{g}$ & 0.107 & EPIA-013B & & \\
\hline SRF-03-02 & 117028 & $\mathrm{R}$ & 0.0493 & $\mathrm{pCi} / \mathrm{g}$ & 0.0825 & EPIA-013B & & 4 \\
\hline SRF-03-03 & 117029 & U & 0.0493 & $\mathrm{pCi} / \mathrm{g}$ & 0.0823 & EPIA-013B & & \\
\hline SRF-03-04 & 117030 & & 0.0683 & $\mathrm{pCi} / \mathrm{g}$ & 0.0443 & EPIA-013B & & \\
\hline SRF-04-01 & 117031 & U & 0.00678 & $\mathrm{pCi} / \mathrm{g}$ & 0.0927 & EPIA-013B & & \\
\hline SRF-04-02 & 117032 & $\mathrm{U}$ & 0.0877 & $\mathrm{pCi} / \mathrm{g}$ & 0.106 & EPIA-013B & & \\
\hline SRF-04-03 & 117033 & $\mathrm{U}$ & 0.089 & $\mathrm{pCi} / \mathrm{g}$ & 0.103 & EPIA-013B & & \\
\hline SRF-05-01 & 117034 & $\mathrm{U}$ & 0.00228 & $\mathrm{pCi} / \mathrm{g}$ & 0.0718 & EPIA-013B & & \\
\hline SRF-05-02 & 117035 & $\mathrm{R}$ & 0.0924 & $\mathrm{pCi} / \mathrm{g}$ & 0.0767 & EPIA-013B & & 4 \\
\hline SRF-05-03 & 117036 & $\mathrm{U}$ & 0.00465 & $\mathrm{pCi} / \mathrm{g}$ & 0.0849 & EPIA-013B & & \\
\hline SRF-06-01 & 117037 & U & 0.0237 & $\mathrm{pCi} / \mathrm{g}$ & 0.0919 & EPIA-013B & & \\
\hline SRF-06-02 & 117038 & $\mathrm{U}$ & 0.0159 & $\mathrm{pCi} / \mathrm{g}$ & 0.0658 & EPIA-013B & & \\
\hline SRF-06-03 & 117039 & U & 0.0565 & $\mathrm{pCi} / \mathrm{g}$ & 0.0904 & EPIA-013B & & \\
\hline SRF-06-03-A & 117040 & U & 0.00505 & $\mathrm{pCi} / \mathrm{g}$ & 0.103 & EPIA-013B & & \\
\hline SRF-07-01 & 117041 & $\mathrm{R}$ & 0.124 & $\mathrm{pCi} / \mathrm{g}$ & 0.106 & EPIA-013B & & 4 \\
\hline SRF-07-02 & 117042 & $\mathrm{U}$ & 0.0868 & $\mathrm{pCi} / \mathrm{g}$ & 0.123 & EPIA-013B & & \\
\hline SRF-07-03 & 117043 & $\mathrm{R}$ & 0.134 & $\mathrm{pCi} / \mathrm{g}$ & 0.109 & EPIA-013B & & 4 \\
\hline SRF-07-03 & 117043 & $\mathrm{U}$ & 0.0271 & $\mathrm{pCi} / \mathrm{g}$ & 0.0842 & EPIA-013B & & \\
\hline \multicolumn{9}{|c|}{$\begin{array}{l}\text { ANALYTE: Lead } \\
\text { Analytical Group: Metals (total recoverable) }\end{array}$} \\
\hline Survey ID & Sample ID & FG & Result & Unit & SsMDL & Method & SC & $\mathbf{E}$ \\
\hline SRF-01-01 & 117018 & $\mathrm{~J}$ & 0.0844 & $\mathrm{mg} / \mathrm{kg}$ & 0.072 & EPA6010B & I & \\
\hline SRF-01-02 & 117019 & $J$ & 0.173 & $\mathrm{mg} / \mathrm{kg}$ & 0.075 & EPA6010B & I & \\
\hline SRF-01-03 & 117020 & U & 0.227 & $\mathrm{mg} / \mathrm{kg}$ & 0.072 & EPA6010B & & \\
\hline SRF-01-04 & 117021 & $\mathrm{~J}$ & 0.146 & $\mathrm{mg} / \mathrm{kg}$ & 0.073 & EPA6010B & I & \\
\hline
\end{tabular}




\begin{tabular}{lllrll}
\hline SRF-01-05 & 117022 & $\mathrm{~J}$ & $0.119 \mathrm{mg} / \mathrm{kg}$ & 0.072 & EPA6010B \\
SRF-02-01 & 117023 & & $0.299 \mathrm{mg} / \mathrm{kg}$ & 0.074 & EPA6010B \\
SRF-02-02 & 117024 & & $0.245 \mathrm{mg} / \mathrm{kg}$ & 0.076 & EPA6010B \\
SRF-02-03 & 117025 & & $1.46 \mathrm{mg} / \mathrm{kg}$ & 0.079 & EPA6010B \\
SRF-02-03-A & 117026 & $\mathrm{~J}$ & $0.189 \mathrm{mg} / \mathrm{kg}$ & 0.075 & EPA6010B \\
SRF-03-01 & 117027 & $\mathrm{~J}$ & $0.0998 \mathrm{mg} / \mathrm{kg}$ & 0.078 & EPA6010B \\
SRF-03-02 & 117028 & & $0.588 \mathrm{mg} / \mathrm{kg}$ & 0.077 & EPA6010B \\
SRF-03-03 & 117029 & $\mathrm{~J}$ & $0.174 \mathrm{mg} / \mathrm{kg}$ & 0.074 & EPA6010B \\
SRF-03-04 & 117030 & $\mathrm{~J}$ & $0.146 \mathrm{mg} / \mathrm{kg}$ & 0.077 & EPA6010B \\
SRF-04-01 & 117031 & & $0.264 \mathrm{mg} / \mathrm{kg}$ & 0.076 & EPA6010B \\
SRF-04-02 & 117032 & $\mathrm{~J}$ & $0.177 \mathrm{mg} / \mathrm{kg}$ & 0.072 & EPA6010B \\
SRF-04-03 & 117033 & $\mathrm{U}$ & $0.248 \mathrm{mg} / \mathrm{kg}$ & 0.078 & EPA6010B \\
SRF-05-01 & 117034 & $\mathrm{~J}$ & $0.109 \mathrm{mg} / \mathrm{kg}$ & 0.077 & EPA6010B \\
SRF-05-02 & 117035 & & $0.306 \mathrm{mg} / \mathrm{kg}$ & 0.075 & EPA6010B \\
SRF-05-03 & 117036 & $\mathrm{U}$ & $0.234 \mathrm{mg} / \mathrm{kg}$ & 0.074 & EPA6010B \\
SRF-06-01 & 117037 & $\mathrm{~J}$ & $0.227 \mathrm{mg} / \mathrm{kg}$ & 0.075 & EPA6010B \\
SRF-06-02 & 117038 & $\mathrm{~J}$ & $0.161 \mathrm{mg} / \mathrm{kg}$ & 0.073 & EPA6010B \\
SRF-06-03 & 117039 & & $0.443 \mathrm{mg} / \mathrm{kg}$ & 0.075 & EPA6010B \\
SRF-06-03-A & 117040 & & $0.498 \mathrm{mg} / \mathrm{kg}$ & 0.078 & EPA6010B \\
SRF-07-01 & 117041 & $\mathrm{~J}$ & $0.0933 \mathrm{mg} / \mathrm{kg}$ & 0.076 & EPA6010B \\
SRF-07-02 & 117042 & $\mathrm{~J}$ & $0.154 \mathrm{mg} / \mathrm{kg}$ & 0.076 & EPA6010B \\
SRF-07-03 & 117043 & & $0.68 \mathrm{mg} / \mathrm{kg}$ & 0.072 & EPA6010B
\end{tabular}

I

I

I

I

I

I

I

I

(1)

ANALYTE: Magnesium

Analytical Group: Metals (total recoverable)

\begin{tabular}{|c|c|c|c|c|c|c|c|}
\hline Survey ID & Sample ID & FG & Result & Unit & ssMDL & Method & SC \\
\hline SRF-01-01 & 117018 & & 380 & $\mathrm{mg} / \mathrm{kg}$ & 0.231 & EPA6010B & \\
\hline SRF-01-02 & 117019 & & 357 & $\mathrm{mg} / \mathrm{kg}$ & 0.242 & EPA6010B & \\
\hline SRF-01-03 & 117020 & & 336 & $\mathrm{mg} / \mathrm{kg}$ & 0.231 & EPA6010B & \\
\hline SRF-01-04 & 117021 & & 475 & $\mathrm{mg} / \mathrm{kg}$ & 0.235 & EPA6010B & \\
\hline SRF-01-05 & 117022 & & 375 & $\mathrm{mg} / \mathrm{kg}$ & 0.233 & EPA6010B & \\
\hline SRF-02-01 & 117023 & & 750 & $\mathrm{mg} / \mathrm{kg}$ & 0.237 & EPA6010B & \\
\hline SRF-02-02 & 117024 & & 677 & $\mathrm{mg} / \mathrm{kg}$ & 0.244 & EPA6010B & \\
\hline SRF-02-03 & 117025 & & 537 & $\mathrm{mg} / \mathrm{kg}$ & 0.254 & EPA6010B & \\
\hline SRF-02-03-A & 117026 & & 1400 & $\mathrm{mg} / \mathrm{kg}$ & 0.24 & EPA6010B & \\
\hline SRF-03-01 & 117027 & & 375 & $\mathrm{mg} / \mathrm{kg}$ & 0.251 & EPA6010B & \\
\hline SRF-03-02 & 117028 & & 278 & $\mathrm{mg} / \mathrm{kg}$ & 0.246 & EPA6010B & \\
\hline SRF-03-03 & 117029 & & 825 & $\mathrm{mg} / \mathrm{kg}$ & 0.237 & EPA6010B & \\
\hline SRF-03-04 & 117030 & & 533 & $\mathrm{mg} / \mathrm{kg}$ & 0.249 & EPA6010B & \\
\hline SRF-04-01 & 117031 & & 558 & $\mathrm{mg} / \mathrm{kg}$ & 0.244 & EPA6010B & \\
\hline SRF-04-02 & 117032 & & 524 & $\mathrm{mg} / \mathrm{kg}$ & 0.233 & EPA6010B & \\
\hline SRF-04-03 & 117033 & & 294 & $\mathrm{mg} / \mathrm{kg}$ & 0.251 & EPA6010B & \\
\hline SRF-05-01 & 117034 & & 381 & $\mathrm{mg} / \mathrm{kg}$ & 0.246 & EPA6010B & \\
\hline SRF-05-02 & 117035 & & 617 & $\mathrm{mg} / \mathrm{kg}$ & 0.242 & EPA6010B & \\
\hline SRF-05-03 & 117036 & & 419 & $\mathrm{mg} / \mathrm{kg}$ & 0.237 & EPA6010B & \\
\hline SRF-06-01 & 117037 & & 1010 & $\mathrm{mg} / \mathrm{kg}$ & 0.242 & EPA6010B & \\
\hline SRF-06-02 & 117038 & & 411 & $\mathrm{mg} / \mathrm{kg}$ & 0.235 & EPA6010B & \\
\hline SRF-06-03 & 117039 & & 463 & $\mathrm{mg} / \mathrm{kg}$ & 0.242 & EPA6010B & \\
\hline SRF-06-03-A & 117040 & & 550 & $\mathrm{mg} / \mathrm{kg}$ & 0.251 & EPA6010B & \\
\hline SRF-07-01 & 117041 & & 382 & $\mathrm{mg} / \mathrm{kg}$ & 0.244 & EPA6010B & \\
\hline SRF-07-02 & 117042 & & 437 & $\mathrm{mg} / \mathrm{kg}$ & 0.244 & EPA6010B & \\
\hline SRF-07-03 & 117043 & & 490 & $\mathrm{mg} / \mathrm{kg}$ & 0.233 & EPA6010B & \\
\hline
\end{tabular}

ANALYTE: Manganese-54

Analytical Group: Radionuclides

\begin{tabular}{lllrllll} 
Survey ID & Sample ID & FG & Result & Unit & ssMDL & Method & SC \\
SRF-01-01 & 117018 & U & -0.0118 & $\mathrm{pCi} / \mathrm{g}$ & 0.0484 & EPIA-013B & \\
SRF-01-02 & 117019 & $\mathrm{U}$ & 0.0133 & $\mathrm{pCi} / \mathrm{g}$ & 0.0516 & EPIA-013B & \\
\hline
\end{tabular}




\begin{tabular}{|c|c|c|c|c|c|c|c|c|}
\hline SRF-01-03 & 117020 & $\mathrm{U}$ & -0.00517 & $\mathrm{pCi} / \mathrm{g}$ & 0.0517 & EPIA-013B & & \\
\hline SRF-01-04 & 117021 & $\mathrm{U}$ & 0.0061 & $\mathrm{pCi} / \mathrm{g}$ & 0.0453 & EPIA-013B & & \\
\hline SRF-01-05 & 117022 & $\mathrm{U}$ & -0.00379 & $\mathrm{pCi} / \mathrm{g}$ & 0.0436 & EPIA-013B & & \\
\hline SRF-01-05 & 117022 & $\mathrm{U}$ & 0.0103 & $\mathrm{pCi} / \mathrm{g}$ & 0.0434 & EPIA-013B & & \\
\hline SRF-02-01 & 117023 & $\mathrm{U}$ & 0.0257 & $\mathrm{pCi} / \mathrm{g}$ & 0.0584 & EPIA-013B & & \\
\hline SRF-02-02 & 117024 & $\mathrm{U}$ & -0.00243 & $\mathrm{pCi} / \mathrm{g}$ & 0.0452 & EPIA-013B & & \\
\hline SRF-02-03 & 117025 & $\mathrm{U}$ & 0.0811 & $\mathrm{pCi} / \mathrm{g}$ & 0.0872 & EPIA-013B & & \\
\hline SRF-02-03-A & 117026 & $\mathrm{U}$ & 0.0215 & $\mathrm{pCi} / \mathrm{g}$ & 0.0693 & EPIA-013B & & \\
\hline SRF-03-01 & 117027 & $\mathrm{U}$ & 0.0347 & $\mathrm{pCi} / \mathrm{g}$ & 0.0649 & EPIA-013B & & \\
\hline SRF-03-02 & 117028 & $\mathrm{U}$ & -0.000495 & $\mathrm{pCi} / \mathrm{g}$ & 0.0498 & EPIA-013B & & \\
\hline SRF-03-03 & 117029 & $\mathrm{U}$ & 0 & $\mathrm{pCi} / \mathrm{g}$ & 0.0569 & EPIA-013B & & \\
\hline SRF-03-04 & 117030 & $\mathrm{U}$ & -0.00853 & $\mathrm{pCi} / \mathrm{g}$ & 0.0303 & EPIA-013B & & \\
\hline SRF-04-01 & 117031 & $\mathrm{U}$ & -0.000791 & $\mathrm{pCi} / \mathrm{g}$ & 0.0488 & EPIA-013B & & \\
\hline SRF-04-02 & 117032 & $\mathrm{U}$ & 0.00577 & $\mathrm{pCi} / \mathrm{g}$ & 0.0586 & EPIA-013B & & \\
\hline SRF-04-03 & 117033 & $\mathrm{U}$ & -0.0036 & $\mathrm{pCi} / \mathrm{g}$ & 0.0714 & EPIA-013B & & \\
\hline SRF-05-01 & 117034 & $\mathrm{U}$ & -0.0143 & $\mathrm{pCi} / \mathrm{g}$ & 0.0467 & EPIA-013B & & \\
\hline SRF-05-02 & 117035 & $\mathrm{U}$ & 0.0105 & $\mathrm{pCi} / \mathrm{g}$ & 0.0599 & EPIA-013B & & \\
\hline SRF-05-03 & 117036 & $\mathrm{U}$ & 0.00248 & $\mathrm{pCi} / \mathrm{g}$ & 0.0543 & EPIA-013B & & \\
\hline SRF-06-01 & 117037 & $\mathrm{U}$ & -0.00667 & $\mathrm{pCi} / \mathrm{g}$ & 0.0644 & EPIA-013B & & \\
\hline SRF-06-02 & 117038 & $\mathrm{U}$ & -0.00517 & $\mathrm{pCi} / \mathrm{g}$ & 0.0365 & EPIA-013B & & \\
\hline SRF-06-03 & 117039 & $\mathrm{U}$ & 0.0255 & $\mathrm{pCi} / \mathrm{g}$ & 0.0711 & EPIA-013B & & \\
\hline \multicolumn{9}{|c|}{ ANALYTE: Manganese-54 (cont.) } \\
\hline Survey ID & Sample ID & FG & Result & Unit & SSMDL & Method & SC & $\mathbf{E}$ \\
\hline SRF-06-03-A & 117040 & $\mathrm{U}$ & -0.00261 & $\mathrm{pCi} / \mathrm{g}$ & 0.0653 & EPIA-013B & & \\
\hline SRF-07-01 & 117041 & $\mathrm{U}$ & 0.0153 & $\mathrm{pCi} / \mathrm{g}$ & 0.0702 & EPIA-013B & & \\
\hline SRF-07-02 & 117042 & $\mathrm{U}$ & -0.0262 & $\mathrm{pCi} / \mathrm{g}$ & 0.0714 & EPIA-013B & & \\
\hline SRF-07-03 & 117043 & $\mathrm{U}$ & -0.000359 & $\mathrm{pCi} / \mathrm{g}$ & 0.0806 & EPIA-013B & & \\
\hline SRF-07-03 & 117043 & $\mathrm{U}$ & 0.0134 & $\mathrm{pCi} / \mathrm{g}$ & 0.0586 & EPIA-013B & & \\
\hline
\end{tabular}

ANALYTE: Manganese

Analytical Group: Metals (total recoverable)

\begin{tabular}{|c|c|c|c|c|c|c|c|c|}
\hline Survey ID & Sample ID & FG & Result & Unit & ssMDL & Method & SC & $\mathbf{E}$ \\
\hline SRF-01-01 & 117018 & & 26.9 & $\mathrm{mg} / \mathrm{kg}$ & 0.323 & EPA6010B & & \\
\hline SRF-01-02 & 117019 & & 66.6 & $\mathrm{mg} / \mathrm{kg}$ & 0.338 & EPA6010B & & \\
\hline SRF-01-03 & 117020 & & 20.1 & $\mathrm{mg} / \mathrm{kg}$ & 0.323 & EPA6010B & & \\
\hline SRF-01-04 & 117021 & & 37 & $\mathrm{mg} / \mathrm{kg}$ & 0.329 & EPA6010B & & \\
\hline SRF-01-05 & 117022 & $\mathrm{~J}$ & 18.5 & $\mathrm{mg} / \mathrm{kg}$ & 0.326 & EPA6010B & $\mathrm{K}$ & I \\
\hline SRF-02-01 & 117023 & & 38 & $\mathrm{mg} / \mathrm{kg}$ & 0.332 & EPA6010B & & \\
\hline SRF-02-02 & 117024 & & 8.99 & $\mathrm{mg} / \mathrm{kg}$ & 0.342 & EPA6010B & & \\
\hline SRF-02-03 & 117025 & & 7.11 & $\mathrm{mg} / \mathrm{kg}$ & 0.355 & EPA6010B & & \\
\hline SRF-02-03-A & 117026 & & 14.3 & $\mathrm{mg} / \mathrm{kg}$ & 0.335 & EPA6010B & & \\
\hline SRF-03-01 & 117027 & & 19.2 & $\mathrm{mg} / \mathrm{kg}$ & 0.351 & EPA6010B & & \\
\hline SRF-03-02 & 117028 & & 76.2 & $\mathrm{mg} / \mathrm{kg}$ & 0.344 & EPA6010B & & \\
\hline SRF-03-03 & 117029 & & 9.96 & $\mathrm{mg} / \mathrm{kg}$ & 0.332 & EPA6010B & & \\
\hline SRF-03-04 & 117030 & & 62.6 & $\mathrm{mg} / \mathrm{kg}$ & 0.348 & EPA6010B & & \\
\hline SRF-04-01 & 117031 & & 56.9 & $\mathrm{mg} / \mathrm{kg}$ & 0.342 & EPA6010B & & \\
\hline SRF-04-02 & 117032 & & 31 & $\mathrm{mg} / \mathrm{kg}$ & 0.326 & EPA6010B & & \\
\hline SRF-04-03 & 117033 & & 1.66 & $\mathrm{mg} / \mathrm{kg}$ & 0.351 & EPA6010B & & \\
\hline SRF-05-01 & 117034 & & 34.4 & $\mathrm{mg} / \mathrm{kg}$ & 0.344 & EPA6010B & & \\
\hline SRF-05-02 & 117035 & & 10.1 & $\mathrm{mg} / \mathrm{kg}$ & 0.338 & EPA6010B & & \\
\hline SRF-05-03 & 117036 & & 4.56 & $\mathrm{mg} / \mathrm{kg}$ & 0.332 & EPA6010B & & \\
\hline SRF-06-01 & 117037 & & 93.4 & $\mathrm{mg} / \mathrm{kg}$ & 0.338 & EPA6010B & & \\
\hline SRF-06-02 & 117038 & & 28.5 & $\mathrm{mg} / \mathrm{kg}$ & 0.329 & EPA6010B & & \\
\hline SRF-06-03 & 117039 & & 5.89 & $\mathrm{mg} / \mathrm{kg}$ & 0.338 & EPA6010B & & \\
\hline SRF-06-03-A & 117040 & & 6.7 & $\mathrm{mg} / \mathrm{kg}$ & 0.351 & EPA6010B & & \\
\hline SRF-07-01 & 117041 & & 18.6 & $\mathrm{mg} / \mathrm{kg}$ & 0.342 & EPA6010B & & \\
\hline SRF-07-02 & 117042 & & 10.1 & $\mathrm{mg} / \mathrm{kg}$ & 0.342 & EPA6010B & & \\
\hline SRF-07-03 & 117043 & & 6.96 & $\mathrm{mg} / \mathrm{kg}$ & 0.326 & EPA6010B & & \\
\hline
\end{tabular}


ANALYTE: Mercury

Analytical Group: Metals (total recoverable)

\begin{tabular}{|c|c|c|c|c|c|c|c|}
\hline Survey ID & Sample ID & FG & Result & Unit & SSMDL & Method & SC \\
\hline SRF-01-01 & 117018 & & 0.0508 & $\mathrm{mg} / \mathrm{kg}$ & 0.00225 & EPA7471A & \\
\hline SRF-01-02 & 117019 & $\mathrm{~J}$ & 0.0285 & $\mathrm{mg} / \mathrm{kg}$ & 0.00196 & EPA7471A & I \\
\hline SRF-01-03 & 117020 & & 0.0706 & $\mathrm{mg} / \mathrm{kg}$ & 0.002 & EPA7471A & \\
\hline SRF-01-04 & 117021 & $\mathrm{~J}$ & 0.0306 & $\mathrm{mg} / \mathrm{kg}$ & 0.00222 & EPA7471A & I \\
\hline SRF-01-05 & 117022 & $\mathrm{~J}$ & 0.0992 & $\mathrm{mg} / \mathrm{kg}$ & 0.00221 & EPA7471A & $\mathrm{L}$ \\
\hline SRF-02-01 & 117023 & & 0.119 & $\mathrm{mg} / \mathrm{kg}$ & 0.00207 & EPA7471A & \\
\hline SRF-02-02 & 117024 & & 0.0532 & $\mathrm{mg} / \mathrm{kg}$ & 0.00212 & EPA7471A & \\
\hline SRF-02-03 & 117025 & $\mathrm{~J}$ & 0.618 & $\mathrm{mg} / \mathrm{kg}$ & 0.00205 & EPA7471A & $\mathrm{L}$ \\
\hline SRF-02-03-A & 117026 & & 0.396 & $\mathrm{mg} / \mathrm{kg}$ & 0.00197 & EPA7471A & \\
\hline SRF-03-01 & 117027 & & 0.268 & $\mathrm{mg} / \mathrm{kg}$ & 0.00205 & EPA7471A & \\
\hline SRF-03-02 & 117028 & & 0.0767 & $\mathrm{mg} / \mathrm{kg}$ & 0.00223 & EPA7471A & \\
\hline SRF-03-03 & 117029 & & 0.261 & $\mathrm{mg} / \mathrm{kg}$ & 0.0141 & EPA7471A & \\
\hline \multicolumn{8}{|c|}{ ANALYTE: Mercury (cont.) } \\
\hline Survey ID & Sample ID & $\mathbf{F G}$ & Result & Unit & SSMDL & Method & SC \\
\hline SRF-03-04 & 117030 & & 0.0569 & $\mathrm{mg} / \mathrm{kg}$ & 0.00225 & EPA7471A & \\
\hline SRF-04-01 & 117031 & & 0.0655 & $\mathrm{mg} / \mathrm{kg}$ & 0.00214 & EPA7471A & \\
\hline SRF-04-02 & 117032 & & 0.151 & $\mathrm{mg} / \mathrm{kg}$ & 0.002 & EPA7471A & \\
\hline SRF-04-03 & 117033 & & 0.222 & $\mathrm{mg} / \mathrm{kg}$ & 0.00213 & EPA7471A & \\
\hline SRF-05-01 & 117034 & & 0.165 & $\mathrm{mg} / \mathrm{kg}$ & 0.00218 & EPA7471A & \\
\hline SRF-05-02 & 117035 & & 0.134 & $\mathrm{mg} / \mathrm{kg}$ & 0.00225 & EPA7471A & \\
\hline SRF-05-03 & 117036 & & 1.25 & $\mathrm{mg} / \mathrm{kg}$ & 0.00214 & EPA7471A & \\
\hline SRF-06-01 & 117037 & & 0.0959 & $\mathrm{mg} / \mathrm{kg}$ & 0.00195 & EPA7471A & \\
\hline SRF-06-02 & 117038 & & 0.134 & $\mathrm{mg} / \mathrm{kg}$ & 0.00219 & EPA7471A & \\
\hline SRF-06-03 & 117039 & & 0.456 & $\mathrm{mg} / \mathrm{kg}$ & 0.0021 & EPA7471A & \\
\hline SRF-06-03-A & 117040 & & 0.474 & $\mathrm{mg} / \mathrm{kg}$ & 0.00194 & EPA7471A & \\
\hline SRF-07-01 & 117041 & & 0.194 & $\mathrm{mg} / \mathrm{kg}$ & 0.00209 & EPA7471A & \\
\hline SRF-07-02 & 117042 & & 0.204 & $\mathrm{mg} / \mathrm{kg}$ & 0.0022 & EPA7471A & \\
\hline SRF-07-03 & 117043 & & 0.543 & $\mathrm{mg} / \mathrm{kg}$ & 0.00224 & EPA7471A & \\
\hline
\end{tabular}

ANALYTE: Methyl ethyl ketone

Analytical Group: Volatile Organic Compounds

\begin{tabular}{|c|c|c|c|c|c|c|c|c|}
\hline Survey ID & Sample ID & FG & Result & Unit & ssMDL & Method & SC & $\mathbf{E}$ \\
\hline SRF-01-01 & 117018 & $\mathrm{U}$ & 25 & $\mu g / \mathrm{kg}$ & 16 & EPA8260B & & \\
\hline SRF-01-02 & 117019 & $\mathrm{~J}$ & 58.2 & $\mu \mathrm{g} / \mathrm{kg}$ & 16 & EPA8260B & $\mathrm{K}$ & $\mathrm{O}$ \\
\hline SRF-01-03 & 117020 & & 54.7 & $\mu \mathrm{g} / \mathrm{kg}$ & 16 & EPA8260B & & \\
\hline SRF-01-04 & 117021 & $\mathrm{~J}$ & 37.8 & $\mu \mathrm{g} / \mathrm{kg}$ & 16 & EPA8260B & $\mathrm{K}$ & $\mathrm{O}$ \\
\hline SRF-01-05 & 117022 & $\mathrm{~J}$ & 34.7 & $\mu g / k g$ & 16 & EPA8260B & $\mathrm{K}$ & $\mathrm{O}$ \\
\hline SRF-02-01 & 117023 & $\mathrm{U}$ & 25 & $\mu \mathrm{g} / \mathrm{kg}$ & 16 & EPA8260B & & \\
\hline SRF-02-02 & 117024 & $\mathrm{U}$ & 25 & $\mu \mathrm{g} / \mathrm{kg}$ & 16 & EPA8260B & & \\
\hline SRF-02-03 & 117025 & $\mathrm{U}$ & 25 & $\mu g / k g$ & 16 & EPA8260B & & \\
\hline SRF-02-03-A & 117026 & $\mathrm{U}$ & 25 & $\mu g / k g$ & 16 & EPA8260B & & \\
\hline SRF-03-01 & 117027 & $\mathrm{U}$ & 25 & $\mu g / \mathrm{kg}$ & 16 & EPA8260B & & \\
\hline SRF-03-02 & 117028 & $\mathrm{U}$ & 25 & $\mu \mathrm{g} / \mathrm{kg}$ & 16 & EPA8260B & & \\
\hline SRF-03-03 & 117029 & $\mathrm{U}$ & 25 & $\mu g / \mathrm{kg}$ & 16 & EPA8260B & & \\
\hline SRF-03-04 & 117030 & $\mathrm{U}$ & 25 & $\mu \mathrm{g} / \mathrm{kg}$ & 16 & EPA8260B & & \\
\hline SRF-04-01 & 117031 & $\mathrm{U}$ & 25 & $\mu g / k g$ & 16 & EPA8260B & & \\
\hline SRF-04-02 & 117032 & $\mathrm{~J}$ & 17 & $\mu \mathrm{g} / \mathrm{kg}$ & 16 & EPA8260B & I & \\
\hline SRF-04-03 & 117033 & $\mathrm{U}$ & 25 & $\mu \mathrm{g} / \mathrm{kg}$ & 16 & EPA8260B & & \\
\hline SRF-05-01 & 117034 & $\mathrm{U}$ & 25 & $\mu \mathrm{g} / \mathrm{kg}$ & 16 & EPA8260B & & \\
\hline SRF-05-02 & 117035 & $\mathrm{U}$ & 25 & $\mu g / k g$ & 16 & EPA8260B & & \\
\hline SRF-05-03 & 117036 & $\mathrm{U}$ & 25 & $\mu \mathrm{g} / \mathrm{kg}$ & 16 & EPA8260B & & \\
\hline
\end{tabular}




\begin{tabular}{|c|c|c|c|c|c|c|c|}
\hline SRF-06-01 & 117037 & $\mathrm{U}$ & 25 & $\mu \mathrm{g} / \mathrm{kg}$ & 16 & EPA8260B & \\
\hline SRF-06-02 & 117038 & U & 25 & $\mu g / \mathrm{kg}$ & 16 & EPA8260B & \\
\hline SRF-06-03 & 117039 & $\mathrm{U}$ & 25 & $\mu g / \mathrm{kg}$ & 16 & EPA8260B & \\
\hline SRF-06-03-A & 117040 & $\mathrm{U}$ & 25 & $\mu g / \mathrm{kg}$ & 16 & EPA8260B & \\
\hline SRF-07-01 & 117041 & U & 25 & $\mu g / \mathrm{kg}$ & 16 & EPA8260B & \\
\hline SRF-07-02 & 117042 & $J$ & 48.6 & $\mu \mathrm{g} / \mathrm{kg}$ & 16 & EPA8260B & $\mathrm{K}$ \\
\hline SRF-07-03 & 117043 & $\mathrm{U}$ & 25 & $\mu \mathrm{g} / \mathrm{kg}$ & 16 & EPA8260B & \\
\hline
\end{tabular}

ANALYTE: Methyl isobutyl ketone

Analytical Group: Volatile Organic Compounds

\begin{tabular}{|c|c|c|c|c|c|c|c|}
\hline Survey ID & Sample ID & FG & Result & Unit & ssMDL & Method & SC \\
\hline SRF-01-01 & 117018 & $\mathrm{U}$ & 25 & $\mu g / \mathrm{kg}$ & 14.6 & EPA8260B & \\
\hline SRF-01-02 & 117019 & $\mathrm{U}$ & 25 & $\mu \mathrm{g} / \mathrm{kg}$ & 14.6 & EPA8260B & \\
\hline SRF-01-03 & 117020 & $\mathrm{U}$ & 25 & $\mu \mathrm{g} / \mathrm{kg}$ & 14.6 & EPA8260B & \\
\hline \multicolumn{8}{|c|}{ ANALYTE: Methyl isobutyl ketone (cont.) } \\
\hline Survey ID & Sample ID & FG & Result & Unit & ssMDL & Method & SC \\
\hline SRF-01-04 & 117021 & $\mathrm{U}$ & 25 & $\mu \mathrm{g} / \mathrm{kg}$ & 14.6 & EPA8260B & \\
\hline SRF-01-05 & 117022 & $\mathrm{U}$ & 25 & $\mu g / \mathrm{kg}$ & 14.6 & EPA8260B & \\
\hline SRF-02-01 & 117023 & $\mathrm{U}$ & 25 & $\mu g / \mathrm{kg}$ & 14.6 & EPA8260B & \\
\hline SRF-02-02 & 117024 & $\mathrm{U}$ & 25 & $\mu g / \mathrm{kg}$ & 14.6 & EPA8260B & \\
\hline SRF-02-03 & 117025 & $\mathrm{U}$ & 25 & $\mu g / \mathrm{kg}$ & 14.6 & EPA8260B & \\
\hline SRF-02-03-A & 117026 & $\mathrm{U}$ & 25 & $\mu \mathrm{g} / \mathrm{kg}$ & 14.6 & EPA8260B & \\
\hline SRF-03-01 & 117027 & $\mathrm{U}$ & 25 & $\mu \mathrm{g} / \mathrm{kg}$ & 14.6 & EPA8260B & \\
\hline SRF-03-02 & 117028 & $\mathrm{U}$ & 25 & $\mu g / \mathrm{kg}$ & 14.6 & EPA8260B & \\
\hline SRF-03-03 & 117029 & $\mathrm{U}$ & 25 & $\mu \mathrm{g} / \mathrm{kg}$ & 14.6 & EPA8260B & \\
\hline SRF-03-04 & 117030 & $\mathrm{U}$ & 25 & $\mu g / \mathrm{kg}$ & 14.6 & EPA8260B & \\
\hline SRF-04-01 & 117031 & $\mathrm{U}$ & 25 & $\mu g / \mathrm{kg}$ & 14.6 & EPA8260B & \\
\hline SRF-04-02 & 117032 & $\mathrm{U}$ & 25 & $\mu g / \mathrm{kg}$ & 14.6 & EPA8260B & \\
\hline SRF-04-03 & 117033 & $\mathrm{U}$ & 25 & $\mu \mathrm{g} / \mathrm{kg}$ & 14.6 & EPA8260B & \\
\hline SRF-05-01 & 117034 & $\mathrm{U}$ & 25 & $\mu g / \mathrm{kg}$ & 14.6 & EPA8260B & \\
\hline SRF-05-02 & 117035 & $\mathrm{U}$ & 25 & $\mu g / \mathrm{kg}$ & 14.6 & EPA8260B & \\
\hline SRF-05-03 & 117036 & $\mathrm{U}$ & 25 & $\mu g / \mathrm{kg}$ & 14.6 & EPA8260B & \\
\hline SRF-06-01 & 117037 & $\mathrm{U}$ & 25 & $\mu \mathrm{g} / \mathrm{kg}$ & 14.6 & EPA8260B & \\
\hline SRF-06-02 & 117038 & $\mathrm{U}$ & 25 & $\mu g / \mathrm{kg}$ & 14.6 & EPA8260B & \\
\hline SRF-06-03 & 117039 & $\mathrm{U}$ & 25 & $\mu g / \mathrm{kg}$ & 14.6 & EPA8260B & \\
\hline SRF-06-03-A & 117040 & $\mathrm{U}$ & 25 & $\mu g / \mathrm{kg}$ & 14.6 & EPA8260B & \\
\hline SRF-07-01 & 117041 & $\mathrm{U}$ & 25 & $\mu g / \mathrm{kg}$ & 14.6 & EPA8260B & \\
\hline SRF-07-02 & 117042 & $\mathrm{U}$ & 25 & $\mu \mathrm{g} / \mathrm{kg}$ & 14.6 & EPA8260B & \\
\hline SRF-07-03 & 117043 & $\mathrm{U}$ & 25 & $\mu \mathrm{g} / \mathrm{kg}$ & 14.6 & EPA8260B & \\
\hline
\end{tabular}

ANALYTE: Neptunium-239

Analytical Group: Radionuclides

\begin{tabular}{|c|c|c|c|c|c|c|c|}
\hline Survey ID & Sample ID & FG & Result & Unit & ssMDL & Method & SC \\
\hline SRF-01-01 & 117018 & U & -0.00946 & $\mathrm{pCi} / \mathrm{g}$ & 0.222 & EPIA-013B & \\
\hline SRF-01-02 & 117019 & $\mathrm{U}$ & 0.087 & $\mathrm{pCi} / \mathrm{g}$ & 0.174 & EPIA-013B & \\
\hline SRF-01-03 & 117020 & $\mathrm{U}$ & -0.0623 & $\mathrm{pCi} / \mathrm{g}$ & 0.208 & EPIA-013B & \\
\hline SRF-01-04 & 117021 & $\mathrm{U}$ & 0.028 & $\mathrm{pCi} / \mathrm{g}$ & 0.184 & EPIA-013B & \\
\hline SRF-01-05 & 117022 & $\mathrm{U}$ & -0.0297 & $\mathrm{pCi} / \mathrm{g}$ & 0.183 & EPIA-013B & \\
\hline SRF-01-05 & 117022 & $\mathrm{U}$ & -0.0625 & $\mathrm{pCi} / \mathrm{g}$ & 0.168 & EPIA-013B & \\
\hline SRF-02-01 & 117023 & U & 0.0165 & $\mathrm{pCi} / \mathrm{g}$ & 0.234 & EPIA-013B & \\
\hline SRF-02-02 & 117024 & $\mathrm{U}$ & -0.0467 & $\mathrm{pCi} / \mathrm{g}$ & 0.174 & EPIA-013B & \\
\hline SRF-02-03 & 117025 & $\mathrm{U}$ & -0.0876 & $\mathrm{pCi} / \mathrm{g}$ & 0.269 & EPIA-013B & \\
\hline SRF-02-03-A & 117026 & $\mathrm{U}$ & 0.114 & $\mathrm{pCi} / \mathrm{g}$ & 0.277 & EPIA-013B & \\
\hline SRF-03-01 & 117027 & $\mathrm{U}$ & 0.0519 & $\mathrm{pCi} / \mathrm{g}$ & 0.277 & EPIA-013B & \\
\hline SRF-03-02 & 117028 & $\mathrm{U}$ & 0.0502 & $\mathrm{pCi} / \mathrm{g}$ & 0.207 & EPIA-013B & \\
\hline
\end{tabular}




\begin{tabular}{lllrlrl}
\hline SRF-03-03 & 117029 & $\mathrm{U}$ & -0.117 & $\mathrm{pCi} / \mathrm{g}$ & 0.196 & EPIA-013B \\
SRF-03-04 & 117030 & $\mathrm{U}$ & 0.00155 & $\mathrm{pCi} / \mathrm{g}$ & 0.141 & EPIA-013B \\
SRF-04-01 & 117031 & $\mathrm{U}$ & 0.0669 & $\mathrm{pCi} / \mathrm{g}$ & 0.253 & EPIA-013B \\
SRF-04-02 & 117032 & $\mathrm{U}$ & 0.0289 & $\mathrm{pCi} / \mathrm{g}$ & 0.264 & EPIA-013B \\
SRF-04-03 & 117033 & $\mathrm{U}$ & 0.0212 & $\mathrm{pCi} / \mathrm{g}$ & 0.24 & EPIA-013B \\
SRF-05-01 & 117034 & $\mathrm{U}$ & -0.00259 & $\mathrm{pCi} / \mathrm{g}$ & 0.179 & EPIA-013B \\
SRF-05-02 & 117035 & $\mathrm{U}$ & -0.0482 & $\mathrm{pCi} / \mathrm{g}$ & 0.162 & EPIA-013B \\
SRF-05-03 & 117036 & $\mathrm{U}$ & 0.042 & $\mathrm{pCi} / \mathrm{g}$ & 0.189 & EPIA-013B \\
SRF-06-01 & 117037 & $\mathrm{U}$ & 0.0376 & $\mathrm{pCi} / \mathrm{g}$ & 0.2 & EPIA-013B \\
SRF-06-02 & 117038 & $\mathrm{U}$ & 0.0176 & $\mathrm{pCi} / \mathrm{g}$ & 0.175 & EPIA-013B \\
SRF-06-03 & 117039 & $\mathrm{U}$ & 0.046 & $\mathrm{pCi} / \mathrm{g}$ & 0.257 & EPIA-013B \\
SRF-06-03-A & 117040 & $\mathrm{U}$ & 0.0495 & $\mathrm{pCi} / \mathrm{g}$ & 0.287 & EPIA-013B \\
SRF-07-01 & 117041 & $\mathrm{U}$ & -0.0755 & $\mathrm{pCi} / \mathrm{g}$ & 0.213 & EPIA-013B \\
ANALYTE: Neptunium-239 (cont.) & & & & \\
Survey ID & Sample ID & FG & $\mathbf{R e s u l t}$ & $\mathbf{U n i t}$ & $\mathbf{s s M D L}$ & Method \\
SRF-07-02 & 117042 & $\mathrm{U}$ & 0.0014 & $\mathrm{pCi} / \mathrm{g}$ & 0.304 & EPIA-013B \\
SRF-07-03 & 117043 & $\mathrm{U}$ & -0.162 & $\mathrm{pCi} / \mathrm{g}$ & 0.224 & EPIA-013B \\
SRF-07-03 & 117043 & $\mathrm{U}$ & 0.105 & $\mathrm{pCi} / \mathrm{g}$ & 0.211 & EPIA-013B
\end{tabular}

ANALYTE: Nickel

Analytical Group: Metals (total recoverable)

\begin{tabular}{|c|c|c|c|c|c|c|c|c|}
\hline Survey ID & Sample ID & $\mathbf{F G}$ & Result & Unit & SSMDL & Method & SC & $\mathbf{E}$ \\
\hline SRF-01-01 & 117018 & $\mathrm{U}$ & 0.227 & $\mathrm{mg} / \mathrm{kg}$ & 0.029 & EPA6010B & & \\
\hline SRF-01-02 & 117019 & & 0.276 & $\mathrm{mg} / \mathrm{kg}$ & 0.03 & EPA6010B & & \\
\hline SRF-01-03 & 117020 & $\mathrm{U}$ & 0.227 & $\mathrm{mg} / \mathrm{kg}$ & 0.029 & EPA6010B & & \\
\hline SRF-01-04 & 117021 & $\mathrm{U}$ & 0.231 & $\mathrm{mg} / \mathrm{kg}$ & 0.03 & EPA6010B & & \\
\hline SRF-01-05 & 117022 & $\mathrm{U}$ & 0.229 & $\mathrm{mg} / \mathrm{kg}$ & 0.029 & EPA6010B & & \\
\hline SRF-02-01 & 117023 & $\mathrm{U}$ & 0.373 & $\mathrm{mg} / \mathrm{kg}$ & 0.03 & EPA6010B & $\mathrm{V}$ & \\
\hline SRF-02-02 & 117024 & $\mathrm{U}$ & 0.24 & $\mathrm{mg} / \mathrm{kg}$ & 0.031 & EPA6010B & & \\
\hline SRF-02-03 & 117025 & $\mathrm{U}$ & 0.366 & $\mathrm{mg} / \mathrm{kg}$ & 0.032 & EPA6010B & $\mathrm{V}$ & \\
\hline SRF-02-03-A & 117026 & $\mathrm{U}$ & 0.236 & $\mathrm{mg} / \mathrm{kg}$ & 0.03 & EPA6010B & & \\
\hline SRF-03-01 & 117027 & $\mathrm{U}$ & 0.248 & $\mathrm{mg} / \mathrm{kg}$ & 0.032 & EPA6010B & & \\
\hline SRF-03-02 & 117028 & $\mathrm{U}$ & 0.517 & $\mathrm{mg} / \mathrm{kg}$ & 0.031 & EPA6010B & $\mathrm{V}$ & \\
\hline SRF-03-03 & 117029 & $\mathrm{U}$ & 0.234 & $\mathrm{mg} / \mathrm{kg}$ & 0.03 & EPA6010B & & \\
\hline SRF-03-04 & 117030 & $\mathrm{U}$ & 0.245 & $\mathrm{mg} / \mathrm{kg}$ & 0.031 & EPA6010B & & \\
\hline SRF-04-01 & 117031 & JU & 0.133 & $\mathrm{mg} / \mathrm{kg}$ & 0.031 & EPA6010B & IV & \\
\hline SRF-04-02 & 117032 & JU & 0.145 & $\mathrm{mg} / \mathrm{kg}$ & 0.029 & EPA6010B & IV & \\
\hline SRF-04-03 & 117033 & JU & 0.15 & $\mathrm{mg} / \mathrm{kg}$ & 0.032 & EPA6010B & IV & \\
\hline SRF-05-01 & 117034 & $\mathrm{U}$ & 0.372 & $\mathrm{mg} / \mathrm{kg}$ & 0.031 & EPA6010B & $\mathrm{V}$ & \\
\hline SRF-05-02 & 117035 & $\mathrm{U}$ & 0.238 & $\mathrm{mg} / \mathrm{kg}$ & 0.03 & EPA6010B & & \\
\hline SRF-05-03 & 117036 & $\mathrm{U}$ & 0.234 & $\mathrm{mg} / \mathrm{kg}$ & 0.03 & EPA6010B & & \\
\hline SRF-06-01 & 117037 & JU & 0.181 & $\mathrm{mg} / \mathrm{kg}$ & 0.03 & EPA6010B & IV & \\
\hline SRF-06-02 & 117038 & $\mathrm{U}$ & 0.796 & $\mathrm{mg} / \mathrm{kg}$ & 0.03 & EPA6010B & $\mathrm{V}$ & \\
\hline SRF-06-03 & 117039 & JU & 0.233 & $\mathrm{mg} / \mathrm{kg}$ & 0.03 & EPA6010B & IV & \\
\hline SRF-06-03-A & 117040 & $\mathrm{U}$ & 0.284 & $\mathrm{mg} / \mathrm{kg}$ & 0.032 & EPA6010B & $\mathrm{V}$ & \\
\hline SRF-07-01 & 117041 & $\mathrm{~J}$ & 0.115 & $\mathrm{mg} / \mathrm{kg}$ & 0.031 & EPA6010B & I & \\
\hline SRF-07-02 & 117042 & $\mathrm{~J}$ & 0.115 & $\mathrm{mg} / \mathrm{kg}$ & 0.031 & EPA6010B & I & \\
\hline SRF-07-03 & 117043 & $\mathrm{~J}$ & 0.12 & $\mathrm{mg} / \mathrm{kg}$ & 0.029 & EPA6010B & I & \\
\hline \multicolumn{9}{|c|}{$\begin{array}{l}\text { ANALYTE: Nonvolatile beta } \\
\text { Analytical Group: Radionuclides }\end{array}$} \\
\hline Survey ID & Sample ID & $\mathbf{F G}$ & Result & Unit & SSMDL & Method & SC & $\mathbf{E}$ \\
\hline SRF-01-01 & 117018 & & 5.12 & $\mathrm{pCi} / \mathrm{g}$ & 0.817 & EPIA-001B & & \\
\hline SRF-01-02 & 117019 & & 7.27 & $\mathrm{pCi} / \mathrm{g}$ & 0.579 & EPIA-001B & & \\
\hline SRF-01-03 & 117020 & & 7.32 & $\mathrm{pCi} / \mathrm{g}$ & 1.26 & EPIA-001B & & \\
\hline
\end{tabular}




\begin{tabular}{|c|c|c|c|c|c|c|c|}
\hline SRF-01-04 & 117021 & 7.7 & $\mathrm{pCi} / \mathrm{g}$ & 0.568 & EPIA-001B & & \\
\hline SRF-01-05 & 117022 & 8.22 & $\mathrm{pCi} / \mathrm{g}$ & 2.48 & EPIA-001B & & \\
\hline SRF-01-05 & 117022 & 8.14 & $\mathrm{pCi} / \mathrm{g}$ & 2.57 & EPIA-001B & & \\
\hline SRF-02-01 & 117023 & 7.76 & $\mathrm{pCi} / \mathrm{g}$ & 1.7 & EPIA-001B & & \\
\hline SRF-02-02 & 117024 & 5.87 & $\mathrm{pCi} / \mathrm{g}$ & 1.06 & EPIA-001B & & \\
\hline SRF-02-03 & 117025 & 8.12 & $\mathrm{pCi} / \mathrm{g}$ & 1.04 & EPIA-001B & & \\
\hline SRF-02-03-A & 117026 & 6.29 & $\mathrm{pCi} / \mathrm{g}$ & 1.03 & EPIA-001B & & \\
\hline SRF-03-01 & 117027 & 7.32 & $\mathrm{pCi} / \mathrm{g}$ & 1.75 & EPIA-001B & & \\
\hline SRF-03-02 & 117028 & 7.88 & $\mathrm{pCi} / \mathrm{g}$ & 1.49 & EPIA-001B & & \\
\hline SRF-03-03 & 117029 & 6.13 & $\mathrm{pCi} / \mathrm{g}$ & 1.18 & EPIA-001B & & \\
\hline SRF-03-04 & 117030 & 7.07 & $\mathrm{pCi} / \mathrm{g}$ & 1.98 & EPIA-001B & & \\
\hline \multicolumn{8}{|c|}{ ANALYTE: Nonvolatile beta (cont.) } \\
\hline Survey ID & Sample ID FG & Result & Unit & SSMDL & Method & SC & $\mathbf{E}$ \\
\hline SRF-04-01 & 117031 & 9.76 & $\mathrm{pCi} / \mathrm{g}$ & 1.06 & EPIA-001B & & \\
\hline SRF-04-02 & 117032 & 6.65 & $\mathrm{pCi} / \mathrm{g}$ & 1.75 & EPIA-001B & & \\
\hline SRF-04-03 & 117033 & 7.74 & $\mathrm{pCi} / \mathrm{g}$ & 1.04 & EPIA-001B & & \\
\hline SRF-05-01 & 117034 & 6.39 & $\mathrm{pCi} / \mathrm{g}$ & 1.33 & EPIA-001B & & \\
\hline SRF-05-02 & 117035 & 5.47 & $\mathrm{pCi} / \mathrm{g}$ & 1.8 & EPIA-001B & & \\
\hline SRF-05-03 & 117036 & 5.95 & $\mathrm{pCi} / \mathrm{g}$ & 1.06 & EPIA-001B & & \\
\hline SRF-06-01 & 117037 & 7.33 & $\mathrm{pCi} / \mathrm{g}$ & 1.42 & EPIA-001B & & \\
\hline SRF-06-02 & 117038 & 8.49 & $\mathrm{pCi} / \mathrm{g}$ & 1.31 & EPIA-001B & & \\
\hline SRF-06-03 & 117039 & 6.71 & $\mathrm{pCi} / \mathrm{g}$ & 0.857 & EPIA-001B & & \\
\hline SRF-06-03-A & 117040 & 7.61 & $\mathrm{pCi} / \mathrm{g}$ & 0.932 & EPIA-001B & & \\
\hline SRF-07-01 & 117041 & 7.32 & $\mathrm{pCi} / \mathrm{g}$ & 0.897 & EPIA-001B & & \\
\hline SRF-07-02 & 117042 & 6.33 & $\mathrm{pCi} / \mathrm{g}$ & 1.33 & EPIA-001B & & \\
\hline SRF-07-03 & 117043 & 5.4 & $\mathrm{pCi} / \mathrm{g}$ & 0.642 & EPIA-001B & & \\
\hline SRF-07-03 & 117043 & 5.31 & $\mathrm{pCi} / \mathrm{g}$ & 0.586 & EPIA-001B & & \\
\hline
\end{tabular}

ANALYTE: Plutonium-238

Analytical Group: Radionuclides

\begin{tabular}{|c|c|c|c|c|c|c|c|}
\hline Survey ID & Sample ID & $\mathbf{F G}$ & Result & Unit & SSMDL & Method & SC \\
\hline SRF-01-01 & 117018 & $\mathrm{U}$ & 0.00334 & $\mathrm{pCi} / \mathrm{g}$ & 0.01 & EPIA-012B & \\
\hline SRF-01-02 & 117019 & $\mathrm{U}$ & -0.00105 & $\mathrm{pCi} / \mathrm{g}$ & 0.0231 & EPIA-012B & \\
\hline SRF-01-03 & 117020 & $\mathrm{U}$ & -0.00917 & $\mathrm{pCi} / \mathrm{g}$ & 0.0406 & EPIA-012B & \\
\hline SRF-01-04 & 117021 & $\mathrm{U}$ & 0 & $\mathrm{pCi} / \mathrm{g}$ & 0.0113 & EPIA-012B & \\
\hline SRF-01-05 & 117022 & $\mathrm{U}$ & 0 & $\mathrm{pCi} / \mathrm{g}$ & 0.0183 & EPIA-012B & \\
\hline SRF-02-01 & 117023 & $\mathrm{U}$ & 0.00645 & $\mathrm{pCi} / \mathrm{g}$ & 0.0264 & EPIA-012B & \\
\hline SRF-02-02 & 117024 & $\mathrm{U}$ & 0.00398 & $\mathrm{pCi} / \mathrm{g}$ & 0.028 & EPIA-012B & \\
\hline SRF-02-03 & 117025 & $\mathrm{U}$ & 0.00402 & $\mathrm{pCi} / \mathrm{g}$ & 0.0303 & EPIA-012B & \\
\hline SRF-02-03-A & 117026 & $\mathrm{U}$ & -0.0137 & $\mathrm{pCi} / \mathrm{g}$ & 0.0438 & EPIA-012B & \\
\hline SRF-03-01 & 117027 & $\mathrm{U}$ & -0.00319 & $\mathrm{pCi} / \mathrm{g}$ & 0.0594 & EPIA-012B & \\
\hline SRF-03-02 & 117028 & $\mathrm{U}$ & 0 & $\mathrm{pCi} / \mathrm{g}$ & 0.0129 & EPIA-012B & \\
\hline SRF-03-02 & 117028 & $\mathrm{U}$ & 0 & $\mathrm{pCi} / \mathrm{g}$ & 0.0136 & EPIA-012B & \\
\hline SRF-03-03 & 117029 & $\mathrm{U}$ & -0.00305 & $\mathrm{pCi} / \mathrm{g}$ & 0.024 & EPIA-012B & \\
\hline SRF-03-04 & 117030 & $\mathrm{U}$ & 0 & $\mathrm{pCi} / \mathrm{g}$ & 0.0157 & EPIA-012B & \\
\hline SRF-04-01 & 117031 & $\mathrm{U}$ & -0.00057 & $\mathrm{pCi} / \mathrm{g}$ & 0.0125 & EPIA-012B & \\
\hline SRF-04-02 & 117032 & $\mathrm{U}$ & 0 & $\mathrm{pCi} / \mathrm{g}$ & 0.014 & EPIA-012B & \\
\hline SRF-04-03 & 117033 & $\mathrm{U}$ & 0 & $\mathrm{pCi} / \mathrm{g}$ & 0.00802 & EPIA-012B & \\
\hline SRF-05-01 & 117034 & $\mathrm{U}$ & -0.000653 & $\mathrm{pCi} / \mathrm{g}$ & 0.0144 & EPIA-012B & \\
\hline SRF-05-02 & 117035 & $\mathrm{U}$ & -0.000944 & $\mathrm{pCi} / \mathrm{g}$ & 0.0382 & EPIA-012B & \\
\hline SRF-05-03 & 117036 & $\mathrm{U}$ & -0.00123 & $\mathrm{pCi} / \mathrm{g}$ & 0.0159 & EPIA-012B & \\
\hline SRF-06-01 & 117037 & $\mathrm{U}$ & -0.00168 & $\mathrm{pCi} / \mathrm{g}$ & 0.0218 & EPIA-012B & \\
\hline SRF-06-02 & 117038 & $\mathrm{U}$ & 0 & $\mathrm{pCi} / \mathrm{g}$ & 0.00987 & EPIA-012B & \\
\hline SRF-06-03 & 117039 & $\mathrm{U}$ & 0 & $\mathrm{pCi} / \mathrm{g}$ & 0.00704 & EPIA-012B & \\
\hline SRF-06-03-A & 117040 & $\mathrm{U}$ & 0 & $\mathrm{pCi} / \mathrm{g}$ & 0.00727 & EPIA-012B & \\
\hline SRF-07-01 & 117041 & $\mathrm{U}$ & -0.00284 & $\mathrm{pCi} / \mathrm{g}$ & 0.0224 & EPIA-012B & \\
\hline SRF-07-02 & 117042 & $\mathrm{U}$ & 0.00451 & $\mathrm{pCi} / \mathrm{g}$ & 0.0135 & EPIA-012B & \\
\hline SRF-07-03 & 117043 & $\mathrm{U}$ & -0.000479 & $\mathrm{pCi} / \mathrm{g}$ & 0.0105 & EPIA-012B & \\
\hline
\end{tabular}




\begin{tabular}{|c|c|c|c|c|c|c|c|}
\hline SRF-07-03 & 117043 & $\mathrm{U}$ & 0.00317 & $\mathrm{pCi} / \mathrm{g}$ & 0.013 & EPIA-012B & \\
\hline \multicolumn{8}{|c|}{$\begin{array}{l}\text { ANALYTE: Plutonium- } 239 / 240 \\
\text { Analytical Group: Radionuclides }\end{array}$} \\
\hline Survey ID & Sample ID & FG & Result & Unit & SSMDL & Method & SC \\
\hline SRF-01-01 & 117018 & $\mathrm{U}$ & -0.0048 & $\mathrm{pCi} / \mathrm{g}$ & 0.0286 & EPIA-012B & \\
\hline SRF-01-02 & 117019 & $\mathrm{U}$ & 0 & $\mathrm{pCi} / \mathrm{g}$ & 0.0131 & EPIA-012B & \\
\hline SRF-01-03 & 117020 & $\mathrm{U}$ & -0.00217 & $\mathrm{pCi} / \mathrm{g}$ & 0.0209 & EPIA-012B & \\
\hline SRF-01-04 & 117021 & $\mathrm{U}$ & -0.000901 & $\mathrm{pCi} / \mathrm{g}$ & 0.0198 & EPIA-012B & \\
\hline SRF-01-05 & 117022 & $\mathrm{U}$ & 0.0168 & $\mathrm{pCi} / \mathrm{g}$ & 0.0321 & EPIA-012B & \\
\hline SRF-02-01 & 117023 & $\mathrm{U}$ & -0.00204 & $\mathrm{pCi} / \mathrm{g}$ & 0.0264 & EPIA-012B & \\
\hline SRF-02-02 & 117024 & $\mathrm{U}$ & -0.00116 & $\mathrm{pCi} / \mathrm{g}$ & 0.0151 & EPIA-012B & \\
\hline SRF-02-03 & 117025 & $\mathrm{U}$ & -0.00115 & $\mathrm{pCi} / \mathrm{g}$ & 0.0149 & EPIA-012B & \\
\hline SRF-02-03-A & 117026 & $\mathrm{U}$ & -0.00307 & $\mathrm{pCi} / \mathrm{g}$ & 0.0242 & EPIA-012B & \\
\hline SRF-03-01 & 117027 & $\mathrm{U}$ & 0.00218 & $\mathrm{pCi} / \mathrm{g}$ & 0.0261 & EPIA-012B & \\
\hline SRF-03-02 & 117028 & $\mathrm{U}$ & -0.00103 & $\mathrm{pCi} / \mathrm{g}$ & 0.0227 & EPIA-012B & \\
\hline SRF-03-02 & 117028 & $\mathrm{U}$ & -0.00108 & $\mathrm{pCi} / \mathrm{g}$ & 0.0238 & EPIA-012B & \\
\hline SRF-03-03 & 117029 & $\mathrm{U}$ & -0.00153 & $\mathrm{pCi} / \mathrm{g}$ & 0.0198 & EPIA-012B & \\
\hline SRF-03-04 & 117030 & $\mathrm{U}$ & -0.00753 & $\mathrm{pCi} / \mathrm{g}$ & 0.0448 & EPIA-012B & \\
\hline SRF-04-01 & 117031 & $\mathrm{U}$ & 0 & $\mathrm{pCi} / \mathrm{g}$ & 0.00712 & EPIA-012B & \\
\hline SRF-04-02 & 117032 & $\mathrm{U}$ & -0.00112 & $\mathrm{pCi} / \mathrm{g}$ & 0.0247 & EPIA-012B & \\
\hline SRF-04-03 & 117033 & $\mathrm{U}$ & -0.000641 & $\mathrm{pCi} / \mathrm{g}$ & 0.0141 & EPIA-012B & \\
\hline SRF-05-01 & 117034 & $\mathrm{U}$ & 0 & $\mathrm{pCi} / \mathrm{g}$ & 0.00816 & EPIA-012B & \\
\hline SRF-05-02 & 117035 & $\mathrm{U}$ & -0.00113 & $\mathrm{pCi} / \mathrm{g}$ & 0.0249 & EPIA-012B & \\
\hline SRF-05-03 & 117036 & $\mathrm{U}$ & 0.00451 & $\mathrm{pCi} / \mathrm{g}$ & 0.0135 & EPIA-012B & \\
\hline SRF-06-01 & 117037 & $\mathrm{U}$ & -0.00168 & $\mathrm{pCi} / \mathrm{g}$ & 0.0218 & EPIA-012B & \\
\hline SRF-06-02 & 117038 & $\mathrm{U}$ & -0.00158 & $\mathrm{pCi} / \mathrm{g}$ & 0.0204 & EPIA-012B & \\
\hline SRF-06-03 & 117039 & $\mathrm{U}$ & 0 & $\mathrm{pCi} / \mathrm{g}$ & 0.00703 & EPIA-012B & \\
\hline SRF-06-03-A & 117040 & $\mathrm{U}$ & -0.000581 & $\mathrm{pCi} / \mathrm{g}$ & 0.0128 & EPIA-012B & \\
\hline SRF-07-01 & 117041 & $\mathrm{U}$ & -0.00142 & $\mathrm{pCi} / \mathrm{g}$ & 0.0184 & EPIA-012B & \\
\hline SRF-07-02 & 117042 & $\mathrm{U}$ & -0.00108 & $\mathrm{pCi} / \mathrm{g}$ & 0.0238 & EPIA-012B & \\
\hline SRF-07-03 & 117043 & $\mathrm{U}$ & 0.00199 & $\mathrm{pCi} / \mathrm{g}$ & 0.00598 & EPIA-012B & \\
\hline SRF-07-03 & 117043 & $\mathrm{U}$ & -0.000501 & $\mathrm{pCi} / \mathrm{g}$ & 0.011 & EPIA-012B & \\
\hline
\end{tabular}

ANALYTE: Potassium-40

Analytical Group: Radionuclides

\begin{tabular}{|c|c|c|c|c|c|c|c|c|}
\hline Survey ID & Sample ID & FG & Result & Unit & SsMDL & Method & SC & $\mathbf{E}$ \\
\hline SRF-01-01 & 117018 & & 3.97 & $\mathrm{pCi} / \mathrm{g}$ & 0.611 & EPIA-013B & & \\
\hline SRF-01-02 & 117019 & & 3.51 & $\mathrm{pCi} / \mathrm{g}$ & 0.487 & EPIA-013B & & \\
\hline SRF-01-03 & 117020 & & 4.41 & $\mathrm{pCi} / \mathrm{g}$ & 0.459 & EPIA-013B & & \\
\hline SRF-01-04 & 117021 & & 3.39 & $\mathrm{pCi} / \mathrm{g}$ & 0.456 & EPIA-013B & & \\
\hline SRF-01-05 & 117022 & & 3.64 & $\mathrm{pCi} / \mathrm{g}$ & 0.498 & EPIA-013B & & \\
\hline SRF-01-05 & 117022 & $\mathrm{R}$ & 2.88 & $\mathrm{pCi} / \mathrm{g}$ & 1.37 & EPIA-013B & & 4 \\
\hline SRF-02-01 & 117023 & & 4.23 & $\mathrm{pCi} / \mathrm{g}$ & 0.726 & EPIA-013B & & \\
\hline SRF-02-02 & 117024 & & 2.54 & $\mathrm{pCi} / \mathrm{g}$ & 0.459 & EPIA-013B & & \\
\hline SRF-02-03 & 117025 & & 3.58 & $\mathrm{pCi} / \mathrm{g}$ & 0.695 & EPIA-013B & & \\
\hline SRF-02-03-A & 117026 & & 3.53 & $\mathrm{pCi} / \mathrm{g}$ & 0.739 & EPIA-013B & & \\
\hline SRF-03-01 & 117027 & & 2.47 & $\mathrm{pCi} / \mathrm{g}$ & 0.6 & EPIA-013B & & \\
\hline SRF-03-02 & 117028 & & 3.89 & $\mathrm{pCi} / \mathrm{g}$ & 0.423 & EPIA-013B & & \\
\hline SRF-03-03 & 117029 & & 2.97 & $\mathrm{pCi} / \mathrm{g}$ & 0.556 & EPIA-013B & & \\
\hline SRF-03-04 & 117030 & & 3.84 & $\mathrm{pCi} / \mathrm{g}$ & 0.309 & EPIA-013B & & \\
\hline SRF-04-01 & 117031 & & 3.01 & $\mathrm{pCi} / \mathrm{g}$ & 0.561 & EPIA-013B & & \\
\hline SRF-04-02 & 117032 & & 2.25 & $\mathrm{pCi} / \mathrm{g}$ & 0.646 & EPIA-013B & & \\
\hline SRF-04-03 & 117033 & & 4.01 & $\mathrm{pCi} / \mathrm{g}$ & 0.778 & EPIA-013B & & \\
\hline SRF-05-01 & 117034 & & 3.16 & $\mathrm{pCi} / \mathrm{g}$ & 0.372 & EPIA-013B & & \\
\hline SRF-05-02 & 117035 & & 3.28 & $\mathrm{pCi} / \mathrm{g}$ & 0.44 & EPIA-013B & & \\
\hline SRF-05-03 & 117036 & & 3.56 & $\mathrm{pCi} / \mathrm{g}$ & 0.406 & EPIA-013B & & \\
\hline SRF-06-01 & 117037 & & 2.95 & $\mathrm{pCi} / \mathrm{g}$ & 0.58 & EPIA-013B & & \\
\hline
\end{tabular}


ANALYTE: Potassium-40 (cont.)

\begin{tabular}{|c|c|c|c|c|c|c|c|}
\hline Survey ID & Sample ID & FG & Result & Unit & SsMDL & Method & SC \\
\hline SRF-06-02 & 117038 & & 2.94 & $\mathrm{pCi} / \mathrm{g}$ & 0.389 & EPIA-013B & \\
\hline SRF-06-03 & 117039 & & 2.45 & $\mathrm{pCi} / \mathrm{g}$ & 0.605 & EPIA-013B & \\
\hline SRF-06-03-A & 117040 & & 2.94 & $\mathrm{pCi} / \mathrm{g}$ & 0.69 & EPIA-013B & \\
\hline SRF-07-01 & 117041 & & 2.79 & $\mathrm{pCi} / \mathrm{g}$ & 0.743 & EPIA-013B & \\
\hline SRF-07-02 & 117042 & & 2.8 & $\mathrm{pCi} / \mathrm{g}$ & 0.881 & EPIA-013B & \\
\hline SRF-07-03 & 117043 & & 2.76 & $\mathrm{pCi} / \mathrm{g}$ & 0.818 & EPIA-013B & \\
\hline SRF-07-03 & 117043 & & 2.86 & $\mathrm{pCi} / \mathrm{g}$ & 0.561 & EPIA-013B & \\
\hline
\end{tabular}

ANALYTE: Promethium-144

Analytical Group: Radionuclides

\begin{tabular}{|c|c|c|c|c|c|c|c|}
\hline Survey ID & Sample ID & $\mathbf{F G}$ & Result & Unit & SsMDL & Method & SC \\
\hline SRF-01-01 & 117018 & $\mathrm{U}$ & 0.0151 & $\mathrm{pCi} / \mathrm{g}$ & 0.0622 & EPIA-013B & \\
\hline SRF-01-02 & 117019 & $\mathrm{U}$ & -0.00267 & $\mathrm{pCi} / \mathrm{g}$ & 0.0449 & EPIA-013B & \\
\hline SRF-01-03 & 117020 & $\mathrm{U}$ & -0.0104 & $\mathrm{pCi} / \mathrm{g}$ & 0.0443 & EPIA-013B & \\
\hline SRF-01-04 & 117021 & $\mathrm{U}$ & -0.00147 & $\mathrm{pCi} / \mathrm{g}$ & 0.0413 & EPIA-013B & \\
\hline SRF-01-05 & 117022 & $\mathrm{U}$ & -0.0155 & $\mathrm{pCi} / \mathrm{g}$ & 0.0451 & EPIA-013B & \\
\hline SRF-01-05 & 117022 & $\mathrm{U}$ & 0.0193 & $\mathrm{pCi} / \mathrm{g}$ & 0.0495 & EPIA-013B & \\
\hline SRF-02-01 & 117023 & $\mathrm{U}$ & 0.0247 & $\mathrm{pCi} / \mathrm{g}$ & 0.0659 & EPIA-013B & \\
\hline SRF-02-02 & 117024 & $\mathrm{U}$ & 0.0223 & $\mathrm{pCi} / \mathrm{g}$ & 0.0489 & EPIA-013B & \\
\hline SRF-02-03 & 117025 & $\mathrm{U}$ & 0.015 & $\mathrm{pCi} / \mathrm{g}$ & 0.075 & EPIA-013B & \\
\hline SRF-02-03-A & 117026 & $\mathrm{U}$ & -0.0191 & $\mathrm{pCi} / \mathrm{g}$ & 0.0621 & EPIA-013B & \\
\hline SRF-03-01 & 117027 & $\mathrm{U}$ & 0.03 & $\mathrm{pCi} / \mathrm{g}$ & 0.0621 & EPIA-013B & \\
\hline SRF-03-02 & 117028 & $\mathrm{U}$ & 0.00514 & $\mathrm{pCi} / \mathrm{g}$ & 0.0471 & EPIA-013B & \\
\hline SRF-03-03 & 117029 & $\mathrm{U}$ & -0.00607 & $\mathrm{pCi} / \mathrm{g}$ & 0.0529 & EPIA-013B & \\
\hline SRF-03-04 & 117030 & $\mathrm{U}$ & -0.00494 & $\mathrm{pCi} / \mathrm{g}$ & 0.0308 & EPIA-013B & \\
\hline SRF-04-01 & 117031 & $\mathrm{U}$ & 0.0023 & $\mathrm{pCi} / \mathrm{g}$ & 0.054 & EPIA-013B & \\
\hline SRF-04-02 & 117032 & $\mathrm{U}$ & -0.00452 & $\mathrm{pCi} / \mathrm{g}$ & 0.0713 & EPIA-013B & \\
\hline SRF-04-03 & 117033 & $\mathrm{U}$ & 0.044 & $\mathrm{pCi} / \mathrm{g}$ & 0.0744 & EPIA-013B & \\
\hline SRF-05-01 & 117034 & $\mathrm{U}$ & 0.00753 & $\mathrm{pCi} / \mathrm{g}$ & 0.0472 & EPIA-013B & \\
\hline SRF-05-02 & 117035 & $\mathrm{U}$ & 0.012 & $\mathrm{pCi} / \mathrm{g}$ & 0.0552 & EPIA-013B & \\
\hline SRF-05-03 & 117036 & $\mathrm{U}$ & 0.0193 & $\mathrm{pCi} / \mathrm{g}$ & 0.056 & EPIA-013B & \\
\hline SRF-06-01 & 117037 & $\mathrm{U}$ & -0.00499 & $\mathrm{pCi} / \mathrm{g}$ & 0.0576 & EPIA-013B & \\
\hline SRF-06-02 & 117038 & $\mathrm{U}$ & 0.00184 & $\mathrm{pCi} / \mathrm{g}$ & 0.0391 & EPIA-013B & \\
\hline SRF-06-03 & 117039 & $\mathrm{U}$ & 0.0261 & $\mathrm{pCi} / \mathrm{g}$ & 0.0582 & EPIA-013B & \\
\hline SRF-06-03-A & 117040 & $\mathrm{U}$ & 0.00669 & $\mathrm{pCi} / \mathrm{g}$ & 0.0679 & EPIA-013B & \\
\hline SRF-07-01 & 117041 & $\mathrm{U}$ & 0.00452 & $\mathrm{pCi} / \mathrm{g}$ & 0.0707 & EPIA-013B & \\
\hline SRF-07-02 & 117042 & $\mathrm{U}$ & -0.00318 & $\mathrm{pCi} / \mathrm{g}$ & 0.0755 & EPIA-013B & \\
\hline SRF-07-03 & 117043 & $\mathrm{U}$ & 0.0118 & $\mathrm{pCi} / \mathrm{g}$ & 0.0779 & EPIA-013B & \\
\hline SRF-07-03 & 117043 & $\mathrm{U}$ & 0.0127 & $\mathrm{pCi} / \mathrm{g}$ & 0.0525 & EPIA-013B & \\
\hline
\end{tabular}

ANALYTE: Promethium-146

Analytical Group: Radionuclides

$\begin{array}{lllrlrlr}\text { Survey ID } & \text { Sample ID } & \text { FG } & \text { Result } & \text { Unit } & \text { ssMDL } & \text { Method } & \text { SC } \\ \text { SRF-01-01 } & 117018 & \mathrm{U} & -0.00415 & \mathrm{pCi} / \mathrm{g} & 0.0672 & \text { EPIA-013B } \\ \text { SRF-01-02 } & 117019 & \mathrm{U} & 0.00302 & \mathrm{pCi} / \mathrm{g} & 0.058 & \text { EPIA-013B } \\ \text { SRF-01-03 } & 117020 & \mathrm{U} & -0.0185 & \mathrm{pCi} / \mathrm{g} & 0.0575 & \text { EPIA-013B } \\ \text { SRF-01-04 } & 117021 & \mathrm{U} & 0.00492 & \mathrm{pCi} / \mathrm{g} & 0.0499 & \text { EPIA-013B } \\ \text { SRF-01-05 } & 117022 & \mathrm{U} & -0.00327 & \mathrm{pCi} / \mathrm{g} & 0.0528 & \text { EPIA-013B } \\ \text { SRF-01-05 } & 117022 & \mathrm{U} & -0.0233 & \mathrm{pCi} / \mathrm{g} & 0.0475 & \text { EPIA-013B } \\ \text { SRF-02-01 } & 117023 & \mathrm{U} & -0.044 & \mathrm{pCi} / \mathrm{g} & 0.0655 & \text { EPIA-013B } \\ \text { SRF-02-02 } & 117024 & \mathrm{U} & 0.00314 & \mathrm{pCi} / \mathrm{g} & 0.047 & \text { EPIA-013B }\end{array}$


ANALYTE: Promethium-146 (cont.)

$\begin{array}{lllrlrlr}\text { Survey ID } & \text { Sample ID } & \text { FG } & \text { Result } & \text { Unit } & \text { ssMDL } & \text { Method } & \text { SC } \\ \text { SRF-02-03 } & 117025 & \mathrm{U} & -0.00799 & \mathrm{pCi} / \mathrm{g} & 0.0788 & \text { EPIA-013B } \\ \text { SRF-02-03-A } & 117026 & \mathrm{U} & -0.0416 & \mathrm{pCi} / \mathrm{g} & 0.0706 & \text { EPIA-013B } \\ \text { SRF-03-01 } & 117027 & \mathrm{U} & 0.0264 & \mathrm{pCi} / \mathrm{g} & 0.0695 & \text { EPIA-013B } \\ \text { SRF-03-02 } & 117028 & \mathrm{U} & -0.0187 & \mathrm{pCi} / \mathrm{g} & 0.05 & \text { EPIA-013B } \\ \text { SRF-03-03 } & 117029 & \mathrm{U} & 0.0157 & \mathrm{pCi} / \mathrm{g} & 0.0622 & \text { EPIA-013B } \\ \text { SRF-03-04 } & 117030 & \mathrm{U} & 0.0111 & \mathrm{pCi} / \mathrm{g} & 0.0362 & \text { EPIA-013B } \\ \text { SRF-04-01 } & 117031 & \mathrm{U} & 0.00421 & \mathrm{pCi} / \mathrm{g} & 0.0553 & \text { EPIA-013B } \\ \text { SRF-04-02 } & 117032 & \mathrm{U} & -0.0232 & \mathrm{pCi} / \mathrm{g} & 0.068 & \text { EPIA-013B } \\ \text { SRF-04-03 } & 117033 & \mathrm{U} & 0.0343 & \mathrm{pCi} / \mathrm{g} & 0.0681 & \text { EPIA-013B } \\ \text { SRF-05-01 } & 117034 & \mathrm{U} & -0.0345 & \mathrm{pCi} / \mathrm{g} & 0.0463 & \text { EPIA-013B } \\ \text { SRF-05-02 } & 117035 & \mathrm{U} & 0.00102 & \mathrm{pCi} / \mathrm{g} & 0.0612 & \text { EPIA-013B } \\ \text { SRF-05-03 } & 117036 & \mathrm{U} & -0.0232 & \mathrm{pCi} / \mathrm{g} & 0.0603 & \text { EPIA-013B } \\ \text { SRF-06-01 } & 117037 & \mathrm{U} & -0.0152 & \mathrm{pCi} / \mathrm{g} & 0.0653 & \text { EPIA-013B } \\ \text { SRF-06-02 } & 117038 & \mathrm{U} & -0.0244 & \mathrm{pCi} / \mathrm{g} & 0.0398 & \text { EPIA-013B } \\ \text { SRF-06-03 } & 117039 & \mathrm{U} & -0.0424 & \mathrm{pCi} / \mathrm{g} & 0.0751 & \text { EPIA-013B } \\ \text { SRF-06-03-A } & 117040 & \mathrm{U} & -0.0265 & \mathrm{pCi} / \mathrm{g} & 0.0685 & \text { EPIA-013B } \\ \text { SRF-07-01 } & 117041 & \mathrm{U} & 0.0175 & \mathrm{pCi} / \mathrm{g} & 0.0764 & \text { EPIA-013B } \\ \text { SRF-07-02 } & 117042 & \mathrm{U} & -0.031 & \mathrm{pCi} / \mathrm{g} & 0.0755 & \text { EPIA-013B } \\ \text { SRF-07-03 } & 117043 & \mathrm{U} & 0.0137 & \mathrm{pCi} / \mathrm{g} & 0.0605 & \text { EPIA-013B } \\ \text { SRF-07-03 } & 117043 & \mathrm{U} & 0.000611 & \mathrm{pCi} / \mathrm{g} & 0.0823 & \text { EPIA-013B } \\ & & & & & & \end{array}$

ANALYTE: Ruthenium-106

Analytical Group: Radionuclides

\begin{tabular}{|c|c|c|c|c|c|c|c|}
\hline Survey ID & Sample ID & FG & Result & Unit & SsMDL & Method & SC \\
\hline SRF-01-01 & 117018 & $\mathrm{U}$ & -0.103 & $\mathrm{pCi} / \mathrm{g}$ & 0.457 & EPIA-013B & \\
\hline SRF-01-02 & 117019 & $\mathrm{U}$ & 0.0746 & $\mathrm{pCi} / \mathrm{g}$ & 0.383 & EPIA-013B & \\
\hline SRF-01-03 & 117020 & $\mathrm{U}$ & -0.0132 & $\mathrm{pCi} / \mathrm{g}$ & 0.467 & EPIA-013B & \\
\hline SRF-01-04 & 117021 & $\mathrm{U}$ & -0.0714 & $\mathrm{pCi} / \mathrm{g}$ & 0.364 & EPIA-013B & \\
\hline SRF-01-05 & 117022 & $\mathrm{U}$ & -0.0232 & $\mathrm{pCi} / \mathrm{g}$ & 0.396 & EPIA-013B & \\
\hline SRF-01-05 & 117022 & $\mathrm{U}$ & 0.138 & $\mathrm{pCi} / \mathrm{g}$ & 0.415 & EPIA-013B & \\
\hline SRF-02-01 & 117023 & $\mathrm{U}$ & 0.245 & $\mathrm{pCi} / \mathrm{g}$ & 0.547 & EPIA-013B & \\
\hline SRF-02-02 & 117024 & $\mathrm{U}$ & 0.0177 & $\mathrm{pCi} / \mathrm{g}$ & 0.41 & EPIA-013B & \\
\hline SRF-02-03 & 117025 & $\mathrm{U}$ & 0.0415 & $\mathrm{pCi} / \mathrm{g}$ & 0.699 & EPIA-013B & \\
\hline SRF-02-03-A & 117026 & $\mathrm{U}$ & 0.0977 & $\mathrm{pCi} / \mathrm{g}$ & 0.575 & EPIA-013B & \\
\hline SRF-03-01 & 117027 & $\mathrm{U}$ & -0.0495 & $\mathrm{pCi} / \mathrm{g}$ & 0.514 & EPIA-013B & \\
\hline SRF-03-02 & 117028 & $\mathrm{U}$ & -0.0878 & $\mathrm{pCi} / \mathrm{g}$ & 0.411 & EPIA-013B & \\
\hline SRF-03-03 & 117029 & $\mathrm{U}$ & 0.127 & $\mathrm{pCi} / \mathrm{g}$ & 0.521 & EPIA-013B & \\
\hline SRF-03-04 & 117030 & $\mathrm{U}$ & -0.0324 & $\mathrm{pCi} / \mathrm{g}$ & 0.277 & EPIA-013B & \\
\hline SRF-04-01 & 117031 & $\mathrm{U}$ & -0.27 & $\mathrm{pCi} / \mathrm{g}$ & 0.433 & EPIA-013B & \\
\hline SRF-04-02 & 117032 & $\mathrm{U}$ & 0.261 & $\mathrm{pCi} / \mathrm{g}$ & 0.633 & EPIA-013B & \\
\hline SRF-04-03 & 117033 & $\mathrm{U}$ & -0.101 & $\mathrm{pCi} / \mathrm{g}$ & 0.484 & EPIA-013B & \\
\hline SRF-05-01 & 117034 & $\mathrm{U}$ & 0.0452 & $\mathrm{pCi} / \mathrm{g}$ & 0.449 & EPIA-013B & \\
\hline SRF-05-02 & 117035 & $\mathrm{U}$ & 0.209 & $\mathrm{pCi} / \mathrm{g}$ & 0.506 & EPIA-013B & \\
\hline SRF-05-03 & 117036 & $\mathrm{U}$ & -0.0522 & $\mathrm{pCi} / \mathrm{g}$ & 0.444 & EPIA-013B & \\
\hline SRF-06-01 & 117037 & $\mathrm{U}$ & 0.071 & $\mathrm{pCi} / \mathrm{g}$ & 0.614 & EPIA-013B & \\
\hline SRF-06-02 & 117038 & $\mathrm{U}$ & -0.00601 & $\mathrm{pCi} / \mathrm{g}$ & 0.349 & EPIA-013B & \\
\hline SRF-06-03 & 117039 & $\mathrm{U}$ & -0.371 & $\mathrm{pCi} / \mathrm{g}$ & 0.492 & EPIA-013B & \\
\hline SRF-06-03-A & 117040 & $\mathrm{U}$ & -0.221 & $\mathrm{pCi} / \mathrm{g}$ & 0.586 & EPIA-013B & \\
\hline SRF-07-01 & 117041 & $\mathrm{U}$ & -0.181 & $\mathrm{pCi} / \mathrm{g}$ & 0.583 & EPIA-013B & \\
\hline SRF-07-02 & 117042 & $\mathrm{U}$ & 0.36 & $\mathrm{pCi} / \mathrm{g}$ & 0.751 & EPIA-013B & \\
\hline SRF-07-03 & 117043 & $\mathrm{U}$ & 0.252 & $\mathrm{pCi} / \mathrm{g}$ & 0.497 & EPIA-013B & \\
\hline SRF-07-03 & 117043 & $\mathrm{U}$ & 0.0762 & $\mathrm{pCi} / \mathrm{g}$ & 0.702 & EPIA-013B & \\
\hline
\end{tabular}


ANALYTE: Selenium

Analytical Group: Metals (total recoverable)

\begin{tabular}{|c|c|c|c|c|c|c|}
\hline Survey ID & Sample ID FG & Result & Unit & SSMDL & Method & SC \\
\hline SRF-01-01 & 117018 & 0.904 & $\mathrm{mg} / \mathrm{kg}$ & 0.123 & EPA6010B & \\
\hline SRF-01-02 & 117019 & 1 & $\mathrm{mg} / \mathrm{kg}$ & 0.129 & EPA6010B & \\
\hline SRF-01-03 & 117020 & 0.925 & $\mathrm{mg} / \mathrm{kg}$ & 0.123 & EPA6010B & \\
\hline SRF-01-04 & 117021 & 0.938 & $\mathrm{mg} / \mathrm{kg}$ & 0.125 & EPA6010B & \\
\hline SRF-01-05 & 117022 & 1.02 & $\mathrm{mg} / \mathrm{kg}$ & 0.124 & EPA6010B & \\
\hline SRF-02-01 & 117023 & 0.859 & $\mathrm{mg} / \mathrm{kg}$ & 0.126 & EPA6010B & \\
\hline SRF-02-02 & 117024 & 1.07 & $\mathrm{mg} / \mathrm{kg}$ & 0.13 & EPA6010B & \\
\hline SRF-02-03 & 117025 & 1.27 & $\mathrm{mg} / \mathrm{kg}$ & 0.135 & EPA6010B & \\
\hline SRF-02-03-A & 117026 & 2.85 & $\mathrm{mg} / \mathrm{kg}$ & 0.127 & EPA6010B & \\
\hline SRF-03-01 & 117027 & 0.905 & $\mathrm{mg} / \mathrm{kg}$ & 0.134 & EPA6010B & \\
\hline SRF-03-02 & 117028 & 0.883 & $\mathrm{mg} / \mathrm{kg}$ & 0.131 & EPA6010B & \\
\hline SRF-03-03 & 117029 & 1.13 & $\mathrm{mg} / \mathrm{kg}$ & 0.126 & EPA6010B & \\
\hline SRF-03-04 & 117030 & 1.05 & $\mathrm{mg} / \mathrm{kg}$ & 0.132 & EPA6010B & \\
\hline SRF-04-01 & 117031 & 1.01 & $\mathrm{mg} / \mathrm{kg}$ & 0.13 & EPA6010B & \\
\hline SRF-04-02 & 117032 & 1.15 & $\mathrm{mg} / \mathrm{kg}$ & 0.124 & EPA6010B & \\
\hline SRF-04-03 & 117033 & 0.764 & $\mathrm{mg} / \mathrm{kg}$ & 0.134 & EPA6010B & \\
\hline SRF-05-01 & 117034 & 1.04 & $\mathrm{mg} / \mathrm{kg}$ & 0.131 & EPA6010B & \\
\hline SRF-05-02 & 117035 & 0.88 & $\mathrm{mg} / \mathrm{kg}$ & 0.129 & EPA6010B & \\
\hline SRF-05-03 & 117036 & 1.08 & $\mathrm{mg} / \mathrm{kg}$ & 0.126 & EPA6010B & \\
\hline SRF-06-01 & 117037 & 1.34 & $\mathrm{mg} / \mathrm{kg}$ & 0.129 & EPA6010B & \\
\hline SRF-06-02 & 117038 & 0.941 & $\mathrm{mg} / \mathrm{kg}$ & 0.125 & EPA6010B & \\
\hline SRF-06-03 & 117039 & 0.962 & $\mathrm{mg} / \mathrm{kg}$ & 0.129 & EPA6010B & \\
\hline SRF-06-03-A & 117040 & 1.04 & $\mathrm{mg} / \mathrm{kg}$ & 0.134 & EPA6010B & \\
\hline SRF-07-01 & 117041 & 0.874 & $\mathrm{mg} / \mathrm{kg}$ & 0.13 & EPA6010B & \\
\hline SRF-07-02 & 117042 & 0.845 & $\mathrm{mg} / \mathrm{kg}$ & 0.13 & EPA6010B & \\
\hline SRF-07-03 & 117043 & 1.07 & $\mathrm{mg} / \mathrm{kg}$ & 0.124 & EPA6010B & \\
\hline
\end{tabular}

ANALYTE: Silver

Analytical Group: Metals (total recoverable)

\begin{tabular}{|c|c|c|c|c|c|c|c|}
\hline Survey ID & Sample ID & FG & Result & Unit & SsMDL & Method & SC \\
\hline SRF-01-01 & 117018 & $\mathrm{~J}$ & 0.121 & $\mathrm{mg} / \mathrm{kg}$ & 0.0282 & EPA6010B & I \\
\hline SRF-01-02 & 117019 & $\mathrm{~J}$ & 0.105 & $\mathrm{mg} / \mathrm{kg}$ & 0.0295 & EPA6010B & I \\
\hline SRF-01-03 & 117020 & $\mathrm{U}$ & 0.227 & $\mathrm{mg} / \mathrm{kg}$ & 0.0282 & EPA6010B & \\
\hline SRF-01-04 & 117021 & $\mathrm{~J}$ & 0.0501 & $\mathrm{mg} / \mathrm{kg}$ & 0.0287 & EPA6010B & I \\
\hline SRF-01-05 & 117022 & $\mathrm{U}$ & 0.229 & $\mathrm{mg} / \mathrm{kg}$ & 0.0284 & EPA6010B & \\
\hline SRF-02-01 & 117023 & $\mathrm{U}$ & 0.234 & $\mathrm{mg} / \mathrm{kg}$ & 0.029 & EPA6010B & \\
\hline SRF-02-02 & 117024 & $\mathrm{U}$ & 0.24 & $\mathrm{mg} / \mathrm{kg}$ & 0.0298 & EPA6010B & \\
\hline SRF-02-03 & 117025 & $\mathrm{~J}$ & 0.0969 & $\mathrm{mg} / \mathrm{kg}$ & 0.031 & EPA6010B & I \\
\hline SRF-02-03-A & 117026 & & 0.712 & $\mathrm{mg} / \mathrm{kg}$ & 0.0292 & EPA6010B & \\
\hline SRF-03-01 & 117027 & $\mathrm{U}$ & 0.248 & $\mathrm{mg} / \mathrm{kg}$ & 0.0307 & EPA6010B & \\
\hline SRF-03-02 & 117028 & $\mathrm{U}$ & 0.243 & $\mathrm{mg} / \mathrm{kg}$ & 0.0301 & EPA6010B & \\
\hline SRF-03-03 & 117029 & $\mathrm{~J}$ & 0.067 & $\mathrm{mg} / \mathrm{kg}$ & 0.029 & EPA6010B & I \\
\hline SRF-03-04 & 117030 & $\mathrm{U}$ & 0.245 & $\mathrm{mg} / \mathrm{kg}$ & 0.0304 & EPA6010B & \\
\hline SRF-04-01 & 117031 & $\mathrm{U}$ & 0.24 & $\mathrm{mg} / \mathrm{kg}$ & 0.0298 & EPA6010B & \\
\hline SRF-04-02 & 117032 & $\mathrm{U}$ & 0.229 & $\mathrm{mg} / \mathrm{kg}$ & 0.0284 & EPA6010B & \\
\hline SRF-04-03 & 117033 & $\mathrm{U}$ & 0.248 & $\mathrm{mg} / \mathrm{kg}$ & 0.0307 & EPA6010B & \\
\hline SRF-05-01 & 117034 & $\mathrm{U}$ & 0.243 & $\mathrm{mg} / \mathrm{kg}$ & 0.0301 & EPA6010B & \\
\hline SRF-05-02 & 117035 & $\mathrm{U}$ & 0.238 & $\mathrm{mg} / \mathrm{kg}$ & 0.0295 & EPA6010B & \\
\hline SRF-05-03 & 117036 & $\mathrm{U}$ & 0.234 & $\mathrm{mg} / \mathrm{kg}$ & 0.029 & EPA6010B & \\
\hline SRF-06-01 & 117037 & $\mathrm{U}$ & 0.238 & $\mathrm{mg} / \mathrm{kg}$ & 0.0295 & EPA6010B & \\
\hline SRF-06-02 & 117038 & $\mathrm{U}$ & 0.231 & $\mathrm{mg} / \mathrm{kg}$ & 0.0287 & EPA6010B & \\
\hline SRF-06-03 & 117039 & $\mathrm{~J}$ & 0.0421 & $\mathrm{mg} / \mathrm{kg}$ & 0.0295 & EPA6010B & I \\
\hline SRF-06-03-A & 117040 & $\mathrm{U}$ & 0.248 & $\mathrm{mg} / \mathrm{kg}$ & 0.0307 & EPA6010B & \\
\hline
\end{tabular}


ANALYTE: Silver (cont.)

\begin{tabular}{|c|c|c|c|c|c|c|c|}
\hline Survey ID & Sample ID & FG & Result & Unit & SsMDL & Method & SC \\
\hline SRF-07-01 & 117041 & $\mathrm{~J}$ & 0.0896 & $\mathrm{mg} / \mathrm{kg}$ & 0.0298 & EPA6010B & I \\
\hline SRF-07-02 & 117042 & $\mathrm{~J}$ & 0.082 & $\mathrm{mg} / \mathrm{kg}$ & 0.0298 & EPA6010B & I \\
\hline SRF-07-03 & 117043 & $\mathrm{~J}$ & 0.135 & $\mathrm{mg} / \mathrm{kg}$ & 0.0284 & EPA6010B & IL \\
\hline
\end{tabular}

ANALYTE: Sodium-22

Analytical Group: Radionuclides

\begin{tabular}{|c|c|c|c|c|c|c|c|}
\hline Survey ID & Sample ID & FG & Result & Unit & ssMDL & Method & SC \\
\hline SRF-01-01 & 117018 & $\mathrm{U}$ & -0.00507 & $\mathrm{pCi} / \mathrm{g}$ & 0.0549 & EPIA-013B & \\
\hline SRF-01-02 & 117019 & $\mathrm{U}$ & 0.0191 & $\mathrm{pCi} / \mathrm{g}$ & 0.0649 & EPIA-013B & \\
\hline SRF-01-03 & 117020 & $\mathrm{U}$ & 0.0184 & $\mathrm{pCi} / \mathrm{g}$ & 0.0538 & EPIA-013B & \\
\hline SRF-01-04 & 117021 & $\mathrm{U}$ & 0.00639 & $\mathrm{pCi} / \mathrm{g}$ & 0.0476 & EPIA-013B & \\
\hline SRF-01-05 & 117022 & $\mathrm{U}$ & 0.00542 & $\mathrm{pCi} / \mathrm{g}$ & 0.0503 & EPIA-013B & \\
\hline SRF-01-05 & 117022 & $\mathrm{U}$ & -0.000404 & $\mathrm{pCi} / \mathrm{g}$ & 0.0538 & EPIA-013B & \\
\hline SRF-02-01 & 117023 & $\mathrm{U}$ & -0.00917 & $\mathrm{pCi} / \mathrm{g}$ & 0.0539 & EPIA-013B & \\
\hline SRF-02-02 & 117024 & $\mathrm{U}$ & 0.00668 & $\mathrm{pCi} / \mathrm{g}$ & 0.05 & EPIA-013B & \\
\hline SRF-02-03 & 117025 & $\mathrm{U}$ & 0.0136 & $\mathrm{pCi} / \mathrm{g}$ & 0.0467 & EPIA-013B & \\
\hline SRF-02-03-A & 117026 & $\mathrm{U}$ & -0.0179 & $\mathrm{pCi} / \mathrm{g}$ & 0.0722 & EPIA-013B & \\
\hline SRF-03-01 & 117027 & $\mathrm{U}$ & -0.00353 & $\mathrm{pCi} / \mathrm{g}$ & 0.0672 & EPIA-013B & \\
\hline SRF-03-02 & 117028 & $\mathrm{U}$ & -0.019 & $\mathrm{pCi} / \mathrm{g}$ & 0.0458 & EPIA-013B & \\
\hline SRF-03-03 & 117029 & $\mathrm{U}$ & -0.00506 & $\mathrm{pCi} / \mathrm{g}$ & 0.0567 & EPIA-013B & \\
\hline SRF-03-04 & 117030 & $\mathrm{U}$ & -0.00829 & $\mathrm{pCi} / \mathrm{g}$ & 0.0324 & EPIA-013B & \\
\hline SRF-04-01 & 117031 & $\mathrm{U}$ & -0.0064 & $\mathrm{pCi} / \mathrm{g}$ & 0.0511 & EPIA-013B & \\
\hline SRF-04-02 & 117032 & $\mathrm{U}$ & -0.0205 & $\mathrm{pCi} / \mathrm{g}$ & 0.0777 & EPIA-013B & \\
\hline SRF-04-03 & 117033 & $\mathrm{U}$ & 0.0218 & $\mathrm{pCi} / \mathrm{g}$ & 0.0732 & EPIA-013B & \\
\hline SRF-05-01 & 117034 & $\mathrm{U}$ & -0.00672 & $\mathrm{pCi} / \mathrm{g}$ & 0.0542 & EPIA-013B & \\
\hline SRF-05-02 & 117035 & $\mathrm{U}$ & 0.0351 & $\mathrm{pCi} / \mathrm{g}$ & 0.0614 & EPIA-013B & \\
\hline SRF-05-03 & 117036 & $\mathrm{U}$ & -0.00555 & $\mathrm{pCi} / \mathrm{g}$ & 0.0491 & EPIA-013B & \\
\hline SRF-06-01 & 117037 & $\mathrm{U}$ & -0.0122 & $\mathrm{pCi} / \mathrm{g}$ & 0.0617 & EPIA-013B & \\
\hline SRF-06-02 & 117038 & $\mathrm{U}$ & 0.0294 & $\mathrm{pCi} / \mathrm{g}$ & 0.056 & EPIA-013B & \\
\hline SRF-06-03 & 117039 & $\mathrm{U}$ & 0.00276 & $\mathrm{pCi} / \mathrm{g}$ & 0.0633 & EPIA-013B & \\
\hline SRF-06-03-A & 117040 & $\mathrm{U}$ & 0.0389 & $\mathrm{pCi} / \mathrm{g}$ & 0.0875 & EPIA-013B & \\
\hline SRF-07-01 & 117041 & $\mathrm{U}$ & -0.0104 & $\mathrm{pCi} / \mathrm{g}$ & 0.0719 & EPIA-013B & \\
\hline SRF-07-02 & 117042 & $\mathrm{U}$ & -0.0236 & $\mathrm{pCi} / \mathrm{g}$ & 0.0782 & EPIA-013B & \\
\hline SRF-07-03 & 117043 & $\mathrm{U}$ & -0.00447 & $\mathrm{pCi} / \mathrm{g}$ & 0.0592 & EPIA-013B & \\
\hline SRF-07-03 & 117043 & $\mathrm{U}$ & 0.00507 & $\mathrm{pCi} / \mathrm{g}$ & 0.0852 & EPIA-013B & \\
\hline
\end{tabular}

ANALYTE: Strontium-89/90

Analytical Group: Radionuclides

\begin{tabular}{|c|c|c|c|c|c|c|c|}
\hline Survey ID & Sample ID & $\mathbf{F G}$ & Result & Unit & SSMDL & Method & SC \\
\hline SRF-01-01 & 117018 & $\mathrm{U}$ & 0.428 & $\mathrm{pCi} / \mathrm{g}$ & 0.631 & EPA905 & \\
\hline SRF-01-02 & 117019 & $\mathrm{U}$ & 0.168 & $\mathrm{pCi} / \mathrm{g}$ & 0.826 & EPA905 & \\
\hline SRF-01-03 & 117020 & $\mathrm{U}$ & 0.543 & $\mathrm{pCi} / \mathrm{g}$ & 0.545 & EPA905 & \\
\hline SRF-01-04 & 117021 & $\mathrm{U}$ & 0.532 & $\mathrm{pCi} / \mathrm{g}$ & 0.765 & EPA905 & \\
\hline SRF-01-05 & 117022 & $\mathrm{U}$ & 0.733 & $\mathrm{pCi} / \mathrm{g}$ & 1.15 & EPA905 & \\
\hline SRF-02-01 & 117023 & $\mathrm{U}$ & 0.037 & $\mathrm{pCi} / \mathrm{g}$ & 0.752 & EPA905 & \\
\hline SRF-02-02 & 117024 & $\mathrm{~J}$ & 0.49 & $\mathrm{pCi} / \mathrm{g}$ & 0.48 & EPA905 & I \\
\hline SRF-02-03 & 117025 & $\mathrm{U}$ & 0.427 & $\mathrm{pCi} / \mathrm{g}$ & 0.505 & EPA905 & \\
\hline SRF-02-03-A & 117026 & $\mathrm{U}$ & 0.391 & $\mathrm{pCi} / \mathrm{g}$ & 0.603 & EPA905 & \\
\hline SRF-03-01 & 117027 & $\mathrm{U}$ & 0.675 & $\mathrm{pCi} / \mathrm{g}$ & 0.868 & EPA905 & \\
\hline SRF-03-02 & 117028 & $\mathrm{~J}$ & 0.278 & $\mathrm{pCi} / \mathrm{g}$ & 0.221 & EPA905 & I \\
\hline SRF-03-03 & 117029 & $\mathrm{U}$ & 0.177 & $\mathrm{pCi} / \mathrm{g}$ & 0.198 & EPA905 & \\
\hline
\end{tabular}


ANALYTE: Strontium-89/90 (cont.)

$\begin{array}{lllrlrr}\text { Survey ID } & \text { Sample ID } & \text { FG } & \text { Result } & \text { Unit } & \text { ssMDL } & \text { Method } \\ & & & & & & \\ \text { SRF-03-04 } & 117030 & & 0.361 & \mathrm{pCi} / \mathrm{g} & 0.16 & \text { EPA905 } \\ \text { SRF-04-01 } & 117031 & & 1.81 & \mathrm{pCi} / \mathrm{g} & 0.0918 & \text { EPA905 } \\ \text { SRF-04-02 } & 117032 & & 0.643 & \mathrm{pCi} / \mathrm{g} & 0.15 & \text { EPA905 } \\ \text { SRF-04-03 } & 117033 & & 0.859 & \mathrm{pCi} / \mathrm{g} & 0.0874 & \text { EPA905 } \\ \text { SRF-05-01 } & 117034 & & 0.403 & \mathrm{pCi} / \mathrm{g} & 0.107 & \text { EPA905 } \\ \text { SRF-05-02 } & 117035 & & 0.553 & \mathrm{pCi} / \mathrm{g} & 0.148 & \text { EPA905 } \\ \text { SRF-05-02 } & 117035 & & 0.559 & \mathrm{pCi} / \mathrm{g} & 0.14 & \text { EPA905 } \\ \text { SRF-05-03 } & 117036 & & 0.315 & \mathrm{pCi} / \mathrm{g} & 0.119 & \text { EPA905 } \\ \text { SRF-06-01 } & 117037 & & 0.546 & \mathrm{pCi} / \mathrm{g} & 0.17 & \text { EPA905 } \\ \text { SRF-06-02 } & 117038 & & 0.553 & \mathrm{pCi} / \mathrm{g} & 0.203 & \text { EPA905 } \\ \text { SRF-06-03 } & 117039 & & 0.31 & \mathrm{pCi} / \mathrm{g} & 0.114 & \text { EPA905 } \\ \text { SRF-06-03-A } & 117040 & \mathrm{U} & 0.121 & \mathrm{pCi} / \mathrm{g} & 0.189 & \text { EPA905 } \\ \text { SRF-07-01 } & 117041 & \mathrm{U} & 0.288 & \mathrm{pCi} / \mathrm{g} & 0.537 & \text { EPA905 } \\ \text { SRF-07-02 } & 117042 & \mathrm{U} & 0.364 & \mathrm{pCi} / \mathrm{g} & 0.948 & \text { EPA905 } \\ \text { SRF-07-03 } & 117043 & \mathrm{U} & 0.365 & \mathrm{pCi} / \mathrm{g} & 0.433 & \text { EPA905 } \\ \text { SRF-07-03 } & 117043 & \mathrm{U} & 0.336 & \mathrm{pCi} / \mathrm{g} & 0.466 & \text { EPA905 }\end{array}$

ANALYTE: Styrene

Analytical Group: Volatile Organic Compounds

\begin{tabular}{|c|c|c|c|c|c|c|c|c|}
\hline Survey ID & Sample ID & FG & Result & Unit & ssMDL & Method & SC & $\mathbf{E}$ \\
\hline SRF-01-01 & 117018 & $\mathrm{U}$ & 5 & $\mu g / \mathrm{kg}$ & 1.5 & EPA8260B & & \\
\hline SRF-01-02 & 117019 & $\mathrm{U}$ & 5 & $\mu \mathrm{g} / \mathrm{kg}$ & 1.5 & EPA8260B & & \\
\hline SRF-01-03 & 117020 & $\mathrm{U}$ & 5 & $\mu \mathrm{g} / \mathrm{kg}$ & 1.5 & EPA8260B & & \\
\hline SRF-01-04 & 117021 & $\mathrm{U}$ & 5 & $\mu \mathrm{g} / \mathrm{kg}$ & 1.5 & EPA8260B & & \\
\hline SRF-01-05 & 117022 & $\mathrm{U}$ & 5 & $\mu \mathrm{g} / \mathrm{kg}$ & 1.5 & EPA8260B & & \\
\hline SRF-02-01 & 117023 & $\mathrm{U}$ & 5 & $\mu \mathrm{g} / \mathrm{kg}$ & 1.5 & EPA8260B & & \\
\hline SRF-02-02 & 117024 & $\mathrm{U}$ & 5 & $\mu \mathrm{g} / \mathrm{kg}$ & 1.5 & EPA8260B & & \\
\hline SRF-02-03 & 117025 & $\mathrm{U}$ & 5 & $\mu \mathrm{g} / \mathrm{kg}$ & 1.5 & EPA8260B & & \\
\hline SRF-02-03-A & 117026 & $\mathrm{U}$ & 5 & $\mu \mathrm{g} / \mathrm{kg}$ & 1.5 & EPA8260B & & \\
\hline SRF-03-01 & 117027 & $\mathrm{U}$ & 5 & $\mu \mathrm{g} / \mathrm{kg}$ & 1.5 & EPA8260B & & \\
\hline SRF-03-02 & 117028 & $\mathrm{U}$ & 5 & $\mu \mathrm{g} / \mathrm{kg}$ & 1.5 & EPA8260B & & \\
\hline SRF-03-03 & 117029 & $\mathrm{U}$ & 5 & $\mu \mathrm{g} / \mathrm{kg}$ & 1.5 & EPA8260B & & \\
\hline SRF-03-04 & 117030 & $\mathrm{U}$ & 5 & $\mu \mathrm{g} / \mathrm{kg}$ & 1.5 & EPA8260B & & \\
\hline SRF-04-01 & 117031 & $\mathrm{U}$ & 5 & $\mu \mathrm{g} / \mathrm{kg}$ & 1.5 & EPA8260B & & \\
\hline SRF-04-02 & 117032 & $\mathrm{U}$ & 5 & $\mu \mathrm{g} / \mathrm{kg}$ & 1.5 & EPA8260B & & \\
\hline SRF-04-03 & 117033 & $\mathrm{U}$ & 5 & $\mu \mathrm{g} / \mathrm{kg}$ & 1.5 & EPA8260B & & \\
\hline SRF-05-01 & 117034 & $\mathrm{U}$ & 5 & $\mu \mathrm{g} / \mathrm{kg}$ & 1.5 & EPA8260B & & \\
\hline SRF-05-02 & 117035 & $\mathrm{U}$ & 5 & $\mu \mathrm{g} / \mathrm{kg}$ & 1.5 & EPA8260B & & \\
\hline SRF-05-03 & 117036 & $\mathrm{U}$ & 5 & $\mu \mathrm{g} / \mathrm{kg}$ & 1.5 & EPA8260B & & \\
\hline SRF-06-01 & 117037 & $\mathrm{U}$ & 5 & $\mu \mathrm{g} / \mathrm{kg}$ & 1.5 & EPA8260B & & \\
\hline SRF-06-02 & 117038 & $\mathrm{U}$ & 5 & $\mu \mathrm{g} / \mathrm{kg}$ & 1.5 & EPA8260B & & \\
\hline SRF-06-03 & 117039 & $\mathrm{U}$ & 5 & $\mu \mathrm{g} / \mathrm{kg}$ & 1.5 & EPA8260B & & \\
\hline SRF-06-03-A & 117040 & $\mathrm{U}$ & 5 & $\mu \mathrm{g} / \mathrm{kg}$ & 1.5 & EPA8260B & & \\
\hline SRF-07-01 & 117041 & $\mathrm{U}$ & 5 & $\mu \mathrm{g} / \mathrm{kg}$ & 1.5 & EPA8260B & & \\
\hline SRF-07-02 & 117042 & $\mathrm{U}$ & 5 & $\mu \mathrm{g} / \mathrm{kg}$ & 1.5 & EPA8260B & & \\
\hline SRF-07-03 & 117043 & $\mathrm{U}$ & 5 & $\mu \mathrm{g} / \mathrm{kg}$ & 1.5 & EPA8260B & & \\
\hline \multicolumn{9}{|c|}{$\begin{array}{l}\text { ANALYTE: } 1,1,2,2 \text {-Tetrachloroethane } \\
\text { Analytical Group: Volatile Organic Compounds }\end{array}$} \\
\hline Survey ID & Sample ID & FG & Result & Unit & ssMDL & Method & sC & $\mathbf{E}$ \\
\hline SRF-01-01 & 117018 & $\mathrm{U}$ & 5 & $\mu g / \mathrm{kg}$ & 3 & ЕРA8260B & & \\
\hline SRF-01-02 & 117019 & $\mathrm{U}$ & 5 & $\mu \mathrm{g} / \mathrm{kg}$ & 3 & EPA8260B & & \\
\hline SRF-01-03 & 117020 & $\mathrm{U}$ & 5 & $\mu \mathrm{g} / \mathrm{kg}$ & 3 & EPA8260B & & \\
\hline SRF-01-04 & 117021 & $\mathrm{U}$ & 5 & $\mu \mathrm{g} / \mathrm{kg}$ & 3 & EPA8260B & & \\
\hline
\end{tabular}




$\begin{array}{lllllll}\text { SRF-01-05 } & 117022 & \mathrm{U} & 5 & \mu \mathrm{g} / \mathrm{kg} & 3 & \text { EPA8260B } \\ \text { SRF-02-01 } & 117023 & \mathrm{U} & 5 & \mu \mathrm{g} / \mathrm{kg} & 3 & \text { EPA8260B } \\ \text { SRF-02-02 } & 117024 & \mathrm{U} & 5 & \mu \mathrm{g} / \mathrm{kg} & 3 & \text { EPA8260B } \\ \text { SRF-02-03 } & 117025 & \mathrm{U} & 5 & \mu \mathrm{g} / \mathrm{kg} & 3 & \text { EPA8260B } \\ \text { SRF-02-03-A } & 117026 & \mathrm{U} & 5 & \mu \mathrm{g} / \mathrm{kg} & 3 & \text { EPA8260B } \\ \text { SRF-03-01 } & 117027 & \mathrm{U} & 5 & \mu \mathrm{g} / \mathrm{kg} & 3 & \text { EPA8260B } \\ \text { SRF-03-02 } & 117028 & \mathrm{U} & 5 & \mu \mathrm{g} / \mathrm{kg} & 3 & \text { EPA8260B } \\ \text { SRF-03-03 } & 117029 & \mathrm{U} & 5 & \mu \mathrm{g} / \mathrm{kg} & 3 & \text { EPA8260B } \\ \text { SRF-03-04 } & 117030 & \mathrm{U} & 5 & \mu \mathrm{g} / \mathrm{kg} & 3 & \text { EPA8260B } \\ \text { SRF-04-01 } & 117031 & \mathrm{U} & 5 & \mu \mathrm{g} / \mathrm{kg} & 3 & \text { EPA8260B } \\ \text { SRF-04-02 } & 117032 & \mathrm{U} & 5 & \mu \mathrm{g} / \mathrm{kg} & 3 & \text { EPA8260B } \\ \text { SRF-04-03 } & 117033 & \mathrm{U} & 5 & \mu \mathrm{g} / \mathrm{kg} & 3 & \text { EPA8260B } \\ \text { SRF-05-01 } & 117034 & \mathrm{U} & 5 & \mu \mathrm{g} / \mathrm{kg} & 3 & \text { EPA8260B } \\ \text { SRF-05-02 } & 117035 & \mathrm{U} & 5 & \mu \mathrm{g} / \mathrm{kg} & 3 & \text { EPA8260B } \\ \text { SRF-05-03 } & 117036 & \mathrm{U} & 5 & \mu \mathrm{g} / \mathrm{kg} & 3 & \text { EPA8260B } \\ \text { SRF-06-01 } & 117037 & \mathrm{U} & 5 & \mu \mathrm{g} / \mathrm{kg} & 3 & \text { EPA8260B } \\ \text { SRF-06-02 } & 117038 & \mathrm{U} & 5 & \mu \mathrm{g} / \mathrm{kg} & 3 & \text { EPA8260B } \\ \text { SRF-06-03 } & 117039 & \mathrm{U} & 5 & \mu \mathrm{g} / \mathrm{kg} & 3 & \text { EPA8260B } \\ \text { SRF-06-03-A } & 117040 & \mathrm{U} & 5 & \mu \mathrm{g} / \mathrm{kg} & 3 & \text { EPA8260B } \\ \text { SRF-07-01 } & 117041 & \mathrm{U} & 5 & \mu \mathrm{g} / \mathrm{kg} & 3 & \text { EPA8260B } \\ \text { SRF-07-02 } & 117042 & \mathrm{U} & 5 & \mu \mathrm{g} / \mathrm{kg} & 3 & \text { EPA8260B } \\ \text { SRF-07-03 } & 117043 & \mathrm{U} & 5 & \mu \mathrm{g} / \mathrm{kg} & & \\ & & & & \end{array}$

ANALYTE: Tetrachloroethylene

Analytical Group: Volatile Organic Compounds

\begin{tabular}{|c|c|c|c|c|c|c|c|}
\hline Survey ID & Sample ID & $\mathbf{F G}$ & Result & Unit & SsMDL & Method & SC \\
\hline SRF-01-01 & 117018 & $\mathrm{U}$ & 5 & $\mu g / \mathrm{kg}$ & 2 & EPA8260B & \\
\hline SRF-01-02 & 117019 & $\mathrm{U}$ & 5 & $\mu g / k g$ & 2 & EPA8260B & \\
\hline SRF-01-03 & 117020 & $\mathrm{U}$ & 5 & $\mu g / \mathrm{kg}$ & 2 & EPA8260B & \\
\hline SRF-01-04 & 117021 & $\mathrm{U}$ & 5 & $\mu g / k g$ & 2 & EPA8260B & \\
\hline SRF-01-05 & 117022 & $\mathrm{U}$ & 5 & $\mu \mathrm{g} / \mathrm{kg}$ & 2 & EPA8260B & \\
\hline SRF-02-01 & 117023 & $\mathrm{U}$ & 5 & $\mu g / k g$ & 2 & EPA8260B & \\
\hline SRF-02-02 & 117024 & $\mathrm{U}$ & 5 & $\mu g / \mathrm{kg}$ & 2 & EPA8260B & \\
\hline SRF-02-03 & 117025 & $\mathrm{U}$ & 5 & $\mu g / k g$ & 2 & EPA8260B & \\
\hline SRF-02-03-A & 117026 & $\mathrm{U}$ & 5 & $\mu \mathrm{g} / \mathrm{kg}$ & 2 & EPA8260B & \\
\hline SRF-03-01 & 117027 & $\mathrm{U}$ & 5 & $\mu \mathrm{g} / \mathrm{kg}$ & 2 & EPA8260B & \\
\hline SRF-03-02 & 117028 & $\mathrm{U}$ & 5 & $\mu g / k g$ & 2 & EPA8260B & \\
\hline SRF-03-03 & 117029 & $\mathrm{U}$ & 5 & $\mu \mathrm{g} / \mathrm{kg}$ & 2 & EPA8260B & \\
\hline SRF-03-04 & 117030 & $\mathrm{U}$ & 5 & $\mu g / \mathrm{kg}$ & 2 & EPA8260B & \\
\hline SRF-04-01 & 117031 & $\mathrm{U}$ & 5 & $\mu \mathrm{g} / \mathrm{kg}$ & 2 & EPA8260B & \\
\hline SRF-04-02 & 117032 & $\mathrm{U}$ & 5 & $\mu g / \mathrm{kg}$ & 2 & EPA8260B & \\
\hline SRF-04-03 & 117033 & $\mathrm{U}$ & 5 & $\mu g / \mathrm{kg}$ & 2 & EPA8260B & \\
\hline SRF-05-01 & 117034 & $\mathrm{U}$ & 5 & $\mu g / k g$ & 2 & EPA8260B & \\
\hline SRF-05-02 & 117035 & $\mathrm{U}$ & 5 & $\mu \mathrm{g} / \mathrm{kg}$ & 2 & EPA8260B & \\
\hline SRF-05-03 & 117036 & $\mathrm{U}$ & 5 & $\mu \mathrm{g} / \mathrm{kg}$ & 2 & EPA8260B & \\
\hline SRF-06-01 & 117037 & $\mathrm{U}$ & 5 & $\mu g / \mathrm{kg}$ & 2 & EPA8260B & \\
\hline SRF-06-02 & 117038 & $\mathrm{U}$ & 5 & $\mu \mathrm{g} / \mathrm{kg}$ & 2 & EPA8260B & \\
\hline SRF-06-03 & 117039 & $\mathrm{U}$ & 5 & $\mu \mathrm{g} / \mathrm{kg}$ & 2 & EPA8260B & \\
\hline SRF-06-03-A & 117040 & $\mathrm{U}$ & 5 & $\mu \mathrm{g} / \mathrm{kg}$ & 2 & EPA8260B & \\
\hline \multicolumn{8}{|c|}{ ANALYTE: Tetrachloroethylene (cont.) } \\
\hline Survey ID & Sample ID & $\mathbf{F G}$ & Result & Unit & SsMDL & Method & SC \\
\hline SRF-07-01 & 117041 & $\mathrm{U}$ & 5 & $\mu g / \mathrm{kg}$ & 2 & EPA8260B & \\
\hline SRF-07-02 & 117042 & $\mathrm{U}$ & 5 & $\mu \mathrm{g} / \mathrm{kg}$ & 2 & EPA8260B & \\
\hline SRF-07-03 & 117043 & $\mathrm{U}$ & 5 & $\mu g / \mathrm{kg}$ & 2 & EPA8260B & \\
\hline
\end{tabular}


ANALYTE: Tin-113

Analytical Group: Radionuclides

\begin{tabular}{|c|c|c|c|c|c|c|c|}
\hline Survey ID & Sample ID & FG & Result & Unit & ssMDL & Method & SC \\
\hline SRF-01-01 & 117018 & $\mathrm{U}$ & -0.0116 & $\mathrm{pCi} / \mathrm{g}$ & 0.0698 & EPIA-013B & \\
\hline SRF-01-02 & 117019 & $\mathrm{U}$ & 0.0347 & $\mathrm{pCi} / \mathrm{g}$ & 0.06 & EPIA-013B & \\
\hline SRF-01-03 & 117020 & $\mathrm{U}$ & 0.0118 & $\mathrm{pCi} / \mathrm{g}$ & 0.0694 & EPIA-013B & \\
\hline SRF-01-04 & 117021 & $\mathrm{U}$ & -0.0225 & $\mathrm{pCi} / \mathrm{g}$ & 0.0512 & EPIA-013B & \\
\hline SRF-01-05 & 117022 & $\mathrm{U}$ & -0.00129 & $\mathrm{pCi} / \mathrm{g}$ & 0.0504 & EPIA-013B & \\
\hline SRF-01-05 & 117022 & $\mathrm{U}$ & -0.0245 & $\mathrm{pCi} / \mathrm{g}$ & 0.0483 & EPIA-013B & \\
\hline SRF-02-01 & 117023 & $\mathrm{U}$ & 0.0263 & $\mathrm{pCi} / \mathrm{g}$ & 0.0764 & EPIA-013B & \\
\hline SRF-02-02 & 117024 & $\mathrm{U}$ & -0.00762 & $\mathrm{pCi} / \mathrm{g}$ & 0.0537 & EPIA-013B & \\
\hline SRF-02-03 & 117025 & $\mathrm{U}$ & 0.0399 & $\mathrm{pCi} / \mathrm{g}$ & 0.097 & EPIA-013B & \\
\hline SRF-02-03-A & 117026 & $\mathrm{U}$ & 0.00248 & $\mathrm{pCi} / \mathrm{g}$ & 0.0845 & EPIA-013B & \\
\hline SRF-03-01 & 117027 & $\mathrm{U}$ & -0.0209 & $\mathrm{pCi} / \mathrm{g}$ & 0.0729 & EPIA-013B & \\
\hline SRF-03-02 & 117028 & $\mathrm{U}$ & -0.00207 & $\mathrm{pCi} / \mathrm{g}$ & 0.0579 & EPIA-013B & \\
\hline SRF-03-03 & 117029 & $\mathrm{U}$ & 0.0191 & $\mathrm{pCi} / \mathrm{g}$ & 0.0637 & EPIA-013B & \\
\hline SRF-03-04 & 117030 & $\mathrm{U}$ & -0.0143 & $\mathrm{pCi} / \mathrm{g}$ & 0.0372 & EPIA-013B & \\
\hline SRF-04-01 & 117031 & $\mathrm{U}$ & 0.016 & $\mathrm{pCi} / \mathrm{g}$ & 0.0645 & EPIA-013B & \\
\hline SRF-04-02 & 117032 & $\mathrm{U}$ & 0.0729 & $\mathrm{pCi} / \mathrm{g}$ & 0.0979 & EPIA-013B & \\
\hline SRF-04-03 & 117033 & $\mathrm{U}$ & -0.0179 & $\mathrm{pCi} / \mathrm{g}$ & 0.0713 & EPIA-013B & \\
\hline SRF-05-01 & 117034 & $\mathrm{U}$ & 0.0147 & $\mathrm{pCi} / \mathrm{g}$ & 0.0605 & EPIA-013B & \\
\hline SRF-05-02 & 117035 & $\mathrm{U}$ & 0.013 & $\mathrm{pCi} / \mathrm{g}$ & 0.0605 & EPIA-013B & \\
\hline SRF-05-03 & 117036 & $\mathrm{U}$ & -0.00556 & $\mathrm{pCi} / \mathrm{g}$ & 0.0637 & EPIA-013B & \\
\hline SRF-06-01 & 117037 & $\mathrm{U}$ & 0.00509 & $\mathrm{pCi} / \mathrm{g}$ & 0.0772 & EPIA-013B & \\
\hline SRF-06-02 & 117038 & $\mathrm{U}$ & -0.00714 & $\mathrm{pCi} / \mathrm{g}$ & 0.0529 & EPIA-013B & \\
\hline SRF-06-03 & 117039 & $\mathrm{U}$ & -0.00834 & $\mathrm{pCi} / \mathrm{g}$ & 0.0903 & EPIA-013B & \\
\hline SRF-06-03-A & 117040 & $\mathrm{U}$ & 0.0255 & $\mathrm{pCi} / \mathrm{g}$ & 0.0865 & EPIA-013B & \\
\hline SRF-07-01 & 117041 & $\mathrm{U}$ & -0.0335 & $\mathrm{pCi} / \mathrm{g}$ & 0.0777 & EPIA-013B & \\
\hline SRF-07-02 & 117042 & $\mathrm{U}$ & 0.0498 & $\mathrm{pCi} / \mathrm{g}$ & 0.101 & EPIA-013B & \\
\hline SRF-07-03 & 117043 & $\mathrm{U}$ & 0.0115 & $\mathrm{pCi} / \mathrm{g}$ & 0.0639 & EPIA-013B & \\
\hline SRF-07-03 & 117043 & $\mathrm{U}$ & -0.0125 & $\mathrm{pCi} / \mathrm{g}$ & 0.0884 & EPIA-013B & \\
\hline
\end{tabular}

ANALYTE: Toluene

Analytical Group: Volatile Organic Compounds

\begin{tabular}{|c|c|c|c|c|c|c|c|c|}
\hline Survey ID & Sample ID & FG & Result & Unit & SSMDL & Method & SC & $\mathbf{E}$ \\
\hline SRF-01-01 & 117018 & $\mathrm{~J}$ & 11.3 & $\mu \mathrm{g} / \mathrm{kg}$ & 4.5 & EPA8260B & $\mathrm{K}$ & $\mathrm{O}$ \\
\hline SRF-01-02 & 117019 & $\mathrm{~J}$ & 7.11 & $\mu \mathrm{g} / \mathrm{kg}$ & 4.5 & EPA8260B & $\mathrm{K}$ & $\mathrm{O}$ \\
\hline SRF-01-03 & 117020 & $\mathrm{U}$ & 5 & $\mu g / k g$ & 4.5 & EPA8260B & & \\
\hline SRF-01-04 & 117021 & $\mathrm{U}$ & 5 & $\mu \mathrm{g} / \mathrm{kg}$ & 4.5 & EPA8260B & & \\
\hline SRF-01-05 & 117022 & $\mathrm{~J}$ & 80.2 & $\mu g / k g$ & 4.5 & EPA8260B & $\mathrm{K}$ & $\mathrm{O}$ \\
\hline SRF-02-01 & 117023 & $\mathrm{U}$ & 5 & $\mu \mathrm{g} / \mathrm{kg}$ & 4.5 & EPA8260B & & \\
\hline SRF-02-02 & 117024 & $\mathrm{~J}$ & 5.4 & $\mu \mathrm{g} / \mathrm{kg}$ & 4.5 & EPA8260B & $\mathrm{K}$ & $\mathrm{O}$ \\
\hline SRF-02-03 & 117025 & $\mathrm{U}$ & 5 & $\mu \mathrm{g} / \mathrm{kg}$ & 4.5 & EPA8260B & & \\
\hline SRF-02-03-A & 117026 & $\mathrm{U}$ & 5 & $\mu \mathrm{g} / \mathrm{kg}$ & 4.5 & EPA8260B & & \\
\hline SRF-03-01 & 117027 & $\mathrm{U}$ & 5 & $\mu g / \mathrm{kg}$ & 4.5 & EPA8260B & & \\
\hline SRF-03-02 & 117028 & & 6.11 & $\mu \mathrm{g} / \mathrm{kg}$ & 4.5 & EPA8260B & & \\
\hline SRF-03-03 & 117029 & & 5.14 & $\mu g / \mathrm{kg}$ & 4.5 & EPA8260B & & \\
\hline \multicolumn{9}{|c|}{ ANALYTE: Toluene (cont.) } \\
\hline Survey ID & Sample ID & FG & Result & Unit & SSMDL & Method & SC & $\mathbf{E}$ \\
\hline SRF-03-04 & 117030 & & 9.57 & $\mu g / \mathrm{kg}$ & 4.5 & EPA8260B & & \\
\hline SRF-04-01 & 117031 & $\mathrm{~J}$ & 5.69 & $\mu \mathrm{g} / \mathrm{kg}$ & 4.5 & EPA8260B & $\mathrm{K}$ & $\mathrm{O}$ \\
\hline SRF-04-02 & 117032 & $\mathrm{U}$ & 5 & $\mu g / \mathrm{kg}$ & 4.5 & EPA8260B & & \\
\hline SRF-04-03 & 117033 & $\mathrm{U}$ & 5 & $\mu \mathrm{g} / \mathrm{kg}$ & 4.5 & EPA8260B & & \\
\hline SRF-05-01 & 117034 & $\mathrm{U}$ & 5 & $\mu g / \mathrm{kg}$ & 4.5 & EPA8260B & & \\
\hline SRF-05-02 & 117035 & $\mathrm{U}$ & 5 & $\mu \mathrm{g} / \mathrm{kg}$ & 4.5 & EPA8260B & & \\
\hline SRF-05-03 & 117036 & $\mathrm{U}$ & 5 & $\mu \mathrm{g} / \mathrm{kg}$ & 4.5 & EPA8260B & & \\
\hline
\end{tabular}




\begin{tabular}{|c|c|c|c|c|c|c|c|c|}
\hline SRF-06-01 & 117037 & $\mathrm{~J}$ & 17.4 & $\mu g / \mathrm{kg}$ & 4.5 & EPA8260B & $\mathrm{K}$ & $\mathrm{O}$ \\
\hline SRF-06-02 & 117038 & $\mathrm{U}$ & 5 & $\mu \mathrm{g} / \mathrm{kg}$ & 4.5 & EPA8260B & & \\
\hline SRF-06-03 & 117039 & $\mathrm{U}$ & 5 & $\mu \mathrm{g} / \mathrm{kg}$ & 4.5 & EPA8260B & & \\
\hline SRF-06-03-A & 117040 & & 18.3 & $\mu \mathrm{g} / \mathrm{kg}$ & 4.5 & EPA8260B & & \\
\hline SRF-07-01 & 117041 & $\mathrm{U}$ & 5 & $\mu \mathrm{g} / \mathrm{kg}$ & 4.5 & EPA8260B & & \\
\hline SRF-07-02 & 117042 & $\mathrm{U}$ & 5 & $\mu \mathrm{g} / \mathrm{kg}$ & 4.5 & EPA8260B & & \\
\hline SRF-07-03 & 117043 & $\mathrm{U}$ & 5 & $\mu \mathrm{g} / \mathrm{kg}$ & 4.5 & EPA8260B & & \\
\hline
\end{tabular}

ANALYTE: 1,1,1-Trichloroethane

Analytical Group: Volatile Organic Compounds

\begin{tabular}{|c|c|c|c|c|c|c|c|}
\hline Survey ID & Sample ID & $\mathbf{F G}$ & Result & Unit & SSMDL & Method & SC \\
\hline SRF-01-01 & 117018 & $\mathrm{U}$ & 5 & $\mu g / \mathrm{kg}$ & 0.5 & EPA8260B & \\
\hline SRF-01-02 & 117019 & $\mathrm{U}$ & 5 & $\mu \mathrm{g} / \mathrm{kg}$ & 0.5 & EPA8260B & \\
\hline SRF-01-03 & 117020 & $\mathrm{U}$ & 5 & $\mu g / k g$ & 0.5 & EPA8260B & \\
\hline SRF-01-04 & 117021 & $\mathrm{U}$ & 5 & $\mu \mathrm{g} / \mathrm{kg}$ & 0.5 & EPA8260B & \\
\hline SRF-01-05 & 117022 & $\mathrm{U}$ & 5 & $\mu g / k g$ & 0.5 & EPA8260B & \\
\hline SRF-02-01 & 117023 & $\mathrm{U}$ & 5 & $\mu \mathrm{g} / \mathrm{kg}$ & 0.5 & EPA8260B & \\
\hline SRF-02-02 & 117024 & $\mathrm{U}$ & 5 & $\mu g / k g$ & 0.5 & EPA8260B & \\
\hline SRF-02-03 & 117025 & $\mathrm{U}$ & 5 & $\mu g / \mathrm{kg}$ & 0.5 & EPA8260B & \\
\hline SRF-02-03-A & 117026 & $\mathrm{U}$ & 5 & $\mu g / k g$ & 0.5 & EPA8260B & \\
\hline SRF-03-01 & 117027 & $\mathrm{U}$ & 5 & $\mu g / \mathrm{kg}$ & 0.5 & EPA8260B & \\
\hline SRF-03-02 & 117028 & $\mathrm{U}$ & 5 & $\mu \mathrm{g} / \mathrm{kg}$ & 0.5 & EPA8260B & \\
\hline SRF-03-03 & 117029 & $\mathrm{U}$ & 5 & $\mu g / \mathrm{kg}$ & 0.5 & EPA8260B & \\
\hline SRF-03-04 & 117030 & $\mathrm{U}$ & 5 & $\mu \mathrm{g} / \mathrm{kg}$ & 0.5 & EPA8260B & \\
\hline SRF-04-01 & 117031 & $\mathrm{U}$ & 5 & $\mu g / k g$ & 0.5 & EPA8260B & \\
\hline SRF-04-02 & 117032 & $\mathrm{U}$ & 5 & $\mu g / \mathrm{kg}$ & 0.5 & EPA8260B & \\
\hline SRF-04-03 & 117033 & $\mathrm{U}$ & 5 & $\mu \mathrm{g} / \mathrm{kg}$ & 0.5 & EPA8260B & \\
\hline SRF-05-01 & 117034 & $\mathrm{U}$ & 5 & $\mu g / k g$ & 0.5 & EPA8260B & \\
\hline SRF-05-02 & 117035 & $\mathrm{U}$ & 5 & $\mu \mathrm{g} / \mathrm{kg}$ & 0.5 & EPA8260B & \\
\hline SRF-05-03 & 117036 & $\mathrm{U}$ & 5 & $\mu g / \mathrm{kg}$ & 0.5 & EPA8260B & \\
\hline SRF-06-01 & 117037 & $\mathrm{U}$ & 5 & $\mu \mathrm{g} / \mathrm{kg}$ & 0.5 & EPA8260B & \\
\hline SRF-06-02 & 117038 & $\mathrm{U}$ & 5 & $\mu \mathrm{g} / \mathrm{kg}$ & 0.5 & EPA8260B & \\
\hline SRF-06-03 & 117039 & $\mathrm{U}$ & 5 & $\mu \mathrm{g} / \mathrm{kg}$ & 0.5 & EPA8260B & \\
\hline SRF-06-03-A & 117040 & $\mathrm{U}$ & 5 & $\mu g / \mathrm{kg}$ & 0.5 & EPA8260B & \\
\hline SRF-07-01 & 117041 & $\mathrm{U}$ & 5 & $\mu \mathrm{g} / \mathrm{kg}$ & 0.5 & EPA8260B & \\
\hline SRF-07-02 & 117042 & $\mathrm{U}$ & 5 & $\mu \mathrm{g} / \mathrm{kg}$ & 0.5 & EPA8260B & \\
\hline SRF-07-03 & 117043 & $\mathrm{U}$ & 5 & $\mu \mathrm{g} / \mathrm{kg}$ & 0.5 & EPA8260B & \\
\hline
\end{tabular}

ANALYTE: 1,1,2-Trichloroethane

Analytical Group: Volatile Organic Compounds

\begin{tabular}{|c|c|c|c|c|c|c|c|c|}
\hline Survey ID & Sample ID & FG & Result & Unit & SSMDL & Method & SC & $\mathbf{E}$ \\
\hline SRF-01-01 & 117018 & $\mathrm{U}$ & 5 & $\mu g / \mathrm{kg}$ & 1.2 & EPA8260B & & \\
\hline SRF-01-02 & 117019 & $\mathrm{U}$ & 5 & $\mu g / \mathrm{kg}$ & 1.2 & EPA8260B & & \\
\hline SRF-01-03 & 117020 & $\mathrm{U}$ & 5 & $\mu \mathrm{g} / \mathrm{kg}$ & 1.2 & EPA8260B & & \\
\hline \multicolumn{9}{|c|}{ ANALYTE: $1,1,2$-Trichloroethane (cont.) } \\
\hline Survey ID & Sample ID & FG & Result & Unit & SSMDL & Method & SC & $\mathbf{E}$ \\
\hline SRF-01-04 & 117021 & $\mathrm{U}$ & 5 & $\mu g / k g$ & 1.2 & EPA8260B & & \\
\hline SRF-01-05 & 117022 & $\mathrm{U}$ & 5 & $\mu g / \mathrm{kg}$ & 1.2 & EPA8260B & & \\
\hline SRF-02-01 & 117023 & $\mathrm{U}$ & 5 & $\mu g / \mathrm{kg}$ & 1.2 & EPA8260B & & \\
\hline SRF-02-02 & 117024 & $\mathrm{U}$ & 5 & $\mu \mathrm{g} / \mathrm{kg}$ & 1.2 & EPA8260B & & \\
\hline SRF-02-03 & 117025 & $\mathrm{U}$ & 5 & $\mu g / \mathrm{kg}$ & 1.2 & EPA8260B & & \\
\hline SRF-02-03-A & 117026 & $\mathrm{U}$ & 5 & $\mu g / k g$ & 1.2 & EPA8260B & & \\
\hline SRF-03-01 & 117027 & $\mathrm{U}$ & 5 & $\mu \mathrm{g} / \mathrm{kg}$ & 1.2 & EPA8260B & & \\
\hline SRF-03-02 & 117028 & $\mathrm{U}$ & 5 & $\mu \mathrm{g} / \mathrm{kg}$ & 1.2 & EPA8260B & & \\
\hline SRF-03-03 & 117029 & $\mathrm{U}$ & 5 & $\mu \mathrm{g} / \mathrm{kg}$ & 1.2 & EPA8260B & & \\
\hline
\end{tabular}




\begin{tabular}{lllllll}
\hline SRF-03-04 & 117030 & $\mathrm{U}$ & 5 & $\mu \mathrm{g} / \mathrm{kg}$ & 1.2 & EPA8260B \\
SRF-04-01 & 117031 & $\mathrm{U}$ & 5 & $\mu \mathrm{g} / \mathrm{kg}$ & 1.2 & EPA8260B \\
SRF-04-02 & 117032 & $\mathrm{U}$ & 5 & $\mu \mathrm{g} / \mathrm{kg}$ & 1.2 & EPA8260B \\
SRF-04-03 & 117033 & $\mathrm{U}$ & 5 & $\mu \mathrm{g} / \mathrm{kg}$ & 1.2 & EPA8260B \\
SRF-05-01 & 117034 & $\mathrm{U}$ & 5 & $\mu \mathrm{g} / \mathrm{kg}$ & 1.2 & EPA8260B \\
SRF-05-02 & 117035 & $\mathrm{U}$ & 5 & $\mu \mathrm{g} / \mathrm{kg}$ & 1.2 & EPA8260B \\
SRF-05-03 & 117036 & $\mathrm{U}$ & 5 & $\mu \mathrm{g} / \mathrm{kg}$ & 1.2 & EPA8260B \\
SRF-06-01 & 117037 & $\mathrm{U}$ & 5 & $\mu \mathrm{g} / \mathrm{kg}$ & 1.2 & EPA8260B \\
SRF-06-02 & 117038 & $\mathrm{U}$ & 5 & $\mu \mathrm{g} / \mathrm{kg}$ & 1.2 & EPA8260B \\
SRF-06-03 & 117039 & $\mathrm{U}$ & 5 & $\mu \mathrm{g} / \mathrm{kg}$ & 1.2 & EPA8260B \\
SRF-06-03-A & 117040 & $\mathrm{U}$ & 5 & $\mu \mathrm{g} / \mathrm{kg}$ & 1.2 & EPA8260B \\
SRF-07-01 & 117041 & $\mathrm{U}$ & 5 & $\mu \mathrm{g} / \mathrm{kg}$ & 1.2 & EPA8260B \\
SRF-07-02 & 117042 & $\mathrm{U}$ & 5 & $\mu \mathrm{g} / \mathrm{kg}$ & 1.2 & EPA8260B \\
SRF-07-03 & 117043 & $\mathrm{U}$ & 5 & $\mu \mathrm{g} / \mathrm{kg}$ & 1.2 & EPA8260B
\end{tabular}

ANALYTE: Trichloroethylene

Analytical Group: Volatile Organic Compounds

\begin{tabular}{|c|c|c|c|c|c|c|c|c|}
\hline Survey ID & Sample ID & FG & Result & Unit & SSMDL & Method & SC & $\mathbf{E}$ \\
\hline SRF-01-01 & 117018 & $\mathrm{U}$ & 5 & $\mu \mathrm{g} / \mathrm{kg}$ & 1.35 & EPA8260B & & \\
\hline SRF-01-02 & 117019 & $\mathrm{U}$ & 5 & $\mu g / \mathrm{kg}$ & 1.35 & EPA8260B & & \\
\hline SRF-01-03 & 117020 & U & 5 & $\mu g / \mathrm{kg}$ & 1.35 & EPA8260B & & \\
\hline SRF-01-04 & 117021 & $\mathrm{U}$ & 5 & $\mu g / \mathrm{kg}$ & 1.35 & EPA8260B & & \\
\hline SRF-01-05 & 117022 & U & 5 & $\mu \mathrm{g} / \mathrm{kg}$ & 1.35 & EPA8260B & & \\
\hline SRF-02-01 & 117023 & $\mathrm{U}$ & 5 & $\mu g / \mathrm{kg}$ & 1.35 & EPA8260B & & \\
\hline SRF-02-02 & 117024 & $\mathrm{U}$ & 5 & $\mu \mathrm{g} / \mathrm{kg}$ & 1.35 & EPA8260B & & \\
\hline SRF-02-03 & 117025 & $\mathrm{U}$ & 5 & $\mu g / \mathrm{kg}$ & 1.35 & EPA8260B & & \\
\hline SRF-02-03-A & 117026 & $\mathrm{U}$ & 5 & $\mu g / \mathrm{kg}$ & 1.35 & EPA8260B & & \\
\hline SRF-03-01 & 117027 & $\mathrm{U}$ & 5 & $\mu g / \mathrm{kg}$ & 1.35 & EPA8260B & & \\
\hline SRF-03-02 & 117028 & $\mathrm{U}$ & 5 & $\mu g / \mathrm{kg}$ & 1.35 & EPA8260B & & \\
\hline SRF-03-03 & 117029 & $\mathrm{U}$ & 5 & $\mu g / \mathrm{kg}$ & 1.35 & EPA8260B & & \\
\hline SRF-03-04 & 117030 & U & 5 & $\mu g / \mathrm{kg}$ & 1.35 & EPA8260B & & \\
\hline SRF-04-01 & 117031 & U & 5 & $\mu g / \mathrm{kg}$ & 1.35 & EPA8260B & & \\
\hline SRF-04-02 & 117032 & $\mathrm{U}$ & 5 & $\mu \mathrm{g} / \mathrm{kg}$ & 1.35 & EPA8260B & & \\
\hline SRF-04-03 & 117033 & U & 5 & $\mu g / \mathrm{kg}$ & 1.35 & EPA8260B & & \\
\hline SRF-05-01 & 117034 & $\mathrm{U}$ & 5 & $\mu \mathrm{g} / \mathrm{kg}$ & 1.35 & EPA8260B & & \\
\hline SRF-05-02 & 117035 & $\mathrm{U}$ & 5 & $\mu \mathrm{g} / \mathrm{kg}$ & 1.35 & EPA8260B & & \\
\hline SRF-05-03 & 117036 & $\mathrm{U}$ & 5 & $\mu g / \mathrm{kg}$ & 1.35 & EPA8260B & & \\
\hline SRF-06-01 & 117037 & U & 5 & $\mu \mathrm{g} / \mathrm{kg}$ & 1.35 & EPA8260B & & \\
\hline SRF-06-02 & 117038 & U & 5 & $\mu g / \mathrm{kg}$ & 1.35 & EPA8260B & & \\
\hline SRF-06-03 & 117039 & U & 5 & $\mu \mathrm{g} / \mathrm{kg}$ & 1.35 & EPA8260B & & \\
\hline SRF-06-03-A & 117040 & $\mathrm{U}$ & 5 & $\mu g / \mathrm{kg}$ & 1.35 & EPA8260B & & \\
\hline SRF-07-01 & 117041 & U & 5 & $\mu g / \mathrm{kg}$ & 1.35 & EPA8260B & & \\
\hline SRF-07-02 & 117042 & $\mathrm{U}$ & 5 & $\mu g / \mathrm{kg}$ & 1.35 & EPA8260B & & \\
\hline SRF-07-03 & 117043 & $\mathrm{U}$ & 5 & $\mu \mathrm{g} / \mathrm{kg}$ & 1.35 & EPA8260B & & \\
\hline \multicolumn{9}{|c|}{$\begin{array}{l}\text { ANALYTE: Tritium } \\
\text { Analytical Group: Radionuclides }\end{array}$} \\
\hline Survey ID & Sample ID & FG & Result & Unit & ssMDL & Method & SC & $\mathbf{E}$ \\
\hline SRF-01-01 & 117018 & $\mathrm{U}$ & 0 & $\mathrm{pCi} / \mathrm{g}$ & 0.157 & EPIA-002B & & \\
\hline SRF-01-02 & 117019 & $\mathrm{U}$ & 0.0751 & $\mathrm{pCi} / \mathrm{g}$ & 0.171 & EPIA-002B & & \\
\hline SRF-01-03 & 117020 & U & 0.0205 & $\mathrm{pCi} / \mathrm{g}$ & 0.132 & EPIA-002B & & \\
\hline SRF-01-04 & 117021 & $\mathrm{U}$ & 0.0632 & $\mathrm{pCi} / \mathrm{g}$ & 0.144 & EPIA-002B & & \\
\hline SRF-01-04 & 117021 & U & 0.0155 & $\mathrm{pCi} / \mathrm{g}$ & 0.142 & EPIA-002B & & \\
\hline SRF-01-05 & 117022 & $\mathrm{U}$ & -0.0433 & $\mathrm{pCi} / \mathrm{g}$ & 0.214 & EPIA-002B & & \\
\hline SRF-02-01 & 117023 & $\mathrm{U}$ & -0.0229 & $\mathrm{pCi} / \mathrm{g}$ & 0.0984 & EPIA-002B & & \\
\hline SRF-02-02 & 117024 & $\mathrm{U}$ & -0.0264 & $\mathrm{pCi} / \mathrm{g}$ & 0.107 & EPIA-002B & & \\
\hline SRF-02-03 & 117025 & U & -0.0238 & $\mathrm{pCi} / \mathrm{g}$ & 0.162 & EPIA-002B & & \\
\hline SRF-02-03-A & 117026 & U & -0.0247 & $\mathrm{pCi} / \mathrm{g}$ & 0.13 & EPIA-002B & & \\
\hline
\end{tabular}




\begin{tabular}{|c|c|c|c|c|c|c|}
\hline SRF-03-01 & 117027 & $\mathrm{U}$ & 0 & $\mathrm{pCi} / \mathrm{g}$ & 0.144 & EPIA-002B \\
\hline SRF-03-01 & 117027 & $\mathrm{U}$ & -0.01 & $\mathrm{pCi} / \mathrm{g}$ & 0.137 & EPIA-002B \\
\hline SRF-03-02 & 117028 & $\mathrm{U}$ & 0.0312 & $\mathrm{pCi} / \mathrm{g}$ & 0.145 & EPIA-002B \\
\hline SRF-03-03 & 117029 & $\mathrm{U}$ & -0.0231 & $\mathrm{pCi} / \mathrm{g}$ & 0.179 & EPIA-002B \\
\hline SRF-03-04 & 117030 & $\mathrm{U}$ & 0.151 & $\mathrm{pCi} / \mathrm{g}$ & 0.175 & EPIA-002B \\
\hline SRF-04-01 & 117031 & & 4.07 & $\mathrm{pCi} / \mathrm{g}$ & 0.127 & EPIA-002B \\
\hline SRF-04-02 & 117032 & & 1.31 & $\mathrm{pCi} / \mathrm{g}$ & 0.155 & EPIA-002B \\
\hline SRF-04-03 & 117033 & $\mathrm{U}$ & 0.105 & $\mathrm{pCi} / \mathrm{g}$ & 0.116 & EPIA-002B \\
\hline SRF-05-01 & 117034 & & 3.32 & $\mathrm{pCi} / \mathrm{g}$ & 0.127 & EPIA-002B \\
\hline SRF-05-02 & 117035 & & 0.425 & $\mathrm{pCi} / \mathrm{g}$ & 0.108 & EPIA-002B \\
\hline SRF-05-03 & 117036 & & 1.28 & $\mathrm{pCi} / \mathrm{g}$ & 0.112 & EPIA-002B \\
\hline SRF-06-01 & 117037 & $\mathrm{~J}$ & 0.151 & $\mathrm{pCi} / \mathrm{g}$ & 0.12 & EPIA-002B \\
\hline SRF-06-02 & 117038 & $\mathrm{~J}$ & 0.262 & $\mathrm{pCi} / \mathrm{g}$ & 0.146 & EPIA-002B \\
\hline SRF-06-03 & 117039 & $\mathrm{~J}$ & 0.13 & $\mathrm{pCi} / \mathrm{g}$ & 0.0974 & EPIA-002B \\
\hline SRF-06-03-A & 117040 & $\mathrm{~J}$ & 0.178 & $\mathrm{pCi} / \mathrm{g}$ & 0.103 & EPIA-002B \\
\hline SRF-07-01 & 117041 & $\mathrm{U}$ & 0.0187 & $\mathrm{pCi} / \mathrm{g}$ & 0.17 & EPIA-002B \\
\hline SRF-07-02 & 117042 & $\mathrm{~J}$ & 0.229 & $\mathrm{pCi} / \mathrm{g}$ & 0.19 & EPIA-002B \\
\hline SRF-07-03 & 117043 & $\mathrm{U}$ & 0 & $\mathrm{pCi} / \mathrm{g}$ & 0.0844 & EPIA-002B \\
\hline
\end{tabular}

ANALYTE: Uranium-233/234

Analytical Group: Radionuclides

\begin{tabular}{|c|c|c|c|c|c|c|c|}
\hline Survey ID & Sample ID & FG & Result & Unit & SSMDL & Method & SC \\
\hline SRF-01-01 & 117018 & $\mathrm{U}$ & 0.00283 & $\mathrm{pCi} / \mathrm{g}$ & 0.0205 & EPIA-011B & \\
\hline SRF-01-02 & 117019 & $\mathrm{U}$ & 0.0232 & $\mathrm{pCi} / \mathrm{g}$ & 0.0256 & EPIA-011B & \\
\hline SRF-01-03 & 117020 & $\mathrm{U}$ & 0.00685 & $\mathrm{pCi} / \mathrm{g}$ & 0.023 & EPIA-011B & \\
\hline SRF-01-04 & 117021 & $\mathrm{U}$ & -0.00341 & $\mathrm{pCi} / \mathrm{g}$ & 0.0268 & EPIA-011B & \\
\hline SRF-01-05 & 117022 & $\mathrm{U}$ & -0.00837 & $\mathrm{pCi} / \mathrm{g}$ & 0.061 & EPIA-011B & \\
\hline SRF-02-01 & 117023 & $\mathrm{U}$ & 0.0164 & $\mathrm{pCi} / \mathrm{g}$ & 0.0277 & EPIA-011B & \\
\hline SRF-02-02 & 117024 & $\mathrm{U}$ & 0.00679 & $\mathrm{pCi} / \mathrm{g}$ & 0.0144 & EPIA-011B & \\
\hline SRF-02-03 & 117025 & $\mathrm{U}$ & 0.00548 & $\mathrm{pCi} / \mathrm{g}$ & 0.0203 & EPIA-011B & \\
\hline SRF-02-03-A & 117026 & $\mathrm{U}$ & -0.00104 & $\mathrm{pCi} / \mathrm{g}$ & 0.0265 & EPIA-011B & \\
\hline SRF-03-01 & 117027 & $\mathrm{U}$ & 0.000518 & $\mathrm{pCi} / \mathrm{g}$ & 0.047 & EPIA-011B & \\
\hline SRF-03-02 & 117028 & $\mathrm{U}$ & 0.0145 & $\mathrm{pCi} / \mathrm{g}$ & 0.0256 & EPIA-011B & \\
\hline SRF-03-02 & 117028 & $\mathrm{U}$ & 0.0194 & $\mathrm{pCi} / \mathrm{g}$ & 0.0314 & EPIA-011B & \\
\hline SRF-03-03 & 117029 & $\mathrm{U}$ & 0.000119 & $\mathrm{pCi} / \mathrm{g}$ & 0.0224 & EPIA-011B & \\
\hline SRF-03-04 & 117030 & $\mathrm{U}$ & 0.00156 & $\mathrm{pCi} / \mathrm{g}$ & 0.0457 & EPIA-011B & \\
\hline SRF-04-01 & 117031 & $\mathrm{U}$ & 0.0154 & $\mathrm{pCi} / \mathrm{g}$ & 0.0222 & EPIA-011B & \\
\hline SRF-04-02 & 117032 & $\mathrm{U}$ & 0.00409 & $\mathrm{pCi} / \mathrm{g}$ & 0.0297 & EPIA-011B & \\
\hline SRF-04-03 & 117033 & $\mathrm{U}$ & 0.00548 & $\mathrm{pCi} / \mathrm{g}$ & 0.0167 & EPIA-011B & \\
\hline SRF-05-01 & 117034 & $\mathrm{U}$ & 0.0156 & $\mathrm{pCi} / \mathrm{g}$ & 0.0195 & EPIA-011B & \\
\hline SRF-05-02 & 117035 & $\mathrm{U}$ & 0.0104 & $\mathrm{pCi} / \mathrm{g}$ & 0.0408 & EPIA-011B & \\
\hline SRF-05-03 & 117036 & $\mathrm{U}$ & 0.000186 & $\mathrm{pCi} / \mathrm{g}$ & 0.0219 & EPIA-011B & \\
\hline SRF-06-01 & 117037 & $\mathrm{R}$ & 0.019 & $\mathrm{pCi} / \mathrm{g}$ & 0.00949 & EPIA-011B & \\
\hline \multicolumn{8}{|c|}{ ANALYTE: Uranium-233/234 (cont.) } \\
\hline Survey ID & Sample ID & FG & Result & Unit & SSMDL & Method & SC \\
\hline SRF-06-02 & 117038 & $\mathrm{U}$ & -0.000488 & $\mathrm{pCi} / \mathrm{g}$ & 0.03 & EPIA-011B & \\
\hline SRF-06-03 & 117039 & $\mathrm{U}$ & 0.00849 & $\mathrm{pCi} / \mathrm{g}$ & 0.0138 & EPIA-011B & \\
\hline SRF-06-03-A & 117040 & $\mathrm{U}$ & 0.00294 & $\mathrm{pCi} / \mathrm{g}$ & 0.016 & EPIA-011B & \\
\hline SRF-07-01 & 117041 & $\mathrm{U}$ & 0.00216 & $\mathrm{pCi} / \mathrm{g}$ & 0.0252 & EPIA-011B & \\
\hline SRF-07-02 & 117042 & $\mathrm{U}$ & 0.00153 & $\mathrm{pCi} / \mathrm{g}$ & 0.0449 & EPIA-011B & \\
\hline SRF-07-03 & 117043 & $\mathrm{U}$ & $7.1 \mathrm{E}-05$ & $\mathrm{pCi} / \mathrm{g}$ & 0.0134 & EPIA-011B & \\
\hline SRF-07-03 & 117043 & $\mathrm{U}$ & 0.00995 & $\mathrm{pCi} / \mathrm{g}$ & 0.0131 & EPIA-011B & \\
\hline
\end{tabular}


ANALYTE: Uranium-235

Analytical Group: Radionuclides

\begin{tabular}{|c|c|c|c|c|c|c|}
\hline Survey ID & Sample ID & FG & Result & Unit & SsMDL & Method \\
\hline SRF-01-01 & 117018 & $\mathrm{U}$ & -0.000545 & $\mathrm{pCi} / \mathrm{g}$ & 0.0221 & EPIA-011B \\
\hline SRF-01-02 & 117019 & $\mathrm{U}$ & 0.00651 & $\mathrm{pCi} / \mathrm{g}$ & 0.0195 & EPIA-011B \\
\hline SRF-01-03 & 117020 & $\mathrm{U}$ & -0.00258 & $\mathrm{pCi} / \mathrm{g}$ & 0.0203 & EPIA-011B \\
\hline SRF-01-04 & 117021 & $\mathrm{U}$ & 0.000996 & $\mathrm{pCi} / \mathrm{g}$ & 0.0247 & EPIA-011B \\
\hline SRF-01-05 & 117022 & $\mathrm{U}$ & -0.00575 & $\mathrm{pCi} / \mathrm{g}$ & 0.0453 & EPIA-011B \\
\hline SRF-02-01 & 117023 & $\mathrm{U}$ & 0.000961 & $\mathrm{pCi} / \mathrm{g}$ & 0.0239 & EPIA-011B \\
\hline SRF-02-02 & 117024 & $\mathrm{U}$ & -0.000914 & $\mathrm{pCi} / \mathrm{g}$ & 0.0178 & EPIA-011B \\
\hline SRF-02-03 & 117025 & $\mathrm{U}$ & 0.00205 & $\mathrm{pCi} / \mathrm{g}$ & 0.0142 & EPIA-011B \\
\hline SRF-02-03-A & 117026 & $\mathrm{U}$ & -0.00188 & $\mathrm{pCi} / \mathrm{g}$ & 0.0181 & EPIA-011B \\
\hline SRF-03-01 & 117027 & $\mathrm{U}$ & 0.0045 & $\mathrm{pCi} / \mathrm{g}$ & 0.0327 & EPIA-011B \\
\hline SRF-03-02 & 117028 & $\mathrm{U}$ & 0.00799 & $\mathrm{pCi} / \mathrm{g}$ & 0.024 & EPIA-011B \\
\hline SRF-03-02 & 117028 & $\mathrm{U}$ & -0.00198 & $\mathrm{pCi} / \mathrm{g}$ & 0.0257 & EPIA-011B \\
\hline SRF-03-03 & 117029 & $\mathrm{U}$ & 0.00322 & $\mathrm{pCi} / \mathrm{g}$ & 0.0281 & EPIA-011B \\
\hline SRF-03-04 & 117030 & $\mathrm{U}$ & 0.000174 & $\mathrm{pCi} / \mathrm{g}$ & 0.0328 & EPIA-011B \\
\hline SRF-04-01 & 117031 & $\mathrm{U}$ & 0.00399 & $\mathrm{pCi} / \mathrm{g}$ & 0.012 & EPIA-011B \\
\hline SRF-04-02 & 117032 & $\mathrm{U}$ & -0.00473 & $\mathrm{pCi} / \mathrm{g}$ & 0.0319 & EPIA-011B \\
\hline SRF-04-03 & 117033 & $\mathrm{U}$ & 0.00183 & $\mathrm{pCi} / \mathrm{g}$ & 0.0127 & EPIA-011B \\
\hline SRF-05-01 & 117034 & $\mathrm{U}$ & -0.00187 & $\mathrm{pCi} / \mathrm{g}$ & 0.018 & EPIA-011B \\
\hline SRF-05-02 & 117035 & $\mathrm{U}$ & 0.00417 & $\mathrm{pCi} / \mathrm{g}$ & 0.0303 & EPIA-011B \\
\hline SRF-05-03 & 117036 & $\mathrm{U}$ & 0.00177 & $\mathrm{pCi} / \mathrm{g}$ & 0.0123 & EPIA-011B \\
\hline SRF-06-01 & 117037 & $\mathrm{U}$ & 0 & $\mathrm{pCi} / \mathrm{g}$ & 0.00952 & EPIA-011B \\
\hline SRF-06-02 & 117038 & $\mathrm{U}$ & -0.00796 & $\mathrm{pCi} / \mathrm{g}$ & 0.0362 & EPIA-011B \\
\hline SRF-06-03 & 117039 & $\mathrm{U}$ & 0.00398 & $\mathrm{pCi} / \mathrm{g}$ & 0.00597 & EPIA-011B \\
\hline SRF-06-03-A & 117040 & $\mathrm{U}$ & 0.0023 & $\mathrm{pCi} / \mathrm{g}$ & 0.00691 & EPIA-011B \\
\hline SRF-07-01 & 117041 & $\mathrm{U}$ & 0.00196 & $\mathrm{pCi} / \mathrm{g}$ & 0.0136 & EPIA-011B \\
\hline SRF-07-02 & 117042 & $\mathrm{U}$ & 0.000171 & $\mathrm{pCi} / \mathrm{g}$ & 0.0322 & EPIA-011B \\
\hline SRF-07-03 & 117043 & $\mathrm{U}$ & 0.000142 & $\mathrm{pCi} / \mathrm{g}$ & 0.0168 & EPIA-011B \\
\hline SRF-07-03 & 117043 & $\mathrm{U}$ & 0.00521 & $\mathrm{pCi} / \mathrm{g}$ & 0.00997 & EPIA-011B \\
\hline
\end{tabular}

ANALYTE: Uranium-238

Analytical Group: Radionuclides

\begin{tabular}{|c|c|c|c|c|c|c|c|c|}
\hline Survey ID & Sample ID & FG & Result & Unit & ssMDL & Method & SC & $\mathbf{E}$ \\
\hline SRF-01-01 & 117018 & $\mathrm{U}$ & 0.00685 & $\mathrm{pCi} / \mathrm{g}$ & 0.0169 & EPIA-011B & & \\
\hline SRF-01-02 & 117019 & $\mathrm{R}$ & 0.024 & $\mathrm{pCi} / \mathrm{g}$ & 0.0229 & EPIA-011B & & 4 \\
\hline SRF-01-03 & 117020 & $\mathrm{U}$ & -0.00118 & $\mathrm{pCi} / \mathrm{g}$ & 0.023 & EPIA-011B & & \\
\hline SRF-01-04 & 117021 & $\mathrm{R}$ & 0.0142 & $\mathrm{pCi} / \mathrm{g}$ & 0.0106 & EPIA-011B & & 4 \\
\hline SRF-01-05 & 117022 & $\mathrm{U}$ & -0.0043 & $\mathrm{pCi} / \mathrm{g}$ & 0.0415 & EPIA-011B & & \\
\hline SRF-02-01 & 117023 & $\mathrm{U}$ & 0.000959 & $\mathrm{pCi} / \mathrm{g}$ & 0.0238 & EPIA-011B & & \\
\hline SRF-02-02 & 117024 & $\mathrm{R}$ & 0.0119 & $\mathrm{pCi} / \mathrm{g}$ & 0.0109 & EPIA-011B & & 4 \\
\hline SRF-02-03 & 117025 & $\mathrm{U}$ & 0.0014 & $\mathrm{pCi} / \mathrm{g}$ & 0.0167 & EPIA-011B & & \\
\hline \multicolumn{9}{|c|}{ ANALYTE: Uranium-238 (cont.) } \\
\hline Survey ID & Sample ID & FG & Result & Unit & SSMDL & Method & SC & $\mathbf{E}$ \\
\hline SRF-02-03-A & 117026 & $\mathrm{U}$ & 0.0078 & $\mathrm{pCi} / \mathrm{g}$ & 0.0078 & EPIA-011B & & \\
\hline SRF-03-01 & 117027 & $\mathrm{R}$ & 0.0248 & $\mathrm{pCi} / \mathrm{g}$ & 0.0228 & EPIA-011B & & 4 \\
\hline SRF-03-02 & 117028 & $\mathrm{U}$ & 0.0125 & $\mathrm{pCi} / \mathrm{g}$ & 0.0239 & EPIA-011B & & \\
\hline SRF-03-02 & 117028 & $\mathrm{U}$ & 0.0114 & $\mathrm{pCi} / \mathrm{g}$ & 0.0217 & EPIA-011B & & \\
\hline SRF-03-03 & 117029 & $\mathrm{U}$ & 0.00226 & $\mathrm{pCi} / \mathrm{g}$ & 0.0157 & EPIA-011B & & \\
\hline SRF-03-04 & 117030 & $\mathrm{U}$ & 0.0109 & $\mathrm{pCi} / \mathrm{g}$ & 0.0269 & EPIA-011B & & \\
\hline SRF-04-01 & 117031 & $\mathrm{U}$ & 0.000723 & $\mathrm{pCi} / \mathrm{g}$ & 0.0204 & EPIA-011B & & \\
\hline SRF-04-02 & 117032 & $\mathrm{U}$ & -0.00189 & $\mathrm{pCi} / \mathrm{g}$ & 0.0244 & EPIA-011B & & \\
\hline SRF-04-03 & 117033 & $\mathrm{U}$ & 0.00605 & $\mathrm{pCi} / \mathrm{g}$ & 0.0149 & EPIA-011B & & \\
\hline SRF-05-01 & 117034 & $\mathrm{U}$ & 0.00775 & $\mathrm{pCi} / \mathrm{g}$ & 0.00775 & EPIA-011B & & \\
\hline SRF-05-02 & 117035 & $\mathrm{U}$ & 0.0131 & $\mathrm{pCi} / \mathrm{g}$ & 0.0277 & EPIA-011B & & \\
\hline
\end{tabular}




\begin{tabular}{lllrlrr}
\hline SRF-05-03 & 117036 & $\mathrm{U}$ & 0.00408 & $\mathrm{pCi} / \mathrm{g}$ & 0.0122 & EPIA-011B \\
SRF-06-01 & 117037 & $\mathrm{U}$ & 0.00949 & $\mathrm{pCi} / \mathrm{g}$ & 0.00949 & EPIA-011B \\
SRF-06-02 & 117038 & $\mathrm{U}$ & -0.00281 & $\mathrm{pCi} / \mathrm{g}$ & 0.0288 & EPIA-011B \\
SRF-06-03 & 117039 & $\mathrm{U}$ & 0.00198 & $\mathrm{pCi} / \mathrm{g}$ & 0.00595 & EPIA-011B \\
SRF-06-03-A & 117040 & $\mathrm{U}$ & 0.00404 & $\mathrm{pCi} / \mathrm{g}$ & 0.0121 & EPIA-011B \\
SRF-07-01 & 117041 & $\mathrm{U}$ & 0.000821 & $\mathrm{pCi} / \mathrm{g}$ & 0.0232 & EPIA-011B \\
SRF-07-02 & 117042 & $\mathrm{U}$ & 0.00221 & $\mathrm{pCi} / \mathrm{g}$ & 0.0265 & EPIA-011B \\
SRF-07-03 & 117043 & $\mathrm{U}$ & 0.00135 & $\mathrm{pCi} / \mathrm{g}$ & 0.00937 & EPIA-011B \\
SRF-07-03 & 117043 & $\mathrm{U}$ & 0.00143 & $\mathrm{pCi} / \mathrm{g}$ & 0.00994 & EPIA-011B
\end{tabular}

ANALYTE: Vinyl acetate

Analytical Group: Volatile Organic Compounds

\begin{tabular}{|c|c|c|c|c|c|c|c|}
\hline Survey ID & Sample ID & $\mathbf{F G}$ & Result & Unit & SSMDL & Method & SC \\
\hline SRF-01-01 & 117018 & $\mathrm{U}$ & 75 & $\mu g / \mathrm{kg}$ & 10.5 & EPA8260B & \\
\hline SRF-01-02 & 117019 & $\mathrm{U}$ & 75 & $\mu \mathrm{g} / \mathrm{kg}$ & 10.5 & EPA8260B & \\
\hline SRF-01-03 & 117020 & $\mathrm{U}$ & 75 & $\mu g / \mathrm{kg}$ & 10.5 & EPA8260B & \\
\hline SRF-01-04 & 117021 & $\mathrm{U}$ & 75 & $\mu \mathrm{g} / \mathrm{kg}$ & 10.5 & EPA8260B & \\
\hline SRF-01-05 & 117022 & $\mathrm{U}$ & 75 & $\mu \mathrm{g} / \mathrm{kg}$ & 10.5 & EPA8260B & \\
\hline SRF-02-01 & 117023 & $\mathrm{U}$ & 75 & $\mu \mathrm{g} / \mathrm{kg}$ & 10.5 & EPA8260B & \\
\hline SRF-02-02 & 117024 & $\mathrm{U}$ & 75 & $\mu g / \mathrm{kg}$ & 10.5 & EPA8260B & \\
\hline SRF-02-03 & 117025 & $\mathrm{U}$ & 75 & $\mu g / \mathrm{kg}$ & 10.5 & EPA8260B & \\
\hline SRF-02-03-A & 117026 & $\mathrm{U}$ & 75 & $\mu \mathrm{g} / \mathrm{kg}$ & 10.5 & EPA8260B & \\
\hline SRF-03-01 & 117027 & $\mathrm{U}$ & 75 & $\mu \mathrm{g} / \mathrm{kg}$ & 10.5 & EPA8260B & \\
\hline SRF-03-02 & 117028 & $\mathrm{U}$ & 75 & $\mu \mathrm{g} / \mathrm{kg}$ & 10.5 & EPA8260B & \\
\hline SRF-03-03 & 117029 & $\mathrm{U}$ & 75 & $\mu \mathrm{g} / \mathrm{kg}$ & 10.5 & EPA8260B & \\
\hline SRF-03-04 & 117030 & $\mathrm{U}$ & 75 & $\mu \mathrm{g} / \mathrm{kg}$ & 10.5 & EPA8260B & \\
\hline SRF-04-01 & 117031 & $\mathrm{U}$ & 75 & $\mu \mathrm{g} / \mathrm{kg}$ & 10.5 & EPA8260B & \\
\hline SRF-04-02 & 117032 & $\mathrm{U}$ & 75 & $\mu \mathrm{g} / \mathrm{kg}$ & 10.5 & EPA8260B & \\
\hline SRF-04-03 & 117033 & $\mathrm{U}$ & 75 & $\mu g / \mathrm{kg}$ & 10.5 & EPA8260B & \\
\hline SRF-05-01 & 117034 & $\mathrm{U}$ & 75 & $\mu g / \mathrm{kg}$ & 10.5 & EPA8260B & \\
\hline SRF-05-02 & 117035 & $\mathrm{U}$ & 75 & $\mu \mathrm{g} / \mathrm{kg}$ & 10.5 & EPA8260B & \\
\hline SRF-05-03 & 117036 & $\mathrm{U}$ & 75 & $\mu g / \mathrm{kg}$ & 10.5 & EPA8260B & \\
\hline SRF-06-01 & 117037 & $\mathrm{U}$ & 75 & $\mu \mathrm{g} / \mathrm{kg}$ & 10.5 & EPA8260B & \\
\hline SRF-06-02 & 117038 & $\mathrm{U}$ & 75 & $\mu g / \mathrm{kg}$ & 10.5 & EPA8260B & \\
\hline SRF-06-03 & 117039 & $\mathrm{U}$ & 75 & $\mu \mathrm{g} / \mathrm{kg}$ & 10.5 & EPA8260B & \\
\hline SRF-06-03-A & 117040 & $\mathrm{U}$ & 75 & $\mu \mathrm{g} / \mathrm{kg}$ & 10.5 & EPA8260B & \\
\hline SRF-07-01 & 117041 & $\mathrm{U}$ & 75 & $\mu \mathrm{g} / \mathrm{kg}$ & 10.5 & EPA8260B & \\
\hline SRF-07-02 & 117042 & $\mathrm{U}$ & 75 & $\mu \mathrm{g} / \mathrm{kg}$ & 10.5 & EPA8260B & \\
\hline SRF-07-03 & 117043 & $\mathrm{U}$ & 75 & $\mu \mathrm{g} / \mathrm{kg}$ & 10.5 & EPA8260B & \\
\hline
\end{tabular}

ANALYTE: Xylenes

Analytical Group: Volatile Organic Compounds

\begin{tabular}{|c|c|c|c|c|c|c|c|c|}
\hline Survey ID & Sample ID & $\mathbf{F G}$ & Result & Unit & SSMDL & Method & SC & $\mathbf{E}$ \\
\hline SRF-01-01 & 117018 & $\mathrm{~J}$ & 25.8 & $\mu g / \mathrm{kg}$ & 5 & EPA8260B & $\mathrm{K}$ & $\mathrm{O}$ \\
\hline SRF-01-02 & 117019 & $\mathrm{~J}$ & 33.6 & $\mu g / \mathrm{kg}$ & 5 & EPA8260B & $\mathrm{K}$ & $\mathrm{O}$ \\
\hline SRF-01-03 & 117020 & $\mathrm{U}$ & 25 & $\mu \mathrm{g} / \mathrm{kg}$ & 5 & EPA8260B & & \\
\hline SRF-01-04 & 117021 & $\mathrm{~J}$ & 16 & $\mu g / \mathrm{kg}$ & 5 & EPA8260B & IK & $\mathrm{O}$ \\
\hline SRF-01-05 & 117022 & $\mathrm{~J}$ & 255 & $\mu \mathrm{g} / \mathrm{kg}$ & 5 & EPA8260B & $\mathrm{K}$ & $\mathrm{O}$ \\
\hline SRF-02-01 & 117023 & $\mathrm{~J}$ & 14.2 & $\mu \mathrm{g} / \mathrm{kg}$ & 5 & EPA8260B & IK & $\mathrm{O}$ \\
\hline SRF-02-02 & 117024 & $\mathrm{~J}$ & 34.3 & $\mu \mathrm{g} / \mathrm{kg}$ & 5 & EPA8260B & $\mathrm{K}$ & $\mathrm{O}$ \\
\hline SRF-02-03 & 117025 & $\mathrm{~J}$ & 13.1 & $\mu g / \mathrm{kg}$ & 5 & EPA8260B & IK & $\mathrm{O}$ \\
\hline SRF-02-03-A & 117026 & $\mathrm{U}$ & 25 & $\mu g / k g$ & 5 & EPA8260B & & \\
\hline SRF-03-01 & 117027 & $\mathrm{~J}$ & 5.89 & $\mu g / k g$ & 5 & EPA8260B & I & \\
\hline SRF-03-02 & 117028 & & 25.1 & $\mu \mathrm{g} / \mathrm{kg}$ & 5 & EPA8260B & & \\
\hline SRF-03-03 & 117029 & $\mathrm{~J}$ & 10.9 & $\mu g / k g$ & 5 & EPA8260B & I & \\
\hline SRF-03-04 & 117030 & & 50 & $\mu \mathrm{g} / \mathrm{kg}$ & 5 & EPA8260B & & \\
\hline SRF-04-01 & 117031 & $\mathrm{~J}$ & 25 & $\mu \mathrm{g} / \mathrm{kg}$ & 5 & EPA8260B & $\mathrm{K}$ & $\mathrm{O}$ \\
\hline SRF-04-02 & 117032 & $\mathrm{~J}$ & 20 & $\mu \mathrm{g} / \mathrm{kg}$ & 5 & EPA8260B & I & \\
\hline
\end{tabular}




\begin{tabular}{lllrlllll}
\hline SRF-04-03 & 117033 & $\mathrm{~J}$ & 8.61 & $\mu \mathrm{g} / \mathrm{kg}$ & 5 & EPA8260B & IK & $\mathrm{O}$ \\
SRF-05-01 & 117034 & $\mathrm{~J}$ & 5.99 & $\mu \mathrm{g} / \mathrm{kg}$ & 5 & EPA8260B & IK & O \\
SRF-05-02 & 117035 & $\mathrm{~J}$ & 11.5 & $\mu \mathrm{g} / \mathrm{kg}$ & 5 & EPA8260B & $\mathrm{I}$ & \\
SRF-05-03 & 117036 & $\mathrm{~J}$ & 8.05 & $\mu \mathrm{g} / \mathrm{kg}$ & 5 & EPA8260B & $\mathrm{IK}$ & $\mathrm{O}$ \\
SRF-06-01 & 117037 & $\mathrm{~J}$ & 51.3 & $\mu \mathrm{gg} / \mathrm{kg}$ & 5 & EPA8260B & $\mathrm{K}$ & $\mathrm{O}$ \\
SRF-06-02 & 117038 & $\mathrm{U}$ & 25 & $\mu \mathrm{kg}$ & 5 & EPA8260B & & \\
SRF-06-03 & 117039 & $\mathrm{~J}$ & 5.67 & $\mu \mathrm{kg} / \mathrm{kg}$ & 5 & EPA8260B & $\mathrm{IK}$ & $\mathrm{O}$ \\
SRF-06-03-A & 117040 & & 32.4 & $\mu \mathrm{g} / \mathrm{kg}$ & 5 & EPA8260B & & \\
SRF-07-01 & 117041 & $\mathrm{~J}$ & 5.55 & $\mu \mathrm{g} / \mathrm{kg}$ & 5 & EPA8260B & $\mathrm{IK}$ & $\mathrm{O}$ \\
SRF-07-02 & 117042 & $\mathrm{~J}$ & 15.9 & $\mu \mathrm{g} / \mathrm{kg}$ & 5 & EPA8260B & $\mathrm{IK}$ & $\mathrm{O}$ \\
SRF-07-03 & 117043 & $\mathrm{~J}$ & 5.87 & $\mu \mathrm{gg} / \mathrm{kg}$ & 5 & EPA8260B & $\mathrm{IK}$ & $\mathrm{O}$
\end{tabular}

ANALYTE: Yttrium-88

Analytical Group: Radionuclides

\begin{tabular}{|c|c|c|c|c|c|c|c|}
\hline Survey ID & Sample ID & FG & Result & Unit & ssMDL & Method & SC \\
\hline SRF-01-01 & 117018 & $\mathrm{U}$ & 0.00144 & $\mathrm{pCi} / \mathrm{g}$ & 0.0803 & EPIA-013B & \\
\hline SRF-01-02 & 117019 & $\mathrm{U}$ & -0.00316 & $\mathrm{pCi} / \mathrm{g}$ & 0.0656 & EPIA-013B & \\
\hline SRF-01-03 & 117020 & $\mathrm{U}$ & 0.0122 & $\mathrm{pCi} / \mathrm{g}$ & 0.0775 & EPIA-013B & \\
\hline SRF-01-04 & 117021 & $\mathrm{U}$ & -0.0146 & $\mathrm{pCi} / \mathrm{g}$ & 0.0485 & EPIA-013B & \\
\hline SRF-01-05 & 117022 & $\mathrm{U}$ & 0.0119 & $\mathrm{pCi} / \mathrm{g}$ & 0.0574 & EPIA-013B & \\
\hline SRF-01-05 & 117022 & $\mathrm{U}$ & -0.00547 & $\mathrm{pCi} / \mathrm{g}$ & 0.0586 & EPIA-013B & \\
\hline SRF-02-01 & 117023 & $\mathrm{U}$ & 0.0152 & $\mathrm{pCi} / \mathrm{g}$ & 0.0764 & EPIA-013B & \\
\hline SRF-02-02 & 117024 & $\mathrm{U}$ & 0.00638 & $\mathrm{pCi} / \mathrm{g}$ & 0.0576 & EPIA-013B & \\
\hline SRF-02-03 & 117025 & $\mathrm{U}$ & -0.0388 & $\mathrm{pCi} / \mathrm{g}$ & 0.0872 & EPIA-013B & \\
\hline SRF-02-03-A & 117026 & $\mathrm{U}$ & 0.0237 & $\mathrm{pCi} / \mathrm{g}$ & 0.0977 & EPIA-013B & \\
\hline SRF-03-01 & 117027 & $\mathrm{U}$ & -0.00252 & $\mathrm{pCi} / \mathrm{g}$ & 0.0679 & EPIA-013B & \\
\hline SRF-03-02 & 117028 & $\mathrm{U}$ & 0.0177 & $\mathrm{pCi} / \mathrm{g}$ & 0.0548 & EPIA-013B & \\
\hline SRF-03-03 & 117029 & $\mathrm{U}$ & -0.00236 & $\mathrm{pCi} / \mathrm{g}$ & 0.0693 & EPIA-013B & \\
\hline SRF-03-04 & 117030 & $\mathrm{U}$ & 0.00828 & $\mathrm{pCi} / \mathrm{g}$ & 0.0436 & EPIA-013B & \\
\hline SRF-04-01 & 117031 & $\mathrm{U}$ & 0.0136 & $\mathrm{pCi} / \mathrm{g}$ & 0.0622 & EPIA-013B & \\
\hline SRF-04-02 & 117032 & $\mathrm{U}$ & -0.0251 & $\mathrm{pCi} / \mathrm{g}$ & 0.0715 & EPIA-013B & \\
\hline SRF-04-03 & 117033 & $\mathrm{U}$ & 0.0328 & $\mathrm{pCi} / \mathrm{g}$ & 0.103 & EPIA-013B & \\
\hline SRF-05-01 & 117034 & $\mathrm{U}$ & 0.0016 & $\mathrm{pCi} / \mathrm{g}$ & 0.0479 & EPIA-013B & \\
\hline SRF-05-02 & 117035 & $\mathrm{U}$ & 0.0083 & $\mathrm{pCi} / \mathrm{g}$ & 0.0739 & EPIA-013B & \\
\hline SRF-05-03 & 117036 & $\mathrm{U}$ & 0.00973 & $\mathrm{pCi} / \mathrm{g}$ & 0.0779 & EPIA-013B & \\
\hline SRF-06-01 & 117037 & $\mathrm{U}$ & -0.00179 & $\mathrm{pCi} / \mathrm{g}$ & 0.0683 & EPIA-013B & \\
\hline SRF-06-02 & 117038 & $\mathrm{U}$ & -0.00464 & $\mathrm{pCi} / \mathrm{g}$ & 0.0551 & EPIA-013B & \\
\hline SRF-06-03 & 117039 & $\mathrm{U}$ & -0.0151 & $\mathrm{pCi} / \mathrm{g}$ & 0.0678 & EPIA-013B & \\
\hline \multicolumn{8}{|c|}{ ANALYTE: Yttrium-88 (cont.) } \\
\hline Survey ID & Sample ID & FG & Result & Unit & ssMDL & Method & SC \\
\hline SRF-06-03-A & 117040 & $\mathrm{U}$ & -0.00322 & $\mathrm{pCi} / \mathrm{g}$ & 0.0956 & EPIA-013B & \\
\hline SRF-07-01 & 117041 & $\mathrm{U}$ & -0.0196 & $\mathrm{pCi} / \mathrm{g}$ & 0.098 & EPIA-013B & \\
\hline SRF-07-02 & 117042 & $\mathrm{U}$ & -0.00231 & $\mathrm{pCi} / \mathrm{g}$ & 0.114 & EPIA-013B & \\
\hline SRF-07-03 & 117043 & $\mathrm{U}$ & -0.00343 & $\mathrm{pCi} / \mathrm{g}$ & 0.0863 & EPIA-013B & \\
\hline SRF-07-03 & 117043 & $\mathrm{U}$ & 0.00984 & $\mathrm{pCi} / \mathrm{g}$ & 0.0743 & EPIA-013B & \\
\hline
\end{tabular}

ANALYTE: Zinc-65

Analytical Group: Radionuclides

$\begin{array}{lllrlrlll}\text { Survey ID } & \text { Sample ID } & \text { FG } & \text { Result } & \text { Unit } & \text { ssMDL } & \text { Method } & \text { SC } & \text { E } \\ & & & & & & & \\ \text { SRF-01-01 } & 117018 & \mathrm{U} & -0.0125 & \mathrm{pCi} / \mathrm{g} & 0.139 & \text { EPIA-013B } & \\ \text { SRF-01-02 } & 117019 & \mathrm{U} & -0.00192 & \mathrm{pCi} / \mathrm{g} & 0.116 & \text { EPIA-013B } & \\ \text { SRF-01-03 } & 117020 & \mathrm{U} & -0.0219 & \mathrm{pCi} / \mathrm{g} & 0.114 & \text { EPIA-013B } & \\ \text { SRF-01-04 } & 117021 & \mathrm{U} & 0.0615 & \mathrm{pCi} / \mathrm{g} & 0.114 & \text { EPIA-013B } & \\ \text { SRF-01-05 } & 117022 & \mathrm{U} & 0.0308 & \mathrm{pCi} / \mathrm{g} & 0.111 & \text { EPIA-013B } & \\ \text { SRF-01-05 } & 117022 & \mathrm{U} & 0.00905 & \mathrm{pCi} / \mathrm{g} & 0.0994 & \text { EPIA-013B } & \end{array}$




\begin{tabular}{|c|c|c|c|c|c|c|}
\hline SRF-02-01 & 117023 & $\mathrm{U}$ & 0.109 & $\mathrm{pCi} / \mathrm{g}$ & 0.122 & EPIA-013B \\
\hline SRF-02-02 & 117024 & $\mathrm{U}$ & -0.0305 & $\mathrm{pCi} / \mathrm{g}$ & 0.104 & EPIA-013B \\
\hline SRF-02-03 & 117025 & $\mathrm{U}$ & 0.0773 & $\mathrm{pCi} / \mathrm{g}$ & 0.151 & EPIA-013B \\
\hline SRF-02-03-A & 117026 & $\mathrm{U}$ & -0.0228 & $\mathrm{pCi} / \mathrm{g}$ & 0.138 & EPIA-013B \\
\hline SRF-03-01 & 117027 & $\mathrm{U}$ & -0.0121 & $\mathrm{pCi} / \mathrm{g}$ & 0.128 & EPIA-013B \\
\hline SRF-03-02 & 117028 & $\mathrm{U}$ & 0.0849 & $\mathrm{pCi} / \mathrm{g}$ & 0.125 & EPIA-013B \\
\hline SRF-03-03 & 117029 & $\mathrm{U}$ & -0.0198 & $\mathrm{pCi} / \mathrm{g}$ & 0.106 & EPIA-013B \\
\hline SRF-03-04 & 117030 & $\mathrm{U}$ & 0.0138 & $\mathrm{pCi} / \mathrm{g}$ & 0.0811 & EPIA-013B \\
\hline SRF-04-01 & 117031 & $\mathrm{U}$ & -0.0117 & $\mathrm{pCi} / \mathrm{g}$ & 0.115 & EPIA-013B \\
\hline SRF-04-02 & 117032 & $\mathrm{U}$ & -0.0767 & $\mathrm{pCi} / \mathrm{g}$ & 0.158 & EPIA-013B \\
\hline SRF-04-03 & 117033 & $\mathrm{U}$ & -0.0511 & $\mathrm{pCi} / \mathrm{g}$ & 0.138 & EPIA-013B \\
\hline SRF-05-01 & 117034 & $\mathrm{U}$ & -0.0392 & $\mathrm{pCi} / \mathrm{g}$ & 0.108 & EPIA-013B \\
\hline SRF-05-02 & 117035 & $\mathrm{U}$ & 0.0243 & $\mathrm{pCi} / \mathrm{g}$ & 0.138 & EPIA-013B \\
\hline SRF-05-03 & 117036 & $\mathrm{U}$ & -0.0397 & $\mathrm{pCi} / \mathrm{g}$ & 0.108 & EPIA-013B \\
\hline SRF-06-01 & 117037 & $\mathrm{U}$ & 0.0543 & $\mathrm{pCi} / \mathrm{g}$ & 0.157 & EPIA-013B \\
\hline SRF-06-02 & 117038 & $\mathrm{U}$ & -0.0389 & $\mathrm{pCi} / \mathrm{g}$ & 0.0855 & EPIA-013B \\
\hline SRF-06-03 & 117039 & $\mathrm{U}$ & -0.0666 & $\mathrm{pCi} / \mathrm{g}$ & 0.12 & EPIA-013B \\
\hline SRF-06-03-A & 117040 & $\mathrm{U}$ & 0.00423 & $\mathrm{pCi} / \mathrm{g}$ & 0.168 & EPIA-013B \\
\hline SRF-07-01 & 117041 & $\mathrm{U}$ & -0.00653 & $\mathrm{pCi} / \mathrm{g}$ & 0.168 & EPIA-013B \\
\hline SRF-07-02 & 117042 & $\mathrm{U}$ & 0.0628 & $\mathrm{pCi} / \mathrm{g}$ & 0.201 & EPIA-013B \\
\hline SRF-07-03 & 117043 & $\mathrm{U}$ & -0.00123 & $\mathrm{pCi} / \mathrm{g}$ & 0.187 & EPIA-013B \\
\hline SRF-07-03 & 117043 & $\mathrm{U}$ & -0.0264 & $\mathrm{pCi} / \mathrm{g}$ & 0.123 & EPIA-013B \\
\hline
\end{tabular}

ANALYTE: Zinc

Analytical Group: Metals (total recoverable)

$\begin{array}{ll}\text { Survey ID } & \text { Sample } \\ \text { SRF-01-01 } & 117018 \\ \text { SRF-01-02 } & 117019 \\ \text { SRF-01-03 } & 117020 \\ \text { SRF-01-04 } & 117021 \\ \text { SRF-01-05 } & 117022 \\ \text { SRF-02-01 } & 117023 \\ \text { SRF-02-02 } & 117024 \\ \text { SRF-02-03 } & 117025 \\ \text { SRF-02-03-A } & 117026 \\ \text { SRF-03-01 } & 117027\end{array}$

ANALYTE: Zinc (cont.)

$\begin{array}{ll}\text { Survey ID } & \text { Sample ID } \\ & \text { FG } \\ \text { SRF-03-02 } & 117028 \\ \text { SRF-03-03 } & 117029 \\ \text { SRF-03-04 } & 117030 \\ \text { SRF-04-01 } & 117031 \\ \text { SRF-04-02 } & 117032 \\ \text { SRF-04-03 } & 117033 \\ \text { SRF-05-01 } & 117034 \\ \text { SRF-05-02 } & 117035 \\ \text { SRF-05-03 } & 117036 \\ \text { SRF-06-01 } & 117037 \\ \text { SRF-06-02 } & 117038 \\ \text { SRF-06-03 } & 117039 \\ \text { SRF-06-03-A } & 117040 \\ \text { SRF-07-01 } & 117041 \\ \text { SRF-07-02 } & 117042 \\ \text { SRF-07-03 } & 117043\end{array}$

$\begin{array}{rr}\text { Result } & \text { Unit } \\ & \\ 14.1 & \mathrm{mg} / \mathrm{kg} \\ 15.2 & \mathrm{mg} / \mathrm{kg} \\ 6.96 & \mathrm{mg} / \mathrm{kg} \\ 19.5 & \mathrm{mg} / \mathrm{kg} \\ 17.9 & \mathrm{mg} / \mathrm{kg} \\ 23.6 & \mathrm{mg} / \mathrm{kg} \\ 18.6 & \mathrm{mg} / \mathrm{kg} \\ 12.6 & \mathrm{mg} / \mathrm{kg} \\ 21.5 & \mathrm{mg} / \mathrm{kg} \\ 12.4 & \mathrm{mg} / \mathrm{kg}\end{array}$

$\begin{array}{rl}\text { Result } & \text { Unit } \\ 16.3 & \mathrm{mg} / \mathrm{kg} \\ 23.8 & \mathrm{mg} / \mathrm{kg} \\ 36.2 & \mathrm{mg} / \mathrm{kg} \\ 22.2 & \mathrm{mg} / \mathrm{kg} \\ 16.9 & \mathrm{mg} / \mathrm{kg} \\ 5.98 & \mathrm{mg} / \mathrm{kg} \\ 15.7 & \mathrm{mg} / \mathrm{kg} \\ 24 & \mathrm{mg} / \mathrm{kg} \\ 10.6 & \mathrm{mg} / \mathrm{kg} \\ 28 & \mathrm{mg} / \mathrm{kg} \\ 17.9 & \mathrm{mg} / \mathrm{kg} \\ 11.5 & \mathrm{mg} / \mathrm{kg} \\ 18 & \mathrm{mg} / \mathrm{kg} \\ 20.8 & \mathrm{mg} / \mathrm{kg} \\ 24.6 & \mathrm{mg} / \mathrm{kg} \\ 15.1 & \mathrm{mg} / \mathrm{kg}\end{array}$

SSMDL

0.168

0.176

0.168

0.171

0.17

0.173

0.178

0.185

0.175

0.184

$\begin{aligned} \text { ssMDL } & \text { Method } \\ 0.18 & \text { EPA6010B } \\ 0.173 & \text { EPA6010B } \\ 0.181 & \text { EPA6010B } \\ 0.178 & \text { EPA6010B } \\ 0.17 & \text { EPA6010B } \\ 0.184 & \text { EPA6010B } \\ 0.18 & \text { EPA6010B } \\ 0.176 & \text { EPA6010B } \\ 0.173 & \text { EPA6010B } \\ 0.176 & \text { EPA6010B } \\ 0.171 & \text { EPA6010B } \\ 0.176 & \text { EPA6010B } \\ 0.184 & \text { EPA6010B } \\ 0.178 & \text { EPA6010B } \\ 0.178 & \text { EPA6010B } \\ 0.17 & \text { EPA6010B }\end{aligned}$

EPA6010B
SC E EPA6010B EPA6010B

EPA6010B

EPA6010B

EPA6010B

EPA6010B

EPA6010B

EPA6010B

EPA6010B 
ANALYTE: Zirconium-95

Analytical Group: Radionuclides

\begin{tabular}{|c|c|c|c|c|c|c|c|}
\hline Survey ID & Sample ID & FG & Result & Unit & SsMDL & Method & SC \\
\hline SRF-01-01 & 117018 & $\mathrm{U}$ & 0.0173 & $\mathrm{pCi} / \mathrm{g}$ & 0.13 & EPIA-013B & \\
\hline SRF-01-02 & 117019 & $\mathrm{U}$ & 0.0348 & $\mathrm{pCi} / \mathrm{g}$ & 0.112 & EPIA-013B & \\
\hline SRF-01-03 & 117020 & $\mathrm{U}$ & -0.0247 & $\mathrm{pCi} / \mathrm{g}$ & 0.112 & EPIA-013B & \\
\hline SRF-01-04 & 117021 & $\mathrm{U}$ & 0.0114 & $\mathrm{pCi} / \mathrm{g}$ & 0.105 & EPIA-013B & \\
\hline SRF-01-05 & 117022 & $\mathrm{U}$ & 0.00348 & $\mathrm{pCi} / \mathrm{g}$ & 0.101 & EPIA-013B & \\
\hline SRF-01-05 & 117022 & $\mathrm{U}$ & 0.00184 & $\mathrm{pCi} / \mathrm{g}$ & 0.0993 & EPIA-013B & \\
\hline SRF-02-01 & 117023 & $\mathrm{U}$ & 0.0612 & $\mathrm{pCi} / \mathrm{g}$ & 0.138 & EPIA-013B & \\
\hline SRF-02-02 & 117024 & $\mathrm{U}$ & 0.0125 & $\mathrm{pCi} / \mathrm{g}$ & 0.0955 & EPIA-013B & \\
\hline SRF-02-03 & 117025 & $\mathrm{U}$ & 0.00407 & $\mathrm{pCi} / \mathrm{g}$ & 0.164 & EPIA-013B & \\
\hline SRF-02-03-A & 117026 & $\mathrm{U}$ & 0.0143 & $\mathrm{pCi} / \mathrm{g}$ & 0.143 & EPIA-013B & \\
\hline SRF-03-01 & 117027 & $\mathrm{U}$ & 0.0463 & $\mathrm{pCi} / \mathrm{g}$ & 0.136 & EPIA-013B & \\
\hline SRF-03-02 & 117028 & $\mathrm{U}$ & 0.0159 & $\mathrm{pCi} / \mathrm{g}$ & 0.109 & EPIA-013B & \\
\hline SRF-03-03 & 117029 & $\mathrm{U}$ & -0.079 & $\mathrm{pCi} / \mathrm{g}$ & 0.109 & EPIA-013B & \\
\hline SRF-03-04 & 117030 & $\mathrm{U}$ & 0.00882 & $\mathrm{pCi} / \mathrm{g}$ & 0.0676 & EPIA-013B & \\
\hline SRF-04-01 & 117031 & $\mathrm{U}$ & 0.0125 & $\mathrm{pCi} / \mathrm{g}$ & 0.117 & EPIA-013B & \\
\hline SRF-04-02 & 117032 & $\mathrm{U}$ & 0.0651 & $\mathrm{pCi} / \mathrm{g}$ & 0.173 & EPIA-013B & \\
\hline SRF-04-03 & 117033 & $\mathrm{U}$ & -0.0114 & $\mathrm{pCi} / \mathrm{g}$ & 0.139 & EPIA-013B & \\
\hline SRF-05-01 & 117034 & $\mathrm{U}$ & -0.00215 & $\mathrm{pCi} / \mathrm{g}$ & 0.109 & EPIA-013B & \\
\hline SRF-05-02 & 117035 & $\mathrm{U}$ & -0.00859 & $\mathrm{pCi} / \mathrm{g}$ & 0.127 & EPIA-013B & \\
\hline SRF-05-03 & 117036 & $\mathrm{U}$ & 0.00296 & $\mathrm{pCi} / \mathrm{g}$ & 0.119 & EPIA-013B & \\
\hline SRF-06-01 & 117037 & $\mathrm{U}$ & 0.0643 & $\mathrm{pCi} / \mathrm{g}$ & 0.154 & EPIA-013B & \\
\hline SRF-06-02 & 117038 & $\mathrm{U}$ & 0.0417 & $\mathrm{pCi} / \mathrm{g}$ & 0.0947 & EPIA-013B & \\
\hline SRF-06-03 & 117039 & $\mathrm{U}$ & 0.0122 & $\mathrm{pCi} / \mathrm{g}$ & 0.136 & EPIA-013B & \\
\hline SRF-06-03-A & 117040 & $\mathrm{U}$ & 0.0199 & $\mathrm{pCi} / \mathrm{g}$ & 0.147 & EPIA-013B & \\
\hline SRF-07-01 & 117041 & $\mathrm{U}$ & 0.054 & $\mathrm{pCi} / \mathrm{g}$ & 0.184 & EPIA-013B & \\
\hline SRF-07-02 & 117042 & $\mathrm{U}$ & 0.13 & $\mathrm{pCi} / \mathrm{g}$ & 0.188 & EPIA-013B & \\
\hline SRF-07-03 & 117043 & $\mathrm{U}$ & -0.0442 & $\mathrm{pCi} / \mathrm{g}$ & 0.101 & EPIA-013B & \\
\hline SRF-07-03 & 117043 & $\mathrm{U}$ & -0.0093 & $\mathrm{pCi} / \mathrm{g}$ & 0.176 & EPIA-013B & \\
\hline
\end{tabular}


Table D.9 Results for Blanks Sorted by Analyte

ANALYTE: Acetone

Analytical Group: Volatile Organic Compounds

$\begin{array}{lllrlrll}\text { Survey ID } & \text { Sample ID } & \text { FG } & \text { Result } & \text { Unit } & \text { ssMDL } & \text { Method } & \text { SC } \\ \text { SRF-TB-01-B } & 117044 & \text { U } & 5 & \mu \mathrm{g} / \mathrm{L} & 3.7 & \text { EPA8260B } \\ \text { SRF-TB-02-B } & 117045 & \mathrm{U} & 5 & \mu \mathrm{g} / \mathrm{L} & 3.7 & \text { EPA8260B } & \text { EPA8260B }\end{array}$

ANALYTE: Benzene

Analytical Group: Volatile Organic Compounds

$\begin{array}{lllclccc}\text { Survey ID } & \text { Sample ID } & \text { FG } & \text { Result } & \text { Unit } & \text { ssMDL } & \text { Method } & \text { SC } \\ \text { SRF-TB-01-B } & 117044 & \mathrm{U} & 1 & \mu \mathrm{g} / \mathrm{L} & 0.3 & \text { EPA8260B } & \\ \text { SRF-TB-02-B } & 117045 & \mathrm{U} & 1 & \mu \mathrm{g} / \mathrm{L} & 0.3 & \text { EPA8260B } & \\ \text { SRF-TB-03-B } & 117046 & \mathrm{U} & 1 & \mu \mathrm{g} / \mathrm{L} & 0.3 & \text { EPA8260B }\end{array}$

ANALYTE: Bromodichloromethane

Analytical Group: Volatile Organic Compounds

\begin{tabular}{|c|c|c|c|c|c|c|c|}
\hline Survey ID & Sample ID & FG & Result & Unit & ssMDL & Method & SC \\
\hline SRF-TB-01-B & 117044 & $\mathrm{U}$ & 1 & $\mu g / L$ & 0.4 & EPA8260B & \\
\hline SRF-TB-02-B & 117045 & $\mathrm{U}$ & 1 & $\mu g / L$ & 0.4 & EPA8260B & \\
\hline SRF-TB-03-B & 117046 & $\mathrm{U}$ & 1 & $\mu \mathrm{g} / \mathrm{L}$ & 0.4 & ЕРA8260B & \\
\hline
\end{tabular}

ANALYTE: Bromoform

Analytical Group: Volatile Organic Compounds

$\begin{array}{lllrlcrc}\text { Survey ID } & \text { Sample ID } & \text { FG } & \text { Result } & \text { Unit } & \text { ssMDL } & \text { Method } & \text { SC } \\ \text { SRF-TB-01-B } & 117044 & \mathrm{U} & 1 & \mu \mathrm{g} / \mathrm{L} & 0.4 & \text { EPA8260B } \\ \text { SRF-TB-02-B } & 117045 & \mathrm{U} & 1 & \mu \mathrm{g} / \mathrm{L} & 0.4 & \text { EPA8260B } & \\ \text { SRF-TB-03-B } & 117046 & \mathrm{U} & 1 & \mu \mathrm{g} / \mathrm{L} & 0.4 & \text { EPA8260B }\end{array}$

ANALYTE: Bromomethane (Methyl bromide)

Analytical Group: Volatile Organic Compounds

$\begin{array}{lllclccc}\text { Survey ID } & \text { Sample ID } & \text { FG } & \text { Result } & \text { Unit } & \text { ssMDL } & \text { Method } & \text { SC } \\ \text { SRF-TB-01-B } & 117044 & \mathrm{U} & 1 & \mu \mathrm{g} / \mathrm{L} & 0.3 & \text { EPA8260B } & \\ \text { SRF-TB-02-B } & 117045 & \mathrm{U} & 1 & \mu \mathrm{g} / \mathrm{L} & 0.3 & \text { EPA8260B } & \text { EPA8260B }\end{array}$

ANALYTE: Carbon disulfide

Analytical Group: Volatile Organic Compounds

$\begin{array}{lllrlccc}\text { Survey ID } & \text { Sample ID } & \text { FG } & \text { Result } & \text { Unit } & \text { ssMDL } & \text { Method } & \text { SC } \\ \text { SRF-TB-01-B } & 117044 & \text { U } & 5 & \mu \mathrm{g} / \mathrm{L} & 1.8 & \text { EPA8260B } & \\ \text { SRF-TB-02-B } & 117045 & \mathrm{U} & 5 & \mu \mathrm{g} / \mathrm{L} & 1.8 & \text { EPA8260B } & \end{array}$


ANALYTE: Carbon disulfide (cont.)

$\begin{array}{lllrrrrrr}\text { Survey ID } & \text { Sample ID } & \text { FG } & \text { Result } & \text { Unit } & \text { ssMDL } & \text { Method } & \text { SC } & \text { E } \\ \text { SRF-TB-03-B } & 117046 & \text { U } & 5 & \mu \mathrm{g} / \mathrm{L} & 1.8 & \text { EPA8260B } & \end{array}$

ANALYTE: Carbon tetrachloride

Analytical Group: Volatile Organic Compounds

\begin{tabular}{|c|c|c|c|c|c|c|c|}
\hline Survey ID & Sample ID & $\mathbf{F G}$ & Result & Unit & SSMDL & Method & SC \\
\hline SRF-TB-01-B & 117044 & $\mathrm{U}$ & 1 & $\mu g / L$ & 0.2 & EPA8260B & \\
\hline SRF-TB-02-B & 117045 & $\mathrm{U}$ & 1 & $\mu g / L$ & 0.2 & EPA8260B & \\
\hline SRF-TB-03-B & 117046 & $\mathrm{U}$ & 1 & $\mu \mathrm{g} / \mathrm{L}$ & 0.2 & EPA8260B & \\
\hline
\end{tabular}

ANALYTE: Chlorobenzene

Analytical Group: Volatile Organic Compounds

$\begin{array}{lllrlcrc}\text { Survey ID } & \text { Sample ID } & \text { FG } & \text { Result } & \text { Unit } & \text { ssMDL } & \text { Method } & \text { SC } \\ \text { SRF-TB-01-B } & 117044 & \mathrm{U} & 1 & \mu \mathrm{g} / \mathrm{L} & 0.3 & \text { EPA8260B } & \\ \text { SRF-TB-02-B } & 117045 & \mathrm{U} & 1 & \mu \mathrm{g} / \mathrm{L} & 0.3 & \text { EPA8260B } & \text { EPA8260B }\end{array}$

ANALYTE: Chloroethane

Analytical Group: Volatile Organic Compounds

$\begin{array}{lllclccc}\text { Survey ID } & \text { Sample ID } & \text { FG } & \text { Result } & \text { Unit } & \text { ssMDL } & \text { Method } & \text { SC } \\ \text { SRF-TB-01-B } & 117044 & \mathrm{U} & 1 & \mu \mathrm{g} / \mathrm{L} & 0.3 & \text { EPA8260B } & \\ \text { SRF-TB-02-B } & 117045 & \mathrm{U} & 1 & \mu \mathrm{g} / \mathrm{L} & 0.3 & \text { EPA8260B } & \\ \text { SRF-TB-03-B } & 117046 & \mathrm{U} & 1 & \mu \mathrm{g} / \mathrm{L} & 0.3 & \text { EPA8260B }\end{array}$

ANALYTE: Chloroethene (Vinyl chloride)

Analytical Group: Volatile Organic Compounds

\begin{tabular}{|c|c|c|c|c|c|c|c|}
\hline Survey ID & Sample ID & FG & Result & Unit & SSMDL & Method & SC \\
\hline SRF-TB-01-B & 117044 & $\mathrm{U}$ & 1 & $\mu g / L$ & 0.4 & EPA8260B & \\
\hline SRF-TB-02-B & 117045 & $\mathrm{U}$ & 1 & $\mu g / L$ & 0.4 & EPA8260B & \\
\hline SRF-TB-03-B & 117046 & $\mathrm{U}$ & 1 & $\mu \mathrm{g} / \mathrm{L}$ & 0.4 & EPA8260B & \\
\hline
\end{tabular}

ANALYTE: Chloroform

Analytical Group: Volatile Organic Compounds

\begin{tabular}{|c|c|c|c|c|c|c|c|}
\hline Survey ID & Sample ID & FG & Result & Unit & SSMDL & Method & SC \\
\hline SRF-TB-01-B & 117044 & $\mathrm{U}$ & 1 & $\mu g / L$ & 0.7 & EPA8260B & \\
\hline SRF-TB-02-B & 117045 & $\mathrm{U}$ & 1 & $\mu g / L$ & 0.7 & EPA8260B & \\
\hline SRF-TB-03-B & 117046 & $\mathrm{U}$ & 1 & $\mu \mathrm{g} / \mathrm{L}$ & 0.7 & EPA8260B & \\
\hline
\end{tabular}

ANALYTE: Chloromethane (Methyl chloride)

Analytical Group: Volatile Organic Compounds

$\begin{array}{lllccccc}\text { Survey ID } & \text { Sample ID } & \text { FG } & \text { Result } & \text { Unit } & \text { ssMDL } & \text { Method } & \text { SC } \\ \text { SRF-TB-01-B } & 117044 & \mathrm{U} & 1 & \mu \mathrm{g} / \mathrm{L} & 0.2 & \text { EPA8260B } & \\ \text { SRF-TB-02-B } & 117045 & \mathrm{U} & 1 & \mu \mathrm{g} / \mathrm{L} & 0.2 & \text { EPA8260B } & \end{array}$




$\begin{array}{lllllll}\text { SRF-TB-03-B } & 117046 & \mathrm{U} & 1 & \mu \mathrm{g} / \mathrm{L} & 0.2 & \text { EPA8260B }\end{array}$

ANALYTE: Dibromochloromethane

Analytical Group: Volatile Organic Compounds

$\begin{array}{lllrlrlrl}\text { Survey ID } & \text { Sample ID } & \text { FG } & \text { Result } & \text { Unit } & \text { ssMDL } & \text { Method } & \text { SC } & \text { E } \\ \text { SRF-TB-01-B } & 117044 & \text { U } & 1 & \mu g / L & 0.3 & \text { EPA8260B } & \\ \text { SRF-TB-02-B } & 117045 & \text { U } & 1 & \mu g / L & 0.3 & \text { EPA8260B } & \\ \text { SRF-TB-03-B } & 117046 & \text { U } & 1 & \mu g / L & 0.3 & \text { EPA8260B } & \end{array}$

ANALYTE: 1,1-Dichloroethane

Analytical Group: Volatile Organic Compounds

$\begin{array}{lllrlrlrl}\text { Survey ID } & \text { Sample ID } & \text { FG } & \text { Result } & \text { Unit } & \text { ssMDL } & \text { Method } & \text { SC } & \text { E } \\ \text { SRF-TB-01-B } & 117044 & \mathrm{U} & 1 & \mu \mathrm{g} / \mathrm{L} & 0.4 & \text { EPA8260B } & \\ \text { SRF-TB-02-B } & 117045 & \mathrm{U} & 1 & \mu \mathrm{g} / \mathrm{L} & 0.4 & \text { EPA8260B } & \\ \text { SRF-TB-03-B } & 117046 & \mathrm{U} & 1 & \mu \mathrm{g} / \mathrm{L} & 0.4 & \text { EPA8260B } & \end{array}$

ANALYTE: 1,2-Dichloroethane

Analytical Group: Volatile Organic Compounds

$\begin{array}{lllrlrlrl}\text { Survey ID } & \text { Sample ID } & \text { FG } & \text { Result } & \text { Unit } & \text { ssMDL } & \text { Method } & \text { SC } & \text { E } \\ \text { SRF-TB-01-B } & 117044 & \mathrm{U} & 1 & \mu \mathrm{g} / \mathrm{L} & 0.2 & \text { EPA8260B } & \\ \text { SRF-TB-02-B } & 117045 & \mathrm{U} & 1 & \mu \mathrm{g} / \mathrm{L} & 0.2 & \text { EPA8260B } & \\ \text { SRF-TB-03-B } & 117046 & \mathrm{U} & 1 & \mu \mathrm{g} / \mathrm{L} & 0.2 & \text { EPA8260B } & \end{array}$

ANALYTE: cis-1,2-Dichloroethylene

Analytical Group: Volatile Organic Compounds

$\begin{array}{lllrlrrrr}\text { Survey ID } & \text { Sample ID } & \text { FG } & \text { Result } & \text { Unit } & \text { ssMDL } & \text { Method } & \text { SC } & \text { E } \\ \text { SRF-TB-01-B } & 117044 & \mathrm{U} & 1 & \mu \mathrm{g} / \mathrm{L} & 0.7 & \text { EPA8260B } & \\ \text { SRF-TB-02-B } & 117045 & \mathrm{U} & 1 & \mu \mathrm{g} / \mathrm{L} & 0.7 & \text { EPA8260B } & \\ \text { SRF-TB-03-B } & 117046 & \mathrm{U} & 1 & \mu \mathrm{g} / \mathrm{L} & 0.7 & \text { EPA8260B }\end{array}$

ANALYTE: 1,1-Dichloroethylene

Analytical Group: Volatile Organic Compounds

\begin{tabular}{|c|c|c|c|c|c|c|c|}
\hline Survey ID & Sample ID & FG & Result & Unit & ssMDL & Method & SC \\
\hline SRF-TB-01-B & 117044 & U & 1 & $\mu \mathrm{g} / \mathrm{L}$ & 0.7 & EPA8260B & \\
\hline SRF-TB-02-B & 117045 & U & 1 & $\mu \mathrm{g} / \mathrm{L}$ & 0.7 & EPA8260B & \\
\hline SRF-TB-03-B & 117046 & $\mathrm{U}$ & 1 & $\mu \mathrm{g} / \mathrm{L}$ & 0.7 & EPA8260B & \\
\hline \multicolumn{8}{|c|}{$\begin{array}{l}\text { ANALYTE: trans-1,2-Dichloroethylene } \\
\text { Analytical Group: Volatile Organic Compounds }\end{array}$} \\
\hline Survey ID & Sample ID & FG & Result & Unit & ssMDL & Method & SC \\
\hline SRF-TB-01-B & 117044 & $\mathrm{U}$ & 1 & $\mu \mathrm{g} / \mathrm{L}$ & 0.7 & EPA8260B & \\
\hline SRF-TB-02-B & 117045 & $\mathrm{U}$ & 1 & $\mu \mathrm{g} / \mathrm{L}$ & 0.7 & EPA8260B & \\
\hline SRF-TB-03-B & 117046 & $\mathrm{U}$ & 1 & $\mu \mathrm{g} / \mathrm{L}$ & 0.7 & EPA8260B & \\
\hline
\end{tabular}


ANALYTE: Dichloromethane (Methylene chloride)

Analytical Group: Volatile Organic Compounds

$\begin{array}{lllrlrrrr}\text { Survey ID } & \text { Sample ID } & \text { FG } & \text { Result } & \text { Unit } & \text { ssMDL } & \text { Method } & \text { SC } & \text { E } \\ \text { SRF-TB-01-B } & 117044 & \text { JU } & 4.16 & \mu \mathrm{g} / \mathrm{L} & 1.2 & \text { EPA8260B } & \text { IV } \\ \text { SRF-TB-02-B } & 117045 & \text { JU } & 3.99 & \mu g / L & 1.2 & \text { EPA8260B } & \text { IV } \\ \text { SRF-TB-03-B } & 117046 & \text { JU } & 3.39 & \mu g / L & 1.2 & \text { EPA8260B } & \text { IV } & \end{array}$

ANALYTE: 1,2-Dichloropropane

Analytical Group: Volatile Organic Compounds

$\begin{array}{lllrlrlrl}\text { Survey ID } & \text { Sample ID } & \text { FG } & \text { Result } & \text { Unit } & \text { ssMDL } & \text { Method } & \text { SC } & \text { E } \\ \text { SRF-TB-01-B } & 117044 & \mathrm{U} & 1 & \mu \mathrm{g} / \mathrm{L} & 0.2 & \text { EPA8260B } & \\ \text { SRF-TB-02-B } & 117045 & \mathrm{U} & 1 & \mu \mathrm{g} / \mathrm{L} & 0.2 & \text { EPA8260B } & \\ \text { SRF-TB-03-B } & 117046 & \mathrm{U} & 1 & \mu \mathrm{g} / \mathrm{L} & 0.2 & \text { EPA8260B } & \end{array}$

ANALYTE: cis-1,3-Dichloropropene

Analytical Group: Volatile Organic Compounds

$\begin{array}{lllrlrrrr}\text { Survey ID } & \text { Sample ID } & \text { FG } & \text { Result } & \text { Unit } & \text { ssMDL } & \text { Method } & \text { SC } & \text { E } \\ \text { SRF-TB-01-B } & 117044 & \mathrm{U} & 1 & \mu \mathrm{g} / \mathrm{L} & 0.3 & \text { EPA8260B } & \\ \text { SRF-TB-02-B } & 117045 & \mathrm{U} & 1 & \mu \mathrm{g} / \mathrm{L} & 0.3 & \text { EPA8260B } & \\ \text { SRF-TB-03-B } & 117046 & \mathrm{U} & 1 & \mu \mathrm{g} / \mathrm{L} & 0.3 & \text { EPA8260B } & \end{array}$

ANALYTE: trans-1,3-Dichloropropene

Analytical Group: Volatile Organic Compounds

$\begin{array}{lllrlrrrr}\text { Survey ID } & \text { Sample ID } & \text { FG } & \text { Result } & \text { Unit } & \text { ssMDL } & \text { Method } & \text { SC } & \text { E } \\ \text { SRF-TB-01-B } & 117044 & \mathrm{U} & 1 & \mu \mathrm{g} / \mathrm{L} & 0.3 & \text { EPA8260B } & \\ \text { SRF-TB-02-B } & 117045 & \mathrm{U} & 1 & \mu \mathrm{g} / \mathrm{L} & 0.3 & \text { EPA8260B } & \\ \text { SRF-TB-03-B } & 117046 & \mathrm{U} & 1 & \mu \mathrm{g} / \mathrm{L} & 0.3 & \text { EPA8260B } & \end{array}$

ANALYTE: Ethylbenzene

Analytical Group: Volatile Organic Compounds

$\begin{array}{lllrlrlrl}\text { Survey ID } & \text { Sample ID } & \text { FG } & \text { Result } & \text { Unit } & \text { ssMDL } & \text { Method } & \text { SC } & \text { E } \\ \text { SRF-TB-01-B } & 117044 & \mathrm{U} & 1 & \mu \mathrm{g} / \mathrm{L} & 0.3 & \text { EPA8260B } & \\ \text { SRF-TB-02-B } & 117045 & \mathrm{U} & 1 & \mu \mathrm{g} / \mathrm{L} & 0.3 & \text { EPA8260B } & \\ \text { SRF-TB-03-B } & 117046 & \mathrm{U} & 1 & \mu \mathrm{g} / \mathrm{L} & 0.3 & \text { EPA8260B } & \end{array}$

ANALYTE: 2-Hexanone

Analytical Group: Volatile Organic Compounds

$\begin{array}{lllrlrlrl}\text { Survey ID } & \text { Sample ID } & \text { FG } & \text { Result } & \text { Unit } & \text { ssMDL } & \text { Method } & \text { SC } & \text { E } \\ \text { SRF-TB-01-B } & 117044 & \mathrm{U} & 5 & \mu \mathrm{g} / \mathrm{L} & 3.2 & \text { EPA8260B } & \\ \text { SRF-TB-02-B } & 117045 & \mathrm{U} & 5 & \mu \mathrm{g} / \mathrm{L} & 3.2 & \text { EPA8260B } & \\ \text { SRF-TB-03-B } & 117046 & \mathrm{U} & 5 & \mu \mathrm{g} / \mathrm{L} & 3.2 & \text { EPA8260B } & \end{array}$


ANALYTE: Methyl ethyl ketone

Analytical Group: Volatile Organic Compounds

$\begin{array}{lllrlcrc}\text { Survey ID } & \text { Sample ID } & \text { FG } & \text { Result } & \text { Unit } & \text { ssMDL } & \text { Method } & \text { SC } \\ \text { SRF-TB-01-B } & 117044 & \text { U } & 10 & \mu \mathrm{g} / \mathrm{L} & 5.9 & \text { EPA8260B } \\ \text { SRF-TB-02-B } & 117045 & \mathrm{U} & 10 & \mu \mathrm{g} / \mathrm{L} & 5.9 & \text { EPA8260B } & \\ \text { SRF-TB-03-B } & 117046 & \mathrm{U} & 10 & \mu \mathrm{g} / \mathrm{L} & 5.9 & \text { EPA8260B }\end{array}$

ANALYTE: Methyl isobutyl ketone

Analytical Group: Volatile Organic Compounds

$\begin{array}{lllrlcrc}\text { Survey ID } & \text { Sample ID } & \text { FG } & \text { Result } & \text { Unit } & \text { ssMDL } & \text { Method } & \text { SC } \\ \text { SRF-TB-01-B } & 117044 & \mathrm{U} & 5 & \mu \mathrm{g} / \mathrm{L} & 1.6 & \text { EPA8260B } \\ \text { SRF-TB-02-B } & 117045 & \mathrm{U} & 5 & \mu \mathrm{g} / \mathrm{L} & 1.6 & \text { EPA8260B } & \\ \text { SRF-TB-03-B } & 117046 & \mathrm{U} & 5 & \mu \mathrm{g} / \mathrm{L} & 1.6 & \text { EPA8260B }\end{array}$

ANALYTE: Styrene

Analytical Group: Volatile Organic Compounds

$\begin{array}{lllclclc}\text { Survey ID } & \text { Sample ID } & \text { FG } & \text { Result } & \text { Unit } & \text { ssMDL } & \text { Method } & \text { SC } \\ \text { SRF-TB-01-B } & 117044 & \mathrm{U} & 1 & \mu \mathrm{g} / \mathrm{L} & 0.2 & \text { EPA8260B } & \\ \text { SRF-TB-02-B } & 117045 & \mathrm{U} & 1 & \mu \mathrm{g} / \mathrm{L} & 0.2 & \text { EPA8260B } & \text { EPA8260B }\end{array}$

ANALYTE: 1, 1,2,2-Tetrachloroethane

Analytical Group: Volatile Organic Compounds

$\begin{array}{lllrlrll}\text { Survey ID } & \text { Sample ID } & \text { FG } & \text { Result } & \text { Unit } & \text { ssMDL } & \text { Method } & \text { SC } \\ \text { SRF-TB-01-B } & 117044 & \mathrm{U} & 1 & \mu \mathrm{g} / \mathrm{L} & 0.5 & \text { EPA8260B } & \\ \text { SRF-TB-02-B } & 117045 & \mathrm{U} & 1 & \mu \mathrm{g} / \mathrm{L} & 0.5 & \text { EPA8260B } & \\ \text { SRF-TB-03-B } & 117046 & \mathrm{U} & 1 & \mu \mathrm{g} / \mathrm{L} & 0.5 & \text { EPA8260B }\end{array}$

ANALYTE: Tetrachloroethylene

Analytical Group: Volatile Organic Compounds

\begin{tabular}{|c|c|c|c|c|c|c|c|}
\hline Survey ID & Sample ID & $\mathbf{F G}$ & Result & Unit & SSMDL & Method & SC \\
\hline SRF-TB-01-B & 117044 & $\mathrm{U}$ & 1 & $\mu g / L$ & 0.7 & EPA8260B & \\
\hline SRF-TB-02-B & 117045 & $\mathrm{U}$ & 1 & $\mu g / L$ & 0.7 & EPA8260B & \\
\hline SRF-TB-03-B & 117046 & $\mathrm{U}$ & 1 & $\mu g / L$ & 0.7 & EPA8260B & \\
\hline
\end{tabular}

ANALYTE: Toluene

Analytical Group: Volatile Organic Compounds

\begin{tabular}{|c|c|c|c|c|c|c|c|}
\hline Survey ID & Sample ID & FG & Result & Unit & SSMDL & Method & SC \\
\hline SRF-TB-01-B & 117044 & $\mathrm{U}$ & 1 & $\mu g / L$ & 0.5 & EPA8260B & \\
\hline SRF-TB-02-B & 117045 & $\mathrm{U}$ & 1 & $\mu g / L$ & 0.5 & EPA8260B & \\
\hline SRF-TB-03-B & 117046 & $\mathrm{U}$ & 1 & $\mu \mathrm{g} / \mathrm{L}$ & 0.5 & EPA8260B & \\
\hline
\end{tabular}


ANALYTE: 1,1,1-Trichloroethane

Analytical Group: Volatile Organic Compounds

$\begin{array}{lllclclc}\text { Survey ID } & \text { Sample ID } & \text { FG } & \text { Result } & \text { Unit } & \text { ssMDL } & \text { Method } & \text { SC } \\ \text { SRF-TB-01-B } & 117044 & \mathrm{U} & 1 & \mu \mathrm{g} / \mathrm{L} & 0.2 & \text { EPA8260B } & \text { EPA8260B } \\ \text { SRF-TB-02-B } & 117045 & \mathrm{U} & 1 & \mu \mathrm{g} / \mathrm{L} & 0.2 & \text { EPA8260B }\end{array}$

ANALYTE: 1,1,2-Trichloroethane

Analytical Group: Volatile Organic Compounds

\begin{tabular}{|c|c|c|c|c|c|c|c|}
\hline Survey ID & Sample ID & FG & Result & Unit & SSMDL & Method & SC \\
\hline SRF-TB-01-B & 117044 & $\mathrm{U}$ & 1 & $\mu g / L$ & 0.4 & EPA8260B & \\
\hline SRF-TB-02-B & 117045 & $\mathrm{U}$ & 1 & $\mu \mathrm{g} / \mathrm{L}$ & 0.4 & EPA8260B & \\
\hline SRF-TB-03-B & 117046 & $\mathrm{U}$ & 1 & $\mu \mathrm{g} / \mathrm{L}$ & 0.4 & EPA8260B & \\
\hline
\end{tabular}

ANALYTE: Trichloroethylene

Analytical Group: Volatile Organic Compounds

$\begin{array}{lllrlcrl}\text { Survey ID } & \text { Sample ID } & \text { FG } & \text { Result } & \text { Unit } & \text { ssMDL } & \text { Method } & \text { SC } \\ \text { SRF-TB-01-B } & 117044 & \mathrm{U} & 1 & \mu \mathrm{g} / \mathrm{L} & 0.6 & \text { EPA8260B } \\ \text { SRF-TB-02-B } & 117045 & \mathrm{U} & 1 & \mu \mathrm{g} / \mathrm{L} & 0.6 & \text { EPA8260B } & \\ \text { SRF-TB-03-B } & 117046 & \mathrm{U} & 1 & \mu \mathrm{g} / \mathrm{L} & 0.6 & \text { EPA8260B }\end{array}$

ANALYTE: Xylenes

Analytical Group: Volatile Organic Compounds

$\begin{array}{lllclccc}\text { Survey ID } & \text { Sample ID } & \text { FG } & \text { Result } & \text { Unit } & \text { ssMDL } & \text { Method } & \text { SC } \\ \text { SRF-TB-01-B } & 117044 & \mathrm{U} & 2 & \mu \mathrm{g} / \mathrm{L} & 1.1 & \text { EPA8260B } & \\ \text { SRF-TB-02-B } & 117045 & \mathrm{U} & 2 & \mu \mathrm{g} / \mathrm{L} & 1.1 & \text { EPA8260B } & \\ \text { SRF-TB-03-B } & 117046 & \mathrm{U} & 2 & \mu \mathrm{g} / \mathrm{L} & 1.1 & \text { EPA8260B } & \end{array}$


This page was left blank intentionally. 


\section{Appendix E: Data Management/Administrative Files}

This section discusses the generation, processing, and storage of paper and computer records produced during the completion of this project. The processing of the materials follows methodology prescribed by EGG EPD/EMS.

EGG establishes administrative files for each project that are maintained as permanent SRS records. The following materials are placed in the administrative files:

- Bound sample-collection logbooks

- Original copies of the chain-of-custody forms

- Case narratives from the laboratories

- Data-validation logbooks

- Data-entry cover sheets for data files created by EGG

- Copies of all correspondence between data validation personnel and the laboratories

EGG generates computer data files from paper records using double-entry methods to ensure a low transcription error rate. Computer data files for analytical data also are received from the laboratories. Changes to the data are recorded in the data-validation logbook, approved by the project technical manager, and confirmed through a computer program by comparing the modified and original data files. EGG stores the final computer data files, original source files, and computer listings of all changes made to the original files in the administrative files.

Access to the administrative files remains with the manager of EGG. Paper materials eventually will be transferred to permanent box storage; however, EGG plans to make the final data files available to SRS computer users indefinitely. Please contact the manager of EGG to obtain access to this data. 
This page was left blank intentionally. 


\section{Appendix F: Glossary}

Acceptance limits: The range of acceptable analytical results established by a laboratory for spike recovery, duplicate precision, and contamination levels.

Accuracy: The closeness of agreement between an observed value and an accepted reference value.

Activity: The rate of decay of a radioactive source at a given time.

Advisory range: The limits of acceptable analytical results established by the provider of certified quality control standards.

Aliquot: A measured portion of a sample taken for analysis.

Analysis: The separation and determination of the component parts or a specific property or element contained within a sample. The determination of the concentration or activity of an analyte contained within a sample.

Analysis date/time: The date and military time (24-hour clock) of the start of analysis on a prepared sample.

Analyte: The specific compound, element, isotope, or radionuclide of interest that an analyst seeks to determine.

Analytical batch: A group of samples of the same matrix analyzed together using the same method and containing the required number of method blanks, matrix spike samples, laboratory control samples, and duplicate samples.

Analytical narrative: A portion of the data package that includes laboratory, contract, and sample identification. The analytical narrative also contains descriptive documentation of any problems encountered in processing the samples, along with corrective action taken and problems resolved.

Analytical sample: Any solution or medium introduced into an instrument on which an analysis is performed, excluding instrument calibration standards, calibration verification standards, and calibration blanks.

Appendix IX analytes: The list of analytes specified in Appendix IX of theCode of Federal Regulations, Title 40, Part 264 that is required by the Resource Conservation and Recovery Act.

Aquifer: A geologic formation, group of formations, or part of a formation capable of yielding groundwater to wells or springs.

Associated sample: Any sample that is related to another sample or quality control sample. Some examples are the parent sample of a matrix spike, a sample in a preparation batch, a split sample, a duplicate sample, and a sample related to a blank.

Batch: A group of samples prepared at the same time, in the same location, using the same method.

Becquerel: One Becquerel is a rate of one disintegration per second and is equal to $2.7 \times 10^{-1}$ Curie. 
Blank: An artificial sample designed to monitor the introduction of artifacts into the measurement process. For aqueous samples, deionized water is used as the blank matrix. A universal matrix does not exist for solid samples; therefore, no matrix blank is routinely used. There are several types of blanks that monitor a variety of processes.

- A Field Blank for Soils consists of DI water or dry quartz sand placed in a stainless steel bowl and exposed to the atmosphere while the associated sample is being taken. The field blank is shipped to the laboratory with the samples. The field blank measures contamination introduced during sample collection.

- A Field Blank for Groundwater is a sample container filled with DI water and capped in the WSRC well building. It is taken to the field during sampling, but is not opened. It is shipped to the laboratory with the samples to be analyzed.

- A Rinsate or Equipment Blank is deionized water poured over or through sample collection devices that tests for cleanliness of sampling equipment.

- A Trip Blank is shipped to the laboratory in the same cooler with the sample containers for volatile organic analytes. It provides a test for contamination from sample preservation and transport as well as sample storage, preparation, and analysis.

- A Method Blank is prepared in the laboratory and analyzed exactly as the samples it accompanies. It is a test for contamination in laboratory sample preparation and analyses.

These blanks are handled by the laboratory as actual samples; however, they are not to be used for matrix spike or duplicate samples.

\section{Blank spike (BS): See Laboratory Control Sample.}

Calibration: The establishment of an instrument response curve or mathematical correlation based on the measured response of known concentrations of an analyte or group of analytes.

Calibration blank: A volume of deionized water in the same matrix as the calibration standards, an empty planchet, or the instrument geometry that is analyzed to establish the instrument accuracy at the low end of the calibration curve.

Calibration verification: The periodic analysis of one or more standards independent of the calibration standards to verify the accuracy and stability of the initial instrument calibration.

Cation exchange capacity: The sum of exchangeable cations a soil can absorb expressed in milliequivalents per 100 grams of soil. The cation exchange capacity is determined by sampling to the depth of solid waste placement and analyzing by the summation method for distinctly acid soils or the sodium acetate method for neutral, calcareous, or saline soils.

Certified value: The known concentration of an analyte in a reference sample as affirmed by the preparer.

Chain-of-custody (COC) form: A document designed to trace the custody of a sample(s) from the point of origin to final disposition, with the intent of legally proving that custody remained intact and that tampering or substitutions were precluded.

Codes: Qualification codes, or modifiers, are used to convey additional information about an analytical result in data format AN98.

- Functional Guideline (FG) Codes describe the analytical result. 
- STORET Codes describe issues arising during the analytical process.

- EMS Codes contain additional information to aid the data validators in qualifyng the data.

See Appendix $D$ for additional information and the specific qualifier codes used.

Coefficient of variation (CV): The standard deviation as a percent of the arithmetic mean.

Comparability: The degree of confidence with which one set of data can be compared to a related set of data.

Completeness: A measure of the amount of valid data obtained from a measurement system relative to the amount that was expected to be obtained under current, normal conditions.

Comprehensive Environmental Response, Compensation, and Liability Act (CERCLA):A federal law passed in 1980 and periodically modified by the Superfund Amendments and Reauthorization Acts passed by the U.S. Congress. The acts created a special tax that goes into a trust fund, commonly known as Superfund, to investigate and clean up abandoned or uncontrolled hazardous waste sites. Under the program, the EPA has the following options:

- $\quad$ pay for the site cleanup when parties responsible for the contamination cannot be located or are unwilling or unable to perform the work

- take legal action to force parties responsible for site contamination to clean up the site or repay the federal government for the cost of the cleanup

Concentration: The relative fraction of one substance in another, normally expressed in weight percent, in volume percent, as a weight per volume ratio, or as a weight per weight ratio.

Confidence limits: The limits within which, at some specified level of probability, the true value of a result lies.

Confined aquifer: An aquifer bounded above and below by impermeable beds or by beds of distinctly lower permeability than the aquifer itself. Also, an aquifer containing confined groundwater.

Congener: Any one particular member of a class of chemical substances. A specific congener is denoted by a unique chemical structure e.g., 2,3,7,8-tetrachlorodibenzofuran.

Contaminant: A chemical or biological substance that is harmful if it is incorporated into, onto, or ingested by organisms, consumers of organisms, or users of the environment.

Continuing calibration blank (CCB): A blank analyzed periodically during an analytical run to detect carryover contamination.

Continuing calibration verification (CCV): The periodic analysis of one or more standards during an analytical run to verify that the initial calibration continues to be valid.

Contract-required quantitation limit (CRQL): The lowest required concentration of an analyte the laboratory may report for a specific contract. The contract-required quantitation limit is usually greater than the method detection limit.

Control limits: A range within which specified measurement results must fall to be compliant. Control limits may be mandatory, requiring corrective action if exceeded, or advisory, requiring that noncompliant data be flagged. 
Correlation coefficient (r): A numeric value (r) that indicates the degree of dependence between two variables (concentration vs. response). The more dependent they are, the closer the value is to one. The correlation coefficient is determined on the basis of the least squares function.

Custody: Immediate charge, control, or possession exercised by a competent authority on a sample.

Data quality objectives: The objectives for precision, accuracy, representativeness, completeness, comparability, and detection levels that are required to support the intended use of the data.

Definitive data: Data for which the accuracy of the concentration level and the identity of the analyte of interest are assessed. Definitive data are determined for samples considered essential in the decisionmaking process.

Deionized (DI) water: Water from which all charged species or ionizable organic and inorganic salts are removed by means of ion exchange.

Detection: The act of measuring the quantity of a property, compound, or element contained in a sample.

Dilution factor: The mathematical factor by which a sample is diluted to bring the concentration of an analyte in the sample within the analytical range of the instrument (e.g., $1 \mathrm{~mL}$ of sample $+9 \mathrm{~mL}$ of solvent $=1: 10$ dilution, or a dilution factor of 10 ).

Dry weight: The weight of a sample based on percent solids or the weight after drying in an oven for a specified time period at a specified temperature.

Electronic data deliverable (EDD): The summary of analytical results on machine- readable media received from a laboratory.

EMS Code: See Codes.

Environmental sample record: Any groundwater, soil, surface water, vegetation, trip blank, or field blank sample record. Laboratory duplicate records are not considered environmental sample records.

Estimated quantitation limit (EQL): The minimum concentration of a substance that can be reliably measured and reported within specified limits of precision and accuracy during routine laboratory operating conditions.

FG Code: See Codes.

Field blank: See Blank

Field duplicate: A second sample taken at the same time as the primary sample and sent to the laboratory for analysis as an unknown. The field duplicate is used to determine the precision of the entire sampling and analytical process.

Field sample: The material received at the laboratory to be analyzed that is contained in single or multiple containers and identified by a unique WSRC sample identification number.

Frequency: The specified intervals between samples in which quality control samples or standards must be analyzed during an analytical run.

Functional Guideline Code (FG Code): See Codes.

Groundwater: Water in a saturated zone or stratum beneath the surface of land. 
Half-life: The time required for one-half of the initial number of radioactive nuclei of a specific isotopic species to undergo radioactive decay.

Holding time: The maximum amount of time allowed for samples to be held from sample collection to laboratory analysis.

Independent standard: A laboratory-prepared standard solution that is composed of analytes from a different source than those used in the standards for the initial calibration.

Initial calibration: The analysis of standards containing varying concentration levels of the analytes of interest in order to establish the ratio of concentration vs. response across the working range of the analytical technique. The initial calibration is used to define the linearity and dynamic range of response of the detector to the target analytes.

Initial calibration blank (ICB): A blank analyzed immediately after the initial calibration verification but before the analytical run to detect carryover contamination.

Initial calibration verification (ICV): The analysis of one or more standards immediately after the initial calibration but before the analytical run to verify that the initial calibration was valid.

Instrument detection limit (IDL): The minimum concentration of an analyte that can be measured and reported with $99 \%$ confidence that the analyte concentration is greater than zero. It is determined from analysis of a deionized water blank containing the analyte.

Interference check sample (ICS): Two solutions, one consisting of interferents only (ICSA) and one consisting of interferents mixed with the analytes (ICSAB), that are analyzed consecutively to verify the interelement and background correction factors for inductively coupled plasma metal analyses.

Interferents: Substances that affect the analysis for the element of interest.

Interlaboratory comparison: The comparison of results from split samples analyzed by two laboratories.

Internal standard: A chemically inert compound not expected to occur in an environ-mental sample that is added to a sample prior to analysis; it is used as an instrument response check.

Intralaboratory comparison: The comparison of duplicate results from a single sample analyzed by a laboratory.

Isomer: One of two or more chemical substances having the same elementary percentage composition and molecular weight but differing in structure and properties.

Isotope: One of a number of specific atoms with identical atomic numbers but with discrete atomic weights, or similarly specific atoms whose nuclei have the same number of protons but different numbers of neutrons.

Laboratory control sample (LCS): A control sample of known composition. Aqueous and solid laboratory control samples are analyzed using the same sample preparation, reagents, and analytical methods employed for the samples received.

Laboratory data records review (LDRR): The investigation of technical data validation issues that are not adequately addressed by the computer checking of EDDs, the review of the analytical narratives, and the review of the COC forms. The review is conducted at the laboratory using the laboratory's instrument logs, raw analytical data, and other pertinent documentation. 
Laboratory duplicate: A second aliquot of a homogenized sample that is analyzed as an individual sample, using the same procedure. This duplicate is used to determine the precision of the method.

Laboratory ID: The identifier assigned by the laboratory for its internal use during the analytical process.

Linear range, linear dynamic range:The concentration range over which the calibration curve remains linear.

Lowest practical quantitation limit (LPQL): The lowest concentration of an analyte that can be reliably achieved within specified limits of precision and accuracy during routine laboratory operating conditions.

Matrix: The predominant material of which the sample to be analyzed is composed. For the purpose of this document, a sample matrix is either water or soil/sediment.

Matrix interference: The influence of the sample matrix or sample components upon the ability to quantitatively measure compounds in environmental samples.

Matrix spike/matrix spike duplicate: A first and second aliquot of a matrix fortified (spiked) with a known quantity of analyte(s) and subjected to the entire analytical procedure in order to determine the appropriateness of the method for the matrix by measuring accuracy (recovery) and precision (relative percent difference).

Maximum contaminant level (MCL): The maximum permissible level of a contaminant in a specified medium.

Mean relative difference (MRD): The average of the absolute difference of the results from two laboratories for the $i^{\text {th }}$ sample that is expressed as a percentage of the mean of both laboratories.

\section{Method blank: See Blank.}

Method detection limit (MDL): The minimum concentration of an analyte that can be measured and reported with $99 \%$ confidence that the analyte concentration is greater than zero. It is determined from analysis of a sample in a given matrix containing the analyte.

Minimum detectable activity (MDA): The smallest quantity of a radionuclide that can be detected in a sample with a $95 \%$ confidence level.

Nuclide: General term applied to all isotopes of all elements including stable and radio-active forms. Nuclides are not considered isotopes; rather, a given nuclide is characterized by the number of neutrons and protons contained in the atomic nuclei of that species.

Null hypothesis: A statement that can be tested statistically to determine if there is a significant difference between duplicate/split results.

Parts per billion (ppb)/Parts per million (ppm): Units commonly used to express low concentrations of contaminants. For example, 5 micrograms of benzene in 1 kilogram of soil is $5 \mathrm{ppb} ; 3$ milligrams of nitrate in 1 liter of water is 3 ppm.

Percent moisture: An approximation of the amount of water in a soil/sediment sample determined by drying an aliquot of sample at a specified temperature until constant weight is achieved.

Percent recovery: A measure of recovery that is calculated as the measured value relative to the true value, expressed as a percent. 
Percent solids: The proportion of solid in a soil/sediment sample determined by the percent moisture procedure.

Performance evaluation sample: A sample of known composition that may be provided by the EPA or WSRC for laboratory analysis that is used to evaluate laboratory performance.

PicoCurie (pCi): The unit of radiation equivalent to $3.7 \times 10^{2}$ disintegrations per second.

Precision: The agreement or repeatability of a set of replicate results among themselves, usually expressed in terms of the deviation of a set of results from the arithmetic mean. Precision may be qualified in terms of possible sources of variability, replicability, repeatability, and reproducibility.

Preparation log: An official record of the sample preparation.

Preservative: A chemical compound added to a sample or a physical process (such as cooling) to prevent or slow decomposition or degradation of a target analyte. Physical and chemical preservation may be used in tandem to prevent sample deterioration.

Primary laboratory: The laboratory having a contract with WSRC to perform a specific set of analyses for field-generated samples, duplicates, and blanks. A primary laboratory may subcontract this work to other laboratories.

Qualitative analysis: An analysis to determine the presence or absence of a target analyte.

Quality assurance plan: The documented data quality objectives and the actions to be performed to meet those objectives.

Quality control (QC): The performance of actions that provide a way to control and measure the characteristics of measurement equipment and processes to meet the established data quality objectives.

Quantitative analysis: An analysis to measure or determine the amount of a target compound or analyte within the limits of defined precision and accuracy requirements.

Radionuclide: Any radioactive isotope of an element.

Range: The difference between the maximum and minimum values within a set of values.

RCRA Facility Investigation/Remedial Investigation (RFI/RI) program:The EPA-regulated investigation of a solid-waste management site, including CERCLA and hazardous-substance regulations, with regard to its potential impact on the environment.

Recovery: A determination of accuracy of the analytical procedure made by comparing measured values for a reference or fortified (spiked) sample against the known true reference or spike values.

Reference detection limit (RDL): The detection limit chosen to allow comparison of multiple analyses with different detection limits. For this report, the individual detection limits of at least $90 \%$ of the analyses are less than the reference detection limit.

Relative percent difference (RPD): A measure of precision that is calculated as the absolute value of the difference between two results, relative to their arithmetic mean, expressed as a percent.

Reporting limit: The value reported in the result field when an analyte is not detected. 
Reproducibility: The precision of measurements of the same sample at different laboratories using the same protocols.

Resolution: The degree to which two signal peaks are separated. Resolution is calculated by dividing the height of the valley between the peaks by the height of the peak being resolved, multiplied by 100 .

Resource Conservation and Recovery Act (RCRA): A 1976 federal law that established a regulatory system to define and track hazardous wastes from the time of generation to disposal. The law requires safe and secure procedures to be used in treating, transporting, storing, and disposing of hazardous substances.

\section{Rinsate blank: See Blank}

Run: A continuous analytical sequence consisting of prepared samples and all associated quality control measurements.

Sample: A portion of material to be analyzed that is contained in single or multiple containers and identified by a unique sample number.

Sample ID: A unique identification number designated by WSRC for each sample. The WSRC sample ID appears on the sample chain of custody that documents information on that sample.

Sample matrix: All of the chemical components and physical characteristics of a sample other than the parameter of interest.

Sample-specific estimated quantitation limit (ssEQL):The estimated quantitation limit multiplied by factors of concentration, dilution, aliquot size, and percent solids.

Sediment: The unconsolidated inorganic and organic material that is suspended in and being transported by surface water or that has settled out and has deposited into beds.

Self-absorption: The internal absorption of radiation by material in which radioactive atoms are located.

Sensitivity: The ability of a measurement system to detect and accurately quantitate a parameter at a critical level within a specific sample matrix. The critical level may be a regulatory maximum contaminant level (MCL), MDA, or risk-based exposure level.

Significance of probability: The probability of observing a statistical value as significant as, or even more significant than, the value actually observed.

Soil: All unconsolidated materials normally found on or near the surface of the earth including, but not limited to, silts, clays, sands, gravel, and small rocks.

Solvent: Liquid that is capable of dissolving another substance.

Split sample: A second aliquot of a sample, generated in the field, that is sent to a secondary laboratory for analysis; a split sample serves as a quality control check.

Standard deviation: The measurement of dispersion about a mean value of a series of observations expressed in the same units as the mean value.

STORET Code: See Codes.

Surface water: All water that is open to the atmosphere and subject to surface runoff. 
Surrogate: A chemically inert organic compound similar in composition and test performance to an analyte of interest added to samples prior to analysis as a quality control check.

Surrogate recovery: A quality control measurement to monitor unusual matrix effects, gross sample processing errors, etc. It is evaluated for acceptance by determining whether the measured concentration falls within the statistical acceptance limits established by the laboratory.

Target analyte list (TAL): The list of inorganic analytes required by CERCLA.

Target compound list (TCL): The list of organic analytes required by CERCLA.

Technical holding time: The storage time allowed between sample collection and sample analysis when designated preservation and storage techniques are employed. This is determined by the elapsed time in days from the date and time of collection to the date and time of sample preparation and analysis.

- $\quad$ Technical holding time $=($ sample analysis date and time - sample collection date and time $)$.

Tentatively identified compound (TIC): Chromatographic peak in volatile and semivolatile fraction analysis that is not a target analyte, surrogate, or internal standard.

Time: When required to record time on any deliverable item, time is expressed as military time, i.e., on a 24-hour clock.

Toxicity characteristic leaching procedure (TCLP):An analytical extraction procedure used to determine the mobility of both inorganic and organic analytes present in solid, liquid, and multiphasic wastes.

Tracer: A quantity of a unique radioisotope of the same element added to a sample, chemically prepared, or separated and counted. The quantity of tracer measured is compared to the quantity of target radioactive element measured, and the target quantity is calculated on the basis of unity with the tracer concentration.

Trip blank: See Blank

Wet weight: The weight of a sample aliquot including moisture (undried). 